GÖTTINGER ZENTRUM

FÜR BIODIVERSITÄTSFORSCHUNG UND ÖKOLOGIE

- GÖTTINGEN CENTRE FOR BIODIVERSITY AND ECOLOGY -

\title{
Phylogeography in sexual and parthenogenetic European Oribatida
}

Dissertation zur Erlangung des akademischen Grades eines Doctor rerum naturalium an der Georg-August Universität Göttingen

vorgelegt von

Dipl. Biol. Martin Julien Rosenberger

aus

Langen, Hessen

Referent: Prof. Dr. Stefan Scheu

Koreferent: PD Dr. Mark Maraun

Tag der Einreichung: 21 Oktober 2010

Tag der mündlichen Prüfung: 


\section{Curriculum Vitae}

\section{$\underline{\text { Personal data }}$}

Name:

Address:

Date of Birth:

Place of Birth:

\section{Education}

1987-1991

$1991-2000$

2000-2006

2006-2007

2007-2008

2008-2009

Since 2009

2009-2010
Martin Julien Rosenberger

Brandenburgerstrasse 53, 63329 Egelsbach

October $31^{\text {st }} 1980$

Langen (Hessen)

Wilhelm Leuschner Primary School, Egelsbach

Abitur at Dreieich-Schule, Langen

Study of Biology at Darmstadt University of Technology, Germany

Diploma thesis: "Postglaziale Kolonisation von Zentraleuropa durch parthenogenetische (Platynothrus peltifer) und sexuelle (Steganacarus magnus) Hornmilben (Oribatida)" at Darmstadt University of Technology, Germany under supervision of Dipl. Biol. Katja Domes and Prof. Dr. S. Scheu

Scientific assistant at Darmstadt University of Technology, Germany

Scientific officer Darmstadt University of Technology, Germany

PhD student at the Georg August University, Göttingen, Germany at the J. F. Blumenbach Insitute of Zoology and Anthropology under supervision of Prof. Dr. S. Scheu

Scientific officer at the Georg August University, Göttingen, Germany

\section{$\underline{\text { International Congress Contributions }}$}

March 2008

March 2009

April 2009
Annual mesofauna meeting Vienna

talk

DZG PhD meeting, Munich

talk

Annual mesofauna meeting, Goettingen

talk 
Curriculum Vitae

March $2010 \quad$ Annual mesofauna meeting, Innsbruck

talk

\section{Other Experiences}

April/May 2007/08 Supervision of the alternative practical course for 'Spezielle Zoologie' at Darmstadt University of Technology, Germany

February $2010 \quad$ Supervision of the practical course 'Evolutionary Ecology' at Georg August University Göttingen, Germany

\section{Further supervision}

Jennifer Wilhein "Intraspezifische genetische Varianz bei Arten der Gattung Eupteryx

(Cicadellidae/Typhlocybinae) in Deutschland“, Diploma thesis 2009

Darmstadt University of Technology, Germany

Valerie Biewener "Thechniques in Molecular Biology", research intership 2009/10 at Georg August University Göttingen, Germany

Erik-Thor Hagenah “Thechniques in Molecular Biology", research intership 2009/10 at Georg August University Göttingen, Germany

Helge von Saltzwedel "Ecological niche differentiation of the parthenogenetic oribatid mite Oppiella nova (Acari, Oribatida) investigated by molecular markers", Diploma thesis 2010 Darmstadt University of Technology, Germany 


\section{Table of Contents}

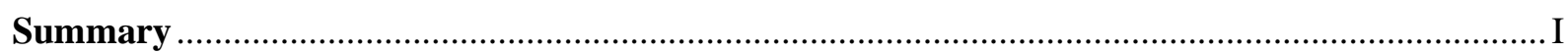

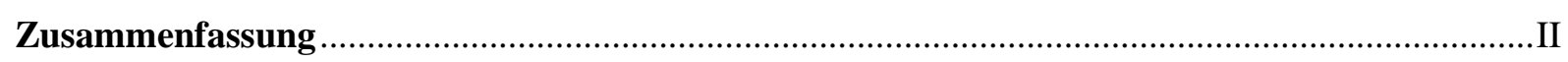

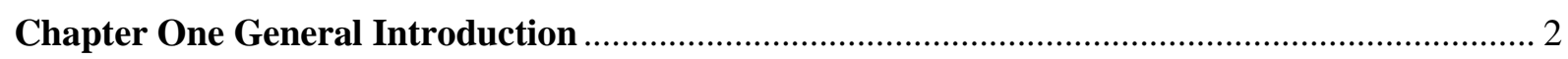

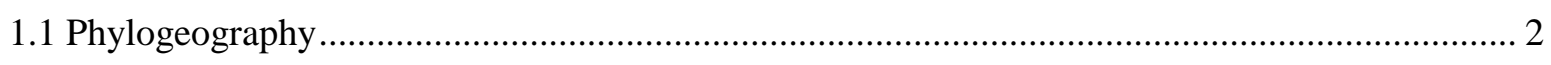

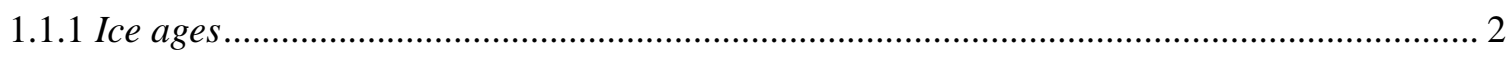

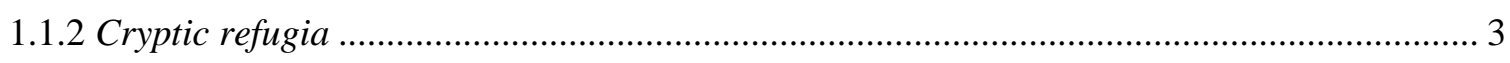

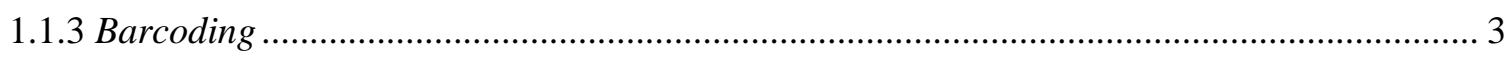

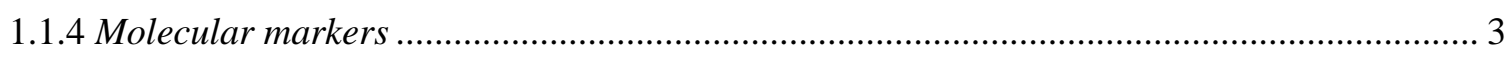

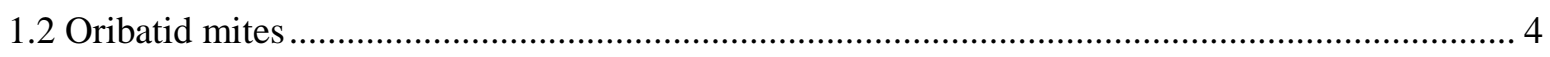

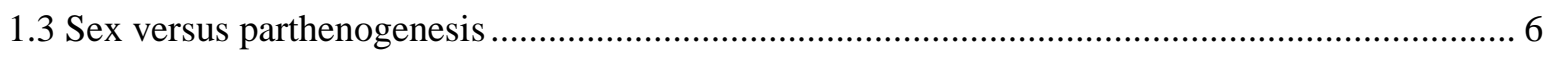

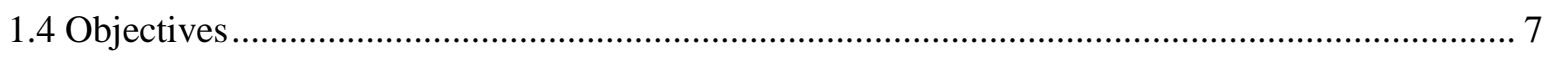

Chapter Two Genetic diversity in a soil living microarthropod species: Cryptic species and reconstruction of the evolution of genetic complexity ............................................................ 8

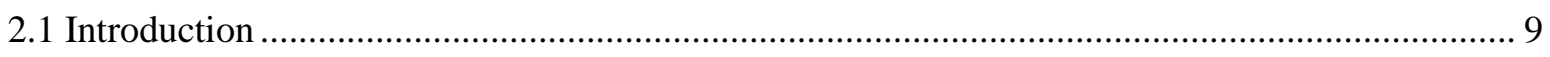

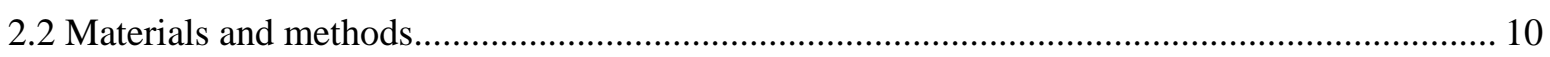

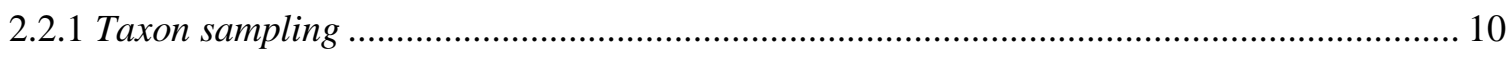

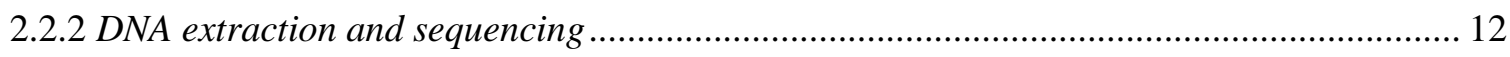

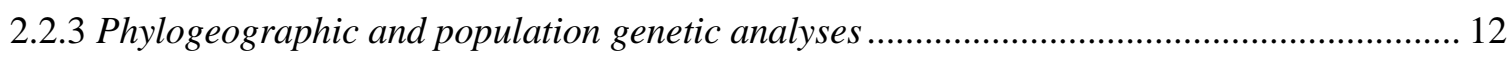

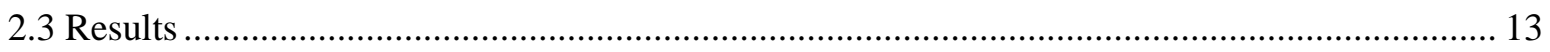

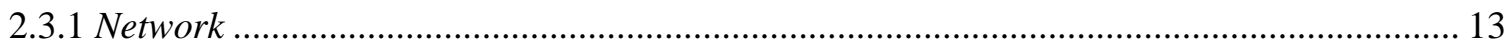

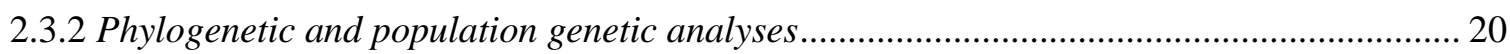

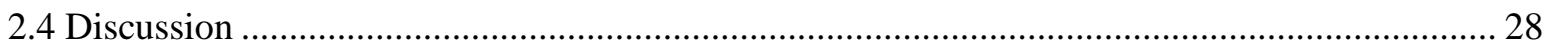

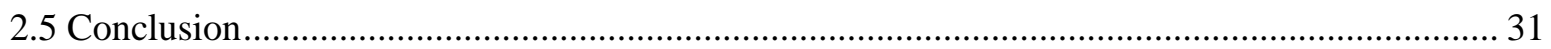

Chapter Three Is there a cryptic species complex in the oribatid mite Steganacarus magnus

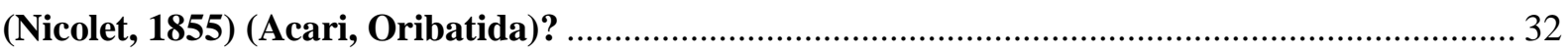

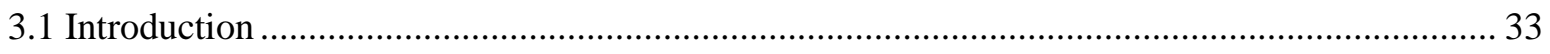

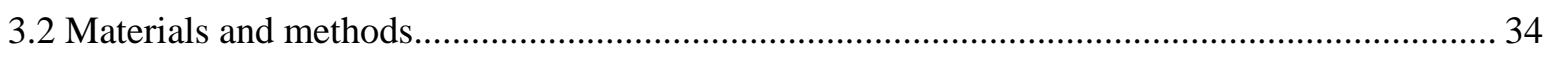

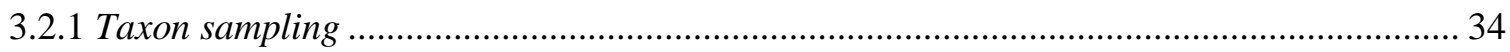

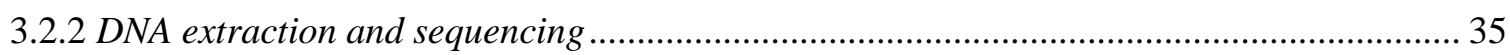

3.2.3 Phylogenetic, population genetic and statistical analyses .................................................... 36

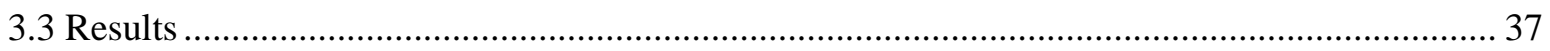

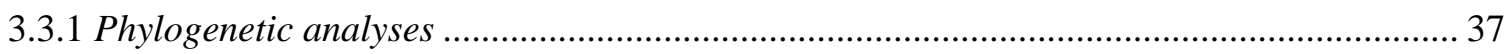

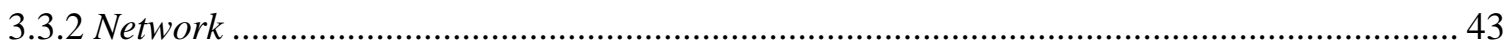

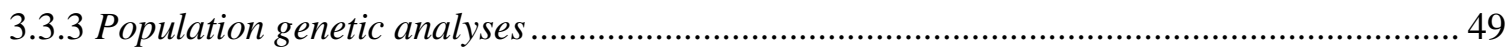

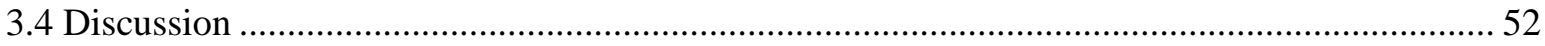


Table of Contents

3.5 Conclusions

Chapter Four Differential colonization of Europe by sexual and asexual oribatid mite species: post- and pre- ice age events 54

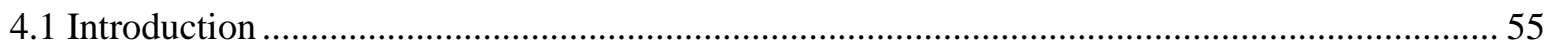

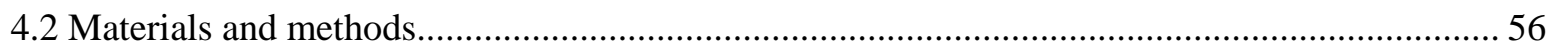

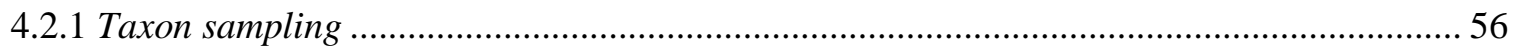

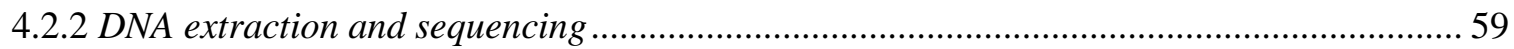

4.2.3 Phylogeographic, population genetic and statistical analyses .......................................... 59

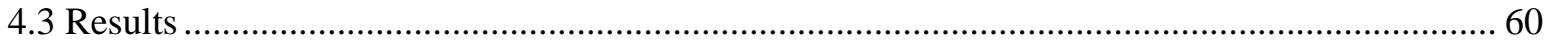

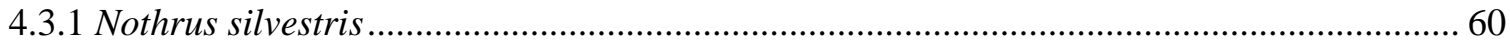

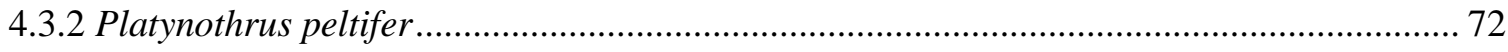

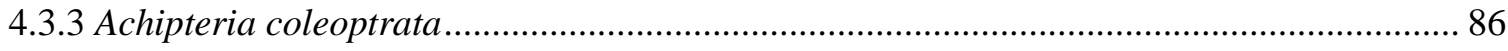

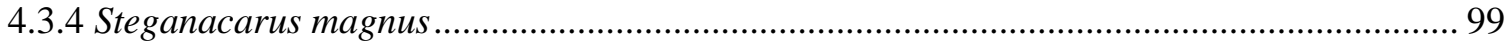

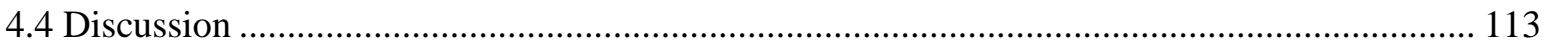

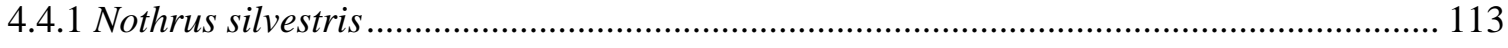

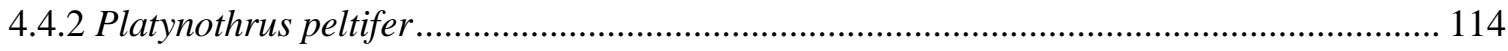

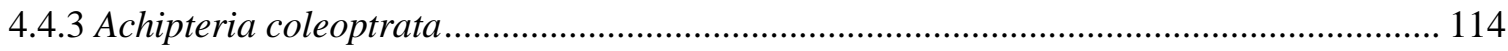

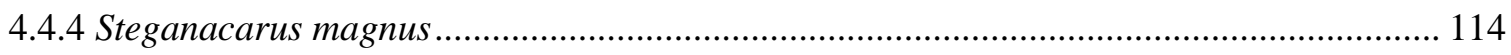

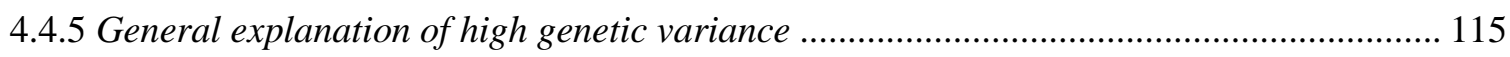

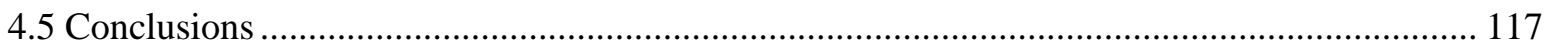

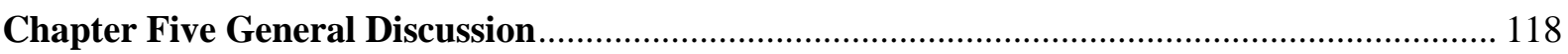

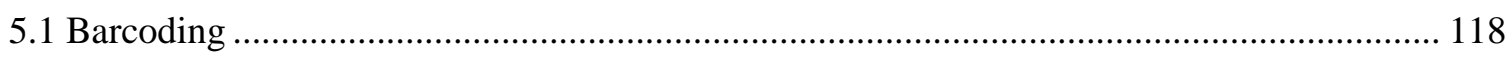

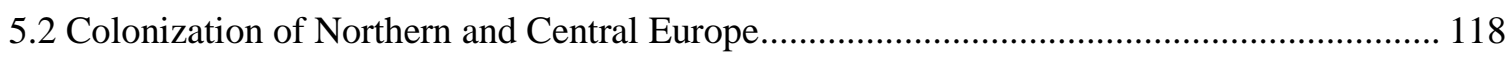

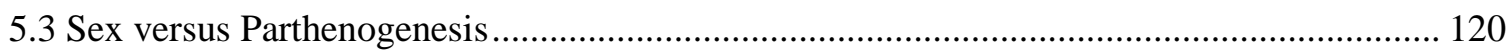

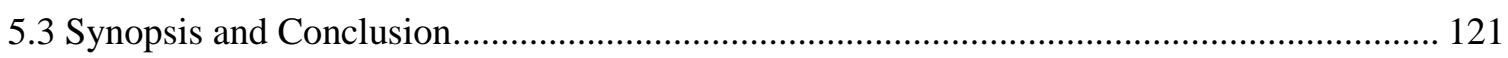

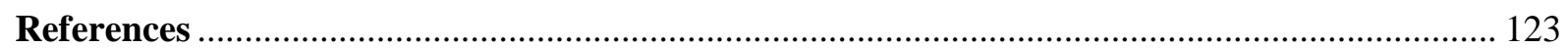

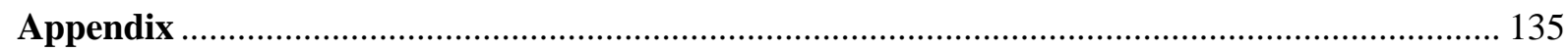

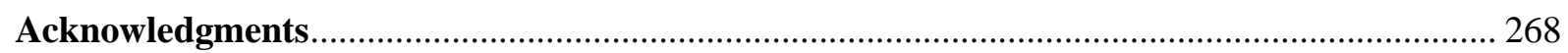

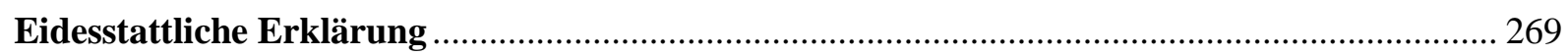




\section{Summary}

Oribatid mites (Acari, Oribatida) are a species rich group which may form the oldest group of Chelicerata, as indicated by fossils from Devonian sediment ( $\sim 380$ million years) and molecular clock data ( 570 million years). They are ubiquitous soil living arthropods and important decomposers. In oribatid mites parthenogenesis is common, about $10 \%$ of the individuals reproduce parthenogenetically, and they likely radiated while being parthenogenetic. Their high abundance (up to 400.000 individuals per square meter in temperate and boreal forest soils) and their species richness (10.000 described species) render oribatid mites ideal model organisms to answer evolutionary and ecological questions. Using molecular markers I investigated the genetic diversity of two sexual and two parthenogenetic European oribatid mite species.

(1) Genetic diversity in soil living microarthropods

Pleistocene glaciations shaped the genetic and species diversity of Europe. Using the mitochondrial gene of the cytochrome $\mathrm{c}$ oxidase (COI) I investigated the genetic structure of the soil living oribatid mite species Steganacarus magnus. The high intraspecific genetic variance of COI at nucleotide (32\% uncorrected p-distance) and protein level (5\% uncorrected p-distance) suggests that the climatic change had no strong influence on S. magnus. It survived the last ice ages in cryptic refugia, radiated in the Miocene or earlier and colonized Europe after the last ice age from cryptic refugia.

\section{(2) Cryptic species complex in Steganacarus magnus}

Intraspecific variance of mitochondrial DNA higher than $3 \%$ indicates a cryptic species complex. The high intraspecific distance in COI of the oribatid mite species S. magnus (up to 32\%) indicates the existence of cryptic species. Using one mitochondrial (COI) and one nuclear marker (elongation factor 1 alpha; ef $1 \alpha$ ) I investigated if there is a cryptic species complex in $S$. magnus. The results suggest that $S$. magnus does not comprise a cryptic species complex; phylogenetic trees of the genes studied were different indicating recombination between lineages.

(3) Post- and pre-glacial colonization of Europe by sexual and parthenogenetic oribatid mite species

Belowground organisms could have survived low temperatures undamaged and therefore survived the ice age in cryptic refugia. Using the molecular marker COI I investigated the colonization events of two sexual (Achipteria coleoptrata, S. magnus) and two parthenogenetic (Nothrus silvestris, Platynothrus peltifer) European oribatid mite species. Each oribatid mite species showed a different colonization pattern of Europe. A. coleoptrata, S. magnus and $P$. peltifer had high nucleotide divergences (19\% A. coleoptrata, $31 \%$ S. magnus, $20 \%$ P. peltifer) but only the two sexual oribatid mite species also had high protein divergences (3\% A. coleoptrata, 4\% S. magnus). N. silvestris had low nucleotide (2\%) and protein (0\%) divergences. The results indicate that A. coleoptrata, S. magnus and $P$. peltifer radiated in the Miocene and survived the Pleistocene ice ages in cryptic refugia. IN contrast, $N$. silvestris did not survive the ice age in cryptic refugia but colonized Central and Northern Europe thereafter and radiated in the Holocene. The high protein variance in sexual and the low variance in parthenogenetic oribatid mites provided hints on mechanisms responsible for the maintenance of sexual reproduction. 


\section{Zusammenfassung}

Oribatiden (Hornmilben) sind eine sehr alte und artenreiche Gruppe der Chelicerata; Fossilfunde aus dem frühen Devon (vor 380 Millionen Jahren) belegen den Ursprung der Gruppe. Molekulare Datierungen weisen jedoch auf eine weitaus ältere Entstehung der Oribatiden im Präkambrium hin (vor 570 Millionen Jahren). Auffällig bei Hornmilben ist, dass Parthenogenese weit verbreitet ist; ca. $10 \%$ der Oribatidenarten reproduzieren parthenogenetisch und radiieren sogar, wobei aber nur 1\% aller Metazoen sich parthenogenetisch reproduzieren. Sie kommen ubiquitär in Bodensystemen vor und sind wichtige Zersetzer. Der Artenreichtum (10.000 beschriebene Arten) und ihre hohe Abundanz (bis zu 400.000 Individuen pro Quadratmeter in bodensauren Wäldern der temperierten Breiten) machen Oribatiden zu idealen Modellorganismen für evolutionsbiologische und ökologische Fragestellungen. In dieser Arbeit wurde die genetische Diversität zweier sexueller und zweier parthenogenetischer europäischer Oribatiden mit molekularen Markern untersucht.

\section{(1) Genetische Diversität von im Boden lebendem Mikroarthropoden}

Der Artenreichtum und die genetische Diversität Europas wurden durch die letzte Eiszeit stark beeinflusst. Mit Hilfe des mitochondrialen Gens der Cytochromoxidase I (COI) wurden die Auswirkungen auf die im Boden lebende Hornmilbe Steganacarus magnus untersucht. Die hohe intraspezifische Varianz der COI auf Nukleotid- (32\% unkorrigierte p-Distanz) und Proteinebene (5\% unkorrigierte p-Distanz) zeigen deutlich, dass die pleistozäne Vereisung auf die im Boden lebende Art S. magnus keinen starken Einflüsse ausgeübt hat. Die hohe Nukleotidvarianz weist daraufhin, dass diese Art die Eiszeit in kryptischen Refugien überdauert hat, wogegen sie im Miozän oder sogar schon früher diversifiziert ist und sich im Holozän weiter verbreitet hat.

\section{(2) Kryptischer Artenkomplex in Steganacarus magnus}

Über 3\% innerartliche Varianz in mitochondrialer DNS weist auf einen kryptischen Artenkomplex hin. Die innerartliche Varianz bei der sexuellen Oribatide S. magnus lag bei bis zu 32\%, was auf einen kryptischen Artenkomplex hindeutet. Um festzustellen, ob es sich tatsächlich um einen kryptischen Artenkomplex handelt, wurden COI und das nukleare Gen des Elongationsfaktors 1 alpha (ef $1 \alpha)$ untersucht. Das Ergebnis zeigte keinen kryptischen Artenkomplex in S. magnus, da sich die Topologien der phylogenetischen Bäume unterschieden, was darauf hindeutet, dass Individuen der verschiedenen mitochondrialen Linien untereinander Nachkommen produzieren.

(3) Unterschiedliche vor- und nacheiszeitlichen Kolonisation von Europa durch sexuelle und parthenogenetische Oribatiden

Bodenorganismen können niedrige Temperaturen über einen längeren Zeitraum unbeschadet überdauern und sind daher nur wenig von den Eiszeiten beeinflusst worden. Um herauszufinden, ob parthenogenetische und sexuelle Bodenorganismen sich in ihren nacheiszeitlichen Kolonisationsmustern unterscheiden, wurde der mitochondriale Marker COI von zwei parthenogenetischen (Nothrus silvestris, Platynothrus peltifer) und zwei sexuellen Oribatidenarten (Achipteria coleoptrata, S. magnus) europaweit untersucht. Jede Oribatidenart zeigte ein anderes Muster der Kolonisation von Europa. A. coleoptrata, S. magnus und P. peltifer wiesen eine hohe Nukleotidvarianz auf (19\% A. coleoptrata, $31 \%$ S. magnus, $20 \%$ P. peltifer), wobei nur die sexuellen Arten auch eine hohe Proteinvarianz hatten ( $3 \%$ A. coleoptrata, $4 \%$ S. magnus). N. silvestris zeigte eine geringe Nukleotid- $(2 \%)$ und Proteinvarianz $(0 \%)$. Die hohe Nukleotidvarianz weist auf einen präglazialen Ursprung hin (mit einer Radiation im Miozän) während die geringe Nukleotidvarianz auf 


\section{Zusammenfassung}

einen postglazialen Ursprung hinweist (und eine Radiation im Holozän). Die hohe Proteinvarianz bei sexuellen und die niedrige Proteinvarianz bei parthenogenetischen Oribatiden bietet Hinweise auf Mechanismen für die Aufrechterhaltung sexueller Reproduktion. 


\section{Chapter One}

\section{General Introduction}

\subsection{Phylogeography}

Phylogeography explores the phylogenetic and geographical origin of genetic lineages of a taxon thereby combining biogeography, population genetics and phylogenetic analyses.

\subsubsection{Ice ages}

Since the beginning of the Quaternary about 3 million years ago the climate in Europe oscillated several times from cold (ice age) to warmer periods (interglacial) (Hewitt 2000, 2004). These ice ages shaped the biodiversity of the European flora and fauna and were named after their maximum extension (Biber, Donau, Günz, Saale and Weichsel). The last ice age began 115.000 years and ended about 10.000 years before present. The ice sheet covered North Europe including Northern Germany, Poland and the Baltic States; glaciers also covered part of the Alps, the Pyrenees, the Carpathians, the Apennines and the Balkan. Steppe, tundra and permafrost expanded in Central Europe (Fig. 1). The living space for animals and plants shrunk to areas south of the Balkan, the Alps and the Pyrenees (Hewitt and Ibrahim 2001). In the past twenty years, several phylogeographic studies investigated the role of these refugia for populations and the current distribution of species by analyzing genetic data in a spatial context (Beheregaray 2008).

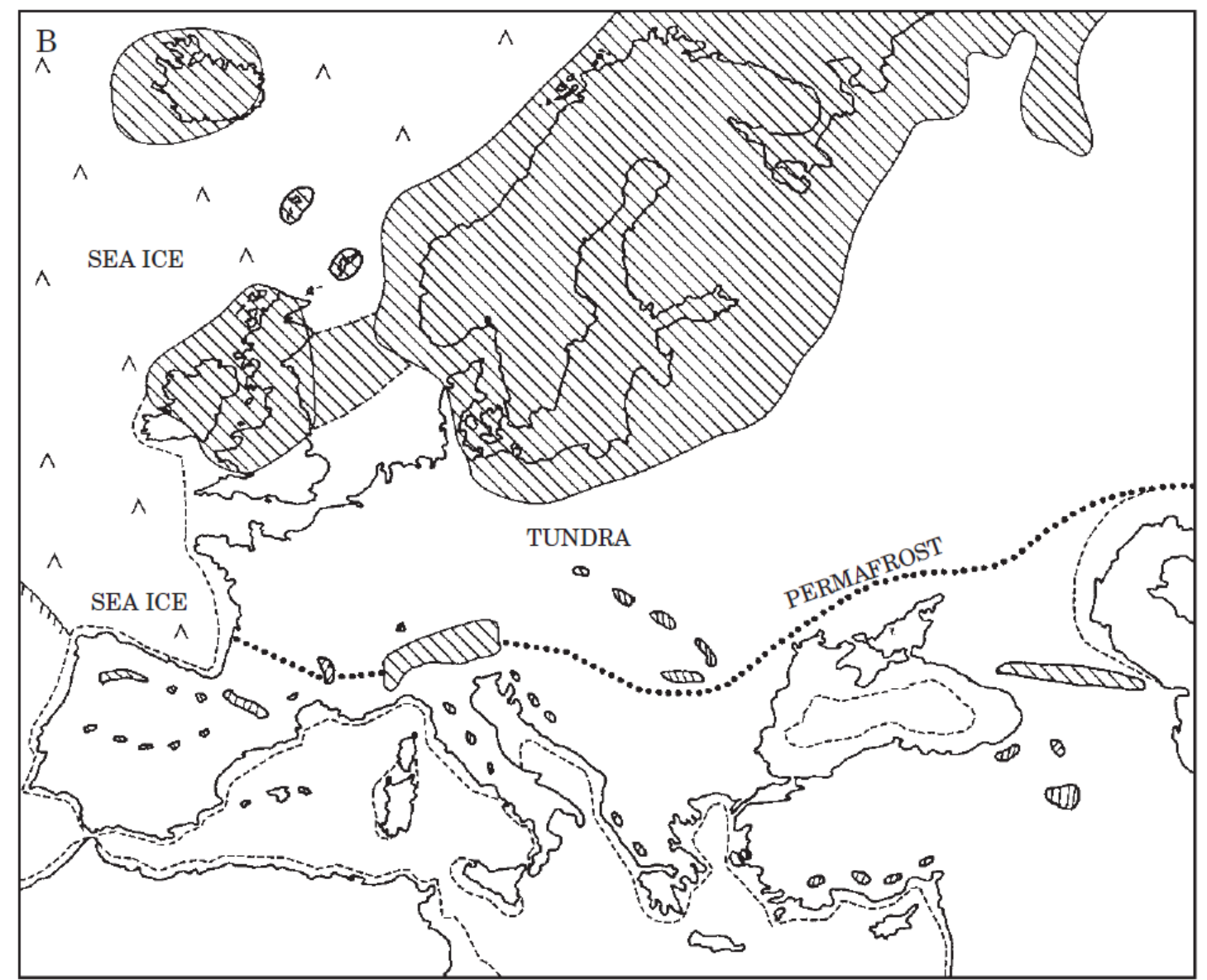

Figure 1: Map of Europe at the last glacial maximum (20.000 years before present). The shaded area represents the ice cover and the dotted line the Permafrost border (from Hewitt 1999). 


\subsubsection{Cryptic refugia}

Cryptic refugia were areas in Central and Northern Europe, which were not covered with an ice sheet or had permafrost soil at the last glacial maximum (LGM) 20.000 years ago. Stewart and Lister (2001) recently discussed the existence of these Northern refugia for European biota. Their theory of cryptic refugia is supported by studies using pollen, molecular and radiocarbon analyses (Willis et al. 2000, Stewart and Lister 2001, Verovnik et al. 2005, Tollefsrud et al. 2008). However, these areas are difficult to detect, since presumably they were small, geographically isolated or located in wind protected valleys.

Survival of populations in central refugia implies that species expanding from southern refugia met populations that experienced a different demographic and ecological history for some period of time. Determining the extent of refugia at higher latitudes and their genetic contribution to present day populations is important to understand migration rates and the role of local adaptations to gene flow among populations, both of which are important factors to understand speciation processes.

\subsubsection{Barcoding}

DNA barcoding is a molecular technique which revolutionized taxonomy by allowing to delineate species on the basis of molecules. For DNA barcoding of metazoan animals a short gene fragment ( $660 \mathrm{bp})$ of the mitochondrial cytochrome c oxidase gene is commonly used (Hebert et al. 2003a-b, 2004 a-b, Ball et al. 2005, Hajibabaei et al. 2007). A divergence of over 3\% in the nucleotide sequences is taken as indication for the existence of distinct species (Hebert et al. 2003a). The mitochondrial genome is maternally inherited and evolves with a faster rate than the nuclear genome (Wolstenholme 1992, Boore 1999). This makes mitochondrial genes a perfect tool for DNA barcoding.

\subsubsection{Molecular markers}

In this study I used the mitochondrial gene of the cytochrome $\mathrm{c}$ oxidase subunit I and the nuclear gene of the elongation factor $1 \alpha$. Both genes were used in several phylogeographic studies (Danforth et al. 1999, Verovnik et al. 2005) and in phylogenetic studies in oribatid mites (Heethoff et al. 2007, Domes et al. 2007a, Laumann et al. 2007, Dabert et al. 2010).

\section{Cytochrome c oxidase subunit I}

The enzyme cytochrome c oxidase (COI) is a large transmembrane protein complex and is located in the mitochondrial membrane in eukaryotes and in the cell membrane in prokaryotes. It is a hemecopper oxidase (Saraste 1990) and the terminal energy transfer enzyme in the respiratory chain (Mitchell 1966). It catalyzes the electron transfer from cytochrome c to molecular oxygen thereby reducing oxygen to water. The enzyme complex consists of 13 subunits in mammals and the catalytic center is located in the subunit I (Kadenbach and Stroh 1984; Blenkinsop et al. 1996; Arnold and Kadenbach 1997). However, only the subunits I-III are located in the mitochondrion, the other subunits are located in the nucleus. The subunit I of the cytochrome c oxidase consists of twelve transmembrane helices and contains two heme (heme a and heme $\left.\mathrm{a}_{3}\right)$ and one copper $\left(\mathrm{Cu}_{\mathrm{B}}\right)$ centers (Fig. 2). 


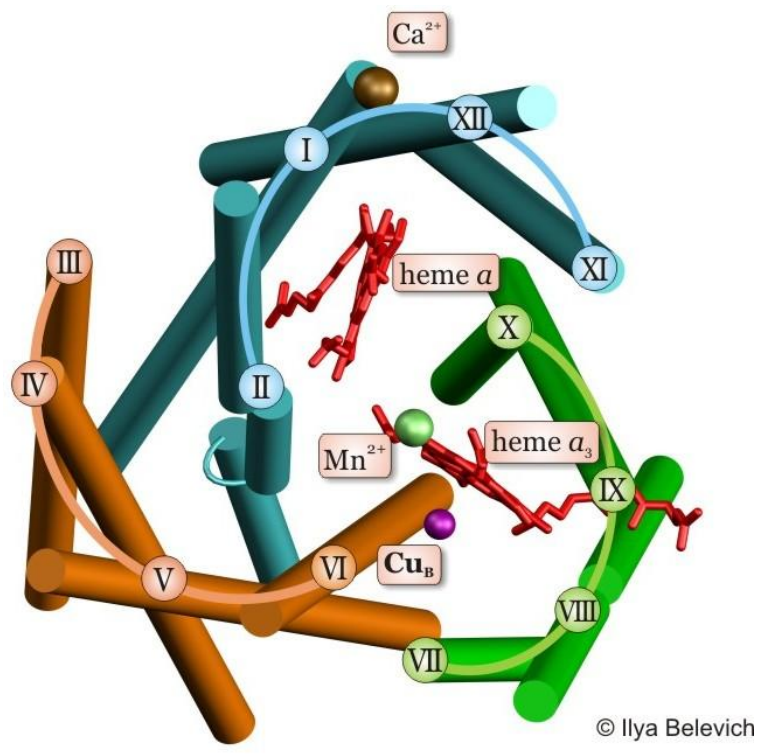

Figure 2: Model of the subunit one of the cytochrome c oxidase complex. The twelve helices are brown, green and blue. The catalytic center contains the two heme groups (red) and one copper atom (magenta).

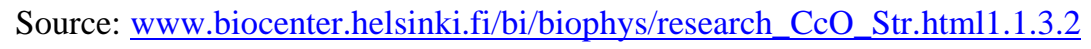

\section{Elongation factor $1 \alpha$}

The gene of the elongation factor $1 \alpha(e f 1 \alpha)$ is located in the nucleus and is highly conserved (Keeling and Inagaki 2004). It is a single copy gene with a length of 1,430 bp (Klompen 2000) and a member of GTPase superfamily (Baldauf et al. 1996, Keeling et al. 1998, Keeling and Inagaki 2004). The ef $1 \alpha$ protein is associated with the ribosomes at the protein biosynthesis and catalyses the binding between the incoming amino acid and the growing polypeptide chain. It builds a binary complex with GTP which is associated with the aminoacyl-tRNA. This complex binds under GTP hydrolysis to the ribosome and thereby the elongation factor and GDP $+\mathrm{P}_{\mathrm{i}}$ is set free.

\subsection{Oribatid mites}

Acari are one of the oldest, most abundant and diverse arthropod groups (Walter and Proctor 1999). Over 42,000 species are clustered in three major groups, the Opilioacaridae (20 species), the Parasitiformes (>10,000 species) and the Acariformes (>30,000 species) (Krantz 1978, Evans 1992, Walter and Proctor 1999, Krantz and Walter 2009). The Acariformes are grouped into Prostigmata, Astigmata, Oribatida and the paraphyletic Endeostigmata (O'Connor 1984, Walter 2001). The oribatid mites are the largest subgroup of the Acariformes with 10,000 described species (Schatz 2002, Subias 2004), but estimated numbers range from 50,000 (Travé et al. 1996) to 100,000 species (Schatz 2002). Oribatid mites are grouped into six subgroups, the two basal groups Palaesomata and Enarthronota, the small group Parhyposomata, the paraphyletic "Mixonomata", the "Desmonomata" with mainly parthenogenetic taxa and the higher Oribatida the Circumdehiscentiae (=Brachypylina) (Grandjean 1953, 1965, 1969). Fossils of oribatid mites were found in Devonian sediments 380 million years ago (mya) (Shear et al.1984, Norton et al. 1988a) but the origin of this group is dated back to 440 mya (Lindquist 1984) and molecular clock analyses date the origin of the Oribatida back to 570 mya (Schaefer et al. 2010).

Oribatid mites are soil-dwelling detritivorous and fungivourous microarthropods (Maraun and Scheu 2000, Schneider et al. 2004). Some species also prey on nematodes (Muraoka and Ishibashi 1976, Schneider et al. 2004, K. Heidemann unpublished data). In forests of the temperate and boreal zone they reach densities of up to 400,000 individuals per square meter (Maraun and Scheu 2000). 


\section{Chapter 1 General Introduction}

\section{Achipteria coleoptrata (Linné, 1758)}

The sexual oribatid mite species Achipteria coleoptrata (Oribatida, Brachypylina) (Fig. 3) has a size of 530-650 $\mu \mathrm{m}$, is distributed throughout the Holarctic and lives in wet meadows and forests (Weigmann 2006, Subias 2009). In the laboratory it feeds on bark algae (Desmococcus vulgaris), grasses, herb litter and fungi (Hubert et al. 2001). The generation time is one year (Luxton 1981). Fossilis of Achipteriidae are known from Jurassic and Baltic amber (Labandeira et al. 1997, Krantz and Walter 2009).
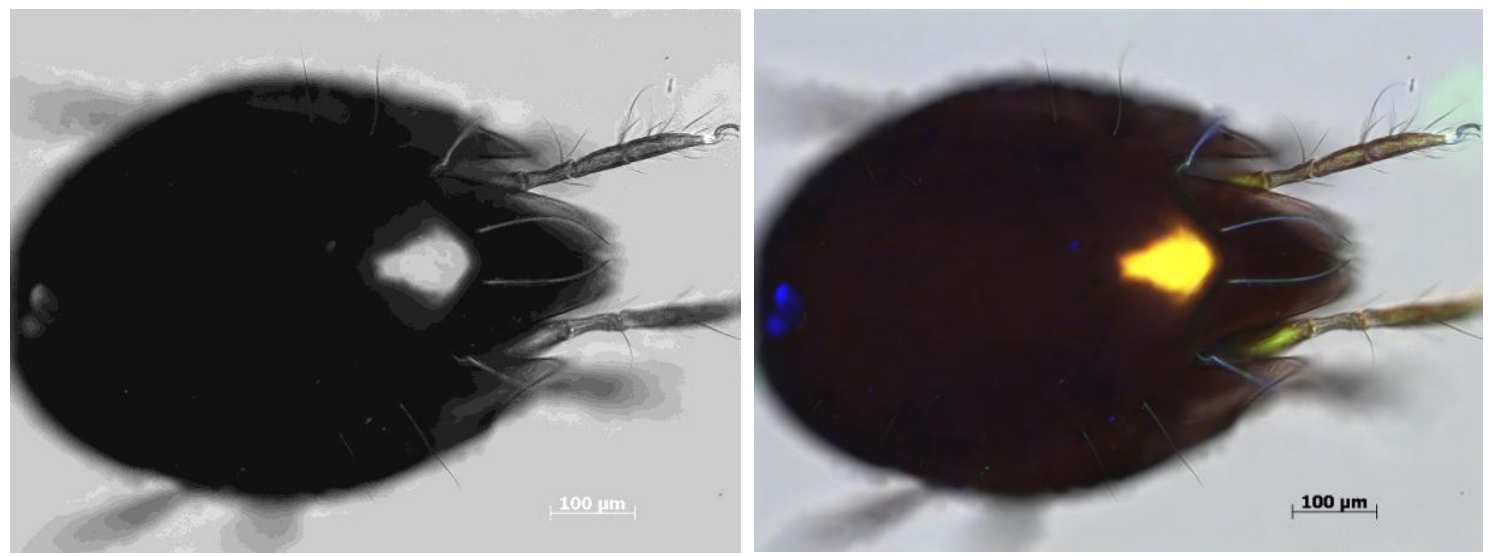

Figure 3: Scanning microscope image of Achipteria coleoptrata (left: black and white, right: fluorescence).

\section{Steganacarus magnus (Nicolet, 1855)}

The sexual oribatid mite species Steganacarus magnus (Oribatida, "Mixonomata") (Fig. 4) has a size of 700-1200 $\mu \mathrm{m}$ (Weigmann 2006) and is distributed throughout the Palearctic (Subias 2009). S. magnus has a generation time of one year from egg to adult (Webb 1977, 1989). Larval stages and nymphs live endophagous in lignified plant tissues. It is cold resistant, adult individuals can survive temperatures of up to $-12^{\circ} \mathrm{C}$ and juveniles of up to $-14^{\circ} \mathrm{C}$ undamaged (Webb and Block 1993); its supercooling point ranges between $-7^{\circ} \mathrm{C}$ and $-38^{\circ} \mathrm{C}$ (Block 1979, Krantz and Walter 2009). S. magnus is also tolerant against drought and heat (Siepel 1996). Generally, S. magnus functions as primary decomposer (Schneider et al. 2004) but he may also feed on nematodes (K. Heidemann unpublished data).
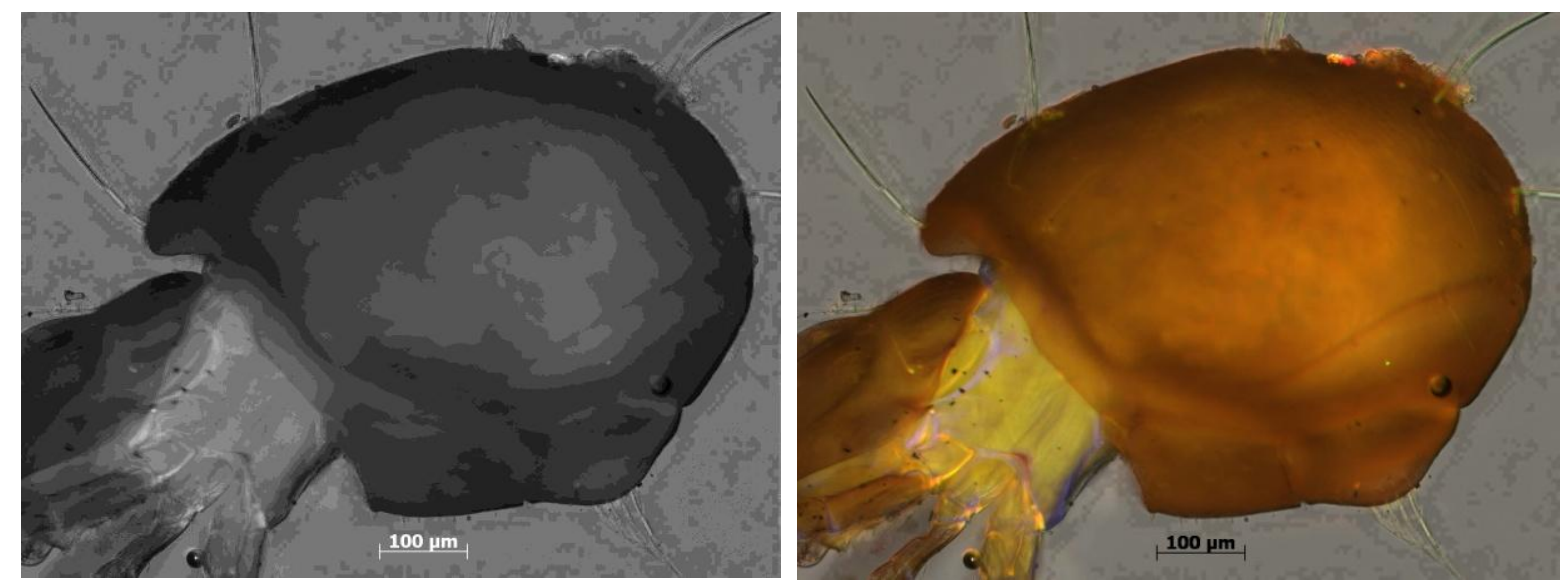

Figure 4: Scanning microscope image of Steganacarus magnus (left: black and white, right: fluorescence).

Nothrus silvestris (Nicolet, 1855)

The parthenogenetic oribatid mite species Nothrus silvestris (Oribatida, "Desmonomata") (Fig. 5) has a size of 710-810 $\mu \mathrm{m}$ and is distributed in acidic soils and mesophilic forests in the Holarctic and 
Neotropis (Weigmann 2006, Subias 2009). It is assumed that N. silvestris is a primary decomposer and feeds on decaying plants and fungi (Siepel 1990). Recent analysis showed that N. silvestris also preys on nematodes (K. Heidemann unpublished data).
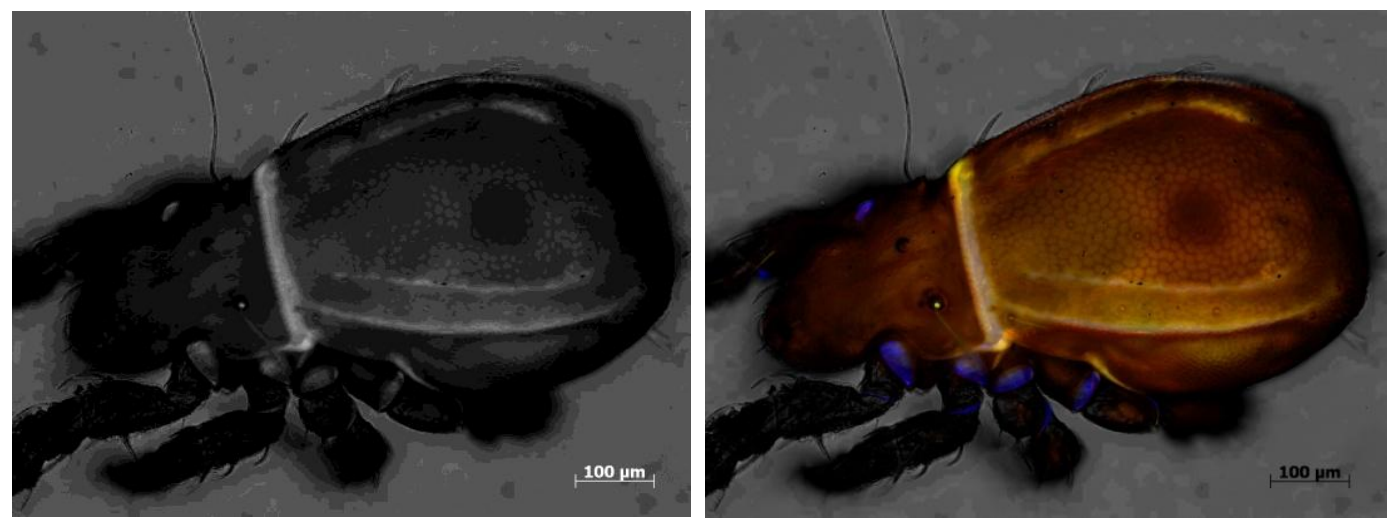

Figure 5: Scanning microscope image from Nothrus silvestris (left: black and white, right: fluorescence).

\section{Platynothrus peltifer (C. L. Koch, 1839)}

The parthenogenetic oribatid mite species Platynothrus peltifer (Oribatida, Desmonomata) (Fig. 6) is a cosmopolitan species (Subias 2009) with a size of 770-980 $\mu \mathrm{m}$. It occurs in various terrestrial (Weigmann 2006) but also in freshwater and benthic habitats (Schatz and Gerecke 1996, Krantz and Walter 2009). P. peltifer is tolerant against salt, heat, drought (Siepel 1996) and metal contamination from smelters (Zaitsev and Van Straalen 2001). It is assumed that P. peltifer is a primary decomposer and feeds on decaying plant material and fungi (Siepel 1990, Schneider et al. 2004) but it also preys on nematodes (K. Heidemann unpublished data). The generation time of $P$. peltifer is one year in temperate European forests (Weigmann 1975, Schenker 1986, Krantz and Walter 2009).
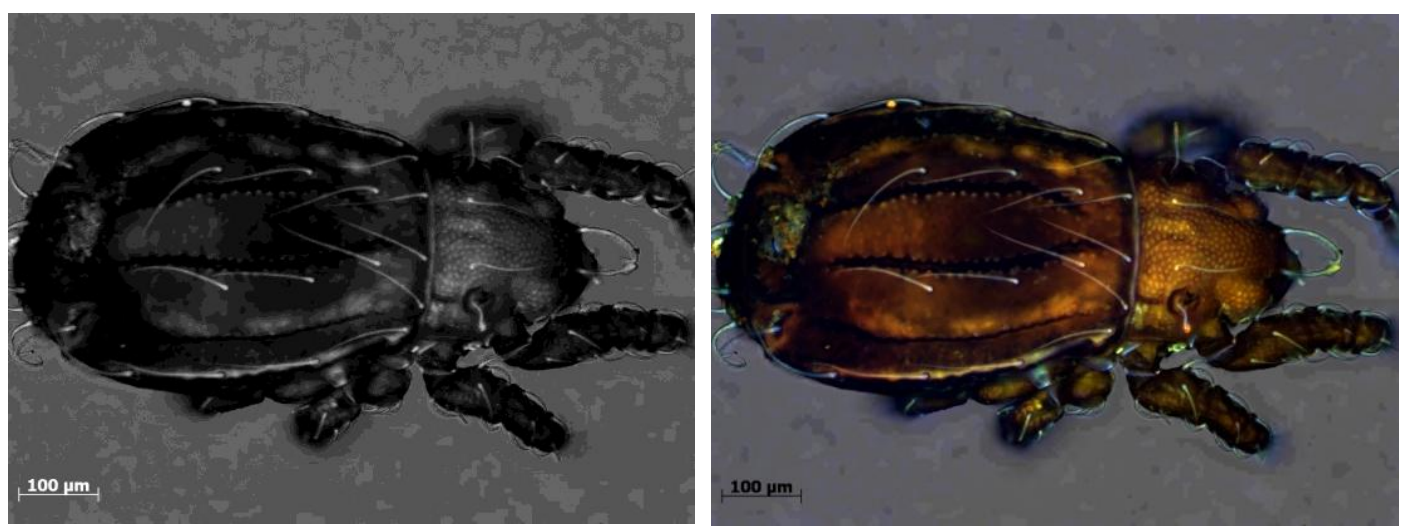

Figure 6: Scanning microscope image of Platynothrus peltifer (left: black and white, right: fluorescence).

\subsection{Sex versus parthenogenesis}

Sexual reproduction is the most widespread reproductive mechanism in animals; >99\% of all metazoan taxa reproduce sexually (Bell 1982). Parthenogenetic reproduction is characterized by developing offspring from unfertilized eggs (Hughes 1989). Parthenogenesis includes different genetic mechanisms such as deuterotoky, arrhenotoky, pseudo-arrhenotoky and thelytoky (Bell 1982, Hughes 1989). Species reproducing by deuterotoky produce males and females from reduced eggs and diploidy is restored postmeiotically. Arrhenotoky is a reproductive mode where females develop from fertilized eggs and males from unfertilized eggs. In pseudo-arrhenotoky males and females develop from fertilized eggs and males become haploid later. These three mechanisms are not truly parthenogenetic, i.e. unisexual, since both sexes are needed and produced. In contrast, thelytoky is 
characterized by the development of female offspring from unfertilized eggs and is called the "true" parthenogenesis as no males occur. Thelytoky is divided into two different forms, apomixis and automixis. In apomixis meiosis is suppressed and the offspring is genetically identical to the mother. In automixis meiosis is retained and the diploid status is restored -premeiotically- or by post-meiotic processes (Maynard Smith 1978, Hughes 1989), such as terminal or central fusion of meiotic products (Suomalainen et al. 1987, Stenberg and Saura 2009). In central fusion automixis the two central polar nuclei fuse (i.e. fusion of non sister chromatids). The maternal heterozygosity is restored, if the mother was heterozygous. In terminal fusion automixis the egg nucleus fuses with the second polar nucleus (i.e. fusion of sister chromatids) which results in homozygous offspring by a heterozygous mother (Wrench et al. 1994, Stenberg and Saura 2009, Heethoff et al. 2009). Parthenogenetic oribatid mites such as $P$. peltifer reproduce by automixis and restore diploidy by terminal fusion (Taberly 1987, Heethoff et al. 2009).

Nonsexual species have a number of advantages compared to sexual species, e.g. the twofold advantage of not producing males (Williams 1975, Maynard Smith, 1978, 1998, Tagg et al. 2005), faster colonization of habitats and easier establishment of new populations (Williams 1975; Bell 1982; Scheu and Schulz 1996; Lindberg and Bengtsson 2005, Schön 2007). However, for long-term survival sexual reproduction appears to be indispensible to counteract the accumulation of detrimental mutations (Mullers ratchet, Muller 1964; Mutational Load Theory, Kondrashov 1988, Butlin et al. 1999). However, this view is challenged by the existence of "ancient asexuals" (Maynard Smith 1978), which radiated while being parthenogenetic as is assumed to be in the case in the bdelloid rotifers with $\sim 360$ species (Welch and Messelson 2000), darwinulid ostracods with 36 species (Martens et al. 2003) and oribatid mites with more than 400 species (Norton et al. 1988b, Palmer and Norton 1990, Norton and Palmer 1991, Maraun et al. 2003).

\subsection{Objectives}

The present work investigated evolutionary aspects in parthenogenetic and sexual oribatid mites using molecular markers. Chapter II investigated the postglacial colonization of the sexual oribatid mite species $S$. magnus in Europe. I expected that $S$. magnus survived the last ice age in cryptic refugia and colonized Central and Northern Europe from there, since S. magnus is cold tolerant, small and has low dispersal ability.

Chapter III based on the high genetic diversity in COI in S. magnus (Chapter II) which could be a result of a cryptic species complex. To investigate if $S$. magnus comprises a cryptic species complex, $\mathrm{mt}(C O I)$ and nuclear $(e f 1 \alpha)$ were sequenced from the same individual from regions with different $\mathrm{mt}$ lineages.

In Chapter IV I investigated the postglacial colonization of two sexual (A. coleoptrata and S. magnus) and two parthenogenetic (N. silvestris and P. peltifer) oribatid mites species in Europe to detect cryptic refugia and to develop general postglacial colonization patterns for soil living oribatid mites based on the $\mathrm{mt}$ gene $\mathrm{COI}$. I expected that parthenogenetic species have the advantage of faster colonization and that the genetic diversity of $\mathrm{COI}$ is similar in parthenogenetic and sexual oribatid mite species since mitochondria inherited maternally. 


\section{Chapter Two \\ Genetic diversity in a soil living \\ microarthropod species: Cryptic species and reconstruction of the evolution of genetic complexity}

\section{Summary}

Since the beginning of the Quaternary ( $\sim 3$ million years ago) ice ages shaped the biodiversity of Europe. The living space for many aboveground species shrunk to areas south of the Alps, the Pyrenees and the Balkans. If this was also true for soil living animals is unknown. I analyzed 180 individuals of the soil living oribatid mite species Steganacarus magnus from 47 locations in Europe and Central Asia using the mitochondrial gene of the cytochrome c oxidase (COI). The 180 sequences constituted 111 haplotypes for the nucleotide and 67 haplotypes for the protein. The maximum difference between populations was $31.8 \%$ in the nucleotide and $4.7 \%$ in the protein. Three different COI main lineages exist in S. magnus and coexist in the same sample of several Northern European locations. I conclude that the soil living oribatid mite S. magnus did not go through bottlenecks during the last ice ages contrasting the situation in aboveground animals and plants. The results suggest that individuals survived in multiple cryptic Northern refugia in Northern Germany, Poland and Scandinavia. The high genetic divergences between populations of $S$. magnus likely resulted at least from radiation in the Miocene ( 20 mya). 


\subsection{Introduction}

Climatic changes during the past 2.6 million years caused considerable range shifts in species' distributions in the Northern hemisphere and led to contractions and expansions in population sizes in both warm and cold adapted species. The effects of the last glacial maximum (LGM - 18-22 kya) on species' diversity has been intensively investigated for warm adapted, aboveground and freshwater animals and plants (Beheregaray 2008). The overall pattern has been characterized by genetic richness in Southern European countries and genetic paucity in Northern Europe (Hewitt 1999, Hewitt and Ibrahim 2001). This pattern supports the hypothesis that most taxa retreated during ice ages to refugia south of the Alps, whereas populations expanded rapidly into higher latitudes with post-glacial climatic warming (Hewitt 2000). Presumably, the Iberian Peninsula, Italy, Greece and the Balkan served as major glacial refuge areas where retreating populations met and escaped hostile climatic conditions in Central and Northern Europe.

Extending this view, radiocarbon (Stewart and Lister 2001), pollen (Willis et al. 2000; Tollefsrud et al. 2008, Kelly et al. 2010) and molecular data (Pfenninger et al. 2003; Verovnik et al. 2005; Tollefsrud et al. 2008) indicate that isolated areas in Central and Northern Europe also acted as refuges for species of the temperate and boreal zone (Bilton et al. 1998; Willis et al. 2000; Stewart and Lister 2001; Stewart 2003). These cryptic refugia (Stewart and Lister 2001; Provan and Bennett 2008), however, are difficult to detect since they presumably were small, geographically isolated or located in wind protected valleys or on ice free Nunataks (Schmitt 2009). Cryptic refugia resemble climatic islands in which conditions are more favorable than in surrounding areas and describes a species' distribution range during its contraction phase at glacial maxima (Stewart and Lister 2001; Stewart et al. 2010). Expansions and contractions of populations into refugia significantly affected the evolution and genetic variation of species (Stewart et al. 2010). Therefore, understanding the contribution of cryptic refugia during glacial and interglacial phases is important for understanding speciation, biodiversity and intraspecific variance of species of the Northern Hemisphere.

Oribatid mites (Acari, Oribatida) are among the most diverse and abundant soil living arthropods with about 10,000 described species (Schatz 2002) and up to 400,000 individuals per square meter of forest soil (Maraun and Scheu 2000). Fossils of oribatid mites were found in Devonian sediments (380 my ago; Shear at al. 1984, Norton et al. 1988a) and the origin of oribatid mites dates back to 440 my (Lindquist 1984) or even 570 my according to molecular dating (Schaefer et al. 2010).

The soil living oribatid mite species Steganacarus magnus (Nicolet, 1855) serves as excellent model organism to investigate the existence of cryptic refugia in Central and Northern Europe. This species requires ligneous plant tissues for development as larval stages and nymphs live endophagous in coniferous needles or leave petioles (Norton 1994). The dependence on host plants is rather unspecific and relatively loose as S. magnus predominantly feeds on dead organic material (Schneider et al. 2004). This implies that this species could persist in geographically isolated areas during glacial phases as long as habitat tolerances were met and plants producing ligneous needles or leaves were present. Presumably, S. magnus was directly affected by changing climatic conditions in its natural distribution range, as the species has a palearctic distribution (Subías 2009) and very limited dispersal abilities (Salomone et al. 2002). However, adults and juveniles survive temperatures of $-12^{\circ} \mathrm{C}$ to $-14^{\circ} \mathrm{C}$ (Webb and Block 1993) and the super-cooling point for this species is between $-7^{\circ} \mathrm{C}$ and $-38^{\circ} \mathrm{C}$ (Block 1979; Krantz and Walter 2009). Tolerance against cold temperatures, drought and heat (Siepel 1996; Krantz and Walter 2009) is common in oribatid mite species (R.A. Norton; personal communication). However, the distribution of $S$. magnus suggests that the species is not strictly cold-adapted but evolved a broad temperature tolerance. Another important character for using $S$. magnus as model organism to infer cryptic refugia is its small body size. ( 1.2 $\mathrm{mm}$; Weigmann 2006). Small species are 
more likely to persist in cryptic refugia as their population density and habitat capacity exceeds that of large species (Stewart et al. 2010). Finally, S. magnus is relatively easy to sample, the species is common in temperate forests where it occurs in relatively high densities.

To detect intraspecific genetic diversity and to reconstruct phylogenetic relationships among haplotypes, I analyzed nucleotide and protein sequences of cytochrome oxidase I (COI) of 180 individuals of $S$. magnus from 44 sampling sites across Europe and three sampling sites in Central Asia. Sampling in Central Europe was extensive in order to detect cryptic refugia which were proposed for Central and Northern Europe (Stewart and Lister 2001; Tollefsrud et al. 2008). I expected that lineages which persisted in Central refugia express isolated haplotypes that are distinct from populations from Southern Europe and Central Asia and that genetic diversity within this species' range is highest in these former refuge areas (Stewart et al. 2010).

\subsection{Materials and methods}

\subsubsection{Taxon sampling}

Steganacarus magnus was sampled from 47 locations of Europe, Russia and China (Fig. 7, described in more detail in Table 1). Specimens were extracted from leaf litter using heat (Macfadyen 1961, Kempson et al. 1963). Animals were identified under a stereomicroscope, determined after Weigmann (2006) and stored in $75 \%$ ethanol at $-20^{\circ} \mathrm{C}$ until preparation.

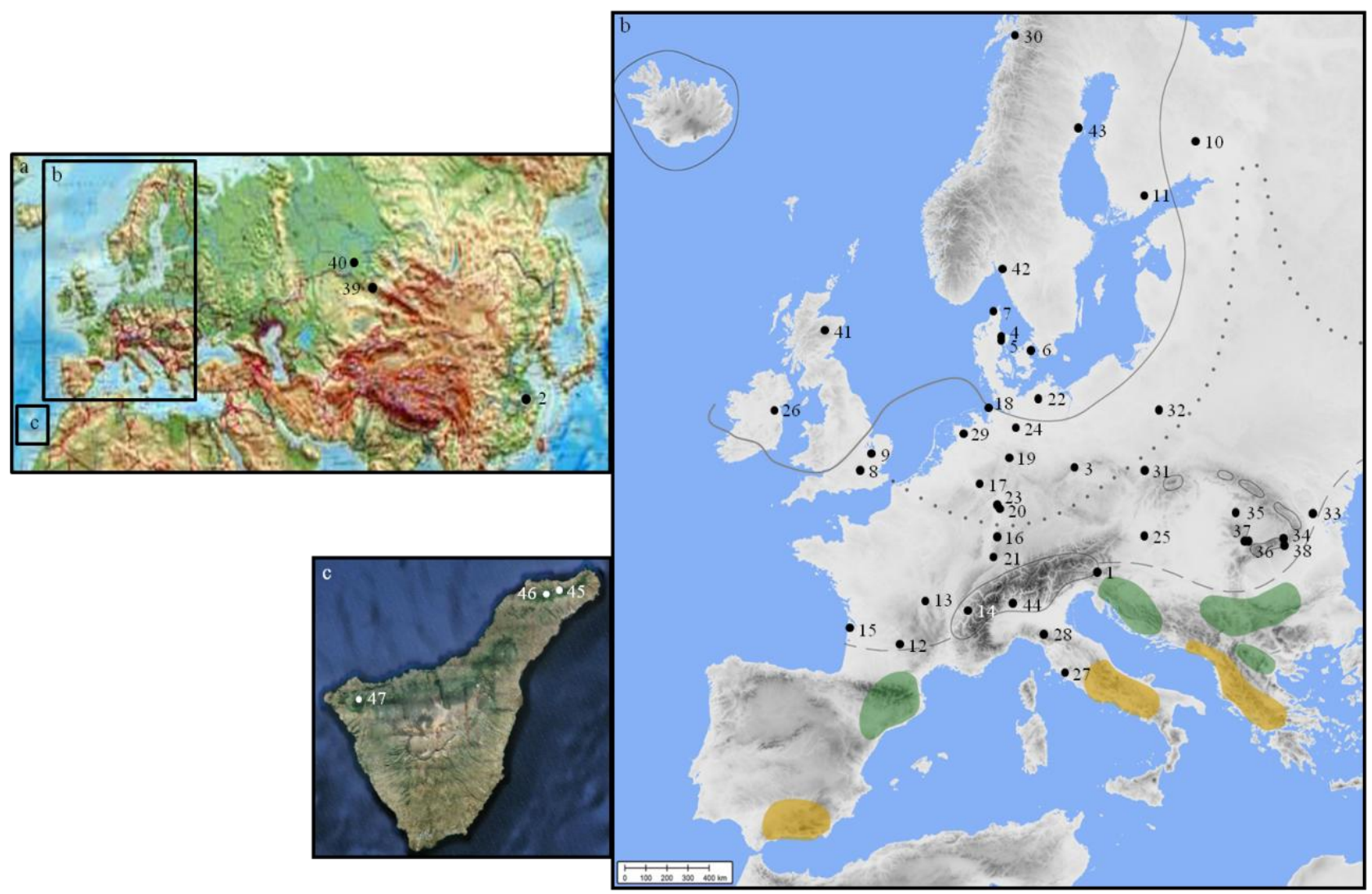

Figure 7: (a) Map of Europe and Asia (source: http:Ilwww.kelt.de), black dots with numbers are the sampling points; (b) Map of Europe from the last glacial maximum (modified from Hewitt 1999). Black dots with numbers represent the sampling points of oribatid mites used in this study, green shading marks refugia of coniferous trees and yellow shading those of deciduous trees, grey lines mark the expansion of glaciers, the dotted line represents the polar desert climate border and the dashed line the tundra and permafrost border (Hewitt and Ibrahim 2001, Alexander Kartographie 2006); (c) Map of Tenerife (source: Google Earth), white dots with number are the sampling points. 
Table 1: Localities where Steganacarus magnus was sampled [ln: location number, country, location, coordinates, abbreviations of sampling locations (code) and name of collector (for details see Acknowledgements)]. Each location is marked by a colour symbol.

\begin{tabular}{|c|c|c|c|c|c|c|}
\hline $\ln$ & country & location & coordinates & codle & colour & litter collector \\
\hline 1 & Austria & Villach & $46.57^{\circ} 13.85^{\circ}$ & SM_A_1.1-2 & O & K. Domes-Wehner \\
\hline 2 & China & Nanjing & $32.06^{\circ} 118.78^{\circ}$ & SM_CHINA_1.1-2 & 0 & J. Shan \\
\hline 3 & Czech Republic & Decin & $50.78^{\circ} 14.23^{\circ}$ & SM_CZ_1.1-5 & $\oplus$ & M. Rosenberger \\
\hline 4 & Demark & Arhus 2 & $56.11^{\circ} 10.21^{\circ}$ & SM_DK_4.1-3 & (1) & T. Bilde \\
\hline 5 & & Arhus 1 & $56.13^{\circ} 10.19^{\circ}$ & SM_DK_3.1-3 & $\ominus$ & T. Bilde \\
\hline 6 & & Copenhagen & $55.68^{\circ} 12.58^{\circ}$ & SM_DK_1.1-3 & $\Theta$ & N. Eisenhauer \\
\hline 7 & & Hjørring & $57.48^{\circ} 9.96^{\circ}$ & SM_DK_2.1-5 & 0 & M. Rosenberger \\
\hline 8 & England & Ascot & $51.40^{\circ}-0.68^{\circ}$ & SM_GB_2.1-2 & $\oplus$ & A. Milcu \\
\hline 9 & & Bedford & $52.07^{\circ}-0.44^{\circ}$ & SM_GB_1.1-5 & 0 & A. Milcu \\
\hline 10 & Finland & Joensulu & $62.58^{\circ} 29.73^{\circ}$ & SM_FIN_2.1 & $\oplus$ & C. Platner \\
\hline 11 & & Lahti & $60.99^{\circ} 25.65^{\circ}$ & SM_FIN_- $1.1-3$ & ○ & H. Setela \\
\hline 12 & France & Haute Loire & $44.99^{\circ} 3.86^{\circ}$ & SM_F_t $\overline{4} .1-2$ & 0 & A. Jousset \\
\hline 13 & & Loire & $45.56^{\circ} \quad 4.79^{\circ}$ & SM_F_2.1-4 & ○ & M. Maraun \\
\hline 14 & & Mont Blanc & $45.82^{\circ} 6.74^{\circ}$ & SM_F_1.1-6 & O & A. Jousset \\
\hline 15 & & Saint Isidore & $45.27^{\circ}-1.09^{\circ}$ & SM_F_3.1-5 & 0 & C. Digl \\
\hline 16 & Germany & Black Forest & $48.89^{\circ} 8.43^{\circ}$ & SM_D_- 6.1 & 0 & K. Heidemann \\
\hline 17 & & Bonn & $50.84^{\circ} 7.14^{\circ}$ & SM_D_9.1-4 & (1) & R. Koller \\
\hline 18 & & Cuxhaven & $53.86^{\circ} 8.65^{\circ}$ & SM_D_8.1-3 & $\oplus$ & K. Heidemann \\
\hline 19 & & Goettingen & $51.53^{\circ} 9.96^{\circ}$ & SM_D_2.1-9 & 0 & K. Domes-Wehner \\
\hline 20 & & Kranichstein & $49.89^{\circ} 8.69^{\circ}$ & SM_D_1.1-14 & 0 & M. Rosenberger \\
\hline 21 & & Lake Constance & $47.71^{\circ} 9.37^{\circ}$ & SM_D_3.1-3 & O & K. Heidemann \\
\hline 22 & & Mecklen. Seenplatte & $53.57^{\circ} 12.33^{\circ}$ & SM_D_4.1-9 & ○ & I. Schaefer \\
\hline 23 & & Moerfelden & $49.96^{\circ} 8.55^{\circ}$ & SM_D_5.1-3 & O & K. Domes-Wehner \\
\hline 24 & & Uelzen & $52.97^{\circ} 10.52^{\circ}$ & SM_D_7.1-4 & 0 & H. Treptow \\
\hline 25 & Hungary & Piliscaba & $47.62^{\circ} 18.83^{\circ}$ & SM_HUN_1.1 & $\oplus$ & C. Csjui \\
\hline 26 & Ireland & Swords & $53.45^{\circ}-6.22^{\circ}$ & SM_IRL_1.1-2 & $\ominus$ & B. Eitzinger \\
\hline 27 & Italy & Grosseto & $42.63^{\circ} 11.11^{\circ}$ & SM_I_1.1-10 & ○ & M. Maraun \\
\hline 28 & & Parma & $44.20^{\circ} 10.34^{\circ}$ & SM_I_2.1-4 & O & M. Maraun \\
\hline 29 & Netherlands & Wageningen & $51.97^{\circ} \quad 5.70^{\circ}$ & SM_N̄̄- $1.1-3$ & $\Theta$ & O. Butenschoen \\
\hline 30 & Norway & Narvik & $68.44^{\circ} 17.40^{\circ}$ & SM_N_1.1-3 & $\Theta$ & O. Butenschoen \\
\hline 31 & Poland & Krakow & $50.04^{\circ} 19.84^{\circ}$ & SM_PL_1.1-6 & 0 & S. Scheu \\
\hline 32 & & Warsaw & $52.33^{\circ} 20.76^{\circ}$ & SM_PL_2.1-3 & 0 & A. Uvarov \\
\hline 33 & Romania & Bagau & $46.55^{\circ} 26.72^{\circ}$ & SM_RUM_3.1-4 & 0 & T. Pasca \\
\hline 34 & & Busteni & $45.41^{\circ} 25.55^{\circ}$ & SM_RUM_5.1-4 & (1) & C. Ivanescu \\
\hline 35 & & Cluj & $46.77^{\circ} 23.52^{\circ}$ & SM_RUM_4.1-3 & $\oplus$ & T. Pasca \\
\hline 36 & & Sibiu 1 & $45.64^{\circ} 23.74^{\circ}$ & SM_RUM_1.1-3 & 0 & S. Scheu \\
\hline 37 & & Sibiu 2 & $45.65^{\circ} 23.70^{\circ}$ & SM_RUM_2.1-4 & O & S. Scheu \\
\hline 38 & & Sinaia & $45.35^{\circ} 25.56^{\circ}$ & SM_RUM_6.1-3 & $\theta$ & C. Ivanescu \\
\hline 39 & Russia & Altai Mountains & $51.73^{\circ} 85.76^{\circ}$ & SM_RUS_1.1-4 & O & M. Ackermann \\
\hline 40 & & Novosibirsk & $54.97^{\circ} 83.39^{\circ}$ & SM_RUS_2.1-5 & $\oplus$ & A. Uvarov \\
\hline 41 & Scotland & Braemar & $57.00^{\circ}-3.40^{\circ}$ & SM_GB_3.1-3 & 0 & A. Jousset \\
\hline 42 & Sweden & Stroemstad & $58.87^{\circ} 11.14^{\circ}$ & SM_S_2.1-3 & O & G. Kalinkat \\
\hline 43 & & Umea & $63.83^{\circ} 20.29^{\circ}$ & SM_S_1.1 & ○ & O. Butenschoen \\
\hline 44 & Switzerland & Locarno & $46.17^{\circ} 8.77^{\circ}$ & SM_CH_1.1 & (1) & M. Scheu \\
\hline 45 & Tenerife & Anaga Mountains 1 & $28.55^{\circ}-16.20^{\circ}$ & SM_E_2.1 & $\oplus$ & S. Scheu \\
\hline 46 & & Anaga Mountains 2 & $28.53^{\circ}-16.27^{\circ}$ & SM_E_3.1-4 & O & S. Scheu \\
\hline 47 & & Teno Mountains & $28.32^{\circ}-16.83^{\circ}$ & SM E 1.1-2 & 0 & S. Scheu \\
\hline
\end{tabular}




\subsubsection{DNA extraction and sequencing}

Genomic DNA was extracted from single individual using the DNeasy ${ }^{\circledR}$ Blood and Tissue Kit (Qiagen; Hilden, Germany) following the manufacturer's protocol for animal tissue. A 514 bp region of cytochrome c oxidase subunit I (COI) was amplified using the primers COIarch1 (5'GGTCAACAAATCATAAAGAYATYGG-3') and COIarch2 (5'TAAACTTCAGGGTGACCAAAAAATCA-3') (Heethoff et al. 2007), the HotStarTaq ${ }^{\circledR}$ Master Mix Kit (Qiagen; Hilden, Germany) and the SuperHot Taq Mastermix (Genaxxon), respectively. The polymerase chain reaction (PCR) contained $0.5 \mu \mathrm{l}$ of each primer (100 pmol/ $\mu \mathrm{l}), 1 \mu 1 \mathrm{MgCl}_{2}(25 \mathrm{mM})$, $12.5 \mu \mathrm{l}$ of $\mathrm{HotStarTaq}^{\circledR}$ Master Mix (1.25 units HotStarTaq ${ }^{\circledR}$ polymerase, $100 \mu \mathrm{M}$ of each dNTP and $7.5 \mathrm{mM} \mathrm{MgCl}{ }_{2}$ buffer solution; Qiagen, Germany) or SuperHot Taq Mastermix [2.5 units SuperHot Taq polymerase, $10 \mu \mathrm{M}$ of each dNTP and buffer solution (20 mM Tris-HCI (pH 8.3), $100 \mathrm{mM} \mathrm{KCl,}$ $0.1 \mathrm{mM}$ EDTA, $1 \mathrm{mM}$ DTT, 50\% glycerol, $0.5 \%$ Nonidet P40 and 0.5\% Tween 20), Genaxxon; Ulm, Germany] containing polymerase, $3 \mu$ template DNA and filled up to a total reaction volume of 25 $\mu 1$ with RNase free water. PCR parameters included a 15 min step at $95^{\circ} \mathrm{C}$ for polymerase activation followed by 36 cycles with $30 \mathrm{~s}$ at $94^{\circ} \mathrm{C}$ for denaturation, $60 \mathrm{~s}$ at $51^{\circ} \mathrm{C}$ for primer annealing and $60 \mathrm{~s}$ at $72^{\circ} \mathrm{C}$ for elongation and a final $10 \mathrm{~min}$ step for elongation at $72^{\circ} \mathrm{C}$. PCR products were visualized on $1 \%$ agarose gel and purified using the QIAquick ${ }^{\circledR}$ PCR Purification Kit (Qiagen; Hilden, Germany) following the manufacturer's protocol. The purified PCR products were sequenced in both directions by Macrogen Inc. (Seoul, Korea) and the Department of Experimental Phycology and Culture Collection of Algae (Georg-August University Göttingen, Germany).

\subsubsection{Phylogeographic and population genetic analyses}

Nucleotide sequences were edited and translated into amino acids using the invertebrate mitochondrial code implemented in SEQUENCHER v4.9 (Gene Codes) and aligned with ClustalX v1.81 (Thompson et al. 1997) using multiple alignment parameters: 10.0 for gap opening and 0.1 for gap extension for the nucleotide, default settings for the amino acids dataset. Phylogenetic trees were generated with Beast v1.5.4 (Drummond and Rambaut 2007), MrBayes v3.1.2 (Ronquist and Huelsenbeck 2003) and PAUP* (Swofford 1999) using the NJ algorithm without and with model of sequence evolution to identify monophyletic clusters in the dataset. The model of sequence evolution was estimated with Modeltest3.6 (Posada and Crandall 1998) in PAUP* and in MrModeltest (Nylander 2004) for the Bayesian analysis. The best fit model was GTR $+\mathrm{I}+\mathrm{G}$ for both analyses. Model parameters were nst=6 and rates=invgamma for nucleotide sequences in BEAST and MrBayes, mtrev for the protein in Beast and aamodel=equalin for the protein alignment in MrBayes. Outgroups were Hypochthonius rufulus (C.L. Koch, 1835) (Oribatida, Enarthronota) and Rhysotritia duplicata (Grandjean, 1953) (Oribatida, Mixonomata). The Markov Chain Monte Carlo was run for ten million generations and sampled every $1000^{\text {th }}$ generation, the $50 \%$ majority consensus tree excluded the first $2,500,000$ trees (burnin of $25 \%$ ).

Standard diversity indices for the nucleotide sequences [haplotype number $\left(\mathrm{N}_{\mathrm{h}}\right)$, haplotype diversity $\left(\mathrm{H}_{\mathrm{d}}\right.$, ) nucleotide diversity $\left(\Pi_{\mathrm{n}}\right)$, number of variable $\left(\mathrm{N}_{\mathrm{vs}}\right)$ and invariable sites $\left(\mathrm{N}_{\mathrm{is}}\right)$, parsimony informative sites $\left(\mathrm{N}_{\text {pars }}\right)$, the number of singletons $\left(\mathrm{N}_{\mathrm{s}}\right)$ and the total number of substitutions $\left.\left(\mathrm{N}_{\mathrm{m}}\right)\right]$ and the McDonald-Kreitman (MK) test, to detect selection, were calculated in DNASP v5.0 (Rozas et al. 2003). The McDonald-Kreitman test can detect selection by examining the distribution of synonymous and non-synonymous substitutions among populations; it is robust against demographic and recombination events (McDonald and Kreitman 1991). The geographical structure of genetic diversity among and within populations and geographical clades were calculated with ARLEQUIN v3.01 (Excoffier et al. 2005) using analysis of molecular variance (AMOVA, 16,000 permutations). Only populations with two and more individuals were included in the analysis. Estimates for demographic 
expansion (Tajima's D and Fu's $\mathrm{F}_{\mathrm{s}}$ neutrality tests) and pairwise differences ( $\mathrm{F}_{\mathrm{ST}} 10,000$ permutations) were also calculated in ARLEQUIN I calculating 10,000 permutations to test for significance.

The dataset exceeded the connection limit of TCS (Clement et al. 2000); therefore a parsimony based median-joining haplotype network (Bandelt et al. 1999) was generated in NETWORK v4.5 (FluxusTechnology, Suffolk, UK) with default settings for nucleotide sequences and amino acids.

\subsection{Results}

A total of 182 individuals (180 S. magnus, one R. duplicata and one H. rufulus) from 47 localities in 19 countries were sequenced. The amplified COI fragments were $514 \mathrm{bp}$ long and coded for 171 amino acids. The frequency of bases declined in the order 45.1, 25.7, 14.7 and $14.5 \%$ for T, A, C and G, respectively. The 180 S. magnus COI sequences formed 111 haplotypes for the nucleotide $(61.7 \%)$ (Fig. 8) with 310 variable (60.7\%); 275 of these were parsimony informative sites (Table A1). The amino acids formed 67 haplotypes (37.2\%) (Fig. 9). The haplotype diversity $\left(\mathrm{H}_{\mathrm{d}}\right)$ was very high with 0.98 in the nucleotide sequences.

\subsubsection{Network}

In the nucleotide haplotype network (Fig. 8) seven haplotypes were shared by individuals from different locations (D_1/D_2/D_9/F_2/I_1/I_2, D_1/D_2/D_9/DK_3/DK_4/I_2, D_1/I_1, D_8/GB_1/NL_1, D_4/DK_2, F_3/GB_1 and GB_3/NL_1). Sixteen haplotypes comprised two or more individuals from the same location (A_1.1-2, CZ_1.1-2, CHINA_1.1-2, D_2.6-7, D_4.12/D_4.6-7, D_5.1-3, D_7.2/D_7.4, F_1.1/F_1.4-6, F_3.3/F_3.5, F_4.1-2, FIN_1.1-2, N_1.1/N_1.3, PL_2.1/PL_2.3, RUM_1.1-2, RUM_3.1-2/RUM_3.4 and RUS_2.1-4). The other 88 haplotypes were single individuals.

The nucleotide haplotype network formed 30 subclades and eight isolated individual haplotypes which were separated each by high numbers of substitution steps. The different subclades formed three main clades (black, blue and red).

The black main clade consisted of 14 subclades, two isolated single individual haplotypes each from one location (GB_1.4, RUM_6.3) and one isolated haplotype of two individuals from one Chinese location (CHINA_1.1-2).

Subclade 1 comprised three smaller subclades 1a-c. Subclade 1a comprised eight haplotypes from seven locations [single individual haplotypes: one from one German location (D_1.12), one from another German location (D_2.2), one from one Danish location (DK_3.1), one from one Italian location (I_1.1), two from on French location (F_2.2, F_2.4); haplotypes of more than one individual: one haplotype of two individuals from one German location (D_2.6-7),.one of 13 individuals from six locations (two individuals from each one German location (D_1.1, D_9.1), five individuals from another German location (D_2.1, D_2.4-5, D_2.8-9), two individuals from one French location (F_2.1, F_2.3), three individuals from one Italian location (I_1.4, I_1.9-10), one individual from another Italian location (I_2.4)]. The most abundant haplotype was the centre of the subclade 1a, the other seven surrounding haplotypes were connected by one or two substitution steps to the Central haplotype.

Subclade $1 \mathrm{~b}$ comprised six single individual haplotypes from four locations [two from one German location (D_1.5, D_1.14), two each from one location (D_9.2, I_1.2), two from one Italian location (I_2.1, I_2.2)] and one haplotype of six individuals from two locations [one individual from one German (D_1.2), five individuals from one Italian (I_1.3, I_1.5-8)]. Five single individual haplotypes were connected by one substitution step to the most abundant haplotype. The single individual I_2.2 was connected by six substitution steps to the most abundant haplotype. 
Subclade 1c comprised four single individual haplotypes from three locations [two from one German (D_1.7, D_1.13), two each from one Danish (DK_1.3, DK_4.3)] and one haplotype of 15 individuals from six locations [seven individuals from one German (D_1.3-4, D_1.6, D_1.8-11), two individuals each one (D_2.3, I_2.3), two individuals from a third German (D_9.3-4), two individuals from one Danish (DK_3.2-3), two individuals from another Danish DK_4.1-2)]. Three single individuals were separated from the most abundant haplotype by one substitution step. The single individual haplotype D_1.13 was separated from the single individual haplotype D_1.7 by eleven substitution steps.

Subclade 2 comprised five single individual haplotypes from two locations on Tenerife [one from one location (E_2.1), four from another location (E_3.1-3.4)]. Subclade 3 comprised two single individuals from one location on Tenerife (E_1.1-2). Subclades 2 and 3 were separated from each other by 71 substitution steps. Subclade 4 comprised of three single individual haplotypes from one German location (D_3.1-3).

Subclade 5 comprised of two smaller subclades $5 \mathrm{a}$ and $5 \mathrm{~b}$. Subclade $5 \mathrm{a}$ comprised nine haplotypes from six locations [single individual haplotypes: three individuals each from one location (GB_1.2, GB_3.1, NL_1.2), two individuals from one English (GB_2.1-2), two individuals from one Irish (IRL_1.1-2); haplotypes of more than one individual: one haplotype of two individuals from two locations (F_3.1, GB_1.3), one haplotype of three individuals from two locations (GB_3.2-3 and NL_1.1)]. Subclade $5 \mathrm{~b}$ comprised two haplotypes from three locations [one single individual haplotype from one German (D_8.3), one haplotype of five individuals from three locations (D_8.1-2, GB_1.1, GB_1.5, NL_1.3)].

Subclade 6 comprised three haplotypes from one French location [two single individuals (F_3.2, F_3.4), haplotype of two individuals (F_3.3, F_3.5)]. Subclade 7 comprised one haplotype of three individuals from one German location (D_5.1-3). Subclade 8 comprised six single individual haplotypes from two Romanian locations (RUM_5.1-4 and RUM_6.1-2). Subclade 9 comprised two haplotypes from one Polish location [one single individual haplotype (PL_2.2), one haplotype of two individuals (PL_2.1 and PL_2.3). Subclade 10 comprised six haplotypes from two Romanian locations [five single individual haplotypes from two locations (RUM_1.3 and RUM_2.1-4), haplotype of two individuals from one location (RUM_1.1-2)]. Subclade 11 comprised two single individual haplotypes each from one Scandinavian location (FIN_2.1, S_2.2).

The blue main clade formed three subclades and one isolated haplotype of two individuals from one Norwegian location (N_1.1, N_1.3). Subclade 12 comprised five haplotypes from three locations [single individual haplotypes: two haplotypes each from one location (D_4.5, HUN_1.1), two haplotypes from one Danish location (DK_1.1-2); haplotypes of more than one individual: one of four individuals one German location (D_4.1-2, 4.6-7)]. Subclade 13 comprised three haplotypes from two locations [two single individual haplotypes from two locations (CZ_1.5, F_1.2), haplotype of two individuals from one Czech location (CZ_1.1-2)]. Subclade 14 comprised five single individual haplotypes from four locations [three haplotypes each from one location (CZ_1.4, D_6.1, S_2.1), two haplotypes from one Polish location (PL_1.1, 1.3)].

The red main clade comprised nine subclades, six single individual haplotypes each from one location (D_4.4, D_5.5, F_1.3, RUM_3.3, RUS_1.4, RUS_2.5), two single individual haplotypes from one German location (D_7.1, 7.3) and two haplotypes of two individuals each from one location (D_7.2, D_7.4; A_1.1-2).

Subclade 15 comprised five haplotypes from five locations [single individual haplotypes: two each from one location (CH_1.1, S_2.3), two haplotypes from one Polish location (Pl_1.4-5); one haplotype of eight individuals from two locations (DK_2.1-5, D_4.3, 4.8-9). Subclade 16 comprised four haplotypes from three locations [single individual haplotypes: one from one German location (D_5.4), two from one Polish location (PL_1.2, 1.6); one haplotype of two individuals from one French location (F_4.1-2)]. Subclade 17 comprised three haplotypes from two Scandinavian locations [single 
individual haplotypes: two each from one location (FIN_1.3, S_1.1); one haplotype of two individuals from one Finish location (FIN_1.1-2)]. Subclade 18 comprised one haplotype of four individuals from on French location (F_1.1 and F_1.4-6). Subclade 19 consisted of two smaller subclades 19a and 19b each from one Romanian and one single individual haplotype from one Romanian location (RUM_3.3). Subclade 19a comprised three single individual haplotypes from one Romanian location (RUM_4.1-3). Subclade 19b comprised one haplotype of three individuals from one Romanian location (RUM_3.1-2, 3.4). Subclade 20 comprised two single individual haplotypes each from one location (CZ_1.3 and N_1.2). Subclade 21 comprised three single individual haplotypes from one Russian location (RUS_1.1-3) and subclade 22 one haplotype of four individuals from another Russian location (RUS_2.1-4).

The southwest (subclades 2, 3), the southeast (subclades 8, 10, 19) and the Far East (subclade 21, 22, CHINA) refuge areas had no directly linkage to the subclades of Central and Northern Europe. Only the Italian locations were linked to Central French, Central German and Northern Danish locations. All other Central and Northern subclades were separated by large numbers of substitution steps to each other. 


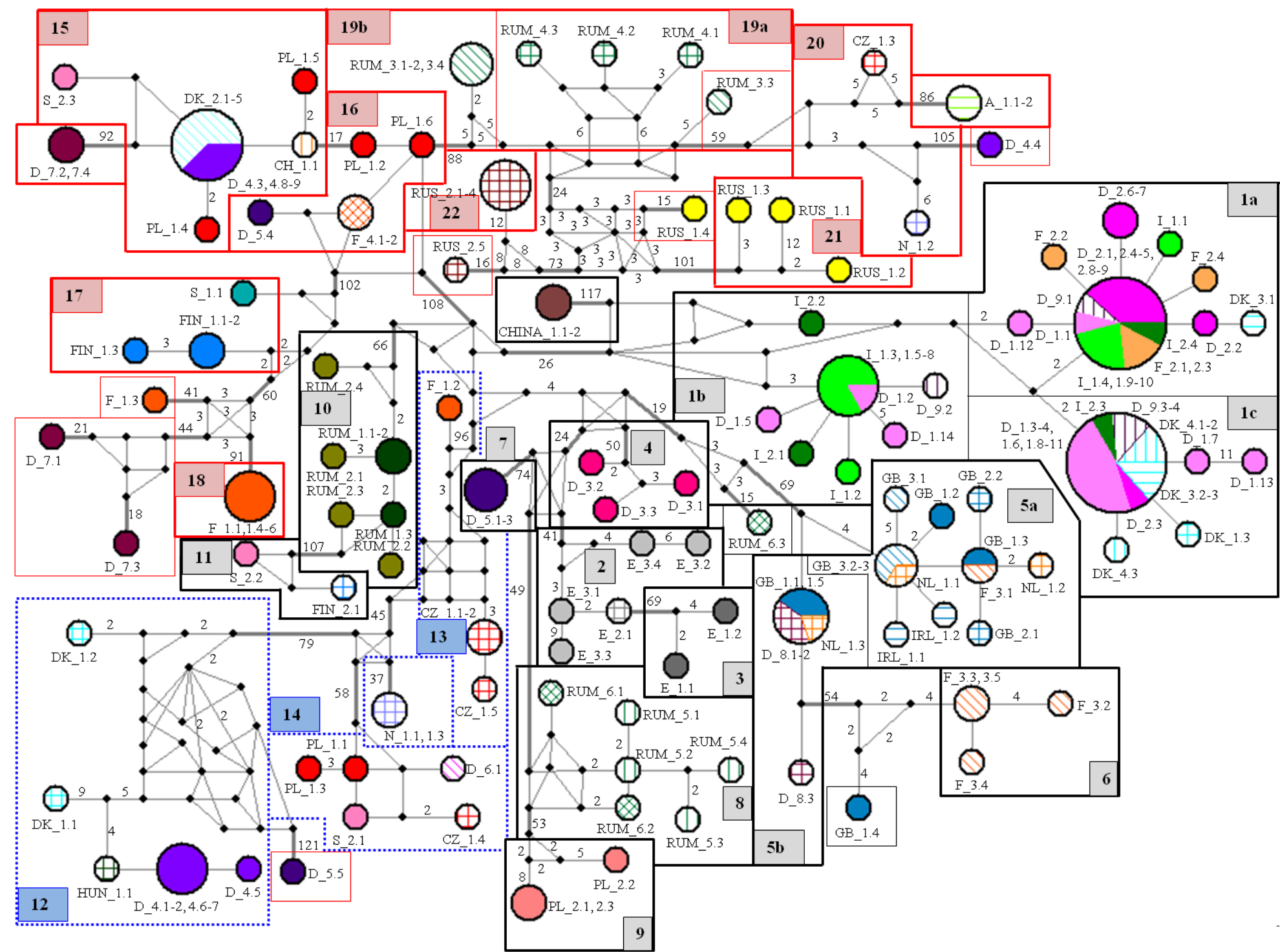

Figure 8: Median-joining haplotype network for the COI nucleotide of 111 haplotypes from Steganacarus magnus. Each location has a specific colour marking. The size of the circles is proportional to the number of sequences per haplotype. Numbers on the lines represent the number of substitution steps separating the haplotypes (no number: only one substitution step between haplotypes). Major subclades are marked by boxes. 
The protein haplotype network (Fig. 9) comprised 67 haplotypes and 19 subclades, five single individual haplotypes each from one location (D_1.13, D_4.4, D_5.5, F_1.3, RUM_6.3), two single haplotypes from one German location (D_7.1, 7.3) and two haplotypes of two individuals each from one location (A_1.1-2, CHINA_1.1-2) which were separated by five or more amino acid changes ( $3 \%$ divergence).

Subclade 1 comprised eight haplotypes from nine locations [single individual haplotypes: two each from one location (DK_4.3, I_2.2), two from one Italian location (I_1.1-2), two from one German location (D_1.13-14); haplotypes of more than one individual: one haplotype of ten individuals from five locations (three individuals each from one location (D_9.2, DK_1.3, I_2.1), two individuals from one German location (D_1.2, D_1.5), five individuals from one Italian location (I_1.3, 1.5-8)), one haplotype of 35 individuals from eight locations (ten individuals from one German location (D_1.1, 1.3-4, 1.6-12), nine individuals from another German location (D_2.1-9), three individuals from a third German location (D_9.1, 9.3-4), three individuals from one Danish location (DK_3.1-3), two individuals from another Danish location (DK_4.-2), four individuals from one French location (F_2.1-4), three individuals from one Italian location (I_1.4,1.9-10), one individual from another Italian location (I_2.3-4))].

Subclades 2 and 3 fused and comprised four haplotypes from three locations on Tenerife [single individual haplotypes: two from one location (E_3.2-3); haplotypes of more than one individual: one haplotype of two individuals from one location (E_1.1-2), one haplotype of three individuals from two locations (E_2.1, E_3.1, 3.4)]. Subclade 4 comprised one haplotype of three individuals of one German location (D_3.1-3). Subclades 5, 6 and the isolated individual GB_1.4 fused and comprised eight haplotypes from five locations [single individual haplotypes: three each from one location (D_8.3, GB_2.1, GB_3.1), two from one French location (F_3.2, 3.4); haplotypes of more than one individual: one of three individuals from two locations (F_3.3, 3.5; GB_1.4), one of four individuals each from one location (F_3.1, GB_1.3, GB_2.2, NL_1.2), one of eleven individuals from five locations (two individuals from one German (D_8.1-2), two individuals from one Scottish (GB_3.2-3), two individuals from one Irish (IRL_2.1-2), two individuals from one Dutch (NL_1.1, NL_1.3) three individuals from one British (GB_1.1-2, 1.5))].

Subclade 7 was identical with the nucleotide network. Subclades 8 and 9 fused and comprised four individuals [single individual haplotypes: two each from one location (PL_2.2, RUM_5.1); haplotypes of more than one individual: one of two individuals form one Polish location (PL_2.1, 2.3), one of five individuals from two Romanian locations (RUM_5.2-4, RUM_6.1-2)]. Subclade 10 comprised three haplotypes from two Romanian locations [single individual haplotypes: two from one Romanian (RUM_2.2, 2.4); one of five individuals from two Romanian locations (RUM_1.1-3, RUM_2.1, 2.3)]. Subclade 11 had no changes to the nucleotide network.

Subclade 12 comprised four haplotypes from three locations [single individual haplotypes: one from one German (D_4.5), two from one Danish (DK_1.1-2); one of five individuals from two locations (D_4.1-2, 4.6-7, HUN_1.1)]. Subclade 13 and the isolated Norwegian haplotype (N_1.1, 1.3) fused and comprised three haplotypes from five locations [single individual haplotype: one from one Polish (PL_1.3); haplotypes of more than one individual: one of two individuals from one Norwegian location ( $\mathrm{N} \_1.1$ and $\mathrm{N} \_1.3$ ), one of four individuals each from one location (CZ_1.4, D_6.1, PL_1.1, S_2.1). Subclade 14 comprised two haplotypes from two locations [one single individual haplotype from one Czech (CZ_1.5), one of three individuals from two locations (CZ_1.1-2, F_1.2)].

Subclades 15 and 16 fused and comprised six haplotypes from seven locations [single individual haplotypes: two individuals each from one location (D_5.4, S_2.3), two from one Polish location (PL_1.4-5); haplotypes of more than one individual: one of four individuals from two locations (F_4.1-2, PL_1.2, 1.6), one of nine individuals from three locations $(\mathrm{CH}$ _1.1, D_4.3, 4.8-9, DK_2.15)]. The protein haplotype network of subclades 17 and 18 was identical to that of the nucleotide 
network. Subclades 19, 20, the isolated Austrian haplotype (A_1.1-2) and the isolated haplotype RUS_1.4 fused and comprised four haplotypes from six locations [single individual haplotype: one from one Romanian (RUM_3.3); haplotypes of more than one individual: one of two individuals from one Austrian location (A_1.1-2), one of two individuals from two locations (CZ_1.3, N_1.2), one of seven individuals from three locations (RUM_3.1-2, 3.4, RUM_4.1-3, RUS_1.4)]. Subclade 21 was identical to that of the nucleotide network. Subclade 22 and the isolated haplotype (RUS_2.5) fused and comprised two haplotypes [one single individual (RUS_2.5), one haplotype of four individuals from one Russian location (RUS_2.1-4)].

The Chinese and the two German (D_7) subclades of the protein haplotype network were identical to the nucleotide haplotype network. The Chinese subclade was next to subclade 19/20 and not to subclade 1 as in the nucleotide network. The isolated individual D_1.13 was separated from the most abundant haplotype of the largest subclade by seven amino acid changes (more than $4 \%$ differences). In the nucleotide network the D_1.13 haplotype was separated from the D_1.7 haplotype by eleven substitution steps (2.1\% divergence). Haplotypes of individuals from the same location existed in different subclades in the nucleotide and in the protein (CZ_1, D_4, D_5, N_1, RUM_6, RUS_1 and S_2). Among these subclades there were large numbers of substitution steps in the nucleotide and several amino acid changes in the protein. 


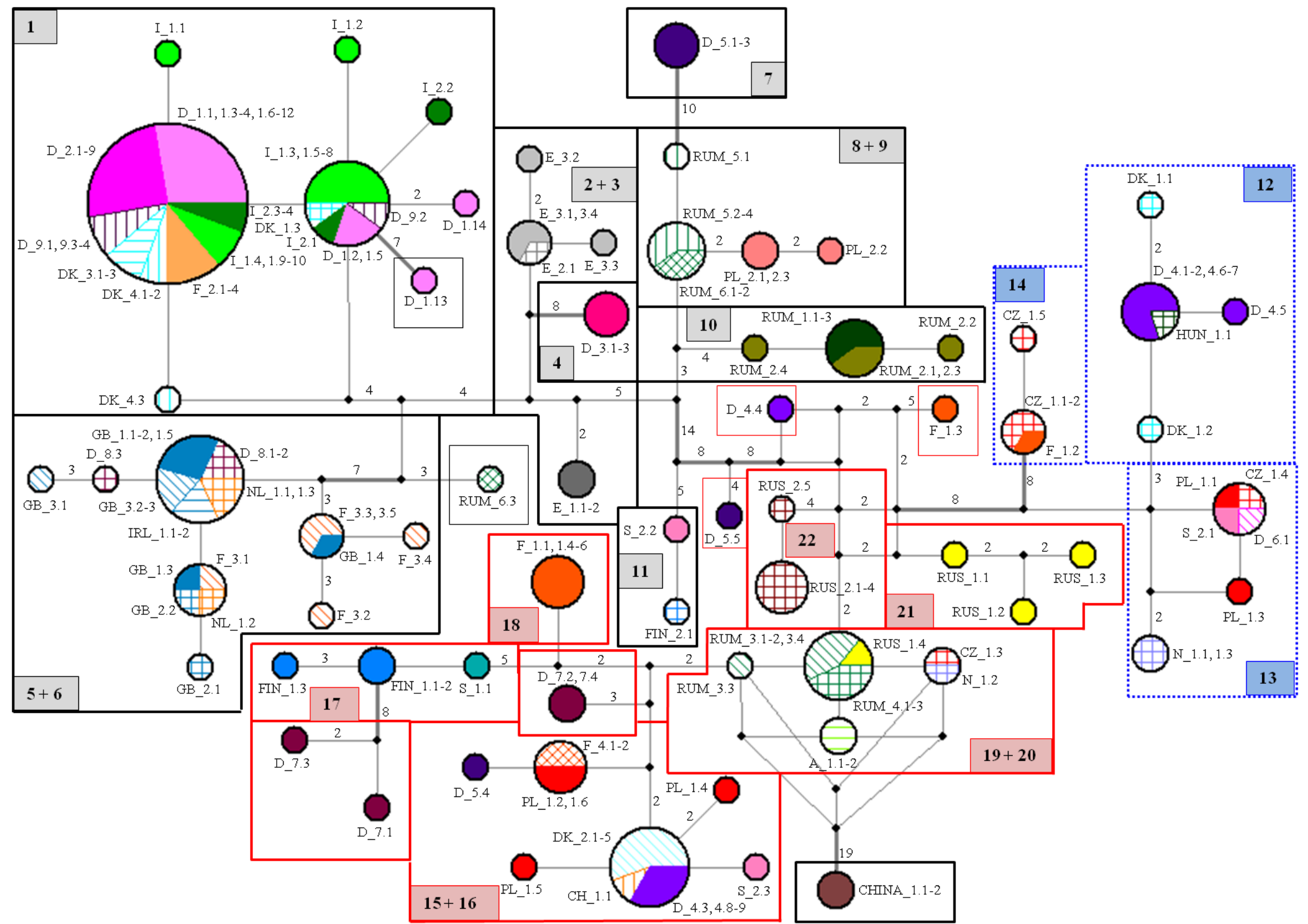

Figure 9: Median-joining haplotype network for the COI protein of 67 haplotypes from Steganacarus magnus. Each location has a specific colour marking. The size of the circles is proportional to the number of sequences per haplotype. Numbers on the lines represent the number of substitution steps separating the haplotypes (no number: only one amino acid change between haplotypes). Major subclades are marked by boxes. 


\subsubsection{Phylogenetic and population genetic analyses}

In the Bayesian phylogenetic analysis of the nucleotide in BEAST the three main clades were monophyletic and supported by maximum posterior probabilities (Fig. 10). The blue and the black clade formed sister clades. In the other three phylogenetic analyses (NJ without and with model of sequence evolution and MrBayes) the black and blue clades were paraphyletic (Fig. A1-4). In all phylogenetic analyses the Chinese individuals were in the black clade which was supported by high bootstrap values and posterior probabilities. The red clade was only monophyletic in the Bayesian analysis of BEAST and paraphyletic in the other analyses. In the phylogenetic analyses of the protein the three main clades were monophyletic (Fig. 11, Fig. A5-7) and supported by high bootstrap and posterior probabilities. The Chinese individuals formed the basal group of the $S$. magnus protein sequences.

The minimum and maximum average pairwise differences for the nucleotide sequences between populations were $0.3 \%$ (D_2/F_2) and 31.8\% (RUM_5/RUS_1) (Table 3; for the protein see Table A1). Excluding Russia and China from the analysis the maximum average pairwise difference was $30.2 \%$ (A_1/D_3). Within populations the minimum and maximum average pairwise differences were $0 \%$ (A_1, CHINA_1, DK_2 and F_4) and $25.8 \%$ (S_2). For the protein the minimum and maximum average pairwise differences between populations were $0 \%$ (A_1/RUM_3/RUM_4, CHINA_1/F_4, D_2/DK_3/DK4/F_2/RUM_1, D_9/RUM_1, GB_1/GB_2/IRL_1/NL_1, I_1/RUM_1, I_2/RUM_1) and 4.7\% (D_3/CHINA_1); excluding Russia and China from the analysis the maximum average pairwise difference was $4.2 \%$ (FIN_1/PL_2). The minimum and maximum average pairwise differences within population were 0\% (A_1, CHINA_1, D_2, D_3, DK_2, DK_3, DK_4, E_1, E_3, F_2, F_4, FIN_1, GB_1, GB_2, IRL_1, NL_1, RUM_1, RUM_3 and RUM_4) and 2.1\% (D_5) for the protein (Table A2). 


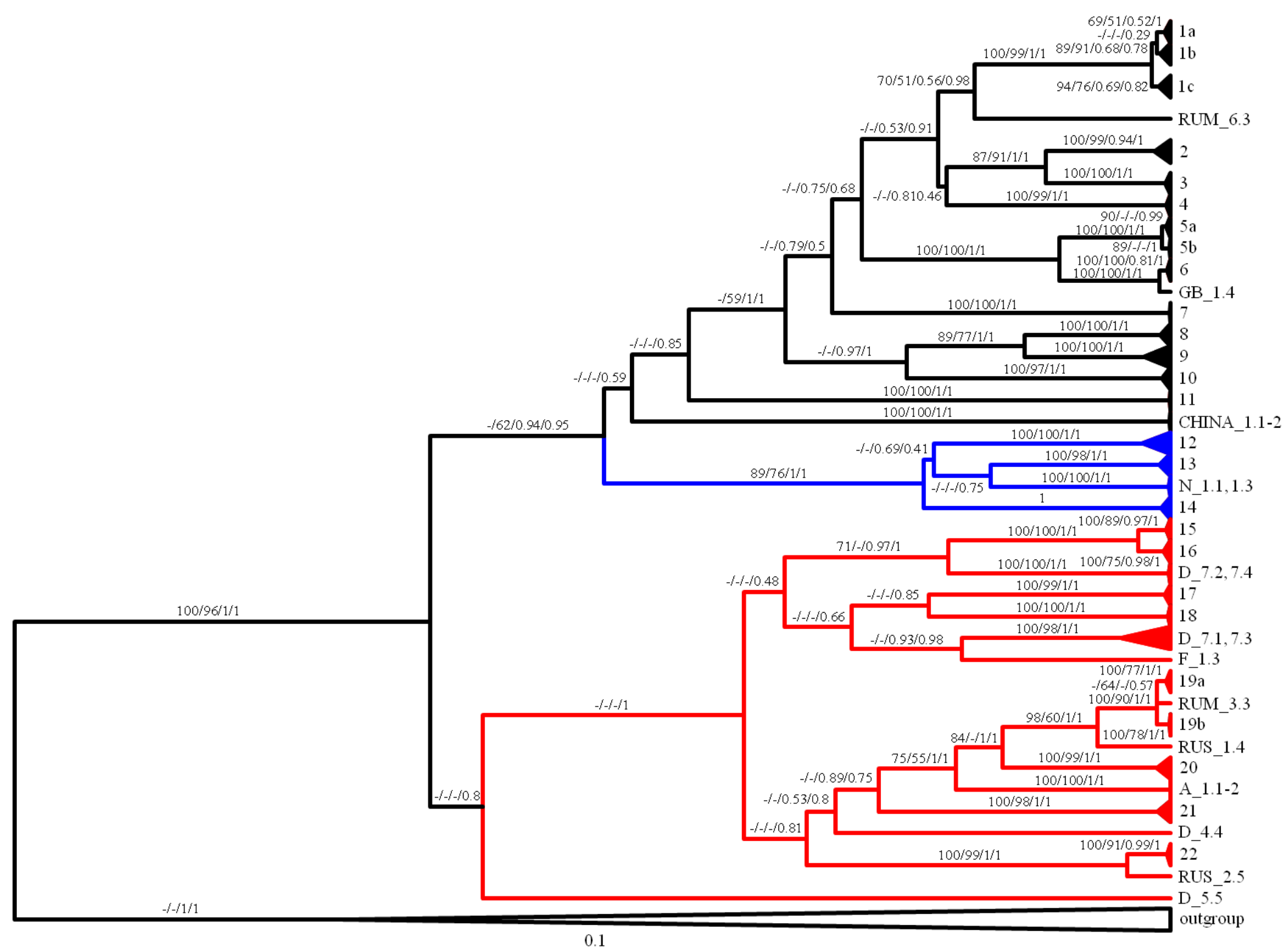

Figure 10: Bayesian phylogeny after 10x $10^{6}$ generations from the $180 \mathrm{COI}$ nucleotide sequences of Steganacarus magnus with Beast v1.5.4. Outgroups were Hypochthonius rufulus and Rhysotritia duplicata. Numbers on the branches are bootstrap values from NJ without and with evolution model (GTR $+\mathrm{I}+\mathrm{G})$ analysis and posterior probabilities from MrBayes and Beast. Branch colours represent the different clades (black, blue, red). Numbers at the end represent the different subclades as explained in Table 2. 


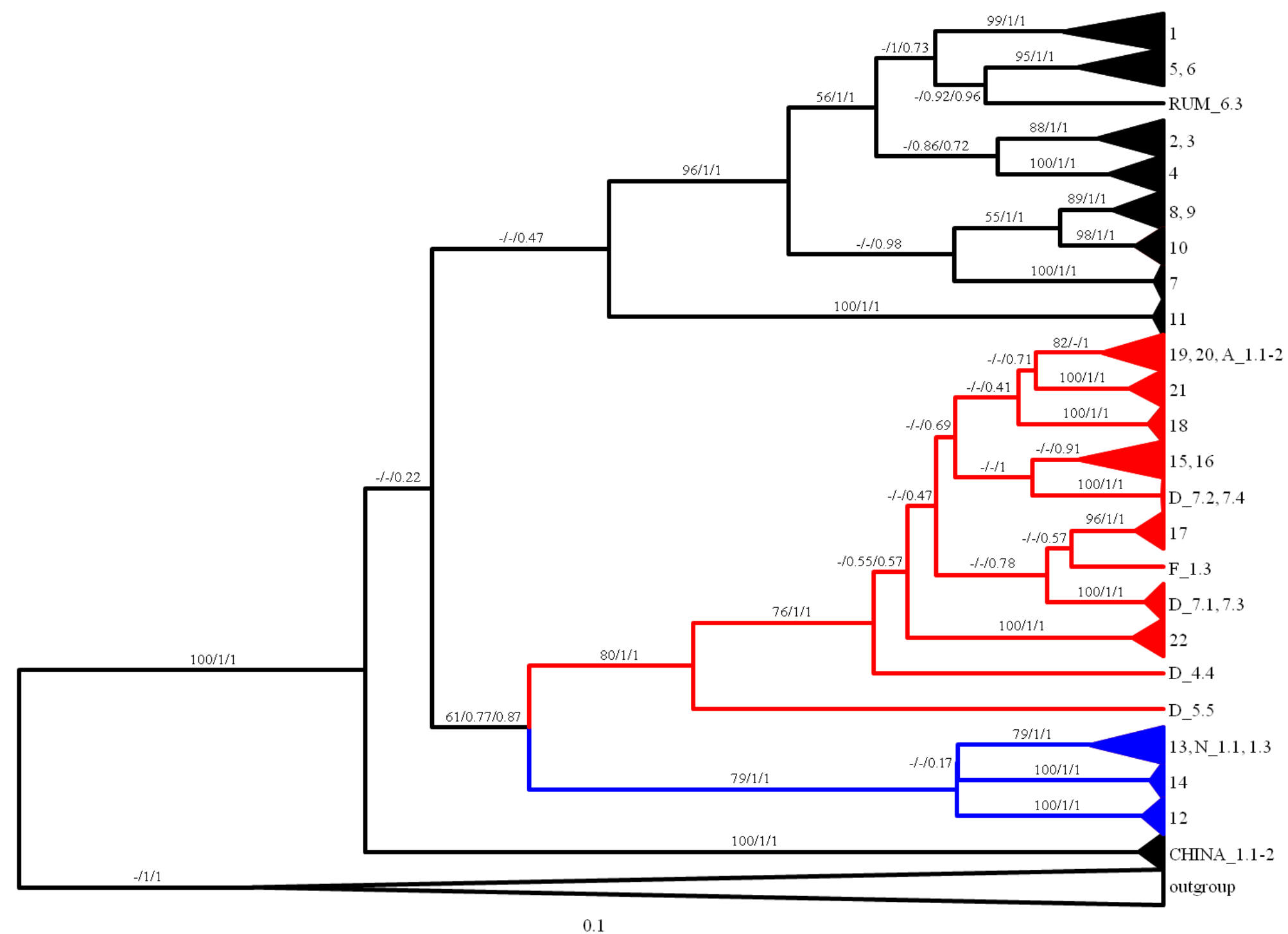

Figure 11: Bayesian phylogeny after 10x1 $10^{6}$ generations with Beast v1.5.4 based on 180 COI protein sequences of Steganacarus magnus. Outgroups were Hypochthonius rufulus and Rhysotritia duplicata. Numbers on the branches are bootstrap values from $\mathrm{NJ}$ analysis and posterior probabilities from MrBayes and Beast. Branch colours represent the different clades (black, blue, red). Numbers at the end represent the different subclades as explained in Table 2. 
Table 2: Subclades of Bayesian phylogenetic trees of Steganacarus magnus from the phylogenetic trees based on COI nucleotide sequences (\#ind=number of individuals, $\mathrm{pp}=$ posterior probabilities, sampling site, individuals and ind. pop.=quantity of individuals from the population).

\section{Clade I}

\begin{tabular}{|c|c|c|c|c|c|c|}
\hline Subclade & $\#$ ind & pp & \multicolumn{2}{|c|}{ sampling site } & individuals & ind. pop. \\
\hline 1 & 53 & 1 & 9 & & & \\
\hline \multirow[t]{7}{*}{ 1a } & 21 & 1 & KW & D_1 & 1,12 & $2 / 14$ \\
\hline & & & Goettingen & D_2 & $1-2,4-9$ & $8 / 9$ \\
\hline & & & Bonn & D_9 & 1 & $1 / 4$ \\
\hline & & & Arhus 1 & DK_3 & 1 & $1 / 3$ \\
\hline & & & Loire & F_2 & $1-4$ & $4 / 4$ \\
\hline & & & Grosseto & I_1 & $1,4,9-10$ & $4 / 10$ \\
\hline & & & Parma & I_2 & 4 & $1 / 4$ \\
\hline \multirow[t]{4}{*}{$1 b$} & 12 & 0.82 & KW & D_1 & $2,5,14$ & $3 / 14$ \\
\hline & & & Bonn & D_9 & 2 & $1 / 4$ \\
\hline & & & Grosseto & I_1 & $2-3,5-8$ & $6 / 10$ \\
\hline & & & Parma & I_2 & $1-2$ & $2 / 4$ \\
\hline \multirow[t]{7}{*}{$1 c$} & 20 & 0.78 & KW & D_1 & $3-46-11$ & $8 / 14$ \\
\hline & & & Goettingen & D_2 & 3 & $1 / 9$ \\
\hline & & & Bonn & D_9 & $3-4$ & $2 / 4$ \\
\hline & & & Parma & I_2 & 3 & $1 / 4$ \\
\hline & & & Copenhagen & DK_1 & 1 & $3 / 3$ \\
\hline & & & Arhus 1 & DK_3 & $2-3$ & $2 / 3$ \\
\hline & & & Arhus 2 & DK_4 & $1-3$ & $3 / 3$ \\
\hline isol. ind. & 1 & & Sinaia & RUM_6 & 3 & $1 / 3$ \\
\hline \multirow[t]{2}{*}{2} & 5 & 1 & Anaga Mountains 1 & E_2 & 1 & $1 / 1$ \\
\hline & & & Anaga Mountains 2 & E_3 & 4 & $4 / 4$ \\
\hline 3 & 2 & 1 & Teno Mountains & E_1 & 2 & $2 / 2$ \\
\hline 4 & 3 & 1 & Lake Constance & D_3 & $1-3$ & $3 / 3$ \\
\hline 5 & 18 & 1 & & & & \\
\hline \multirow[t]{6}{*}{$5 \mathbf{a}$} & 12 & 0.99 & St. Isidore & F_3 & 1 & $1 / 5$ \\
\hline & & & Bedford & GB_1 & $2-3$ & $2 / 5$ \\
\hline & & & Ascot & GB_2 & $1-2$ & $2 / 2$ \\
\hline & & & Braemar & GB_3 & $1-3$ & $3 / 3$ \\
\hline & & & Swords & IRL_1 & $1-2$ & $2 / 2$ \\
\hline & & & Wageningen & NL_1 & $1-2$ & $2 / 2$ \\
\hline \multirow[t]{3}{*}{$5 b$} & 6 & 1 & Cuxhaven & D_8 & $1-3$ & $3 / 3$ \\
\hline & & & Bedford & GB_1 & 1,5 & $2 / 5$ \\
\hline & & & Wageningen & NL_1 & 3 & $1 / 3$ \\
\hline 6 & 4 & 1 & St. Isidore & F_3 & $2-5$ & $4 / 5$ \\
\hline isol. ind. & 1 & & Bedford & GB_1 & 4 & $1 / 5$ \\
\hline 7 & 3 & 1 & Moerfelden & D_5 & $1-3$ & $3 / 5$ \\
\hline \multirow[t]{2}{*}{8} & 6 & 1 & Busteni & RUM_5 & $1-4$ & $4 / 4$ \\
\hline & & & Sinaia & RUM_6 & $1-2$ & $2 / 3$ \\
\hline 9 & 3 & 1 & Warsaw & PL_2 & $1-3$ & $3 / 3$ \\
\hline \multirow[t]{2}{*}{10} & 7 & 1 & Sibiu 1 & RUM_1 & $1-3$ & $3 / 3$ \\
\hline & & & Sibiu 2 & RUM_2 & $1-4$ & $4 / 4$ \\
\hline \multirow[t]{2}{*}{11} & 2 & 1 & Joensuu & FIN_2 & 1 & $1 / 1$ \\
\hline & & & Stroemstad & S_2 & 2 & $1 / 3$ \\
\hline isol. ind & 2 & 1 & Nanjing & CHINA_1 & $1-2$ & $2 / 2$ \\
\hline
\end{tabular}


Table 2 continued

Clade II

\begin{tabular}{ccccccc}
\hline Subclade & \# ind & pp & \multicolumn{2}{c}{ sampling site } & individuals & ind. pop. \\
\hline $\mathbf{1 2}$ & $\mathbf{8}$ & $\mathbf{1}$ & Meckl. Seenpl. & D_4 & $1-2,5-7$ & $5 / 9$ \\
& & & Copenhagen & DK_1 & $1-2$ & $2 / 3$ \\
& & & Piliscaba & HUN_1 & 1 & $1 / 1$ \\
$\mathbf{1 3}$ & $\mathbf{4}$ & $\mathbf{1}$ & Decin & CZ_1 & $1-2,5$ & $3 / 5$ \\
& & & Mont Blanc & F_1 & 2 & $1 / 6$ \\
isol. ind. & $\mathbf{2}$ & $\mathbf{1}$ & Narvik & N_1 & 1,3 & $2 / 3$ \\
$\mathbf{1 4}$ & $\mathbf{5}$ & $\mathbf{1}$ & Decin & CZ_1 & 4 & $1 / 5$ \\
& & & Black Forest & D_6 & 1 & $1 / 1$ \\
& & & Krakow & PL_1 & 1,3 & $2 / 6$ \\
& & & Stroemstad & S_2 & 1 & $1 / 3$ \\
isol. ind. & $\mathbf{1}$ & & Moerfelden & D_5 & 1 & $1 / 5$ \\
\hline
\end{tabular}

Clade

III

\begin{tabular}{|c|c|c|c|c|c|c|}
\hline \multirow{2}{*}{$\frac{\text { Subclade }}{15}$} & \multirow{2}{*}{$\frac{\# \text { ind }}{12}$} & \multirow{2}{*}{$\frac{p p}{1}$} & \multicolumn{2}{|c|}{ sampling site } & \multirow{2}{*}{$\begin{array}{c}\text { individuals } \\
1\end{array}$} & \multirow{2}{*}{$\begin{array}{c}\text { ind. pop. } \\
1 / 1\end{array}$} \\
\hline & & & Logarno & CH_1 & & \\
\hline & & & Meckl. Seenpl. & D_4 & $3,8-9$ & $3 / 9$ \\
\hline & & & Hjorring & DK_2 & $1-5$ & $5 / 5$ \\
\hline & & & Krakow & $\mathrm{PL}_{-} 1$ & $4-5$ & $2 / 6$ \\
\hline & & & Stroemstad & S_2 & 3 & $1 / 3$ \\
\hline \multirow[t]{3}{*}{16} & 5 & 1 & Moerfelden & D_5 & 4 & $1 / 5$ \\
\hline & & & Haute Loire & F_4 & $1-2$ & $2 / 2$ \\
\hline & & & Krakow & PL_1 & 2,6 & $2 / 6$ \\
\hline isol. ind. & 2 & 1 & Uelzen & D_7 & 2,4 & $2 / 4$ \\
\hline \multirow{2}{*}{17} & 4 & 1 & Lahti & FIN_1 & $1-3$ & $3 / 3$ \\
\hline & & & Umea & S_1 & 1 & $1 / 1$ \\
\hline 18 & 4 & 1 & Mont Blanc & F_1 & $1,4-6$ & $4 / 6$ \\
\hline isol. ind. & 2 & 1 & Uelzen & D_7 & 1,3 & $2 / 4$ \\
\hline isol. ind. & 1 & & Mont Blanc & F_1 & 3 & $1 / 6$ \\
\hline 19 & 7 & 1 & 2 & & & \\
\hline $19 a$ & 3 & 1 & Cluj Napoca & RUM_4 & $1-3$ & $3 / 3$ \\
\hline isol. ind. & 1 & & Bagau & RUM_3 & 3 & $1 / 4$ \\
\hline $19 b$ & 3 & 1 & Bagau & RUM_3 & $1-2,4$ & $3 / 4$ \\
\hline isol. ind. & 1 & & Altai Mountains & RUS_1 & 4 & $1 / 4$ \\
\hline \multirow[t]{2}{*}{20} & 2 & 1 & Narvik & N_1 & 2 & $1 / 3$ \\
\hline & & & Decin & CZ_1 & 3 & $1 / 5$ \\
\hline isol. ind. & 2 & 1 & Villach & A_1 & $1-2$ & $2 / 2$ \\
\hline 21 & 3 & 1 & Altai Mountains & RUS_1 & $1-3$ & $3 / 4$ \\
\hline isol. ind. & 1 & & Meckl. Seenpl. & D_ $\overline{4}$ & 4 & $1 / 9$ \\
\hline 22 & 4 & 1 & Novosibirsk & RUS_2 & $1-4$ & $4 / 4$ \\
\hline isol. ind. & 1 & & Novosibirsk & RUS_2 & 5 & $1 / 5$ \\
\hline isol. ind. & 1 & & Moerfelden & D_5 & 5 & $1 / 5$ \\
\hline
\end{tabular}


Chapter 2 Genetic diversity in soil

Table 3: Mean percentage pairwise differences of uncorrected p-distances for the COI nucleotide of Steganacarus magnus from 41 locations. The diagonal represents the within population differences (bold), among population differences are below the diagonal. Bold numbers in red are the minimum and maximum differences within and among populations. Bold numbers in pink are the maximum divergences excluding individuals from China and Russia. Locations with less than two individuals were excluded from the analysis.

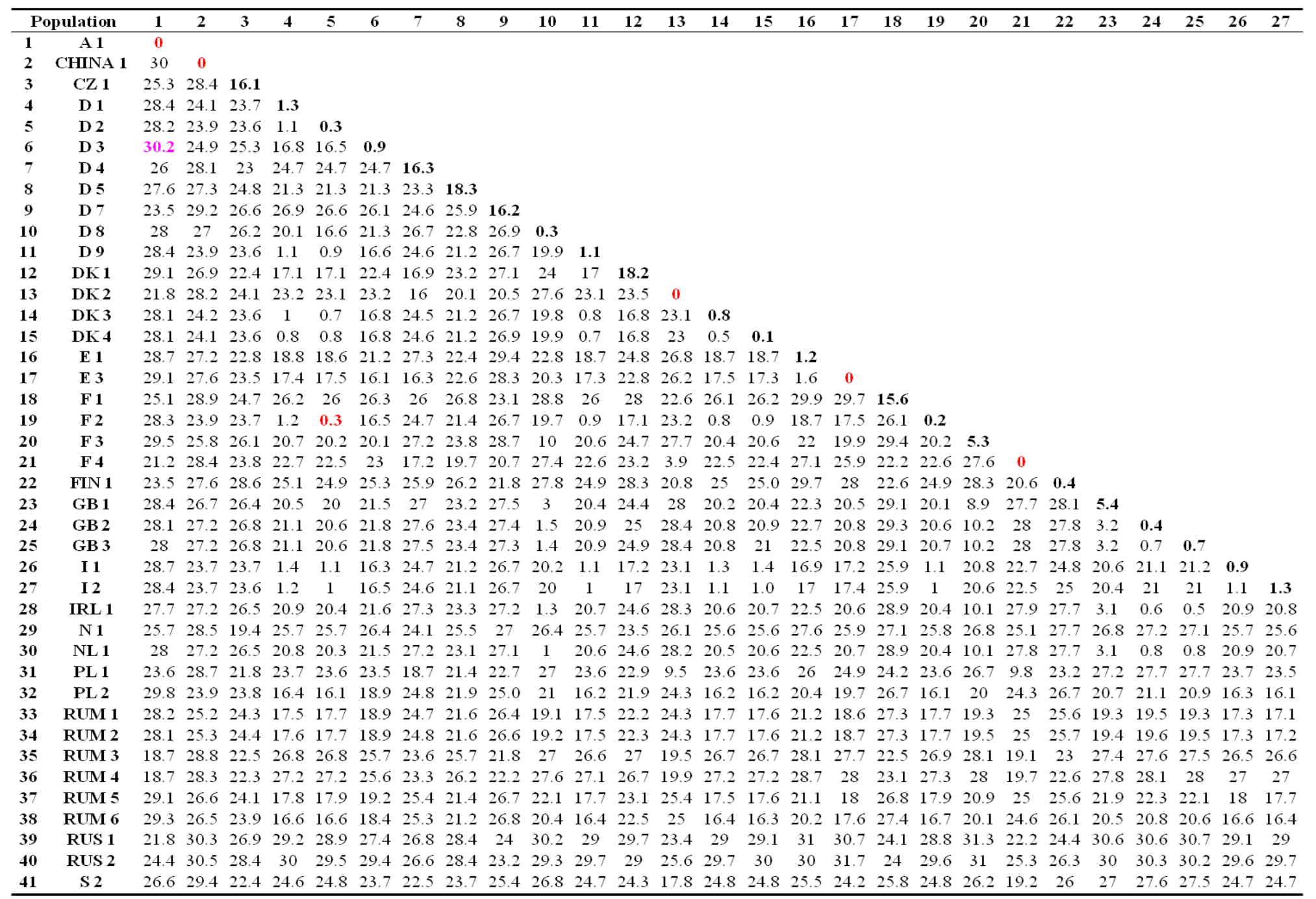


Table 3 continued

\begin{tabular}{cccccccccccccccc}
\hline \multicolumn{2}{c}{ Population } & $\mathbf{2 8}$ & $\mathbf{2 9}$ & $\mathbf{3 0}$ & $\mathbf{3 1}$ & $\mathbf{3 2}$ & $\mathbf{3 3}$ & $\mathbf{3 4}$ & $\mathbf{3 5}$ & $\mathbf{3 6}$ & $\mathbf{3 7}$ & $\mathbf{3 8}$ & $\mathbf{3 9}$ & $\mathbf{4 0}$ & $\mathbf{4 1}$ \\
\hline $\mathbf{2 8}$ & IRL 1 & $\mathbf{0 . 4}$ & & & & & & & & & & & & & \\
$\mathbf{2 9}$ & N 1 & 26.8 & $\mathbf{1 8}$ & & & & & & & & & & & & \\
$\mathbf{3 0}$ & NL 1 & 0.7 & 26.8 & $\mathbf{1}$ & & & & & & & & & & & \\
$\mathbf{3 1}$ & PL 1 & 27.6 & 24.1 & 27.5 & $\mathbf{1 4 . 4}$ & & & & & & & & & & \\
$\mathbf{3 2}$ & PL 2 & 20.9 & 25.1 & 21 & 23.9 & $\mathbf{2}$ & & & & & & & & & \\
$\mathbf{3 3}$ & RUM 1 & 19.4 & 26.7 & 19.3 & 24 & $\mathbf{1 6}$ & $\mathbf{0 . 3}$ & & & & & & & & \\
$\mathbf{3 4}$ & RUM 2 & 19.5 & 26.8 & 19.4 & 24 & $\mathbf{1 6}$ & 0.6 & $\mathbf{1 . 1}$ & & & & & & & \\
$\mathbf{3 5}$ & RUM 3 & 27.4 & 23.7 & 27.2 & 21.3 & 27.2 & 27.9 & 27.8 & $\mathbf{1 . 8}$ & & & & & & \\
$\mathbf{3 6}$ & RUM 4 & 27.9 & 23.3 & 27.8 & 21.7 & 27.8 & 27.7 & 27.7 & 3 & $\mathbf{0 . 9}$ & & & & & \\
$\mathbf{3 7}$ & RUM 5 & 22 & 25.1 & 22 & 25.1 & 12.8 & 16.1 & 16.3 & 29.4 & 29.3 & $\mathbf{0 . 6}$ & & & & \\
$\mathbf{3 8}$ & RUM 6 & 20.5 & 25.1 & 20.4 & 24.6 & 14.6 & 16.4 & 16.6 & 28.7 & 28.6 & 6.1 & $\mathbf{1 1 . 6}$ & & & \\
$\mathbf{3 9}$ & RUS 1 & 30.5 & 27.3 & 30.4 & 24.9 & 30.7 & 30.4 & 30.6 & 19.5 & 20 & $\mathbf{3 1 . 8}$ & 31.2 & $\mathbf{1 2 . 7}$ & & \\
$\mathbf{4 0}$ & RUS 2 & 29.9 & 28.2 & 29.9 & 26.6 & 29.3 & 27.8 & 28.1 & 23 & 22.7 & 30.2 & 30.4 & 23.5 & $\mathbf{2 . 8}$ & \\
$\mathbf{4 1}$ & S 2 & 26.5 & 24.8 & 27.3 & 17.9 & 24.5 & 22.7 & 22.8 & 24.5 & 24.5 & 24.5 & 24.3 & 27.8 & 27.1 & $\mathbf{2 5 . 8}$ \\
\hline
\end{tabular}

The results of the AMOVA showed that the nucleotide variation among countries (16.2\%), the variation among samples within countries $(57.5 \%)$ and the variation within samples $(26.4 \%)$ were significant and high (Table 2). Also, the protein variation among samples within countries $(67.7 \%)$ and the variation within samples $(28 \%)$ were significant and high. In contrast, variation among countries $(4.2 \%)$ was not significant (Table 4$)$. The neutrality test (Tajima's D) was only significant for two populations in the nucleotide (F_3 Tajima's $\mathrm{D}=-1.26, \mathrm{p}=0.0033$ and RUS_2 Tajima's $\mathrm{D}=-1.25$, $\mathrm{p}=0.0081$ ) (Table A3) and for three populations in the protein $\left(\mathrm{CZ} \_1 \mathrm{D}=-1.4, \mathrm{p}<0.01, \mathrm{D} \_1 \mathrm{D}=-1.54\right.$, $\mathrm{p}=0.04$ and I_2 $\mathrm{D}=-2.86, \mathrm{p}<0.01$ ) (Table A3). Fu's $\mathrm{F}_{\mathrm{s}}$ were not significant for the nucleotide dataset but significant for one population (I_2 FS $=-0.32, p=0.04$ ) in the protein dataset (Table A3 and A4).

Table 4: Results of the AMOVA on the variation in nucleotide and protein sequences among countries, among populations, within populations and in total. Each population was considered as a separate group. Populations with less than two individuals were excluded from the analysis. Significant level is p<0.05 (d.f.: degree of freedom). Black letters are for the nucleotide and red letters are for the protein.

\begin{tabular}{lccccc}
\hline \multicolumn{1}{c}{ Source of variance } & d.f. & sum of squares & variance components & percent of total variation & fixation indices \\
\hline among countries & $16 / 16$ & $3668.05 / 87.71$ & $9.16 \mathrm{Va}^{*} / 0.07 \mathrm{Va}$ & $16.15 / 4.24$ & $\mathrm{FCT}: 0.16^{*} / 0.04$ \\
among populations & $24 / 24$ & $3797.55 / 126.74$ & $32.6 \mathrm{Vb}^{*} / 1.1 \mathrm{Vb}^{*}$ & $57.5 / 67.74$ & $\mathrm{FSC}: 0.69^{*} / 0.71^{*}$ \\
within population & $133 / 133$ & $1986.6 / 60.42$ & $14.94 \mathrm{Vc}^{*} / 0.45 \mathrm{Vc} *$ & $26.35 / 28.02$ & FST:0.74*/0.72* \\
total & $173 / 173$ & $9452.2 / 273.87$ & $56.7 / 1.62$ & & \\
\hline
\end{tabular}

The McDonald-Kreitman test showed that the differences between 233 of 820 population comparisons (28.4\%) were significant (Table 5; for more details see Table A5). All computed neutrality indices were 0 (Table A6) and indicated purifying selection. 
Chapter 2 Genetic diversity in soil

Table 5: Results of the McDonald-Kreitman test of Steganacarus magnus from 41 locations (38 European, 3 East Asian). *. $0.01<\mathrm{P}<0.05$ (90 populations), **: $0.001<\mathrm{P}<0.01$ (80 populations), ***: $\mathrm{P}<0.001$ (63 populations)

\begin{tabular}{|c|c|c|c|c|c|c|c|c|c|c|c|c|c|c|c|c|c|c|c|c|c|c|c|c|c|c|c|c|}
\hline \multicolumn{2}{|c|}{ Population } & \multirow[t]{2}{*}{1} & \multirow[t]{2}{*}{2} & \multirow[t]{2}{*}{3} & \multirow[t]{2}{*}{4} & \multirow[t]{2}{*}{5} & \multirow[t]{2}{*}{6} & \multirow[t]{2}{*}{7} & \multirow[t]{2}{*}{8} & \multirow[t]{2}{*}{9} & \multirow[t]{2}{*}{10} & \multirow[t]{2}{*}{11} & \multirow[t]{2}{*}{12} & \multirow[t]{2}{*}{13} & 14 & 15 & 16 & 17 & 18 & 19 & 20 & 21 & 22 & 23 & 24 & 25 & 26 & 27 \\
\hline $\mathbf{1}$ & A 1 & & & & & & & & & & & & & & & & & & & & & & & & & & & \\
\hline 2 & CHINA 1 & - & & & & & & & & & & & & & & & & & & & & & & & & & & \\
\hline 3 & CZ 1 & * & * & & & & & & & & & & & & & & & & & & & & & & & & & \\
\hline 4 & D 1 & - & - & * & & & & & & & & & & & & & & & & & & & & & & & & \\
\hline 5 & D 2 & - & - & * & - & & & & & & & & & & & & & & & & & & & & & & & \\
\hline 6 & D 3 & - & - & * & * & - & & & & & & & & & & & & & & & & & & & & & & \\
\hline 7 & D 4 & - & $* *$ & - & $* *$ & ** & * & & & & & & & & & & & & & & & & & & & & & \\
\hline 8 & D 5 & $*$ & - & - & - & - & - & - & & & & & & & & & & & & & & & & & & & & \\
\hline 9 & D 7 & - & $* * *$ & - & $* *$ & **** & $* * *$ & - & - & & & & & & & & & & & & & & & & & & & \\
\hline 10 & D 8 & - & - & $* *$ & $* *$ & - & - & $* *$ & - & $* * *$ & & & & & & & & & & & & & & & & & & \\
\hline 11 & D 9 & - & - & $* *$ & - & - & - & $* *$ & - & **** & - & & & & & & & & & & & & & & & & & \\
\hline 12 & DK 1 & - & - & - & - & - & *** & - & - & - & $* *$ & - & & & & & & & & & & & & & & & & \\
\hline 13 & DK 2 & - & - & * & - & - & - & - & - & - & - & - & - & & & & & & & & & & & & & & & \\
\hline 14 & DK 3 & - & - & * & - & - & - & $* *$ & - & **** & - & - & - & - & & & & & & & & & & & & & & \\
\hline 15 & DK 4 & - & - & * & - & - & - & $* *$ & - & *** & - & - & - & - & - & & & & & & & & & & & & & \\
\hline 16 & E 1 & - & - & * & * & - & - & - & - & **** & - & - & **** & - & - & - & & & & & & & & & & & & \\
\hline 17 & E 3 & - & - & $* *$ & * & - & - & * & - & $* * *$ & - & - & $* *$ & - & - & - & - & & & & & & & & & & & \\
\hline 18 & F 1 & - & * & - & * & * & $* *$ & - & - & - & $* *$ & $* *$ & - & * & * & * & $* *$ & $* *$ & & & & & & & & & & \\
\hline 19 & F 2 & - & - & * & - & - & - & $* *$ & - & **** & - & - & - & - & - & - & - & - & * & & & & & & & & & \\
\hline 20 & F 3 & $* *$ & $*$ & $* *$ & - & - & - & $* * *$ & - & **** & - & - & $*$ & $*$ & - & - & - & - & $* *$ & - & & & & & & & & \\
\hline 21 & F 4 & - & - & * & - & - & - & - & - & - & - & - & - & - & - & - & - & - & * & - & $* *$ & & & & & & & \\
\hline 22 & FIN 1 & $* *$ & $*$ & - & - & - & - & - & $*$ & - & $*$ & - & - & $* *$ & - & $* *$ & - & - & $*$ & - & - & $* *$ & & & & & & \\
\hline 23 & GB 1 & **** & $* * *$ & $* * *$ & - & - & - & $* * *$ & - & **** & - & - & - & $* * *$ & - & - & - & - & $* * *$ & - & - & $* * *$ & $* *$ & & & & & \\
\hline 24 & GB 2 & - & - & $* *$ & $* *$ & - & - & $* *$ & - & $* * *$ & - & - & $* *$ & - & - & - & - & - & $* *$ & - & - & - & $*$ & - & & & & \\
\hline 25 & GB 3 & * & $*$ & $*$ & $* * *$ & - & - & $* *$ & - & $* * *$ & $*$ & - & $* *$ & $*$ & - & $* * *$ & - & $*$ & $*$ & $* *$ & - & $*$ & $* *$ & - & - & & & \\
\hline 26 & I 1 & - & - & * & - & - & - & $* *$ & - & $* * *$ & - & - & - & - & - & - & - & - & $*$ & - & - & - & - & - & - & $* *$ & & \\
\hline 27 & I 2 & - & - & $*$ & - & - & - & $* *$ & - & **** & - & - & - & - & - & - & - & - & $*$ & - & - & - & - & - & - & - & - & \\
\hline 28 & IRL 1 & - & - & $* *$ & $* *$ & - & - & $* *$ & - & **** & - & - & *** & - & - & - & - & - & *** & - & - & - & - & - & - & - & - & - \\
\hline 29 & N 1 & $* *$ & - & - & - & - & - & - & $*$ & - & $*$ & - & - & $* *$ & - & - & - & $*$ & - & - & $* *$ & $* *$ & $*$ & $* * *$ & $*$ & - & - & - \\
\hline 30 & NL 1 & - & - & * & $* *$ & - & - & $* *$ & - & **** & - & - & $*$ & - & - & - & - & - & ** & - & - & - & - & - & - & - & - & - \\
\hline 31 & PL 1 & $*$ & - & - & - & - & - & - & - & $*$ & - & - & - & - & - & - & - & $*$ & - & - & * & - & $*$ & $* *$ & - & - & - & - \\
\hline 32 & PL 2 & - & - & ** & - & - & - & * & - & **** & - & - & - & - & - & - & - & - & *** & - & - & - & - & - & - & - & - & - \\
\hline 33 & RUM 1 & - & - & - & $*$ & - & - & - & $*$ & $* *$ & - & - & ** & - & - & - & - & - & * & - & - & - & - & - & - & * & - & - \\
\hline 34 & RUM 2 & - & - & - & - & - & - & - & - & $* *$ & - & - & $* *$ & - & - & - & - & - & * & - & - & - & - & - & - & - & - & - \\
\hline 35 & RUM 3 & - & - & - & - & - & * & - & - & - & - & - & - & - & - & - & - & - & - & - & $* * *$ & - & - & $* * *$ & - & - & - & - \\
\hline 36 & RUM 4 & - & - & - & - & $*$ & $* *$ & - & $*$ & - & - & $*$ & - & - & * & - & $*$ & - & * & - & $* * *$ & - & - & $* * *$ & - & - & - & - \\
\hline 37 & RUM 5 & - & - & * & - & - & - & * & - & **** & - & - & - & - & - & - & - & - & ** & - & - & - & - & - & - & * & - & - \\
\hline 38 & RUM 6 & - & - & $* *$ & - & - & - & $* *$ & - & $* * *$ & - & - & - & - & - & - & * & - & ** & - & - & - & - & - & - & - & - & - \\
\hline 39 & RUS 1 & - & **** & - & $* *$ & $* * *$ & **** & - & - & - & **** & **** & - & - & $* * *$ & **** & **** & **** & $*$ & $* * *$ & $* * *$ & - & - & **** & **** & **** & $* * *$ & **** \\
\hline 40 & RUS 2 & - & $* *$ & - & - & $* *$ & $* * *$ & - & - & - & $*$ & $* *$ & - & - & $* *$ & $*$ & $* *$ & $*$ & - & $* *$ & $*$ & - & - & $* * *$ & $*$ & - & $* * *$ & $* *$ \\
\hline 41 & S 2 & $*$ & - & - & - & - & - & - & - & - & - & - & - & - & - & - & - & - & - & - & - & - & $*$ & - & - & - & - & - \\
\hline
\end{tabular}


Table 5 continued

\begin{tabular}{ccccccccccccccc}
\hline Population & 28 & 29 & 30 & 31 & 32 & 33 & 34 & 35 & 36 & 37 & 38 & 39 & 40 \\
\hline 29 & N 1 & $*$ & & & & & & & & & & & & \\
30 & NL 1 & - & $*$ & & & & & & & & & & & \\
31 & PL 1 & - & $* *$ & - & & & & & & & & & & \\
32 & PL 2 & - & - & - & - & & & & & & & & & \\
33 & RUM 1 & - & - & - & - & - & & & & & & & & \\
34 & RUM 2 & - & - & - & - & - & - & & & & & & & \\
$\mathbf{3 5}$ & RUM 3 & - & $* *$ & - & - & - & - & - & & & & & & \\
$\mathbf{3 6}$ & RUM 4 & - & $* *$ & - & - & - & - & - & - & & & & & \\
37 & RUM 5 & - & - & - & - & - & - & - & - & - & & & & \\
38 & RUM 6 & - & - & - & - & - & - & $*$ & - & - & - & & & \\
39 & RUS 1 & $* * *$ & $*$ & $* * *$ & - & $* * *$ & $* * *$ & $* * *$ & $*$ & - & $* * *$ & $* * *$ & & \\
$\mathbf{4 0}$ & RUS 2 & $* *$ & - & $*$ & - & $*$ & $* *$ & $* *$ & - & - & $*$ & - & - & \\
$\mathbf{4 1}$ & S 2 & - & - & - & - & - & - & - & - & - & - & - & - & - \\
\hline
\end{tabular}

\subsection{Discussion}

The structure of the population of $S$. magnus in Europe is genetically exceptionally complex. Remarkably, the high genetic variance in COI nucleotides of up to $31.8 \%$ did not only result from variations of the four times degenerated third codon position (Baker 2000), rather, variations in the first and second codon positions were also high. Fourfold degenerate sites do not affect the protein sequence and are assumed to have a slower saturation and less sensitivity to transversion-transition bias (Li 1993). These variations applied to both Southern and Northern populations and therefore were not confirming to the pattern of Southern richness and Northern paucity in aboveground animals and plants (Hewitt 1999, Hewitt and Ibrahim 2001). The extensive variation in both the COI nucleotide and the protein in populations from Central and Northern Europe suggests that $S$. magnus survived Pleistocene glaciations in cryptic refugia. The lack of star-like haplotypes (one big haplotype with several smaller nearly connected haplotypes) in the median-joining network (Fig. 8) resulted from deep splits of the several COI lineages which must have originated before the Pleistocene. Only in one COI lineage star-like haplotypes indicated postglacial re-colonisation of Central Europe (Avise 1994, 2004, Conroy and Cook 2000, Jolly et al. 2005, Mortimer and van Vuuren 2007); individuals from Italy, France, Germany and Denmark shared the same haplotype in subclade 1a. Survival in cryptic refugia is supported by the fact that individuals from locations which are geographically close together (D_1/D_5) or from the same location (CZ_1, D_4, D_5, DK_1, F_1, N_1, PL_1 and S_2) were genetically very different.

Coexistence of very different genetic lineages of S. magnus at the same location suggests that these lineages do not exclude each other. Results of the two different analyses for detecting mitochondrial clusters of S. magnus (Fig. 8, 9 and Fig. 10, 11) were identical. The substitution rate of the mitochondrial COI gene of Steganacarus has been estimated as $2.15 \%$ per million years (Salomone et al. 2002). This rate suggests that major radiations of lineages of $S$. magnus occurred in the Miocene or earlier (>20 my ago). However, nucleotide sequences of mtDNA will be saturated after 10-20 my (Avise 2004, Schaefer 2009) and with such highly saturated sequences, it becomes difficult to estimate divergence times even if the sequences evolved clock-like (Heethoff et al. 2007). Time estimates therefore have to be interpreted with some caution, but that such large genetic distances (up to 31.8\%) evolved over at least twenty million years seems to be certain. Representatives of the three lineages (black, blue and red) survived in multiple Northern refugia, including Sweden, Finland, Norway, Great Britain, the Netherlands, Germany, Poland, Czech Republic, Austria and at Nunataks (Schmitt 2009) such as the Mont Blanc region (Fig. 12). Schmitt (2009) postulated a genetic linkage between the North-eastern Alps and the North-western Carpathians. I only found indications for such a linkage in 
the protein in specimens from the Eastern Alps and the Carpathians, however, and in the nucleotide these specimens were separated by a large number of substitutions.

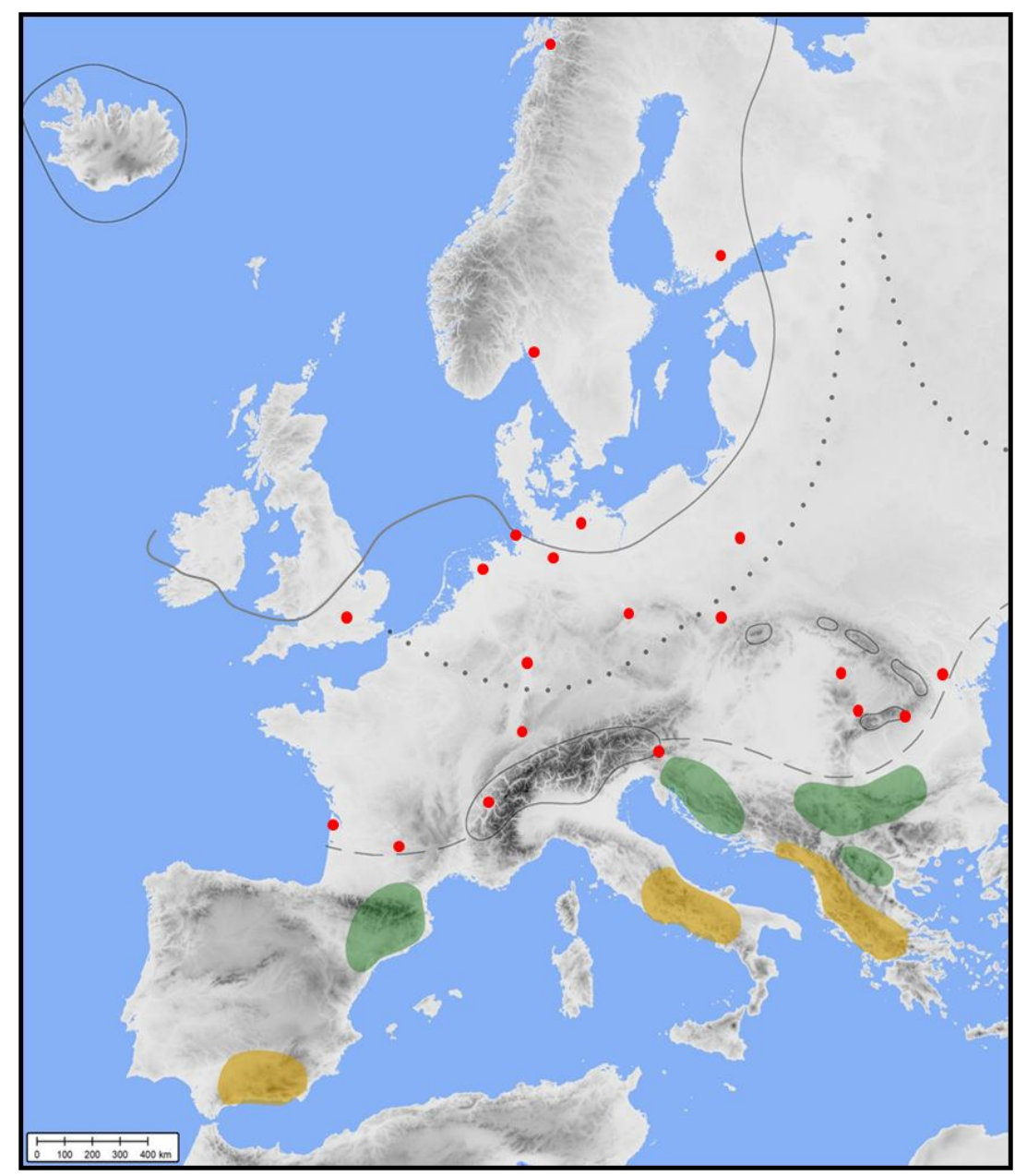

Figure 12: Map of Europe at the last glacial maximum. Red dots represent cryptic refugia as indicated by results of this study. Green areas represent refugia of coniferous trees, yellow areas refugia of deciduous trees; grey lines depict the maximum expansion of glaciers, the dotted line the border of arctic vegetation and the dashed line the border of tundra vegetation and permafrost (modified from Hewitt 1999, Hewitt and Ibrahim 2001, Alexander Kartographie 2006).

The phylogenetic analyses of the nucleotide and the protein were largely consistent, however, the blue clade changed its sister clade in the protein. In the nucleotide the black clade formed the sister clade of the blue, whereas in the protein it was the red clade. This shift in the clade arrangement likely resulted from the four times degenerated third codon position in the mitochondrial code (Xia et al. 1996, Baker 2000). Substitutions on these positions were synonymous and had no effect on the protein structure and therefore varied strongly. This variation in synonymous substitutions renders phylogenetic analyses difficult resulting in wrong phylogenetic relationships. The protein phylogeny is much more conservative and therefore more trustworthy e.g., back mutations are unlikely to be included.

This is the first study investigating nucleotide and protein divergences in COI. The exceptionally high molecular divergence in COI nucleotide and protein indicates that $S$. magnus comprises a cryptic species, as in barcoding literature sequence divergences in the COI fragment of $>3 \%$ is sufficient to describe new species )Hebert et al 2003a-b, 2004b Hogg and Hebert 2004, Johnson et al. 2008). The translated nucleotide sequence of the investigated COI fragment is fully functional and amplification of COI pseudogenes can therefore be excluded (Bensasson et al. 2001, Williams and Knowlton 2001, 
Song et al. 2008, Buhay 2009). Intraspecific COI divergences typically is less than $2 \%$, whereas interspecific divergences generally exceeds $4 \%$ (Peek et al. 1997, Johnson et al. 2008) The COI gene is highly conserved at the protein level (Johnson et al. 2008) and the high protein divergence (up to $4.7 \%$ ) strongly indicates the existence of cryptic species in S. magnus. Gene duplications and heteroplasmy of the COI gene are excluded, since the complete mitochondrion of S. magnus was sequenced by Domes et al. 2008 and Domes-Wehner 2009 and neither gene duplications nor heteroplasmy were detected. In contrast to Hurst and Jiggins (2005) the high genetic variance is not an effect of inherited symbionts such as Wolbachia or Cardinium, since none of these symbionts has ever been found in S. magnus. However, Wolbachia and Cardinium can trigger parthenogenesis and were found in an unidentified oribatid mite (Cordaux et al. 2001) and in some populations of Oppiella nova (Weeks et al. 2003).

To study the structure of a cryptic species complex of $S$. magnus in more detail analyses including several nuclear genes from the same individual need to be investigated to detect recombination of several mitochondrial lineages (e.g. using the elongation factor $1 \alpha$ and heat shock protein). Individuals of S. magnus are morphologically coherent, characters indicative of a species complex are lacking, suggesting that there is gene flow, i.e. S. magnus forms a biological species. There are variations in body size and colour but these are unlikely due to be based on genetic differences but rather to variations in habitat characteristics and food availability (Weigmann 2006).

High mobility of species may contribute to genetic dissimilarity of specimens occurring locally (Schäffer et al. 2010). For the dispersal of soil microarthropods wind and water are likely to be important, but not common in oribatid mites (Seyd 1962, Starý and Block 1998). Oribatid mite species might attach to nest-dwelling vertebrates (Miko and Stanko 1991, Lebedeva et al. 2006); another species of the genus Steganacarus (Steganacarus striculus C.L. Koch, 1836) has been found in bird plumage; in fact, several oribatid mite species are distributed by birds (Lebedeva et al. 2006) and same taxa showed active dispersal by phoresy on arthropods (Norton 1980, Townsend et al. 2008). Wind dispersal (Hawes et al. 2007), e.g. of eggs deposited in litter materials or as aerial plankton (Washburn and Washburn 1984), is possible but not known for this species and not common in oribatid mites (Seyd 1962, Starý and Block 1998). S. magnus is unlikely to be dispersed easily. At a single site or at locations close to each other genetically very different individuals exist, separated from each other by high numbers of substitutions. In contrast to the high nucleotide divergence the protein divergence is low. Explanations for the lower protein variance are the four times degenerated third codon position (Xia et al. 1996, Baker 2000) and the fact that the COI is under purifying selection (Li et al. 1985). Purifying selection eliminates non-synonymous substitutions and tolerates synonymous substitutions (Xia et al. 1996). However, non-synonymous substitutions take place in the COI gene of S. magnus and non-synonymous substitutions could be present in a small number since $60 \%$ of the amino acids of the COI barcoding region do not interact with another protein (Ballard and Melvin 2010).

High frost tolerance of S. magnus (Block 1979, Webb and Block 1993) likely contributed to the high genetic diversity in S. magnus as this may have allowed several lineages to survive Pleistocene glaciations in Central and Northern Europe. Thereafter, representatives of other lineages arrived contributing to the local coexistence of very different genetic lineages. Dispersal by man colonizing Central and Northern Europe in the Holocene likely contributed to the high genetic variance in local populations (Dlugosh and Parker 2008).

As stated above, major splits in S. magnus likely occurred in the Tertiary, presumably before the Miocene with the diversity of the lineages increasing fastly. At the beginning of the Miocene ( 23 Ma) the climate and vegetation in Europe and Asia changed. The evergreen forests dominating in the early and mid Triassic changed to coniferous and deciduous forests and the climate became dryer and cooler 
(Mai 1989, 1991, 1996, Kvaček 2000). Today, early to mid Miocene evergreen forests only exist in the western Mediterranean such as the Canary Islands and East Asia (Kvaček 2000, Kvaček et al. 2000). Radiation in S. magnus in the Miocene may be related to these vegetation changes. In fact, $S$. magnus dominates in forests of deciduous and conifer trees and may have dispersed with the expansion of temperate and boreal forest in the Miocene. Then, during the Pleistocene cooling $S$. magnus survived in various glacial refugia from where it started to expand with the recent Holocene warming and expansions of temperate and boreal forest.

High genetic differences in COI nucleotide sequences were also found in other species of Steganacarus (up to 28.6\%; Salomone et al. 2002) and other arthropods, such as the oribatid mites Platynothrus peltifer (up to 25\%; Heethoff et al. 2007), Scutovertex sculptus (up to 11\%; Schäffer et al. 2010) the springtail Friesea grisea (17.7\%; Torricelli et al. 2010), the harvestman Aoraki denticulata (up to 19.2\%; Boyer et al. 2007) and the intertidal copepod Tigriopus californicus (up to 23\%; Edmands 2001). High genetic variation in soil and marine animals but not in those living above the ground may indicate that species in these habitats stay more constant but accumulate neutral mutations whereas species above the ground evolved more vigorously and radiated, presumably parallel to that of higher plants. In fact, the radiation of the most diverse group insects, such as Diptera, Chrysomelidae (Coleoptrata) and Aculeata (Hymenoptera), occurred in the Neozoic (Grimaldi and Engel 2005). In contrast, radiation in soil organisms, such as oribatid mites and Collembola occurred much earlier (Schaefer 2009, Schaefer et al. 2010, Schäffer et al. 2010, Torricelli et al. 2010). However, to prove whether this scenario applies in general other soil living animals, such as Collembola, nematodes and earthworms, need to be investigated. This will also prove if other soil animal taxa also survived the Pleistocene glaciation in refugia in Central and Northern Europe as is the case in oribatid mites.

\subsection{Conclusion}

The results of this study suggest that microarthropods in soil, such as the oribatid mite species $S$. magnus, survived the Pleistocene glaciations in cryptic refugia contrasting the pattern proposed for the majority of aboveground animals and plants. A multitude of lineages presumably survived in refugia including Scandinavia, Central and Eastern Europe, and also in Alpine regions of Austria. Remarkably, today individuals of very different COI lineages coexist at localities which are geographically close together or even at the same locality. The exceptionally high genetic variation in S. magnus suggests that the major lineages of this species split in the Tertiary and radiated with the expansion of the temperate and boreal forests in the Miocene. With cooling in the Pleistocene populations shrunk but survived in multiple refugia in Northern and Central Europe, and also in Southern locations. From these refugia they expanded with warming following the temperate and boreal forests colonizing Central and Northern Europe in the Holocene. With this expansion representatives of very different lineages met and colonized the same or geographically close locations. To support this scenario nuclear genes, such as elongation factor $1 \alpha$, need to be investigated to allow reconstruction of mixing processes due to sexual reproduction. 


\section{Chapter Three}

\section{Is there a cryptic species complex in the oribatid mite Steganacarus magnus (Nicolet, 1855) (Acari, Oribatida)?}

\section{Summary}

DNA barcoding is a popular tool for species determination and delineation in molecular ecology and the mitochondrial cytochrome c oxidase (COI) gene is most common marker used for barcoding and detection of cryptic species. However, several studies revealed problems when using mitochondrial DNA for barcoding approaches due to nuclear amplified pseudogenes, symbionts and gene duplications. The soil-living oribatid mite Steganacarus magnus is a member of a morphological difficult to determine species complex and displays extraordinary high genetic diversity among populations in Europe (Chapter II). In this study, 77 individuals of 14 populations throughout Europe were analyzed to clarify if $S$. magnus consists of cryptic species or if gene-flow is maintained between individuals. To preclude the above mentioned problems and to detect sexual recombination between individuals the nuclear elongation factor 1 alpha $($ ef $1 \alpha)$, a conservative single copy gene, and the COI were sequenced from 37 individuals of the oribatid mite $S$. magnus. Phylogenetic trees generated with nucleotide and protein sequences of both genes revealed that sexual reproduction occurred between individuals with distinct mitochondrial lineages. The maximum of the uncorrected mean pairwise differences of the COI sequences between the populations was $28.4 \%$ in the nucleotide and $3.1 \%$ in the protein. The maximum of the pairwise differences ef $1 \alpha$ sequences were $19.6 \%$ in the nucleotide and $3.7 \%$ in the protein. Despite this high variability the results suggest that $S$. magnus does not consist of cryptic species and that the high genetic diversity is not maintained by speciation processes, but by neutral evolution at the third codon position in the mitochondrial gene (COI). The results support the hypothesis that the soil-system is evolutionary old and has endured ecological and climatic changes for tens of millions years. However, if sexual reproduction of different lineages combines different ecotypes and therefore provides selective advantage over other lineages remains to be tested. 


\subsection{Introduction}

DNA barcoding is a well established tool for discovery of new species and biodiversity assessment without using morphology (Ball et al. 2005, Hebert et al. 2003a, Hajibabaei et al. 2007, Dasmahaptra et al. 2010). For invertebrates, the first $650 \mathrm{bp}$ of the 5 '-end of the mitochondrial gene cytochrome $\mathrm{c}$ oxidase (COI) has become the most widely used marker ("Folmer fragment", Folmer et al. 1994, Hebert et al. 2003a-b, 2004a-b, Hajibabaei et al. 2006, Rock et al. 2008, Derycke et al. 2010). For vertebrates another mitochondrial (mt) gene, part of cytochrome b, is used (Taberlet et al. 1994, Bilton et al. 1998, Conroy and Cook 2000, Ursenbacher et al. 2006). The method of DNA barcoding revolutionized taxonomy and species delineation of undescribed species and of species which are difficult to determine morphologically; further, it has been successful in detecting cryptic species, overlooked by traditional taxonomic methods (Burns et al. 2007). However, the method received much criticism based on theoretical (Hickerson et al. 2006), methodological (Will and Rubinoff 2004, Cameron et al. 2006, Song et al. 2008) and empirical grounds (Hurst and Jiggins 2005).

Oribatid mites (Acari, Oribatida) are among the most diverse and abundant soil living arthropods with about 10,000 described species (Schatz 2002) and up to 400,000 individuals per square meter of forest soil (Maraun and Scheu 2000). Fossils of oribatid mites were found in Devonian sediments (380 my ago; Shear at al. 1984, Norton et al. 1988a) and the origin of oribatid mites dates back to 440 my (Lindquist 1984) or even 570 my according to molecular dating (Schaefer et al. 2010).

The soil-living oribatid mite Steganacarus magnus is a member of a morphologically difficult to determine species complex (Steganacaridae, Weigmann 2006) and displays extraordinary high genetic diversity among populations in Europe (up to 31.8\%; Chapter II). The extraordinary high genetic diversity indicates a cryptic species complex in S. magnus. Reasons for this high genetic diversity are that many morphologically identical species live together in a small area (cryptic species complex) or neutral evolution, i.e. large population size of S. magnus and polymorphism established coincidentally. One way to overcome problems with using mtDNA as barcoding gene is to compare mitochondrial markers with nuclear markers (Roe and Sperling 2007, Moniz and Kaczmarska 2009, Cicconardi et al. 2010, Leo et al. 2010) to investigate recombination between different mitochondrial lineages and to detect cryptic species (co-cladogenesis of both genes). The same phylogenetic tree in both genes suggests that the species in fact comprises a cryptic species complex. In contrast, different phylogenetic trees in the mtDNA and the nuclear gene provide evidence for recombination and sex and therefore indicate that lineages studied originated from a single biological species.

Mitochondria are maternally inherited cell organelles with a circular genome of about 14-19 kp in bilaterian organisms (Wolstenholme 1992, Boore 1999). The mitochondrial genome evolves with a faster rate than the nuclear genome (Chantangsi et al. 2007) and 'reproduces' without sexual recombination. Migration, speciation and splitting events that occurred several million years ago can be detected even in morphologically coherent taxa.

To investigate if $S$. magnus comprises a cryptic species complex, mt (COI) and nuclear genes (ef $1 a)$ were sequenced from the same individuals. Sampling in regions with different mt lineages (Chapter II) was extended (Northern Germany, Poland, Czech Republic). In total, 77 individuals of eleven countries throughout Europe were analyzed. 


\subsection{Materials and methods}

\subsubsection{Taxon sampling}

Throughout Europe, 77 individuals of Steganacarus magnus (Oribatida, Mixonomata) were sampled from 14 locations in eleven countries (Fig. 13, Table 6). Specimens were extracted from leaf litter using life extraction by heat (Macfadyen 1961, Kempson et al. 1963). Hypochthonius rufulus (Oribatida, Enarthronota) was sampled as outgroup taxon. Animals were identified under a stereomicroscope, determined after Weigmann (2006) and stored in $75 \%$ ethanol at $-20^{\circ} \mathrm{C}$ until DNA extraction.

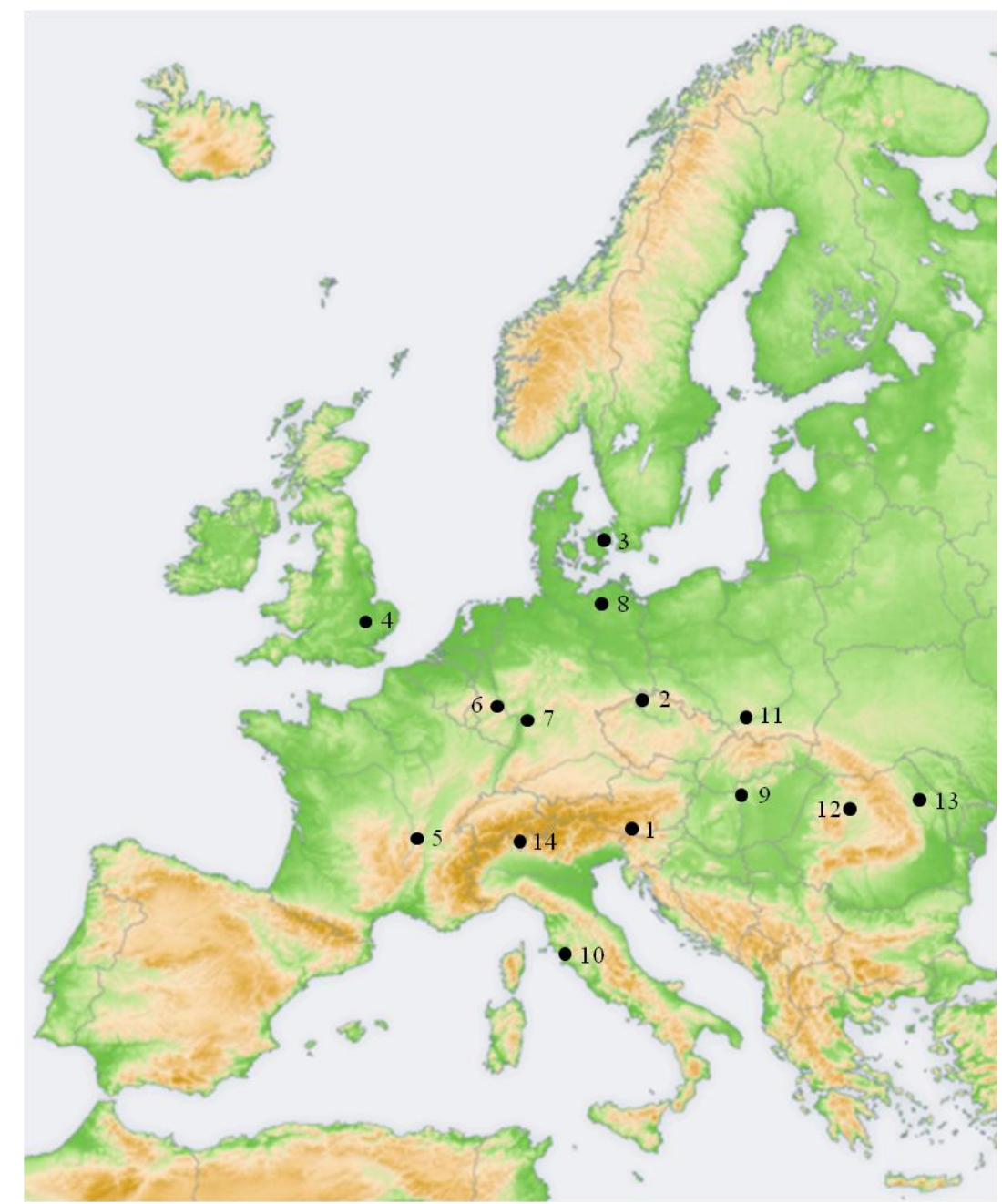

Figure 13: Map of Europe (http://de.wikipedia.org/wiki/Europa). Black dots with numbers are the sampling points of Steganacarus magnus. 
Table 6: Localities where Steganacarus magnus was sampled [ln: location number, country, location, coordinates, abbreviations of sampling locations (code), number of sequenced individuals per gene (COI, ef $1 \alpha)$ ] and name of collector (for details see Acknowledgements). Each location is marked by a colour symbol.

\begin{tabular}{|c|c|c|c|c|c|c|c|c|}
\hline $\ln$ & country & location & coordinates & code & $\mathrm{COI}$ & $e f 1 \alpha$ & colour & litter collector \\
\hline 1 & Austria & Villach & $46.57^{\circ} 13.85^{\circ}$ & A 1 & $1-2$ & 3 & $\ominus$ & K. Domes-Wehner \\
\hline 2 & Czech Republic & Decin & $50.78^{\circ} 14.23^{\circ}$ & $\mathrm{CZ} 1$ & $1-5$ & $1-2$ & $\oplus$ & M. Rosenberger \\
\hline 3 & Denmark & Copenhagen & $55.68^{\circ} 12.58^{\circ}$ & DK 1 & $1-3$ & 2 & $\Theta$ & N. Eisenhauer \\
\hline 4 & England & Bedford & $52.07^{\circ}-0.44^{\circ}$ & GB 1 & $1-5$ & $2-6$ & O & A. Milcu \\
\hline 5 & France & Loire & $45.56^{\circ} 4.79^{\circ}$ & F 1 & $1-4$ & 1,5 & $\bigcirc$ & M. Maraun \\
\hline 6 & Germany & Colonge & $50.83^{\circ} 7.18^{\circ}$ & D 3 & - & 1 & 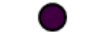 & M. Maraun \\
\hline 7 & & Kranichstein & $49.89^{\circ} 8.69^{\circ}$ & D 1 & $1-14$ & $3,13,15-18$ & 0 & M. Rosenberger \\
\hline 8 & & Mecklen. Seenplatte & $53.57^{\circ} 12.33^{\circ}$ & D 2 & $1-9$ & $3,6-9$ & O & I. Schaefer \\
\hline 9 & Hungary & Piliscaba & $47.62^{\circ} 18.83^{\circ}$ & HUN1 & 1 & 1 & $\oplus$ & C. Csjui \\
\hline 10 & Italy & Grosseto & $42.63^{\circ} 11.11^{\circ}$ & I 1 & $1-10$ & $4-7$ & ○ & M. Maraun \\
\hline 11 & Poland & Krakow & $50.04^{\circ} 19.84^{\circ}$ & PL 1 & $1-6$ & $1-2,5,7$ & ○ & S. Scheu \\
\hline 12 & Romania & Bagau & $46.55^{\circ} 26.72^{\circ}$ & RUM 1 & $1-4$ & $1-2$ & 0 & T. Pasca \\
\hline 13 & & Cluj & $46.77^{\circ} 23.52^{\circ}$ & RUM 2 & $1-3$ & 1,3 & $\oplus$ & T. Pasca \\
\hline 14 & Switzerland & Locarno & $46.17^{\circ} 8.77^{\circ}$ & $\mathrm{CH} 1$ & 1 & 1 & (1) & M. Scheu \\
\hline
\end{tabular}

\subsubsection{DNA extraction and sequencing}

Genomic DNA was extracted from single individuals using the DNeasy ${ }^{\circledR}$ Blood and Tissue Kit (Qiagen; Hilden, Germany) for animal tissue following the manufacturer's protocol.

In total, two PCR reactions of 76 individuals were performed using the primers 40.71F (5'-TCN TTY AAR TAY GCN TGG GT-3') and 52RC (5'-CCD ATY TTR TAN ACR TCY TG-3') for elongation factor-1 a (Klompen 2000) and COIarch1 (5'-GGT CAA CAA ATC ATA AAG AYA TYG G-3') and COIarch2 (5'-TAA ACT TCA GGG TGA CCA AAA AAT CA-3') (Heethoff et al. 2007) for COI, amplifying fragments of $598 \mathrm{bp}$ and $463 \mathrm{bp}$, respectively.

The PCR contained $0.5 \mu 1$ of each primer $(100 \mathrm{pmol} / \mu \mathrm{l}), 1 \mu \mathrm{l} \mathrm{\textrm {MgCl } _ { 2 } ( 2 5 \mathrm { mM } ) , 1 2 . 5 \mu \mathrm { l } \text { of HotStarTaq }}{ }^{\circledR}$ Master Mix (1.25 units HotStarTaq ${ }^{\circledR}$ polymerase, $100 \mu \mathrm{M}$ of each dNTP and $7.5 \mathrm{mM} \mathrm{MgCl}{ }_{2}$ buffer solution; Qiagen, Germany) or SuperHot Taq Mastermix [2.5 units SuperHot Taq polymerase, $10 \mu \mathrm{M}$ of each dNTP and buffer solution (20 mM Tris-HCI (pH 8.3), $100 \mathrm{mM} \mathrm{KCl}, 0.1 \mathrm{mM}$ EDTA, $1 \mathrm{mM}$ DTT, 50\% glycerol, $0.5 \%$ Nonidet P40 and 0.5\% Tween 20), Genaxxon; Ulm, Germany] containing polymerase, $5 \mu \mathrm{l}$ template DNA for ef $1 \alpha$ and $3 \mu \mathrm{l}$ for $\mathrm{COI}$; reaction volumes were filled up to $25 \mu \mathrm{l}$ with RNase free water. PCR parameters for $\mathrm{COI}$ included a $15 \mathrm{~min}$ step at $95^{\circ} \mathrm{C}$ for polymerase activation followed by 36 cycles of $30 \mathrm{~s}$ at $94^{\circ} \mathrm{C}$ for denaturation, $60 \mathrm{~s}$ at $51^{\circ} \mathrm{C}$ for primer annealing and $60 \mathrm{~s}$ at $72^{\circ} \mathrm{C}$ for elongation and a final $10 \mathrm{~min}$ step for elongation at $72^{\circ} \mathrm{C}$ parameters. PCR parameters for ef $1 \alpha$ included a $15 \mathrm{~min}$ step at $95^{\circ} \mathrm{C}$ for polymerase activation followed by 9 cycles of $50 \mathrm{~s}$ at $95^{\circ} \mathrm{C}$ for denaturation, $70 \mathrm{~s}$ at $46^{\circ} \mathrm{C}$ for primer annealing and $120 \mathrm{~s}$ at $72^{\circ} \mathrm{C}$ for elongation, followed by 34 cycles of $50 \mathrm{~s}$ at $95^{\circ} \mathrm{C}$ for denaturation, $70 \mathrm{~s}$ at $50^{\circ} \mathrm{C}$ for primer annealing and $120 \mathrm{~s}$ at $72^{\circ} \mathrm{C}$ for elongation and a final $10 \mathrm{~min}$ step for elongation at $72^{\circ} \mathrm{C}$. PCR programme are given in Table 7. PCR products were visualized on $1 \%$ agarose gel and purified using the QIAquick ${ }^{\circledR}$ PCR Purification Kit (Qiagen; Hilden, Germany) following the manufacturer's protocol. The purified PCR products were sequenced in both directions by Macrogen Inc. (Seoul, Korea). 
Table 7: PCR conditions of the elongation factor $1 \alpha$ (ef $1 \alpha)$ and of the cytochrome c oxidase subunit I (COI).

\begin{tabular}{lccccc}
\hline & \multicolumn{2}{c}{ ef $\mathbf{1 \alpha}$} & \multicolumn{2}{c}{ COI } \\
& temperature $\left({ }^{\circ} \mathbf{C}\right)$ & time & temperature $\left({ }^{\circ} \mathbf{C}\right)$ & time \\
\hline initial denaturation step & 95 & $15 \mathrm{~min}$ & 95 & $15 \mathrm{~min}$ \\
denaturation & 95 & $50 \mathrm{sec}$ & 94 & $30 \mathrm{sec}$ \\
annealing & 46 & $70 \mathrm{sec}$ & $1 \mathrm{~min}$ & $60 \mathrm{sec}$ \\
elongation & 72 & $2 \mathrm{~min}$ & 72 & $60 \mathrm{sec}$ \\
number of cycles & 9 & & & 36 & \\
denaturation & 95 & $50 \mathrm{sec}$ & - & - \\
annealing & 50 & $70 \mathrm{sec}$ & - & - \\
elongation & 72 & $2 \mathrm{~min}$ & - & - \\
number of cycles & & & & - & \\
final elongation & 74 & & $10 \mathrm{~min}$ & 72 & $10 \mathrm{~min}$ \\
\hline
\end{tabular}

\subsubsection{Phylogenetic, population genetic and statistical analyses}

Nucleotide sequences were edited and translated into amino acids using the invertebrate mitochondrial and the nuclear standard eukaryotic codes for $\mathrm{COI}$ and ef $1 \alpha$ implemented in SEQUENCHER v4.9 (Gene Codes) (starting at the third position). Ambiguous positions were corrected using electropherogramms and corrected sequences were aligned with ClustalX v1.81 (Thompson et al. 1997), using multiple alignment parameters: 10.0 for gap opening and 0.1 for gap extension for the nucleotide, default settings for the amino acid dataset. Phylogenetic trees were generated with Beast v1.5.4 (Drummond and Rambaut 2007), MrBayes v3.1.2 (Ronquist and Huelsenbeck 2003) and Neighbor-Joining analyses without and with model of sequence evolution in PAUP* (Swofford 1999) to identify monophyletic clusters in the dataset. The best model of sequence evolution was estimated with Modeltest3.6 (Posada and Crandall 1998) for PAUP* and MrModeltest (Nylander 2004) for the Bayesian analysis. The best fit model for the $\mathrm{COI}$ dataset was $\mathrm{GTR}+\mathrm{I}+\mathrm{G}$ for the analyses and $\mathrm{K} 80+\mathrm{G}$ for MrBayes and $\mathrm{TrNef}+\mathrm{G}$ for PAUP*. Model parameters were $n s t=6$ and rates=invgamma for $\mathrm{COI}$ nucleotide sequences and $n s t=2$ and rates=gamma for ef $1 \alpha$ nucleotide sequences. All protein alignments in MrBayes were analyzed with aamodel=equalin and in Beast with mtrev for mitochondrial protein and WAG for nuclear protein sequences. One individual of Hypochthonius rufulus (C. L. Koch 1835) (Oribatida, Enarthronota) was selected as outgroup for S. magnus. The Markov Chain Monte Carlo was run for ten million generations and sampled every $1000^{\text {th }}$ generation, the 50\% majority consensus tree excluded the first 2,500,000 trees (burnin of 25\%) for MrBayes. Burnin for the Bayesian approach in Beast was 1000 (burnin of 10\%).

Standard diversity indices (haplotype number $\left(\mathrm{N}_{\mathrm{h}}\right)$, haplotype diversity $\left(\mathrm{H}_{\mathrm{d}}\right.$, ) nucleotide diversity $\left(\Pi_{\mathrm{n}}\right)$, number of variable $\left(\mathrm{N}_{\mathrm{vs}}\right)$ and invariable sites $\left(\mathrm{N}_{\mathrm{is}}\right)$, parsimony informative sites $\left(\mathrm{N}_{\mathrm{pars}}\right)$, the number of singletons $\left(\mathrm{N}_{\mathrm{s}}\right)$ and the McDonald-Kreitman $(\mathrm{MK})$ test, to detect selection, were calculated with DNASP v5.0 (Rozas et al. 2003). The McDonald-Kreitman test detects selection by examining the distribution of synonymous and non-synonymous substitutions among populations; it is robust against demographic and recombination events (McDonald and Kreitman 1991). The geographical structure of genetic diversity among and within populations and geographical clades were calculated with ARLEQUIN v3.01 (Excoffier et al. 2005) using analysis of molecular variance (AMOVA, 16.000 permutations). Only populations with two and more individuals were included for the analysis. Estimates for demographic expansion (Tajima's D and Fu's $F_{s}$ neutrality tests) and pairwise differences ( $\mathrm{F}_{\mathrm{ST}} 10.000$ permutations) were also calculated in ARLEQUIN. Significance was calculated with 10.000 permutations for nucleotide sequences and amino acids for both genes. 
A parsimony based median-joining haplotype network (Bandelt et al. 1999) was generated in NETWORK v4.5 (Fluxus-Technology, Suffolk, UK) with default settings for nucleotide sequences and amino acids of both genes.

\subsection{Results}

Amplification of ef $1 \alpha$ proved to be difficult; both genes were amplified from 28 individuals, from 39 individuals only $C O I$ and from 9 individuals only ef $1 \alpha$ was amplified.

The 67 COI sequences consisted of 47 haplotypes (70.2\%) for the nucleotide (Fig. 18) with 309 variable (51.8\%) and 277 (46.4\%) parsimony informative sites (Table A7), the amino acid sequences of COI consisted of 29 haplotypes (43.3\%) (Fig. 19). Variance in the 37 ef $1 \alpha$ sequences was even higher, the nucleotide dataset consisted of 35 haplotypes (94.6\%) (Fig. 20) with 218 variable (47.3\%) and 127 (27.5\%) parsimony informative sites (Table A8); the protein formed 33 haplotypes (89.2\%) (Fig. 21). Haplotype diversity $\left(\mathrm{H}_{\mathrm{d}}\right)$ of the nucleotide sequences was high being 0.980 in $\mathrm{COI}$ and 0.997 in $e f 1 \alpha$.

\subsubsection{Phylogenetic analyses}

Phylogenetic trees (NJ without and with model of sequence evolution, MrBayes and Beast, Fig. A811) for the $C O I$ nucleotide sequences uniformly separated three monophyletic clades. One clade contained populations from Italy, Germany, France, Great Britain and one individual from Denmark (black clade), the second comprised most individuals from Eastern Europe, i.e. Czech Republic, Hungary, eastern Germany and eastern Denmark (blue clade), and the third included all populations from southern east Europe (Romania, Austria and Switzerland) as well as four individuals from eastern Germany and one individual from Czech Republic (red clade) (Fig. 14). The black clade was well supported in all analyses, with $97 \%$ and $93 \%$ bootstrap support for NJ without and with model of sequence evolution, respectively and posterior probabilities of 1 for the two Bayesian approaches (MrBayes and Beast). The blue clade was also well supported with respective bootstrap values of $98 \%$ and $88 \%$ and posterior probabilities of 1 in the Bayesian analyses. The red was only recovered in the Bayesian analyses with posterior probabilities of 1 but was paraphyletic in the NJ analyses. In all phylogenetic analyses the black and the blue clade formed sister clades and this was supported by high bootstrap values and posterior probabilities $(94 \%, 91 \%, 1$ and 0.87$)$.

The phylogenetic trees based on the amino acid sequences of $C O I$ confirmed the three main clades with high statistical support (Fig. 15, A12-14), but in contrast to the trees based on nucleotide sequences, the blue and the red clade grouped as sister clades and the blue clade became paraphyletic in the Bayesian approach of MrBayes. However, in all other analyses the blue clade was monophyletic and supported by a high bootstrap values and posterior probabilities (76\% and 1). 
Chapter 3 Cryptic species complex

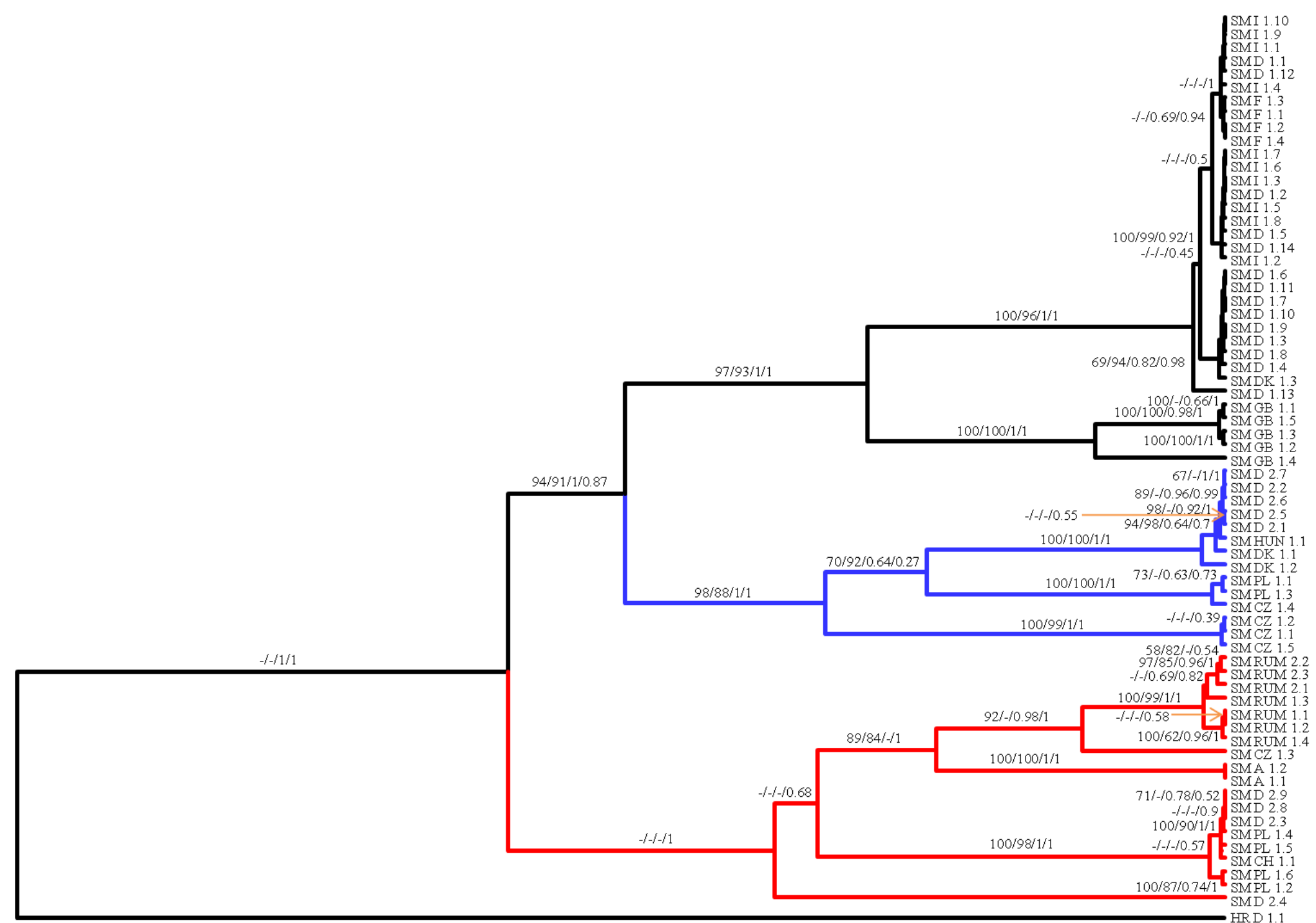

Figure 14: Bayesian phylogeny after $10 \times 10^{6}$ generations with Beast v1.5.4 based on 67 COI nucleotide sequences and Hypochthonius rufulus as outgroup (HR_D_1.1). Branch colours show the different clades (black, blue, red). Numbers on branches are bootstrap values from NJ analysis without and with evolution model (TVM+I+G) and posterior probabilities from MrBayes and Beast. 
Chapter 3 Cryptic species complex

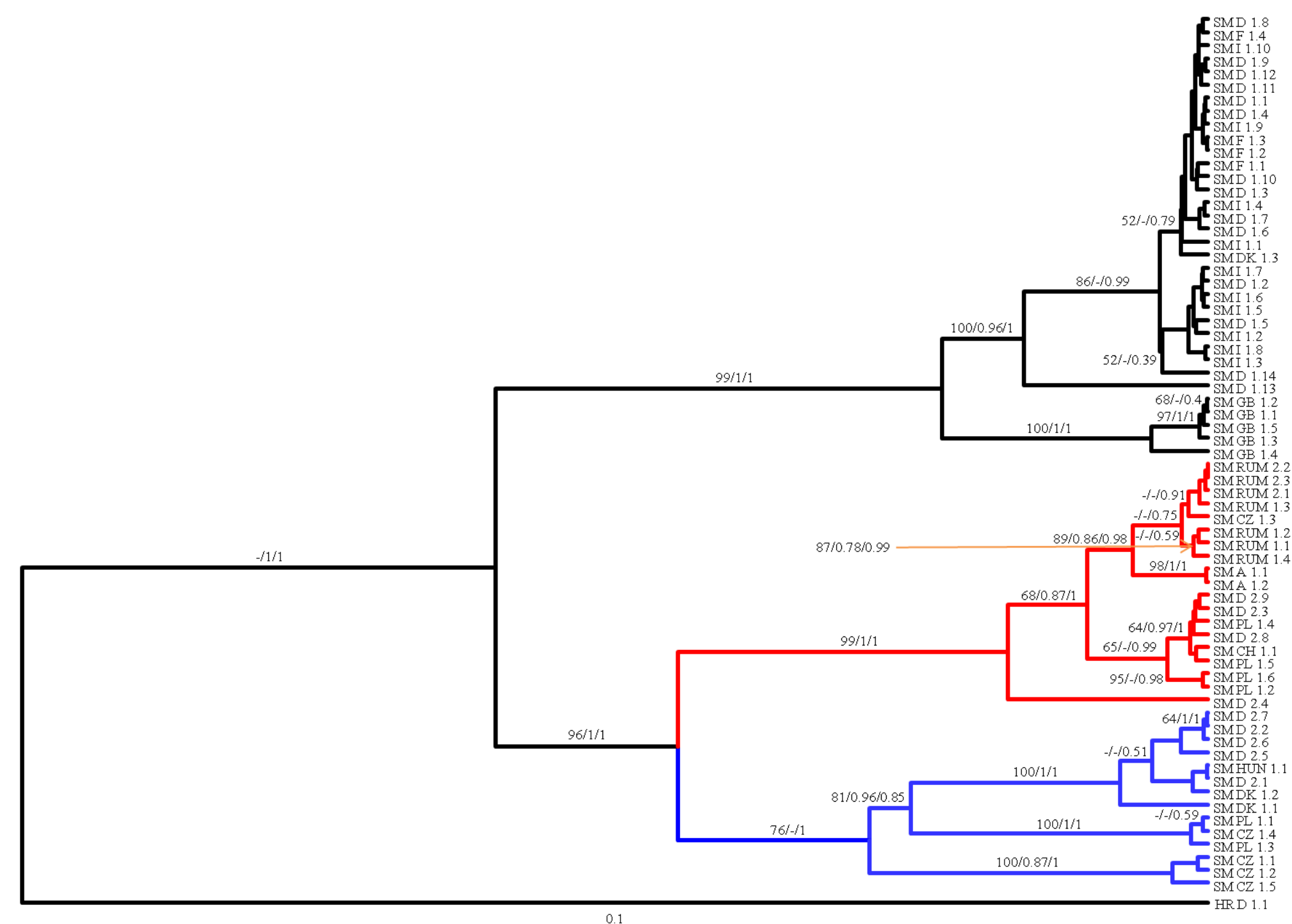

Figure 15: Bayesian phylogeny after 10x1 $10^{6}$ generations with Beast v1.5.4 based on 67 COI protein sequences and Hypochthonius rufulus as outgroup (HR_D_1.1). Branch colours show the different clades (black, blue, red). Numbers on branches are bootstrap values from NJ analysis without evolution model and posterior probabilities from MrBayes and Beast. 
The phylogenetic tree based on the nucleotide sequences of ef $1 \alpha$ (Fig. 16, A15-18) recovered the same monophyletic clades as those formed in the analysis of the COI gene. Again, the black clade was very well supported by high bootstrap values and posterior probabilities of $100 \%$ and 1 , respectively. The blue clade was also well supported by bootstrap values of $93 \%$ and $88 \%$ and posterior probabilities of 0.95 and 1, however, it included one individual of the red clade. The other individuals of the red clade also formed one clade which was supported with high bootstrap values and posterior probabilities $(91 \%, 63 \%,-$ and 1$)$.

Importantly, one ef $1 \alpha$ haplotype (PL_1.2) that was in the red clade in the COI trees, was recovered in a well supported blue subclade (bootstrap values of $99 \%$ and $100 \%$ and posterior probabilities of 0.99 and 1).

The three main clades in the phylogenetic analyses based on the amino acid sequences of the ef $1 \alpha$ were paraphyletic and weakly supported (Fig. 17). However, the haplotypes in the paraphyletic subclades corresponded to the subclades in the other phylogenetic trees (Fig. A19-21). Notably, in accordance with the nucleotide based trees of ef $1 \alpha$, haplotype PL_1.2 clustered within the blue subclade.

The red and the blue clade only formed sister clades in the Bayesian approach of Beast which were supported by a posterior probability of 1 . In the other phylogenetic analyses the black clade formed the sister clade of the blue (Fig. 17, A18). In the other phylogenetic approaches the black clade formed the sister clade of the blue (Fig. A15-17), or the red clade was paraphyletic (Fig. A17). In each of the three phylogenetic trees (NJ, MrBayes and Beast) of the ef $1 \alpha$ protein the black, blue and red clades were paraphyletic (Fig. 17, Fig. A19-21). 
Chapter 3 Cryptic species complex

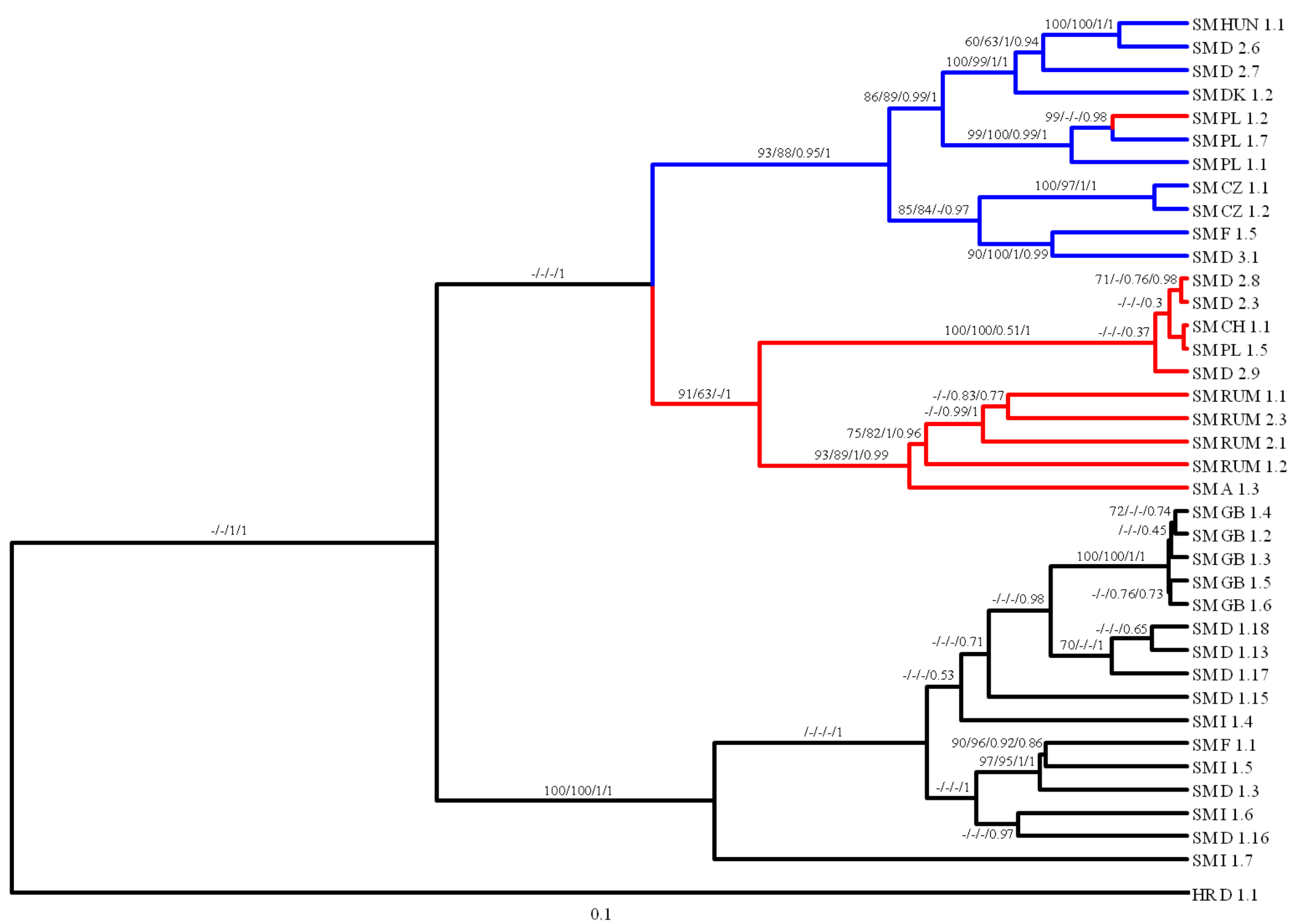

Figure 16: Bayesian phylogeny after 10x10 $0^{6}$ generations with Beast v1.5.4 based on 37 ef $1 \alpha$ nucleotide sequences and Hypochthonius rufulus (HR_D_1.1) as outgroup. Numbers on the branches are bootstrap values from $\mathrm{NJ}$ analysis without and with model of sequence evolution $(\operatorname{TrNef}+\mathrm{G})$ and posterior probabilities from MrBayes and Beast. Branch colours show the different clades as identified in phylogenies based on COI sequences (black, blue, red; Figs. 14, A8-11). 


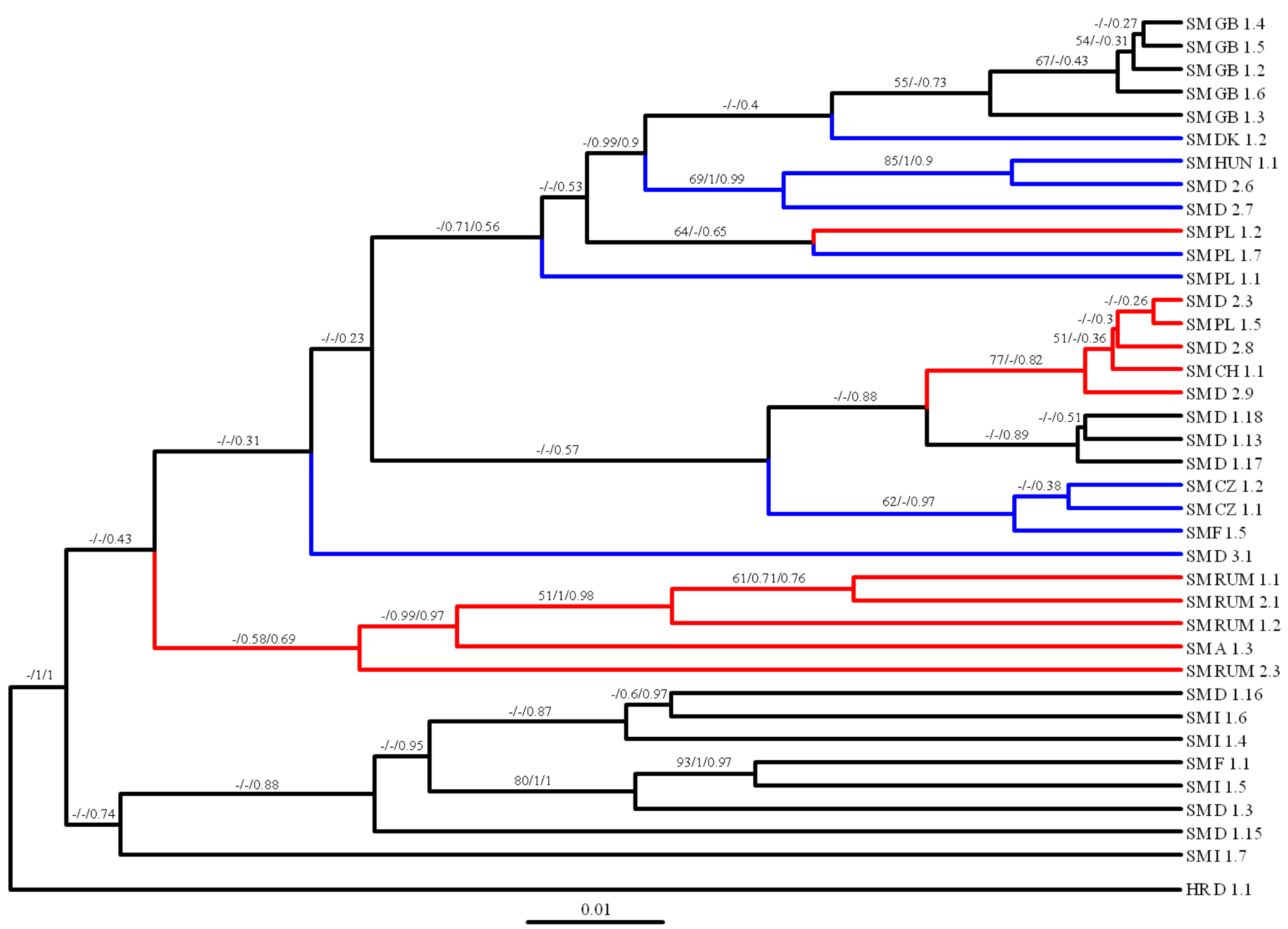

Figure 17: Bayesian phylogeny after $10 \times 10^{6}$ generations with Beast v1.5.4 based on 37 ef l $\alpha$ protein sequences and Hypochthonius rufulus (HR_D_1.1) as outgroup. Numbers on the branches are bootstrap values from NJ analysis without model of sequence evolution and posterior probabilities from MrBayes and Beast. Branch colours show the different clades as identified in phylogenies based on COI sequences (black, blue, red; Figs. 15, A12-14). 


\subsubsection{Network}

All Network analyses recovered the three main clades (black, blue and red) from the phylogenetic trees representing three distinct mitochondrial lineages (Fig. 18, 19). The nucleotide dataset of $C O I$ consisted of 47 haplotypes and the three main clades were separated by 127 and 130 numbers of substitution steps (Fig. 18).

The black clade consisted of 34 sequences representing 20 haplotypes. As in the phylogenetic analyses, this clade comprised populations of Central Europe (Central Germany, France, Great Britain), Italy and one individual from Denmark. All individuals in this clade were closely related, except for the population from Great Britain that was separated from the other haplotypes by $>110$ substitution steps. Individuals from France, Italy and Germany shared identical or closely related haplotypes.

The blue clade comprised 14 individuals with twelve haplotypes from Central-East Europe, four individuals with four haplotypes from Czech Republic, five individuals with three haplotypes from northeast Germany, a single individual from Hungary, two individuals with different haplotypes from Denmark and two individuals with two haplotypes from Poland. The high number of haplotypes indicates that the mitochondrial lineages in these populations have been separated for a longer time than those in populations of the black clade, which is also demonstrated by long branches in the phylogenetic tree (Fig. 14).

The red clade consisted of 15 haplotypes comprising the same 19 individuals forming the red clade in the phylogenetic tree (Fig. 14); the individuals originated from Southeast Europe and four individuals from Northeast Germany, four individuals from Poland and one individual from the Czech Republic.

There were only three individuals sharing the same haplotype (D_2, RUM_1 and A_1) all other haplotypes were isolated from each other by high numbers of substitution steps and long branches in the phylogenetic tree. In addition to the genetic distance between lineages within the main clades, the three main clades were separated by substantial distances; the black clade was separated from the blue clade by at least 127 substitution steps and the blue clade was separated by at least 130 substitution steps from the red clade. 


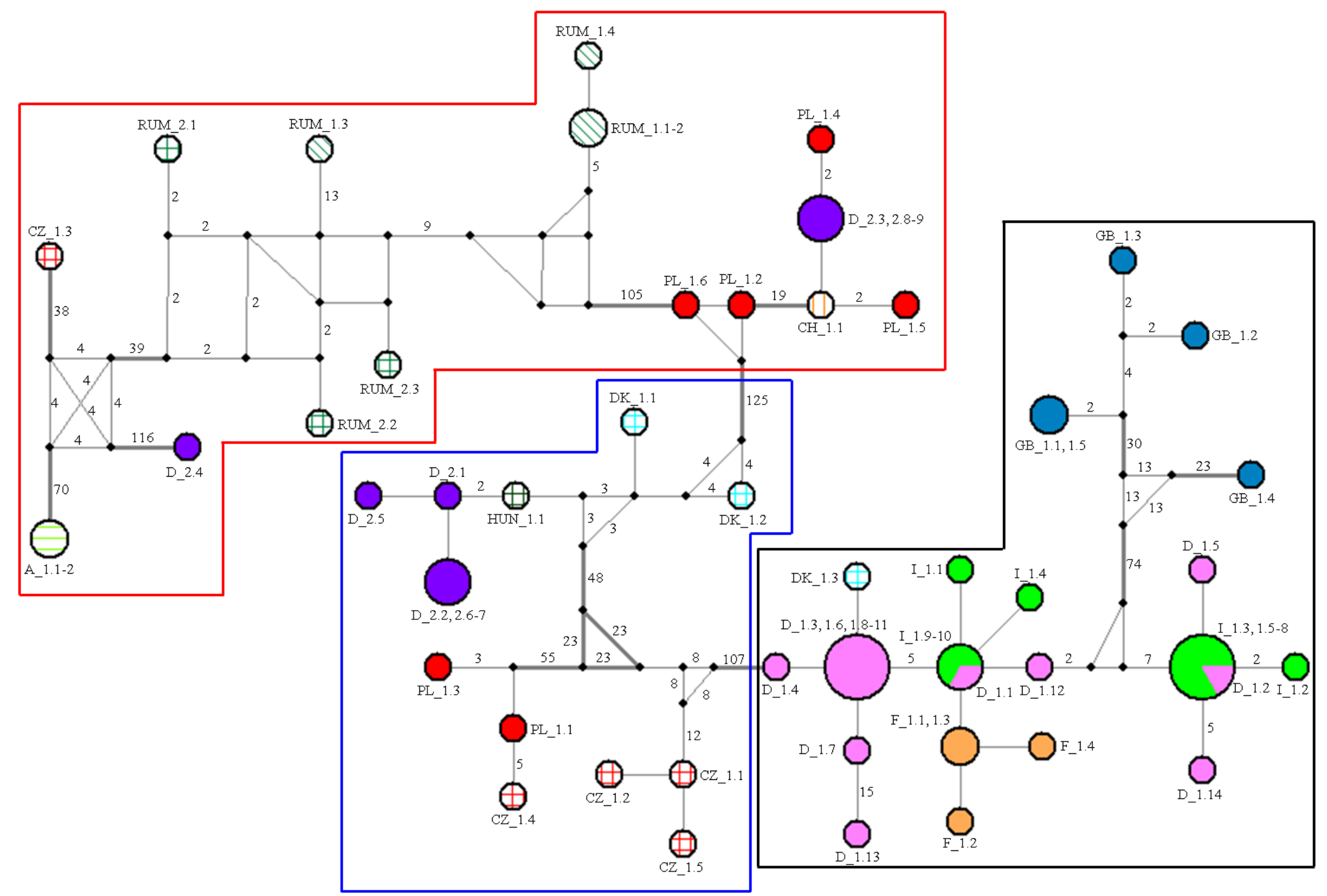

Figure 18: Median-joining haplotype network of 47 COI nucleotide haplotypes from Steganacarus magnus. Each location has a specific colour marking. The size of the circles is proportional to the number of sequences per haplotype. Numbers on the lines represent the number of substitution steps separating the haplotypes (no number: only one substitution step between haplotypes). Major subclades are marked by boxes. 
The COI haplotype network based on the protein formed 29 haplotypes; several subclades recovered in the nucleotide network collapsed, as different nucleotide sequences coded for the same protein (Fig. 19).

The 20 nucleotide haplotypes that were connected in the black clade represented only ten haplotypes in the protein network. Similar to the nucleotide network, haplotypes from Great Britain were separated from all other haplotypes in the black clade by several substitution steps (eleven amino acid exchanges). All individuals from France shared the most common haplotype that also included individuals from Central Germany and Italy (I_1.9-10). The individual from Denmark (DK_1.3) shared the second most common haplotype in the black clade.

In the blue clade, the twelve nucleotide haplotypes formed nine amino acid haplotypes; four individuals from Germany (D_2.1-2, 2.6-7) and one individual of Hungary (HUN_1.1) shared the protein sequence of the most common haplotype in this clade. One haplotype was shared by one Polish (PL_1.1) and one Czech (CZ_1.4) individual. The other seven haplotypes were formed by single individuals from four locations [two haplotypes each from one location (D_2.5 and PL_1.3), two haplotypes from one Danish location (DK_1.1-2) and three haplotypes from one Czech location (CZ_1.1-2, 1.5)].

In the red clade, the 15 nucleotide haplotypes merged to ten. Three nucleotide haplotypes from one Romanian population shared the protein haplotype with two individuals from another Romanian population (RUM_1.1-2 and RUM_2.1-3). Further, the individual from Switzerland was identical to one haplotype from Northeast Germany. However, as in the nucleotide network protein haplotypes from individuals from Poland were distinct and were included in the blue (PL_1.1, 1.3) and the red clade (PL_1.2, 1.4-6).

Generally, the black clade was separated from the blue clade by at least 28 amino acid chances and the blue clade was separated from the red clade by at least 16 amino acid changes.

In the ef $1 \alpha$ nucleotide network the 37 sequences represented 35 haplotypes in the nucleotide merged to 33 haplotypes in the protein network (Fig. 20). In both datasets the same three main clades (black, blue and red) were recovered as in the COI network and the phylogenetic trees with one exception. Individual PL_1.2 of the red mitochondrial lineage in the COI dataset swapped position to the blue clade. In all three clades individuals that shared the same mitochondrial haplotype in the COI network, had isolated haplotypes in the ef $1 \alpha$ nucleotide network (black clade: I_1.5, I_1.6, I_1.7, GB_1.2 and GB_1.4; blue clade: D_2.6 and D_2.7; red clade: D_2.8, D_2.9, RUM_1.1 and RUM_1.2). 


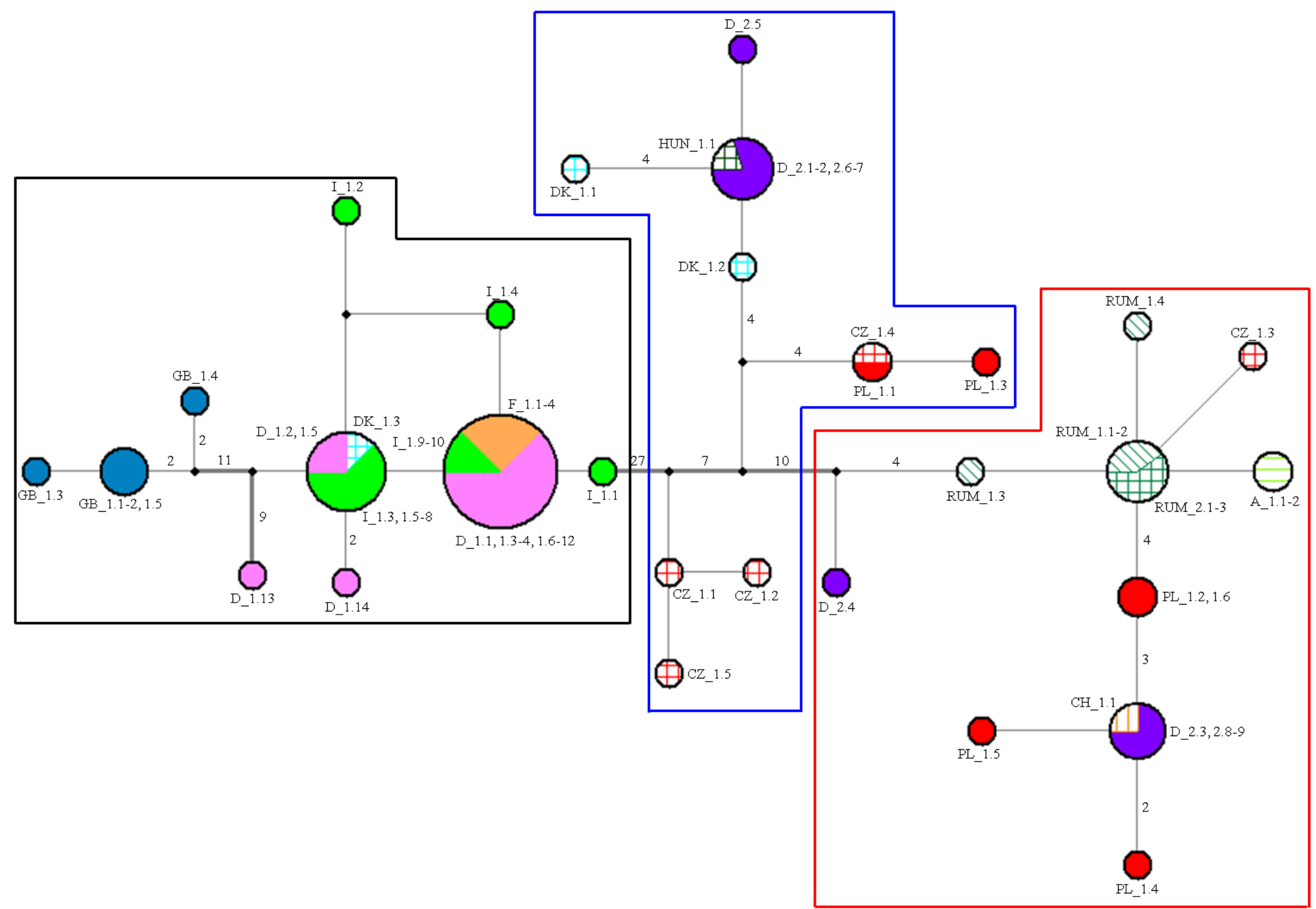

Figure 19: Median-joining haplotype network of 29 COI protein haplotypes from Steganacarus magnus. Each location has a specific colour marking. The size of the circles is proportional to the number of sequences per haplotype. Numbers on the lines represent the number of substitution steps separating the haplotypes (no number: only one amino acid change between haplotypes). Major subclades are marked by boxes. 
Chapter 3 Cryptic species complex

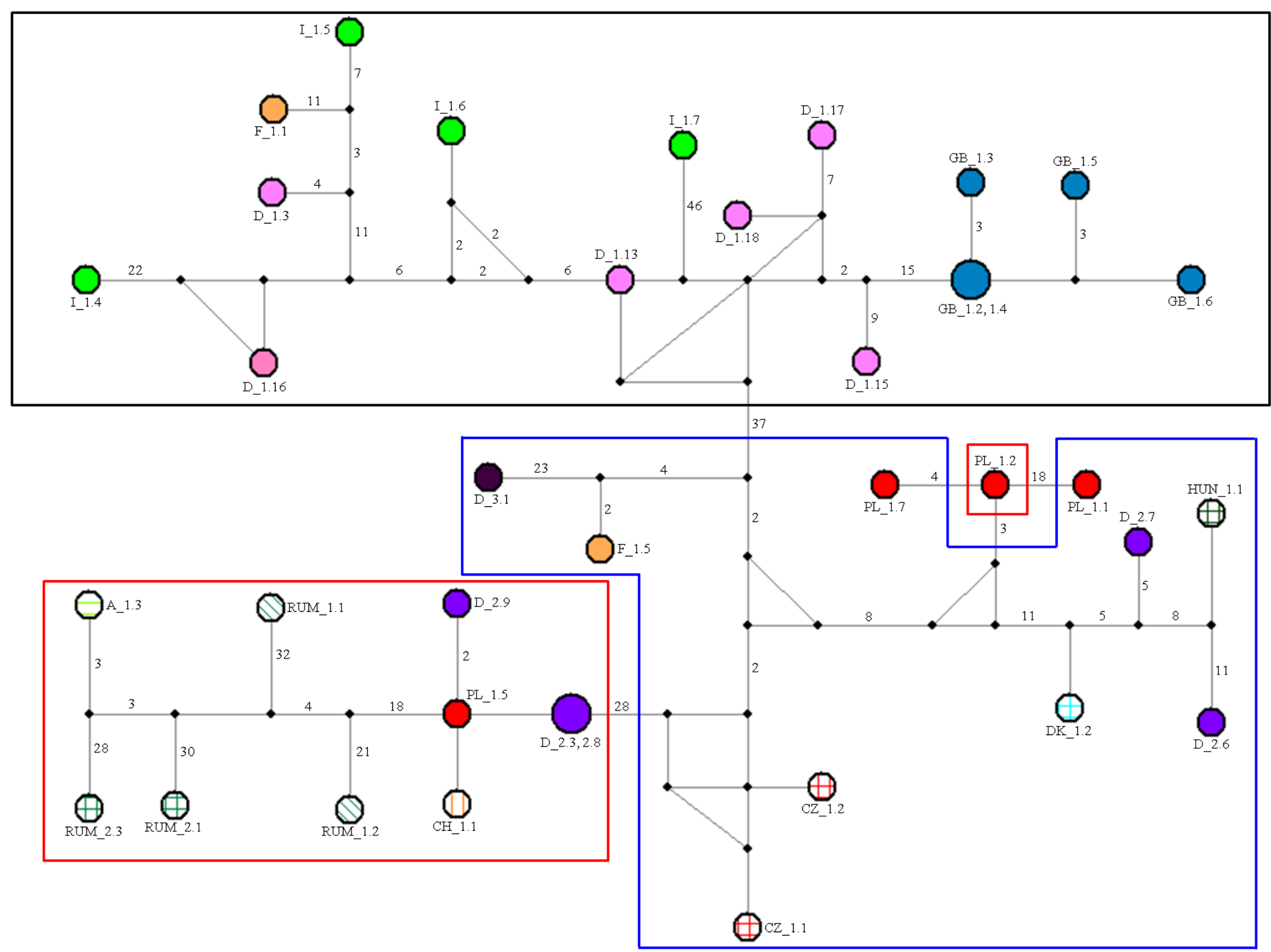

Figure 20: Median-joining haplotype network of the 35 ef $1 \alpha$ nucleotide haplotypes from Steganacarus magnus. Each location has a specific colour marking. The size of the circles is proportional to the number of sequences per haplotype. Numbers on the lines represent the number of substitution steps separating the haplotypes (no number: only one substitution step between haplotypes). Major subclades are marked by boxes. 


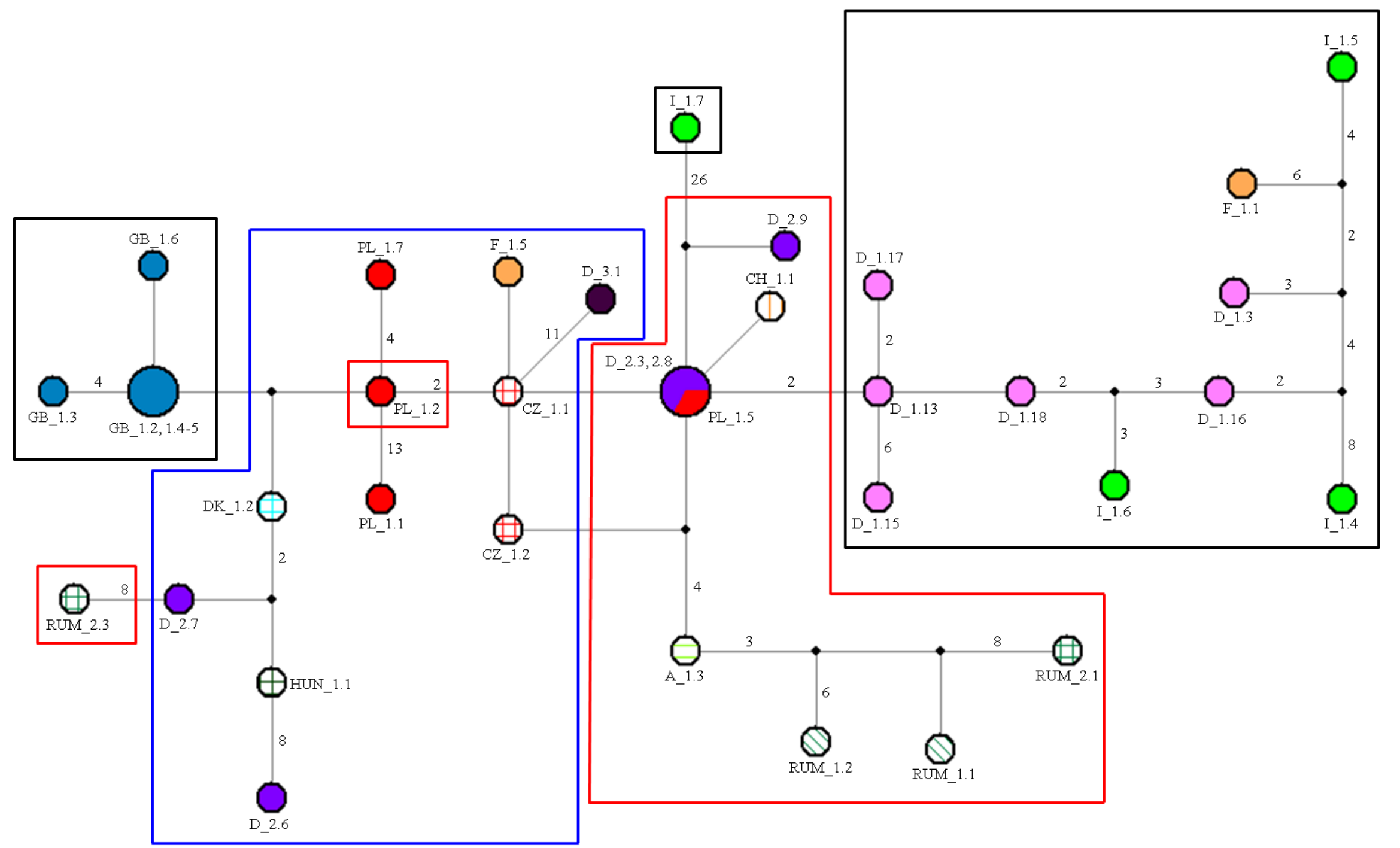

Figure 21: Median-joining haplotype network of the 33 ef $1 \alpha$ protein haplotypes from Steganacarus magnus. Each location has a specific colour marking. The size of the circles is proportional to the number of sequences per haplotype. Numbers on the lines represent the number of substitution steps separating the haplotypes (no number: only one amino acid change between haplotypes). Major subclades are marked by boxes. 


\subsubsection{Population genetic analyses}

The minimum and maximum mean average pairwise uncorrected p-differences for the nucleotide sequences between populations were $1.4 \%$ (F_1/I_1) and $28.4 \%$ (A_1/DK_1) in COI and $0.6 \%$ (D_1/I_1) and $19.6 \%$ in ef $1 \alpha$ (I_1/RUM_2) (Table 8, 9). The minimum and maximum mean average pairwise uncorrected p-differences for the protein sequences among populations were in COI $0 \%$ (A_1/RUM_2) and 3.1\% (A_1/D_1, D_1/RUM_1, D_1/RUM_2, I_1/RUM_1 and I_1/RUM_2) and for ef $1 \alpha 0.5 \%$ (CZ_1/D_2) and 3.7\% (I_1/PL_1) (Table A9, A10).

Within populations the minimum and maximum mean average pairwise uncorrected p-differences of the nucleotide sequences were $0 \%$ (A_1) and $18.2 \%$ (DK_1) in COI and 0.7\% (CZ_1) and 14\% (F_1) in ef $1 \alpha$ (Table 8,9). For the protein the minimum and maximum mean average pairwise uncorrected p-differences within populations were 0\% (A_1, F_1, GB_1 and RUM_2) and 1.7\% (DK_1) in COI and $0 \%$ (CZ_1, RUM_2) and 3.1\% (I_1) in ef $1 \alpha$ (Table A9, A10).

The average uncorrected p-distances in the $C O I$ nucleotide within populations and among populations were $6.8 \%$ and $22.1 \%$, respectively. Respective p distances in the protein were $0.5 \%$ and $1.9 \%$. The average uncorrected p-distances in the ef $1 \alpha$ nucleotide were $6.4 \%$ within and $12.2 \%$ among populations and in the protein respective values were $1 \%$ and $1.6 \%$.

Table 8: Mean percentage pairwise differences of uncorrected p-distances for the COI nucleotide of Steganacarus magnus from eleven locations. The diagonal is the within population differences (bold) and below the diagonal the among population differences. Bold letters in red are the minimum and maximum differences within and among the populations. Locations with less than two individuals were excluded from the analysis.

\begin{tabular}{ccccccccccccc}
\hline Population & $\mathbf{1}$ & $\mathbf{2}$ & $\mathbf{3}$ & $\mathbf{4}$ & $\mathbf{5}$ & $\mathbf{6}$ & $\mathbf{7}$ & $\mathbf{8}$ & $\mathbf{9}$ & $\mathbf{1 0}$ & $\mathbf{1 1}$ \\
\hline $\mathbf{1}$ & A_1 & $\mathbf{0}$ & & & & & & & & & & \\
$\mathbf{2}$ & CZ_1 & 24.7 & $\mathbf{1 5 . 8}$ & & & & & & & & & \\
$\mathbf{3}$ & D_1 & 27.9 & 23.5 & $\mathbf{1 . 5}$ & & & & & & & & \\
$\mathbf{4}$ & D_2 & 25.1 & 22.5 & 24.6 & $\mathbf{1 6}$ & & & & & & & \\
$\mathbf{5}$ & DK_1 & $\mathbf{2 8 . 4}$ & 22 & 17 & 16.8 & $\mathbf{1 8 . 2}$ & & & & & & \\
$\mathbf{6}$ & F_1 & 27.6 & 23.3 & 1.5 & 24.4 & 17 & $\mathbf{0 . 2}$ & & & & & \\
$\mathbf{7}$ & GB_1 & 27.9 & 25.6 & 20.5 & 26.2 & 23.8 & 20 & $\mathbf{5 . 3}$ & & & & \\
$\mathbf{8}$ & I_1 & 28 & 23.6 & 1.7 & 24.4 & 17.1 & $\mathbf{1 . 4}$ & 20.4 & $\mathbf{1 . 1}$ & & & \\
$\mathbf{9}$ & PL_1 & 23 & 21.5 & 23.9 & 18.5 & 23 & 23.6 & 27.2 & 23.8 & $\mathbf{1 4 . 3}$ & & \\
$\mathbf{1 0}$ & RUM_1 & 19.1 & 22.3 & 26.4 & 23.4 & 26.3 & 26.3 & 26.8 & 26.3 & 21.7 & $\mathbf{1 . 7}$ & \\
$\mathbf{1 1}$ & RUM_2 & 19.2 & 22.2 & 26.9 & 23.1 & 26.1 & 26.7 & 27.2 & 26.9 & 22 & 2.8 & $\mathbf{1 . 1}$ \\
\hline
\end{tabular}


Table 9: Mean percentage pairwise differences of uncorrected p-distances for the ef $1 \alpha$ nucleotide of Steganacarus magnus from nine locations. The diagonal is the within population differences (bold) and below the diagonal among population differences. Bold letters in red are the minimum and maximum differences within and among the populations. Locations with less than two individuals were excluded from the analysis.

\begin{tabular}{lcccccccccc}
\hline Population & $\mathbf{1}$ & $\mathbf{2}$ & $\mathbf{3}$ & $\mathbf{4}$ & $\mathbf{5}$ & $\mathbf{6}$ & $\mathbf{7}$ & $\mathbf{8}$ & $\mathbf{9}$ \\
\hline $\mathbf{1}$ & CZ_1 & $\mathbf{0 . 7}$ & & & & & & & & \\
$\mathbf{2}$ & D_1 & 11.1 & $\mathbf{3 . 7}$ & & & & & & & \\
$\mathbf{3}$ & D_2 & 7.2 & 13.7 & $\mathbf{7 . 1}$ & & & & & & \\
$\mathbf{4}$ & F_1 & 8.6 & 8.6 & 12.6 & $\mathbf{1 4}$ & & & & & \\
$\mathbf{5}$ & GB_1 & 11.7 & 5.8 & 13.6 & 10.4 & $\mathbf{0 . 7}$ & & & & \\
$\mathbf{6}$ & I_1 & 13.8 & $\mathbf{0 . 6}$ & 16.1 & 10.9 & 9.3 & $\mathbf{9 . 1}$ & & & \\
$\mathbf{7}$ & PL_1 & 5.7 & 12.9 & 7.8 & 11.3 & 12.2 & 15.3 & $\mathbf{6 . 6}$ & & \\
$\mathbf{8}$ & RUM_1 & 12.2 & 16.2 & 11.8 & 15.4 & 16 & 18.3 & 12.4 & $\mathbf{7 . 6}$ & \\
$\mathbf{9}$ & RUM_2 & 13.5 & 17.4 & 12.2 & 16.4 & 16.6 & $\mathbf{1 9 . 6}$ & 13 & 8.2 & $\mathbf{8 . 4}$ \\
\hline
\end{tabular}

The results of the AMOVA showed that the nucleotide variation among populations within countries (COI 59.9\%, ef $1 \alpha 54.5 \%$ ) and the variation within samples (COI 32.1\%, ef $1 \alpha 46.8 \%$ ) were significant and high (Table 10,11). Also, the protein variation among populations within countries (COI 72.1\%, ef $1 \alpha 42.4 \%$ ) and the variation within samples (COI 27.3\%, ef $1 \alpha 61.9 \%$ ) were significant and high. The neutrality tests (Tajima's D and Fu's $\mathrm{F}_{\mathrm{s}}$ ) were not significant for the COI nucleotide (Table A11). Tajima's D was only significant for one population in the COI protein (Table A12) (D_1: $\mathrm{D}=-1.54, \mathrm{p}$-value=0.043) and ef $1 \alpha$ nucleotide (Table A13) (PL_1: $\mathrm{D}=-0.869$, pvalue $=0.027)$, respectively. The neutrality test of $\mathrm{Fu}\left(\mathrm{Fu}^{\prime} \mathrm{s}_{\mathrm{s}}\right)$ was only significant for one population in the ef $1 \alpha$ protein dataset (Table A14) (D_2: FS=-1.79; FS p-value $=0.021$ ).

Table 10: AMOVA table on variations among countries and within populations in nucleotide (black) and protein (red) sequences of COI. Each population was considered as a separate group. Populations with less than two individuals were excluded from the analysis. Significant level is $\mathrm{p}<0.05$ (d.f.: degree of freedom).

\begin{tabular}{lccccc}
\hline Source of variance & d.f. & Sum of squares & Variance components & Percent of total variation & Fixation indices \\
\hline Among countries & $8 / 8$ & $1986.28 / 62.29$ & $4.88 \mathrm{Va} / 0.01 \mathrm{Va}$ & $7.95 / 0.63$ & $\mathrm{FCT}: 0.08 / 0.01$ \\
Among populations within groups & $2 / 2$ & $569.03 / 20.44$ & $36.81 \mathrm{Vb} * / 1.35 \mathrm{Vb} *$ & $59.91 / 72.08$ & $\mathrm{FSC}: 0.65^{*} / 0.73^{*}$ \\
Within populations & $54 / 54$ & $1066.31 / 27.59$ & $19.75 \mathrm{Vc} * / 0.51 \mathrm{Vc} *$ & $32.14 / 27.28$ & $\mathrm{FST}: 0.68^{*} / 0.73^{*}$ \\
total & $64 / 64$ & $3621.62 / 110.31$ & $61.44 / 1.87$ & & \\
\hline
\end{tabular}

Table 11: AMOVA table on variations among countries and within populations in nucleotide (black) and protein (red) sequences in ef $1 \alpha$. Each population was considered as a separate group. Populations with less than two individuals were excluded from the analysis. Significant level is $\mathrm{p}<0.05$ (d.f.: degree of freedom).

\begin{tabular}{lccccc}
\hline Source of variance & d.f. & Sum of squares & Variance components & Percent of total variation & Fixation indices \\
\hline Among countries & $6 / 6$ & $371.95 / 14.71$ & $-0.35 \mathrm{Va} /-0.05 \mathrm{Va}$ & $-1.28 /-4.11$ & $\mathrm{FCT}:-0.01 /-0.04$ \\
Among populations within groups & $3 / 3$ & $136.98 / 5.73$ & $14.93 \mathrm{Vb}^{*} / 0.55 \mathrm{Vb}^{*}$ & $54.49 / 42.41$ & $\mathrm{FSC}: 0.54^{*} / 0.41^{*}$ \\
Within populations & $23 / 23$ & $294.98 / 18.62$ & $12.83 \mathrm{Vc} * / 0.81 \mathrm{Vc} *$ & $46.79 / 61.9$ & $\mathrm{FST}: 0.53^{*} / 0.38^{*}$ \\
Total & $31 / 31$ & $803.91 / 39.06$ & $61.44 / 1.31$ & & \\
\hline
\end{tabular}

The McDonald-Kreitman test showed that the differences in the COI gene between seven of 55 populations $(12.7 \%)$ were significant, between other two of 55 populations $(3.6 \%)$ the differences 
were highly significant and between seven of 55 populations (12.7\%) they were very highly significant (Table 12, for more details see Table A15). All computed neutrality indices were 0 (Table A17).

The McDonald-Kreitman test showed that the differences in the ef $1 \alpha$ gene between two of 36 populations $(5.6 \%)$ were significant, between other three of 36 populations $(8.3 \%)$ the differences were highly significant and between 14 of 36 populations (38.9\%) they were very highly significant (Table 13, Table A16). All computed neutrality indices were 0 (Table A18) indicating purifying selection.

Table 12: Results of the McDonald-Kreitman test of the COI gene of Steganacarus magnus. The differences between seven populations are significant $\left({ }^{*} 0.01<\mathrm{P}<0.05\right)$, between other nine population highly significant $(* * 0.001<\mathrm{P}<0.01, * * * \mathrm{P}<0.001)$.

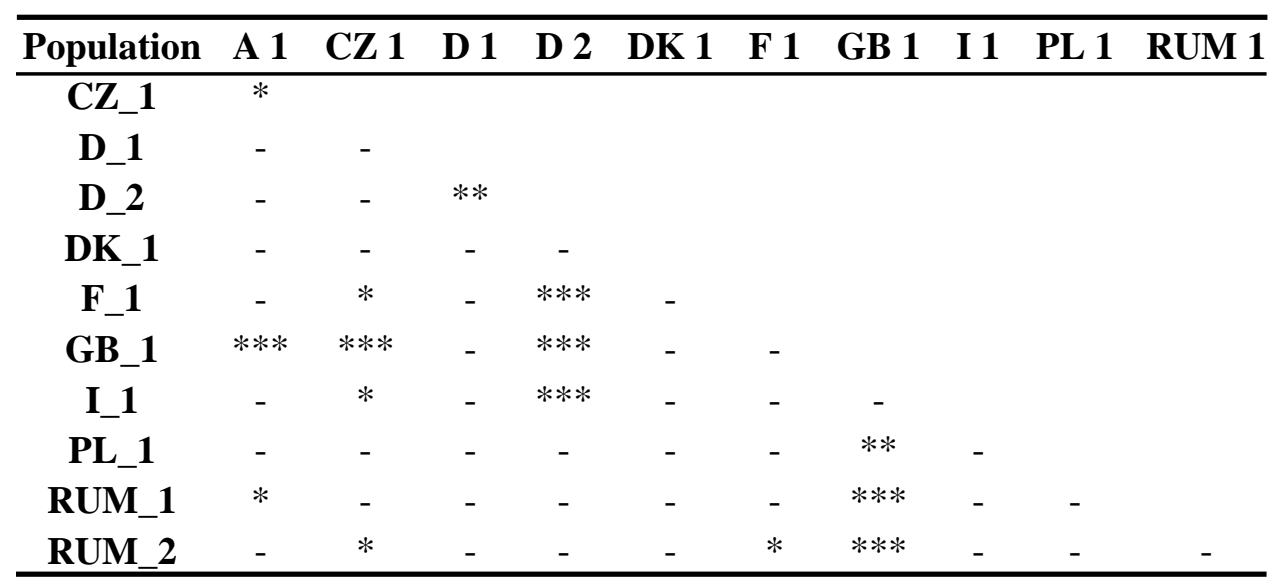

Table 13: Results of the McDonald-Kreitman test of the ef $1 \alpha$ gene of Steganacarus magnus. The differences between two populations were significant $(* 0.01<\mathrm{P}<0.05)$, between other 17 populations highly significant $(* * 0.001<\mathrm{P}<0.01, * * * \mathrm{P}<0.001)$.

\begin{tabular}{ccccccccc}
\hline Population & CZ 1 & D 1 & D 2 & F 1 & GB 1 & I 1 & PL 1 & RUM 1 \\
\hline D_1 & $* * *$ & & & & & & & \\
D_2 & - & $* * *$ & & & & & & \\
F_1 & - & - & - & & & & & \\
GB_1 & $* *$ & - & $* * *$ & - & & & & \\
I_1 & $* * *$ & - & $* * *$ & - & - & & & \\
PL_1 & - & $* * *$ & - & - & $* * *$ & $* * *$ & & \\
RUM_1 & - & $* * *$ & - & - & $*$ & $* * *$ & - & \\
RUM_2 & $* * *$ & $* * *$ & $* *$ & $* *$ & $* * *$ & $* * *$ & $*$ & - \\
\hline
\end{tabular}




\subsection{Discussion}

Commonly, a threshold of $>3 \%$ genetic distance in the standard barcoding gene COI is taken as evidence for new species (Hebert et al. 2003a-b, 2004a-b), but this delineation often does not hold for invertebrates (Edmands 2001, Heethoff et al. 2007, Boyer et al. 2007, Rich et al. 2008, Torricelli et al. 2010).

In the oribatid mite S. magnus, high genetic differences among (31.8\%) and within (25.8\%) populations in Europe indicate the existence of cryptic species (Chapter 2). All COI and ef $1 \alpha$ sequences are translatable into proteins without stop codons. The high variance therefore did not result from COI pseudogenes (Bensasson et al. 2001, Williams and Knowlton 2001, Song et al. 2008, Buhay 2009). Gene duplications and heteroplasmy (Pohl et al. 2009) of the COI gene also can be excluded as the full mitochondrial genome of S. magnus has been sequenced (Domes et al. 2008, Domes-Wehner 2009) and no gene duplications and no heteroplasmy have been found. In contrast to Hurst and Jiggins (2005) the high genetic variance did not result from inherited symbionts such as Wolbachia or Cardinium, since none of these symbionts has been found in S. magnus. However, Wolbachia and Cardinium can trigger parthenogenesis and were found in an unidentified oribatid mite (Cordaux et al. 2001) and in some populations of Oppiella nova (Weeks et al. 2003).

Comparing phylogenetic relationships of the mitochondrial $\mathrm{COI}$ and the nuclear single copy gene ef $1 \alpha$ provides evidence for gene flow, i.e. sexual recombination, between individuals of different mitochondrial lineages. Individuals collected from Poland (PL_1) carry two mitochondrial and two nuclear lineages that cluster in two separate clades (red and blue) in all phylogenetic analyses. In contrast, one individual in these populations (PL_1.2) carries the red mitochondrial lineage but a nuclear haplotype of the blue lineage, a pattern that likely resulted from sexual reproduction between a female from the red mitochondrial lineage and a male from the blue nuclear lineage. Further evidence for recombination is provided by the protein dataset of the two genes and therefore is unlikely to be an artifact, e.g. due the fourfold degenerated mitochondrial code that tolerates high variance in the third codon position (Xia et al. 1996, Baker 2000). The phylogenetic signal from the protein supports the evidence for recombination, as the protein code is more conserved and reflects neutral and non-neutral substitutions. The linkage between the nucleus and the mitochondrion did not break and the communication between them is still functional; $40 \%$ of the amino acids of the COI barcoding region interact with the nuclear protein of the cytochrome oxidase complex IV (Ballard and Melvin 2010) and non-synonymous substitutions are likely to affect this interaction. Non-synonymous substitutions could be present in a small number since $60 \%$ of the amino acids of the COI barcoding region do not interact with another protein (Ballard and Melvin 2010).

Replication cycles and the genetic code of mitochondria are beyond the control of the nucleus. However, cooperation between nuclear and mitochondrial encoded genes is necessary for a fully functional respiratory chain. Nuclear and mitochondrial genes therefore are closely linked, as mutations in one of the two may disturb their interaction. The high genetic distances among lineages but the presence of gene flow among individuals of different mitochondrial lineages indicates that substitutions accumulate easily in the mitochondrial gene if they are neutral. Population sizes of $S$. magnus must be very large to maintain this high genetic variance at small and large geographic scales. S. magnus has poor dispersal abilities, phoresy and dispersal by wind or birds are unknown (Seyd 1962). However, long generation cycles of up to three years, relatively long life spans and pronounced cold tolerance are characteristic for this species (Webb 1977, 1989, Webb and Block 1993). The high genetic diversity at small scale supports the idea that European populations of $S$. magnus are very old and comprise several relict populations that survived the last ice age and other climatic changes in Central Europe (Chapter 2). The substitution rate for the COI gene of S. magnus is known (2.15\% per million year; Salomone et al. 2002); taking this substitution rate the mitochondrial lineages from 
Poland with an intraspecific variance of $14.3 \%$ (Table 8) separated >30 mya: Remarkably, they coexist on very small scale allowing sexual reproduction and gene flow among these lineages. This, however, is only possible if the majority of variance is neutral ensuring that the interaction between nuclear and mitochondrial coded units of the cytochrome complex I remain functional and offspring therefore viable. The genus Steganacarus includes twelve European species but discrimination of species is difficult (Weigmann 2006). Each of the species varied in body size and colour and this variation changes with age and environment (R.A. Norton, pers. comm.). However, chaetotaxy, prodorsum and notogaster structure are distinct in $S$. magnus allowing unequivocal identification of this species. This morphological distinctness is contrasted by very high genetic variation in the $C O I$ gene where the third codon position which is in full saturation, suggesting that $C O I$ is unsuitable for barcoding in this oribatid mite species.

In contrast to Schaefer et al. (2006) and Laumann et al. (2007), the intraspecific divergence of the ef $1 \alpha$ sequences in S. magnus was very high (up to $20 \%$ ). Both of these studies used individuals from one location and did not sample over a large scale as done in the present study.

High genetic differences in $\mathrm{COI}$ nucleotide sequences were also found in species of the genus Steganacarus from the Canary Islands (up to 28.6\%; Salomone et al. 2002) and other arthropods such as the pan-Antarctic springtail Friesea grisea (17.7\%; Torricelli et al. 2010), the harvestman Aoraki denticulata in New Zealand (up to 19.2\%; Boyer et al. 2007) and the intertidal copepod Tigriopus californicus in North America (up to 23\%; Edmands 2001). Potentially, soil and marine arthropods are more flexible in handling substitutions than aboveground organisms $(0.2-2.5 \%$ in the pine shoot beetle Tomicus piniperda, Ritzerow et al. 2004, 0-2.6\% in the snake Vipera berus, Ursenbacher et al. 2006, $1.5 \%$ in the snail Trochulus villosus, Dépraz et al. 2008).

The high local genetic variance in S. magnus may be due to sexual reproduction and recombination of different ecotypes (Meyers and Bull 2002). However, if sexual reproduction of different lineages combines different ecotypes and therefore provides selective advantage over other lineages remain to be tested.

\subsection{Conclusions}

High genetic distances characterize populations of the oribatid mite species S. magnus in Europe. This species probably survived the last ice ages in cryptic refugia in Central and Northern Europe. However, S. magnus does not represent a cryptic species complex as gene-flow between lineages that separated tens of millions of years ago still exists. Phylogenetic analyses of the mitochondrial COI and the nuclear ef $1 \alpha$ gene, both sequenced from the same individual, suggest recombination of the nuclear genome and the maternal inherited mitochondrial genome.

This is remarkable, as $40 \%$ of the amino acids of the COI barcoding region interact with the nuclear coded cytochrome oxidase complex IV (Ballard and Melvin 2010) and non-synonymous substitutions are likely to change this interaction. Sexual recombination may buffer variations in the mitochondria coded subunits of the cytochrome I protein complex by providing recombinant individuals with matching nuclear coded subunits ensuring functionality of the protein complex. To support this hypothesis, analyses of the mitochondrial genome of sexual and parthenogenetic species are needed. If true, sexual species should have more non-synonymous substitutions in the mitochondria encoded cytochrome oxidase subunits than parthenogenetic species. 


\section{Chapter Four}

\section{Differential colonization of Europe by sexual and asexual oribatid mite species: post- and pre- ice age events}

\section{Summary}

Since the beginning of the Quaternary ( $\sim 3$ million years ago) ice ages shaped the biodiversity of Europe. The living space for aboveground species shrunk to areas south of the Alps, the Pyrenees and the Balkans. Whether this also applies to soil living animals, however, is unknown. Soil living animals include a high number of parthenogenetic species and parthenogenetic organisms have the advantage of faster colonization of new habitats than sexual species. I analyzed two parthenogenetic (Nothrus silvestris and Platynothrus peltifer) and two sexual (Achipteria coleoptrata and Steganacarus magnus) oribatid mite species and their colonization of Europe using the cytochrome c oxidase $(C O I)$ as molecular marker. I expected that species in Central Europe that were not affected by glaciation constitute isolated haplotypes that are distinct from populations of Southern Europe and Central Asia and that genetic diversity of these species is highest in refuge areas. The four oribatid mite species showed different colonization patterns. N. silvestris was highly affected by the last ice age. Only one clonal lineage exists and all European locations studied grouped in one haplotype. However, the origin of this clonal lineage remains unclear. Two possible options of re-colonisation of Central Europe exist, a Romanian or a Southern French refuge area. The genotype distribution of the other three species suggests that they were not affected by Quaternary glaciation. The second parthenogenetic oribatid mite species $P$. peltifer constitute several separated Scandinavian clusters with a maximum pairwise nucleotide difference of $20.4 \%$ but no differences in the protein. The two sexual oribatid mite species, A. coleoptrata and S. magnus, also showed very high nucleotide variation (up to $19.4 \%$ and $31.3 \%$ in A. coleoptrata and S. magnus, respectively) and also high protein variation (up to $2.6 \%$ and $4.2 \%$ in $A$. coleoptrata and $S$. magnus, respectively). Haplotype networks were dominated by single individual haplotypes and several clusters separated by a large number of substitutions. Phylogenetic trees of $P$. peltifer, A. coleoptrata and $S$. magnus were characterized by deep splits. The results suggest that these species radiated in the Miocene or earlier and survived in multiple refugia in Northern and Central Europe; potential Southern refugia (Iberian Peninsula, Italy and the Balkan) were not closely linked to the Northern or Central clusters. In A. coleoptrata haplotype clusters of Northern locations were separated by large numbers of substitution steps. The haplotype of $S$. magnus suggest that pre- and post-glacial splits exist. Generally, however, pre Quaternary splits dominated the haplotype networks of A. coleoptrata, P. peltifer and S. magnus. Cryptic refugia were detected in the Alps for P. peltifer and A. coleoptrata, and in Scandinavia, Northern, Central and South Germany, Poland, Czech Republic and Great Britain in A. coleoptrata, P. peltifer and S. magnus. 


\subsection{Introduction}

For the past 2.6 million years (my), the climate in Europe oscillated between warm and cold periods (Hewitt 2000, Hewitt 2004, Mosbrugger et al. 2005) resulting in expansion - contraction scenarios for most living organisms (Hewitt 1999, Provan and Bennett 2008). During glacial periods, warm adapted animals and plants either went extinct or retreated to more favorable sites, whereas during interglacials they expanded from refugia. These oscillations shaped the genetic structure of the European fauna and flora which has been characterized as "Southern richness and Northern purity" (Hewitt 1999, Hewitt and Ibrahim 2001, Schmitt 2007). Genetic diversity is high in populations that live in former glacial refugia, whereas populations are genetically homogenous in areas which were re-colonised from refugial populations during interglacial periods. The Iberian Peninsula, Italy, Greece and the Balkans are well established refugia for many European species (Hewitt 1999, Steinfratz et al. 2000, Ursenbacher et al. 2006). Several phylogeographic studies on freshwater and terrestrial organisms confirmed the importance of Mediterranean refugia (Beheregaray 2008). Central Asia also contributed to the re-colonization of Central and Northern Europe in some species such as the brown bear (Ursus arctos; Hewitt 1999). The three most common patterns of re-colonization were delineated form the pattern in the common grasshopper (Chorthippus parallelus), the brown bear (U. arctos) and the hedgehog (Erinaceus spp.) (Hewitt and Ibrahim 2001).

Radiocarbon (Stewart and Lister 2001), pollen (Willis et al. 2000, Tollefsrud et al. 2008) and molecular (Pfenninger et al. 2003, Verovnik et al. 2005, Tollefsrud et al. 2008) data suggest that isolated areas in Central and Northern Europe also functioned as refugial areas. These cryptic refugia (Stewart and Lister 2001, Provan and Bennett 2008) are difficult to detect because presumably they were small, geographically isolated or located in wind protected valleys or ice free Nunataks (Stewart and Cooper 2008, Schmitt 2009).

Survival of populations in Central refugia implies that species expanding from Southern refugia met populations with different demographic and ecological history. Identifying Central and evaluating their contribution to the gene pool of present day populations is important for understanding the genetic structure of populations and their adaptation to local conditions, both important factors for speciation processes. Until now, phylogeographic studies on the postglacial colonization of Central and Northern Europe were restricted exclusively to aboveground species (Hewitt 1999, Hewitt and Ibrahim 2001, Ursenbacher et al. 2006), there is no information on belowground species and therefore it remains unknown if the identified patterns also apply to soil animals which typically are much smaller than those aboveground (Whitfield 2005).

Oribatid mites are soil-dwelling predominantly detritivorous and fungivourous microarthropods (Maraun and Scheu 2000, Schneider et al. 2004) and fossils of oribatid mites date back to Devonian sediments 380 million years ago (mya) (Shear et al.1984, Norton et al. 1988a), but the origin of this group is dated back to 440 mya (Lindquist 1984) and to even 570 mya using molecular clock analysis (Schaefer et al. 2010). They reach densities of up to 400,000 individuals per square meters in temperate and boreal forest soils (Maraun and Scheu 2000) and 80\% of the individuals reproduce by parthenogenesis (Domes-Wehner 2009).

To investigate if sexual and asexual oribatid mite species colonized Europe differently after the last glaciations, a mt gene (COI) was sequenced from two sexual and two parthenogenetic oribatid mite species of Europe, since parthenogenetic species have a number of advantages compared to sexual species, e.g. the twofold advantage of not producing males (Williams 1975, Maynard Smith, 1978, 1998, Tagg et al. 2005), faster colonization of habitats and easier establishment of new populations (Williams 1975; Bell 1982; Scheu and Schulz 1996; Lindberg and Bengtsson 2005, Schön 2007) and they coexist in the same habitat with sexual species (Domes-Wehner 2009). In total 558 individuals (100 N. silvestris, 160 P. peltifer, 141 A. coleoptrata and 157 S. magnus) from 65 locations in Europe and two east Russian locations were analyzed. I assumed that the $\mathrm{mt}$ variance is similar in 
Chapter 4 Sex vs parthenogenesis

parthenogenetic and sexual species since mitochondria are inherited maternally. Sampling in Central Europe was extensive in order to detect cryptic refugia which were proposed for Central and Northern Europe (Stewart and Lister 2001; Tollefsrud et al. 2008). I expected lineages which survived in Central refugia to contain isolated haplotypes that are distinct from populations from Southern Europe and Central Asia and that genetic diversity within this species' range is highest in these former refugial areas (Stewart et al. 2010).

\subsection{Materials and methods}

\subsubsection{Taxon sampling}

Oribatid mites were sampled from 65 locations in Europe and two locations in Russia (Fig. 22, described in more detail in Table 14). A. coleoptrata was sampled from 40 locations, $N$. silvestris from 26 locations, $P$. peltifer from 41 locations and S. magnus from 37 locations. Specimens were extracted from leaf litter using life extraction along a heat gradient (Macfadyen 1961, Kempson et al. 1963). Carabodes femoralis, Carabodes marginatus, Hypochthonius rufulus, Nothrus palustris and Rhysotritia duplicata were sampled as outgroups for phylogenetic analyses. Animals were identified under a stereomicroscope, determined after Weigmann (2006) and stored in $75 \%$ ethanol at $-20^{\circ} \mathrm{C}$ until preparation. 


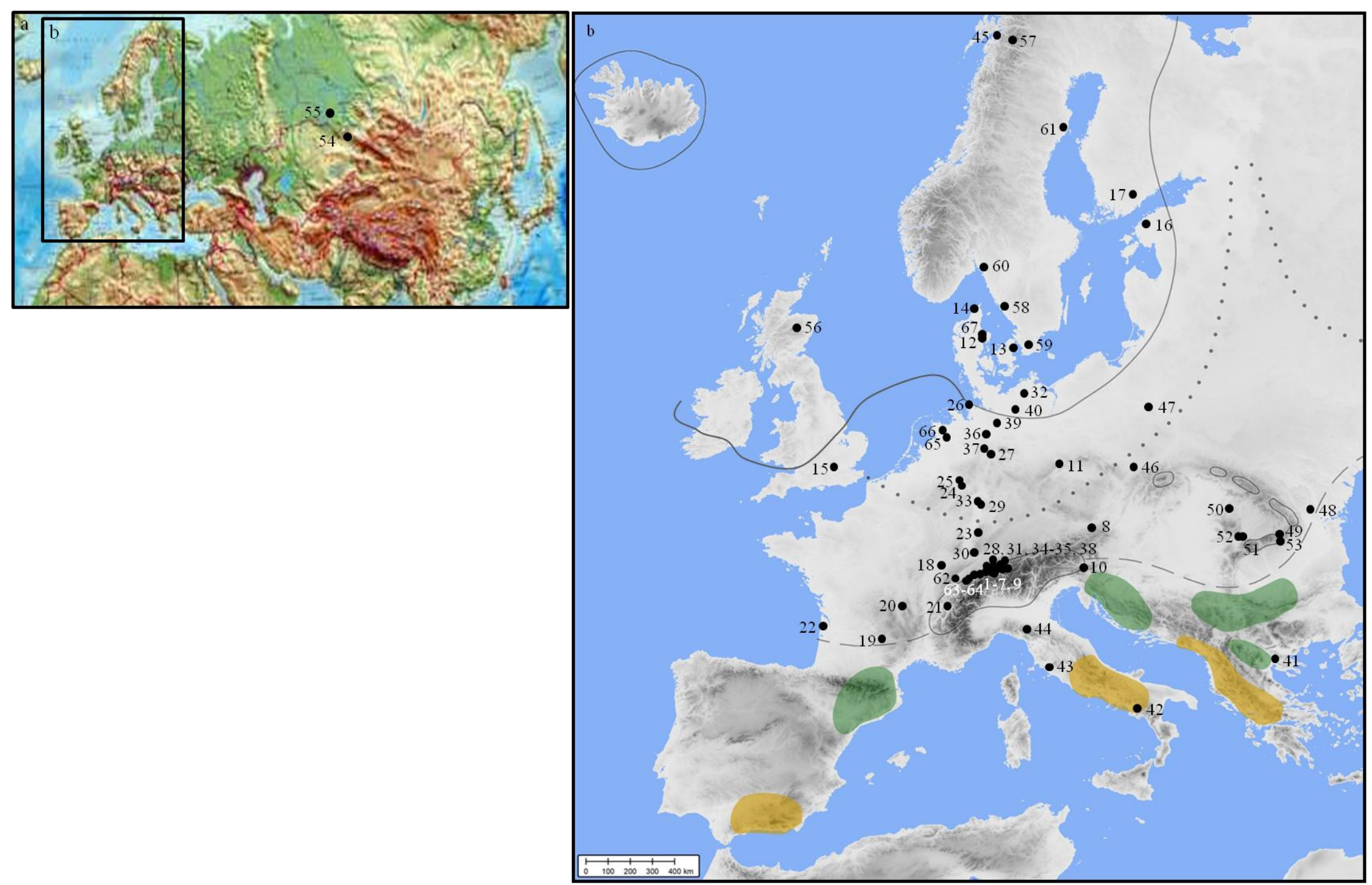

Figure 22: (a) Map of Europe and Russia and (b) map of Europe from the last glacial maximum (modified after Hewitt 1999). Black dots with numbers represent the sampling points of oribatid mites used in this study, green shading marks refugia of coniferous trees and yellow shading those of deciduous trees, grey lines mark the expansion of glaciers, the dotted line represents the polar desert climate border and the dashed line the tundra and permafrost border (Hewitt and Ibrahim 2001, Alexander Kartographie 2006). 
Table 14: Localities where oribatid mites analyzed in this study were sampled [ln: location number, country, location, coordinates, abbreviations of sampling locations (code) and name of collector (for details see Acknowledgements)]. Each location is marked by a colour symbol (AC=Achipteria coleoptrata, NS=Nothrus silvestris, $\mathrm{PP}=$ Platynothrus peltifer, $\mathrm{SM}=$ Steganacarus magnus, outgroups: $\mathrm{CF}=$ Carabodes femoralis, $\mathrm{CM}=$ Carabodes marginatus, $\mathrm{HR}=$ Hypochthonius rufulus, $\mathrm{NP}=$ Nothrus palustris and $\mathrm{RD}=$ Rhysotritia duplicata).

\begin{tabular}{|c|c|c|c|c|c|c|c|}
\hline In & country & location & coordinates & code & species & colour & litter collector \\
\hline 1 & Austria & Bregenz & $47.49^{\circ} 9.70^{\circ}$ & A 2 & PP & 0 & I. Schaefer \\
\hline 2 & & Glacier & $47.17^{\circ} 10.53^{\circ}$ & A 9 & PP & 0 & I. Schaefer \\
\hline 3 & & Hittisau & $47.46^{\circ} 9.95^{\circ}$ & A 3 & $\mathrm{AC}, \mathrm{PP}$ & O & I. Schaefer \\
\hline 4 & & Holzgau & $47.29^{\circ} 10.33^{\circ}$ & A 4 & PP & ○ & I. Schaefer \\
\hline 5 & & Imst & $47.24^{\circ} 10.69^{\circ}$ & A 8 & $\mathrm{AC}$ & O & B. Eitzinger \\
\hline 6 & & Landeck & $47.13^{\circ} 10.57^{\circ}$ & A 6 & $\mathrm{AC}$ & 0 & I. Schaefer \\
\hline 7 & & Memminger Huette & $47.21^{\circ} 10.46^{\circ}$ & A 5 & $\mathrm{AC}, \mathrm{PP}$ & 0 & I. Schaefer \\
\hline 8 & & Roggendorf & $48.20^{\circ} 15.37^{\circ}$ & A 7 & $\mathrm{AC}$ & $\ominus$ & E. Latz \\
\hline 9 & & Stuiben & $47.52^{\circ} 10.18^{\circ}$ & A 10 & PP & ○ & I. Schaefer \\
\hline 10 & & Villach & $46.57^{\circ} 13.85^{\circ}$ & A 1 & PP, SM & $\ominus$ & K. Domes-Wehner \\
\hline 11 & Czech Republic & Decin & $50.78^{\circ} 14.23^{\circ}$ & $\mathrm{CZ} 1$ & $\mathrm{SM}$ & $\oplus$ & M. Rosenberger \\
\hline 12 & Denmark & Arhus & $56.11^{\circ} 10.21^{\circ}$ & DK 3 & $\mathrm{AC}, \mathrm{PP}, \mathrm{SM}$ & O & T. Bilde \\
\hline 13 & & Copenhagen & $55.68^{\circ} 12.58^{\circ}$ & DK 1 & SM & ○ & N. Eisenhauer \\
\hline 14 & & Hjørring & $57.48^{\circ} 9.96^{\circ}$ & DK 2 & $\mathrm{AC}, \mathrm{PP}, \mathrm{SM}$ & O & M. Rosenberger \\
\hline 15 & England & Ascot & $51.40^{\circ}-0.68^{\circ}$ & GB 1 & $\mathrm{AC}, \mathrm{NS}, \mathrm{PP}, \mathrm{SM}$ & $\bigoplus$ & A. Milcu \\
\hline 16 & Estonia & Tallin & $59.43^{\circ} 24.69^{\circ}$ & EST 1 & $\mathrm{AC}$ & & A. Micic \\
\hline 17 & Finland & Lahti & $60.99^{\circ} 25.65^{\circ}$ & FIN 1 & $\mathrm{AC}, \mathrm{NS}, \mathrm{PP}, \mathrm{SM}$ & & H. Setela \\
\hline 18 & France & Brunstatt & $47.71^{\circ} 7.32^{\circ}$ & F 5 & $\mathrm{PP}$ & 0 & B. Lerb \\
\hline 19 & & Haute Loire & $44.99^{\circ} 3.86^{\circ}$ & F 4 & $\mathrm{AC}, \mathrm{SM}$ & $\theta$ & A. Jousset \\
\hline 20 & & Loire & $45.56^{\circ} 4.79^{\circ}$ & F 2 & $\mathrm{AC}, \mathrm{SM}$ & O & M.Maraun \\
\hline 21 & & Mont Blanc & $45.82^{\circ} 6.74^{\circ}$ & F 1 & $\mathrm{AC}, \mathrm{PP}, \mathrm{SM}$ & & A. Jousset \\
\hline 22 & & Saint Isidore & $45.27^{\circ}-1.09^{\circ}$ & F 3 & NS, PP, SM & 0 & C. Digl \\
\hline 23 & Germany & Black Forest & $48.89^{\circ} 8.43^{\circ}$ & D 6 & NS, PP, SM & 0 & K. Heidemann \\
\hline 24 & & Bonn & $50.84^{\circ} 7.14^{\circ}$ & D 9 & $\mathrm{AC}, \mathrm{NS}, \mathrm{PP}, \mathrm{SM}$ & (I) & R. Koller \\
\hline 25 & & Colonge & $50.83^{\circ} 7.18^{\circ}$ & D 10 & $\mathrm{AC}, \mathrm{NS}$ & & M.Maraun \\
\hline 26 & & Cuxhaven & $53.86^{\circ} 8.65^{\circ}$ & D 8 & AC, NS, PP, SM & $\oplus$ & K. Heidemann \\
\hline 27 & & Goettingen & $51.53^{\circ} 9.96^{\circ}$ & D 2 & $\mathrm{AC}, \mathrm{NS}, \mathrm{PP}, \mathrm{SM}$ & & K. Schneider \\
\hline 28 & & Gunzestied & $47.51^{\circ} 10.23^{\circ}$ & D 13 & $\mathrm{AC}$ & (1) & I. Schaefer \\
\hline 29 & & Kranichstein & $49.89^{\circ} 8.69^{\circ}$ & D 1 & $\mathrm{AC}, \mathrm{NS}, \mathrm{PP}, \mathrm{SM}$ & & M. Rosenberger \\
\hline 30 & & Lake Constance & $47.71^{\circ} 9.37^{\circ}$ & D 3 & $\mathrm{AC}, \mathrm{NS}, \mathrm{PP}, \mathrm{SM}$ & & K. Heidemann \\
\hline 31 & & Langenwang & $47.43^{\circ} 10.28^{\circ}$ & D 15 & $\mathrm{AC}, \mathrm{PP}$ & ค & I. Schaefer \\
\hline 32 & & Mecklen. Seenplatte & $53.57^{\circ} 12.33^{\circ}$ & D 4 & NS, PP, SM & & I. Schaefer \\
\hline 33 & & Moerfelden & $49.96^{\circ} 8.55^{\circ}$ & D 5 & $\mathrm{AC}, \mathrm{PP}, \mathrm{SM}$ & & K. Domes-Wehner \\
\hline 34 & & Rubi & $47.43^{\circ} 10.28^{\circ}$ & D 17 & NS, PP & (8) & I. Schaefer \\
\hline 35 & & Steineberg & $47.52^{\circ} 10.19^{\circ}$ & D 14 & $\mathrm{AC}$ & Q & I. Schaefer \\
\hline 36 & & Steinhuder Meer & $52.48^{\circ} 9.38^{\circ}$ & D 18 & NS, PP & & E. Latz \\
\hline 37 & & Solling & $51.76^{\circ} 9.57^{\circ}$ & D 16 & NS & $\Theta$ & K. Schneider \\
\hline 38 & & Sonthofen & $47.46^{\circ} 10.22^{\circ}$ & D 12 & $\mathrm{AC}, \mathrm{PP}$ & 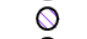 & I. Schaefer \\
\hline 39 & & Uelzen & $52.97^{\circ} 10.52^{\circ}$ & D 7 & NS, PP, SM & & H. Treptow \\
\hline 40 & & Wittmoor & $53.19^{\circ} 11.83^{\circ}$ & D 11 & $\mathrm{AC}, \mathrm{NS}$ & 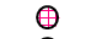 & K. Schneider \\
\hline 41 & Greece & Thessaloniki & $40.64^{\circ} 22.97^{\circ}$ & GR 1 & $\mathrm{AC}$ & & M. Tsiafaoli \\
\hline 42 & Italy & Felitto & $40.37^{\circ} 15.24^{\circ}$ & I 3 & $\mathrm{AC}$ & 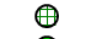 & G. Humpert \\
\hline 43 & & Grosseto & $42.63^{\circ} 11.11^{\circ}$ & I 1 & $\mathrm{AC}, \mathrm{PP}, \mathrm{SM}$ & & M.Maraun \\
\hline 44 & & Parma & $44.20^{\circ} 10.34^{\circ}$ & I 2 & $\mathrm{AC}, \mathrm{SM}$ & & M.Maraun \\
\hline 45 & Norway & Narvik & $68.44^{\circ} 17.40^{\circ}$ & N 1 & $\mathrm{PP}, \mathrm{SM}$ & 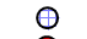 & O. Butenschoen \\
\hline 46 & Poland & Krakov & $50.04^{\circ} 19.84^{\circ}$ & PL 1 & $\mathrm{AC}, \mathrm{SM}$ & & S. Scheu \\
\hline 47 & & Warsaw & $52.33^{\circ} 20.76^{\circ}$ & PL2 & $\mathrm{AC}, \mathrm{NS}, \mathrm{PP}, \mathrm{SM}$ & & A. Uvarov \\
\hline 48 & Romania & Bagau & $46.55^{\circ} 26.72^{\circ}$ & RUM 3 & SM & 0 & T. Pasca \\
\hline 49 & & Busteni & $45.41^{\circ} 25.55^{\circ}$ & RUM 5 & $\mathrm{AC}, \mathrm{NS}, \mathrm{PP}, \mathrm{SM}$ & (1) & C. Ivanescu \\
\hline 50 & & Cluj & $46.77^{\circ} 23.52^{\circ}$ & RUM 4 & SM & $\oplus$ & T. Pasca \\
\hline 51 & & Sibiu 1 & $45.64^{\circ} 23.74^{\circ}$ & RUM 1 & $\mathrm{AC}, \mathrm{NS}, \mathrm{SM}$ & & S. Scheu \\
\hline 52 & & Sibiu 2 & $45.65^{\circ} 23.70^{\circ}$ & RUM 2 & $\mathrm{AC}, \mathrm{NS}, \mathrm{PP}, \mathrm{SM}$ & & S. Scheu \\
\hline 53 & & Sinaia & $45.35^{\circ} 25.56^{\circ}$ & RUM 6 & AC, PP, SM & & C. Ivanescu \\
\hline 54 & Russia & Altai Mountains & $51.73^{\circ} 85.76^{\circ}$ & RUS 1 & SM & 0 & M. Ackermann \\
\hline 55 & & Novosibirsk & $54.97^{\circ} 83.39^{\circ}$ & RUS 2 & $\mathrm{AC}, \mathrm{SM}$ & $\oplus$ & A. Uvarov \\
\hline 56 & Scotland & Braemar & $57.00^{\circ}-3.40^{\circ}$ & GB 2 & $\mathrm{AC}, \mathrm{NS}, \mathrm{PP}, \mathrm{SM}$ & & A. Jousset \\
\hline 57 & Sweden & Abisko & $68.35^{\circ} 18.82^{\circ}$ & S 4 & $\mathrm{PP}$ & & O. Butenschoen \\
\hline 58 & & Gothenburg & $57.67^{\circ} 11.97^{\circ}$ & S 5 & PP & & P. Pachl \\
\hline 59 & & Malmoe & $56.00^{\circ} 13.14^{\circ}$ & S 3 & NS & & N. Lindberg \\
\hline 60 & & Stroemstad & $58.87^{\circ} 11.14^{\circ}$ & S 2 & $\mathrm{AC}, \mathrm{PP}, \mathrm{SM}$ & 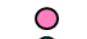 & G. Kalinkat \\
\hline 61 & & Umeå & $63.83^{\circ} 20.29^{\circ}$ & S 1 & $\mathrm{PP}, \mathrm{SM}$ & & O. Butenschoen \\
\hline 62 & Switzerland & Basel & $47.50^{\circ} \quad 7.59^{\circ}$ & $\mathrm{CH} 1$ & $\mathrm{AC}$ & & M. Maraun \\
\hline 63 & & Buesingen & $47.69^{\circ} 8.69^{\circ}$ & $\mathrm{CH} 2$ & NS & $\oplus$ & P. Pachl \\
\hline 64 & & Rorschach & $47.47^{\circ} 9.53^{\circ}$ & $\mathrm{CH} 3$ & $\mathrm{AC}, \mathrm{NS}, \mathrm{PP}$ & (1) & I. Schaefer \\
\hline 65 & The Netherlands & Hoge Veluwe & $52.02^{\circ} 5.87^{\circ}$ & NL 2 & NS, PP & $\ominus$ & M. Maraun \\
\hline 66 & & Wageningen & $51.97^{\circ} \quad 5.70^{\circ}$ & NL 1 & NS, PP, SM & $\oplus$ & O. Butenschoen \\
\hline 67 & Denmark & Arhus Stadion & $56.13^{\circ} 10.19^{\circ}$ & DK 4 & NP & & T. Bilde \\
\hline 27 & Germany & Goettingen & $51.53^{\circ} 9.96^{\circ}$ & D 2 & NP & & M. Rosenberger \\
\hline 29 & & Kranichstein & $49.89^{\circ} 8.69^{\circ}$ & D 1 & $\mathrm{CF}, \mathrm{CM}, \mathrm{HR}, \mathrm{RD}$ & & M. Rosenberger \\
\hline 47 & Poland & Warsaw & $52.33^{\circ} 20.76^{\circ}$ & PL2 & NP & & A. Uvarov \\
\hline
\end{tabular}




\subsubsection{DNA extraction and sequencing}

Genomic DNA was extracted from single individuals using the DNeasy ${ }^{\circledR}$ Blood and Tissue Kit (Qiagen; Hilden, Germany) following the manufacturer's protocol for animal tissue. For A. coleoptrata a $512 \mathrm{bp}$, for $N$. silvestris a $581 \mathrm{bp}$ and for $S$. magnus a $531 \mathrm{bp}$ region of cytochrome c oxidase subunit I (COI) was amplified using the primers COIarch1 (5'GGTCAACAAATCATAAAGAYATYGG-3') and COIarch2 (5'TAAACTTCAGGGTGACCAAAAAATCA-3') (Heethoff et al. 2007) and the HotStarTaq ${ }^{\circledR}$ Master Mix Kit (1.25 units HotStarTaq ${ }^{\circledR}$ polymerase, $100 \mu \mathrm{M}$ of each dNTP and $7.5 \mathrm{mM} \mathrm{MgCl}_{2}$ buffer solution; Qiagen; Hilden, Germany) and the SuperHot Taq Mastermix [2.5 units SuperHot Taq polymerase, $10 \mu \mathrm{M}$ of each dNTP and buffer solution (20 mM Tris-HCI (pH 8.3), $100 \mathrm{mM} \mathrm{KCl}, 0.1$ mM EDTA, $1 \mathrm{mM}$ DTT, 50\% glycerol, 0.5\% Nonidet P40 and 0.5\% Tween 20), Genaxxon; Ulm, Germany], respectively. For $P$. peltifer a 558 bp region of cytochrome c oxidase subunit I (COI) was amplified using the primers COIarch1 (5'-GGTCAACAAATCATAAAGAYATYGG-3') and Plat 52 (5'-ATAAATCCTAAGGATCATAGC-3') (Domes-Wehner 2009). The polymerase chain reaction (PCR) contained $0.5 \mu \mathrm{l}$ of each primer $(100 \mathrm{pmol} / \mu \mathrm{l}), 1 \mu 1 \mathrm{MgCl}_{2}(25 \mathrm{mM}), 12.5 \mu 1$ of HotStarTaq ${ }^{\circledR}$ Master Mix or SuperHot Taq Mastermix containing polymerase, $3 \mu 1$ template DNA and filled up to $25 \mu 1$ with RNase free water. PCR parameters included a $15 \mathrm{~min}$ step at $95^{\circ} \mathrm{C}$ for polymerase activation followed by 36 cycles of $30 \mathrm{~s}$ at $94^{\circ} \mathrm{C}$ for denaturation, $60 \mathrm{~s}$ at $51^{\circ} \mathrm{C}$ for primer annealing and $60 \mathrm{~s}$ at $72^{\circ} \mathrm{C}$ for elongation and a final $10 \mathrm{~min}$ step for elongation at $72^{\circ} \mathrm{C}$. The PCR products were visualized by $1 \%$ agarose gel electrophoresis and purified using the QIAquick ${ }^{\circledR}$ PCR Purification Kit (Qiagen; Hilden, Germany) following the manufacturer's protocol. The purified PCR products were sequenced in both directions by Macrogen Inc. (Seoul, Korea) and the Department of Experimental Phycology and Culture Collection of Algae (Georg-August-University Göttingen, Germany).

\subsubsection{Phylogeographic, population genetic and statistical analyses}

Nucleotide sequences were edited and translated into amino acids using the invertebrate mitochondrial code implemented in SEQUENCHER v4.9 (Gene Codes) and aligned with ClustalX v1.81 (Thompson et al. 1997) using multiple alignment parameters: 10.0 for gap opening and 0.1 for gap extension for the nucleotide, default settings for the amino acids dataset. Phylogenetic trees were generated with Beast v1.5.4 (Drummond and Rambaut 2007), MrBayes v3.1.2 (Ronquist and Huelsenbeck 2003) and PAUP* (Swofford 1999) with the NJ analyses without and with model of sequence evolution to identify monophyletic clusters in the dataset. The best model of sequence evolution was estimated with Modeltest 3.6 (Posada and Crandall 1998) for PAUP* and MrModeltest (Nylander 2004) for the Bayesian analyses. The best fit model was GTR $+\mathrm{I}+\mathrm{G}$ for both analyses. Model parameters were nst=6 rates=invgamma for nucleotide sequences of S. magnus. For $N$. silvestris the best fit model was $\mathrm{HKY}+\mathrm{G}$ in both analyses and model parameters were $\mathrm{nst}=2$ rates=gamma for the nucleotide. The best fit model for PAUP* was $\mathrm{HKY}+\mathrm{G}$ and for MrBayes $\mathrm{GTR}+\mathrm{G}$ for the nucleotide dataset of P. peltifer. Model parameters were nst $=6$ rates $=$ gamma for nucleotide sequences of $P$. peltifer . The best fit model for PAUP* was TVM+I+G for MrBayes $\mathrm{GTR}+\mathrm{I}+\mathrm{G}$ for the nucleotide dataset of $A$. coleoptrata. Model parameters were nst=6 rates=invgamma for nucleotide sequences of $A$. coleoptrata. All protein alignments were analyzed with aamodel=equalin in MrBayes and mtrev in Beast. One individual of H. rufulus (C.L. Koch, 1835) (Oribatida, Enarthronota) and R. duplicata (Grandjean, 1953) (Oribatida, Mixonomata) were used as outgroups for P. peltifer and S. magnus. For A. coleoptrata outgroups were one individual of $C$. femoralis (Nicolet, 1855) (Oribatida, Brachypylina) and C. marginatus (Michael, 1884) (Oribatida, Brachypylina); for N. silvestris four individuals of $N$. palustris (C.L. Koch, 1839) (Oribatida, Desmonomata) were used as outgroup. The Markov Chain Monte Carlo was run for ten million generations and sampled every $1000^{\text {th }}$ generation, 
the 50\% majority consensus tree excluded the first 2,500,000 trees (burnin of 25\%) in MrBayes and $10 \%$ burnin in Beast.

Standard diversity indices for the nucleotide sequences [haplotype number $\left(\mathrm{N}_{\mathrm{h}}\right)$, haplotype diversity $\left(\mathrm{H}_{\mathrm{d}}\right.$, nucleotide diversity $\left(\Pi_{\mathrm{n}}\right)$, number of variable $\left(\mathrm{N}_{\mathrm{vs}}\right)$ and invariable sites $\left(\mathrm{N}_{\mathrm{is}}\right)$, parsimony informative sites $\left(\mathrm{N}_{\text {pars }}\right)$, the number of singletons $\left(\mathrm{N}_{\mathrm{s}}\right)$ and the total number of substitutions $\left(\mathrm{N}_{\mathrm{m}}\right)$ ] and the McDonald-Kreitman (MK) test, to detect selection, were calculated with DNASP v5.0 (Rozas et al. 2003). In the McDonald-Kreitman test selection is detected by examining the distribution of synonymous and non-synonymous substitutions among populations; the test is robust against demographic and recombination events (McDonald and Kreitman 1991). The geographical structure of genetic diversity among and within populations and geographical clades were calculated with ARLEQUIN v3.01 (Excoffier et al. 2005) using analysis of molecular variance (AMOVA, 16.000 permutations); populations with less than two sampled individuals were excluded from the AMOVA. Estimates for demographic expansion (Tajima's D and Fu's $F_{s}$ neutrality tests) and pairwise differences ( $\mathrm{F}_{\text {ST }} 10.000$ permutations) were also calculated in ARLEQUIN. I calculated significance with 10.000 permutations.

The dataset exceeded the connection limit of TCS (Clement et al. 2000); therefore a parsimony based median-joining haplotype network (Bandelt et al. 1999) was generated in NETWORK v4.5 (FluxusTechnology; Suffolk, UK) with default settings for nucleotide sequences and amino acids.

To investigate the effect of the number of sample locations and the richness of haplotypes Jackknife rarefaction curves were calculated using the program EstimateSWin820 with default settings for the four nucleotide and protein alignments (Colwell, 1994-2004. EstimateS: statistical estimation of species richness and shared species from samples (http://viceroy.eeb.uconn.edu/estimates).

Geographical distances of the populations were generated by GenAlEx6 (Peakal and Smouse 2006). I analyzed the associations of genetic differentiation at mitochondrial markers with geographical distances using the Mantel test implemented in the program Isolation by Distances version 1.52 (IBD 1.52, Bohonak 2002) or with Isolation by Distances Web Service version 3.16 (http://ibdws.sdsu.edu/ ibdws/) (Jensen et al. 2005) with default settings for the four oribatid mites. Graphical constructions were generated with Statistica 7 (Stat Soft Inc, 2002).

Delimitation of mtDNA clusters on the COI trees with the generalized mixed Yule coalescent method (GMYC) (Pons et al. 2006, Fontaneto et al. 2007, Papadopoulou et al. 2009) were done with the R package 'splits' (SPecies LImits by Threshold Statistics, http://r-forge.r-project.org/projects/splits/) in R 2.11.1 (R Development Core Team 2010, http://www.R-project.org) for the four oribatid mite species. Ultrametric trees were generated with the strict clock method in Beast.

\subsection{Results}

\subsubsection{Nothrus silvestris}

A total of 104 individuals (100 individuals of $N$. silvestris and four individuals of $N$. palustris as outgroup) from 26 locations in ten countries were sequenced. The sequences contained $29.1 \% \mathrm{~A}, 25 \%$ $\mathrm{C}, 17.6 \% \mathrm{G}$ and $28.4 \% \mathrm{~T}$. The $100 \mathrm{COI}$ nucleotide sequences consisted of 25 haplotypes for the nucleotide (25\%) (Fig. 23) with 45 variable sites (7.75\%); 21 of these were parsimony informative (Table A19). The protein consisted of eight haplotypes (8\%) in the network (Fig. 24). The haplotype diversity $\left(\mathrm{H}_{\mathrm{d}}\right)$ was high with 0.67 in the nucleotide. 


\section{Haplotype networks}

The nucleotide haplotype network formed four subclades and two isolated one individual haplotypes which were separated each by seven or more substitution steps (Fig. 23). The isolated haplotypes comprised only one individual from Germany and one from France (D_4.5, F_3.4). Subclade 1 comprised nine haplotypes with a starlike arrangement. Six haplotypes comprised one individual from different locations (one English GB_1.3, one Romanian RUM_1.3, one Swedish S_3.2, one Swiss CH_3.2 and two German D_6.2 and D_16.1). Two individuals from different locations in Germany (D_4.2/D_17.2) and four individuals from one location in Romania also were of the same haplotype (RUM_5.1-4). The last haplotype comprised 57 individuals from 19 locations of South-east, Central and Northern Europe [twelve individuals are one Polish location (PL_2.1-5, 2.7-13); four individuals each from one Finish (FIN_1.1-4), one Romanian (RUM_2.1-4) and three German locations (D_1.1-4, D_9.1-4 and D_18.1-4); three individuals each from one Dutch (NL_1.1-3) and two German locations (D_8.1-3 and D_16.2-4); two individuals each from one English (GB_1.2, 1.4), one German (D_4.4, 4.7), one Scottish (GB_2.1, 2.4), one Romanian (RUM_1.1-2), one Swedish (S_3.1, 3.4) and one Swiss (CH_2.1-2) location; the other four individuals were from one Dutch (NL_2.2), one Swiss (CH_3.1) and two German locations(D_3.1 and D_17.3)]. Subclade 2 comprised four haplotypes with a starlike arrangement (one big haplotype with three surrounding haplotypes). All three satellite haplotypes were from the same German location (D_4). Two of the three satellite haplotypes comprised one individual (D_4.6 and D_4.8). The third satellite haplotype comprised two individuals (D_4.1 and D_4.3). The fourth haplotype comprised ten individuals from seven locations (CH_2, D_6, D_7, D_10, GB_1, NL_1 and S_3) [four individuals were from the same German location (D_7.1-4); the other six were from six different locations from Central and Northern Europe (one Dutch NL_2.1, one English GB_1.1, one Swedish S_3.3 one Swiss CH_2.3 and two German D_6.1 and D_10.1)]. Subclade 3 comprised seven haplotypes from four locations, two German (D_2 and D_11), one French (F_3) and one Polish (PL_2). One haplotype comprised four individuals from two German locations (D_2.2/D_11.1/D_11.3-4). Another haplotype comprised two individuals from the same location (F_3.1-2). The other five haplotypes comprised one individual [two comprised individuals from one German location (D_2.1 and D_2.4); one comprised one individual from another German location (D_11.2); one comprised one Polish individual (PL_2.6) and one comprised one French individual (F_3.3)]. Subclade 4 comprised three haplotypes from two locations in Germany (D_2 and D_17) and one location in Scotland (GB_2) [one comprised two individuals of the same haplotype, one from a German (D_2.3) and one from a Scottish location (GB_2.3); two comprised one individual each from a German and Scottish location (D_17.1, GB_2.2)].

The protein haplotype network formed eight haplotypes with a starlike arrangement (Fig. 24). Five haplotypes comprised one individual from four locations [one English (GB_1.3), one Scottish /GB_2.2) and two German (D_4.6, 4.8 and D_17.1)]. One comprised four individuals from three German locations (D_2.1, 2.4, D_11.2 and D_16.1). One comprised twelve individuals from eight locations [four individuals from one German location (D_7.1-4); two individuals from another German location (D_4.1 and 4.3); six individuals from six different locations (one Dutch NL_2.1, one English GB_1.1, one Swedish S_3.3, one Swiss CH_2.3 and two German D_6.1 and D_10.1)]. The Central haplotype comprised 79 individuals from 24 locations [all 13 Polish individuals (PL_2.1-13), all eleven Romanian individuals from the three locations (RUM_1.1-3, RUM_2.1-4 and RUM_5.1-4), all four French individuals (F_3.1-4), all four Finish individuals (FIN_1.1-4), 31 individuals from eleven German locations (D_1.1-4, D_2.2-3, D_3.1, D_4.2, 4.4-5, 4.7, D_6.2, D_8.1-3, D_9.1-4, D_11.1, 11.3-4, D_16.2-4. D_17.2-3 and D_19.1-4), five individuals from two locations in Great Britain (GB_1.2, 1.4 and GB_2.1 and 2.3-4), four individuals from two Dutch locations (NL_1.1-3 and NL_2.2), four individuals from two Swiss locations (CH_2.1-2 and $\left.\mathrm{CH}_{-} 3.1-2\right)$ and three 
Chapter 4 Sex versus parthenogenesis

individuals from one Swedish location (S_3.1-2,3.4)]. The other haplotypes were connected by one or two amino acid changes to the Central haplotype.

\section{Phylogenetic and population genetic analyses}

All phylogenetic analyses (NJ with and without model of sequence evolution, MrBayes and Beast) showed a maximum supported monophyletic group of $N$. silvestris with four subclades and two isolated individuals in the nucleotide (Fig. 25, A22-25). The four subclades were supported by high bootstrap values and posterior probabilities. Only the arrangement of the four subclades was variable in the analyses. The individuals which built these clades are presented in Table 15. In the phylogenetic trees of the protein all sequences were grouped in one highly supported monophyletic clade (Fig. 26, A26-28).

The minimum and maximum mean average pairwise differences for the nucleotide sequences between populations were $0 \%$ (D_1/D_8, D_1/D_9, D_1/D_16, D_1/D_18, D_1/FIN_1, D_1/NL_1, D_1/RUM_2, D_8/D_9, D_8/D_16, D_8/D_18, D_8/FIN_1, D_8/NL_1，D_8/RUM_2, D_9/D_16, D_9/D_18, D_9/FIN_1, D_9/NL_1, D_9/RUM_2, D_16/D_18, D_16/FIN1, D_16/NL_1, D_16/RUM_2, D_18/FIN_1, D_18/NL_1, D_18/RUM_2, FIN_1/NL_1，FIN_1/RUM_2 and NL_1/RUM_2) and $1.8 \%$ (CH_3/D_2). Within populations the minimum and maximum mean average pairwise differences were 0\% (D_1, D_7, D_8, D_9, D_18, FIN_1, NL_1, RUM_2 and RUM_5) and $1.7 \%$ (D_6) (Table 16). For the protein the minimum and maximum mean average pairwise differences between populations were $0 \%$ (all populations except for GB_2) and $0.13 \%$ (each population to population GB_2). The minimum and maximum mean average pairwise differences within population were $0 \%$ (all populations except for GB_2) and $0.26 \%$ (GB_2) for the protein (Table A20).

The results of the AMOVA showed that the nucleotide variation among samples within countries (53.9\%) and the variation within samples $(57.2 \%)$ were significant and high. In contrast, variation among countries was not significant (Table 17). 


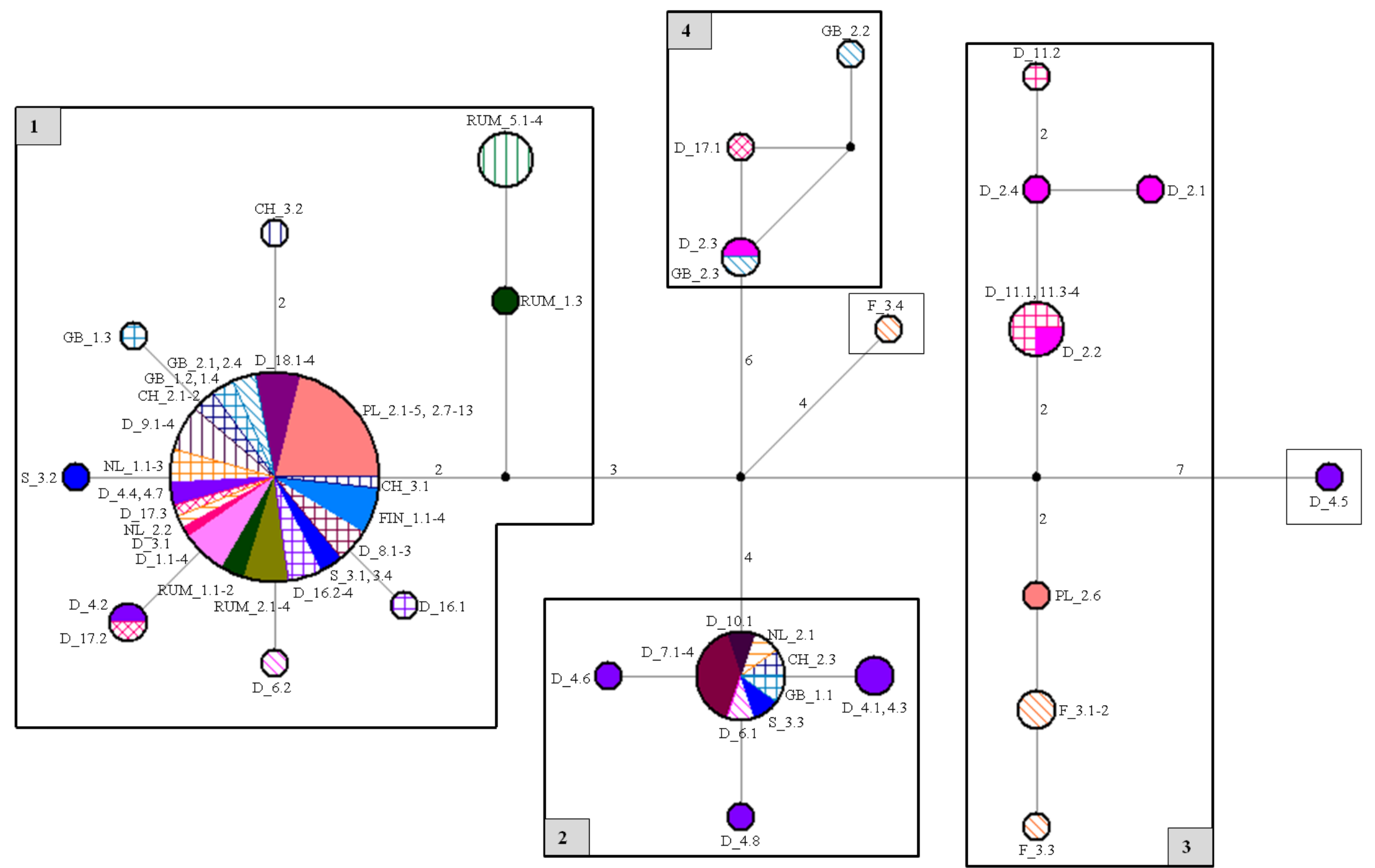

Figure 23: Median-joining haplotype network for the COI nucleotide of 25 haplotypes from Nothrus silvestris. Each location has a specific colour marking. The size of the circles is proportional to the number of sequences per haplotype. Numbers on the lines represent the number of substitution steps separating the haplotypes (no number: only one substitution step between haplotypes). Major subclades are marked by boxes. 


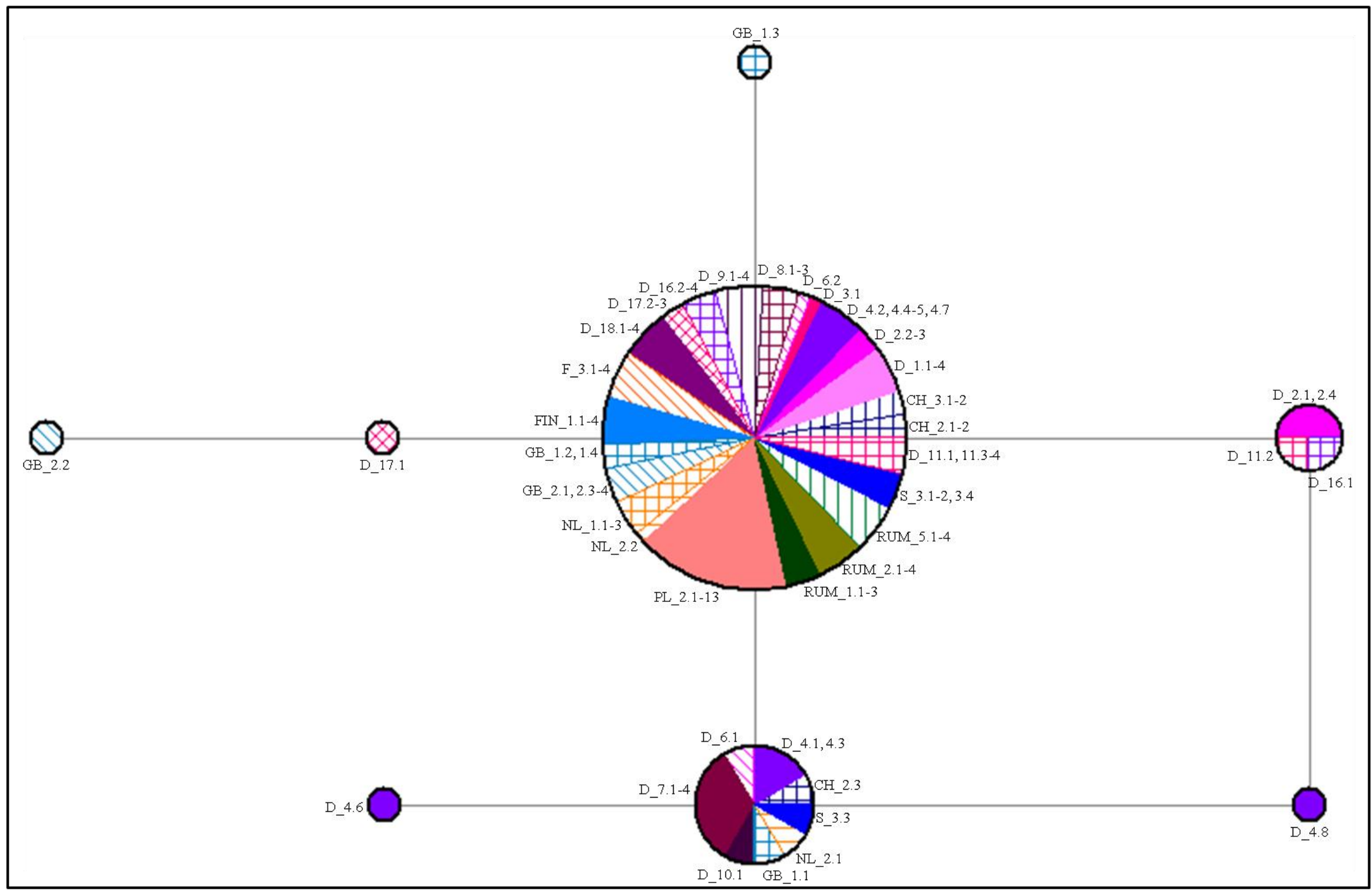

Figure 24: Median-joining haplotype network for the COI protein of eight haplotypes from Nothrus silvestris. Each location has a specific colour marking. The size of the circles is proportional to the number of sequences per haplotype. Numbers on the lines represent the number of substitution steps separating the haplotypes (no number: only one amino acid change between haplotypes). 
Chapter 4 Sex versus parthenogenesis

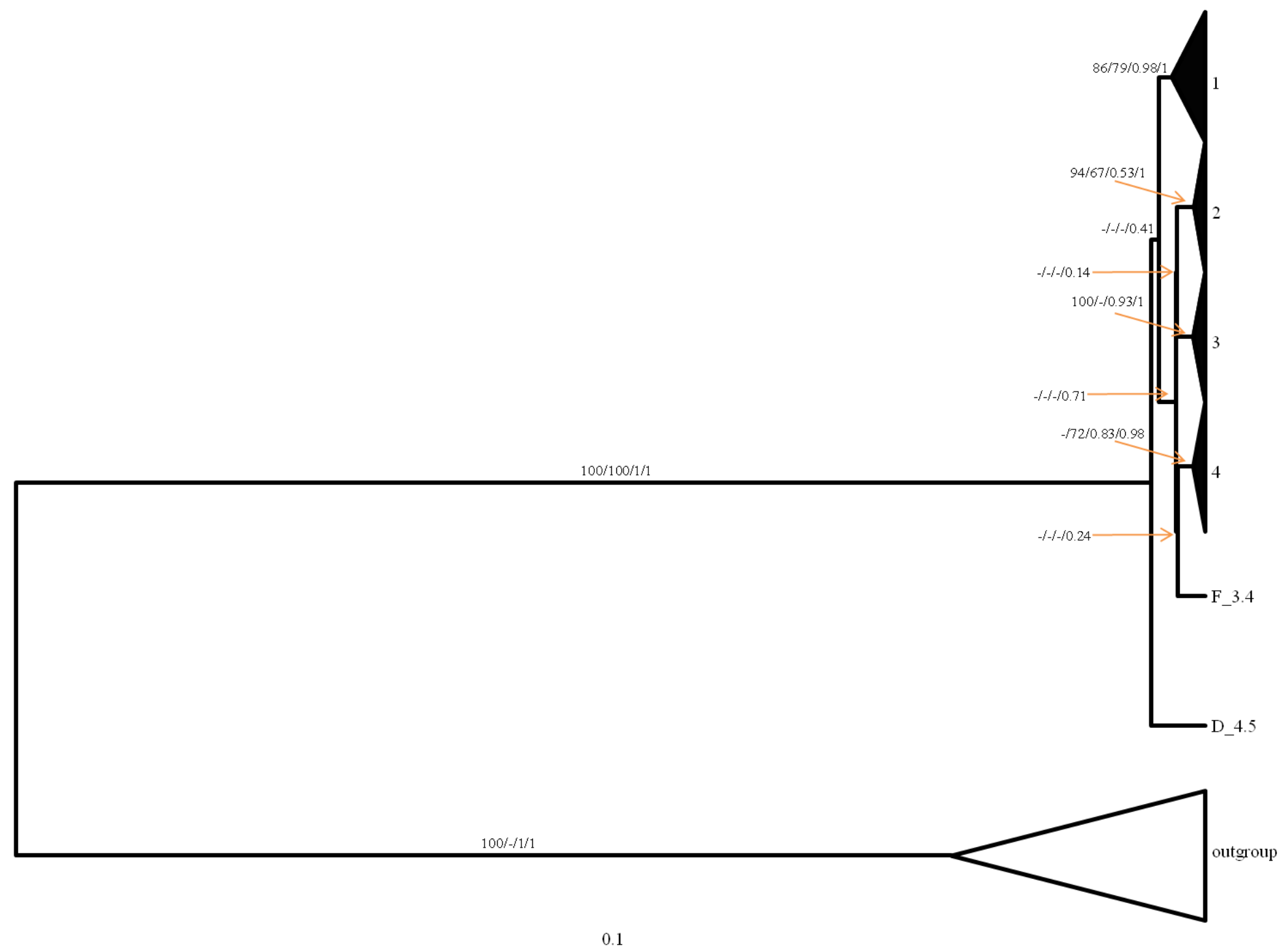

Figure 25: Bayesian phylogeny after 10x $10^{6}$ generations from the $100 \mathrm{COI}$ nucleotide sequences of Nothrus silvestris with Beast v1.5.4. Outgroups are four individuals of $N$. palustris from different locations. Numbers on the branches are bootstrap values from $\mathrm{NJ}$ without and with evolution model (HKY+G) analysis and posterior probabilities from MrBayes and Beast. Tip numbers are the different subclades and explained in Table 15. 


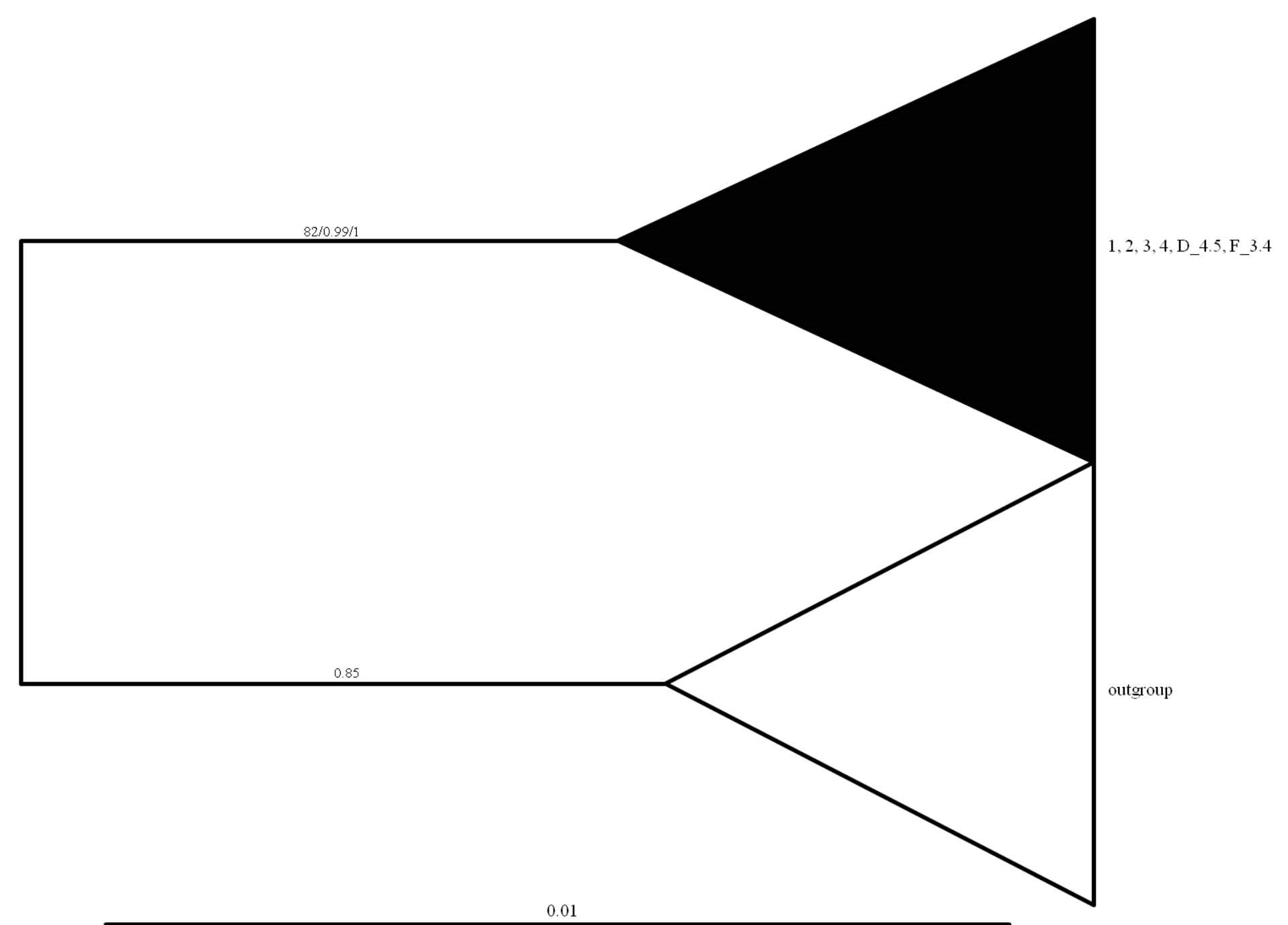

Figure 26: Bayesian phylogeny after 10x1 $10^{6}$ generations from the $100 \mathrm{COI}$ protein sequences of Nothrus silvestris with Beast v1.5.4. Outgroups are four individuals of $N$. palustris from different locations. Numbers on the branches are bootstrap values from $\mathrm{NJ}$ and posterior probabilities from MrBayes and Beast. Tip numbers are the different subclades and explained in Table 15. 
Table 15: Subclades of Bayesian phylogenetic trees of Nothrus silvestris based on COI nucleotide sequences (\#ind=number or individuals, $\mathrm{pp}=$ posterior probabilities, sampling sites with abbreviations, individuals and ind. pop.=quantity of individuals from the population).

\begin{tabular}{|c|c|c|c|c|c|c|}
\hline \multirow{2}{*}{$\frac{\text { Subclade }}{1}$} & \multirow{2}{*}{$\frac{\# \text { ind }}{69}$} & \multirow{2}{*}{$\frac{p p}{1}$} & \multicolumn{2}{|c|}{ sampling sites } & \multirow{2}{*}{$\frac{\text { individuals }}{1-2}$} & \multirow{2}{*}{$\frac{\text { ind. pop. }}{2 / 3}$} \\
\hline & & & Buesingen & CH_2 & & \\
\hline & & & Rorschach & CH_3 & $1-2$ & $2 / 2$ \\
\hline & & & KW & D_1 & $1-4$ & $4 / 4$ \\
\hline & & & Lake Constance & D_3 & 1 & $1 / 1$ \\
\hline & & & Meckl. Seenplatte & D_4 & $2,4,7$ & $3 / 8$ \\
\hline & & & Black Forrest & D_6 & 2 & $1 / 2$ \\
\hline & & & Cuxhaven & D_8 & $1-3$ & $3 / 3$ \\
\hline & & & Bonn & D_9 & $1-4$ & $4 / 4$ \\
\hline & & & Solling & D_16 & $1-4$ & $4 / 4$ \\
\hline & & & Rubi & D_17 & $2-3$ & $2 / 3$ \\
\hline & & & Steinhuder Meer & D_18 & $1-4$ & $4 / 4$ \\
\hline & & & Lahti & FIN_1 & $1-4$ & $4 / 4$ \\
\hline & & & Ascot & GB_1 & $2-4$ & $3 / 4$ \\
\hline & & & Braemar & GB_2 & 1,4 & $2 / 4$ \\
\hline & & & Wageningen & NL_1 & 3 & $3 / 3$ \\
\hline & & & Hoge Veluwe & $\mathrm{NL} \_2$ & 2 & $1 / 2$ \\
\hline & & & Warsaw & PL_2 & $1-5,7-13$ & $12 / 13$ \\
\hline & & & Sibiu_1 & RUM_1 & $1-3$ & $3 / 3$ \\
\hline & & & Sibiu_2 & RUM_2 & $1-4$ & $4 / 4$ \\
\hline & & & Busteni & RUM_5 & $1-4$ & $4 / 4$ \\
\hline & & & Malmoe & S_3 & $1-2,4$ & $3 / 4$ \\
\hline \multirow[t]{7}{*}{2} & 14 & 1 & Buesingen & CH_2 & 3 & $1 / 3$ \\
\hline & & & Meckl. Seenplatte & D_4 & $1,3,6,8$ & $4 / 8$ \\
\hline & & & Black Forrest & D_6 & 1 & $1 / 2$ \\
\hline & & & Uelzen & D_7 & $1-4$ & $4 / 4$ \\
\hline & & & Cologne & D_10 & 1 & $1 / 1$ \\
\hline & & & Ascot & GB_1 & $2-4$ & $3 / 4$ \\
\hline & & & Hoge Veluwe & NL_2 & 1 & $1 / 2$ \\
\hline \multirow[t]{3}{*}{3} & 4 & 1 & Goettingen & D_2 & 3 & $1 / 4$ \\
\hline & & & Rubi & D_17 & 1 & $1 / 3$ \\
\hline & & & Braemar & GB_2 & $2-3$ & $2 / 4$ \\
\hline \multirow[t]{4}{*}{4} & 11 & 0.98 & Goettingen & D_2 & $1-2,4$ & $3 / 4$ \\
\hline & & & Wittmoor & D_11 & $1-4$ & $4 / 4$ \\
\hline & & & Saint Isidore & F_3 & $1-3$ & $3 / 4$ \\
\hline & & & Warsaw & PL_2 & 6 & $1 / 13$ \\
\hline isol. ind. & 1 & & Saint Isidore & F_3 & 4 & $1 / 4$ \\
\hline isol. ind. & 1 & & Meckl. Seenplatte & D_4 & 5 & $1 / 8$ \\
\hline
\end{tabular}

Table 17: AMOVA table on variations among countries and within populations in nucleotide (black) and protein (red) sequences of COI of Nothrus silvestris. Each population was considered as separate groups. Populations with less than two individuals were excluded from the analysis. Significance level is p<0.05 (d.f.: degree of freedom).

\begin{tabular}{lccccc}
\hline \multicolumn{1}{c}{ Source of variance } & d.f. & sum of squares & variance components percent of total variation & fixation indices \\
\hline among countries & $8 / 8$ & $41.18 / 0.12$ & $-0.28 \mathrm{Va} / 0 \mathrm{Va}$ & $-11.15 / 9.41$ & $\mathrm{FCT}:-0.11 / 0.09$ \\
among populations & $15 / 15$ & $96.49 / 0.11$ & $1.36 \mathrm{Vb}^{*} / 0 \mathrm{Vb}$ & $53.93 /-8.21$ & $\mathrm{FSC}: 0.49^{*} /-0.09$ \\
within population & $73 / 73$ & $105.37 / 0.75$ & $1.44 \mathrm{Vc} * / 0.01 \mathrm{Vc}$ & $57.22 / 98.74$ & $\mathrm{FST}: 0.43^{*} / 0.01$ \\
total & $96 / 96$ & $243.04 / 0.98$ & $2.52 / 0.01041$ & & \\
\hline
\end{tabular}




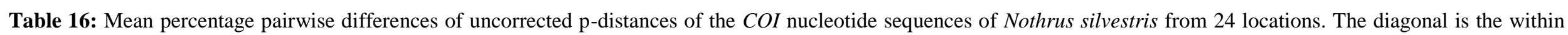

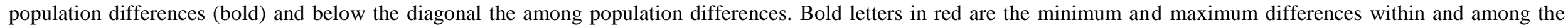
populations. Locations with less than two individuals were excluded from the analysis.

\begin{tabular}{|c|c|c|c|c|c|c|c|c|c|c|c|c|c|c|c|c|c|c|c|c|c|c|c|c|c|}
\hline \multicolumn{2}{|c|}{ Population } & \multirow{2}{*}{$\begin{array}{l}1 \\
1\end{array}$} & \multirow[t]{2}{*}{2} & \multirow[t]{2}{*}{3} & \multirow[t]{2}{*}{4} & \multirow[t]{2}{*}{5} & \multirow[t]{2}{*}{6} & \multirow[t]{2}{*}{7} & \multirow[t]{2}{*}{8} & \multirow[t]{2}{*}{9} & \multirow[t]{2}{*}{10} & \multirow[t]{2}{*}{11} & \multirow[t]{2}{*}{12} & \multirow[t]{2}{*}{13} & \multirow[t]{2}{*}{14} & \multirow[t]{2}{*}{15} & \multirow[t]{2}{*}{16} & \multirow[t]{2}{*}{17} & \multirow[t]{2}{*}{18} & \multirow[t]{2}{*}{19} & \multirow[t]{2}{*}{20} & \multirow[t]{2}{*}{21} & \multirow[t]{2}{*}{22} & \multirow[t]{2}{*}{23} & 24 \\
\hline 1 & $\mathrm{CH} 2$ & & & & & & & & & & & & & & & & & & & & & & & & \\
\hline 2 & CH 3 & 0.7 & 0.3 & & & & & & & & & & & & & & & & & & & & & & \\
\hline 3 & D 1 & 0.5 & 0.2 & 0 & & & & & & & & & & & & & & & & & & & & & \\
\hline 4 & D 2 & 1.6 & 1.8 & 1.6 & $\mathbf{1}$ & & & & & & & & & & & & & & & & & & & & \\
\hline 5 & D 4 & 1.1 & 1.3 & 1.1 & 1.7 & 1.4 & & & & & & & & & & & & & & & & & & & \\
\hline 6 & D 6 & 0.9 & 1 & 0.9 & 1.6 & 1.1 & 1.7 & & & & & & & & & & & & & & & & & & \\
\hline 7 & D 7 & 1 & 1.7 & 1.6 & 1.5 & 1 & 0.9 & 0 & & & & & & & & & & & & & & & & & \\
\hline 8 & D 8 & 0.5 & 0.2 & 0 & 1.6 & 1.1 & 0.9 & 1.6 & 0 & & & & & & & & & & & & & & & & \\
\hline 9 & D 9 & 0.5 & 0.2 & 0 & 1.6 & 1.1 & 0.9 & 1.6 & 0 & 0 & & & & & & & & & & & & & & & \\
\hline 10 & D 11 & 1.5 & 1.7 & 1.5 & 0.6 & 1.5 & 1.5 & 1.3 & 1.5 & 1.5 & 0.3 & & & & & & & & & & & & & & \\
\hline 11 & D 16 & 0.6 & 0.2 & 0 & 1.6 & 1.2 & 0.9 & 1.6 & 0 & 0 & 1.5 & 0.1 & & & & & & & & & & & & & \\
\hline 12 & D 17 & 1.1 & 0.9 & 0.8 & 1.6 & 1.5 & 1.3 & 1.7 & 0.8 & 0.8 & 1.7 & 0.8 & 1.5 & & & & & & & & & & & & \\
\hline 13 & D 18 & 0.5 & 0.2 & 0 & 1.6 & 1.1 & 0.9 & 1.6 & 0 & 0 & 1.5 & 0 & 0.8 & 0 & & & & & & & & & & & \\
\hline 14 & F 3 & 1.4 & 1.5 & 1.3 & 1.1 & 1.6 & 1.5 & 1.4 & 1.3 & 1.3 & 0.9 & 1.4 & 1.6 & 1.3 & 0.8 & & & & & & & & & & \\
\hline 15 & FIN 1 & 0.5 & 0.2 & 0 & 1.6 & 1.1 & 0.9 & 1.6 & 0 & 0 & 1.5 & 0 & 0.8 & 0 & 1.3 & 0 & & & & & & & & & \\
\hline 16 & GB 1 & 0.8 & 0.8 & 0.6 & 1.6 & 1.1 & 0.9 & 1.1 & 0.6 & 0.6 & 1.5 & 0.6 & 1.1 & 0.6 & 1.5 & 0.6 & 1.2 & & & & & & & & \\
\hline 17 & GB 2 & 1.3 & 1.2 & 1 & 1.6 & 1.6 & 1.5 & 1.7 & 1 & 1 & 1.7 & 1.1 & 1.1 & 1 & 1.6 & 1 & 1.3 & 1.4 & & & & & & & \\
\hline 18 & NL 1 & 0.5 & 0.2 & 0 & 1.6 & 1.1 & 0.9 & 1.6 & 0 & 0 & 1.5 & 0 & 0.8 & 0 & 1.3 & 0 & 0.6 & 1 & 0 & & & & & & \\
\hline 19 & NL 2 & 0.8 & 1 & 0.8 & 1.6 & 1 & 0.9 & 0.8 & 0.8 & 0.8 & 1.4 & 0.8 & 1.2 & 0.8 & 1.4 & 0.8 & 0.8 & 1.4 & 0.8 & 1.6 & & & & & \\
\hline 20 & PL 2 & 0.6 & 0.3 & 0.1 & 1.6 & 1.1 & 0.9 & 1.5 & 0.1 & 0.1 & 1.5 & 0.1 & 0.2 & 0.1 & 1.3 & 0.1 & 0.6 & 1.1 & 0.1 & 0.8 & 0.2 & & & & \\
\hline 21 & RUM 1 & 0.6 & 0.3 & 0.2 & 1.6 & 1.2 & 0.9 & 1.5 & 0.2 & 0.2 & 1.5 & 0.2 & 0.8 & 0.2 & 1.4 & 0.2 & 0.7 & 1.1 & 0.2 & 0.8 & 0.2 & 0.3 & & & \\
\hline 22 & RUM 2 & 0.5 & 0.2 & 0 & 1.6 & 1.1 & 0.9 & 1.6 & 0 & 0 & 1.5 & 0 & 0.8 & 0 & 1.3 & 0 & 0.6 & 1 & 0 & 0.8 & 0.1 & 0.2 & 0 & & \\
\hline 23 & RUM 5 & 1 & 0.9 & 0.7 & 1.6 & 1.4 & 1.2 & 1.6 & 0.7 & 0.5 & 1.5 & 0.7 & 1.2 & 0.7 & 1.6 & 0.7 & 1 & 1.4 & 0.7 & 1.1 & 0.7 & 0.5 & 0.7 & 0 & \\
\hline 24 & S 3 & 0.7 & 0.6 & 0.4 & 1.6 & 1.1 & 0.9 & 1.2 & 0.4 & 0.4 & 1.5 & 0.5 & 1 & 0.4 & 1.4 & 0.4 & 0.8 & 1.3 & 0.4 & 0.8 & 0.5 & 0.6 & 0.4 & 1 & 0.9 \\
\hline
\end{tabular}


The neutrality test of Tajima's D and Fu's FS neither were significant for the nucleotide nor for the protein (Table A21 and A22). The McDonald-Kreitman test was not significant for the whole dataset of N. silvestris (Table 19, for more detail Table A23). All neutrality indices which were computed were $\geq 0$ (Table A24) and indicated purifying selection.

None of the estimated rarefaction curves of the nucleotide and the protein levelled off (Fig. 27 and 28). The Jackknife rarefaction curves ended at 60 haplotypes for the nucleotide and 17 haplotypes for the protein. The unique rarefaction curves ended at 17 haplotypes for the nucleotide and five haplotypes for the protein. The observed rarefaction curves (Sobs Mao Tau) ended at 25 haplotypes for the nucleotide and eight haplotypes for the protein.

The results of the Mantel test showed no evidence for isolation by distance in $N$. silvestris $\left[\mathrm{R}^{2}=0.0066\right.$, $\mathrm{p}=0.27$ (Fig. A29) and $\mathrm{R}^{2}=0.0005, \mathrm{p}=0.411$ for $\log 10$ transformed geographical distance (Fig. 29) using 1000 randomizations].

The 'Generalized Mixed Yule Coalescent Model' (gmyc) had a significantly better fit in the single and the multiple analysis than the null model (Table 28). The model identified seven distinct mtDNA (COI) clusters for the single analysis and 19 for the multiple analysis. The clusters of the single and multiple analyses differed from the clusters which were delimited by the phylogenetic analyses (Fig. A30 and 31). The phylogenetic clade I was split in three distinct clusters and the phylogenetic clade IV was split in two distinct clusters in the single method. In the multiple method clade I was split into 14 distinct clusters, clade III shrunk to a two individual cluster and clade IV was split into three distinct clusters.

Table 18: Results of the generalized mixed Yule coalescent analyses of the four oribatid mite species (Nothrus silvestris, Platynothrus peltifer, Achipteria coleoptrata, Steganacarus magnus; $\log \mathrm{L}$ null=likelihood of the null model, $\log$ L GMYC=likelihood of the GMYC model, No. of clusters=number of GMYC clusters corresponding to the optimized threshold with confidence limits. Asterisks indicate the significance as assessed by the likelihood ratio test $* * * P \leq 0.001, * * P \leq 0.005, * P<0.01)$.

\begin{tabular}{llccc}
\hline Species & method & $\log \mathbf{L}($ null) & $\log$ L $($ GMYC) & No. of clusters \\
\hline N. silvestris & single & 950.0625 & 959.1814 & $7(2-19)^{* * *}$ \\
& multiple & 950.0625 & 960.703 & $19(7-22)^{* *}$ \\
P. peltifer & single & 1481.659 & 1497.187 & $6(4-12)^{* * *}$ \\
& multiple & 1481.659 & 1497.978 & $6(4-6)^{* * *}$ \\
A. coleoptrata & single & 1071.225 & 1074.535 & $5(1-13) \mathrm{ns}$ \\
& multiple & 1071.225 & 1076.376 & $30(5-31) \mathrm{ns}$ \\
S. magnus & single & 1123.333 & 1175.131 & $21(21-23)^{* * *}$ \\
& multiple & 1123.333 & 1177.836 & $26(22-26)^{* * *}$ \\
\hline
\end{tabular}


Chapter 4 Sex versus parthenogenesis

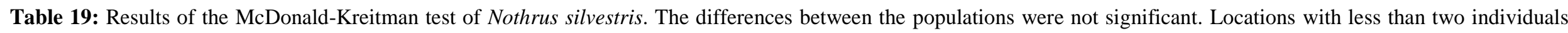
were excluded.

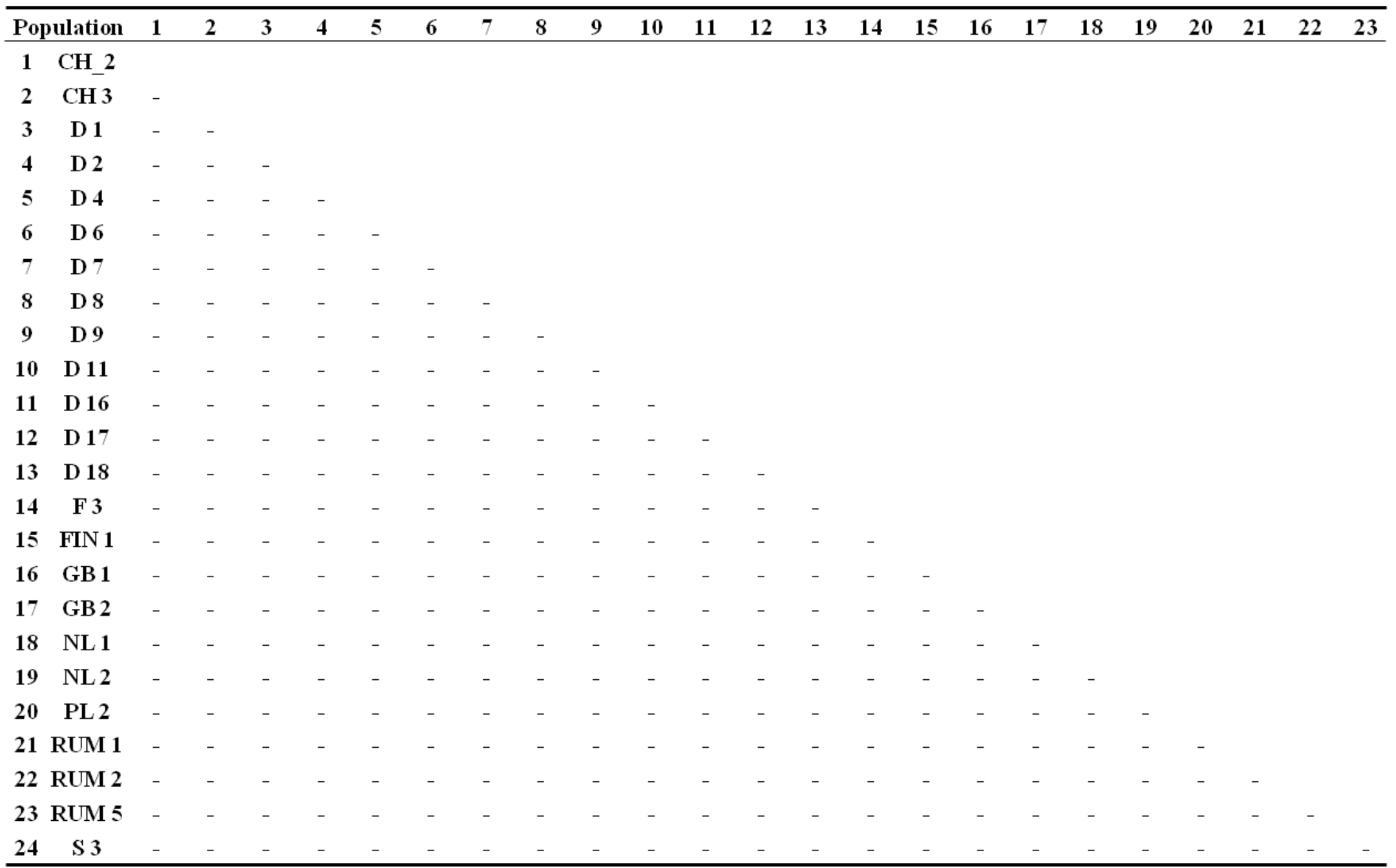


Chapter 4 Sex versus parthenogenesis

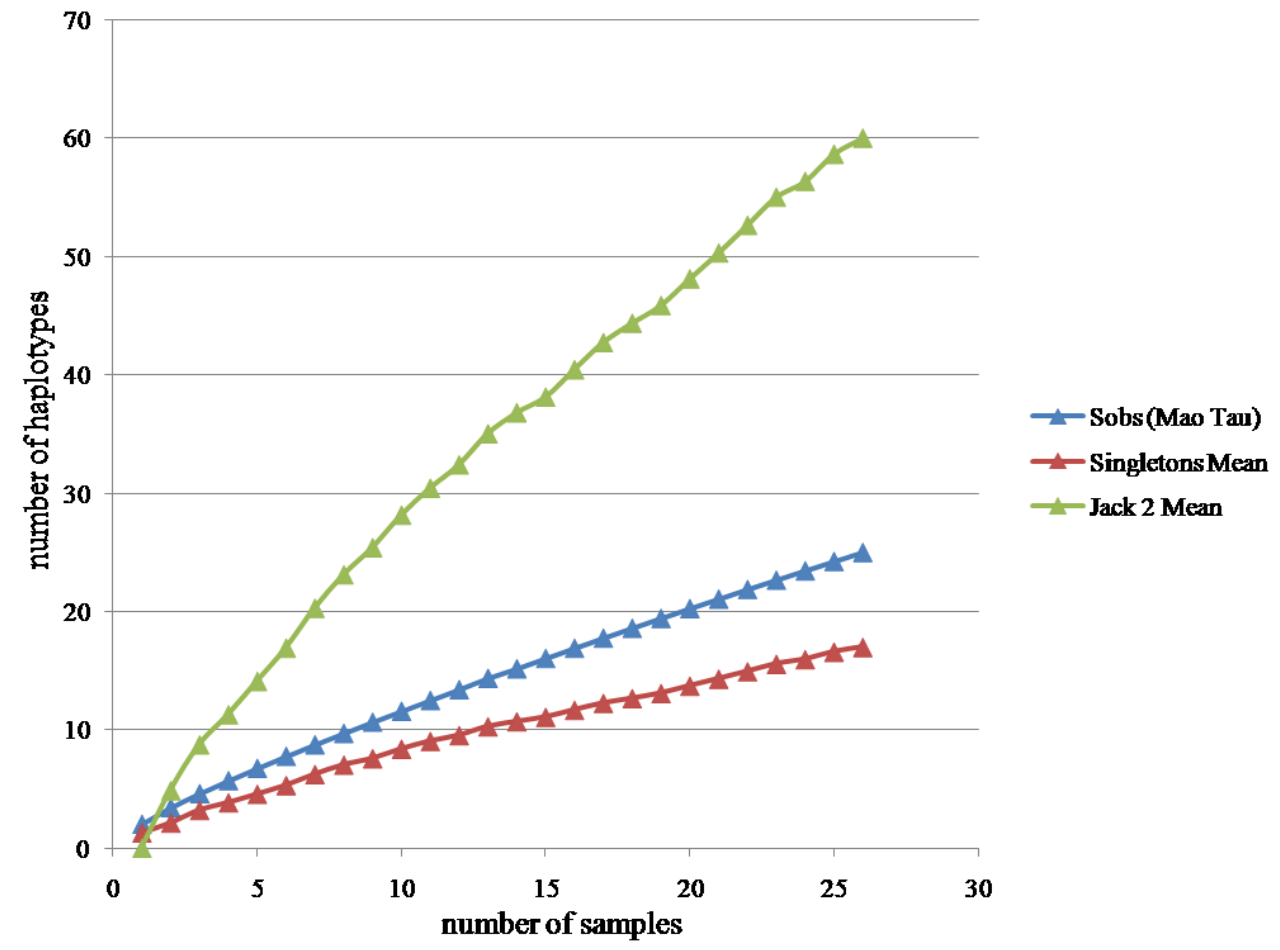

Figure 27: Sample based rarefaction analysis of haplotypes of the COI gene of Nothrus silvestris. Jack 2 Mean, second-order Jackknife richness estimator, Singletons, numbers of haplotypes each present in only one sample, Sob (Mao Tau) is the observed haplotype richness.

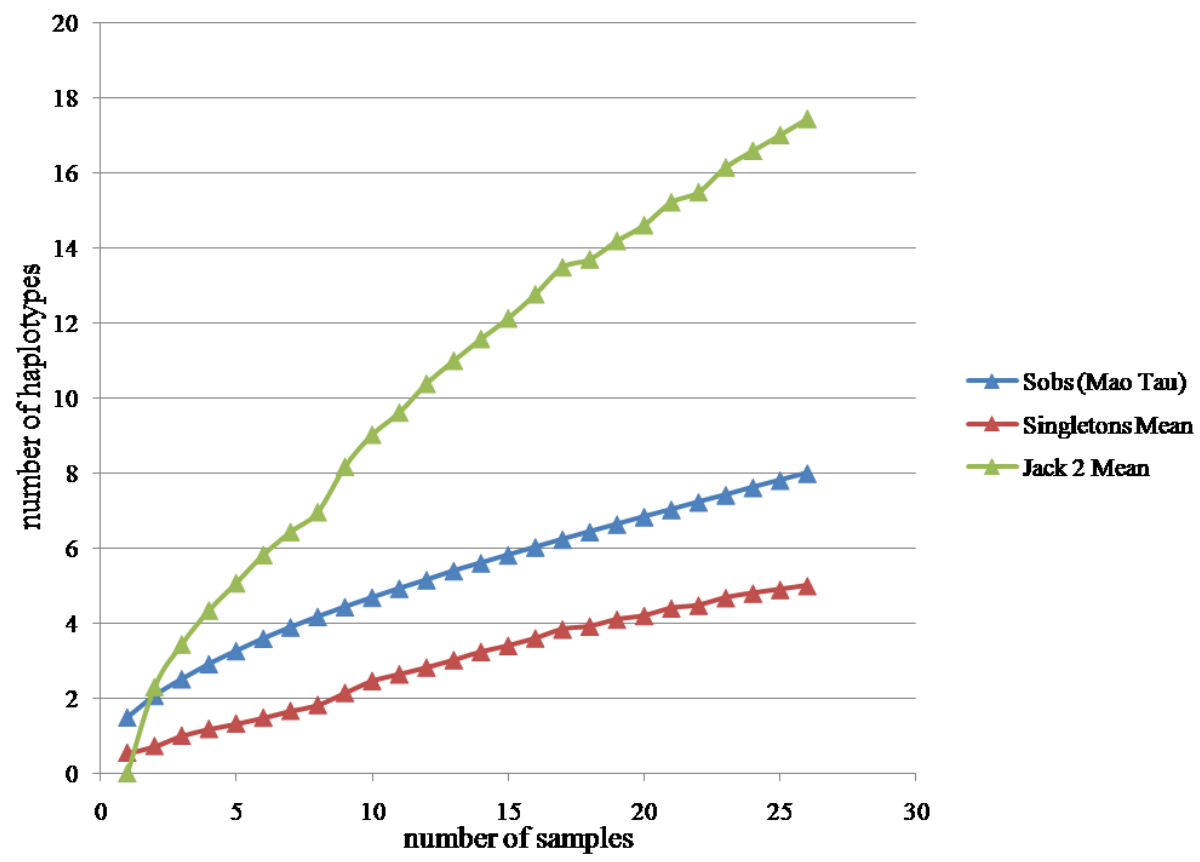

Figure 28: Sample based rarefaction analysis of haplotypes of the COI protein of Nothrus silvestris. Jack 2 Mean, second-order Jackknife richness estimator, Singletons, numbers of haplotypes each present in only one sample, Sob (Mao Tau) is the observed haplotype richness. 


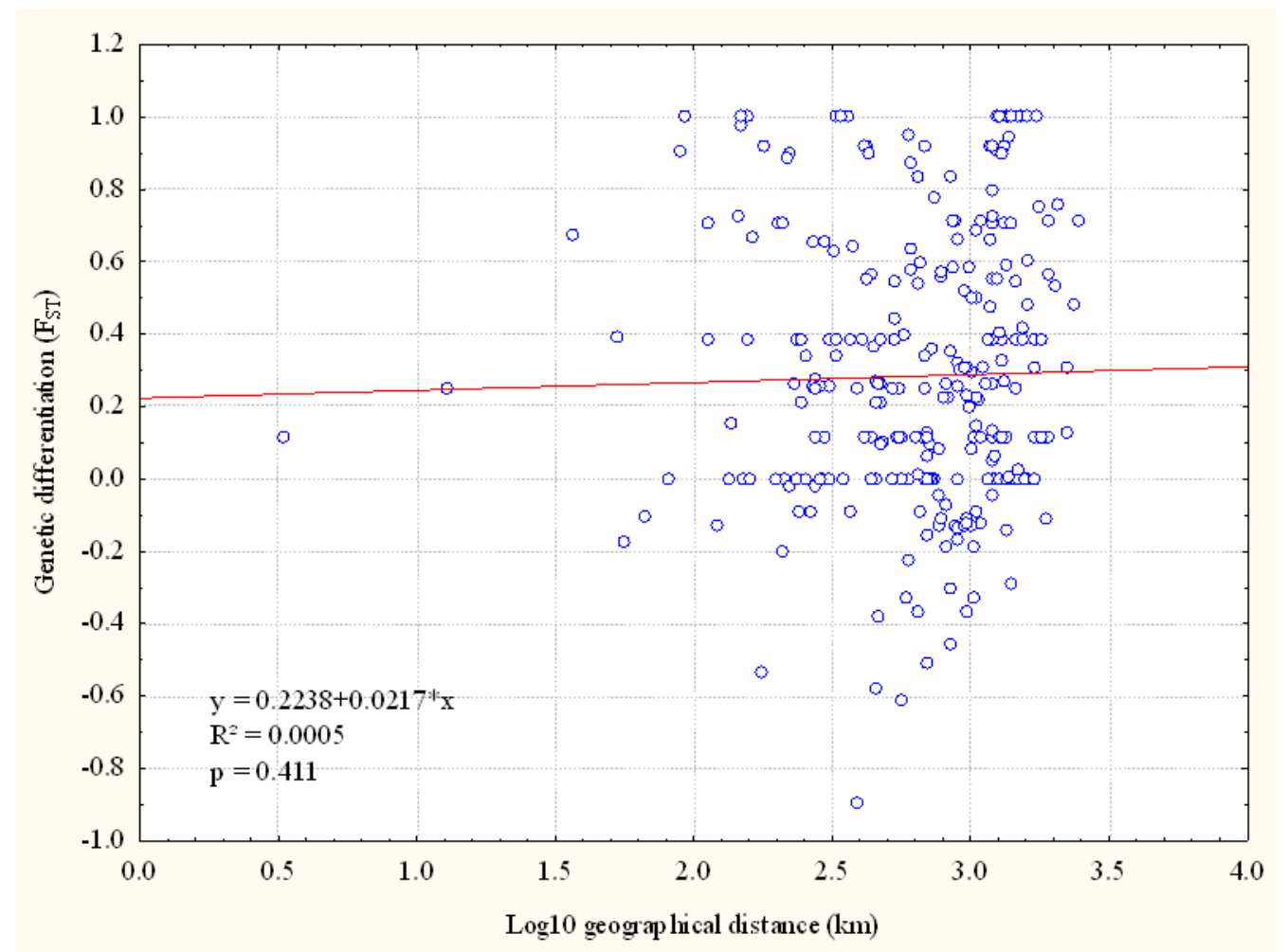

Figure 29: Linear regression of $\log 10$ geographical distances (kilometers) vs. genetic differentiations $\left(\mathrm{F}_{\mathrm{ST}}\right)$ based on COI nucleotide sequences of Nothrus silvestris. The regression is not significant using 1000 randomizations.

\subsubsection{Platynothrus peltifer}

A total of 162 individuals (160 individuals of $P$. peltifer, one individual of $H$. rufulus and one individual of $R$. duplicata as outgroup) from 41 locations in 14 countries were sequenced. The sequences contained $24.7 \% \mathrm{~A}, 25.6 \% \mathrm{C}, 18.5 \% \mathrm{G}$ and $31.2 \% \mathrm{~T}$. The $160 \mathrm{COI}$ sequences consisted of 75 haplotypes for the nucleotide (46.9\%) (Fig. 30) with 196 variable sites (35.3\%) and 172 parsimony informative sites (Table A25). The 160 sequences of the protein consisted of 18 haplotypes $(11.3 \%)$ (Fig. 31). Haplotypes diversity $\left(H_{d}\right)$ was very high with 0.98 in the nucleotide.

\section{Haplotype networks}

The nucleotide haplotype network formed six subclades and one isolated individual haplotype from a Swedish location (S_4.2) which were separated each by 16 or more substitution steps (Fig. 30). Subclade 1a comprised 20 single individual haplotypes and five haplotypes which comprised two to nine individuals from the same or different locations [single individual haplotypes: five from one Polish location (PL_2.1, PL_2.4, Pl_2.6, PL_2.8 and PL_2.9), two each from one Austrian (A_1.1-2) and French location (F_5.1 and F_5.3); one from a Danish (DK_2.5) and a Romanian (RUM_2.2) location, nine from six locations in Germany (D_1.3, D_1.4, D_4.1, D_5.1, D_5.2, D_5.4, D_6.5, D_11.1 and D_18.1). Haplotypes of more than one individual: two with two individuals each one Danish (DK_2.3-4) and one Polish (PL_2.5 and PL_2.7) site, one haplotype of four individuals from the one German location (D_6.1-4), one haplotype of seven individuals each one location in Italy (I_1.1), one location in Scotland (GB_2.2-4) and two locations in Germany (D_1.1 and D_2.1-2), one haplotype of nine individuals from one French (F_5.2) and four German locations (D_4.2-4, 4.6, 4.8, D_5.3, D_9.3 and D_18.2)]. Subclade 1b comprised 17 haplotypes from 15 locations [nine single individual haplotypes from seven different locations (one Dutch NL_2.1, one Finish FIN_1.4, one Scottish GB_2.1, one Swedish S_2.5, two Polish PL_2.2 and Pl_2.3 and three German D_8.1, D_8.3 and D_18.3), three haplotypes of two individuals each from one location (F_1.3-4, F_3.1-2 and 
NL_2.2-3) and one haplotype of two individuals from two locations (NL_2.4/S_2.6), one haplotype of three Finish individuals (FIN_1.1-3) and another of three individuals from three different locations (one Austrian A_5.3 and two German D_15.3 and D_17.1), one haplotype of four individuals from the same German location (D_18.4-7), one haplotype of seven individuals from four locations (one Danish DK_2.1-2 and 2.6, one English GB_1.1 and one German D_7.1-3), all other haplotypes of the subclade were connected starlike to the most abundant haplotype]. Subclade 1c comprised five single individual haplotypes from one Swedish location (S_2.1, S_2.2, S_2.3, S_2.4 and S_2.8).

Subclade 2 comprised four single individual haplotypes from four locations [one Austrian (A_5.2), one Norwegian (N_1.2) and two Swedish (S_1.2 and S_4.1)], one haplotype of three Swedish individuals from two locations (S_1.1 and $\left.S_{-} 2.7,2.9\right)$ and one haplotype of five individuals from four locations [one Austrian (A_5.1), one Norwegian (N_1.1) and two Swedish (S_1.3 and S_4.3-4)].

Subclade 3 differed from the other subclades by the high number of identical haplotypes shared by different individuals. Ten of 19 haplotypes were shared by 2-14 individuals and the haplotypes had a starlike arrangement with the haplotype shared by most individuals in the centre. The nine single individual haplotypes were from three German (D_3.1, 3.3, 3.4, D_11.2 and D_12.2), one Austrian (A_9.1 and A_9.5), one Romanian (RUM_6.1) and one Swedish (S_5.3) location. Two haplotypes were shared by two individuals [one by two individuals from the one location in the Netherlands (NL_1.1 and NL_1.3), one by two individuals from two German locations (D_1.2 and D_15.1)]. Three haplotypes were shared by four individuals from two to four locations [one was shared by three individuals from one German location (D_12.3-5) and one individual from an Austrian location (A_3.1), one shared by individuals from three locations, one Austrian (A_4.1-2), one German (D_15.2) and one Swiss (CH_3.3), one was shared by individuals from four locations, one Austrian (A_9.4), one Swedish (S_5.4) and two German (D_4.7 and D_17.3)]. Two haplotypes were shared by five individuals [one from one Austrian location (A_10.1-5), one three locations (two Austrian A_2.12 and A_3.2-3 and one German D_8.4)]. One haplotype was shared by six individuals from five locations [one Romanian (RUM_2.1), one Swedish (S_5.1-2), one Swiss (CH_3.5) and two German (D_3.2 and D_8.2)]. One haplotype was shared by seven individuals from two locations [one Danish (DK_3.1-6), one French (F_1.2)]. One haplotype comprised 14 individuals from nine locations [one English (GB_1.2-3), one Romanian (RUM_5.1), one Swiss (CH_3.1-2, 3.4), two Austrian (A_5.4, A_9.2-3), four German (D_4.5, D_9.1-2, D_12.1, D_17.2)].

Subclade 4 comprised two single individual haplotypes from two locations [one French (F_1.1), one Dutch (NL_1.2)]. Subclade 1a was connected to two other subclades by 24 (subclade 1b) and 95 substitution steps (subclade 3 ). Subclade $1 \mathrm{~b}$ was connected to two other subclades by 16 (subclade 1c) and 24 substitution steps (subclade 1a). Subclade 1c was connected to two other subclades by 16 (subclade 1b) and 71 substitution steps (subclade 2). Subclade 2 was connected to subclade 1c by 71 and to the single individual subclade (S_4.2) by 16 substitution steps. Subclade 3 was connected to two other subclades by 81 (subclade 4) and 95 substitution steps (subclade 1a). Subclade 4 was connected to subclade 3 by 81 substitution steps.

The protein haplotypes network comprised 18 haplotypes with a starlike arrangement (Fig. 31). Four of the 17 satellite haplotypes differed by two and one satelilite haplotype by three amnio acid changes from the Central haplotype. The other twelve satellite haplotype differed by one amino acid change from the Central haplotype. Four satellite haplotypes were shared by two individuals [three from one location, one Austrian (A_1.1-2), one Danish (DK_2.3-4) and one French (F_3.1-2), one from two locations, one German (D_12.2) and one Romanian (RUM_2.2)]. The other 13 satellite haplotypes were single individual haplotypes from nine locations [one Dutch location (NL_2.1), one Norwegian location (N_1.2), one Polish location (PL_2.2, PL_2.9), two Swedish locations (S_2.1, S_2.2, S_2.4, 
S_2.8 and S_4.1) and four German locations (D_3.3, D_6.5, D_8.1 and D_11.1)]. All other 139 individuals from the 40 locations were in the Central haplotype.

\section{Phylogenetic and population genetic analyses}

The phylogenetic analyses (NJ without and with model of sequence evolution, MrBayes and Beast) showed a highly supported monophyletic group of $P$. peltifer with six subclades and one isolated individual in the nucleotide (Fig. 32, A32-35). The clade 1a was paraphyletic in the Bayesian phylogeny (Fig. A34), but the complete clade 1 was monophyletic and highly supported. The six subclades were supported by high bootstrap values and posterior probabilities. The individuals which built these clades are presented in Table 20. In the phylogenetic trees of the protein all sequences were grouped in one highly supported monophyletic clade (Fig. 33, A36-38).

The minimum and maximum mean average pairwise differences for the nucleotide sequences between populations were $0.2 \%$ (A_2/A_3, A_4/CH_3, A_9/CH_3, D_2/F_5, D_7/F_3 and D_7/NL_2) and 20.4\% (DK_3/F_3). Within populations the minimum and maximum mean average pairwise differences were 0\% (A_2, A_4, A_10, D_2, D_7, DK_3 and F_3) and 18.5\% (D_11) (Table 21). For the protein the minimum and maximum mean average pairwise differences between populations were 0\% (all populations except for D_12, NL_2, PL_2 and RUM_2) and 0.41\% (NL_2/RUM_2). The minimum and maximum mean average pairwise differences within population were $0 \%$ (all populations except for D_12, NL_2, PL_2 and RUM_2) and 0.54\% for the protein (RUM_2) (Table A26). 


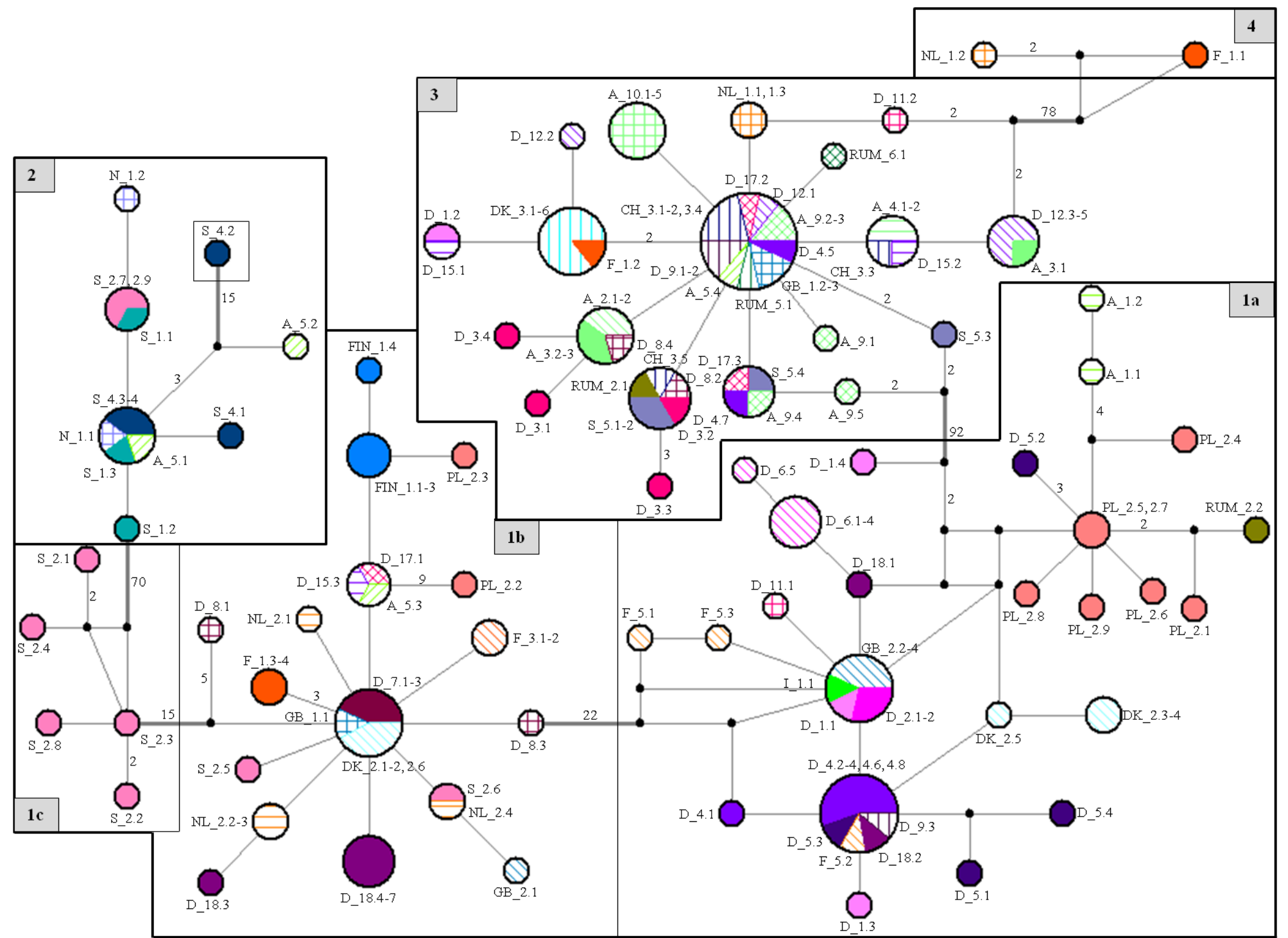

Figure 30: Median-joining haplotype network for the COI nucleotide of 75 haplotypes from Platynothrus peltifer. Each location has a specific colour marking. The size of the circles is proportional to the number of sequences per haplotype. Numbers on the lines represent the number of substitution steps separating the haplotypes (no number: only one substitution step between haplotypes). Major subclades are marked by boxes. 
Chapter 4 Sex versus parthenogenesis

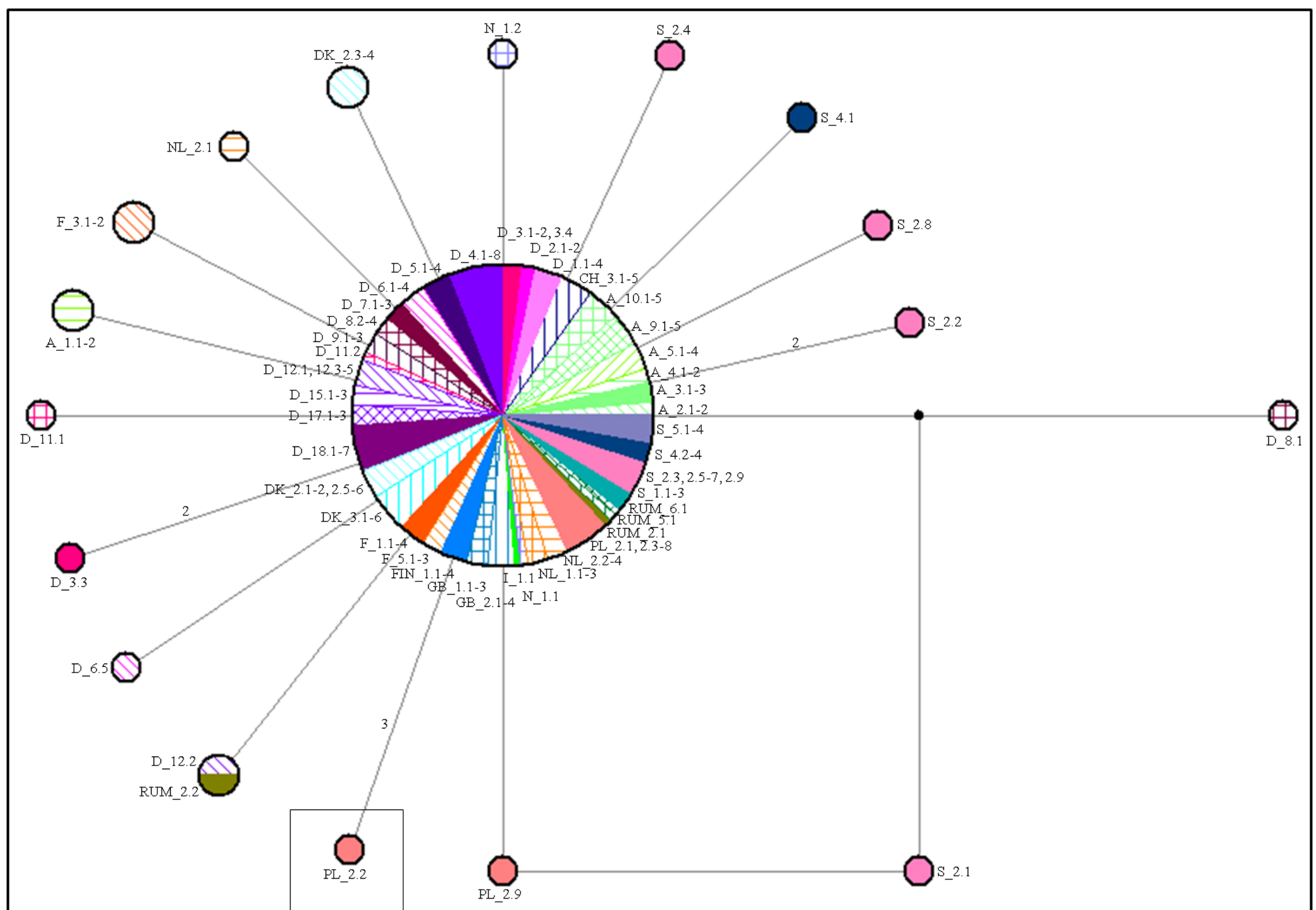

Figure 31: Median-joining haplotype network for the COI protein of 18 haplotypes from Platynothrus peltifer. Each location has a specific colour marking. The size of the circles is proportional to the number of sequences per haplotype. Numbers on the lines represent the number of substitution steps separating the haplotypes (no number: only one amino acid change between haplotypes). 
Chapter 4 Sex versus parthenogenesis

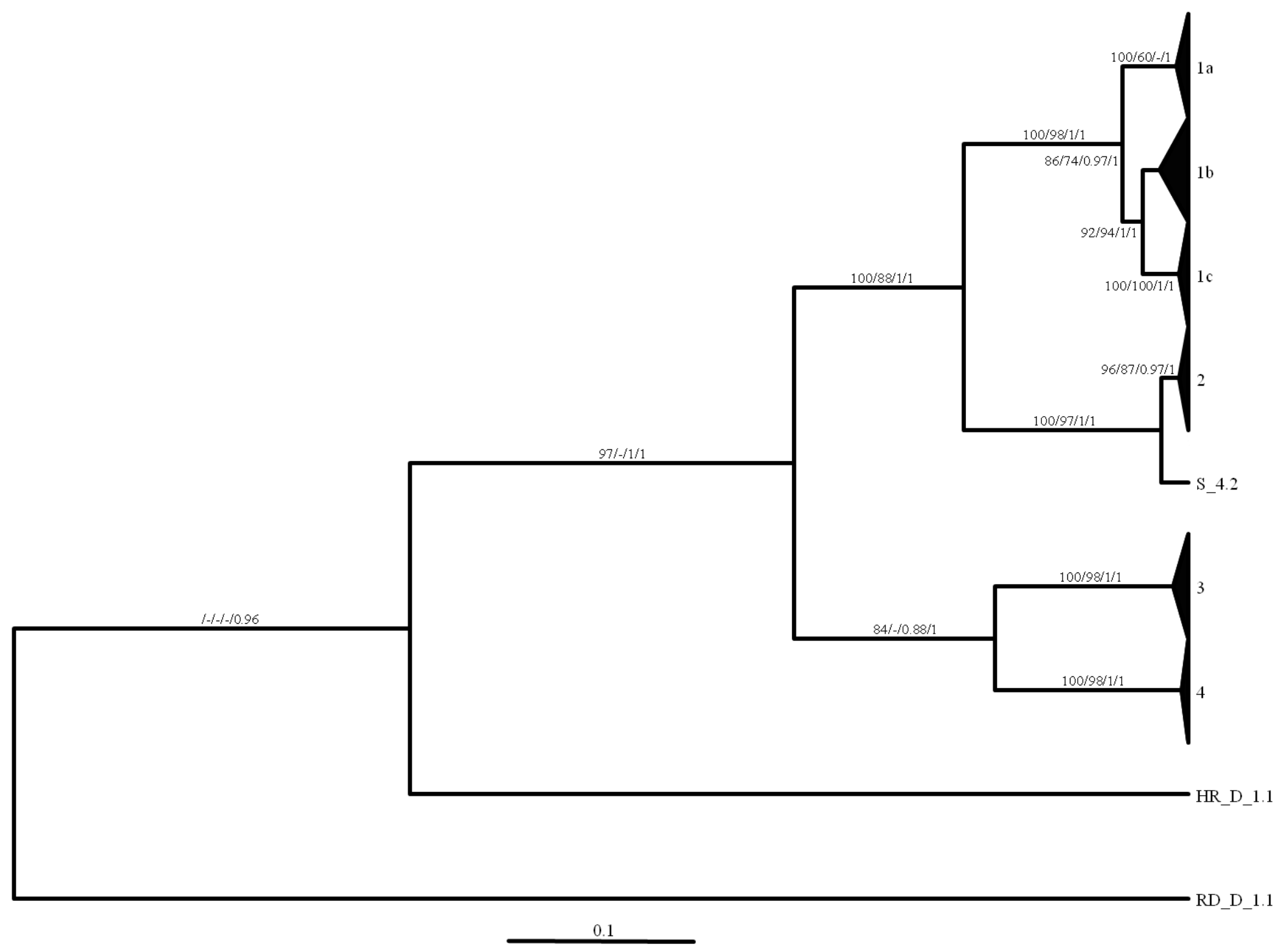

Figure 32: Bayesian phylogeny after $10 \times 10^{6}$ generations from the $160 \mathrm{COI}$ nucleotide sequences of Platynothrus peltifer with Beast v1.5.4. Outgroups are one individual of Hypochthonius rufulus and Rhysotritia duplicata, respectively. Numbers on the branches are bootstrap values from NJ without and with evolution model (HKY $+\mathrm{G})$ analysis and posterior probabilities from MrBayes and Beast. Tip numbers are the different subclades and explained in Table 20. 


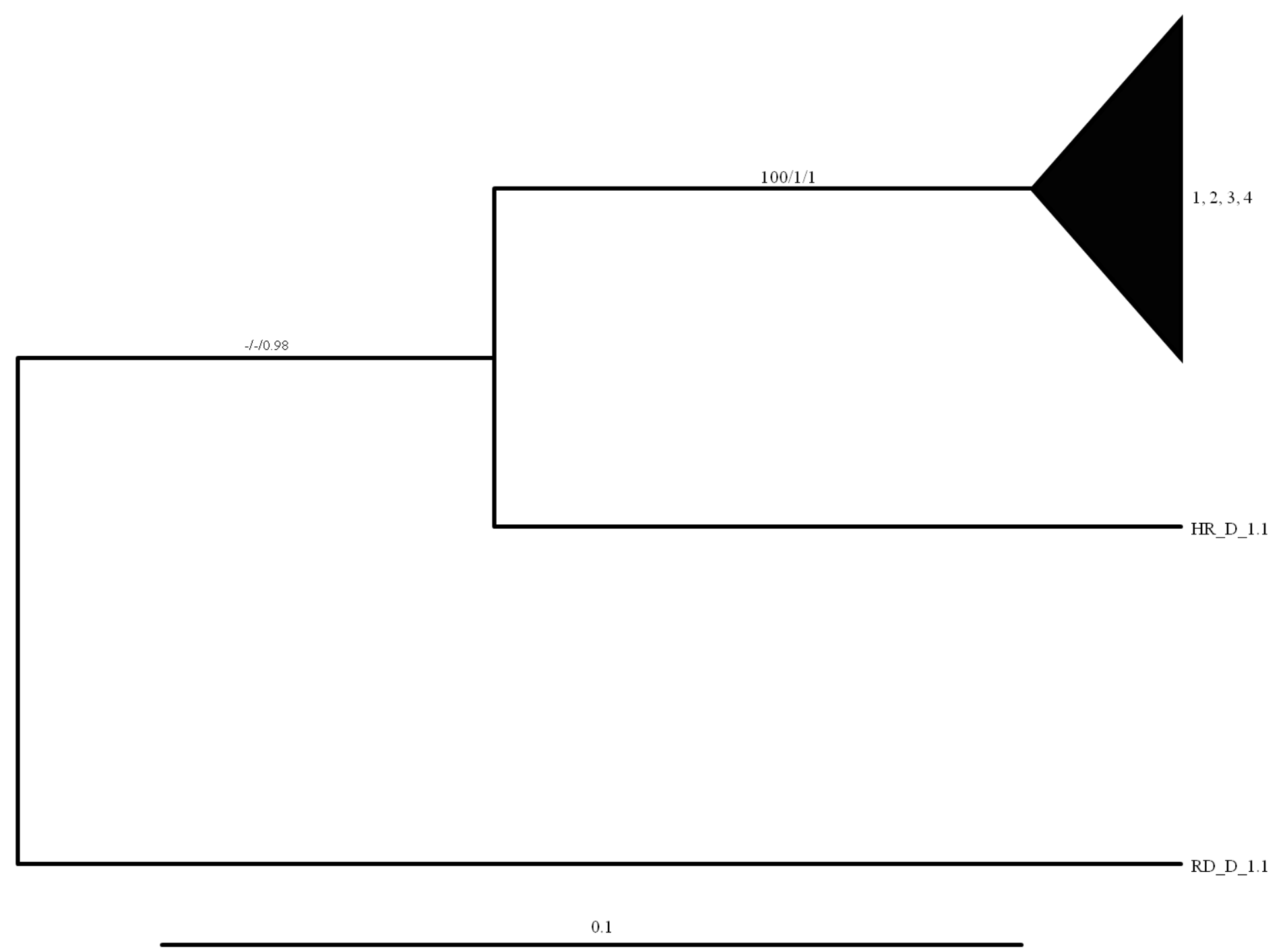

Figure 33: Bayesian phylogeny after $10 \times 10^{6}$ generations from the 160 COI protein sequences of Peltifer peltifer with Beast v1.5.4. Outgroups are one individual of Hypochthonius rufulus and Rhysotritia duplicata, respectively. Numbers on the branches are bootstrap values from NJ and posterior probabilities from MrBayes and Beast. Tip numbers are the different subclades and explained in Table 20. 
Chapter 4 Sex versus parthenogenesis

Table 20: Subclades of Bayesian phylogenetic trees of Platynothrus peltifer based on COI nucleotide sequences (\#ind=number or individuals, pp=posterior probabilities, sampling sites with abbreviations, individuals and ind. pop.=quantity of individuals from the population).

\begin{tabular}{|c|c|c|c|c|c|c|}
\hline Subclade & \# ind & pp & \multicolumn{2}{|c|}{ sampling sites } & individuals & ind. pop \\
\hline 1 & 83 & 1 & 26 & & & \\
\hline \multirow[t]{15}{*}{$1 \mathbf{a}$} & 44 & 1 & Villach & A_1 & $1-2$ & $2 / 2$ \\
\hline & & & Kranichstein & D_1 & $1,3-4$ & $3 / 4$ \\
\hline & & & Goettingen & D_2 & $1-2$ & $2 / 2$ \\
\hline & & & Meckl. Seenplatte & D_4 & $1-4,6,8$ & $6 / 8$ \\
\hline & & & Moerfelden & D_5 & $1-4$ & $4 / 4$ \\
\hline & & & Black Forrest & D_6 & $1-5$ & $5 / 5$ \\
\hline & & & Bonn & D_9 & 3 & $1 / 3$ \\
\hline & & & Wittmoor & D_11 & 1 & $1 / 2$ \\
\hline & & & Steinhuder Meer & D_18 & $1-2$ & $2 / 7$ \\
\hline & & & Hjørring & DK_2 & $3-5$ & $3 / 5$ \\
\hline & & & Brunstatt & F_5 & $1-3$ & $3 / 3$ \\
\hline & & & Braemar & GB_2 & $2-4$ & $3 / 4$ \\
\hline & & & Grosseto & I_1 & 1 & $1 / 1$ \\
\hline & & & Warsaw & PL_2 & $1,4-9$ & $7 / 9$ \\
\hline & & & Sibiu_2 & RUM_2 & 2 & $1 / 2$ \\
\hline \multirow[t]{15}{*}{$1 \mathbf{b}$} & 34 & 1 & Memminger Huette & A_5 & 3 & $1 / 4$ \\
\hline & & & Uelzen & D_7 & $1-3$ & $3 / 3$ \\
\hline & & & Cuxhaven & D_8 & 1,3 & $2 / 4$ \\
\hline & & & Langenwang & D_15 & 3 & $1 / 3$ \\
\hline & & & Rubi & D_17 & 1 & $1 / 3$ \\
\hline & & & Steinhuder Meer & D_18 & $3-7$ & $5 / 7$ \\
\hline & & & Hjørring & DK_2 & $1-2,6$ & $3 / 6$ \\
\hline & & & Mont Blanc & F_1 & $3-4$ & $2 / 4$ \\
\hline & & & Saint Isidore & F_3 & $1-2$ & $2 / 2$ \\
\hline & & & Lahti & FIN_1 & $1-4$ & $4 / 4$ \\
\hline & & & Ascot & GB_1 & 1 & $1 / 3$ \\
\hline & & & Braemar & GB_2 & 1 & $1 / 4$ \\
\hline & & & Hoge Veluwe & NL_2 & $1-4$ & $4 / 4$ \\
\hline & & & Warsaw & PL_2 & $2-3$ & $2 / 9$ \\
\hline & & & Stroemstad & S_2 & $5-6$ & $2 / 9$ \\
\hline 1c & 5 & 1 & Stroemstad & S_2 & $1-4,8$ & $5 / 9$ \\
\hline \multirow[t]{5}{*}{2} & 12 & 1 & Memminger Huette & A_5 & $1-2$ & $2 / 4$ \\
\hline & & & Narvik & N_1 & $1-2$ & $2 / 2$ \\
\hline & & & Umea & S_1 & $1-3$ & $3 / 3$ \\
\hline & & & Stroemstad & S_2 & 7,9 & $2 / 9$ \\
\hline & & & Abisko & S_4 & $1,3-4$ & $3 / 4$ \\
\hline isol. ind. & 1 & & Abisko & S_4 & 2 & $1 / 4$ \\
\hline
\end{tabular}


Chapter 4 Sex versus parthenogenesis

Table 20 continue:

\begin{tabular}{|c|c|c|c|c|c|c|}
\hline Subclade & \# ind & pp & \multicolumn{2}{|c|}{ sampling sites } & individuals & ind. pop. \\
\hline \multirow[t]{24}{*}{$\overline{3}$} & 62 & 1 & Bregenz & A_2 & $1-2$ & $2 / 2$ \\
\hline & & & Hittisau & A_3 & $1-3$ & $3 / 3$ \\
\hline & & & Holzgau & A_4 & $1-2$ & $2 / 2$ \\
\hline & & & Memminger Huette & A_5 & 4 & $1 / 4$ \\
\hline & & & Glacier & A_9 & $1-5$ & $5 / 5$ \\
\hline & & & Stuiben & A_10 & $1-5$ & $5 / 5$ \\
\hline & & & Rorschach & CH_3 & $1-5$ & $5 / 5$ \\
\hline & & & Kranichstein & D_1 & 2 & $1 / 4$ \\
\hline & & & Lake Constance & D_3 & $1-4$ & $4 / 4$ \\
\hline & & & Meckl. Seenplatte & D_4 & 5,7 & $2 / 8$ \\
\hline & & & Cuxhaven & D_8 & 2,4 & $2 / 4$ \\
\hline & & & Bonn & D_9 & $1-2$ & $2 / 3$ \\
\hline & & & Wittmoor & D_11 & 2 & $1 / 2$ \\
\hline & & & Sonthofen & D_12 & $1-5$ & $5 / 5$ \\
\hline & & & Langenwang & D_15 & $1-2$ & $2 / 3$ \\
\hline & & & Rubi & D_17 & $2-3$ & $2 / 3$ \\
\hline & & & Arhus_Morvadsvej & DK_3 & $1-6$ & $6 / 6$ \\
\hline & & & Mont Blanc & F_1 & 2 & $1 / 4$ \\
\hline & & & Ascot & GB_1 & $2-3$ & $2 / 3$ \\
\hline & & & Wageningen & NL_1 & 1,3 & $2 / 3$ \\
\hline & & & Sibiu_2 & RUM_2 & 1 & $1 / 2$ \\
\hline & & & Busteni & RUM_5 & 1 & $1 / 1$ \\
\hline & & & Sinaia & RUM_6 & 1 & $1 / 1$ \\
\hline & & & Gothenburg & S_5 & $1-4$ & $4 / 4$ \\
\hline \multirow[t]{2}{*}{4} & 2 & 1 & Mont Blanc & F_1 & 1 & $1 / 4$ \\
\hline & & & Wageningen & NL_1 1 & 2 & $1 / 3$ \\
\hline
\end{tabular}


Table 21: Mean percent pairwise differences of uncorrected p-distances of the COI nucleotide sequences of Platynothrus peltifer from 39 locations. The diagonal is the within population differences (bold) and below the diagonal the among population differences. Bold letters in red are the minimum and maximum differences within and among the populations. Locations with less than two individuals are excluded from the analysis.

\begin{tabular}{|c|c|c|c|c|c|c|c|c|c|c|c|c|c|c|c|c|c|c|c|c|c|c|c|c|c|c|c|c|c|}
\hline \multicolumn{2}{|c|}{ Population } & \multirow{2}{*}{$\begin{array}{c}1 \\
0.2\end{array}$} & \multirow[t]{2}{*}{2} & \multirow[t]{2}{*}{3} & \multirow[t]{2}{*}{4} & \multirow[t]{2}{*}{5} & \multirow[t]{2}{*}{6} & \multirow[t]{2}{*}{7} & \multirow[t]{2}{*}{8} & \multirow[t]{2}{*}{9} & \multirow[t]{2}{*}{10} & \multirow[t]{2}{*}{11} & \multirow[t]{2}{*}{12} & \multirow[t]{2}{*}{13} & 14 & 15 & 16 & 17 & 18 & 19 & 20 & 21 & 22 & 23 & 24 & 25 & 26 & 27 & 28 \\
\hline 1 & A 1 & & & & & & & & & & & & & & & & & & & & & & & & & & & & \\
\hline 2 & A 2 & 18.2 & 0 & & & & & & & & & & & & & & & & & & & & & & & & & & \\
\hline 3 & A 3 & 18.3 & 0.2 & 0.4 & & & & & & & & & & & & & & & & & & & & & & & & & \\
\hline 4 & A 4 & 18.2 & 0.4 & 0.3 & 0 & & & & & & & & & & & & & & & & & & & & & & & & \\
\hline 5 & A 5 & 12.8 & 14.3 & 14.3 & 14.3 & 14.4 & & & & & & & & & & & & & & & & & & & & & & & \\
\hline 6 & A 9 & 18.3 & 0.3 & 0.4 & 0.3 & 14.4 & 0.3 & & & & & & & & & & & & & & & & & & & & & & \\
\hline 7 & A 10 & 18.6 & 0.4 & 0.4 & 0.4 & 14.4 & 0.3 & 0 & & & & & & & & & & & & & & & & & & & & & \\
\hline 8 & CH 3 & 18.3 & 0.3 & 0.3 & 0.2 & 14.3 & 0.2 & 0.3 & 0.1 & & & & & & & & & & & & & & & & & & & & \\
\hline 9 & D 1 & 5.9 & 13.4 & 13.5 & 13.4 & 13.1 & 13.4 & 13.7 & 13.5 & 9.3 & & & & & & & & & & & & & & & & & & & \\
\hline 10 & D 2 & 1.5 & 17.9 & 18 & 17.9 & 12.5 & 18 & 18.3 & 18 & 4.8 & 0 & & & & & & & & & & & & & & & & & & \\
\hline 11 & D 3 & 18.3 & 0.4 & 0.5 & 0.6 & 14.4 & 0.6 & 0.6 & 0.4 & 13.5 & 18.0 & 0.7 & & & & & & & & & & & & & & & & & \\
\hline 12 & D 4 & 5.9 & 13.5 & 13.6 & 13.5 & 13 & 13.6 & 13.8 & 13.6 & 7.1 & 4.7 & 13.7 & 7.8 & & & & & & & & & & & & & & & & \\
\hline 13 & D 5 & 18.1 & 18.4 & 18 & 18 & 18.1 & 12.7 & 0.6 & 18.1 & 5.2 & 0.6 & 18.1 & 4.9 & 0.9 & & & & & & & & & & & & & & & \\
\hline 14 & D 6 & 1.9 & 17.6 & 17.7 & 17.6 & 12.4 & 17.7 & 18 & 17.7 & 5 & 0.4 & 17.7 & 4.9 & 1 & 0.1 & & & & & & & & & & & & & & \\
\hline 15 & D 7 & 5.7 & 19.7 & 19.8 & 19.7 & 12 & 19.8 & 20.1 & 19.8 & 8.7 & 4.5 & 19.8 & 8.4 & 5.1 & 4.9 & 0 & & & & & & & & & & & & & \\
\hline 16 & D 8 & 12.1 & 9.8 & 9.9 & 9.9 & 13.2 & 9.9 & 10.1 & 9.9 & 11.1 & 11.3 & 10 & 11 & 11.7 & 11.4 & 10.2 & 13.2 & & & & & & & & & & & & \\
\hline 17 & D 9 & 12.8 & 6.1 & 6.2 & 6.1 & 13.7 & 6.1 & 6.2 & 6.1 & 10.6 & 12.1 & 6.3 & 10.6 & 12.3 & 12 & 14.8 & 10.4 & 12.1 & & & & & & & & & & & \\
\hline 18 & D 11 & 10.1 & 9.3 & 9.4 & 9.3 & 13.6 & 9.3 & 9.5 & 9.3 & 9.4 & 9.2 & 9.5 & 9.3 & 9.6 & 9.3 & 12.4 & 10.8 & 9.3 & 18.5 & & & & & & & & & & \\
\hline 19 & D 12 & 18.4 & 0.5 & 0.4 & 0.3 & 14.5 & 0.5 & 0.5 & 0.4 & 13.6 & 18.1 & 0.7 & 13.7 & 18.2 & 17.8 & 20 & 10.1 & 6.3 & 9.5 & 0.4 & & & & & & & & & \\
\hline 20 & D 15 & 14.1 & 6.9 & 6.9 & 6.8 & 13.6 & 6.9 & 7 & 6.8 & 11.8 & 13.5 & 7.1 & 11.9 & 13.8 & 13.4 & 13.3 & 10.1 & 9.1 & 10.4 & 6.9 & 13.4 & & & & & & & & \\
\hline 21 & D 17 & 14.1 & 6.7 & 6.8 & 6.7 & 13.6 & 6.7 & 6.8 & 6.7 & 11.9 & 13.6 & 6.9 & 11.9 & 13.8 & 13.5 & 13.3 & 10 & 9 & 10.3 & 6.9 & 8.9 & 13.1 & & & & & & & \\
\hline 22 & D 18 & 4.7 & 19.1 & 19.1 & 19.1 & 12.2 & 19.2 & 19.4 & 19.2 & 7.7 & 3.4 & 19.2 & 7.4 & 4 & 3.8 & 1.5 & 10.5 & 14 & 11.5 & 19.3 & 13.4 & 13.3 & 2.4 & & & & & & \\
\hline 23 & DK 2 & 3.6 & 18.8 & 18.9 & 18.8 & 12.3 & 18.9 & 19.2 & 18.9 & 6.9 & 2.5 & 18.9 & 6.6 & 18.2 & 2.9 & 2.5 & 10.9 & 13.5 & 10.9 & 19 & 13.5 & 13.5 & 2.7 & 3 & & & & & \\
\hline 24 & DK 3 & 18.4 & 0.5 & 0.6 & 0.5 & 14.5 & 0.5 & 0.5 & 0.4 & 13.4 & 18.1 & 0.8 & 13.7 & 13.4 & 17.8 & 20.3 & 10.3 & 6.3 & 9.5 & 0.5 & 6.9 & 7 & 19.5 & 19.2 & 0 & & & & \\
\hline 25 & F 1 & 12.5 & 13.7 & 13.7 & 13.6 & 14 & 13.7 & 13.8 & 13.6 & 12.5 & 12 & 13.8 & 12.4 & 2.9 & 12 & 10.3 & 12.1 & 13.1 & 13 & 13.7 & 12.5 & 12.5 & 10.8 & 11.2 & 13.8 & 15.7 & & & \\
\hline 26 & F 3 & 5.8 & 19.9 & 20 & 19.9 & 12.2 & 20 & 20.3 & 20 & 8.9 & 4.7 & 20 & 8.6 & 12.3 & 5.1 & 0.2 & 10.4 & 15 & 12.5 & 20.2 & 13.5 & 13.4 & 1.7 & 2.7 & 20.4 & 10.4 & 0 & & \\
\hline 27 & F 5 & 1.8 & 18 & 18 & 18 & 12.6 & 18.1 & 18.3 & 18.1 & 5 & 0.2 & 18.1 & 4.8 & 5.2 & 0.6 & 4.6 & 11.4 & 12.2 & 9.4 & 18.2 & 13.6 & 13.6 & 3.5 & 2.6 & 18.3 & 12.1 & 4.8 & 0.4 & \\
\hline 28 & FIN 1 & 6 & 19.7 & 19.7 & 19.7 & 12 & 19.8 & 20 & 19.8 & 9 & 4.8 & 19.8 & 8.7 & 5.4 & 5.2 & 0.4 & 10.4 & 14.9 & 12.5 & 20 & 13.3 & 13.3 & 1.9 & 2.8 & 20.2 & 10.4 & 0.6 & 4.9 & 0.1 \\
\hline 29 & GB 1 & 14.1 & 6.7 & 6.8 & 6.7 & 13.6 & 6.7 & 6.8 & 6.7 & 11.9 & 13.6 & 6.9 & 11.9 & 1.8 & 13.5 & 13.3 & 10 & 9 & 10.3 & 6.9 & 9.0 & 8.8 & 13.3 & 13.5 & 7 & 12.5 & 13.4 & 13.6 & 13.7 \\
\hline 30 & GB 2 & 2.6 & 18.5 & 18.5 & 18.5 & 12.4 & 18.5 & 18.8 & 18.6 & 5.9 & 1.2 & 18.6 & 5.7 & 2.2 & 1.6 & 3.5 & 11.1 & 12.9 & 10.1 & 18.7 & 13.6 & 13.6 & 3 & 2.6 & 18.7 & 11.6 & 3.7 & 1.4 & 3.8 \\
\hline 31 & N 1 & 13.4 & 18.6 & 18.6 & 18.6 & 8.6 & 18.8 & 18.7 & 18.7 & 15 & 13.7 & 18.6 & 14.9 & 1.8 & 13.4 & 14 & 16.3 & 17 & 16.2 & 18.8 & 17.2 & 17.3 & 14.1 & 13.8 & 18.7 & 16.1 & 14.3 & 13.7 & 14 \\
\hline 32 & NL 1 & 19.1 & 5.4 & 5.4 & 5.3 & 15.5 & 5.3 & 5.3 & 5.2 & 15.3 & 18.8 & 5.5 & 15.4 & 18.8 & 18.4 & 20 & 12.6 & 9.7 & 12 & 5.4 & 10.2 & 10.1 & 19.5 & 19.4 & 5.4 & 13.8 & 20.1 & 18.8 & 19.8 \\
\hline 33 & NL 2 & 5.8 & 19.7 & 19.8 & 19.7 & 12.1 & 19.8 & 20.1 & 19.8 & 8.9 & 4.7 & 19.8 & 8.6 & 5.2 & 5.1 & 0.2 & 10.3 & 14.9 & 12.5 & 20 & 13.4 & 13.3 & 1.6 & 2.7 & 20.3 & 10.4 & 0.4 & 4.8 & 0.6 \\
\hline 34 & PL 2 & 2.4 & 18.3 & 18.3 & 18.3 & 12.6 & 18.3 & 18.6 & 18.4 & 6.2 & 1.8 & 18.4 & 6.1 & 13.6 & 2.2 & 4.3 & 11.4 & 13 & 10.3 & 18.5 & 13.7 & 13.7 & 3.8 & 3.1 & 18.5 & 12 & 4.5 & 2 & 4.5 \\
\hline 35 & RUM 2 & 9.9 & 9.1 & 9.1 & 9.1 & 13.5 & 9.1 & 9.2 & 9 & 9.4 & 9.5 & 9.1 & 9.5 & 9.7 & 9.5 & 12.6 & 10.8 & 9.2 & 9.5 & 9.2 & 10.3 & 10.2 & 11.8 & 11.1 & 9.2 & 13 & 12.8 & 9.7 & 12.8 \\
\hline 36 & S 1 & 13.3 & 18.5 & 18.5 & 18.5 & 8.4 & 18.7 & 18.6 & 18.6 & 14.8 & 13.6 & 18.6 & 14.7 & 7.7 & 13.2 & 13.8 & 16.1 & 16.9 & 16.1 & 18.7 & 17 & 17.1 & 13.8 & 13.6 & 18.6 & 15.9 & 14 & 13.6 & 13.7 \\
\hline 37 & S 2 & 8.2 & 18.9 & 18.9 & 18.9 & 11.4 & 19.0 & 19.2 & 19 & 10.6 & 7.3 & 19 & 10.3 & 18 & 7.5 & 4.9 & 12.1 & 15.2 & 13.3 & 19.1 & 14.4 & 14.4 & 5.8 & 6.3 & 19.3 & 12.3 & 5.1 & 7.4 & 5.2 \\
\hline 38 & S 4 & 13.3 & 18.7 & 18.7 & 18.7 & 8.8 & 18.9 & 18.9 & 18.8 & 14.9 & 13.5 & 18.8 & 14.7 & 13.5 & 13.2 & 13.7 & 16.2 & 16.2 & 17 & 18.9 & 17.1 & 17.3 & 13.8 & 13.5 & 18.9 & 15.9 & 13.9 & 13.5 & 13.7 \\
\hline 39 & S 5 & 18.2 & 0.4 & 0.5 & 0.4 & 14.3 & 0.3 & 0.4 & 0.3 & 13.4 & 17.9 & 0.5 & 13.5 & 13.8 & 17.6 & 19.7 & 9.9 & 6.1 & 9.3 & 0.6 & 6.9 & 6.7 & 19 & 18.7 & 0.6 & 13.6 & 19.9 & 17.9 & 19.6 \\
\hline
\end{tabular}


Table 21continue:

\begin{tabular}{lcccccccccccc}
\hline \multicolumn{2}{c}{ Population } & $\mathbf{2 9}$ & $\mathbf{3 0}$ & $\mathbf{3 1}$ & $\mathbf{3 2}$ & $\mathbf{3 3}$ & $\mathbf{3 4}$ & $\mathbf{3 5}$ & $\mathbf{3 6}$ & $\mathbf{3 7}$ & $\mathbf{3 8}$ & $\mathbf{3 9}$ \\
\hline $\mathbf{2 9}$ & GB 1 & $\mathbf{1 3 . 3}$ & & & & & & & & & & \\
$\mathbf{3 0}$ & GB 2 & 13.6 & $\mathbf{2 . 4}$ & & & & & & & & & \\
$\mathbf{3 1}$ & N 1 & 17.2 & 13.7 & $\mathbf{0 . 5}$ & & & & & & & & \\
$\mathbf{3 2}$ & NL 1 & 10.1 & 19.1 & 18.4 & $\mathbf{1 0}$ & & & & & & & \\
$\mathbf{3 3}$ & NL 2 & 13.3 & 3.6 & 4.5 & 20 & $\mathbf{0 . 3}$ & & & & & & \\
$\mathbf{3 4}$ & PL 2 & 13.7 & 2.5 & 13.9 & 19 & 4.5 & $\mathbf{2 . 7}$ & & & & & \\
$\mathbf{3 5}$ & RUM 2 & 10.2 & 10.4 & 16.0 & 11.9 & 12.7 & 10.1 & $\mathbf{1 7 . 7}$ & & & & \\
$\mathbf{3 6}$ & S 1 & 17 & 13.5 & 0.3 & 18.3 & 13.9 & 13.7 & 15.9 & $\mathbf{0 . 2}$ & & & \\
$\mathbf{3 7}$ & S 2 & 14.3 & 6.8 & 10.6 & 19.3 & 5.1 & 7.4 & 13.4 & 10.4 & $\mathbf{6 . 3}$ & & \\
$\mathbf{3 8}$ & S 4 & 17.1 & 13.5 & 1.1 & 18.5 & 13.8 & 13.6 & 16 & 1 & 10.7 & $\mathbf{1 . 7}$ & \\
$\mathbf{3 9}$ & S 5 & 6.7 & 18.4 & 18.7 & 5.3 & 19.7 & 18.2 & 9 & 18.6 & 18.9 & 18.8 & $\mathbf{0 . 4}$ \\
\hline
\end{tabular}

The results of the AMOVA showed that the nucleotide variation among samples within countries $(57.2 \%)$ and the variation within samples $(36.5 \%)$ were significant and high. In contrast variation among countries was not significant (Table 22).

Table 22: AMOVA table on variations among countries and within populations in nucleotide (black) and protein (red) sequences of COI of Platynothrus peltifer. Each population was considered as separate groups. Populations with less than two individuals were excluded from the analysis. Significance level is $p<0.05$ (d.f.: degree of freedom).

\begin{tabular}{lccccc}
\hline \multicolumn{1}{c}{ Source of variance } & d.f. & sum of squares & variance components & percent of total variation & fixation indices \\
\hline among countries & $11 / 11$ & $1280.79 / 0.73$ & $2.04 \mathrm{Va} / 0.01 \mathrm{Va} *$ & $6.3 / 19.04$ & $\mathrm{FCT}: 0.06 / 0.19$ \\
among populations & $27 / 27$ & $2243.04 / 0.29$ & $18.5 \mathrm{Vb} * / 0 \mathrm{Vb}$ & $57.17 /-14.04$ & $\mathrm{FSC}: 0.61^{*} /-0.17$ \\
within population & $118 / 118$ & $1394.94 / 2.94$ & $11.82 \mathrm{Vc} * / 0.03 \mathrm{Vc}$ & $36.53 / 95$ & $\mathrm{FST}: 0.63^{*} / 0.05$ \\
total & $156 / 156$ & $4918.76 / 3.96$ & $32.36 / 0.03$ & & \\
\hline
\end{tabular}

The neutrality test of Tajima's D was only significant for one population in the nucleotide (D_3 Tajima's $\mathrm{D}=0$, $\mathrm{p}$-value $=0.0359$, Table A27) and was not significant for the protein (Table A28). Fu's FS neutrality test was not significant for both datasets (Table A27 and Table A28).

The results of the McDonald-Kreitman test showed that the differences between 64 of 741 populations were significant $(8.64 \%)$, between other 17 of 741 were high significant $(2.29 \%)$ and between three other of 741 populations were highly significant $(0.41 \%)$ (Table 23 and for detail Table A29). All computed neutrality indices in the McDonald-Kreitman test were $\geq 0$ (Table A30).

None of the calculated rarefaction curves of the nucleotide and the protein reached saturation (Fig. 34 and 35). The Jackknife rarefaction curves ended at 183 haplotypes for the nucleotide and 45 haplotypes for the protein. The unique rarefaction curves ended at 49 haplotypes for the nucleotide and twelve haplotypes for the protein. The observed rarefaction curves (Sobs Mao Tau) ended at 75 haplotypes for the nucleotide and 17 haplotypes for the protein.

The results of the Mantel test indicate isolation by distance in P. peltifer $\left[\mathrm{R}^{2}=0.0629, \mathrm{p}=0.006\right.$; (Fig. A39) and $\mathrm{R}^{2}=0.0524, \mathrm{p}=0.003^{*}$; for $\log$ transformed geographic distances (Fig. 36) using 1000 randomizations], but this explained only $5 \%$ of the variation in the data.

The gmyc model had a significantly better fit in the single and the multiple analysis than the null model (Table 18). For both the single and the multiple analysis the model identified six distinct mtDNA (COI) clusters. The clusters of the single analysis differed from the clusters which were delimited by the phylogenetic analyses in one point; in clade 1c the individual PL_2.2 was excluded from the generated COI cluster (Fig. A40 and A41). 
Chapter 4 Sex versus parthenogenesis

Table 23: Results of the McDonald-Kreitman test of Platynothrus peltifer. The differences between 64 populations were significant $(* 0.01<\mathrm{P}<0.05)$, between other 17 populations high significant $(* * 0.001<\mathrm{P}<0.01)$ and between three populations highly significant $(* * * \mathrm{P}<0.001)$. Locations with less than two individuals were excluded.

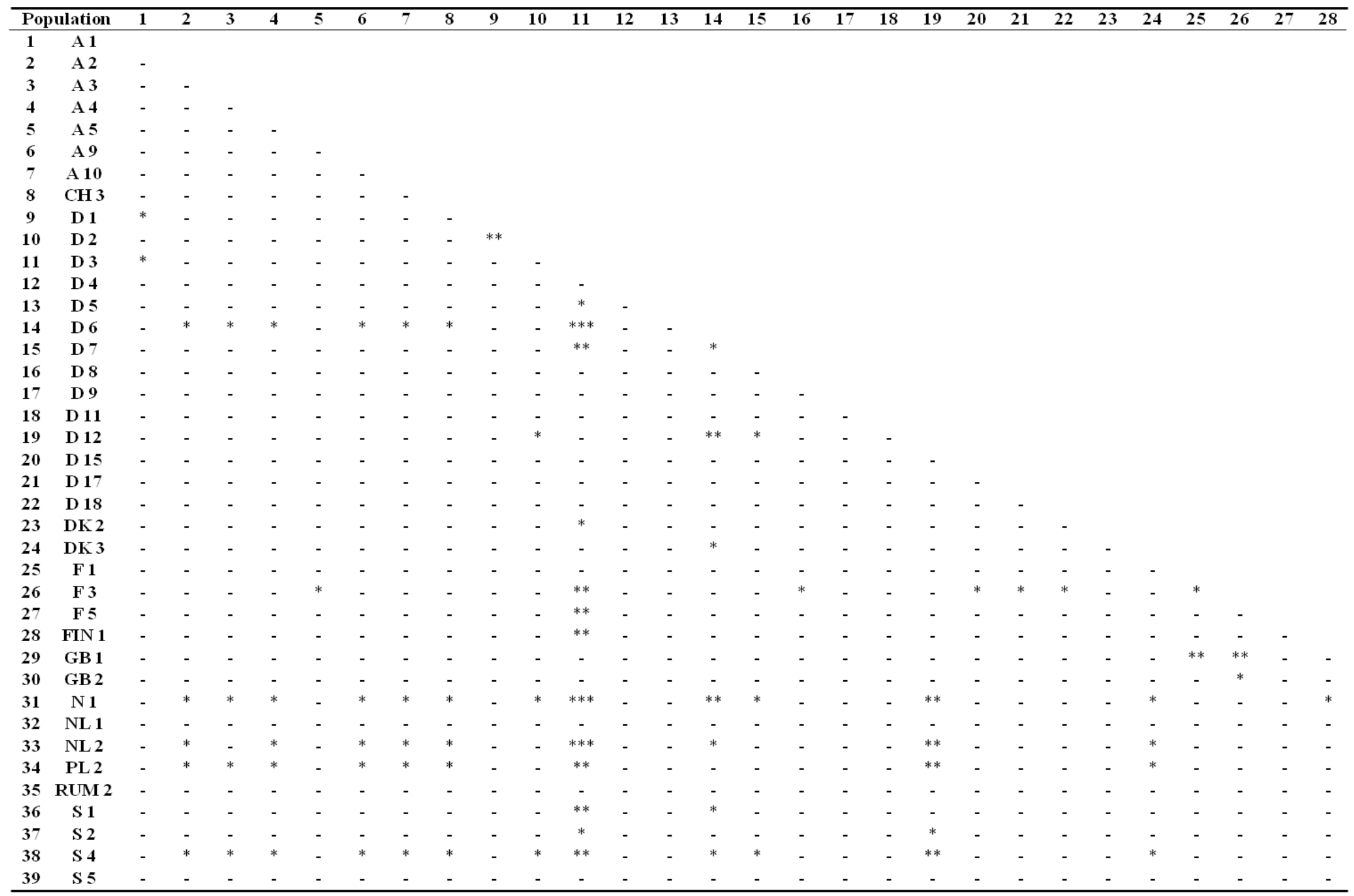


Chapter 4 Sex versus parthenogenesis

Table 23 continue:

\begin{tabular}{|c|c|c|c|c|c|c|c|c|c|c|c|}
\hline \multicolumn{2}{|c|}{ Population } & 29 & 30 & 31 & 32 & 33 & 34 & 35 & 36 & 37 & 38 \\
\hline 30 & GB 2 & - & & & & & & & & & \\
\hline 31 & N 1 & - & - & & & & & & & & \\
\hline 32 & NL 1 & - & - & - & & & & & & & \\
\hline 33 & NL 2 & - & - & *** & - & & & & & & \\
\hline 34 & PL 2 & - & - & $*$ & - & - & & & & & \\
\hline 35 & RUM 2 & - & - & - & - & - & - & & & & \\
\hline 36 & S 1 & - & - & - & - & - & $*$ & - & & & \\
\hline 37 & S 2 & - & - & - & - & - & - & - & - & & \\
\hline 38 & S 4 & - & - & - & - & $*$ & $*$ & - & - & - & \\
\hline 39 & S 5 & - & - & - & - & - & $*$ & - & - & - & $*$ \\
\hline
\end{tabular}

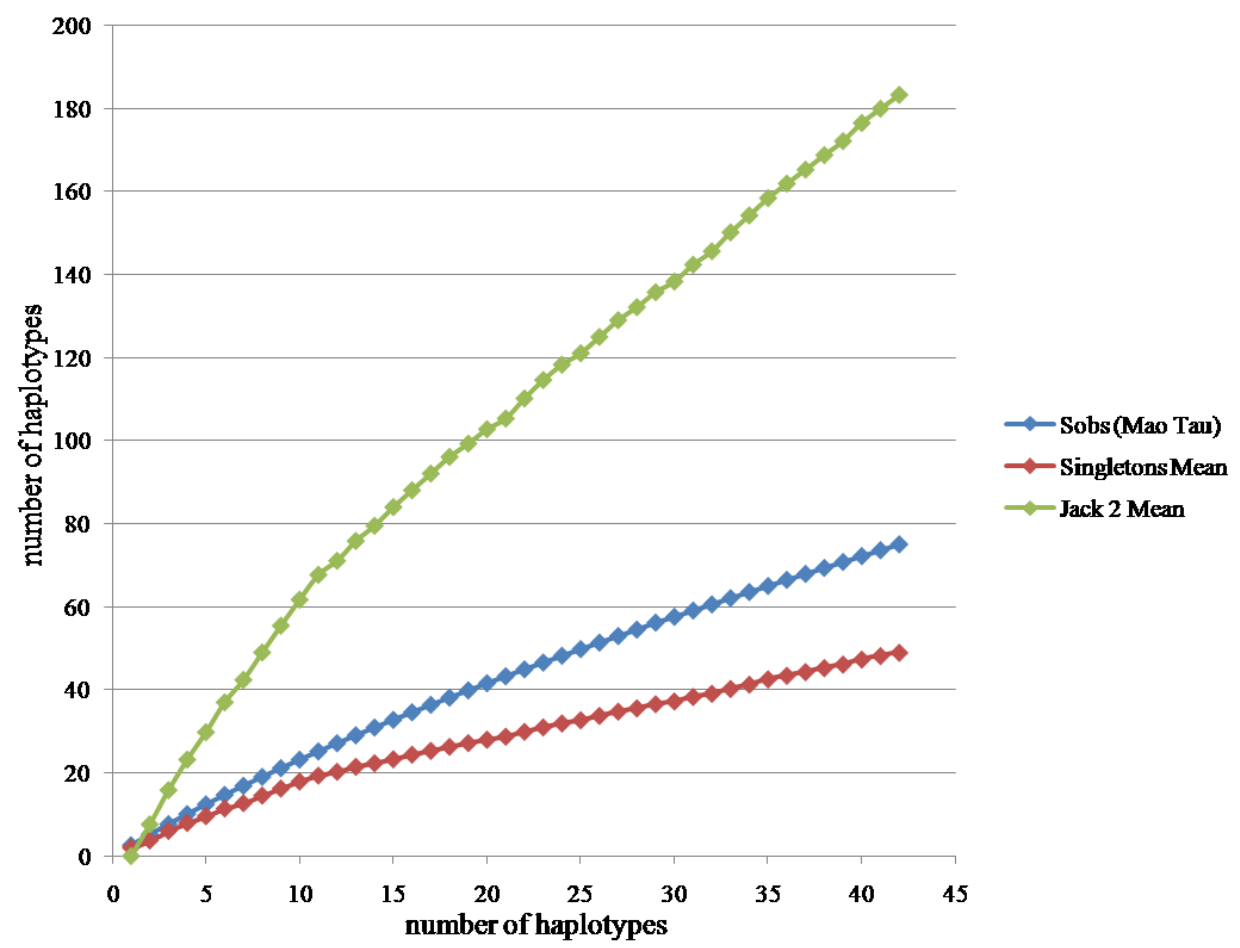

Figure 34: Sample based rarefaction analysis of haplotypes of the COI nucleotide of Platynothrus peltifer. Jack 2 Mean, second-order Jackknife richness estimator, Singletons, numbers of haplotypes each present in only one sample, Sob (Mao Tau) is the observed haplotype richness. 
Chapter 4 Sex versus parthenogenesis

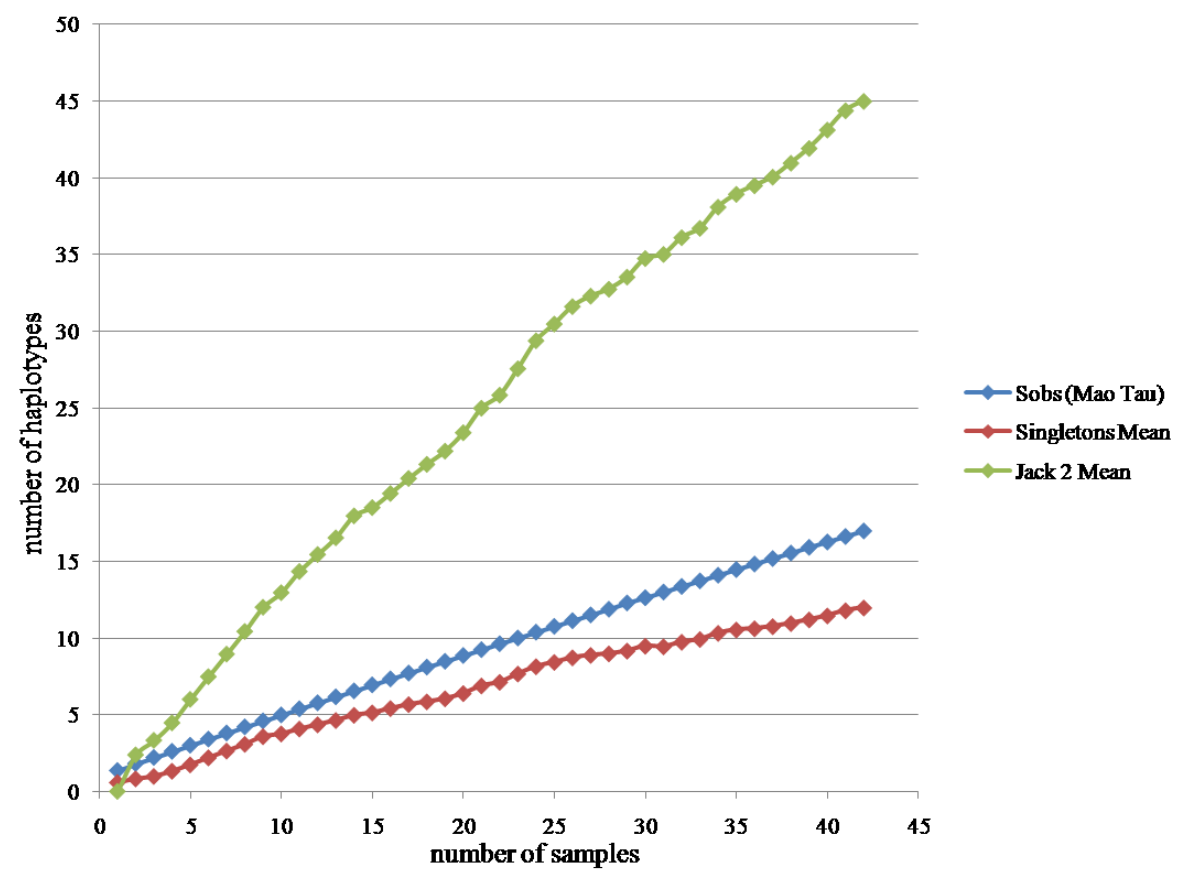

Figure 35: Sample based rarefaction analysis of haplotypes of the COI protein of Platynothrus peltifer. Jack 2 Mean, second-order Jackknife richness estimator, Singletons, numbers of haplotypes each present in only one sample, Sob (Mao Tau) is the observed haplotype richness.

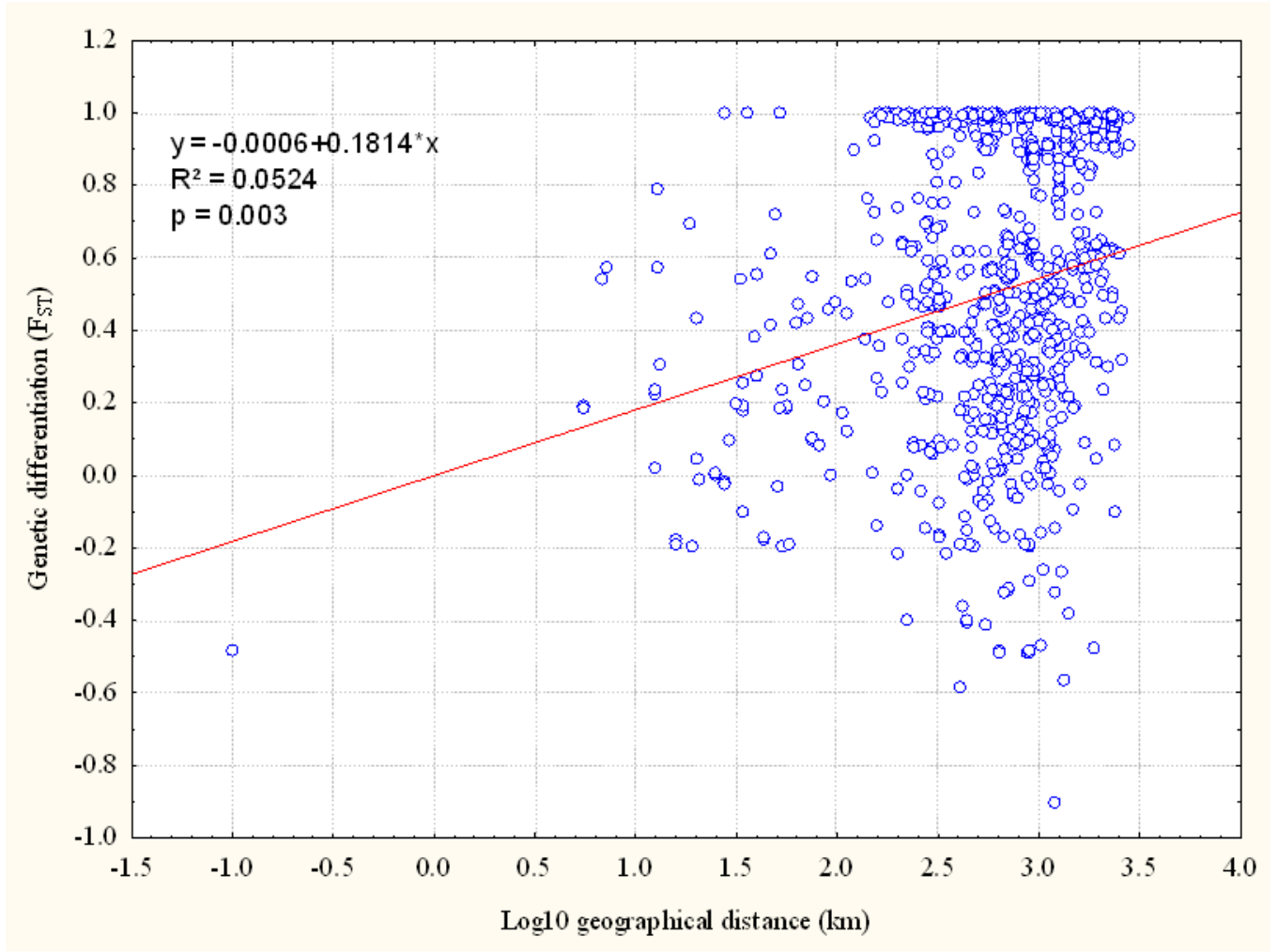

Figure 36: Linear regression of $\log 10$ geographical distances (kilometers) vs. genetic differentiations $\left(\mathrm{F}_{\mathrm{ST}}\right)$ based on COI nucleotide sequences of Platynothrus peltifer. The regression is not significant using 1000 randomizations. 


\subsubsection{Achipteria coleoptrata}

A total of 143 individuals (141 individuals of A. coleoptrata, one individual of $C$. femoralis and one individual of $C$. marginatus as outgroup) from 40 locations in 15 countries were sequenced. The sequences contained $25.2 \% \mathrm{~A}, 17.5 \% \mathrm{C}, 16.4 \% \mathrm{G}$ and $40.9 \% \mathrm{~T}$. The $141 \mathrm{COI}$ sequences consisted of 93 haplotypes for the nucleotide (66\%) (Fig. 37) with 219 variable sites (43.6\%) and 197 parsimony informative sites (Table A31). The protein consisted of 39 haplotypes (27.7\%) in the network (Fig. 38). Haplotype diversity $\left(\mathrm{H}_{\mathrm{d}}\right)$ was very high with 0.98 in the nucleotide.

\section{Haplotype networks}

The nucleotide haplotype network formed 14 subclades and five isolated single individual haplotypes which were separated each by 21 to over 100 substitution steps to the next subclade (Fig. 37). Six subclades were Southern subclades [one Greek (subclade 4a), two Romanian (subclade 1and 5c) and three Italian (subclade 4b-c and haplotype I_3.1)], absolutely. Subclades 4a-c comprised individuals from one location each, [GR_1.1-6 (4a), I_1.1-8 (4c) and I_2.1-4 (4b)]. Subclades 1 and 5c comprised individuals from two Romanian locations [RUM_1/RUM_2 (subclade 5c) and RUM_5/RUM_6 (subclade 1)]. One East-Russian subclade (subclade 3b) and five Central subclades [subclade 2, subclade 5g, seven alpine subclades (subclade $3 \mathrm{a}$, subclade $5 \mathrm{a}-\mathrm{b}, \mathrm{d}$ and three isolated individuals D_12.2, A_7.2, D_15.1)] existed in the network in addition to the six Southern subclades. Five single individual haplotypes each from one location [one Austrian (A_7.1), one Italian (I_3.1), one Polish (PL_1.2) and two German (D_12.2, D_15.1)] were present in the network. Subclades 5a-b comprised two haplotypes [5a comprised two single individual haplotypes from the one German location (D_15.2 and D_15.3), 5b comprised two single individual haplotypes from another German location (D_14.1 and D_14.2)].

Subclade 3a-b comprised two haplotypes with three individuals [subclade $3 \mathrm{a}$ comprised individuals from three Austrian locations (A_3.1, A_5.3, A_8.3), subclade 3b comprised three individuals from one Russian location (RUS_2.1-3)]. Subclades 4a-c and 5e comprised three haplotypes [subclade 4a: two single individual haplotypes and one shared by four individuals from one Greek location (GR_1.1, GR_1.5 and GR_1.2-4/1.6), subclade 4b: two single individual haplotypes and one haplotype shared by individuals from one Italian location (I_2.1, I_2.2 and I_2.3-4), subclade 4c: a single individual haplotype, one haplotype shared by two individuals and one haplotype shared by five individuals from on Italian location (I_1.6, I_1.3/1.7, I_1.1-2/1.4-5/1.8), subclade 5e: two single individual haplotypes from one location in Poland (PL_2.7-2.8) and one haplotype shared by two Finish individuals (FIN_1.1, FIN_1.4)]. Subclade 5c comprised four haplotypes from two Romanian locations (RUM_1, RUM_2) [one haplotype shared by two individuals from both locations (RUM_1.4 and RUM_2.3), one haplotype with two individuals from one location (RUM_1.1-2), one shared by four individuals from two locations (RUM_1.3, RUM_2.1-2, RUM_2.4)].

Two subclades existed in the network with five haplotypes [subclade 1 comprised five single individual haplotypes from two Romanian locations (RUM_5.1-3, RUM_6.1-2), subclade 2 comprised four single individual haplotypes from three locations \{two German locations (D_1.1, D_1.3 and D_5.3), one Polish location (PL_2.1) \} and one haplotype shared by five individuals from two German locations (D_1.2/D_1.4/D_5.1-2/D_5.4)]. Subclade 5f comprised ten haplotypes from six locations [single individual haplotypes: one English (GB_1.1), three Estonian (EST_1.1, EST_1.2, EST_1.3), three Polish (Pl_1.4, PL_2.4, PL_2.6); haplotypes of more than one individual: two haplotypes of two individuals each from one location (one German D_11.1-2, one Polish (PL_1.1, PL1.3), one haplotype of six individuals from three locations, three individuals from one Polish location (PL_2.2-3, PL_2.5), two individuals from the one Finish location (FIN_1.2-3), one individual from an Estonian location (EST_1.4)]. Alpine subclade 5d comprised 16 haplotypes from eight locations [two German (D_12 and D_13), two Swiss (CH_1 and CH_3), four Austrian (A_5, A_6, A_7 and A_8)]. One haplotype 
comprised two individuals from two locations [one Austrian (A_8.2), one Swiss (CH_1.3)] and the other 15 haplotypes were single individual haplotypes from seven locations [four from one Swiss location (CH_1.1, $\left.\mathrm{CH} \_1.2, \mathrm{CH} \_1.4, \mathrm{CH} \_1.5\right)$, two from another Swiss location $\left(\mathrm{CH}_{-} 3.1, \mathrm{CH} \_3.2\right)$, three two German locations (D_12.1, D_12.3, D_14.1), six from four Austrian locations (A_5.1, A_5.2, A_5.4, A_6.1, A_7.1 and A_8.1)]. Subclade 5g was the largest subclade and comprised 21 single individual haplotypes from twelve locations [one Danish (DK_3.2, DK_3.3, DK_3.4), one English (GB_1.2, GB_1.4), one Scottish (GB_2.2), one Swedish (S_2.2), three French (F_1.1, F_1.2, F_2.3, F_2.4, F_2.5, F_2.6, F_4.1), five German (D_2.1, D_3.2, D_3.3, D_8.3, D_9.2, D_10.2, D_10.5)], five haplotypes shared by two individuals each from one location [two French (F_2.1-2, F_4.2-3), three German (D_3.1/3.4, D_8.4/8.6, D_10.1/10.3)], one haplotype shared by three individuals from two locations [one Danish (DK_2.1), one Scottish (GB_2.1/2.3)] and one haplotype of 14 individuals from seven locations [one Danish (DK_3.1), one English (GB_1.3), one Swedish (S_2.1/2.3-6), four German (D_2.2, D_8.1-2/8.5, D_9.1/9.3, D_10.4)].

The protein haplotype network comprised 39 haplotypes with three major starlike arrangements (Fig. 38). The network comprised 27 single individual haplotypes from 21 locations [one Danish (DK_3.2, DK_3.4), one English (GB_1.2), one French (F_2.4), one Greek (GR_1.1), one Italian (I_2.1, I_2.2), one Russian (RUS_2.3), one Swedish (S_2.2), one Swiss (CH_3.2), two Austrian (A_3.1,A_5.1), two Polish (PL_1.2, PL_2.6), two Romanian (RUM_1.4, RUM_5.1, RUM_5.2), seven German locations (D_1.3, D_2.1, D_5.3, D_10.5, D_13.1, D_14.1, D_14.2, D_15.1, D_15.2, D_15.3)], five haplotypes of two individuals from five locations [one French (F_1.1-2), one German (D_8.4/8.6), one Polish (PL_1.1/1.3), one Romanian (RUM_1.1-2), one Russian (RUS_2.1-2)] and two haplotypes of three individuals from two locations [one from two Italian locations (I_2.3-4/I_3.1), one from two Romanian locations (RUM_5.3/RUM_6.1-2)].

\section{Phylogenetic and population genetic analyses}

All phylogenetic analyses (NJ without and with model of sequence evolution, MrBayes and Beast) showed a highly supported monophyletic group of A. coleoptrata with 14 subclades and five isolated individuals (four in MrBayes) in the nucleotide (Fig. 39, A42-45). The 14 subclades were supported by high bootstrap values and posterior probabilities. Only the arrangement of the 14 subclades was variable in the analyses. The individuals which built these clades are presented in Table 24 . In the phylogenetic trees of the protein all sequences are grouped in one highly supported monophyletic clade (Fig. 40, A46-48).

The minimum and maximum mean average pairwise differences for the nucleotide sequences between populations were $0.2 \%$ (D_8/S_2) and 19.4\% (D_14/RUS_2). Excluding Russia the maximum mean average pairwise differences was $18.7 \%$ (D_5/RUM_1 and D_5/RUM_2). Within populations the minimum and maximum mean average pairwise differences were 0\% (D_11) and 12\% (A_8) (Table 25). For the protein the minimum and maximum mean average pairwise differences between populations were $0 \%$ (all populations except for A_5, CH_3, D_2, D_5, D_8, D_10, D_14, D_15, DK_3, GB_1, I_2, PL_1, RUM_1, RUM_2, RUM_5 and RUS_2) and 2.6\% (D_2/D_15). The minimum and maximum mean average pairwise differences within population were $0 \%$ (A_7, A_8, CH_1, D_1, D_3, D_9, D_11, D_12, EST_1, F_1, F_2, F_4, FIN_1, GB_2, GR_1, I_1, PL_2, RUM_6 and $\mathrm{S} \_2$ ) and $2.4 \%$ for the protein (D_15) (Table A32). 


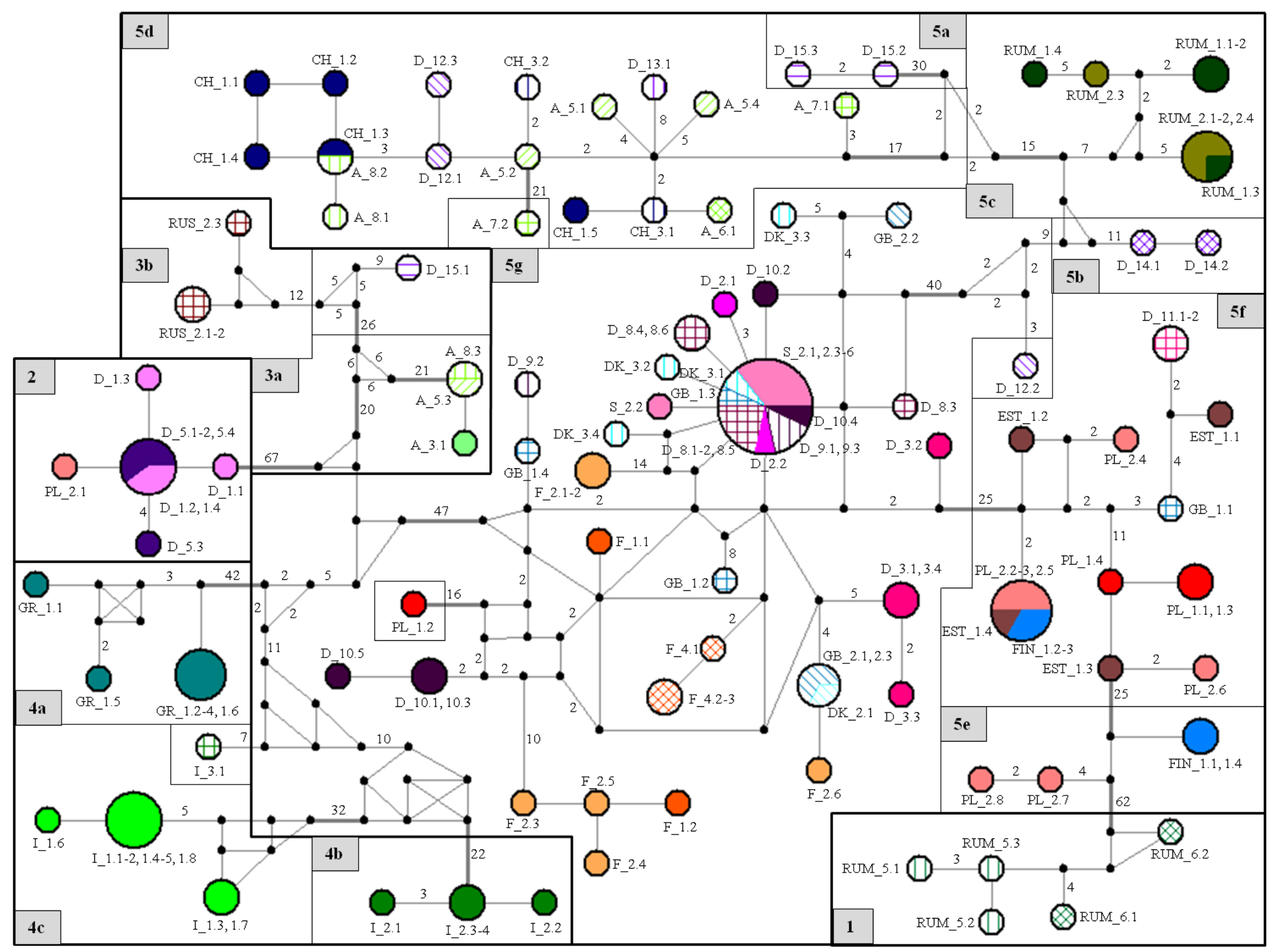

Figure 37: Median-joining haplotype network for the COI nucleotide of 93 haplotypes from Achipteria coleoptrata. Each location has a specific colour marking. The size of the circles is proportional to the number of sequences per haplotype. Numbers on the lines represent the number of substitution steps separating the haplotypes (no number: only one substitution step between haplotypes). Major subclades are marked by boxes. 


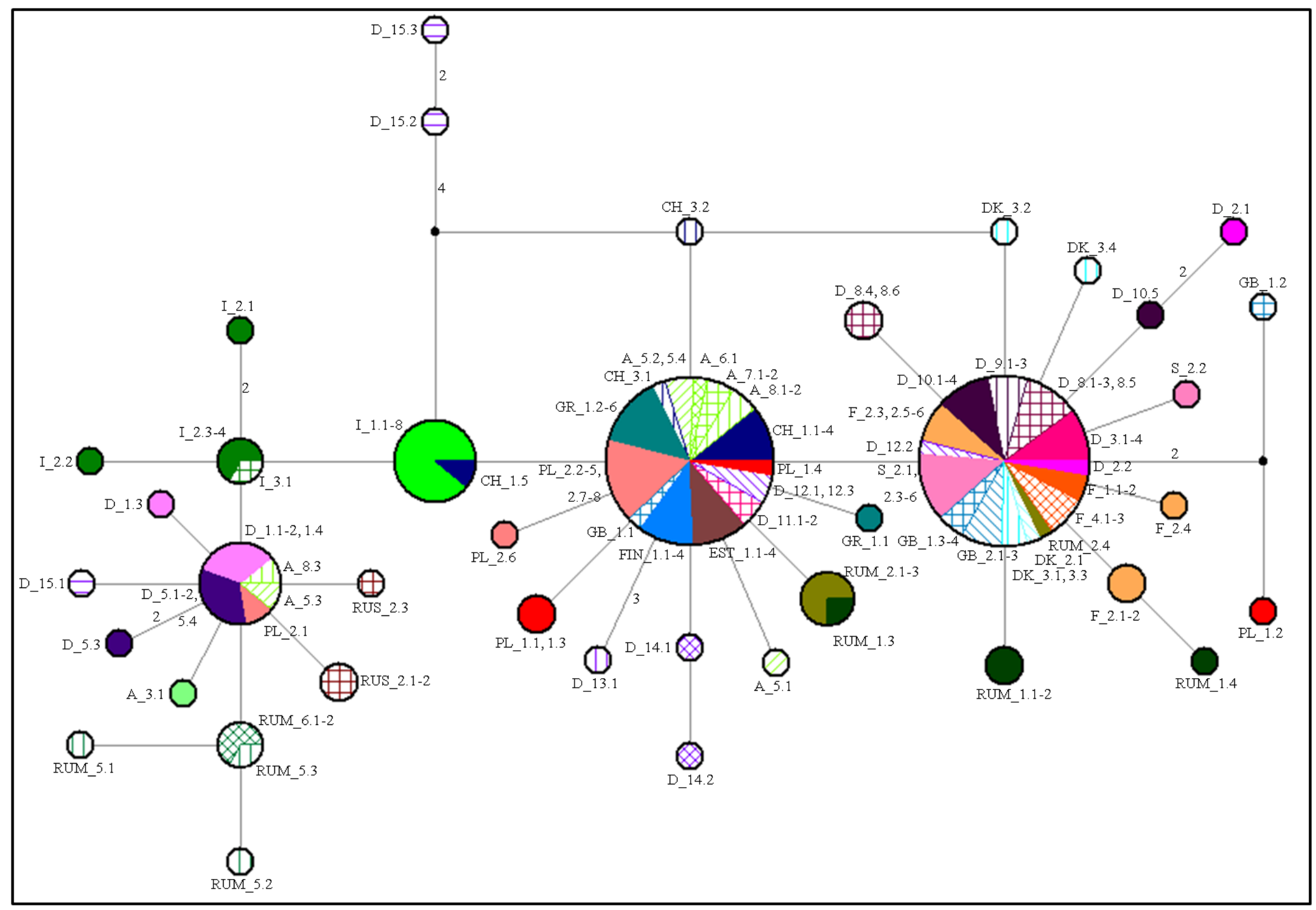

Figure 38: Median-joining haplotype network for the COI protein of 39 haplotypes from Achipteria coleoptrata. Each location has a specific colour marking. The size of the circles is proportional to the number of sequences per haplotype. Numbers on the lines represent the number of substitution steps separating the haplotypes (no number: only one amino acid change between haplotypes). 


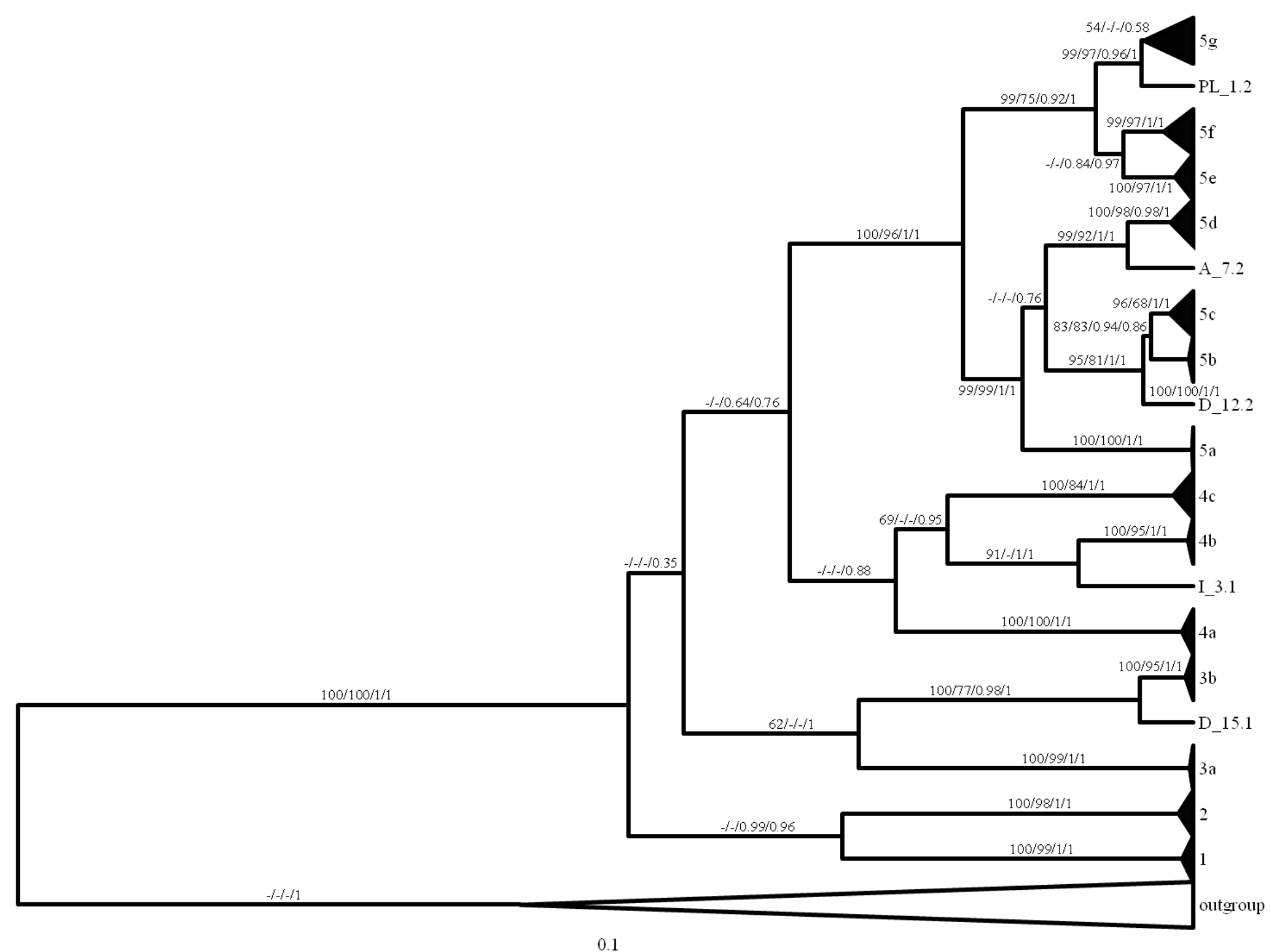

Figure 39: Bayesian phylogeny after $10 \times 10^{6}$ generations from the 141 COI nucleotide sequences of Achipteria coleoptrata with Beast v1.5.4. Outgroups are one individual of Carabodes femoralis and C. marginatus, respectively. Numbers on the branches are bootstrap values from NJ without and with evolution model (GTR+I+G) analysis and posterior probabilities from MrBayes and Beast. Tip numbers are the different subclades and explained in Table 24. 


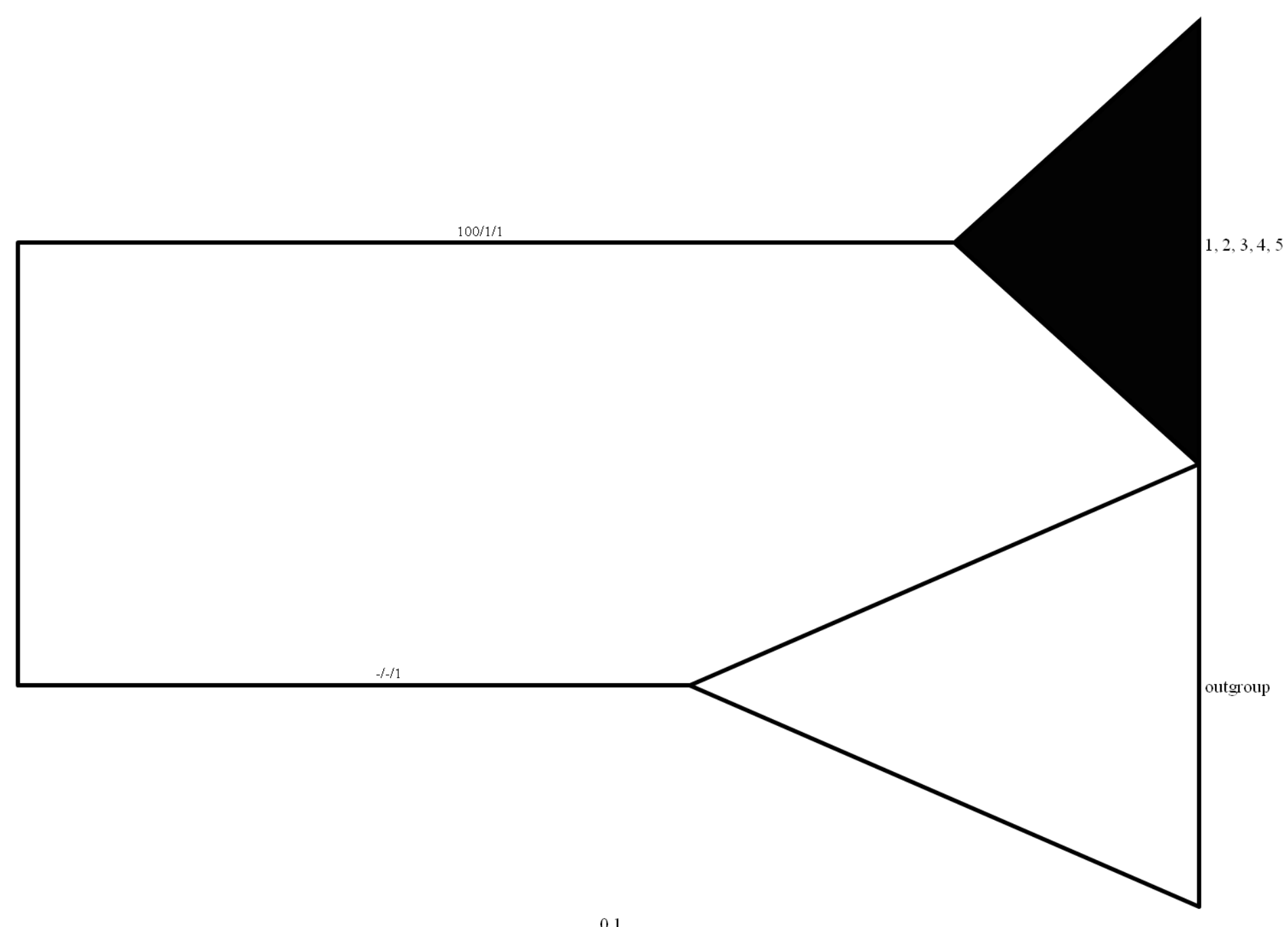

Figure 40: Bayesian phylogeny after 10x $10^{6}$ generations from the 141 COI protein sequences of Achipteria coleoptrata with Beast v1.5.4. Outgroups are one individual of Carabodes femoralis and C. marginatus, respectively. Numbers on the branches are bootstrap values from NJ and posterior probabilities from MrBayes and Beast. Tip numbers are the different subclades and explained in Table 24. 
Table 24: Subclades of Bayesian phylogenetic trees of Achipteria coleoptrata based on COI nucleotide sequences (\#ind=number or individuals, $\mathrm{pp}=$ posterior probabilities, sampling sites with abbreviations, individuals and ind. pop.=quantity of individuals from the population).

\begin{tabular}{|c|c|c|c|c|c|c|}
\hline \multirow{2}{*}{$\frac{\text { Subclade }}{1}$} & \multirow{2}{*}{$\frac{\# \text { ind }}{5}$} & \multirow{2}{*}{$\frac{\text { pp }}{1}$} & \multicolumn{2}{|c|}{ sampling sites } & \multirow{2}{*}{$\frac{\text { individuals }}{1-3}$} & \multirow{2}{*}{$\frac{\text { ind. pop. }}{3 / 3}$} \\
\hline & & & Busteni & RUM_5 & & \\
\hline & & & Sinaia & RUM_6 & $1-2$ & $2 / 2$ \\
\hline \multirow[t]{3}{*}{2} & 9 & 1 & Kranichstein & D_1 & $1-4$ & $4 / 4$ \\
\hline & & & Moerfelden & D_5 & $1-4$ & $4 / 4$ \\
\hline & & & Warsaw & PL_2 & 1 & $1 / 8$ \\
\hline 3 & 7 & 1 & 5 & & & \\
\hline \multirow[t]{3}{*}{$3 \mathbf{a}$} & 3 & 1 & Hittisau & A_3 & 1 & $1 / 1$ \\
\hline & & & Memminger Huette & A_5 & 3 & $1 / 4$ \\
\hline & & & Imst & A_8 & 3 & $1 / 3$ \\
\hline isol. ind. & 1 & & Langenwang & D_15 & 1 & $1 / 3$ \\
\hline $\mathbf{3 b}$ & 3 & 1 & Novosibirsk & RUS_2 & $1-3$ & $3 / 3$ \\
\hline 4 & 19 & 0.88 & 4 & & & \\
\hline $4 a$ & 6 & 1 & Thessaloniki & GR_1 & $1-6$ & $6 / 6$ \\
\hline isol. ind. & 1 & & Felitto & I_3 & 1 & $1 / 1$ \\
\hline $4 b$ & 4 & 1 & Parma & I_2 & $1-4$ & $4 / 4$ \\
\hline $4 c$ & 8 & 1 & Grosseto & I_1 & $1-8$ & $8 / 8$ \\
\hline 5 & 101 & 1 & 30 & & & \\
\hline $5 \mathbf{a}$ & 2 & 1 & Langenwang & D_15 & $2-3$ & $2 / 3$ \\
\hline isol. ind. & 1 & & Sonthofen & D_12 & 2 & $1 / 3$ \\
\hline $5 \mathbf{b}$ & 2 & 1 & Steineberg & D_14 & $1-2$ & $2 / 2$ \\
\hline \multirow[t]{2}{*}{$5 c$} & 8 & 1 & Sibiu_1 & RUM_1 & $1-4$ & $4 / 4$ \\
\hline & & & Sibiu_2 & RUM_2 & $1-4$ & $4 / 4$ \\
\hline isol. ind. & 1 & & Roggendorf & A_7 & 2 & $1 / 2$ \\
\hline \multirow[t]{8}{*}{$5 d$} & 17 & 1 & Memminger Huette & A_5 & $1-2,4$ & $3 / 4$ \\
\hline & & & Landeck & A_6 & 1 & $1 / 1$ \\
\hline & & & Roggendorf & A_7 & 1 & $1 / 2$ \\
\hline & & & Imst & A_8 & $1-2$ & $2 / 3$ \\
\hline & & & Basel & CH_1 & $1-5$ & $5 / 5$ \\
\hline & & & Rorschach & CH_3 & $1-2$ & $2 / 2$ \\
\hline & & & Sonthofen & D_12 & 1,3 & $2 / 3$ \\
\hline & & & Gunzesried & D_13 & 1 & $1 / 1$ \\
\hline \multirow[t]{2}{*}{$5 e$} & 4 & 1 & Lahti & FIN_1 & 1,4 & $2 / 4$ \\
\hline & & & Warsaw & PL_2 & $7-8$ & $2 / 8$ \\
\hline \multirow[t]{6}{*}{$5 f$} & 17 & 1 & Wittmoor & D_11 & $1-2$ & $2 / 2$ \\
\hline & & & Tallin & EST_1 & $1-4$ & $4 / 4$ \\
\hline & & & Lahti & FIN_1 & $2-3$ & $2 / 4$ \\
\hline & & & Ascot & GB_1 & 1 & $1 / 4$ \\
\hline & & & Krakow & PL_1 & $1,3-4$ & $3 / 4$ \\
\hline & & & Warsaw & $\mathrm{PL} \_2$ & $2-6$ & $5 / 8$ \\
\hline isol. ind. & 1 & & Krakow & PL_1 & 2 & $1 / 4$ \\
\hline
\end{tabular}


Chapter 4 Sex versus parthenogenesis

Table 24 continue:

\begin{tabular}{ccccccc}
\hline Subclade & \# ind & pp & sampling sites & & individuals & ind. pop. \\
\hline $\mathbf{5 g}$ & $\mathbf{4 8}$ & $\mathbf{0 . 5 8}$ & Goettingen & D_2 & $1-2$ & $2 / 2$ \\
& & Lake Constance & D_3 & $1-4$ & $4 / 4$ \\
& & Cuxhaven & D_8 & $1-6$ & $6 / 6$ \\
& & Bonn & D_9 & $1-3$ & $3 / 3$ \\
& & Colonge & D_10 & $1-5$ & $5 / 5$ \\
& & Hjørring & DK_2 & 1 & $1 / 1$ \\
& & Arhus_Morvadsvej & DK_3 & $1-4$ & $4 / 4$ \\
& & Mont Blanc & F_1 & $1-2$ & $2 / 2$ \\
& & Loire & F_2 & $1-6$ & $6 / 6$ \\
& & Haute Loire & F_4 & $1-3$ & $3 / 3$ \\
& & Ascot & GB_1 & $2-4$ & $3 / 4$ \\
& & Braemar & GB_2 & $1-3$ & $3 / 3$ \\
& & Stroemstad & S_2 & $1-6$ & $6 / 6$ \\
\hline
\end{tabular}


Table 25: Mean percentage pairwise differences of uncorrected p-distances of the COI nucleotide sequences of Achipteria coleoptrata from 35 locations. The diagonal is the within population differences (bold) and below the diagonal the among population differences. Bold letters in red are the minimum and maximum differences within and among the populations. Bold letters in pink are the maximum differences, if Russia is excluded. Locations with less than two individuals were excluded from the analysis.

\begin{tabular}{|c|c|c|c|c|c|c|c|c|c|c|c|c|c|c|c|c|c|c|c|c|c|c|c|c|c|c|c|c|c|}
\hline \multicolumn{2}{|c|}{ Population } & \multirow{2}{*}{$\frac{1}{9.7}$} & \multirow[t]{2}{*}{2} & \multirow[t]{2}{*}{3} & \multirow[t]{2}{*}{4} & \multirow[t]{2}{*}{5} & \multirow[t]{2}{*}{6} & \multirow[t]{2}{*}{7} & \multirow[t]{2}{*}{8} & \multirow[t]{2}{*}{9} & \multirow[t]{2}{*}{10} & \multirow[t]{2}{*}{11} & \multirow[t]{2}{*}{12} & \multirow[t]{2}{*}{13} & \multirow[t]{2}{*}{14} & \multirow[t]{2}{*}{15} & 16 & 17 & 18 & 19 & 20 & 21 & 22 & 23 & 24 & 25 & 26 & 27 & 28 \\
\hline 1 & A 5 & & & & & & & & & & & & & & & & & & & & & & & & & & & & \\
\hline 2 & A 7 & 6.8 & 4.9 & & & & & & & & & & & & & & & & & & & & & & & & & & \\
\hline 3 & A 8 & 8.2 & 8.1 & 12 & & & & & & & & & & & & & & & & & & & & & & & & & \\
\hline 4 & CH 1 & 5.6 & 3.4 & 6.2 & 0.8 & & & & & & & & & & & & & & & & & & & & & & & & \\
\hline 5 & CH 3 & 5.4 & 3 & 6.8 & 1.3 & 1.2 & & & & & & & & & & & & & & & & & & & & & & & \\
\hline 6 & D 1 & 17.8 & 17.4 & 17.4 & 17.4 & 18 & 0.2 & & & & & & & & & & & & & & & & & & & & & & \\
\hline 7 & D 2 & 13 & 11.8 & 13.1 & 12.2 & 12.4 & 17.1 & 0.6 & & & & & & & & & & & & & & & & & & & & & \\
\hline 8 & D 3 & 13.2 & 11.8 & 13.1 & 12.4 & 12.5 & 17.1 & 1.7 & 1.2 & & & & & & & & & & & & & & & & & & & & \\
\hline 9 & D 5 & 17.9 & 17.5 & 17.5 & 17.5 & 18.1 & 0.3 & 17.2 & 17.1 & 0.4 & & & & & & & & & & & & & & & & & & & \\
\hline 10 & D 8 & 12.7 & 11.5 & 12.8 & 11.9 & 12.1 & 16.9 & 0.4 & 1.5 & 17 & 0.2 & & & & & & & & & & & & & & & & & & \\
\hline 11 & D 9 & 12.8 & 11.6 & 12.8 & 12.1 & 12.3 & 16.9 & 0.7 & 1.6 & 17 & 0.5 & 0.8 & & & & & & & & & & & & & & & & & \\
\hline 12 & D 10 & 13 & 11.6 & 13 & 12.2 & 12.5 & 17 & 1.6 & 2.3 & 17.2 & 1.5 & 1.5 & 1.5 & & & & & & & & & & & & & & & & \\
\hline 13 & D 11 & 12.4 & 10.7 & 13 & 11.6 & 11.3 & 17.2 & 6.4 & 6.6 & 17.3 & 6.1 & 6.1 & 6.4 & 0 & & & & & & & & & & & & & & & \\
\hline 14 & D 12 & 7.1 & 4.8 & 8.1 & 3.5 & 3.5 & 17.1 & 11 & 11.3 & 17.2 & 10.7 & 11 & 11.2 & 10.9 & 5.5 & & & & & & & & & & & & & & \\
\hline 15 & D 14 & 11.5 & 9 & 12.4 & 9 & 8.9 & 18.6 & 12.5 & 12.8 & 18.6 & 12.2 & 12.3 & 12.8 & 11.8 & 7.7 & 0.2 & & & & & & & & & & & & & \\
\hline 16 & D 15 & 13.1 & 12 & 13.3 & 11.9 & 12 & 17.5 & 14.4 & 14.9 & 17.7 & 14.1 & 14.2 & 14.7 & 13 & 12.1 & 13.3 & 11 & & & & & & & & & & & & \\
\hline 17 & DK 3 & 12.9 & 11.8 & 12.9 & 12 & 12.3 & 16.8 & 0.8 & 1.8 & 16.9 & 0.6 & 0.8 & 1.7 & 6.4 & 10.9 & 12.3 & 14.3 & 0.9 & & & & & & & & & & & \\
\hline 18 & EST 1 & 12.9 & 11 & 13.3 & 11.9 & 11.7 & 17.6 & 6.5 & 6.6 & 17.7 & 6.2 & 6.2 & 6.4 & 2 & 11.2 & 12 & 13.6 & 6.4 & 2.4 & & & & & & & & & & \\
\hline 19 & F 1 & 13.6 & 12.2 & 13.5 & 12.9 & 13.1 & 17.4 & 3.2 & 3.7 & 17.5 & 3 & 3 & 2.9 & 7 & 11.7 & 13 & 15 & 3 & 7.3 & 3.9 & & & & & & & & & \\
\hline 20 & F 2 & 13.3 & 12.1 & 13.3 & 12.4 & 12.7 & 17.6 & 3.3 & 3.9 & 17.8 & 3.1 & 3.2 & 3.1 & 7.5 & 11.4 & 12.9 & 15 & 3.2 & 7.5 & 3.1 & 3.4 & & & & & & & & \\
\hline 21 & F 4 & 12.9 & 11.5 & 13 & 11.9 & 12.2 & 16.2 & 0.9 & 1.9 & 16.4 & 0.8 & 1 & 1.7 & 5.8 & 10.7 & 12 & 14.1 & 1.1 & 5.9 & 2.9 & 3.2 & 0.1 & & & & & & & \\
\hline 22 & FIN 1 & 12.4 & 10.5 & 12.6 & 11.1 & 11.1 & 16.5 & 6.3 & 6.5 & 16.7 & 11.9 & 11.9 & 6.3 & 3.9 & 10.7 & 12 & 13.4 & 6.3 & 3.6 & 7.4 & 7.3 & 5.8 & 3.9 & & & & & & \\
\hline 23 & GB 1 & 13.1 & 11.7 & 13.2 & 12.4 & 12.5 & 17.3 & 2.6 & 3.2 & 17.4 & 2.5 & 2.4 & 3.2 & 5 & 11.3 & 12.5 & 14.2 & 2.7 & 5.2 & 4.2 & 4.6 & 2.7 & 5.6 & 4.2 & & & & & \\
\hline 24 & GB 2 & 12.6 & 11.3 & 12.5 & 11.8 & 12 & 16.8 & 1.5 & 2.1 & 16.9 & 1.4 & 1.5 & 2.3 & 5.9 & 10.9 & 12.7 & 14.2 & 1.5 & 5.9 & 3.6 & 3.6 & 1.8 & 5.8 & 3 & 1.7 & & & & \\
\hline 25 & GR 1 & 15.2 & 15.2 & 14.8 & 14.6 & 15.1 & 16 & 14.3 & 14.3 & 16.1 & 14 & 14 & 13.9 & 13.4 & 14.7 & 16.3 & 15.9 & 13.9 & 13.5 & 14.4 & 14.4 & 13.9 & 13.3 & 13.9 & 13.9 & 0.7 & & & \\
\hline 26 & I 1 & 16.5 & 16.4 & 16 & 16.5 & 17.1 & 17.1 & 15.6 & 15.9 & 17.2 & 15.3 & 15.1 & 15.1 & 14 & 17.1 & 17.8 & 16.6 & 15.3 & 14.2 & 15.8 & 15.7 & 15 & 14.4 & 15.3 & 15.5 & 12.8 & 0.6 & & \\
\hline 27 & I 2 & 16.4 & 16.3 & 15.8 & 16.4 & 16.6 & 16 & 15.5 & 15.3 & 16.1 & 15.3 & 15 & 15.2 & 14.3 & 16.5 & 16.7 & 16.2 & 15.1 & 14.5 & 15.4 & 15.8 & 15.6 & 15 & 15.1 & 14.7 & 13.8 & 11.7 & 0.4 & \\
\hline 28 & PL 1 & 13.3 & 11.6 & 13.5 & 12.3 & 12.4 & 18 & 6.1 & 6.4 & 18.1 & 5.9 & 5.9 & 5.8 & 4.2 & 11.4 & 12.7 & 14.3 & 6.1 & 3.8 & 6.8 & 7 & 5.6 & 5.1 & 5.6 & 5.8 & 14.1 & 14.4 & 15.2 & 4.1 \\
\hline 29 & PL 2 & 13.4 & 11.7 & 13.6 & 12.4 & 12.4 & 15.1 & 8 & 8.1 & 15.3 & 7.7 & 7.7 & 7.9 & 5.4 & 11.9 & 12.8 & 14.3 & 7.9 & 5 & 8.8 & 8.9 & 7.5 & 5.2 & 7.2 & 7.5 & 13.9 & 14.7 & 15 & 6.4 \\
\hline 30 & RUM 1 & 11.8 & 9.3 & 12.7 & 9.8 & 9.6 & 18.6 & 11.1 & 11.4 & 18.7 & 10.8 & 11.1 & 11.4 & 11.4 & 8.2 & 5.2 & 13.5 & 10.9 & 11.7 & 11.8 & 11.7 & 11 & 11.7 & 11.4 & 11.2 & 16.5 & 18.4 & 17.9 & 12.6 \\
\hline 31 & RUM 2 & 11.5 & 9.1 & 12.4 & 9.4 & 9.4 & 18.6 & 10.8 & 11.2 & 18.7 & 10.6 & 10.8 & 11.1 & 10.9 & 8 & 5.1 & 13.4 & 10.7 & 11.2 & 11.6 & 11.6 & 10.7 & 11.3 & 11.1 & 10.9 & 16.3 & 18.2 & 17.8 & 12.2 \\
\hline 32 & RUM 5 & 16.3 & 16 & 15.9 & 15.8 & 16.1 & 14.9 & 15.9 & 16 & 15 & 15.7 & 15.6 & 15.1 & 14.2 & 15.7 & 16.8 & 17.1 & 15.7 & 14.8 & 16.1 & 16.2 & 15.4 & 14.2 & 15.6 & 15.6 & 16.9 & 15 & 16.1 & 15.3 \\
\hline 33 & RUM 6 & 16.1 & 15.7 & 15.9 & 15.8 & 15.8 & 14.5 & 15.4 & 15.5 & 14.6 & 15.2 & 15.2 & 14.7 & 13.9 & 15.6 & 16.5 & 17 & 15.3 & 14.4 & 15.7 & 15.8 & 15 & 14 & 15.2 & 15.4 & 16.6 & 14.9 & 15.7 & 14.7 \\
\hline 34 & RUS 2 & 16.8 & 17.4 & 16.2 & 14.4 & 17.6 & 18.6 & 17.1 & 17.7 & 18.7 & 16.9 & 16.8 & 17.2 & 16.6 & 17.3 & 19.4 & 14 & 16.9 & 17 & 17.4 & 17.7 & 16.8 & 16.9 & 16.9 & 17.1 & 16.3 & 16.5 & 17.7 & 17.4 \\
\hline 35 & $\mathrm{~S} 2$ & 12.8 & 11.6 & 12.8 & 11.9 & 12.1 & 16.8 & 0.3 & 1.4 & 16.9 & 0.2 & 0.4 & 1.4 & 6.1 & 10.8 & 12.2 & 14.1 & 0.5 & 6.2 & 3 & 3.1 & 0.7 & 6 & 2.4 & 1.3 & 14 & 15.3 & 15.3 & 5.8 \\
\hline
\end{tabular}


Table 25 continue:

\begin{tabular}{lcccccccc}
\hline Population & 29 & $\mathbf{3 0}$ & $\mathbf{3 1}$ & $\mathbf{3 2}$ & $\mathbf{3 3}$ & $\mathbf{3 4}$ & $\mathbf{3 5}$ \\
\hline $\mathbf{2 9}$ & PL 2 & 7.4 & & & & & & \\
$\mathbf{3 0}$ & RUM 1 & 12.6 & $\mathbf{1 . 6}$ & & & & & \\
$\mathbf{3 1}$ & RUM 2 & 12.2 & 1.5 & $\mathbf{0 . 9}$ & & & & \\
$\mathbf{3 2}$ & RUM 5 & 14.7 & 17.9 & 17.4 & $\mathbf{0 . 5}$ & & & \\
$\mathbf{3 3}$ & RUM 6 & 14.3 & 17.5 & 17.1 & 1 & $\mathbf{1 . 2}$ & & \\
$\mathbf{3 4}$ & RUS 2 & 17.5 & 18.8 & 18.6 & 16.1 & 16.4 & $\mathbf{0 . 4}$ & \\
$\mathbf{3 5}$ & S 2 & 7.7 & 10.8 & 10.6 & 15.5 & 15.1 & 16.8 & $\mathbf{0 . 1}$ \\
\hline
\end{tabular}

The results of the AMOVA showed that the nucleotide variation among countries (24.5\%), among samples within countries $(55.4 \%)$ and the variation within samples $(20.1 \%)$ were significant and high (Table 26).

Table 26: AMOVA table on variations among countries and within populations in nucleotide (black) and protein (red) sequences of COI of Achipteria coleoptrata. Each population was considered as separate groups. Populations with less than two individuals were excluded from the analysis. Significance level is $\mathrm{p}<0.05$ (d.f.: degree of freedom).

\begin{tabular}{lccccc}
\hline \multicolumn{1}{c}{ Source of variance } & d.f. & sum of squares & variance components & percent of total variation & fixation indices \\
\hline among countries & $12 / 12$ & $1891.5 / 4.5$ & $7.71 \mathrm{Va}^{*} /-0.04 \mathrm{Va}$ & $24.51 /-13.31$ & $\mathrm{FCT}: 0.26^{*} /-0.13$ \\
among populations & $20 / 20$ & $1337.35 / 11.75$ & $17.43 \mathrm{Vb}^{*} / 0.11 \mathrm{Vb} *$ & $55.41 / 41.22$ & $\mathrm{FSC}: 0.73^{*} / 0.36^{*}$ \\
within population & $96 / 96$ & $606.18 / 18.88$ & $6.31 \mathrm{Vc}^{*} / 0.2 \mathrm{Vc}$ & $20.08 / 72.09$ & $\mathrm{FST}: 0.8^{*} / 0.28^{*}$ \\
total & $128 / 128$ & $3835.03 / 35.13$ & $31.45 / 0.27$ & & \\
\hline
\end{tabular}

The neutrality tests of Tajima's D and Fu's FS were neither significant for the nucleotide nor for the protein in A. coleoptrata (Table A33 and A34). The results of the McDonald-Kreitman test showed that the differences between 58 of 595 (9.8\%) populations were significant, between other 41 of 595 (6.9\%) were high significant and between 37 of 595 (6.2\%) other populations were highly significant (Table 27 and for detail Table A35). All computed Neutrality indices were $\geq 0$ in the COI nucleotide sequences of A. coleoptrata (Table A36).

None of the calculated rarefaction curves of the nucleotide and the protein reached saturation (Fig. 41 and 42). The Jackknife rarefaction curves ended at 245 haplotypes for the nucleotide and 97 haplotypes for the protein. The unique rarefaction curves ended at 69 haplotypes for the nucleotide and 27 haplotypes for the protein. The observed rarefaction curves (Sobs Mao Tau) ended at 91 haplotypes for the nucleotide and 39 haplotypes for the protein.

The results of the Mantel test indicate isolation by distance in A. coleoptrata $\left[\mathrm{R}^{2}=0.0713, \mathrm{p}=0.004\right.$ (Fig. A49) and $\mathrm{R}^{2}=0.109, \mathrm{p}=0.001$ for $\log$ transformed geographic distances (Fig. 43) using 1000 randomizations], but this explained only $10 \%$ of the variation in the data.

The gmyc model had a better fit but not a significant better fit in the single and the multiple analyses than the null model (Table 18). For the single analysis the model identified five distinct COI clusters and for the multiple analysis 30. The clusters of the single analysis were identical with the phylogenetic main clusters, but differed in their grouping (Fig. A50). The clades 4a-c and clades 5a-g were grouped to one cluster, respectively.

The clusters of the multiple analysis differed from the clusters which were delimited by the phylogenetic analyses (Fig. A50). Phylogenetic clade 1 was not identified in the gmyc model, clade 2 
was split into two distinct clades and the clade 3a included the isolated individual D_15.1. In clade 3b individual A_3.1 was excluded. Clade 4a shrunk to four individuals (GR_1.2-4 and GR_1.6), clade 4b to two individuals (I_2.2-3) and clade 4c was split into three distinct clades with two individuals (I_1.1 and I_1.6, I_1.3 and I_1.7, I_1.5 and I_1.8), respectively. The clade 5b was not identified by the gmyc model, clade 5a was identically in the phylogenetic and the gmyc analysis. The clades 5c-g were split in multiple distinct clusters, (clade $5 \mathrm{~g}$ had nine, clade $5 \mathrm{~d}$ four, clade $5 \mathrm{f}$ three and clades $5 \mathrm{c}$ and $5 \mathrm{e}$ two distinct clusters). 
Chapter 4 Sex versus parthenogenesis

Table 27: Results of the McDonald-Kreitman test of Achipteria coleoptrata. The differences between 58 populations were significant $(* 0.01<\mathrm{P}<0.05)$, between other 41 populations high significant $(* * 0.001<\mathrm{P}<0.01)$ and between 37 populations highly significant $(* * * \mathrm{P}<0.001)$. Locations with less than two individuals were excluded.

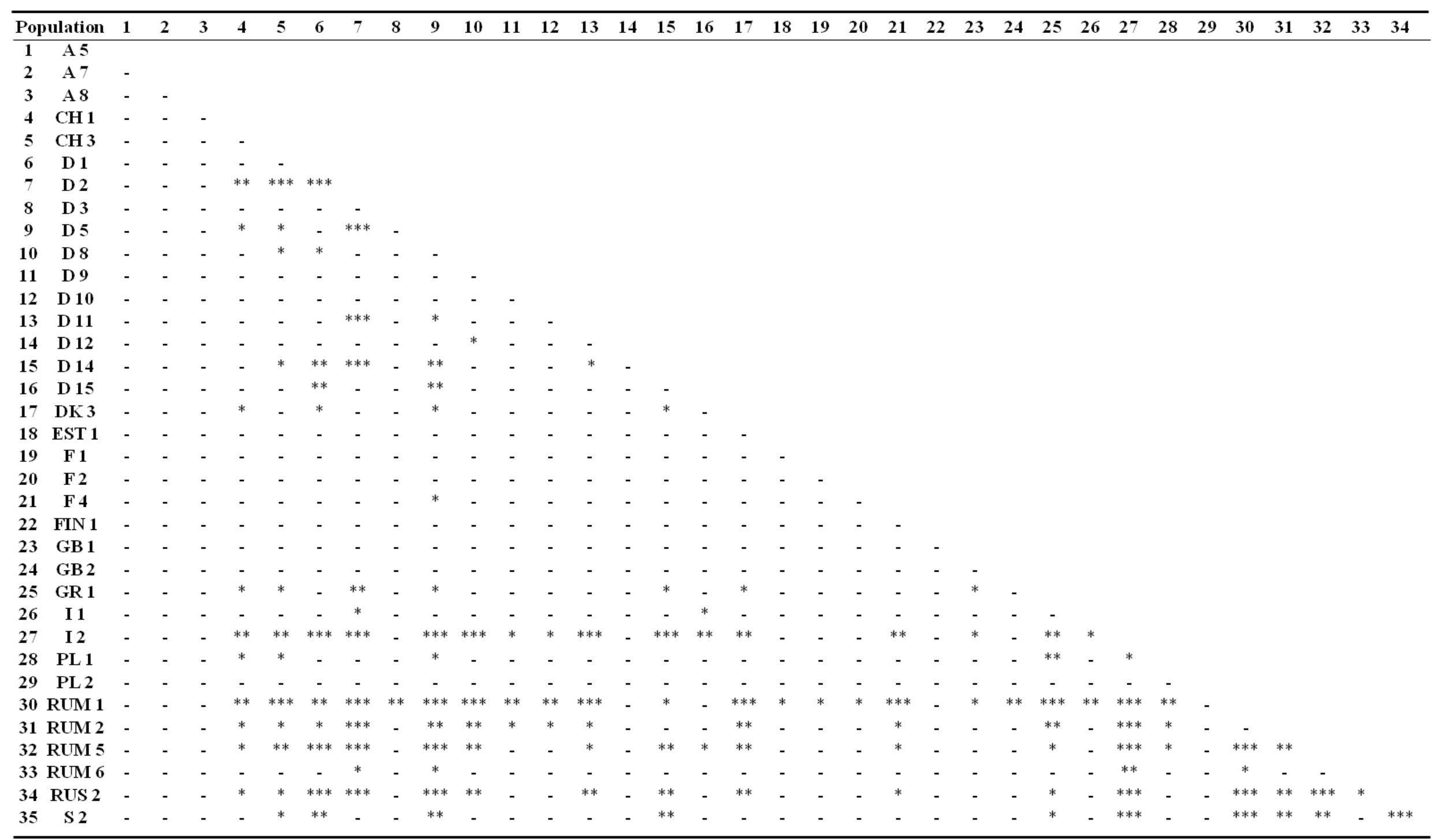


Chapter 4 Sex versus parthenogenesis

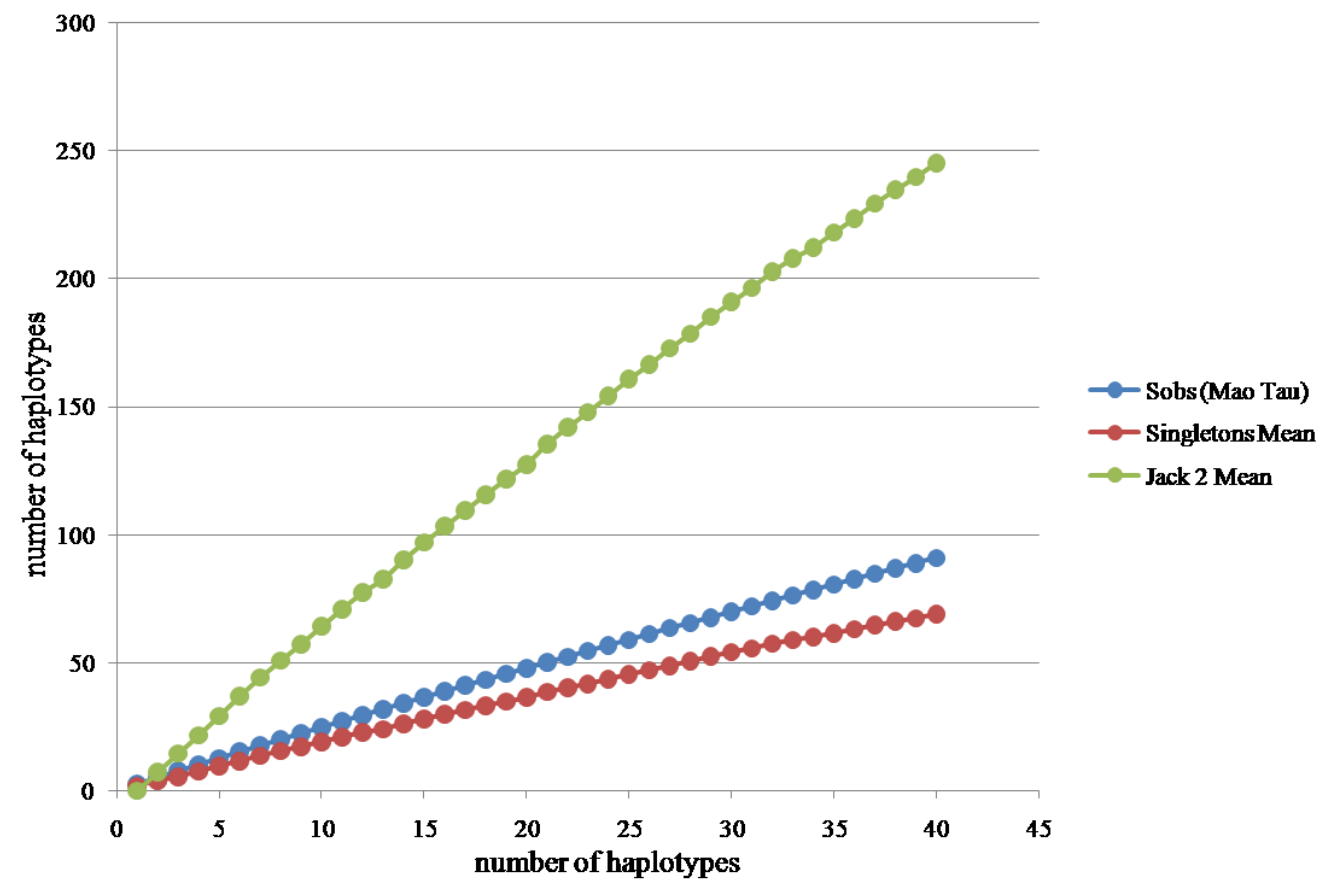

Figure 41: Sample based rarefaction analysis of haplotypes of the COI nucleotide of Achipteria coleoptrata. Jack 2 Mean, second-order Jackknife richness estimator, Singletons, numbers of haplotypes each present in only one sample, Sob (Mao Tau) is the observed haplotype richness.

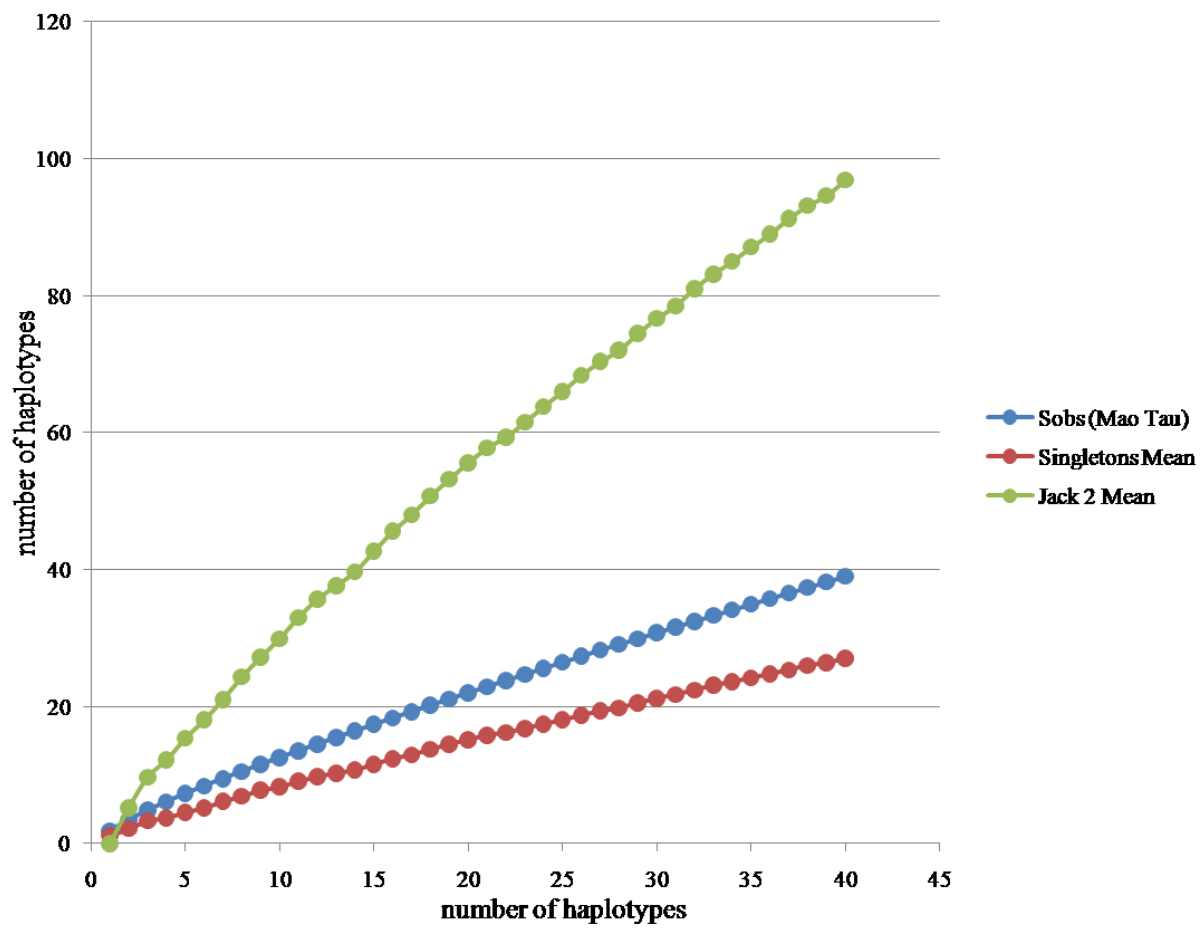

Figure 42: Sample based rarefaction analysis of haplotypes of the COI protein of Achipteria coleoptrata. Jack 2 Mean, second-order Jackknife richness estimator, Singletons, numbers of haplotypes each present in only one sample, Sob (Mao Tau) is the observed haplotype richness. 


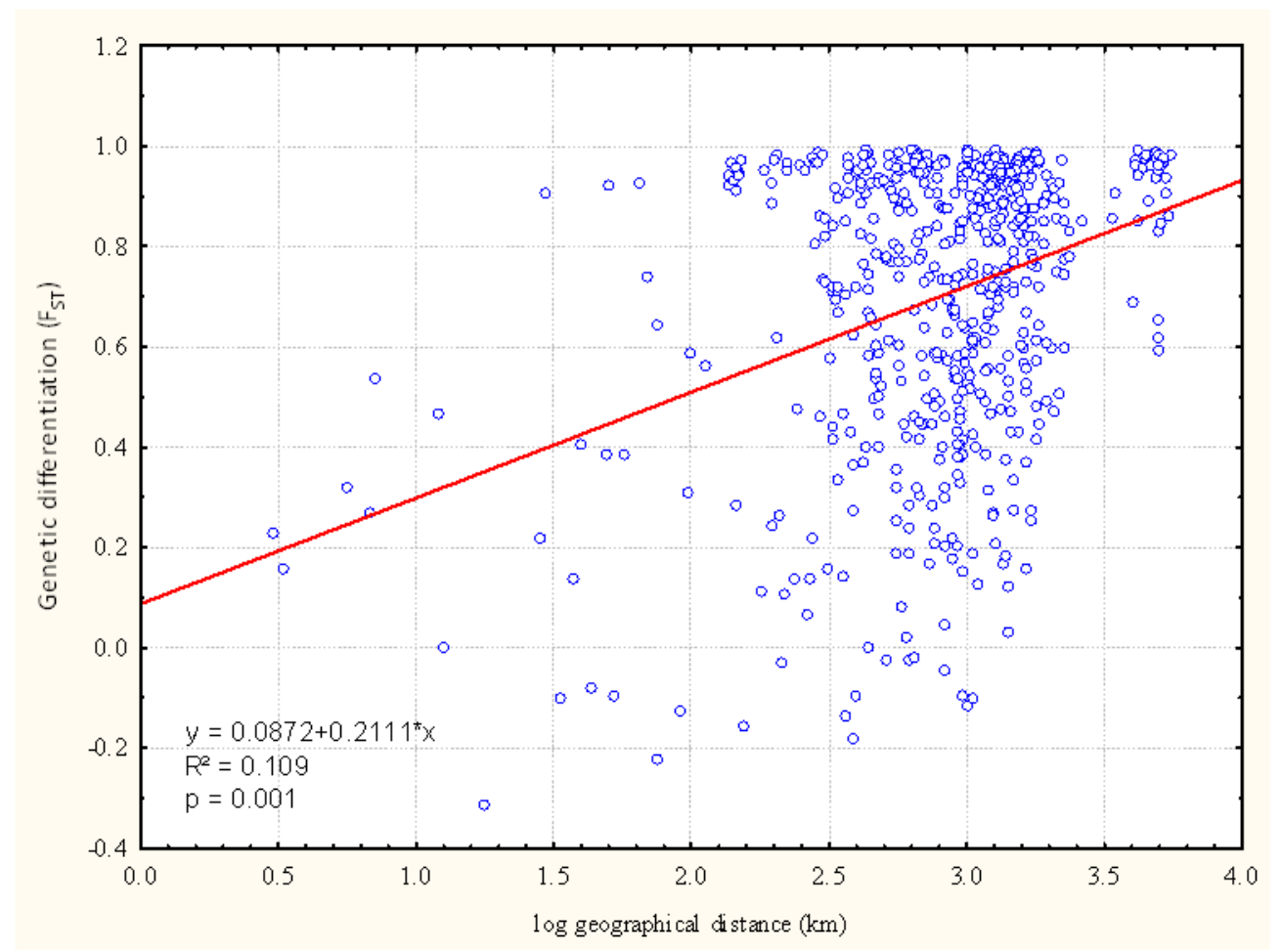

Figure 43: Linear regression of $\log 10$ geographical distances (kilometers) vs. genetic differentiations $\left(\mathrm{F}_{\text {ST }}\right)$ based on COI of Achipteria coleoptrata. Regression is significant using 1000 randomizations.

\subsubsection{Steganacarus magnus}

A total of 159 individuals (157 individuals of $S$. magnus, one individual of $R$. duplicata and one individual of $H$. rufulus) from 37 localities in 15 countries were sequenced. The sequences contained $27.5 \% \mathrm{~A}, 23.4 \% \mathrm{C}, 17.1 \% \mathrm{G}$ and $32 \% \mathrm{~T}$. The $157 \mathrm{COI}$ sequences comprised 96 haplotypes for the nucleotide (61.2\%) (Fig. 44) with 314 variable sites (59.3\%); 276 of these were parsimony informative sites (Table A37). The protein consisted of 59 haplotypes (37.6\%) (Fig. 45). The haplotype diversity $\left(\mathrm{H}_{\mathrm{d}}\right)$ was as high as in the $A$. coleoptrata with 0.98 in the nucleotide sequences.

\section{Haplotype networks}

The nucleotide haplotype network formed three main clades (black, blue dotted and red) with several subclades and isolated haplotypes. The main clades and subclades were separated each by large numbers of substitution steps (Fig. 44).

The black main clade formed eight subclades (subclade 1-8) with a total number of 87 individuals from 20 locations (D_1, D_2, D_3, D_5, D_8, D_9, DK_1, DK_3, F_2, F_3, GB_1, GB_2, I_1, I_2, NL_1, PL_2, RUM_1, RUM_2, RUM_5 and RUM_6) and comprised 49 haplotypes and two isolated single individual haplotypes from two locations (RUM_6 and S_2). Subclade 1 comprised three subclades 1a-c and one single individual haplotype from one German location (D_1.13). Subclade 1a comprised nine haplotypes from six locations [seven single individual haplotypes: two each from one Italian location (I_1.1, I_2.2), two from one French location (F_2.2, 2.4), three from two German locations (D_1.12, D_2.2, 2.8). Haplotypes of more than one individual: one of two individuals from on location in Germany (D_2.6-7), one of twelve individuals from six locations [two individuals from one French location (F_2.1, 2,3); four individuals from two Italian location (I_1.4, 1.9-10, I_2.4); six individuals from three German locations (D_1.1, D_2.1, 2.4-5, 2.9, D_9.1)]. Subclade 1b comprised six haplotypes from four locations [five single individual haplotypes: two each from one Italian location (I_1.2, I_2.1), three from two German locations (D_1.5, 1.14, D_9.2), one haplotype of six 
individuals from two locations (D_1.2, I_1.3, 1.5-8)]. Subclade 1c comprised four haplotypes from six locations [three single individual haplotypes each from one location (D_1.7, DK_1.3, DK_3.3), one haplotype of 13 individuals from five locations \{one from one Italian (I_2.3), two from one Danish (DK_3.1-2), ten from three German (D_1.3-4, 1.6, 1.8-11, D_2.3, D_9.3-4)\}].

Subclade 2 comprised three single individual haplotypes from one German location (D_3.1-3). Subclade 3 comprised eight haplotypes from five locations [single individual haplotypes: one from one German (D_8.3), one from one Dutch (NL_1.2), one from one French (F_3.1), three from two British (GB_1.1-2, GB_2.1). Haplotypes of more than one individual: two of three individuals each of two locations (one of one German and one Dutch location (D_8.1-2, NL_1.3), one of one Scottish and one Dutch location (GB_2.2-3, NL_.11))]. Subclade 4 comprised three haplotypes from one French location [two single individual haplotypes (F_3.2, 3.4) and one haplotype of two individuals (F_3.3, 3.5)]. Subclade 5 comprised one haplotype of three individuals from one German location (D_5.1-3). Subclade 6 comprised six single individual haplotypes from two Romanian locations (RUM_5.1-4, RUM_6.1-2). Subclade 7 comprised two haplotypes from one Polish location [one single individual (PL_2.2), one haplotype of two (PL_2.1, 2.3)]. Subclade 8 comprised six haplotypes from two Romanian locations [single individual haplotypes: one from one Romanian (RUM_1.3), four from another Romanian (RUM_2.1-4); one haplotype of two from one Romanian (RUM_1.1-2)].

The blue dotted main clade formed three subclades (subclade 9-11) with a total number of 16 individuals from seven locations (CZ_1, D_4, D_6, DK_1, F_1, PL_1 and S_2) and comprised 13 haplotypes and one isolated haplotype of two individuals from one Norwegian location (N_1.1, 1.3). Subclade 9 comprised five single individual haplotypes from four locations [one Czech (CZ_1.4), one German (D_6.1), one Swedish (S_2.1), and two Polish (PL_1.1, 1.3)]. Subclade 10 comprised three haplotypes from two locations [two single individual each from one location (CZ_1.5, F_1.2), one of two individuals from one Czech location (CZ_1.1-2)]. Subclade 11 comprised five haplotypes from two locations [single individual haplotypes: two of one Danish location (DK_1.1-2), two of one German location (D_4.1, 4.5); one haplotype of three individuals from one German location (D_4.2, 4.6-7)].

The red main clade formed seven subclades (subclade 12-18) with a total number of 38 individuals from 13 locations (D_4, D_5, DK_2, F_1, F_4, FIN_1, PL_1, RUM_3, RUM_4, RUS_1, RUS_2, S_1 and S_2) and comprised 21 haplotypes, two isolated haplotypes of two individuals each from one location (A_1.1-2 and D_7.2, 7.4) and eight single individual haplotypes from seven locations (CZ_1.3, D_4.4, D_5.5, D_7.1, 7.3, F_1.3, N_1.2 and RUS_1.4).

Subclade 12 comprised two subclades $12 \mathrm{a}-\mathrm{b}$ and one single individual from one Romanian location (RUM_3.3). Subclade 12a comprised three single individuals from one Romanian location (RUM_4.13). Subclade $12 \mathrm{~b}$ comprised one haplotype of three individuals from one Romanian location (RUM_3.1-2, 3.4). Subclade 13 comprised three single individuals from one Russian location (RUS_1.1-3). Subclade 14 comprised one haplotype of four Russian individuals (RUS_2.1-4). Subclade 15 comprised one haplotype of four French individuals (F_1.1, 1.4-6). Subclade 16 comprised three individuals from two Scandinavian locations [two single individuals each from one location (FIN_1.3, S_1.1), one haplotype of two Finish individuals (FIN_1.1-2)]. Subclade 17 comprised four haplotypes from four locations [three single individuals from two locations (PL_1.4-5, S_2.3), one haplotype of eight individuals from two locations (D_4.3, 4.8-9, DK_2.1-5)]. Subclade 18 comprised four haplotypes from three locations [three single individuals from two locations (D_5.4, PL_1.2, 1.6), one haplotype of two from one French location (F_4.1-2)]. 


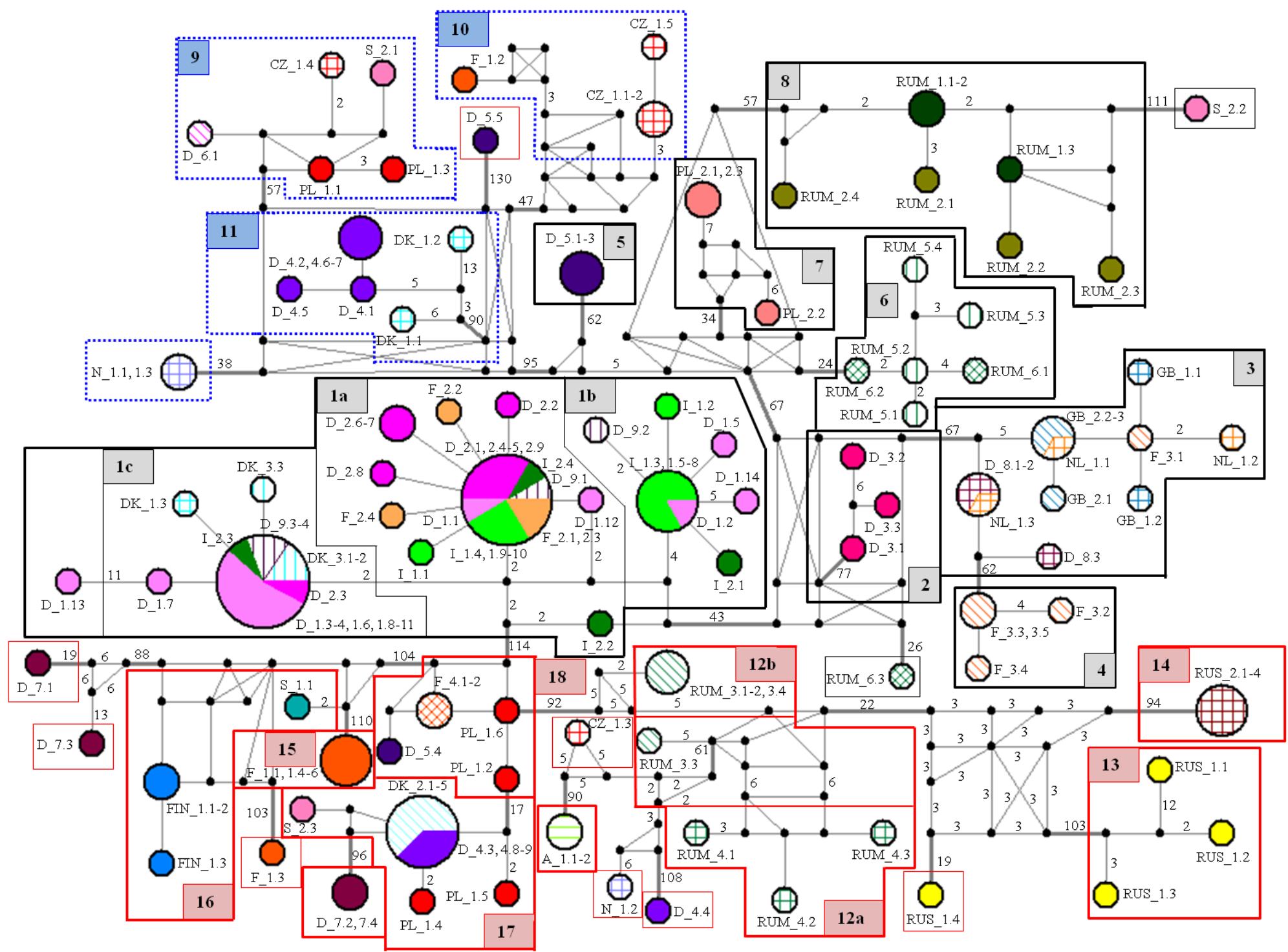

Figure 44: Median-joining haplotype network for the COI nucleotide of 96 haplotypes from Steganacarus magnus. Each location has a specific colour marking. The size of the circles is proportional to the number of sequences per haplotype. Numbers on the lines represent the number of substitution steps separating the haplotypes (no number: only one substitution step between haplotypes). Major subclades are marked by boxes. 
The protein haplotype network consisted of 59 haplotypes and formed the three main clades (black, blue and red), which were separated by large numbers of amino acid changes (Fig. 45).

The black main clade comprised six subclades and two isolated individuals each from one location (RUM_6.3, S_2.2). Subclades 1a-c and the isolated individual (D_1.13) fused two one subclade of four haplotypes [three single individuals from two locations (D_1.13-14, I_1.3), one haplotype of 46 individuals from eight locations]. The three single individuals of subclade 2 fused to one haplotype. Subclades 3 and 4 fused and comprised eight haplotypes from five locations [single individuals: four each of one location (D_8.3, GB_1.1, GB_2.1, NL_1.2), two of one French location (F_3.2, 3.4); haplotypes with more than one individual: one of two French (F_3.3, 3.5), one of eight from five locations (D_8.1-2, F_3.1, GB_1.2, GB_2.2-3, NL_1.1, 1.3)]. Subclade 5 comprised one haplotype of three individuals from one German location (D_5.1-3). Subclades 6 and 7 fused and comprised four haplotypes from three locations [two single individuals each from one location (PL_2.2, RUM_5.1), one haplotype of two from one Polish location (PL_2.1, 2.3), one haplotype of five from two Romanian locations (RUM_5.2-4, RUM_6.1-2)]. Subclade 8 comprised two haplotypes from two Romanian locations [one single individual (RUM_2.4), one of six individuals from two Romanian locations (RUM_1.1-3, RUM_2.1-3)].

The blue clade formed three subclades. Subclade 9 and the isolated haplotype (N_1.1, 1.3) fused and comprised four haplotypes from five locations [two single individuals each from one location (D_6.1, PL_1.3), one haplotype of two individuals from one Norwegian location ( $\left.N_{-} 1.1,1.3\right)$, one haplotype of three individuals from three locations (CZ_1.4, PL_1.1, S_2.1)]. Subclade 10 comprised two haplotypes from two locations [one single individual (CZ_1.5), one haplotype of three individuals from two locations (CZ_1.1-2, F_1.2)]. Subclade 11 comprised five haplotypes from two locations [single individuals: two from one Danish (DK_1.1-2), two from one German (D_4.1, 4.5), one haplotype of three individuals from one German location (D_4.2, 4.6-7)].

The red clade formed six subclades, three isolated single individuals each from one location (D_4.4, D_5.5, F_1.3), one isolated haplotype of two individuals from one German location (D_7.2, 7.4) and two isolated individuals from one German location grouped together (D_7.1, 7.3). Subclade 12a, b, isolated individuals RUM_3.3, CZ_1.3, N_1.2, RUS_1.4 and the haplotype of the two Austrian individuals (A_1.1-2) fused and comprised six haplotypes [three single individuals each from one location (CZ_1.3, N_1.2, RUM_3.3), one haplotype of two individuals from one Austrian location (A_1.1-2), one of three individuals from one Romanian location (RUM_3.1-2, 3.4), one of four individuals from two locations (RUM_4.1-3, RUS_1.4)]. Subclades 13, 14, 15 and 16 had no changes to the nucleotide. Subclade 17 and 18 fused and comprised six haplotypes from six locations [single individuals: two from one Polish location (PL_1.4-5), one from one German (D_5.4), one from one Swedish (S_2.3); haplotype with more than one individual: one of four individuals from two locations (F_4.1-2, PL_1.2, 1.6), one of eight individuals from two locations (D_4.3, 4.8-9, DK_2.1-5)].

Haplotypes of individuals from the same location existed in different subclades like CZ_1, D_4, D_5, F_1, N_1, PL_1 and S_2 and among these subclades there were several high amino acid changes between individuals from the same location $\left(C Z \_1.1 / C Z \_1.4, C Z \_1.4 / C Z 1.5, D \_1.13 / D \_1.14\right.$, D_4.3/D_4.4, D_7.1/D_7.2, D7.2/D_7.3, F_1.1/F_1.3, F_3.1/F_3.2, $\quad$ RUM6.1/RUM6.3, RUS_1.1/RUS_1.4, RUS_1.2/RUS_1.4 and RUS_13/RUS_1.4). 


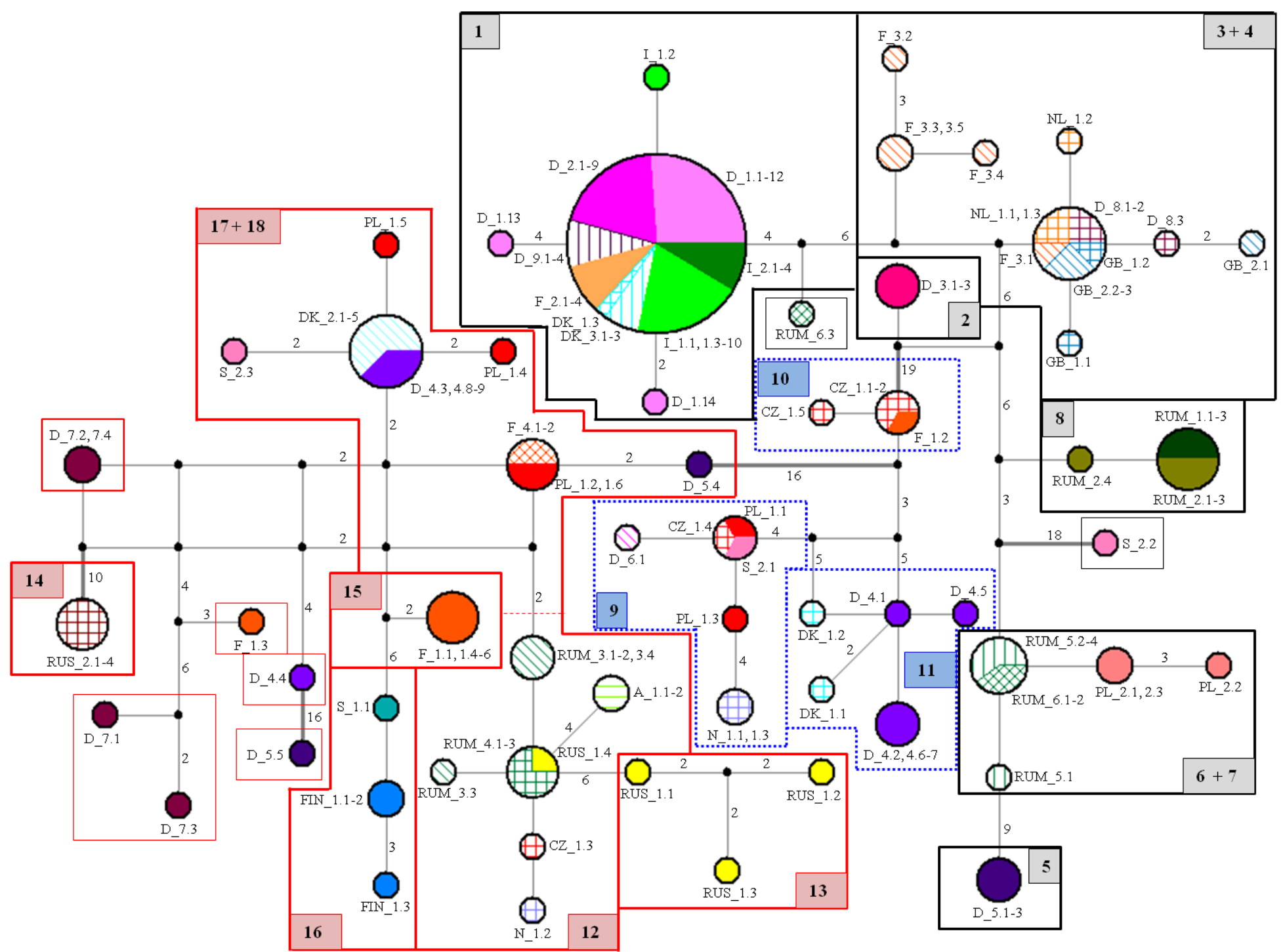

Figure 45: Median-joining haplotype network for the COI protein of 58 haplotypes from Steganacarus magnus. Each location has a specific colour marking. The size of the circles is proportional to the number of sequences per haplotype. Numbers on the lines represent the number of substitution steps separating the haplotypes (no number: only one amino acid change between haplotypes). Major subclades are marked by boxes. 


\section{Phylogenetic and population genetic analyses}

All phylogenetic analyses (NJ without and with model of sequence evolution, ML, MrBayes and Beast) showed a maximum supported monophyletic group of $S$. magnus with three main clades (black, blue and red), 25 subclades and ten isolated individuals in the nucleotide (Fig. 46, A52-56). The three main subclades were supported by high bootstrap values and posterior probabilities. The black and the blue main clade were sister clades and supported by high bootstrap values and posterior probabilities. Only the arrangement of the 14 subclades was variable in the analyses. The different subclades and the individuals which built these clades are explained in Table 28.

In the phylogenetic trees of the protein the arrangement of the three main clades changed (Fig. 47, A57-59). The blue and the red clade were sister clades and supported by high bootstrap values and posterior probabilities. The number of subclades and isolated individuals shrunk from 25 to 17 subclades and from ten to five isolated individuals, respectively. Two subclades 3 and 4, 6 and 7, 9 and N_1.1, 1.3, 17 and 18 in the nucleotide grouped to one subclade in the protein, respectively. Subclade 12, A_1.1-2 and the two isolated individuals CZ_1.3 and N_1.2 grouped in one subclade.

The minimum and maximum mean average pairwise differences for the nucleotide sequences between populations were $0.3 \%$ (D_2/F_2) and 31.3\% (RUM_5/RUS_1). Excluding Russia the maximum mean average pairwise differences was $29.9 \%$ (A_1/D_3). Within populations the minimum and maximum mean average pairwise differences were 0\% (A_1, DK_2, F_4 andRUS_2) and 25.8\% (S_2) (Table 29). For the protein the minimum and maximum mean average pairwise differences between populations were 0\% (A_1/RUM_4, D_2/D_9/DK_3/F_2/I_1/I_2/RUM_1/RUM_2) and 4.2\% (F_3/RUS_1); excluding Russia it was 4\% (A_1/D_3, D_3/DK_2, D_3/F_1, D_3/F_4, D_3/RUM_3 and D_3/RUM_4). The minimum and maximum mean average pairwise differences within population were 0\% (A_1, D_2, D_3, D_9, DK_2, DK_3, F_2, F_4, FIN_1, GB_1, I_1, I_2, RUM_1, RUM_2 and RUS_2) and 2\% for the protein (D_5) (Table A38). 


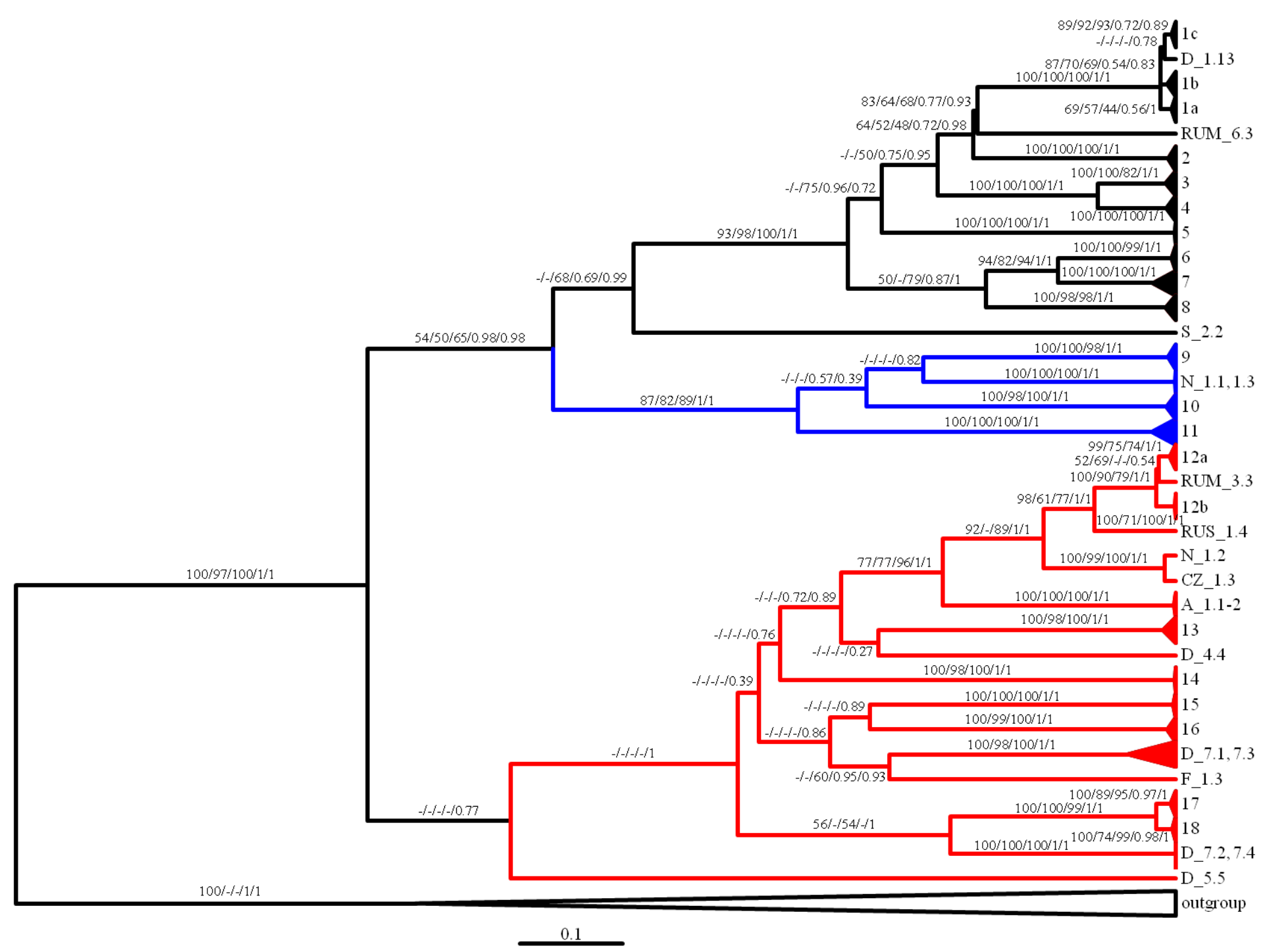

Figure 46: Bayesian phylogeny approach after 10x10 $10^{6}$ generations from the 157 COI nucleotide sequences of Steganacarus magnus. Outgroups are Hypochthonius rufulus and Rhysotritia duplicata. Numbers on the branches are bootstrap values from NJ without and with model of sequence evolution (GTR+I+G) and ML analysis and posterior probabilities from MrBayes and Beast. Coloured branches are the three main clusters (black, blue and red). Tip numbers are the different subclades and explained in Table 28. 


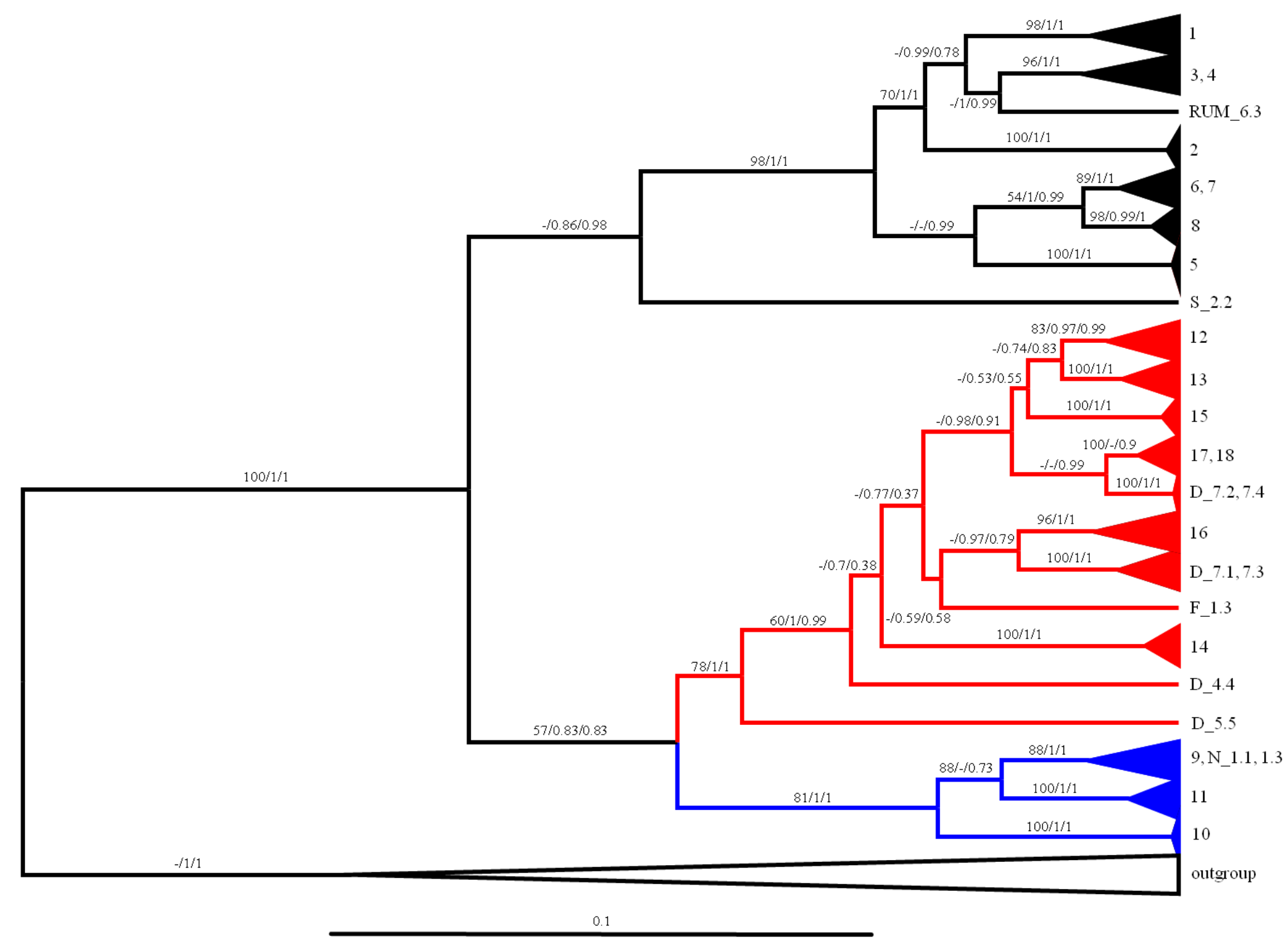

Figure 47: Bayesian phylogeny approach after 10x $10^{6}$ generations from the 157 COI protein sequences of Steganacarus magnus. Outgroups are Hypochthonius rufulus and Rhysotritia duplicata. Numbers on the branches are bootstrap values from NJ analysis and posterior probabilities from MrBayes and Beast. Outgroups are HD_D_1 and RD_D_1.1. Branch colour (black, blue and red) show the different main clades. Tip numbers are the different subclades and explained in Table 28. 
Chapter 4 Sex versus parthenogenesis

Table 28: Subclades of Bayesian phylogenetic trees of Steganacarus magnus based on COI nucleotide sequences (\#ind=number or individuals, $\mathrm{pp}=$ posterior probabilities, sampling sites, individuals and ind. pop.=quantity of individuals from the population).

Clade I

\begin{tabular}{|c|c|c|c|c|c|c|}
\hline Subclade & \# ind & pp & \multicolumn{2}{|c|}{ sampling sites } & individuals & ind. pop. \\
\hline 1 & 49 & 1 & 8 & & & \\
\hline \multirow[t]{6}{*}{$1 \mathbf{a}$} & 20 & 1 & KW & D_1 & 1,12 & $2 / 14$ \\
\hline & & & Goettingen & D_2 & $1-2,4-9$ & $8 / 9$ \\
\hline & & & Bonn & D_9 & 1 & $1 / 4$ \\
\hline & & & Loire & F_2 & $1-4$ & $4 / 4$ \\
\hline & & & Grosseto & I_1 & $1,4,9-10$ & $4 / 10$ \\
\hline & & & Parma & I_2 & 4 & $1 / 4$ \\
\hline \multirow[t]{4}{*}{$1 b$} & 12 & 0.83 & KW & D_1 & $2,5,14$ & $3 / 14$ \\
\hline & & & Bonn & D_9 & 2 & $1 / 4$ \\
\hline & & & Grosseto & I_1 & $2-3,5-8$ & $6 / 10$ \\
\hline & & & Parma & I_2 & $1-2$ & $2 / 4$ \\
\hline \multirow[t]{6}{*}{ 1c } & 16 & 0.89 & KW & D_1 & $3-46-11$ & $8 / 14$ \\
\hline & & & Goettingen & D_2 & 3 & $1 / 9$ \\
\hline & & & Bonn & D_9 & $3-4$ & $2 / 4$ \\
\hline & & & Parma & I_2 & 3 & $1 / 4$ \\
\hline & & & Copenhagen & DK_1 & 1 & $3 / 3$ \\
\hline & & & Arhus & DK_3 & $1-3$ & $3 / 3$ \\
\hline isol. ind. & 1 & 0.78 & KW & D_1 & 13 & $1 / 14$ \\
\hline isol. ind. & 1 & & $\begin{array}{l}\text { Sinaia } \\
\text { Lake }\end{array}$ & RUM_6 & 3 & $1 / 3$ \\
\hline 2 & 3 & 1 & Constance & D_3 & $1-3$ & $3 / 3$ \\
\hline \multirow[t]{5}{*}{3} & 12 & 1 & Cuxhaven & D_8 & $1-3$ & $3 / 3$ \\
\hline & & & St. Isidore & F_3 & 1 & $1 / 5$ \\
\hline & & & Ascot & GB_1 & $1-2$ & $2 / 2$ \\
\hline & & & Braemar & GB_2 & $1-3$ & $3 / 3$ \\
\hline & & & Wageningen & NL_1 & $1-3$ & $3 / 3$ \\
\hline 4 & 4 & 1 & St. Isidore & F_3 & $2-5$ & $3 / 5$ \\
\hline 5 & 3 & 1 & Moerfelden & D_5 & $1-3$ & $3 / 5$ \\
\hline \multirow[t]{2}{*}{6} & 6 & 1 & Busteni & RUM_5 & $1-4$ & $4 / 4$ \\
\hline & & & Sinaia & RUM_6 & $1-2$ & $2 / 3$ \\
\hline 7 & 3 & 1 & Warsaw & PL_2 & $1-3$ & $3 / 3$ \\
\hline \multirow[t]{2}{*}{8} & 7 & 1 & Sibiu_1 & RUM_1 & $1-3$ & $3 / 3$ \\
\hline & & & Sibiu_2 & RUM_2 & $1-4$ & $4 / 4$ \\
\hline isol. ind. & 1 & & Stroemstad & S_2 & 2 & $1 / 3$ \\
\hline
\end{tabular}


Chapter 4 Sex versus parthenogenesis

Table 28 continue:

Clade II

\begin{tabular}{ccccccc}
\hline Subclade & $\#$ ind & pp & \multicolumn{2}{c}{ sampling sites } & individuals & ind. pop. \\
\hline $\mathbf{9}$ & $\mathbf{5}$ & $\mathbf{1}$ & Decin & CZ_1 & 4 & $1 / 5$ \\
& & & Black Forest & D_6 & 1 & $1 / 1$ \\
& & & Krakow & PL_1 & 1,3 & $2 / 6$ \\
& & & Stroemstad & S_2 & 1 & $1 / 3$ \\
isol. ind. & $\mathbf{2}$ & & Narvik & N_1 & 1,3 & $2 / 3$ \\
$\mathbf{1 0}$ & $\mathbf{4}$ & $\mathbf{1}$ & Decin & CZ_1 & $1-2,5$ & $3 / 5$ \\
& \multirow{2}{*}{$\mathbf{4}$} & Mont Blanc & F_1 & 2 & $1 / 6$ \\
$\mathbf{1 1}$ & $\mathbf{7}$ & $\mathbf{1}$ & Meckl. Seenpl. & D_4 & $1-2,5-7$ & $5 / 9$ \\
& & & Copenhagen & DK_1 & $1-2$ & $2 / 3$ \\
\hline
\end{tabular}

Clade III

\begin{tabular}{ccccccc}
\hline Subclade & \# ind & pp & \multicolumn{2}{c}{ sampling sites } & individuals & ind. pop. \\
\hline $\mathbf{1 2}$ & $\mathbf{7}$ & $\mathbf{1}$ & $\mathbf{2}$ & & & \\
$\mathbf{1 2 a}$ & $\mathbf{3}$ & $\mathbf{1}$ & Cluj Napoca & RUM_4 & $1-3$ & $3 / 3$ \\
isol. ind. & $\mathbf{1}$ & & Bagau & RUM_3 & 3 & $1 / 4$ \\
$\mathbf{1 2 b}$ & $\mathbf{3}$ & $\mathbf{1}$ & Bagau & RUM_3 & $1-2,4$ & $3 / 4$ \\
isol. ind. & $\mathbf{1}$ & & Altai Mountains & RUS_1 & 4 & $1 / 4$ \\
isol. ind. & $\mathbf{1}$ & & Narvik & N_1 & 2 & $1 / 3$ \\
isol. ind. & $\mathbf{1}$ & & Decin & CZ_1 & 3 & $1 / 5$ \\
isol. ind. & $\mathbf{2}$ & & Villach & A_1 & $1-2$ & $2 / 2$ \\
$\mathbf{1 3}$ & $\mathbf{3}$ & $\mathbf{1}$ & Altai Mountains & RUS_1 & $1-3$ & $3 / 4$ \\
isol. ind. & $\mathbf{1}$ & & Meckl. Seenpl. & D_4 & 4 & $1 / 9$ \\
$\mathbf{1 4}$ & $\mathbf{4}$ & $\mathbf{1}$ & Novosibirsk & RUS_2 & $1-4$ & $4 / 4$ \\
$\mathbf{1 5}$ & $\mathbf{4}$ & $\mathbf{1}$ & Mont Blanc & F_1 & $1,4-6$ & $4 / 6$ \\
$\mathbf{1 6}$ & $\mathbf{3}$ & $\mathbf{0 . 9 6}$ & Lahti & FIN_1 & $1-3$ & $3 / 3$ \\
isol. ind. & $\mathbf{1}$ & $\mathbf{1}$ & Umea & S_1 & 1 & $1 / 1$ \\
isol. ind. & $\mathbf{1}$ & & Uelzen & D_7 & 1 & $1 / 4$ \\
isol. ind. & $\mathbf{1}$ & & Uelzen & D_7 & 3 & $1 / 4$ \\
isol. ind. & $\mathbf{1}$ & & Mont Blanc & F_1 & 3 & $1 / 6$ \\
$\mathbf{1 7}$ & $\mathbf{1 1}$ & $\mathbf{1}$ & Meckl. Seenpl. & D_4 & $3,8-9$ & $3 / 9$ \\
& & & Hjørring & DK_2 & $1-5$ & $5 / 5$ \\
& & & Krakow & PL_1 & $4-5$ & $2 / 6$ \\
& & & Stroemstad & S_2 & 3 & $1 / 3$ \\
$\mathbf{1 8}$ & $\mathbf{5}$ & $\mathbf{1}$ & Moerfelden & D_5 & 4 & $1 / 5$ \\
& & & Haute Loire & F_4 & $1-2$ & $2 / 2$ \\
& & & Krakow & PL_1 & 2,6 & $2 / 6$ \\
isol. ind. & $\mathbf{2}$ & $\mathbf{1}$ & Uelzen & D_7 & 2,4 & $2 / 4$ \\
isol. ind. & $\mathbf{1}$ & & Moerfelden & D_5 & 5 & $1 / 5$ \\
\hline & & & & & &
\end{tabular}


Table 29: Mean percentage pairwise differences of uncorrected p-distances of the nucleotide of Steganacarus magnus from 35 locations. The diagonal is the within population differences and below the diagonal the among population differences. Bold letters in red are the minimum and maximum differences within and among the populations. Bold letters in pink is the maximum divergences, if Russia is excluded. Locations with less than two individuals are excluded from the analysis.

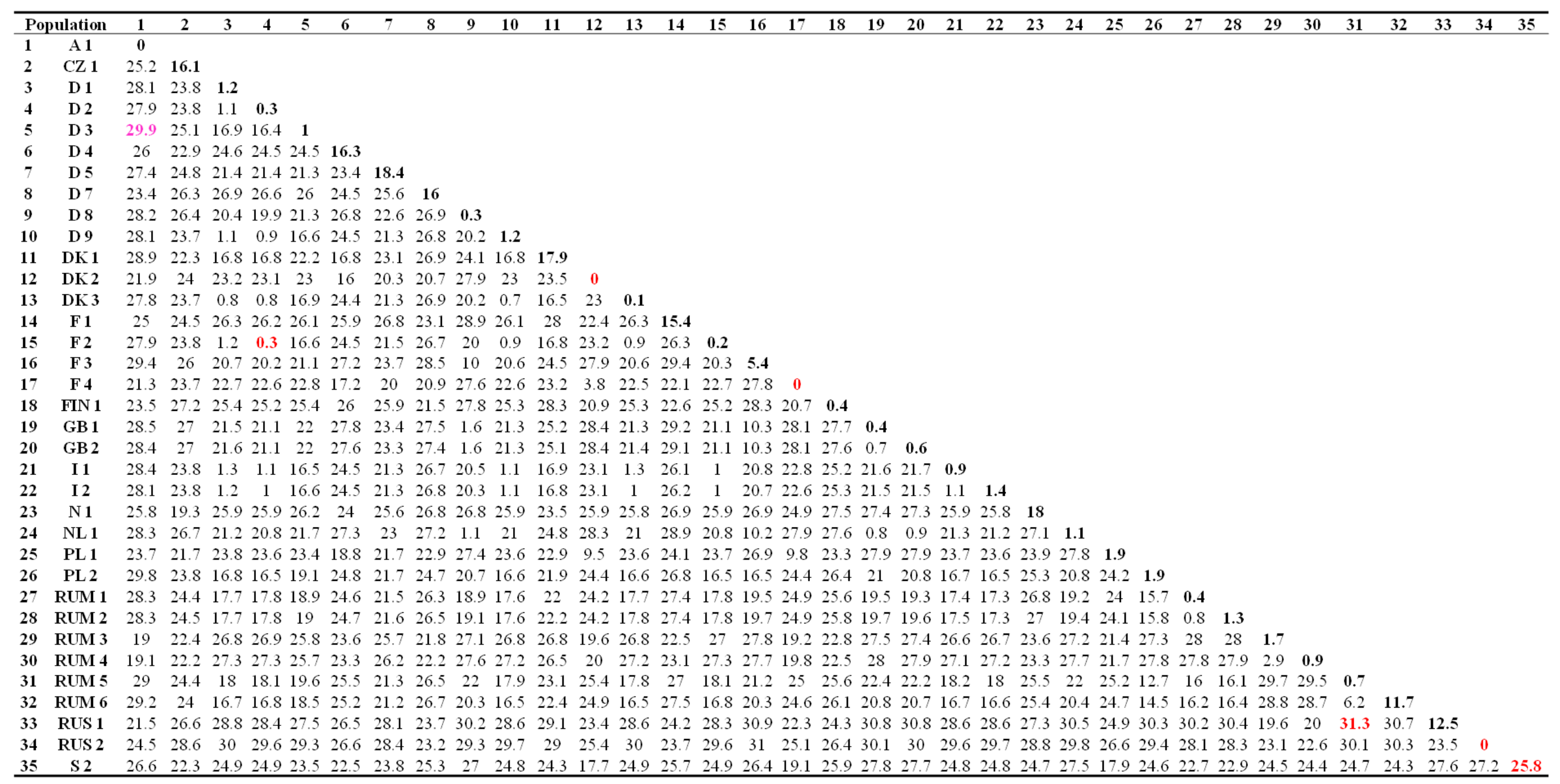


The results of the AMOVA showed that the nucleotide variation among samples within countries $(60.8 \%)$ and the variation within samples $(27.7 \%)$ were significant and high. In contrast, variation among countries was not significant (Table 30). The neutrality test (Tajima's D) was only significant for one population in the nucleotide ( $F \_3$ : Tajima's $D=-1.26296$, $p$-value $=0.0033$ ) and one population in the protein (D_1: Tajima's $D=-1.67053$, p-value $=0.0289$ ) and $F u$ 's $F_{s}$ was neither significant for the nucleotide nor for the protein (Table A39 and A40).

Table 30: AMOVA table on variations among countries and within populations in nucleotide (black) and protein (red) sequences of COI of Steganacarus magnus. Each population was considered as separate groups. Populations with less than two individuals were excluded from the analysis. Significance level is $p<0.05$ (d.f.: degree of freedom).

\begin{tabular}{lccccc}
\hline \multicolumn{1}{c}{ Source of variance } & d.f. & sum of squares & variance components & percent of total variation & fixation indices \\
\hline among countries & $13 / 13$ & $2986.96 / 65$ & $6.7 \mathrm{Va}^{*} /-0.03 \mathrm{Va}$ & $11.49 /-1.6$ & $\mathrm{FCT}: 0.12 /-0.02$ \\
among populations & $21 / 21$ & $3719.61 / 120.15$ & $35.44 \mathrm{Vb}^{*} / 1.16 \mathrm{Vb} *$ & $60.79 / 72.92$ & $\mathrm{FSC:} 0.69^{*} / 0.72^{*}$ \\
within population & $120 / 120$ & $1939.64 / 54.71$ & $16.16 \mathrm{Vc}^{*} / 0.46 \mathrm{Vc}^{*}$ & $27.72 / 28.68$ & $\mathrm{FST}: 0.72^{*} / 0.71^{*}$ \\
total & $154 / 154$ & $8793.72 / 239.86$ & $58.49 / 1.59$ & & \\
\hline
\end{tabular}

The McDonald-Kreitman test showed that the differences between 64 of 595 populations (10.8\%) were significant, between another 64 of 595 populations $(10.8 \%)$ the differences were highly significant and between 41 of 595 populations (6.9\%) they were very highly significant (Table 31, for more detail Table A41). All computed neutrality indices were 0 (Table A42).

None of the calculated rarefaction curves of the nucleotide and the protein reached saturation (Fig. 48 and 49). The Jackknife rarefaction curves ended at 256 haplotypes for the nucleotide and 149 haplotypes for the protein. The unique rarefaction curves ended at 73 haplotypes for the nucleotide and 38 haplotypes for the protein. The observed rarefaction curves (Sobs Mao Tau) ended at 95 haplotypes for the nucleotide and 59 haplotypes for the protein.

The results of the Mantel test indicate no evidence for isolation by distance in S. magnus when geographical distances were not $\log 10$ transformed $\left[\mathrm{R}^{2}=0.0215, \mathrm{p}=0.084\right.$ (Fig. A60), and for $\log 10$ transformed data indicating isolation by distance $\mathrm{R}^{2}=0.0434, \mathrm{p}=0.003$ (Fig. A61) using 1000 randomizations]. Excluding the first three isolated data points from the scatterplot the regression changed little $\mathrm{R}^{2}=0.0272, \mathrm{p}=0.000$ (Fig. 50). However, this explained only $4 \%$ of the variation in the data.

The gmyc model had a significantly better fit in the single and the multiple analyses than the null model (Table 18). The model identified for the single analysis 21 and for the multiple analysis 26 distinct $C O I$ clusters. The clusters of the single analysis were mostly identical with the phylogenetic main clusters (Fig. A62). The clades 1a-c, 12a-b and the isolated individual RUM_3.3 formed single distinct clusters. Clusters 17 and 18 were grouped to one distinct cluster and the two isolated individuals CZ_1.3 and N_1.2 were one distinct cluster. In the multiple analysis of the gmyc model separated seven clades from the phylogenetic tree (in clade 1a the individual I_2.1 was excluded, the isolated individual D_1.13 was included in the clade 1c, individual PL_2.2 and F_1.2 were excluded from their clades, the isolated individual RUM_3.3 was included in clade $12 \mathrm{~b}$ and the clades 3 and 6 persisted of two distinct clusters, respectively) (Fig. A63). 
Chapter 4 Sex versus parthenogenesis

Table 31: Results of the McDonald-Kreitman test. The differences between 64 populations were significant $(* 0.01<\mathrm{P}<0.05)$, between other 64 populations high significant $(* * 0.001<\mathrm{P}<0.01)$ and between 41 populations highly significant $(* * * \mathrm{P}<0.001)$.

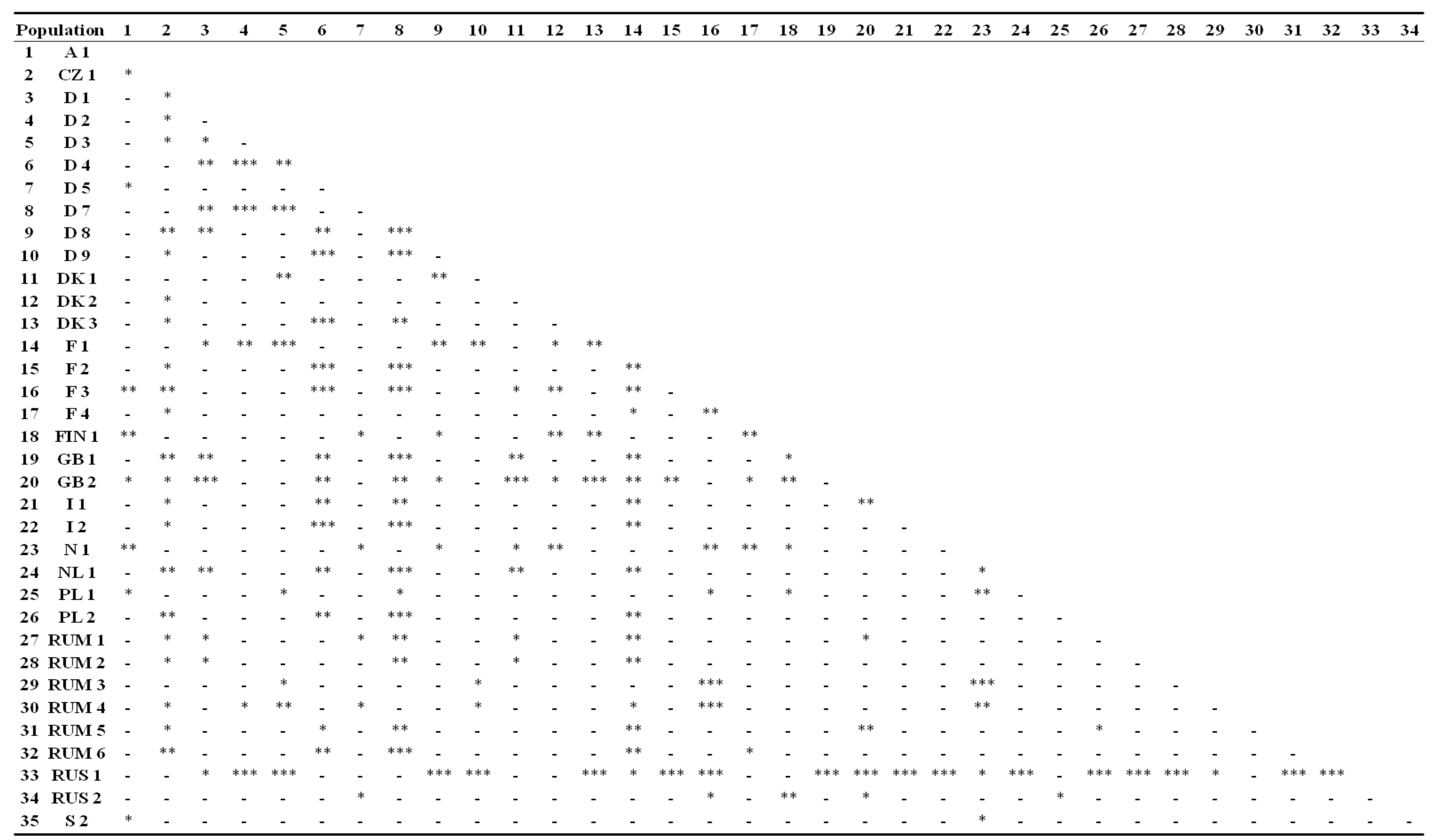


Chapter 4 Sex versus parthenogenesis

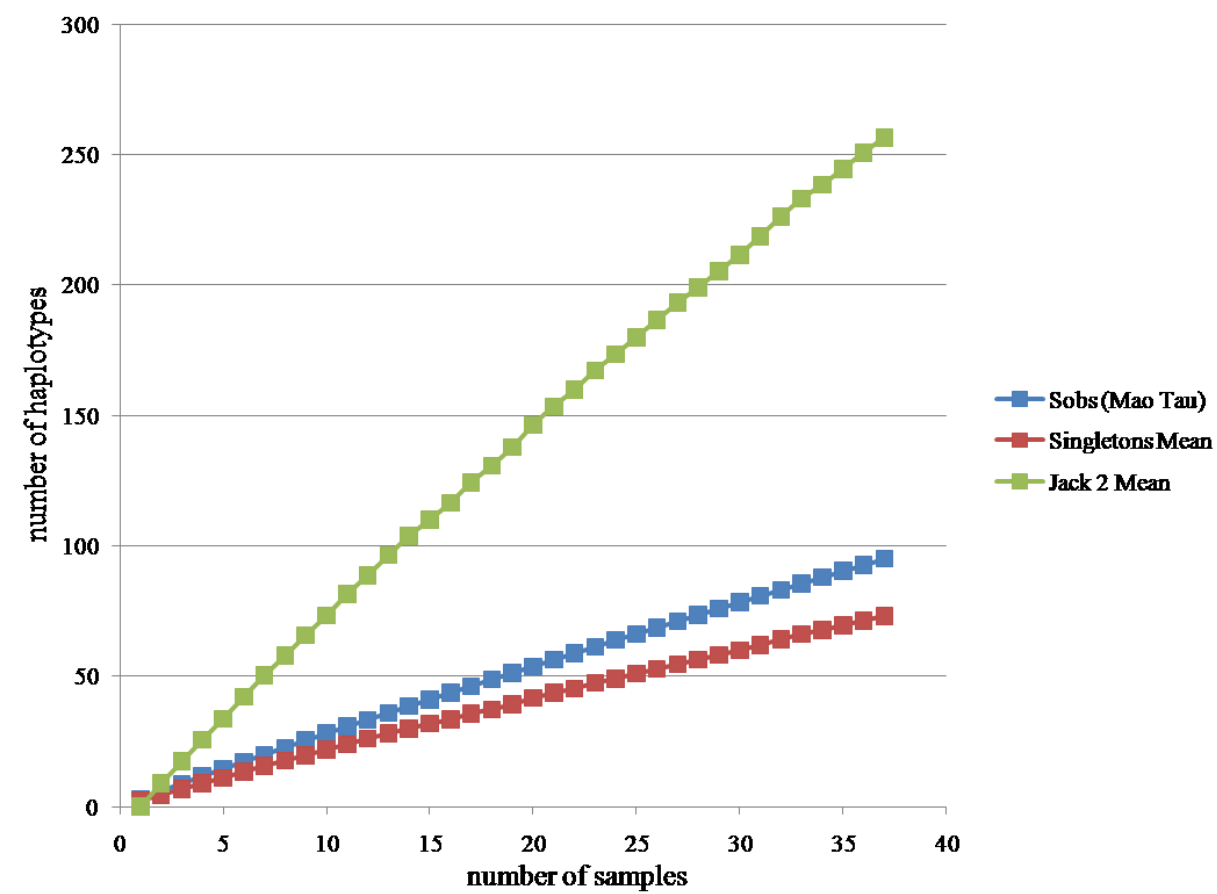

Figure 48: Sample based rarefaction analysis of haplotypes of the COI nucleotide of Steganacarus magnus. Jack 2 Mean, second-order Jackknife richness estimator, Singletons, numbers of haplotypes each present in only one sample, Sob (Mao Tau) is the observed haplotype richness.

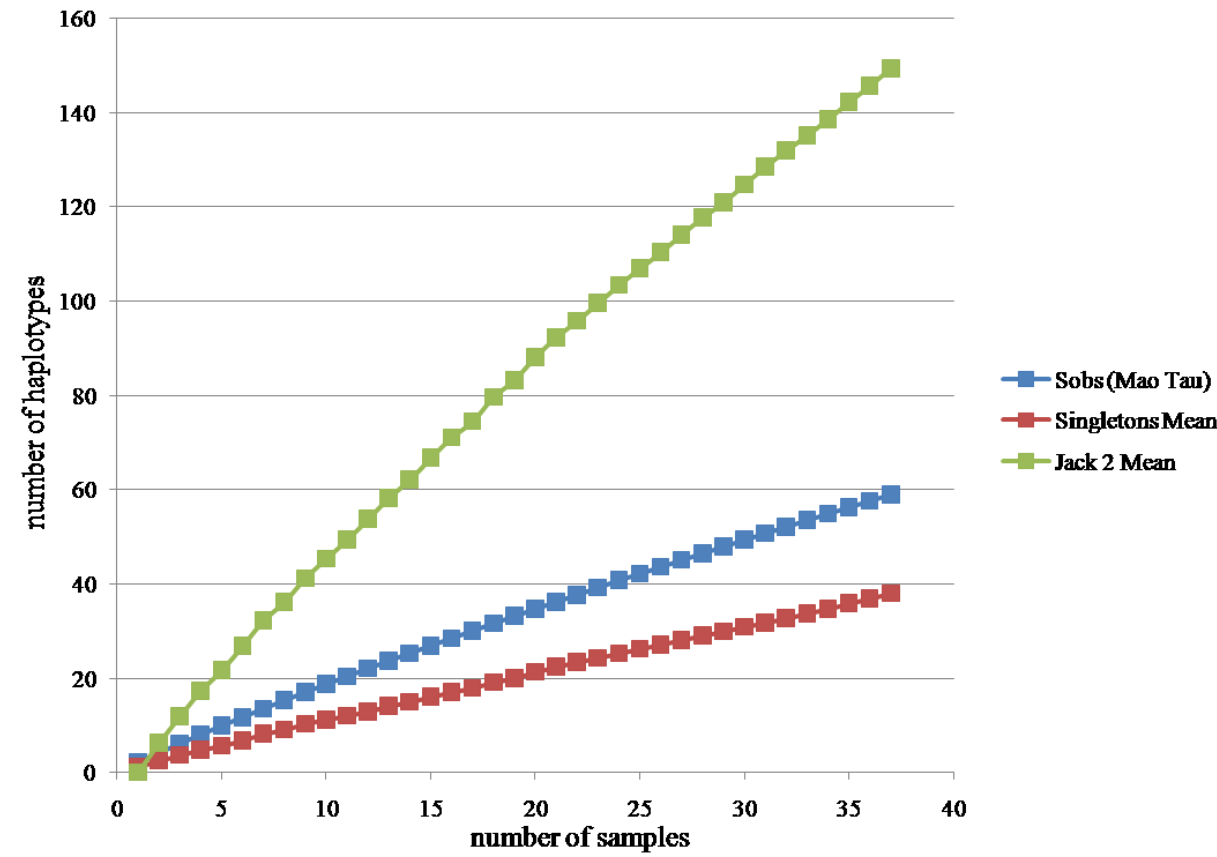

Figure 49: Sample based rarefaction analysis of haplotypes of the COI protein of Steganacarus magnus. Jack 2 Mean, second-order Jackknife richness estimator, Singletons, numbers of haplotypes each present in only one sample, Sob (Mao Tau) is the observed haplotype richness. 


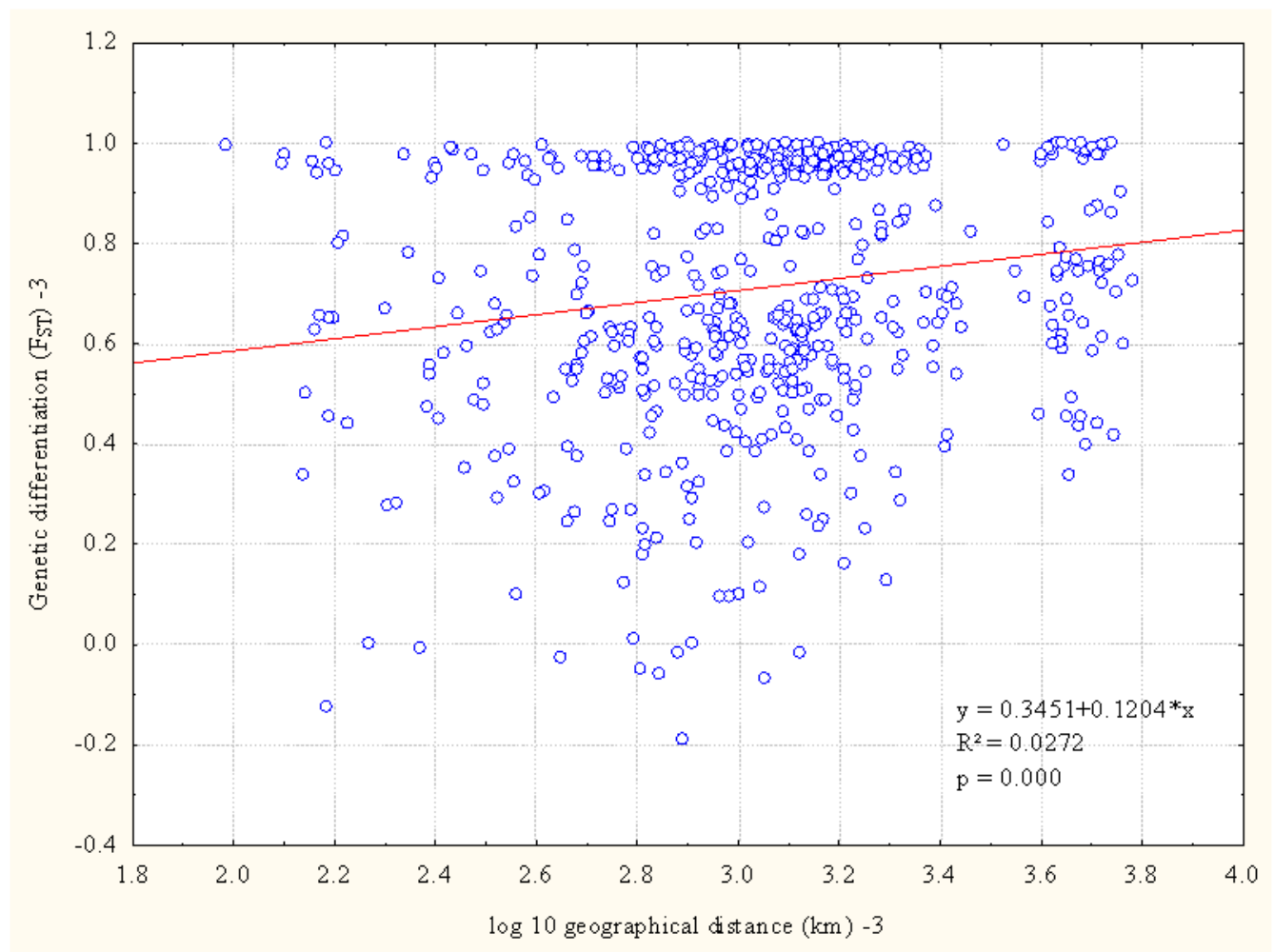

Figure 50: Linear regression of $\log 10$ geographical distances (kilometers) vs. genetic differentiations $\left(\mathrm{F}_{\mathrm{ST}}\right)$ based on COI nucleotide sequences of Steganacarus magnus. The regression is significant using 1000 randomizations. Three isolated data points with no information were excluded. Linear regression of all data points is shown in Fig. A61.

\subsection{Discussion}

In contrast to the common aboveground patterns (Hewitt 1999, 2004, Hewitt and Ibrahim 2001, Habel et al.2009), the genetic structure of three of the four investigated oribatid mites species (A. coleoptrata, S. magnus and P. peltifer) is dominated by deep splits, indicating radiation of the European populations in the Miocene or earlier. Rarefaction plots suggest that the sampling of the four studied oribatid mite species were incomplete and much more haplotypes are to be expected and the genetic complexity is much higher than showed. The results suggest that the paradigm of Southern richness and Northern purity (Hewitt 1999) does not apply to at least three of the four oribatid mite species. Remarkably, each of the four oribatid mite species showed different patterns of colonization of Europe.

\subsubsection{Nothrus silvestris}

The low genetic variance in nucleotide and protein sequences indicates that there is only one clonal lineage of $C O I$ in $N$. silvestris which colonized Central and Northern Europe after the last ice age. The origin of this lineage, however, remains unclear; sequences from the studied countries are almost identical. One possible origin is Romania with colonization of Central and Northern Europe from East to West. Another possible origin is the Southwest Atlantic coast of France below the permafrost border and colonization from West to East. Rarity of $N$. silvestris in Southern Europe suggests that $N$. silvestris is a cold adapted species able to synthesize ethylene glycol (F. Horak, pers. comm.). Today, N. silvestris predominantly occurs in Central Europe (Subias 2004, 2009, Weigmann 2006), its lack in Scandinavia north of Lahti (Finland) may be due to dispersal limitation. Parthenogenetic reproduction has the advantage of faster colonization (Williams 1975; Bell 1982; Scheu and Schulz 1996; Lindberg and Bengtsson 2005, Schön 2007), one individual could establish a new population and that can 
explain the existence of one clonal lineage of $N$. silvestris in Central Europe. In contrast, parthenogenetic oribatid mite species re-colonize litter and soil habitats not faster than sexual oribatid mite species (Domes et al. 2007c) and do not confirm with the theory.

\subsubsection{Platynothrus peltifer}

$P$. peltifer showed a very high genetic variance in the nucleotide but a low variance in the protein. Only synonymous substitutions existed in the parthenogenetic oribatid mite species $P$. peltifer. The existence of several separated Scandinavian clades and the Alpine clade of COI lineages in the nucleotide and the less abundant occurrence in the south suggests that $P$. peltifer survived the last glaciation in cryptic refugia in Central and presumably also Northern Europe. The common pattern of Northern purity and Southern richness (Hewitt and Ibrahim 2001) does not apply to P. peltifer; the genetic diversity in Northern locations even exceeded that in Southern locations. Cryptic refugia for $P$. peltifer likely existed in the Alps, Central and Northern Germany, Poland and Scandinavia.

\subsubsection{Achipteria coleoptrata}

A. coleoptrata showed a very high genetic variance in the nucleotide and in the protein. There were many non-synonymous substitutions which contrasts the only synonymous substitutions in the two parthenogenetic oribatid mites studied. Several geographical groups clustered together based on nucleotide variance. Clusters from the typical Southern refugia (Italy, Balkan and Romania) were not linked to Northern and Central clusters. This and the existence of several separated Central European clusters and one Alpine cluster indicates that similar to $P$. peltifer A. coleoptrata also survived the last glaciations in Northern refugia. Presumably, the Alps, Central and Northern Germany, Poland and Scandinavia likely functioned as cryptic refugia.

\subsubsection{Steganacarus magnus}

S. magnus showed the highest variance in the nucleotide and the protein ever found in one species (Edmands 2001, Boyer et al. 2007, Torricelli et al. 2010; see Chapter III). Non-synonymous substitutions existed in $S$. magnus like in the other sexual oribatid mite A. coleoptrata. Presumably, for $S$. magnus mountains functioned as geographical barrier as indicated by its absence in the Alps. For populations of $S$. magnus of the Canary Islands the substitution rate of $C O I$ has been estimated to be $2.15 \%$ per million years (Salomone et al. 2002). Assuming that this substitution rate also applies to the S. magnus populations investigated in this study, several preglacial splits exist in the European populations of this species. Unexpectedly, S. magnus presumably was little affected by the last ice ages; rather, main radiations of the European lineages of this species took place in the Miocene or the Oligocene. Cold resistance (Webb and Block 1993, Krantz and Walter 2009) and the high genetic divergences suggest that similar to the parthenogenetic P. peltifer and the other sexual oribatid mite species studied, A. coleoptrata, S. magnus survived the last glaciation in cryptic refugia. As indicated by nucleotide and protein variation Southern, Central and Northern Germany, Poland, Czech Republic, Great Britain and Scandinavia functioned as long-term refugia for S. magnus. In cryptic species complexes, as in the skipper butterflies Astraptes fulgerator (Hebert et al. 2004b) and of the genus Perichares (Burns and Janzen 2008), the Crustacean Chydorus sphaericus (Belyaeva and Taylor 2009), COI nucleotide variation may exceed 3\% (Hebert et al. 2003a-b) but this is unlikely because the nuclear and the mitochondrial phylogenetic tree are not identically and a mixture of the different COI lineages existed in the nuclear lineages (Chapter III). 


\subsubsection{General explanation of high genetic variance}

Generally, the results of our study suggest that using mitochondrial nucleotides to delineate species as commonly done (Pons et al. 2006, Fontaneto et al. 2007, Papadopoulou et al. 2009) is misleading in oribatid mites. In A. coleoptrata, $N$. silvestris and S. magnus several identical sequences were grouped in multiple distinct mitochondrial clusters. Only in P. peltifer the cluster delimitation based on $C O I$ nucleotide sequences may be used for identifying species. However, the results for $P$. peltifer showed six distinct mtDNA clusters which may be taken as evidence for the existence of six different species in $P$. peltifer. In each of these species, however, protein sequences of $C O I$ were identical questioning whether $P$. peltifer should be split into several species.

Mitochondria are inherited maternally (Wolstenholme 1992, Boore 1999). Therefore, nucleotide and protein divergence in mitochondria of sexual and parthenogenetic lineages are likely to be the same. In contrast to this assumption, only synonymous substitutions existed in the parthenogenetic species $P$. peltifer whereas in both sexual species studied also non-synonymous substitution existed. This might be due to the fact that in parthenogenetic species mitochondrial genes are fully linked with nuclear genes with non-synonymous substitutions breaking this linkage. This breaking of linked genes may be particularly fatal in cytochrome oxidase genes as the cytochrome oxidase complex is formed by subunits encoded by both mitochondrial and nucleotide genes. Variations in one of these subunits may result in a non-functional protein complex. In sexual species mitochondria and nuclear genomes are not fully linked, due to recombination non-synonymous substitutions presumably occur frequently since ten subunits of the thirteen cytochrome c oxidase subunits are located in the nucleus. Nuclear genes are mixed in every new generation and new cytochrome oxidase combinations are likely to be present. To prove this hypothesis the nuclear subunits of the cytochrome $\mathrm{c}$ oxidase and the other two mitochondrial subunits needs closer investigation.

Isolation by distance may be one reason for the high genetic divergences in P. peltifer, A. coleoptrata and $S$. magnus. However, the high variance in the protein in the two sexual oribatid mite species studied and the mixture of different $C O I$ lineages in the nuclear phylogenetic tree are unlikely caused by isolation by distance. Further, isolation by distance also cannot explain the high genetic divergence in individuals from the same locality and the fact that specimens of close geographical regions are strongly separated genetically and genetically identical to specimens of very distant geographical regions. The results of the isolation by distance analysis suggested that there was no significant relationship between geographic and genetic distance in each of the four oribatid mite species indicating that geographical distance did not affect genetic diversity. In three of the four oribatid mite species (A. coleoptrata, P. peltifer and S. magnus) studied genetic diversity on small geographical scales even exceeded than at large geographical scales.

The rarefaction analyses showed that sampling of haplotypes was very incomplete in each of the four oribatid mite species studied; the rarefaction curves of nucleotide and protein sequences increased in each of the four species.

The high genetic variance in the two sexual oribatid mites A. coleoptrata and $S$. magnus and in the parthenogenetic mite $P$. peltifer is unlikely to result from gene duplication, heteroplasmy, endosymbionts (Hurst and Jiggins 2005) or COI pseudogenes (Bensasson et al. 2001, Williams and Knowlton 2001, Song et al. 2008, Buhay 2009). The complete mitochondrial genomes of S. magnus and $P$. peltifer have been sequenced (Domes et al. 2008, Domes-Wehner 2009) and no gene duplication or heteroplasmy in $\mathrm{COI}$ was found in this or previous studies on oribatid mites. All COI nucleotide sequences of the four oribatid mite species can be translated into functional protein sequences without stop codons. Endosymbionts which indicate parthenogenesis such as Wolbachia (Plantard et al. 1998) and Cardinium (van Wilgenburg et al. 2006) could contaminate DNA and PCR 
samples. Wolbachia was detected in an unidentified oribatid mite species (Cordaux et al. 2001) and in the parthenogenetic oribatid mite species Oppiella nova (Weeks et al. 2003). Further, Cardinium was detected in Oppiella nova (Weeks et al. 2003). In the four oribatid mite species studied no endosymbionts such as Wolbachia and Cardinium have ever been found. Wolbachia and Cardinium endosymbionts are known to induce parthenogenesis (Plantard et al. 1998, van Wilgenburg et al.2006, Bordenstein and Werren 2007). However, A. coleoptrata and S. magnus reproduce sexually whereas P. peltifer is assumed to form an old asexual lineage (Heethoff et al. 2007, Domes et al. 2008, DomesWehner 2009).

At the beginning of the Miocene ( 23 mya) the vegetation and climate changed. The evergreen forests of the Oligocene shrunk and coniferous trees and deciduous trees took over (Mai 1989, 1991, 1996, Kvaček 2000). Potentially, the expansion of these forests was associated by the radiation of oribatid mite species which, according to our data, radiated in Europe during the Miocene or earlier. The expansion of forests of conifers and deciduous trees in Europe may have allowed co-occurring species to colonize new habitats and this typically is associated with radiation events (Hewitt 1999, Castagnoli et al. 2010). Evidence for the radiation of oribatid mite species in the Miocene or earlier are alpine clades of A. coleoptrata and P. peltifer. The rise of the Alps began in the Cretaceous ( 100 Mya) and the last tectonic events occurred in the Oligocene and the Miocene (20 Mya and 6 Mya) (Cederbom $e t$ al. 2004).

The high variance in the COI gene provides evidence for high age, but it also prevents detailed determination of the age of the oribatid mite species studied. Variations in neutral mutations in mitochondrial DNA saturate after about 10-20 million years (Avise 2004). Compared to the nuclear genome mitochondrial genes evolve 10 times faster in Drosophila (Haag-Liautard et al. 2008) and 550 times faster in vertebrates (Lynch 2007) and have a simpler genetic code suggesting that the code is fourfold degenerated and every third position is synonymous (Baker 2000).

The high genetic variance in the protein of the two sexual species A. coleoptrata and S. magnus and the low variance in parthenogenetic species $P$. peltifer were unexpected but may actually be related to the mode of reproduction. Linkage between mitochondrial and nuclear genes in parthenogenetic species is complete. In proteins encoded in part by mitochondrial and nuclear genes, such as the cytochrome oxidase protein complex, variations in protein subunits encoded by mitochondrial genes may be fatal as it may reduce or eliminate the functioning of the protein complex. In sexual species such variations may be less fatal as genes are not closely linked due to outcrossing and recombination. Every generation nuclear genes are mixed, linked genes are broken up and new combinations are assembled. Variations in protein subunits encoded by mitochondrial genes may therefore be buffered by matching variations in the subunits encoded by nuclear genes keeping the protein complex functional.

The investigated fragment of the COI is widely used as barcoding gene (Hebert et al. 2003, 2004a-b, Ball et al. 2005, Hajibabaei et al. 2007) and a divergence of $>3 \%$ is assumed to indicate separate species. The results of our study show that high genetic divergence is inappropriate for separating species in oribatid mites; the gene therefore is not useful for barcoding oribatid mite species. High genetic difference in $C O I$ nucleotide sequences were also found in other lineages of S. magnus (up to 28.6\%; Salomone et al. 2002) and other arthropods, such as the springtail Friesea grisea $(17.7 \%$; Torricelli et al. 2010), the harvestman Aoraki denticulata (up to 19.2\%; Boyer et al. 2007) and the intertidal copepod Tigriopus californicus (up to 23\%; Edmands 2001). Potentially, soil and marine arthropods are able to accumulate high rates of mutations without splitting of species which is not the case in aboveground terrestrial arthropods (Edmands 2001, Boyer et al. 2007, Schaefer 2009, Torricelli et al. 2010). This may be due to the more pronounced changes in the aboveground habitat 
due to the radiation of higher plants and associated coevolving arthropods in the Meso- and Cenozoic (Ehrlich and Raven 1964, Farrell and Mitter 1994). Soil and marine habitats presumably remained more constant allowing the accumulation of high rates of mutations without splitting of species. At least for oribatid mites this scenario is reasonable as the group is ancient (Shear et al.1984, Norton et al. 1988a, Heethoff et al. 2007, Schaefer et al. 2010) and in particular species which are abundant in boreal and temperate forest ecosystems, such as Desmonomata and Mixonomata, changed little for much of the Cenozoic (Palmer and Norton 1992). Further studies including other soil living invertebrates such as Collembola, nematodes and earthworms are needed to prove this assumption.

\subsection{Conclusions}

In contrast to aboveground animals, many oribatid mite species appear to have survived the glaciation of Europe in local refuges. However, colonization of Europe by oribatid mites was not uniform as indicated by the four species studied; rarefaction plots of the four species suggest that sampling of haplotypes was very incomplete. $N$. silvestris was the only species with a similar colonization pattern as documented for aboveground animals and plants; Central and Northern populations were characterized by low genetic variance in nucleotide and protein sequences and re-colonization of Central and Northern Europe likely occurred from Southern refugia but the location of these refugia remained unclear, potential regions include Romania (the Carpathians) and the Southwest Atlantic coast of France. The other three species studied (A. coleoptrata, S. magnus and P. peltifer) presumably were little affected by Pleistocene glaciations as indicated by high genetic divergences in the nucleotide and, in the two sexual species A. coleoptrata and S. magnus, also in the protein. Presumably, these species survived Pleistocene glaciations in cryptic refugia in Central and Northern Europe with the major lineages splitting earlier in the Miocene. The high genetic divergence in these species did not result from gene duplications, heteroplasmy, nuclear pseudogenes, endosymbionts or isolation by distance. Notably, the parthenogenetic species $P$. peltifer differed from the two sexual species in that only synonymous substitutions exist in this species whereas in the sexual species also non-synonymous substitutions occur. The high genetic divergence in the COI protein in sexual species may be linked to the sexual mode of reproduction. Recombination and associated variation in the subunits of the cytochrome oxidase encoded in the nucleus may buffer variations in the subunits of the gene encoded by mitochondrial genes and keep the cytochrome oxidase complex functional. The mitochondrial DNA evolves 10-15 times faster than the nuclear genome. Recombination of the nuclear genome may allow keeping up with these high mutation rates thereby rescuing the function of the protein. To prove this hypothesis variations in the mitochondrial and nuclear subunits of the cytochrome $\mathrm{c}$ oxidase need closer investigation. To estimate the age of the oribatid mite species studies other mitochondrial genes such as $16 \mathrm{~S}$, the other subunits of the cytochrome oxidase and ribosomal genes such as $18 \mathrm{~S}$ need closer investigation. 


\section{Chapter Five}

\section{General Discussion}

Belowground systems are characterized by high abundance and diversity of small animal species of very different phylogenetic affiliation including protozoa, lophotrochozoa (e.g. Annelida and Rotifera) and ecdysozoa (e.g. Crustacea, Insecta, Chelicerata and Nematoda). Therefore, soils harbor a variety of phylogenetically old taxa (Colemann et al. 2004, Schaefer et al. 2010). Soil organisms regulate major processes in ecosystems, such as the nutrient cycling (Scheu 2003), and they interact with the aboveground system (Bardgett 2005, Eisenhauer et al. 2007). Belowground organisms also provide a workscope to investigate evolutionary questions such as the existence and maintenance of sex and colonization of land (Schaefer et al. 2010) and new habitats after the ice ages.

The present study aimed at investigating biogeographical and evolutionary forces in the belowground system, using oribatid mite species as model organisms and molecular markers to detect post-glacial colonization events, cryptic refugia and cryptic species in belowground organisms. Using the intraspecific genetic variation of the mitochondrial cytochrome c oxidase I (COI) of the sexual oribatid mite Steganacarus magnus I investigated cryptic refugia and a possible cryptic species complex (Chapter II). The cryptic species complex in S. magnus was investigated and rejected using nuclear (ef $1 \alpha$ ) and mitochondrial (COI) markers (Chapter III). To detect general post-glacial colonization patterns in oribatid mites, COI of two sexual (Achipteria coleoptrata and S. magnus) and two parthenogenetic (Nothrus silvestris and Platynothrus peltifer) oribatid mite species were investigated (Chapter IV). Oribatid mites are ideal model organisms to detect cryptic refugia since they are small, cold tolerant and have low dispersal abilities.

\subsection{Barcoding}

DNA barcoding is a well established tool for the discovery of new species and revolutionized taxonomy and species delineation of undescribed species and of species which are difficult to determine morphologically (Burns et al. 2007). However, DNA barcoding is discussed controversially on theoretical (Hickerson et al. 2006), methodical (Will and Rubinoff 2004, Cameron et al. 2006, Song et al. 2008) and empirical grounds (Hurst and Jiggins 2005). DNA barcoding is helpful for species taxonomy in groups with few or equivocal morphological characters (Ben-David et al. 2007). Importantly, it allows the determination of juvenile stages which often are hard to identify using morphological characters (Huang et al. 2007). Traditionally, a fragment of the mitochondrial COI gene (position 1-650 bp of the 5'-end of the Folmer fragment; Folmer 1994, Hebert et al. 2003a-b, 2004 a-b, Ball et al. 2005, Hajibabaei et al. 2007) is used for species delineation but in the last years the combined use of mitochondrial and nuclear genes has been advocated (Monaghan et al. 2005, Elias et al. 2007, Sevilla et al. 2007, Dasmahaptra et al. 2010).

Oribatid mites are species rich soil-living arthropods and represent an ecological important group of mainly decomposers but also with predatory, herbi- and lichenivorous species (Muraoka and Ishibashi 1976, Starý and Block 1998, Maraun and Scheu 2000, Schneider et al. 2004, K. Heidemann unpublished data). Species determination can be challenging, since morphological variation in bodysize and color occurs in several species and the species determination of juveniles often is impossible. Due to the high density and diversity of soil invertebrates (up to 400,000 individuals per square meter in temperate forest soils; Maraun and Scheu 2000) and the high numbers of undescribed species, especially in tropical regions, DNA barcoding is likely to be a valuable tool for assessing the number 
of species present and for identifying new species. However, working with molecular methods can be demanding in oribatid mites. Species are small (0.2-1 mm body-size) and the amount of genomic DNA that can be extracted from a single individual is very limited. Additionally, oribatid mites are a phylogenetically old group (Norton et al. 1988a, Schaefer et al. 2010) and mutations in established primer binding regions in different species are frequent (I. Schaefer pers. comm.).

A number of barcoding genes have been tested in oribatid mites. The ribosomal internal transcribed spacer region 1 (ITS1, part of the 5.8/18/28S complex) has been investigated in three species, Heminothrus thori, $N$. silvestris and P. peltifer and interspecific variation was sufficient to distinguish the three species (Heethoff et al. 2000). However, intraspecific and intraindividual variation of the ITS1 region was high, in P. peltifer it ranged between $4.3 \%$ and $4.1 \%$ (Heethoff et al. 2000, Domes 2002), rendering this gene unsuitable for distinguishing species; the threshold proposed for species delineation is $<2.7 \%$ (Hebert et al. 2003a, 2004a). Also, the D3 domain of the ribosomal $28 S$ region has been tested as species marker (Maraun et al. 2003, 2004 Laumann et al. 2007) and no intraindividual or intraspecific variation was found but two closely related species of two different genera (Eupelops hirtus and E. torulosus, Nanhermannia coronata and N. nana; Maraun et al. 2003) had identical sequences; also rendering the $D 3$ domain unsuitable as a barcoding marker (Laumann et al. 2007). The conservative nuclear genes $18 S$, ef $1 \alpha$, heat shock protein (hsp82) and polymerase II (pol II) were applied successfully for phylogenies of oribatid mites (Schaefer et al. 2006, Domes et al. 2007a, 2007b, Laumann et al. 2007, Pachl 2010, Schaefer et al. 2010); closely related species had different DNA sequences, indicating that these genes might be good species markers and promising genes for barcoding. The mitochondrial gene $C O I$ was used successfully in a world wide phylogenetic study of the parthenogenetic oribatid mite species P. peltifer (Heethoff et al. 2007).

In the present study the genes $C O I$ and $e f 1 \alpha$ of four oribatid mite species (A. coleoptrata, N. silvestris $P$. peltifer and S. magnus) were sequenced from 588 individuals sampled all over Europe. Based on this extensive dataset consisting of 581 individuals, ef $1 \alpha, C O I$ and $18 S$ proofed to be unsuitable as DNA barcoding markers due to high intraspecific variance in the species $S$. magnus (31.8\% in COI, Chapter II, $19.6 \%$ in ef $1 \alpha$, Chapter III, and $0.7 \%$ in $18 S$, M. Rosenberger, unpublished), P. peltifer (20.4\% in COI, Chapter IV) and A. coleoptrata (19.4\% in COI, Chapter IV) as well as relatively low interspecific variance between the two closely related taxa S. magnus and Atropacarus spec. of $0.5 \%$ in the $18 S$ gene (M. Rosenberger, unpublished). Intraspecific variance in these genes remain undetected as most phylogenetic studies amplify one sequence from only one individual per species (Dabert et al. 2010, Mutanen et al. 2010).

The conservative gene pol II is a good species marker for oribatid mites and its low mutation rate allows to infer deep phylogenetic splits between species (Pachl 2010), but more analyses with pol II and with polymerase III ( $p o l$ III), another conservative gene, are needed to assess their suitability as barcoding genes in oribatid mites.

The mitochondrial COI gene is the most commonly used marker for barcoding (Hebert et al. 2003a-b, 2004a-b, Ball et al. 2005, Hajibabaei et al. 2007). However, the use of COI as barcoding marker for oribatid mites is problematic due to its high intraspecific variance in the two sexual (Chapter II, III and IV) and in the parthenogenetic oribatid mite species P. peltifer (Heethoff et al. 2007, Chapter IV). Similar to oribatid mites high intraspecific genetic divergence was also found in other soil-living arthropods including the springtails Folsomia spec. (13\%; Hogg and Hebert 2004), F. quadriculata (18.3\%; Schaefer 2009), Ceratophysella denticulata (21.5\%; Schaefer 2009) and Friesea grisea (17.7\%; Torricelli et al. 2010), the harvestman Aoraki denticulata (up to 19.2\%; Boyer et al. 2007) and the intertidal copepod Tigriopus californicus (up to 23\%; Edmands 2001). These and the present study question the use of $\mathrm{COI}$ as barcoding gene in arthropods and indicate that genetic variation in mitochondrial genes is maintained to a higher extend in soil-living and marine arthropods than in vertebrates and above-ground arthropods. 


\subsection{Colonization of Northern and Central Europe}

Studies on aboveground animals and plants confirmed earlier suggestions that Central and Northern Europe were re-colonized via three routes after the last ice-ages (Hewitt 1999, 2004, Hewitt and Ibrahim 2001). The general pattern that few lineages started from countries south of the Alps and recolonized empty habitats in Central and Northern Europe is reflected in the genetic structure of present day populations.

The present study is the first investigating these genetic patterns in soil animals allowing for the first time to compare patterns of above- and belowground invertebrates.

In this study, two sexual (S. magnus and A. coleoptrata) and two parthenogenetic taxa ( $P$. peltifer and $N$. silvestris) were sampled from soil and litter throughout Europe and from three locations in east Russia and China. The genetic patterns of the mitochondrial COI gene, the standard gene for phylogeography, were very complex and indicate the colonization routes of Central and Northern Europe differ from the established routes known from aboveground organisms. Remarkably, the colonization routes of the four investigated oribatid mite species differed. The results suggest that three of the four species studied survived the Pleistocene glaciation of Europe in small refugia in Central and Northern Europe.

In contrast to plant and animal species above the ground, distinct mitochondrial lineages of oribatid mites from Central Europe co-occur in the same habitat or in habitats which are geographically close together. Further, closely related lineages are present at very different geographic locations. In addition, the results proved that the number of haplotypes (haplotype diversity index $=0.67$ in $N$. silvestris and 0.98 in A. coleoptrata, P. peltifer and S. magnus) and the genetic distances between mitochondrial lineages (19-32\%) are large, indicating that lineages of the same species that separated millions of years ago co-exist in one habitat. Presumably, these lineages survived in small populations in cryptic refugia in Central and Northern Europe and successfully spread into other habitats after glaciation.

I hypothesized that parthenogenetic species more quickly colonized Central and Northern Europe after the last ice age. In contrast to this assumption, the genetic pattern of the investigated sexual and parthenogenetic oribatid mite species suggests that the pace of colonization did not correlate with the mode of reproduction and that parthenogenetic species were not more vigorous in colonizing deglaciated regions in Northern Europe after the last ice age. The low genetic variance in the parthenogenetic species as compared to the sexual species indicates that only one or two lineages rerecently colonized Central and Northern Europe. However, the high local genetic variance and genetic distances in the sexual species suggest that several lineages established populations in the past tens of millions of years and likely survived in small Central refugia from where re-colonization started when living conditions improved in the surrounding area.

The parthenogenetic oribatid mite species $P$. peltifer also survived the Pleistocene glaciation of Europe in cryptic refugia as indicated by its high genetic variance (divergence up to $20 \%$ in the nucleotide, see Chapter IV), but the two sexual oribatid mite species studied (A. coleoptrata and S. magnus) presumably survived as long as or even longer as $P$. peltifer in refugia and colonized Central and Northern Europe from there.

The investigated oribatid mite species have long generation times of at least one year from egg to adult (Palmer and Norton 1990) and a relatively low reproductive output with only one to two eggs per year (Domes et al. 2007c, Domes-Wehner 2009). The long generation time, the high abundance in forests, the cold hardiness of $S$. magnus, the dependency on dead lignified plant tissues (larval stages of S. magnus are endophagous) and the low dispersal capabilities render oribatid mites (especially S. magnus) ideal model organisms to detect cryptic refugia. Generally, cryptic refugia are difficult to detect as they may comprise small isolated islands. The dependence on plant material for development and cold hardiness provide $S$. magnus as model-organism ideally suited to reconstruct the 
phylogeographic history of Central Europe. The results suggest that populations of S. magnus endured the ice-ages in isolated areas in Central Europe. Presumably, the species only relies on the presence of organic layers. Constant presence of plants may not be necessary as juvenile stages of S. magnus do not differentiate between living or dead lignified tissues for development.

\subsection{Sex versus Parthenogenesis}

The widespread distribution of sexual reproduction in the animal kingdom despite its high costs (production of two sexes and the dilution of the genome through meiosis) has puzzled evolutionary biologist for decades and is known as "the queen of problems in evolutionary biology" (Bell 1982). Parthenogenetic species are thought to go extinct since they cannot overcome the accumulation of negative substitutions (Maynard Smith1978, Bell 1982, Kondrashov 1993, Butlin 2002, Schön et al. 2008). The existence of so called 'ancient asexual scandals' namely darwinulid ostracods (Martens et al. 2003), bdelloid rotifers (Welch and Meselson 2000) and some taxa of oribatid mites (Maraun et al. 2003, Heethoff et al. 2007, 2009) is still debated. Theoretically, only sexual species radiate and form clusters of species that rarely contain single terminal parthenogenetic offshoots (Barraclough et al. 2003, Birky et al. 2005). Contrasting this pattern, there are two large parthenogenetic clusters in oribatid mites (Desmonomata and Enarthronota) which each likely evolved by radiation of a single parthenogenetic lineage (Maraun et al. 2004). Remarkably, in Desmonomata sex presumably reevolved in Crotoniidae (Domes et al. 2007b). The origin of Desmonomata is dated back to 330 my (Schaefer 2009, Schaefer et al. 2010) suggesting that genes involved in mating and spermatogenesis have been functional or regained functionality after millions of years and generations.

Molecular approaches can be used for testing the long-term absence of sex. The long-term absence of recombination should affect the nuclear genome by accumulation of deleterious mutations (Muller's ratchet; Muller 1964, Mutational Load Theory; Kondrashov 1988). For proving absence of recombination and therefore anciency of parthenogenetic lineages the 'Meselson effect' has been proposed (Welch and Meselson 2000). However, in each of the above mentioned three 'ancient asexual scandals' no Meselson effect has been detected (Butlin 2002, Schaefer et al. 2006, Schön et al. 2008).

The high genetic variances in the nucleotide in three of the four investigated oribatid mite species, $A$. coleoptrata, $P$. peltifer and $S$. magnus, and in the COI protein of the sexual species and the low variance in parthenogenetic species were unexpected but may actually help to explain the maintenance of sexual reproduction. The linkage between mitochondrial and nuclear genes in parthenogenetic species is complete. In proteins encoded in part by mitochondrial and nuclear genes, such as the cytochrome oxidase protein complex, variations in the protein subunits encoded by mitochondrial genes may be fatal as it may reduce or eliminate the functioning of the protein complex. In sexual species such variations may be less fatal as genes are not closely linked due to outcrossing and recombination. Every generation nuclear genes are mixed, linked genes are broken up and new combinations are assembled. Variations in protein subunits encoded by mitochondrial genes may therefore be buffered by matching variations in the subunits encoded by nuclear genes keeping the protein complex functional. This interrelationship may actually be responsible for the existence of sex. In this scenario mixing of nuclear genes by sexual reproduction balances the high non-synonymous mitochondrial mutations. Further studies have to prove this hypothesis by analyzing mitochondrial and nuclear markers in parthenogenetic and sexual species.

\subsection{Synopsis and Conclusion}

The aim of this study was to infer phylogeographic patterns in soil-living arthropods on the basis the abundant and diverse group of oribatid mites. I expected that the extreme climatic conditions of the last glacial maximum (22-18 kya) had a similar impact on oribatid mites as on the already investigated above-ground taxa. The significance of the reproductive mode for re-colonization of empty habitats 
was taken into account as two sexual (A. coleoptrata and $S$. magnus) and two parthenogenetic taxa ( $N$. silvestris and $P$. peltifer), co-occurring in the same habitats, were sampled from forest litter throughout Europe. Molecular analyses concentrated on the standard gene for phylogenetic analyses and DNA barcoding, the mitochondrial COI gene (Folmer fragment, position 1-660). The nuclear encoded single copy gene elongation factor $1 \alpha$ was also sequenced from a subset of the same individuals. In the course of this study it turned out that the well established above-ground re-colonization patterns are only to a limited extent transferable to soil-living oribatid mites.

In general, the genetic variance in oribatid mites throughout Europe is very high and phylogeographic patterns are only at the protein-level clearly comprehensible. On basis of nucleotide sequences, the genetic distance among individuals is unusually high (19-32\%), except for Nothrus silvestris. In the three remaining species the $C O I$ gene appears to be saturated in European populations, indicating that lineages have existed for at least 20-50 million years.

The most complex genetic pattern was found in S. magnus (Chapter II), the species with the highest local genetic diversity and distances among individuals. With phylogenetic analyses, three distinct mitochondrial lineages were identified that even co-existed in the same habitat and probably separated at least 20 mya. This finding suggests a cryptic species complex in S. magnus, e.g. lineages differentiated recently and became reproductively isolated in the same habitat but remained morphologically indistinct. However, it is more likely that the LGM had only a limited effect on $S$. magnus but small populations rather survived in cryptic refugia in Central Europe and that three mitochondrial lineages re-colonized east-central, south-central and south-east of Europe.

A cryptic species complex, however, can be excluded (Chapter III). Phylogenetic analyses based on the mitochondrial COI gene and nuclear elongation factor $1 \alpha$ sequenced from the same individuals showed that sexual reproduction among different mitochondrial lineages occurred. One individual carried the nuclear haplotype of ef $1 \alpha$ from a different mitochondrial lineage, a combination that can only be explained by sexual reproduction between individuals of different mitochondrial lineages both lineages were sampled in this population.

The pattern of high local and global genetic variance and high genetic distance found in S. magnus (Chapter II and III) indicative for pre-glacial diversification and survival in cryptic refugia was confirmed by the other sexual species (A. coleoptrata, Chapter IV).

The populations of the parthenogenetic species however are dominated by only one protein haplotype in $N$. silvestris and $P$. peltifer. This demonstrates that the LGM had a stronger impact on the parthenogenetic species than on the sexual species.

Oribatid mites are perfect model organisms for evolutionary and phylogeographic questions investigated in this study. Results presented in Chapter II showed that the oribatid mite S. magnus survived the last ice ages in cryptic refugia and the high genetic divergence in $C O I$ is not a result of a cryptic species complex (Chapter III). This is supported by the results presented in Chapter IV demonstrating that the last ice age did not shape the biodiversity of belowground organisms and oribatid mites radiated in Miocene or earlier ( 20 mya). Sexual and parthenogenetic oribatid mites survived in Northern and Central Europe and colonized the habitat from there. The high genetic divergences in the protein of sexual species could explain the existence of sex since only in sexual species recombination is present. However, to support these hypotheses several studies with other belowground organisms such as Collembola, earthworms and nematodes must be carried out. 


\section{References}

Alexander Kartographie (2006) Klett Verlag, Leipzig, http://www. klett.de

Arnold S, Kadenbach B (1997) Cell respiration is controlled by ATP, an allosteric inhibitor of cytochrome c oxidase, European Journal of Biochemistry 249, pp 350-354

Avise JC (1994) Molecular markers, natural history and evolution Chapman \& Hall, London

Avise JC (2004) Molecular Markers, Natural History, and Evolution Sinauer Associates, MA; second edition

Baker AJ (2000) Molecular Methods in Ecology Eds. Lawton JH and Likens GE; Blackwell Science Ltd, UK

Baldauf SL, Palmer JD, Doolittle WF (1996) The root of the universal tree and the origin of eukaryotes based on elongation factor phylogeny, Proceedings of the National Academy of Sciences of the United States of America 93, pp 7749-7754

Ball SL, Hebert PDN, Burian SK, Webb JM (2005) Biological identifications of mayflies (Ephemeroptera) using DNA barcodes, Journal of the North American Benthological Society 24, pp 508-524

Ballard JWO, Melvin RG (2010) Linking the mitochondrial genotype to the organismal phenotype, Molecular Ecology 19, pp 1523-1539

Bandelt HJ, Forster P, Rohl A (1999) Median-joining networks for inferring intraspecific phylogenies, Molecular Biology and Evolution 16, pp 37-48

Bardgett R (2005) The Biology of Soil: A Community and Ecosystem Approach. Oxford University Press, New York

Barraclough TG, Birky CW, Burt A (2003) Diversification in sexual and asexual organisms, Evolution 57, pp 2166-2172

Beheregaray LB (2008) Twenty years of phylogeography: the state of the field and the challenges of the southern hemisphere, Molecular Ecology 17, pp 3754-3774

Bell G (1982) The Masterpiece of Nature. The Evolution and Genetics of Sexuality, University of California Press, California

Ben-David T, Melamed S, Gerson U, Morin S (2007) ITS2 sequences as barcodes for identifying and analyzing spider mites (Acari: Tetranychidae), Experimental and Applied Acarology 41, pp 169-181

Bensasson D, Zhang DX, Hartl DL, Hewitt GM (2001) Mitochondrial pseudogenes: evolution's misplaced witnesses, Trends in Ecology \& Evolution 16 (6), pp 314-321

Bilton, D. T., Mirol PM, Mascheretti S, Fredga K, Zima J, Searle JB (1998) Mediterranean Europe as an area of endemism for small mammals rather than a source for northwards postglacial colonization, Proceedings of the Royal Society of London Series B-Biological Sciences 265 (1402), pp 1219-1226

Birky CW, Wolf C, Maughan H, Herbertson L, Henry E (2005) Speciation and selection without sex, Hydrobiologia 546, pp 29-45

Blenkinsop C, Aitken AE, Wilson MT (1996) Physical and functional characterisation of monomeric and dimeric cytochrome $\mathrm{c}$ oxidase, Comparative Biochememistry and Physiology Part B: Biochemistry and Molecular Biology 115, pp 421-428

Block W (1979) Cold tolerance of micro-arthropods from Alaskan taiga, Ecological Entomology 4, pp 103-110

Bohonak AJ (2002) IBD (Isolation By Distance): a program for analyses of isolation by distance. Journal of Heredity 93, pp 153-154

Boore JL (1999) Animal mitochondrial genomes, Nucleic Acids Research 27 (8), pp 1767-1780 
Bordenstein SR, Werren JH (2007) Bidirectional incompatibility among divergent Wolbachia and incompatibility level differences among closely related Wolbachia in Nasonia, Heredity 99, pp 278-287

Boyer SL, Baker JM, Giribet G (2007) Deep genetic divergences in Aoraki denticulata (Arachnida, Opiliones, Cyphophthalmi): a widespread 'mite harvestman' defies DNA taxonomy, Molecular Ecology 16, pp 4999-5016

Buhay JE (2009) "COI-like" sequences are becoming problematic in molecular systematic and DNA barcoding studies, Journal of Crustacean Biology 29 (1), pp 96-110

Burns JM, Janzen DH, Hajibabaei M, Hallwachs W, Hebert PDN (2007) DNA barcodes of closely related (but morphologically and ecologically distinct) species of skipper butterflies (Hesperiidae) can differ by only one to three nucleotides, Journal of the Lepidopterists' Society 61 (3), pp 138-153

Burns JM, Janzen DH (2008) DNA barcodes and cryptic species of skipper butterflies in the genus Perichares in Area de Conservacion Guanacaste, Costa Rica, Proceedings of the National Academy of Sciences of the United States of America 105 (17), pp 6350-6355

Butlin R (2002) The costs and benefits of sex: new insights from old asexual lineages, Nature Review Genetics 3, pp 311-317

Butlin R, Schön I, Martens K (1999) Origin, age and diversity of clones, Journal of Evolutionary Biology 12, pp 1020-1022

Cameron S, Rubinoff D, Kipling W (2006) Who will actually use DNA barcoding and what will it cost? Systematic Biology 55, pp 844-847

Castagnoli M, Lewandowski M, Łabanowski GS, Simoni S, Soika GM (2010) An insight into some relevant aspects concerning eriophyoid mites inhabiting forests, ornamental trees and shrubs, Experimental and Applied Acarology 51, pp 169-189

Cederbom CE, Sinclair HD, Schlunegger F, Rahn MK (2004) Climate-induced rebound and exhumation of the European Alps, Geology 32, pp 709-712

Chantangsi C, Lynn HD, Brandl MT, Cole JC, Hetrick N, Ikonomi P (2007) Barcoding ciliates: a comprehensive study of 75 isolates of the genus Tetrahymena, International Journal of Systematic and Evolutionary Microbiology 57, pp 2412-2425

Cicconardi F, Nardi F, Emerson BC, Frati F, Fanciulli PP (2010) Deep phylogeographic divisions and long-term persistence of forest invertebrates (Hexapoda: Collembola) in the North-Western Mediterranean basin, Molecular Ecology 19 (2), pp 386-400

Clement M, Posada D, Crandall KA (2000) TCS: a computer program to estimate gene genealogies, Molecular Ecology, 9, pp 1657-1659

Colemann DC, Crossley DA Jr., Hendrix PF (2004) Fundamentals of Soil Ecology. Elsevier Academic Press; second edition

Conroy CJ, Cook JA (2000) Phylogeography of a post-glacial colonizer: Microtus longicaudatus (Rodentia: Muridae), Molecular Ecology 9, pp 165-175

Cordaux R, Michel-Salzat A, Bouchon D (2001) Wolbachia infection in crustaceans: novel hosts and potential routes for horizontal transmisson, Journal of Evolutionary Biology 14, pp 237-243

Dabert M, Witalinski W, Kazmierski A, Olszanowski Z, Dabert J (2010) Molecular phylogeny of acariform mites (Acari, Arachnida): Strong conflict between phylogenetic signal and longbranch attraction artifacts, Molecular Phylogenetics and Evolution 56 (1), pp 222-241

Danforth BN, Sauquet H, Packer L (1999) Phylogeny of the bee genus Halictus (Hymenoptera: Halictidae) based on parsimon and likelihood analyses of nuclear EF- $1 \alpha$ sequence data. Molecular Phylogenetics and Evolutoion 13, pp 605-618 
Dasmahapatra KK, Elias M, Hill RI, Hoffman JI, Mallett J (2010) Mitochondrial DNA barcoding detects some species that are real, and some that are not, Molecular Ecology Resources (2), pp 264-273

Dépraz A, Cordellier M, Hausser J, Pfenninger M (2008) Postglacial recolonization at a snail's pace (Trochulus villosus): confronting competing refugia hypotheses using model selection, Molecular Ecology 17, pp 2449-2462

Derycke S, de Ley P, Tandingan de Ley I, Holovachov O, Rigaux A, Moens T (2010) Linking DNA sequences to morphology: cryptic diversity and population genetic structure in the marine nematode Thoracostoma trachygaster (Nematoda, Leptosomatidae), Zoologica Scripta 39, pp 276-289

Dlugosh KM, Parker IM (2008) Invading populations of an ornamental shrub show rapid life history evolution despite genetic bottlenecks, Ecology Letters 11, pp 701-709

Domes K. (2002) Untersuchung der intraindividuellen Variabilität der Internal Transcribed Spacer 1 Region (ITS1) bei der parthenogenetischen Hornmilbe Platynothrus peltifer und der sexuellen Hornmilbe Steganacarus magnus, Research Intership, TU Darmstadt

Domes K, Althammer M, Norton RA, Scheu S, Maraun M (2007a) The phylogenetic relationship between Astigmata and Oribatida (Acari) as indicated by molecular markers, Experimental and Applied Acarology 42 (3), pp 159-171

Domes K, Norton RA, Maraun M. Scheu S (2007b) Re-evolution of sexuality breaks Dollo's law, Proceedings of the National Academy of Science, USA 104, pp 7139-7144

Domes K, Scheu S, Maraun M (2007c) Resources and sex: soil re-colonization by sexual and parthenogenetic oribatid mite species, Pedobiologia 51, pp 1-11

Domes K, Maraun M, Scheu S, Cameron SL (2008) The complete mitochondrial genome of the sexual oribatid mite Steganacarus magnus: genome rearrangements and loss of tRNAs, BMC Genomics 9 (532)

Domes-Wehner K (2009) Parthenogenesis and sexuality in oribatid mites: phylogeny, mitochondrial genome structure and resource dependence, PhD thesis, TU Darmstadt

Drummond AJ, Rambaut A (2007) BEAST: Bayesian evolutionary analysis by sampling trees, $B M C$ Evolutionary Biology 7 (214)

Edmands S (2001) Phylogeography of the intertidal copepod Tigriopus californicus reveals substantially reduced population differentiation at northern latitudes, Molecular Ecology 10, pp 1743-1750

Ehrlich PR, Raven PH (1964) Butterflies and plants: A study in coevolution, Evolution 18, pp 586608

Eisenhauer N, Patsch S, Parkinson D, Scheu S (2007) Invasion of a deciduous forest by earthworms: Changes in soil chemistry, microflora, microarthropods and vegetation, Soil Biology and Biochemistry 39 (5), pp 1099-1110

Elias M, Hill RI, Willmott KR, Dasmahapatra KK, Andrew V. Z. Brower AVZ, Mallet J, Jiggins CD (2007) Limited performance of DNA barcoding in a diverse community of tropical butterflies, Proceedings of the Royal Society B 274, pp 2881-2889

Evans (1992) Principles of acarology Wallingford, Oxon, UK: CAB International

Excoffier L, Laval G, Schneider S (2005) Arlequin (version 3.0): an integrated software package for population genetics data analysis, Evolutionary Bioinformatics Online 1, pp 47-50

Farrell BD, Mitter C (1994) Adaptive Radiation in Insects and Plants: Time and Opportunity, American Zoologist 34, pp 57-69

Folmer O, Black M, Hoeh W, Lutz R, Vrijenhoek R (1994) DNA primers for amplification of mitochondrial cytochrome c oxidase subunit I from diverse metazoan invertebrates, Molecular Marine Biology and Biotechnology 3 (5), pp 294-299 


\section{References}

Fontaneto D, Herniou EA, Boschetti C, Caprioli M, Melone G, Ricci C, Barraclough TG (2007) Independently evolving species in asexual bdelloid rotifers, Plos Biology 5, pp 914921

Grandjean F (1953) Essai de classification des Oribates (Acariens), Bulletin de la Société Zoologique de France 78, pp 421-446

Grandjean F (1965) Complément a mon travail de 1953 sur la classification des oribates, Acarologia 7, pp 713-734

Grandjean F (1969) Considérations sur le classement des Oribates. Leur division en 6 groups majeurs, Acarologia 11, pp 127-153

Grimaldi D and Engel M (2005) Evolution of the insects, Cambridge University Press

Haag-Liautard C, Coffey N, Houle D, Lynch M, Charlesworth B, Keightley PD (2008) Direct estimation of the mitochondrial DNA mutation rate in Drosophila melanogaster, PloS Biology 6, pp 26-36

Habel JC, Dieker P, Schmitt T (2009) Biogeographical connections between the Maghreb and the Mediterranean peninsulas of southern Europe, Biological Journal of the Linnean Society 98, pp 693-703

Hajibabaei M, Janzen DH, Burns JM, Hallwachs W, Hebert PDN (2006) DNA barcodes distinguish species of tropical Lepidoptera, Proceedings of the National Academy of Sciences of the United States of America 103 (4), pp 968-971

Hajibabaei M, Singer GAC, Hebert PDN, Hickey DA (2007) DNA barcoding: how it complements taxonomy, molecular phylogenetics and population genetics, Trends in Genetics 23, pp 167172

Hawes TC, Worland MR, Convey P, Bale JS (2007) Aerial dispersal of springtails on the Antarctic Peninsula: implications for local distribution and demography, Antarctic Science 19 (1), pp 3-10

Hebert PDN, Ratnasingham S, de Waard JR (2003a) Barcoding animal life: cytochrome c oxidase subunit 1 divergences among closely related species, Proceedings of the Royal Society of London Series B-Biological Sciences 270, pp S96-S99

Hebert PDN, Cywinska A, Ball SL, de Waard JR (2003b) Biological identifications through DNA barcodes, Proceedings of the Royal Society of London Series B-Biological Sciences 270 (1512), pp 313-321

Hebert PDN, Stoeckle MY, Zemlak TS, Francis CM (2004a) Identification of birds through DNA barcodes, Plos Biology 2 (10), pp 1657-1663

Hebert PDN, Penton EH, Burns JM, Janzen DH, Hallwachs W (2004b) Ten species in one: $\quad$ DNA barcoding reveals cryptic species in the neotropical skipper butterfly Astraptes fulgerator, Proceedings of the National Academy of Sciences of the United States of America 101 (41), pp 14812-14817

Heethoff M, Maraun M, Scheu S (2000) Genetic variability in ribosomal ITS 1-sequences of the parthenogenetic oribatid mite Platynothrus peltifer (C.L. KOCH, 1839) (Acari: Oribatida), Berichte des naturwissenschaftlich-medizinischen Vereins in Innsbruck 87, pp 339-354

Heethoff M, Domes K, Laumann M, Maraun M, Norton RA, Scheu S (2007) High genetic divergences indicate ancient separation of parthenogenetic lineages of the oribatid mite Platynothrus peltifer (Acari, Oribatida), Journal of Evolutionary Biology 20, pp 392-402

Heethoff M , Norton RA, Scheu S, Maraun M (2009) Parthenogenesis in oribatid mites (Acari, Oribatida): Evolution without sex Lost Sex - The evolutionary biology of parthenogenesis Chapter 12, pp 241-257, Springer

Heidemann K Use of molecular markers for opening the nematode-based food-chain of temperate deciduous forests PhD thesis Georg August University, Goettingen unpublished 
Hewitt GM (1999) Post-glacial re-colonization of European biota, Biological Journal of the Linnean Society 68, pp 87-112

Hewitt GM (2000) The genetic legacy of the Quaternary ice ages, Nature 405, pp 907-913

Hewitt GM (2004) Genetic consequences of climatic oscillations in the Quaternary, Philosophical Transactions of the Royal Society of London Series B-Biological Sciences 359, pp 183-195

Hewitt GM, Ibrahim KM (2001) Inferring glacial refugia and historical migrations with molecular phylogenies, Integrating Ecology and Evolution in a Spatial Context, Chapter13, pp 271-294

Hickerson MJ, Meyer CP, Moritz C (2006) DNA barcoding will often fail to discover new animal species over broad parameter space Systematic Biology 55 (5), pp 729-739

Hogg ID, Hebert PDN (2004) Biological identification of springtails (Hexapoda: Collembola) from the Canadian Arctic, using mitochondrial DNA barcodes, Canadian Journal of Zoology $\mathbf{8 2}$, pp 749-754

Huang J, Xu Q, Sun ZJ, Tang GL, Su ZY (2007) Identifying earthworms through DNA barcodes. Pedobiologia 51, pp 301-309

Hubert J, Žilová M, Pekár S (2001) Feeding preferences and gut contents of three panphytophagous oribatid mites (Acari: Oribatida), European Journal of Soil Biology 37, pp 197-208

Hughes RN (1989) A functional biology of clonal animals. Chapman and Hall, London

Hurst GDD, Jiggins FM (2005) Problems with mitochondrial DNA as a marker in population, phylogeographic and phylogenetic studies: the effects of inherited symbionts, Proceedings of the Royal Society B-Biological Sciences 272 (1572), pp 1525-1534

Jensen JL, Bohonak AJ, Kelly ST (2005) Isolation by distance, web service, BMC Genetics 6

Johnson JB, Warén A, Vrijenhoek RC (2008) DNA barcoding of Lepetodrilus limpets reveals cryptic species, Journal of Shellfish Research 27, pp 43-51

Jolly MT, Jollivet D, Gentil F, Thiebaut E, Viard F (2005) Sharp genetic break between Atlantic and English Channel populations of the polychaete Pectinaria koreni, along the north coast of France, Heredity 94, pp 23-32

Kadenbach B, Stroh A (1984) Different reactivity of carboxylic groups of cytochrome c oxidase polypeptides from pig liver and heart, FEBS Letters 173, pp 374-380

Keeling PJ, Inagaki Y (2004) A class of eukaryotic GTPase with a punctate distribution suggesting multiple functional replacements of translation elongation factor $1 \alpha$, Proceedings of the National Academy of Sciences of the United States of America 101, pp 15380-15385

Keeling PJ, Fast NM, McFadden GI (1998) Evolutionary relationship between translation initiation factor eIF-2g and selenocysteine-specific elongation factor SELB: change of function in translation factors, Journal of Molecular Evolution 47, pp 649-655

Kelly A, Charman DJ, Newnham RM (2010) A Last Glacial Maximum pollen record from Bodmin Moor showing a possible cryptic refugium in southwest England, Journal of Quaternary Science 25, pp 296-308

Kempson D, Llyod M, Ghelardi R (1963) A new extractor for woodland litter, Pedobiologia 3, pp 121

Klompen H (2000) A preliminary assessment of the utility of elongation factor 1alpha in elucidating relationships among basal Mesostigmata, Experimental and Applied Acarology 24, pp 805-820

Kondrashov AS (1988) Deleterious mutations and the evolution of sexual reproduction, Nature 336, pp 435-440

Kondrashov AS (1993) Classification of hypotheses on the advantage of amphimixis, Journal of Heredity 84, pp 372-387

Krantz GW (1978) A manual of acarology 2nd ed, Oregon State University Bookstores, Corvallis 
Krantz GW, Walter DE (2009) A manual of Acarology $3^{\text {rd }}$ ed Texas Tech University Press, Lubbock

Kvaček Z (2000) Climatic oscillations versus environmental changes in the interpretation of Tertiary plant assemblages, Geological Society, London, Special Publications 181; pp 89-94

Kvaček Z, Manchester SR, Schorn HE (2000) Cones, seeds and foliage of Tetraclinis salicornioides (Cupressaceae) from the Oligocene and Miocene of western North America: a geographic extension of the European Tertiary species, International Journal of Plant Science 161, pp 331-344

Labandeira CC, Phillips TL, Norton RA (1997) Oribatid mites and the decomposition of plant tissues in Paleozoic coal-swamp forests, Palaios 12 (2), pp 319-353

Laumann M, Norton RA, Weigmann G, Scheu S, Maraun M, Heethoff M (2007) Speciation in the parthenogenetic oribatid mite genus Tectocepheus (Acari, Oribatida) as indicated by molecular phylogeny, Pedobiologia 51 (2), pp 111-122

Lebedeva NV, Lebedev VD, Melekhina EN (2006) New data on the oribatid mite (Oribatei) fauna of Svalbard, Doklady Biological Sciences 407, pp 182-186

Leo SST, Pybus MJ, Sperling FHA (2010) Deep mitochondrial DNA lineage divergences within Alberta populations of Dermacentor albipictus (Acari: Ixodidae) do not indicate distinct species, Journal of Medical Entomology 47 (4), pp 565-574

Li WH, Wu CI, Luo CC (1985) A new method for estimating synonymous and nonsynonymous rates of nucleotide substitution considering the relative likelihood of nucleotide and codon changes, Molecular Biology and Evolution 2, pp 150-174

Li WH (1993) Unbiased estimation of the rates of synonymous and nonsynonymous substitution, Journal of Molecular Evolution 36: pp 96-99

Lindberg N, Bengtsson J (2005) Population responses of oribatid mites and collembolans after drought, Applied Soil Ecology 28, pp 163-174

Lindquist EE (1984) Current theories on the evolution of major groups of Acari and their relationships with other groups of Arachnida, with consequent implications for their classification, Acarology, VI, pp 28-62

Luxton (1981) Studies on oribatid mites of a Danish beech wood soil. IV Developmental biology Pedobiologia 21, pp 312-340

Lynch M (2007) The origins of genome architecture Sinauer Associates INC, Sunderland, MA

Macfadyen A (1961) Improved funnel-type extractors for soil arthropods, Journal of Animal Ecology 30, pp 171-184

Mai DH (1989) Development and regional differentiation of the European vegetation during the Tertiary, Plant Systematics and Evolution 162, pp 79-91

Mai DH (1991) Palaeofloristic changes in Europe and the confirmation of the ArctotertiaryPalaeotropical geofloral concept, Review of Palaeobotany and Palynology 68, pp 29-36

Mai DH (1996) Tertiäre Vegetationsgeschichte Europas Fischer, Jena

Maraun M, Scheu S (2000) The structure of oribatid mite communities (Acari, Oribatida): patterns, mechanisms and implications for future research, Ecography 23, pp 374-383

Maraun M, Salamon JA, Schneider K, Schaefer M, Scheu S (2003) Oribatid mite and collembolan diversity, density and community structure in a moder beech forest (Fagus sylvatica): effects of mechanical perturbations, Soil Biology \& Biochemistry 35, pp 1387-1394

Maraun M, Heethoff M, Schneider K, Scheu S, Weigmann G, Cianciolo J, Thomas RH, Norton RA (2004) Molecular phylogeny of oribatid mites (Oribatida, Acari): evidence for multiple radiations of parthenogenetic lineages, Experimental and Applied Acarology 33, pp 183-201

Martens K, Rossetti G, Horne D J (2003) How ancient are ancient asexuals? Proceedings of the Royal Society of London B 270, pp 723-729 
Maynard Smith (1978) The Evolution of Sex Cambridge University Press, UK

Maynard Smith (1998) Evolutionary Genetics $2^{\text {nd }}$ Edition, Oxford University Press, Oxford

McDonald JH, Kreitman M (1991) Adaptive protein evolution at the ADH locus in Drosophila, Nature 351, pp 652-654

Meyers LA, Bull JJ (2002) Fighting change with change: adaptive variation in an uncertain world, Trends in Ecology and Evolution 17, pp 551-557

Miko L, Stanko M (1991) Small mammals as carriers of non-parasitic mites (Oribatida Uropodina) In Dusabek F, Bukva V (eds.), Modern Acarology Academica, pp 395-402

Mitchell P (1966): Chemiosmotic coupling in oxidative and photosynthetic phosphorylation, Biological Reviews of the Cambridge Philosophical Society 41, pp 445-502

Monaghan MT, Balke M, Gregory TR, Vogler AP (2005) DNA-based species delineation in tropical beetles using mitochondrial and nuclear markers, Philosophical Transactions of the Royal Society B 360, pp 1925-1933

Moniz MBJ, Kaczmarska I (2009) Barcoding diatoms: Is there a good marker? Molecular Ecology Resources 9, pp 65-74

Mortimer E, van Vuuren BJ (2007) Phylogeography of Eupodes minutus (Acari: Prostigmata) on sub-Antarctic Marion Island reflects the impact of historical events, Polar Biology 30, pp 471476

Mosbrugger V, Utescher T, Dilcher DL. (2005) Cenozoic continental climatic evolution of Central Europe, Proceedings of the National Academy of Sciences of the United States of America, 102, pp 14964-14969

Muller H. J. (1964): The relation of recombination to mutational advance, Mutation Research $\quad \mathbf{1}, \quad \mathrm{pp}$ 2-9

Muraoka M, Ishibashi N (1976) Nematode-feeding mites and their feeding behaviour, Applied Entomology and Zoology 11, pp 1-7

Mutanen M. Wahlberg N, Kaila L (2010) Comprehensive gene and taxon coverage elucidates radiation patterns in moths and butterflies, Proceedings of the Royal Society B - Biological Sciences 277 (1695), pp 2839-2848

Nicolet MH (1855) Histoire naturelle des Acariens qui se trouvent aux environs de Paris, Archives du Museum National d'Histoire Naturelle (Paris) 7, pp 381-482

Norton RA (1980) Observations on phoresy by oribatid mites (Acari: Oribatida), International Journal of Acarology 6, pp 121-130

Norton RA (1994) Evolutionary aspects of oribatid mites life histories and consequences for the origin of the Astigmata Houck MA (ed) Mites: Ecological and Evolutionary Analyses of Life-history Patterns, Chapman and Hall, New York, pp 99-135

Norton RA, Palmer SC (1991) The distribution, mechanisms, and evolutionary significance of parthenogenesis in oribatid mites. In: Schuster R, Murphy PW (eds) The Acari: Reproduction, Development and Life-History Strategies. Chapman and Hall, London, pp. 107-136

Norton RA, Bonamo PM, Grierson JD, Shear WA (1988a) Oribatid mite fossils from a terrestrial Devonian deposit near Gilboa, New York, Journal of Paleontology 62, pp 259-269

Norton RA, Palmer SC, Wang HF (1988b) Parthenogenesis in Nothridae and related groups. In: Channabasavanna GP, Viraktamath CA (eds), Progress in Acarology Volume 1 Oxford and IBH Publishing Co, New Dehli, pp 255-259

Nylander JAA (2004) MrModeltest v2. Program distributed by the author, Evolutionary Biology Centre, Uppsala University

O'Connor BM (1984) Phylogenetic relationship among higher taxa in the Acariformes, with particular reference to the Astigmata In: Grifiths DA, Bowman CE (eds) Acarology VI Vol. 1. Ellis Horwood LTD, Chichester, pp. 19-27 
Pachl P (2010) A conservative genetic marker (RNA Polymerase II) for the resolution of old radiations in oribatid mites (Acari, Oribatida), Diploma thesis TU Darmstadt

Palmer SC, Norton RA (1990) Further experimental proof of thelytokous parthenogenesis in oribatid mites (Acari: Oribatida: Desmonomata), Experimental and Applied Acarology 8, pp 149-159

Palmer SC, Norton RA (1992) Genetic diversity in thelytokous oribatid mites (Acari; Acariformes: Desmonomata), Biochemical Systematics and Ecology 20, pp 219-231

Papadopoulou A, Anastasiou I, Keskin B, Vogler AP (2009) Comparative phylogeography of tenebrionid beetles in the Aegean archipelago: the effect of dispersal ability and habitat preference, Molecular Ecology 18 (11), pp 2503-2517

Peakall R, Smouse PE (2006) GENALEX 6: genetic analysis in Excel. Population genetic software for teaching and research, Molecular Ecology Notes. 6, pp 288-295

Peek AS, Gustafson RG, Lutz RA, Vrijenhoek RC (1997) Evolutionary relationships of deep-sea hydrothermal vent and cold water seep clams (Bivalvia: Vesicomyidae): Results frm mitochondrial cytochrome oxidase subunit I, Marine Biology 130, pp 151-161

Pfenninger M, Posada D, Magnun F (2003) Evidence for survival of Pleistocene climatic changes in Northern refugia by the land snail Trochoidea geyeri (Soós 1926) (Helicellinae, Stylommatophora), BMC Evolutionary Biology, 3

Plantard O, Rasplus J-Y, Mondor G, Le Clainche I, Solignac M (1998) Wolbachia-induced thelytoky in the rose gallwasp Diplolepis sipnosissimae (Giraud) (Hymenoptera: Cynipidae), and its consequences on the genetic structure of its host, Proceedings of the Royal Society of London B 265, pp 1075-1090

Pohl N, Sison-Mangus MP, Yee EN, Liswi SW, Briscoe AD (2009) Impact of duplicate gene copies on phylogenetic analysis and divergence time estimates in butterflies, BMC Evolutionary Biology 9, 99

Pons, J, Barraclough TG, Gomez-Zurita J, Cardoso A, Duran DP, Hazell S, Kamoun S, Sumlin WD, Vogler AP (2006) Sequence-based species delimitation for the DNA taxonomy of undescribed insects, Systematic Biology 55, 595-609

Posada D, Crandall KA (1998) Modeltest: testing the model of DNA substitution, Bioinformatics 14, pp 817-818

Provan J, Bennett KD (2008) Phylogeographic insights into cryptic glacial refugia, Trends in Ecology and Evolution 23, pp 564-571

R Development Core Team (2010) R: A Language and Environment for Statistical Computing, R Foundation for Statistical Computing Vienna, Austria http://www.R-project.org

Rich KA, Thompson JN, Fernandez CC (2008) Diverse historical processes shape deep phylogeographical divergence in the pollinating seed parasite Greya politella, Molecular Ecology 17, pp 2430-2448

Ritzerow S, Konrad H, Stauffer C (2004) Phylogeography of the Eurasian pine shoot beetle Tomicus piniperda (Coleoptera : Scolytidae), European Journal of Entomology 101, pp 13-19

Rock J, Costa FO, Walker DD North AW, Hutchinson WF, Carvalho GR (2008) DNA barcodes of fish of the Scotia Sea, Antarctica indicate priority groups for taxonomic and systematics focus, Antarctic Science 20, pp 253-262.

Roe AD, Sperling FAH (2007) Population structure and species boundary delimitation of cryptic Dioryctria moths: an integrative approach, Molecular Ecology 16 (17), pp 3617-3633

Ronquist F, Huelsenbeck JP (2003) MrBayes 3: Bayesian phylogenetic inference under mixed models, Bioinformatics 19, pp 1572-1574

Rozas J, Sánchez-Del Barrio JC, Messeguer X, Rozas R (2003) DnaSP, DNA polymorphism analyses by the coalescent and other methods, Bioinformatics 19, pp 2496-2497 


\section{References}

Salomone N, Emerson BC, Hewitt GM, Bernini F (2002) Phylogenetic relationships among the Canary Island Steganacaridae (Acari, Oribatida) inferred from mitochondrial DNA sequence data, Molecular Ecology 11 (1), pp 79-89

Saraste M (1990) Structural features of Cytochrome c oxidase Quarterly Reviews of Biophysics. 23, pp 331-366

Schaefer I (2009) Evolutionary processes in oribatid mites at different scales in time as indicated by molecular markers, PhD thesis TU Darmstadt

Schaefer I, Domes K, Heethoff M, Schneider K, Schoen I, Norton RA, Scheu S, Maraun M (2006) No evidence for the 'Meselson effect' in parthenogenetic oribatid mites (Oribatida, Acari), Journal of Evolutionary Biology 19 (1), pp 184-193

Schaefer I, Norton RA, Scheu S, Maraun M (2010) Arthropod colonization of land - linking molecules and fossils in oribatid mites (Acari, Oribatida), Molecular Phylogenetics and Evolution, pp 113-121

Schäffer S, Koblmüller S, Pfingstl, T, Sturmbauer C, Krisper G (2010) Contrasting mitochondrial DNA diversity estimates in Austrian Sctovertex minutus and S. sculptus (Acari, Oribatida, Brachypylina, Scutoverticidae), Pedobiologia 53, pp 203-211

Schatz H (2002) Die Oribatidenliteratur und die beschriebenen Oribatidenarten (1758-2001) - Eine Analyse, Abhandlungen und Berichte des Naturkundemuseums Görlitz 72, pp 37-45

Schatz H, Gerecke R (1996) Hornmilben (Acari, Oribatida) aus Quellen und Quellbächen im Nationalpark Berchtesgaden (Oberbayern) und in den Südlichen Alpen (Trentino, Alto Adige),

Berichte des naturwissenschaftlich-medizinischen Vereins in Innsbruck 83, pp 121-144

Schenker R (1986) Population dynamics of oribatid mites (Acari: Oribatida) in a forest soil ecosystem, Pedobiologia 29, pp 239-246

Scheu S (2003) Effects of earthworms on plant growth: patterns and perspectives. Pedobiologia 47, pp 846-856

Scheu S, Schulz E, (1996) Secondary succession, soil formation and development of diverse a community of oribatids and saprophagous soil macro-invertebrates, Biodiversity and Conservation 5, pp 235-250

Schmitt T (2007) Molecular biogeography of Europe: Pleistocene cycles and postglacial trends, Frontriers in Zoology 4

Schmitt T (2009) Biogeographical and evolutionary importance of the European high mountain systems, Frontiers in Zoology 6

Schneider K, Migge S, Norton RA, Scheu S, Langel R, Reineking A, Maraun M (2004) Trophic niche differentation in soil microarthropods (Oribatida, Acari): evidence from stable isotope ratios $\left({ }^{15} \mathrm{~N} /{ }^{14} \mathrm{~N}\right)$, Soil Biology and Biochemistry 36, pp 1769-1774

Schön I (2007) Did Pleistocene glaciations shape genetic patterns of European ostracods? A phylogeographic analysis of two species with asexual reproduction, Hydrobiologia 575, pp $33-50$

Schön I, Lamatsch DK, Martens K (2008) Lessons to learn from ancient asexuals In: D Lankenau, $R$ Egel (eds) Genome Dynamics and Stability vol 3, Springer Berlin / Heidelberg

Sevillia RG, Diez A, Norén M, Mouchel O, Jérôme M, Verrez-Bagnis V, van Pelt H, Favre Krey L, Krey G,the Fishtrace Consortium, Bautista JM (2007) Primers and polymerase chain reaction conditions for DNA barcoding teleost fish based on the mitochondrial cytochrome $\mathrm{b}$ and nuclear rhodopsin genes, Molecular Ecology Notes 7, pp 730-734

Seyd EL (1962) The moss mites of Kinder Scout, Derbyshire (Acari: Oribatei), Zoological Journal of the Linnean Society 44, pp 585-591 
Shear WA, Bonamo PM, Grierson JD, Rolfe WDI, Smith EL, Norton RA. (1984) Early land animals in North America: evidence from Devonoian age arthropods from Gilboa, New York, Science 224, pp 492-494

Siepel H (1990) Niche relationships between two panphytophagous soil mites, Nothrus silvestris Nicolet (Acari, Oribatida, Nothridae) and Platynothrus peltifer (Koch) (Acari, Oribatida, Camisiidae), Biology and Feritlity of soils 9, pp 139-144

Siepel H (1996) The importance of unpredictable and short-term environmental extremes for biodiversity in oribatid mites, Biodiversity Letters, 3, pp 26-34

Sommer RS, Zachos FE (2009) Fossil evidence and phylogeography of temperate species: 'glacial refugia' and post-glacial recolonization, Journal of Biogeography 36, pp 2013-2020

Song H, Buhay JE, Whiting MF, Crandall KA (2008) Many species in one: DNA barcoding overestimates the number of species when nuclear mitochondrial pseudogenes are coamplified, Proceedings of the National Academy of Sciences of the United States of America 105 (36), pp 13486-13491

Starý J, Block W (1998) Distribution and biogeography of oribatid mites (Acari: Oribatida) in Antarctica, the sub-Antarctic islands and nearby land areas, Journal of Natural History 32, pp 861-894

Steinfartz S, Veith M, Tautz D (2000) Mitochondrial sequence analysis of Salamandra taxa suggests old splits of major lineages and postglacial recolonizations of Central Europe from distinct source populations of Salamandra salamandra, Molecular Ecology, 9, pp 397-410

Stenberg P, Saura A (2009) Cytology of asexual animals Lost Sex The evolutionary biology of parthenogenesis, Chapter 4, pp 63-74, Springer

Stewart, J. R. 2003 Comment on 'Buffered tree population changes in a Quaternary refugium: Evolutionary implications'. Science 299, pp 825

Stewart JR, Lister AM (2001) Cryptic northern refugia and the origins of the modern biota, Trends in Ecology \& Evolution 16, pp 608-613

Stewart JR, Cooper A (2008) Ice Age refugia and Quaternary extinctions: An issue of Quaternary evolutionary palaeoecology, Quaternary Science Reviews 27, pp 2443- 2448

Stewart JR, Lister AM, Barnes I, Dalén L (2010) Refugia revisited: individualistic responses of species in space and time, Proceedings of the Royal Society B 277, pp 661-671

Subias LS (2004) Systematic, synonymic and biogeographical check-list of the oribatid mites (Acariformes, Oribatida) of the world (1748-2002) (Listado sistemático, sinonímico y biogeográfico de los Ácaros Oribátidos (Acarifomes, Oribatida) del mundo (1748-2002)), Graellsia 60, pp 3-305

Subias LS (2009) Listado sistematico, sinonimico y biogeografico de los Acaros Oribatidos (Acariformes, Oribatida) del mundo (excepto fosiles) / Systematic, synonymic and biogeographical check-list of the oribatid mites (Acariformes, Oribatida) of the world (except fossils). http://www.ucm.es/info/zoo/Artropodos/Catalogo.pdf

Suomalainen E, Saura A, Lokki J (1987) Cytology and evolution in parthenogenesis, CRC Press, Boca Raton

Swofford D (1999) PAUP*: phylogenetic analysis using parsimony (and other methods). Version 4.0. Sinauer Associates, Sunderland, Massachusetts

Taberlet P, Fumagalli L, Hausser J (1994) Chromosomal versus mitochondrial-DNA evolution Tracking the evolutionary history of the Southwestern European populations of the SorexAraneus group (Mammalia, Insectivora), Evolution 48 (3), pp 623-636

Taberly G (1987) Recherches sur la parthénogenèse thélytoque de deux espèces d’acariens oríbates: Trhypochthonius tectorum (Berlese) et Platynothrus peltifer (Koch). III. Etude anatomique, histologique et cytologique des femelles parthénogenétiques. Acarologia 28, pp 389-403 
Tagg N, Doncaster CP, Innes DJ (2005) Resource competition between genetically varied and genetically uniform populations of Daphnia pulex (Leydig): does asexual reproduction confer a short-term ecological advantage? Biolgical Journal of the Linnean Society 85, pp 11-123

Thompson JD, Gibson TJ, Plewniak F, Jeanmougin F, Higgins DG (1997) The ClustalX windows interface: flexible strategies for multiple sequence alignment aided by quality analysis tools, Nucleic Acids Research 24, pp 4876-4882

Tollefsrud MM, Kissling R, Gugerli F, Johnsen $\varnothing$, Skrøppa T, Cheddadi R, van der Knapp WO, Latałowa M, Terhürne-Berson R, Litt T, Geburek T, Brochmann C, Sperisen C (2008) Genetic consequences of glacial survival and postglacial colonization in Norway spruce: combined analysis of mitochondrial DNA and fossil pollen, Molecular Ecology 17, pp 4134-4150

Torricelli G, Carapelli A, Convey P, Nardi F, Boore JL, Frati F (2010) High divergence across the whole mitochondrial genome in the "pan-Antarctic" springtail Friesea grisea: Evidence for cryptic species? Gene 449, pp 30-40

Townsend VR, Proud DN, Moore MK, Tibbetts JA, Burns JA, Hunter RK, Lazarowitz SR, Felgenhauer BE (2008) Parasitic and phoretic mites associated with neotropical harvestman from trinidad, Annals of the Entomological Society of America 101, pp1026-1032

Travé J, André HM, Taberly G, Bernini F (1996) Les Acariens Oribates Éditions AGAR et SIALF, Belgique

Ursenbacher S, Carlsson M, Helfer V, Tegelström H, Fumagalli L (2006) Phylogeography and Pleistocene refugia of the adder (Vipera berus) as inferred from mitochondrial DNA sequence data, Molecular Ecology 15, pp 3425-3437

Van Wilgenburg E, Driessen G, Beukeboom LW (2006) Single locus complementary sex determination in Hymenoptera: an "unintelligent" design? Frontiers in Zoology 3, 1

Verovnik R, Sket B, Trontelj P (2005) The colonization of Europe by the freshwater crustacean Asellus aquaticus (Crustacea : Isopoda) proceeded from ancient refugia and was directed by habitat connectivity, Molecular Ecology 14, pp 4355-4369

Walter DE, Proctor HC (1999) Mites: Ecology, Evolution, and Behaviour, University of New South Wales Press

Walter DE (2001) Endemism and cryptogenesis in 'segmented' mites: A review of Australian Alichorhagiidae, Terpnacaridae, Oehserchestidae and Grandjeanicidae (Acari: Sarcoptiformes), Australian Journal of Entomology 40, pp 207-218

Washburn J, Wasburn L (1984) Active aerial dispersal of minute wingless Arthropods: Exploitation of boundary-layer velocity gradients, Science 223, pp 1088-1089

Webb NR (1977) Observations on Steganacarus magnus, general biology and life cycle, Acarologia 19, pp 686-696

Webb (1989) Observations on the life cycle of Steganacarus magnus (Acari, Cryptostigmata), Pedobiologia 33, pp 293-299

Webb NR, Block W (1993) Aspects of cold hardiness in Steganacarus magnus (Acari, Cryptostigmata), Experimental \& Applied Acarology 17, pp 741-748

Weeks AR, Velten R, Stouthamer R (2003) Incidence of a new sex-ratio-distorting endosymbiotic bacterium among arthropods, Proceedings of the Royal Society of London B 270, pp 18571865

Weigmann G (1975) Labor- und Freilanduntersuchungen zur Generationsdauer von Oribatiden (Acari: Oribatei), Pedobiologia 15, pp 133-148

Weigmann G (2006) Hornmilben (Oribatida). Dahl (ed), Tierwelt Deutschlands 76 Goecke and Evers, Keltern

Welch DM, Meselson M (2000) Evidence for the evolution of bdelloid rotifers without sexual reproduction or genetic exchange, Science 288, pp 1211-1215 


\section{References}

Whitfield J (2005) Biogeography: Is everything everywhere? Science 310, pp 960-961

Will KW, Rubinoff D (2004) Myth of the molecule: DNA barcodes for species cannot replace morphology for identification and classification, Cladistics-the International Journal of the Willi Hennig Society 20 (1), pp 47-55

Williams, G.C., 1975. Sex and Evolution, Princeton University Press, Princenton, New Jersey

Williams ST, Knowlton N (2001) Mitochondrial pseudogenes are pervasive and often insidious in the snapping shrimp genus Alpheus, Molecular Biology and Evolution 18 (8), pp 1484-1493

Willis KJ, Rudner E, Sümegi P (2000) The full-glacial forests of central and southeastern Europe, Quaternary Research 53, pp 203-213

Wrench DL, Kethley JB, Norton RA (1994) Cytolgenetics of holokinetic chromosomes and inverted meiosis: keys to the evolutionary success of mites, with generalizations on eukaryotes. In: Houck MA (ed) Mites: Ecological and Evolutionary analyses of lifehistory pattern. Chapman and Hall, New York, pp 282-343

Wolstenholme DR (1992) Animal mitochondrial-DNA - Structure and evolution, International Review of Cytology-a Survey of Cell Biology 141, pp 173-216

Xia X, Hafner MS, Sudman PD (1996) On transition bias in mitochondrial genes of pocket gophers, Journal of Molecular Evolution 43, pp 32-40

Zaitsev AS, van Straalen NM (2001) Species diversity and metal accumulation in oribatid mites (Acari, Oribatida) of forests affected by a metallurgical plant, Pedobiologia 45, pp 467-479 


\section{Appendix}

Table A1: Standard diversity measures of COI nucleotide sequences of Steganacarus magnus. Populations with less than two individuals were excluded (Chapter II).

\begin{tabular}{|c|c|c|c|c|c|c|c|c|c|c|}
\hline population & locality & $\begin{array}{c}\text { sampl } \\
\text { size } \\
\text { n }\end{array}$ & $\begin{array}{l}\text { Ivaribl } \\
\text { sites } \\
\mathrm{N}_{\text {is }}\end{array}$ & $\begin{array}{l}\text { ariabl } \\
\text { sites } \\
\mathrm{N}_{\mathrm{vs}}\end{array}$ & $\begin{array}{l}\text { parsimony } \\
\text { inform. sites } \\
\mathrm{N}_{\text {pars }}\end{array}$ & $\begin{array}{c}\text { number of } \\
\text { singeltons } \\
\mathrm{N}_{\mathrm{s}}\end{array}$ & $\begin{array}{c}\text { number of } \\
\text { haplotypes } \\
N_{h}\end{array}$ & $\begin{array}{r}\text { haplotype } \\
\text { diversity } \\
\mathrm{H}_{\mathbf{d}}\end{array}$ & variance & $\begin{array}{c}\text { nucleotide } \\
\text { diversity } \\
\Pi_{\mathrm{n}}\end{array}$ \\
\hline A_1 & Villach & 2 & 513 & 0 & 0 & 0 & 1 & 0 & 0 & 0 \\
\hline CHINA_1 & Nanjing & 2 & 513 & 0 & 0 & 0 & 1 & 0 & 0 & 0 \\
\hline CZ_1 & Decin & 5 & 346 & 167 & 35 & 132 & 4 & 0.9 & 0.026 & 0.161 \\
\hline D_1 & Kranichstein & 14 & 486 & 27 & 12 & 15 & 8 & 0.769 & 0.014 & 0.013 \\
\hline D_2 & Goettingen & 9 & 508 & 5 & 0 & 5 & 4 & 0.417 & 0.036 & 0.002 \\
\hline D_3 & Lake Const. & 3 & 506 & 7 & 0 & 7 & 3 & 1 & 0.074 & 0.009 \\
\hline D_4 & Meck. Seenpl. & 9 & 326 & 187 & 124 & 63 & 4 & 0.75 & 0.013 & 0.162 \\
\hline D_5 & Moerfelden & 5 & 325 & 188 & 48 & 140 & 3 & 0.7 & 0.048 & 0.183 \\
\hline D_7 & Uelzen & 4 & 382 & 131 & 95 & 36 & 3 & 0.833 & 0.049 & 0.162 \\
\hline D_8 & Cuxhaven & 3 & 511 & 2 & 0 & 2 & 2 & 0.67 & 0.099 & 0.003 \\
\hline D_9 & Bonn & 4 & 502 & 11 & 2 & 9 & 3 & 0.833 & 0.049 & 0.011 \\
\hline DK_1 & Copenhagen & 3 & 377 & 136 & 0 & 136 & 3 & 1 & 0.074 & 0.181 \\
\hline DK_2 & Hjørring & 5 & 513 & 0 & 0 & 0 & 1 & 0 & 0 & 0 \\
\hline DK_3 & Arhus 1 & 3 & 507 & 6 & 0 & 6 & 2 & 0.67 & 0.099 & 0.008 \\
\hline DK_4 & Arhus 2 & 3 & 512 & 1 & 0 & 1 & 2 & 0.67 & 0.099 & 0.001 \\
\hline E_1 & Teno & 2 & 507 & 6 & 0 & 6 & 2 & 1 & 0.25 & 0.012 \\
\hline E_3 & Anaga & 4 & 496 & 17 & 5 & 12 & 4 & 1 & 0.031 & 0.019 \\
\hline F_1 & Mont Blanc & 6 & 327 & 186 & 48 & 138 & 3 & 0.6 & 0.046 & 0.155 \\
\hline F_2 & Loire & 4 & 511 & 2 & 0 & 2 & 3 & 0.833 & 0.049 & 0.002 \\
\hline F_3 & Saint Isidore & 5 & 445 & 68 & 0 & 68 & 4 & 0.9 & 0.026 & 0.053 \\
\hline F_4 & Haute Loire & 2 & 513 & 0 & 0 & 0 & 1 & 0 & 0 & 0 \\
\hline FIN_1 & Lahti & 3 & 510 & 3 & 0 & 3 & 2 & 0.67 & 0.099 & 0.004 \\
\hline GB_1 & Bedford & 5 & 446 & 67 & 5 & 62 & 4 & 0.9 & 0.026 & 0.054 \\
\hline GB_2 & Ascot & 2 & 511 & 2 & 0 & 2 & 2 & 1 & 0.25 & 0.004 \\
\hline GB_3 & Braemar & 3 & 508 & 5 & 0 & 5 & 2 & 0.67 & 0.099 & 0.007 \\
\hline I_1 & Grosseto & 10 & 503 & 10 & 8 & 2 & 4 & 0.711 & 0.014 & 0.009 \\
\hline I_2 & Parma & 4 & 500 & 13 & 2 & 11 & 4 & 1 & 0.031 & 0.013 \\
\hline IRL_1 & Swords & 2 & 511 & 2 & 0 & 2 & 2 & 1 & 0.25 & 0.004 \\
\hline N_1 & Narvik & 3 & 374 & 139 & 0 & 139 & 2 & 0.67 & 0.099 & 0.181 \\
\hline NL_1 & Wageningen & 3 & 505 & 8 & 0 & 8 & 3 & 1 & 0.074 & 0.01 \\
\hline PL_1 & Krakow & 6 & 375 & 138 & 131 & 7 & 6 & 1 & 0.009 & 0.143 \\
\hline $\mathrm{PL}_{-} 2$ & Warsaw & 3 & 498 & 15 & 0 & 15 & 2 & 0.67 & 0.099 & 0.019 \\
\hline RUM_1 & Sibiu 1 & 3 & 511 & 2 & 0 & 2 & 2 & 0.67 & 0.099 & 0.003 \\
\hline RUM_2 & Sibiu 2 & 4 & 502 & 11 & 2 & 9 & 4 & 1 & 0.031 & 0.011 \\
\hline RUM_3 & Bagau & 4 & 495 & 18 & 0 & 18 & 2 & 0.5 & 0.070 & 0.018 \\
\hline RUM_4 & Cluj & 3 & 506 & 7 & 0 & 7 & 3 & 1 & 0.074 & 0.009 \\
\hline RUM_5 & Busteni & 4 & 507 & 6 & 1 & 5 & 4 & 1 & 0.031 & 0.006 \\
\hline RUM_6 & Sinaia & 3 & 425 & 88 & 0 & 88 & 3 & 1 & 0.074 & 0.115 \\
\hline RUS_1 & Altai & 4 & 387 & 126 & 4 & 122 & 4 & 1 & 0.031 & 0.127 \\
\hline RUS_2 & Novosibirsk & 5 & 477 & 36 & 0 & 36 & 2 & 0.4 & 0.056 & 0.028 \\
\hline \multirow[t]{2}{*}{$\mathrm{S}_{-} 2$} & Stroemstad & 3 & 326 & 187 & 0 & 187 & 3 & 1 & 0.074 & 0.257 \\
\hline & all & 174 & 203 & 310 & 275 & 35 & 103 & 0.98 & 0 & 0.212 \\
\hline
\end{tabular}


Appendix

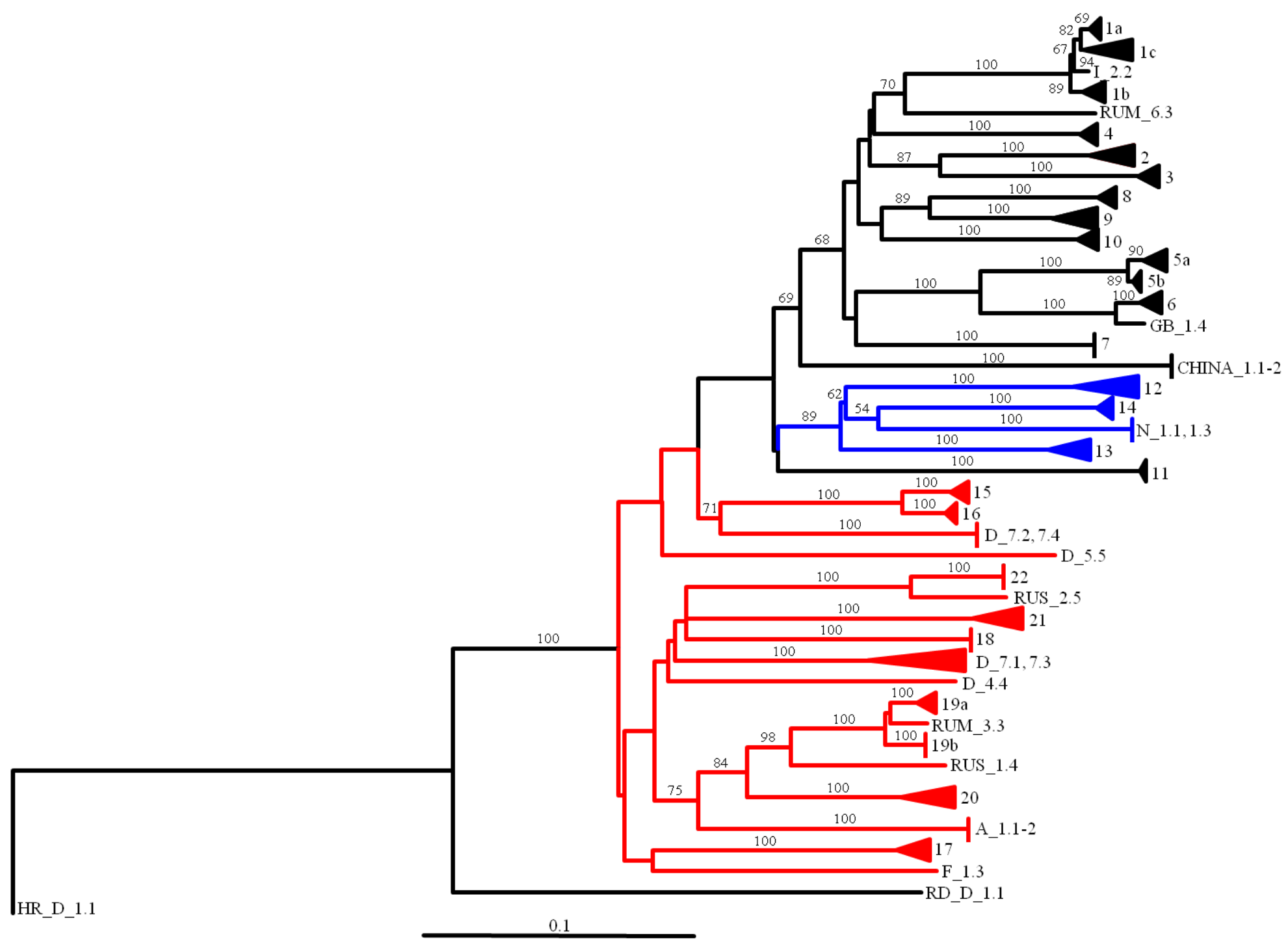

Figure A1: Neighbor-Joining tree of 180 COI nucleotide sequences of Steganacarus magnus. Outgroups are Hypochthonius rufulus (HD_D_1) and Rhysotritia duplicata (RD_D_1.1). Numbers on the branches are bootstrap values of 100,000 pseudo-replicates. Branch colours show the different clades (black, blue, red). Tip numbers are the different subclades and explain in Table 2. 
Appendix

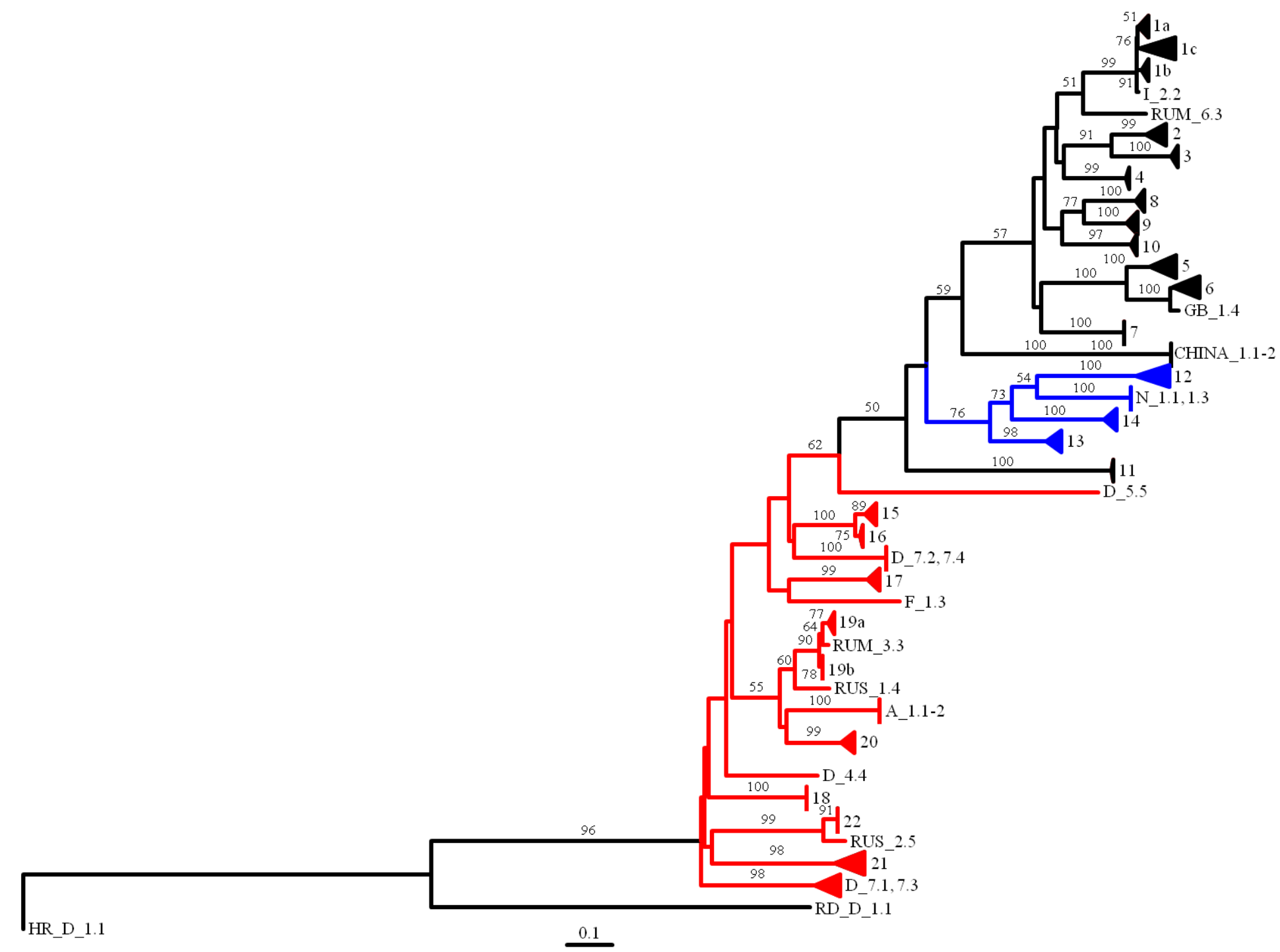

Figure A2: Neighbor-Joining tree of 180 COI nucleotide sequences of Steganacarus magnus with model of sequence evolution GTR+I+G. Outgroups are Hypochthonius rufulus (HD_D_1) and Rhysotritia duplicata (RD_D_1.1). Numbers on the branches are bootstrap values of 100,000 pseudo-replicates. Branch colours show the different clades (black, blue, red). Tip numbers are the different subclades and explain in Table 2. 
Appendix

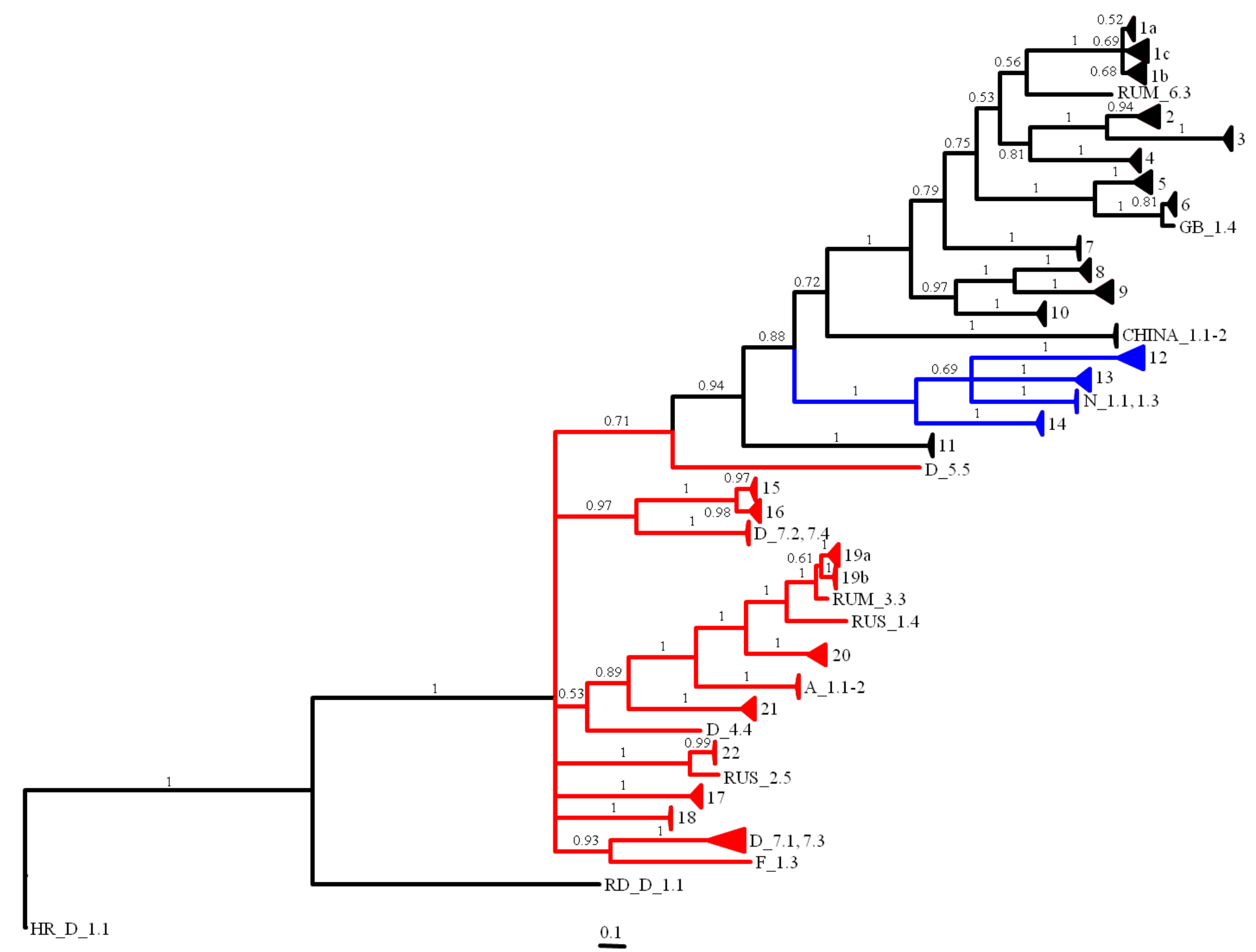

Figure A3: Bayesian tree after 10x10 $10^{6}$ generations from the 180 COI nucleotide sequences of Steganacarus magnus. Split frequencies of 0.013955 and burnin of $25 \%$. Outgroups are Hypochthonius rufulus (HD_D_1) and Rhysotritia duplicata (RD_D_1.1). Numbers on the branches are posterior probabilities. Branch colours show the different clades (black, blue, red). Tip numbers are the different subclades and explain in Table 2. 
Appendix

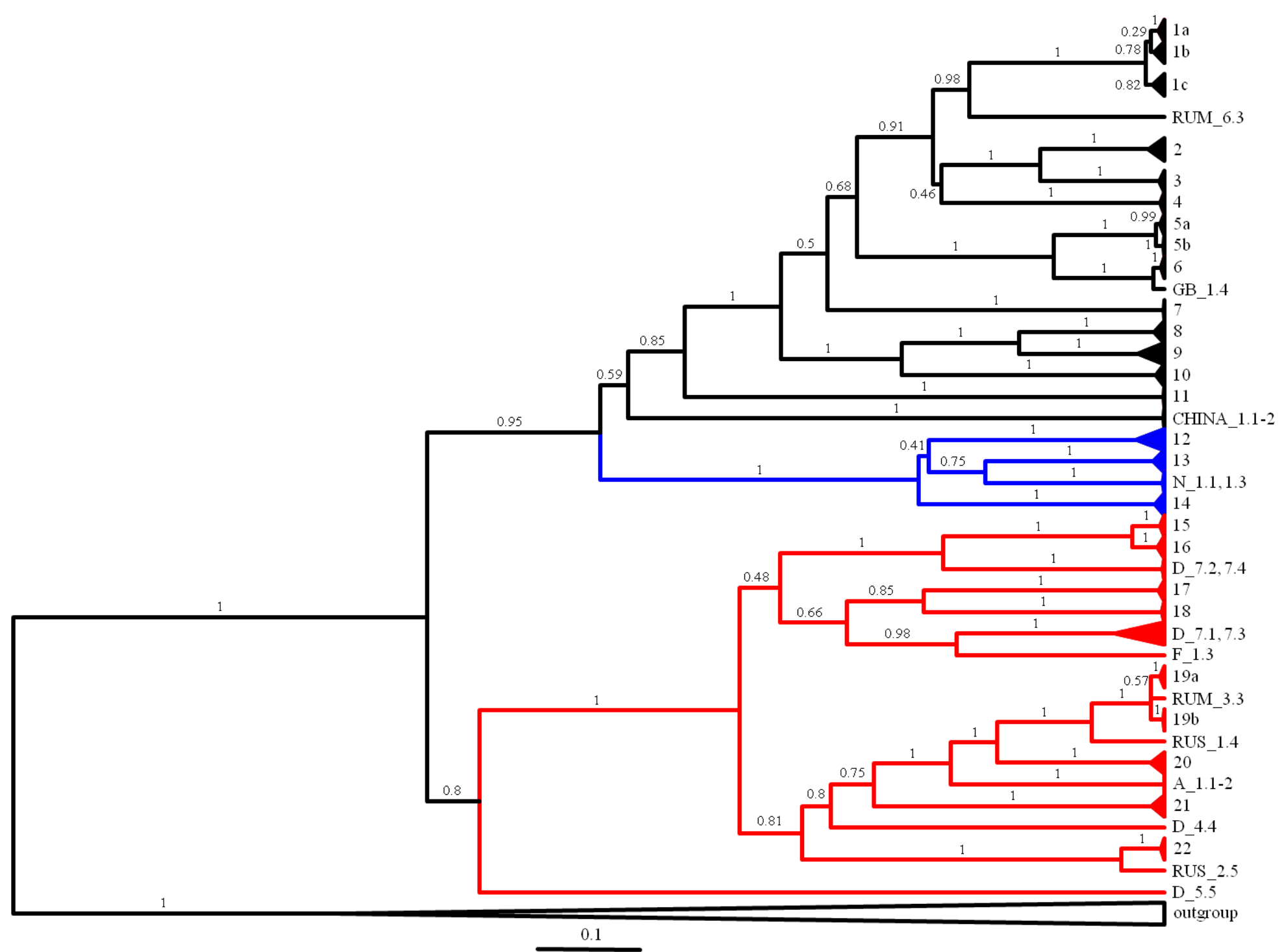

Figure A4: Bayesian phylogeny after 10x10 $0^{6}$ generation with Beast v1.5.4 from the 180 COI nucleotide sequences of Steganacarus magnus. Outgroups are Hypochthonius rufulus (HD_D_1) and Rhysotritia duplicata (RD_D_1.1). Numbers on the branches are posterior probabilities. Branch colours show the different clades (black, blue, red). Tip numbers are the different subclades and explain in Table 2. 
Appendix

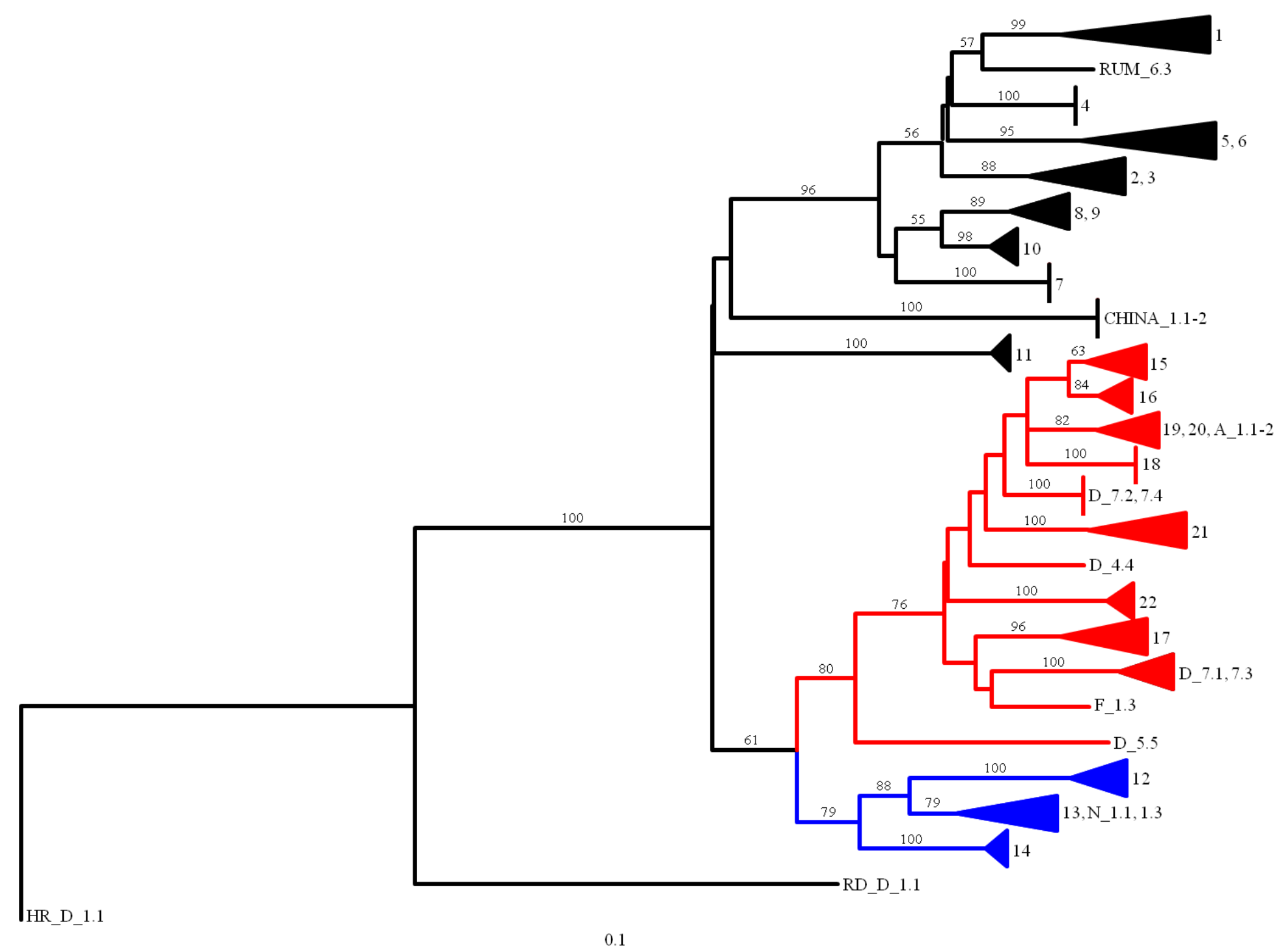

Figure A5: Neighbor-Joining tree of 180 COI protein sequences of Steganacarus magnus. Outgroups are Hypochthonius rufulus (HD_D_1) and Rhysotritia duplicata (RD_D_1.1). Numbers on the branches are bootstrap values of 100,000 pseudo-replicates. Branch colours show the different clades (black, blue, red). Tip numbers are the different subclades and explain in Table 2. 
Appendix

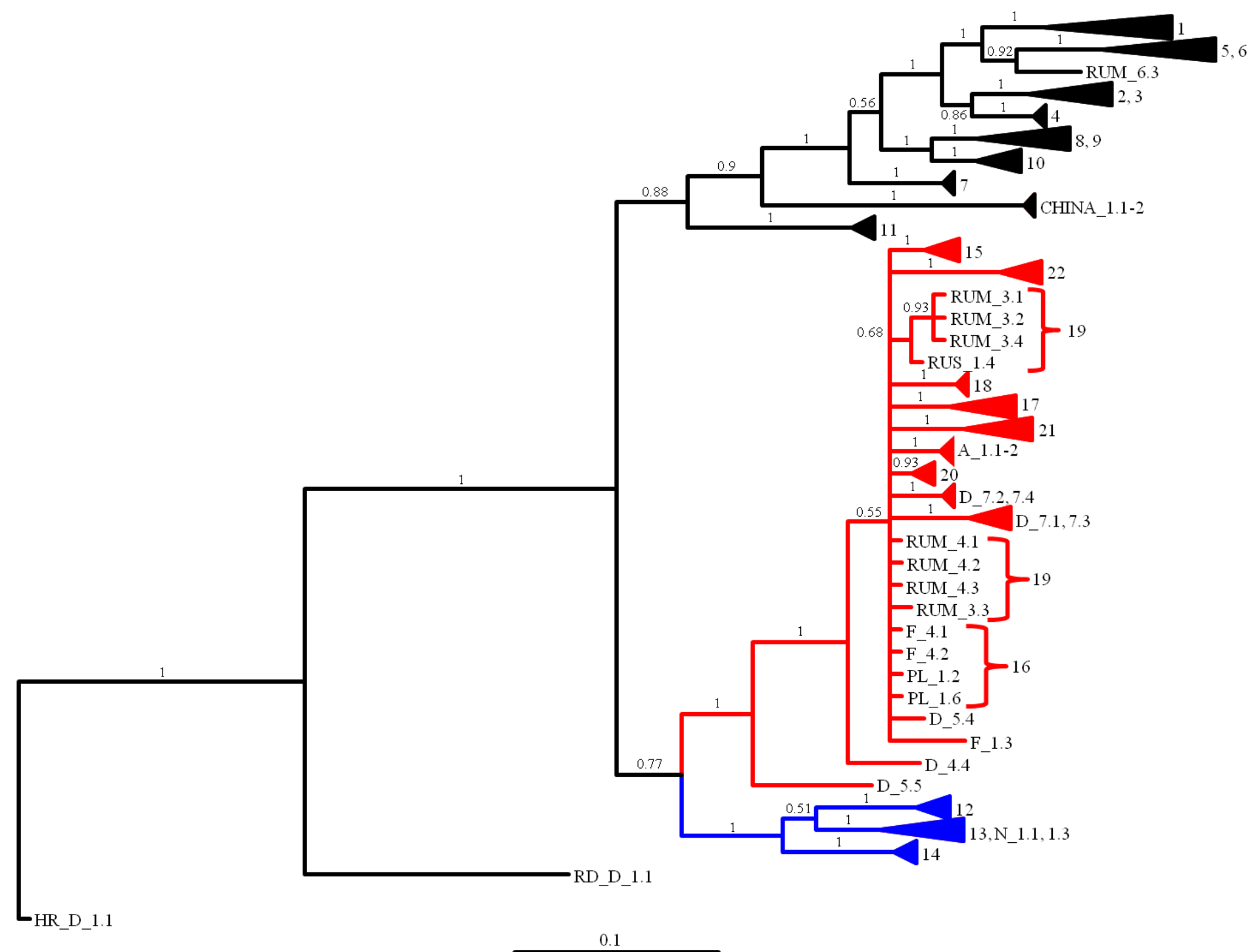

Figure A6: Bayesian tree after 10x10 $0^{6}$ generations from the 180 COI protein sequences of Steganacarus magnus. Split frequencies of 0.008138 and burnin of $25 \%$. Outgroups are Hypochthonius rufulus (HD_D_1) and Rhysotritia duplicata (RD_D_1.1). Numbers on the branches are posterior probabilities. Branch colours show the different clades (black, blue, red). Tip numbers are the different subclades and explain in Table 2. 
Appendix

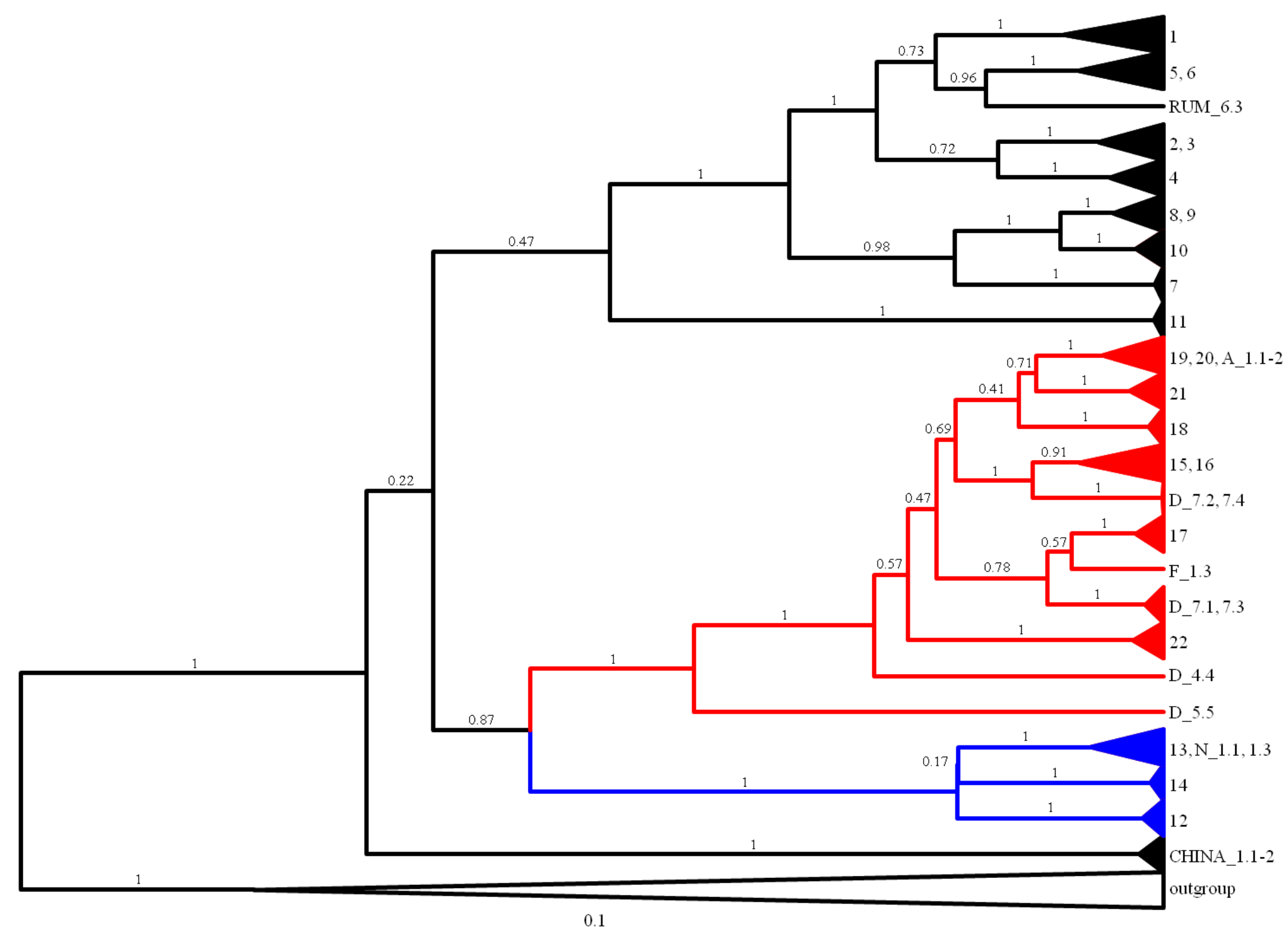

Figure A7: Bayesian phylogeny after 10x10 $0^{6}$ generations with Beast v1.5.4 from the 180 COI protein sequences of Steganacarus magnus. Outgroups are Hypochthonius rufulus (HD_D_1) and Rhysotritia duplicata (RD_D_1.1). Numbers on the branches are posterior probabilities. Branch colours show the different clades (black, blue, red). Tip numbers are the different subclades and explain in Table 2. 


\section{Appendix}

Table A2: Mean percentage pairwise differences of uncorrected p-distances of the protein of Steganacarus magnus from 41 locations in percent. The diagonal is the within population differences (bold) and below the diagonal the among population differences. Bold letters in red are the minimum and maximum differences within and among populations. Bold letters in pink is the maximum divergences, if China and Russia were excluded. Locations with less than two individuals were excluded from the analysis.

\begin{tabular}{|c|c|c|c|c|c|c|c|c|c|c|c|c|c|c|c|c|c|c|c|c|c|c|c|c|c|c|c|c|}
\hline \multicolumn{2}{|c|}{ Population } & 1 & 2 & 3 & 4 & 5 & 6 & 7 & 8 & 9 & 10 & 11 & 12 & 13 & 14 & 15 & 16 & 17 & 18 & 19 & 20 & 21 & 22 & 23 & 24 & 25 & 26 & 27 \\
\hline 1 & A 1 & 0 & & & & & & & & & & & & & & & & & & & & & & & & & & \\
\hline 2 & CHINA 1 & 0.6 & 0 & & & & & & & & & & & & & & & & & & & & & & & & & \\
\hline 3 & $\mathrm{CZ} 1$ & 1.9 & 1.6 & 1.1 & & & & & & & & & & & & & & & & & & & & & & & & \\
\hline 4 & D 1 & 3 & 3.6 & 1.8 & 0.4 & & & & & & & & & & & & & & & & & & & & & & & \\
\hline 5 & D 2 & 2.9 & 3.5 & 1.6 & 0.2 & 0 & & & & & & & & & & & & & & & & & & & & & & \\
\hline 6 & D 3 & 4.1 & 4.7 & 2.8 & 1.3 & 1.2 & 0 & & & & & & & & & & & & & & & & & & & & & \\
\hline 7 & D 4 & 1.7 & 1.5 & 1.2 & 2.3 & 2.2 & 2.7 & 1.2 & & & & & & & & & & & & & & & & & & & & \\
\hline 8 & D 5 & 2.6 & 2.6 & 1.7 & 1.5 & 1.4 & 2.8 & 2.1 & 2.1 & & & & & & & & & & & & & & & & & & & \\
\hline 9 & D 7 & 2.2 & 1.6 & 1.6 & 2.9 & 2.8 & 4 & 1.6 & 2.2 & 1.1 & & & & & & & & & & & & & & & & & & \\
\hline 10 & D 8 & 3.1 & 3.7 & 2.8 & 1.5 & 1.4 & 1.4 & 3.2 & 2.7 & 3.6 & 0.4 & & & & & & & & & & & & & & & & & \\
\hline 11 & D 9 & 2.9 & 3.5 & 1.7 & 0.3 & 0.2 & 1.2 & 2.2 & 1.4 & 2.8 & 1.4 & 0.3 & & & & & & & & & & & & & & & & \\
\hline 12 & DK 1 & 2.7 & 2.9 & 1.6 & 1.6 & 1.6 & 1.8 & 1.5 & 2 & 2.4 & 2.7 & 1.5 & 1.6 & & & & & & & & & & & & & & & \\
\hline 13 & DK 2 & 0.6 & 0 & 1.6 & 3 & 2.9 & 4.1 & 1.4 & 2.3 & 1.3 & 3.1 & 2.9 & 2.7 & 0 & & & & & & & & & & & & & & \\
\hline 14 & DK 3 & 2.9 & 3.5 & 1.6 & 0.2 & 0 & 1.2 & 2.2 & 1.4 & 2.8 & 1.4 & 0.2 & 1.6 & 2.9 & 0 & & & & & & & & & & & & & \\
\hline 15 & DK 4 & 2.9 & 3.5 & 1.6 & 0.2 & 0 & 1.2 & 2.2 & 1.4 & 2.8 & 1.4 & 0.2 & 1.6 & 2.9 & 0 & 0 & & & & & & & & & & & & \\
\hline 16 & E 1 & 2.9 & 4.1 & 2.1 & 0.7 & 0.6 & 1.8 & 2.5 & 2 & 2.8 & 2 & 0.6 & 2 & 2.9 & 0.6 & 0.6 & 0 & & & & & & & & & & & \\
\hline 17 & E 3 & 3.5 & 4.1 & 2.2 & 0.7 & 0.6 & 0.6 & 2.2 & 2.1 & 3.4 & 2 & 0.6 & 1.2 & 3.5 & 0.6 & 0.6 & 1.2 & 0 & & & & & & & & & & \\
\hline 18 & F 1 & 1.6 & 1 & 1.9 & 2.6 & 2.5 & 4.1 & 1.8 & 2.4 & 2.2 & 3.9 & 2.6 & 2.6 & 1 & 2.5 & 2.7 & 3 & 3.5 & 1.5 & & & & & & & & & \\
\hline 19 & F 2 & 2.9 & 3.5 & 1.6 & 0.2 & 0 & 1.2 & 2.2 & 1.4 & 2.8 & 1.4 & 0.2 & 1.6 & 2.9 & 0 & 0 & 0.6 & 0.6 & 2.5 & 0 & & & & & & & & \\
\hline 20 & F 3 & 3.2 & 3.7 & 2.8 & 1.5 & 1.4 & 1.4 & 3.2 & 2.6 & 3.6 & 0.4 & 1.4 & 2.8 & 3.2 & 1.4 & 1.4 & 2 & 2 & 3.9 & 1.4 & 0.5 & & & & & & & \\
\hline 21 & F 4 & 0.6 & 0 & 1.6 & 3 & 2.9 & 4.1 & 1.4 & 2.3 & 1.3 & 3.1 & 2.9 & 2.7 & 0 & 2.9 & 2.9 & 2.9 & 3.5 & 1 & 2.9 & 3.2 & 0 & & & & & & \\
\hline 22 & FIN 1 & 1.2 & 1.2 & 1.3 & 2.4 & 2.3 & 3.5 & 1.3 & 2 & 1 & 2.5 & 2.3 & 2.2 & 0.6 & 2.3 & 2.3 & 2.3 & 2.9 & 1.6 & 2.3 & 2.6 & 0.6 & 0 & & & & & \\
\hline 23 & GB 1 & 2.9 & 3.5 & 2.6 & 1.3 & 1.2 & 1.2 & 3 & 2.5 & 3.4 & 0.2 & 1.2 & 2.5 & 2.9 & 1.2 & 1.2 & 1.8 & 1.8 & 3.7 & 1.2 & 0.2 & 2.9 & 2.3 & 0 & & & & \\
\hline 24 & GB 2 & 2.9 & 3.5 & 2.6 & 1.3 & 1.2 & 1.2 & 3 & 2.5 & 3.4 & 0.2 & 1.2 & 2.5 & 2.9 & 1.2 & 1.2 & 1.8 & 1.8 & 3.7 & 1.2 & 0.2 & 2.9 & 2.3 & 0 & 0 & & & \\
\hline 25 & GB 3 & 3.1 & 3.7 & 2.8 & 1.5 & 1.4 & 1.4 & 3.2 & 2.7 & 3.6 & 0.3 & 1.4 & 2.7 & 3.1 & 1.4 & 1.4 & 2 & 2 & 3.9 & 1.4 & 0.4 & 3.1 & 2.5 & 0.2 & 0.2 & 0.4 & & \\
\hline 26 & I 1 & 2.9 & 3.5 & 1.8 & 0.4 & 0.3 & 1.2 & 2.2 & 1.4 & 2.8 & 1.4 & 0.3 & 1.4 & 2.9 & 0.3 & 0.3 & 0.6 & 0.6 & 2.6 & 0.3 & 1.4 & 2.9 & 2.3 & 1.2 & 1.2 & 1.4 & 0.3 & \\
\hline 27 & I 2 & 2.9 & 3.5 & 1.7 & 0.3 & 0.2 & 1.2 & 2.2 & 1.4 & 2.7 & 1.4 & 0.2 & 1.5 & 2.9 & 0.2 & 0.2 & 0.6 & 0.6 & 2.6 & 0.2 & 1.4 & 2.9 & 2.3 & 1.2 & 1.2 & 1.4 & 0.2 & 0.2 \\
\hline 28 & IRL 1 & 2.9 & 3.5 & 2.6 & 1.3 & 1.2 & 1.2 & 3 & 2.5 & 3.4 & 0.2 & 1.2 & 2.5 & 2.9 & 1.2 & 1.2 & 1.8 & 1.8 & 3.7 & 1.2 & 0.2 & 2.9 & 2.3 & 0 & 0 & 0.2 & 1.2 & 1.2 \\
\hline 29 & N 1 & 1.8 & 1.6 & 1.3 & 2.3 & 2.2 & 3.3 & 1.6 & 2.2 & 1.8 & 3.1 & 2.2 & 2 & 1.8 & 2.2 & 2.2 & 2.5 & 2.7 & 2 & 2.2 & 3.2 & 1.8 & 1.6 & 2.9 & 2.9 & 3.1 & 2.2 & 2.2 \\
\hline 30 & NL 1 & 2.9 & 3.5 & 2.6 & 1.3 & 1.2 & 1.2 & 3 & 2.5 & 3.4 & 0.2 & 1.2 & 2.5 & 2.9 & 1.2 & 1.2 & 1.8 & 1.8 & 3.7 & 1.2 & 0.2 & 2.9 & 2.3 & 0 & 0 & 0.2 & 1.2 & 1.2 \\
\hline 31 & PL 1 & 1 & 0.6 & 1.3 & 2.4 & 2.3 & 3.5 & 1.3 & 2 & 1.4 & 2.9 & 2.3 & 2.2 & 0.6 & 2.3 & 2.3 & 2.5 & 2.9 & 1.2 & 2.3 & 3 & 0.6 & 0.6 & 2.7 & 2.7 & 2.9 & 2.3 & 2.3 \\
\hline 32 & PL 2 & 3.7 & 4.3 & 2.3 & 0.7 & 0.6 & 0.8 & 2.2 & 2.2 & 3.3 & 2.2 & 0.6 & 2.8 & 1.2 & 0.6 & 0.7 & 1.4 & 0.2 & 3.4 & 0.6 & 2.2 & 3.7 & 4.2 & 2 & 2 & 2.2 & 0.6 & 0.6 \\
\hline 33 & RUM 1 & 2.9 & 3.5 & 1.6 & 0.1 & 0 & 1.2 & 2.2 & 1.5 & 2.5 & 1.4 & 0 & 1.4 & 2.9 & 0 & 0 & 0.6 & 0.6 & 2.9 & 0 & 1.4 & 2.9 & 2.3 & 1.2 & 1.2 & 1.4 & 0 & 0 \\
\hline 34 & RUM 2 & 3.2 & 3.8 & 1.8 & 0.2 & 0.2 & 1.2 & 2.2 & 1.8 & 2.8 & 1.7 & 0.2 & 1.3 & 3.2 & 0.2 & 0.2 & 0.9 & 0.6 & 3 & 0.2 & 1.7 & 3.2 & 2.6 & 1.5 & 1.5 & 1.7 & 0.2 & 0.2 \\
\hline 35 & RUM 3 & 0 & 0.6 & 1.9 & 3 & 2.9 & 4.1 & 1.6 & 2.6 & 1.9 & 3.1 & 2.9 & 2.7 & 0.6 & 2.9 & 2.9 & 2.9 & 3.5 & 2 & 2.9 & 3.2 & 0.6 & 1.2 & 2.9 & 2.9 & 3.1 & 2.9 & 2.9 \\
\hline 36 & RUM 4 & 0 & 0.6 & 1.9 & 3 & 2.9 & 4.1 & 1.6 & 2.6 & 1.9 & 3.1 & 2.9 & 2.7 & 0.6 & 2.9 & 2.9 & 2.9 & 3.5 & 2 & 2.9 & 3.2 & 0.6 & 1.2 & 2.9 & 2.9 & 3.1 & 2.9 & 2.9 \\
\hline 37 & RUM 5 & 3.1 & 3.7 & 1.8 & 0.2 & 0.2 & 1.3 & 2.3 & 1.6 & 2.6 & 1.5 & 0.2 & 1.5 & 3.1 & 0.2 & 0.2 & 0.7 & 0.7 & 3 & 0.2 & 1.5 & 3.1 & 2.5 & 1.3 & 1.3 & 1.5 & 0.2 & 0.2 \\
\hline 38 & RUM 6 & 2.7 & 3.3 & 1.6 & 0.5 & 0.4 & 1.8 & 2.1 & 1.7 & 2.6 & 1.6 & 0.4 & 1.5 & 2.7 & 0.4 & 0.5 & 1.2 & 1.2 & 2.6 & 0.4 & 1.6 & 2.7 & 2.2 & 1.4 & 1.4 & 1.6 & 0.4 & 0.4 \\
\hline 39 & RUS 1 & 1.9 & 2.5 & 2.5 & 2.4 & 2.3 & 4 & 2.1 & 2.7 & 2.9 & 4.3 & 2.3 & 2.3 & 2.5 & 2.3 & 2.5 & 2.8 & 3.4 & 2.5 & 2.3 & 4.3 & 2.5 & 2.6 & 4.1 & 4.1 & 4.3 & 2.3 & 2.3 \\
\hline 40 & RUS 2 & 3.4 & 1.9 & 2.2 & 2.4 & 2.3 & 4.1 & 2.9 & 2.5 & 1.7 & 3.7 & 2.3 & 2.9 & 3 & 2.3 & 2.3 & 2.3 & 2.9 & 3 & 2.3 & 3.7 & 3 & 2.5 & 3.5 & 3.5 & 3.7 & 2.3 & 2.3 \\
\hline 41 & S 2 & 1.4 & 1.4 & 0.9 & 1.9 & 1.8 & 2.9 & 1.2 & 1.7 & 1.5 & 2.7 & 1.8 & 1.6 & 1.2 & 1.8 & 1.8 & 2.2 & 2.3 & 1.5 & 1.8 & 2.8 & 1.2 & 1 & 2.5 & 2.5 & 2.7 & 1.8 & 1.8 \\
\hline
\end{tabular}


Appendix

Table A2continue:

\begin{tabular}{lccccccccccccccc}
\hline \multicolumn{2}{c}{ Population } & $\mathbf{2 8}$ & $\mathbf{2 9}$ & $\mathbf{3 0}$ & $\mathbf{3 1}$ & $\mathbf{3 2}$ & $\mathbf{3 3}$ & $\mathbf{3 4}$ & $\mathbf{3 5}$ & $\mathbf{3 6}$ & $\mathbf{3 7}$ & $\mathbf{3 8}$ & $\mathbf{3 9}$ & $\mathbf{4 0}$ & $\mathbf{4 1}$ \\
\hline $\mathbf{2 8}$ & IRL 1 & $\mathbf{0}$ & & & & & & & & & & & & & \\
$\mathbf{2 9}$ & N 1 & 2.9 & $\mathbf{1 . 6}$ & & & & & & & & & & & & \\
$\mathbf{3 0}$ & NL 1 & $\mathbf{0}$ & 2.9 & $\mathbf{0}$ & & & & & & & & & & & \\
$\mathbf{3 1}$ & PL 1 & 2.7 & $\mathbf{1 . 6}$ & 2.7 & $\mathbf{0 . 9}$ & & & & & & & & & & \\
$\mathbf{3 2}$ & PL 2 & 2 & 2.8 & 2.0 & 3.1 & $\mathbf{0 . 4}$ & & & & & & & & & \\
$\mathbf{3 3}$ & RUM 1 & 1.2 & 2.2 & 1.2 & 2.3 & 0.8 & 0 & & & & & & & & \\
$\mathbf{3 4}$ & RUM 2 & 1.5 & 2.3 & 1.5 & 2.6 & 0.7 & 0.3 & $\mathbf{0 . 6}$ & & & & & & & \\
$\mathbf{3 5}$ & RUM 3 & 2.9 & 1.6 & 2.9 & 1 & 3.7 & 2.9 & 3.2 & 0 & & & & & & \\
$\mathbf{3 6}$ & RUM 4 & 2.9 & 1.6 & 2.9 & 1 & 3.7 & 2.9 & 3.2 & $\mathbf{0}$ & $\mathbf{0}$ & & & & & \\
$\mathbf{3 7}$ & RUM 5 & 1.3 & 2.3 & 1.3 & 2.5 & 0.9 & 0.2 & 0.4 & 3.1 & 3.1 & $\mathbf{0 . 3}$ & & & & \\
$\mathbf{3 8}$ & RUM 6 & 1.4 & 2.1 & $\mathbf{1 . 4}$ & 2.2 & 1.2 & 0.6 & 0.8 & 2.7 & 2.7 & 0.7 & $\mathbf{1 . 2}$ & & & \\
$\mathbf{3 9}$ & RUS 1 & 4.1 & 2.2 & 4.1 & 2.3 & 3.3 & 2.8 & 2.9 & 2.3 & 2.3 & 2.9 & 2.6 & $\mathbf{1 . 8}$ & & \\
$\mathbf{4 0}$ & RUS 2 & 3.5 & 2.7 & 3.5 & 2.4 & 3.1 & 2.3 & 2.6 & 3.4 & 3.4 & 2.5 & 2.5 & 3.5 & $\mathbf{0 . 2}$ & \\
$\mathbf{4 1}$ & S 2 & 2.5 & $\mathbf{1 . 2}$ & 2.5 & $\mathbf{1}$ & 2.5 & 1.8 & 2 & 1.4 & 1.4 & 1.9 & $\mathbf{1 . 8}$ & 2 & $\mathbf{2 . 4}$ & $\mathbf{1 . 2}$ \\
\hline
\end{tabular}


Appendix

Table A3: Tajima's D and Fu's FS neutrality tests of Steganacarus magnus COI nucleotide sequences. Bold red letters show significance $(\mathrm{P}<0.05)$.

\begin{tabular}{|c|c|c|c|c|c|c|c|c|c|c|c|c|c|c|c|c|c|c|c|c|c|}
\hline Neutrality tests & A 1 & CHINA 1 & $\mathrm{CZ} 1$ & D 1 & D 2 & D 3 & D 4 & D 5 & D 7 & D 8 & D 9 & DK 1 & DK 2 & DK 3 & DK 4 & E 1 & E 3 & F 1 & F 2 & F 3 & F 4 \\
\hline \multicolumn{22}{|l|}{ Tajima's D test } \\
\hline Samplesize & 2 & 2 & 5 & 14 & 9 & 3 & 9 & 5 & 4 & 3 & 4 & 3 & 5 & 3 & 3 & 2 & 4 & 6 & 4 & 5 & 2 \\
\hline$S$ & 0 & 0 & 168 & 27 & 6 & 7 & 188 & 188 & 131 & 2 & 11 & 137 & 0 & 6 & 1 & 6 & 18 & 187 & 2 & 68 & 0 \\
\hline $\mathrm{Pi}$ & 0 & 0 & 82.9 & 6.44 & 1.5 & 4.67 & 83.53 & 94.1 & 83 & 1.33 & 5.83 & 93.67 & 0 & 4 & 0.67 & 6 & 10.17 & 79.93 & 1 & 27 & 0 \\
\hline Tajima's D & 0 & 0 & 0.21 & -1.03 & -1.4 & 0 & 1.08 & 0.33 & 1.69 & 0 & -0.28 & 6523804.68 & 0 & 0 & 0 & 0 & 0.36 & -0.16 & -0.71 & -1.26 & 0 \\
\hline Tajima's D p-value & 1 & 1 & 0.61 & 0.15 & 0.08 & 0.76 & 0.9 & 0.64 & 0.93 & 0.94 & 0.55 & 1 & 1 & 0.78 & 0.99 & 1 & 0.73 & 0.46 & 0.28 & 0.003 & 1 \\
\hline
\end{tabular}

\section{Fu's FS test}

Real no. of alleles

Orig. no. of alleles

Theta pi

Exp.no. of alleles

FS

FS p-value

$\begin{array}{ccccccccc}4 & 8 & 4 & 3 & 4 & 3 & 3 & 2 & 3 \\ 4 & 8 & 4 & 3 & 4 & 3 & 3 & 2 & 3 \\ 82.9 & 6.44 & 1.5 & 4.67 & 83.53 & 94.1 & 83 & 1.33 & 5.83 \\ 4.88 & 7.79 & 3.4 & 2.52 & 8.6 & 4.9 & 3.93 & 1.97 & 3.26 \\ 5.38 & 0.27 & -0.21 & 0.31 & 15.28 & 9.82 & 6.5 & 1.06 & 1.75 \\ 0.96 & 0.55 & 0.4 & 0.38 & 1 & 1 & 0.98 & 0.58 & 0.75\end{array}$

3
3
93.67
2.97
3.43
0.58

\begin{tabular}{|c|c|c|c|c|c|c|}
\hline 2 & 2 & 2 & 4 & 3 & 3 & 4 \\
\hline 2 & 2 & 2 & 4 & 3 & 3 & 4 \\
\hline 4 & 0.67 & 6 & 10.17 & 79.93 & 1 & 27.2 \\
\hline 2.47 & 1.65 & 1.86 & 3.52 & 5.82 & 2.08 & 4.67 \\
\hline 2.64 & 0.20 & 1.79 & 0.35 & 12.09 & -0.89 & 3.31 \\
\hline 0.84 & 0.40 & 0.52 & 0.35 & 1.00 & 0.09 & 0.88 \\
\hline
\end{tabular}

\begin{tabular}{|c|c|c|c|c|c|c|c|c|c|c|c|c|c|c|c|c|c|c|c|}
\hline Neutrality tests & GB 1 & GB 2 & GB 3 & I 1 & I 2 & IRL 1 & N 1 & NL 1 & PL 1 & PL 2 & RUM 1 & RUM 2 & RUM 3 & RUM 4 & RUM 5 & RUM 6 & RUS 1 & RUS 2 & $\mathrm{~S} 2$ \\
\hline \multicolumn{20}{|l|}{ Tajima's D test } \\
\hline Samplesize & 5 & 2 & 3 & 10 & 4 & 2 & 3 & 3 & 6 & 3 & 3 & 4 & 4 & 3 & 4 & 3 & 4 & 5 & 3 \\
\hline$S$ & 67 & 2 & 5 & 10 & 13 & 2 & 139 & 8 & 139 & 15 & 2 & 11 & 18 & 7 & 6 & 89 & 127 & 36 & 188 \\
\hline $\mathrm{Pi}$ & 27.8 & 2 & 3.33 & 4.67 & 6.83 & 2 & 92.67 & 5.33 & 73.8 & 10 & 1.33 & 5.83 & 9 & 4.67 & 3.17 & 59.67 & 65.5 & 14.4 & 132.67 \\
\hline Tajima's D & -1.03 & 0 & 0 & 1.42 & -0.37 & 0 & 0 & 0 & 1.38 & 0 & 0 & -0.28 & -0.85 & 0 & -0.31 & 1447938.65 & -0.57 & -1.25 & 14872900.98 \\
\hline Tajima's D p-value & 0.13 & 1 & 0.8 & 0.94 & 0.52 & 1 & 0.82 & 0.74 & 0.96 & 0.7 & 0.93 & 0.55 & 0.07 & 0.76 & 0.55 & 1.00 & 0.39 & 0.01 & 1.00 \\
\hline \multicolumn{20}{|l|}{ Fu's FS test } \\
\hline Real no. of alleles & 4 & 2 & 2 & 4 & 4 & 2 & 2 & 3 & 6 & 2 & 2 & 4 & 2 & 3 & 4 & 3 & 4 & 2 & 3 \\
\hline Orig. no. of alleles & 4 & 2 & 2 & 4 & 4 & 2 & 2 & 3 & 6 & 2 & 2 & 4 & 2 & 3 & 4 & 3 & 4 & 2 & 3 \\
\hline Theta_pi & 27.8 & 2 & 3.33 & 4.67 & 6.83 & 2 & 92.67 & 5.33 & 73.8 & 10 & 1.33 & 5.83 & 9 & 4.67 & 3.17 & 59.67 & 65.5 & 14.4 & 132.67 \\
\hline Exp. no. of alleles & 4.67 & 1.67 & 2.39 & 5.7 & 3.34 & 1.67 & 2.97 & 2.57 & 5.81 & 2.74 & 1.97 & 3.26 & 3.47 & 2.52 & 2.89 & 2.95 & 3.91 & 4.42 & 2.98 \\
\hline $\mathrm{FS}$ & 3.35 & 0.69 & 2.36 & 2.77 & -0.12 & 0.69 & 8.4 & 0.46 & 1.52 & 4.17 & 1.06 & -0.32 & 5.39 & 0.31 & -1.16 & 2.98 & 2.36 & 8.12 & 3.78 \\
\hline FS p-value & 0.88 & 0.37 & 0.81 & 0.91 & 0.27 & 0.37 & 0.99 & 0.39 & 0.49 & 0.93 & 0.59 & 0.22 & 0.98 & 0.38 & 0.09 & 0.57 & 0.55 & 1 & 0.6 \\
\hline
\end{tabular}


Appendix

Table A3 continue:

\begin{tabular}{lcc}
\hline Neutrality tests & mean & s.d. \\
\hline Tajima's D test & & \\
Samplesize & 4.24 & 2.43 \\
S & 49.76 & 66.94 \\
Pi & 27.14 & 37.55 \\
Tajima's D & 557186.37 & 2515606.36 \\
Tajima's D p-value & 0.70 & 0.32 \\
& & \\
Fu's FS test & & \\
Real no. of alleles & 2.95 & 1.36 \\
Orig.no. of alleles & 2.95 & 1.36 \\
Theta_pi & 27.14 & 37.55 \\
Exp.no. of alleles & 3.18 & 1.88 \\
FS & 2.72 & 3.63 \\
FS p-value & N.A. & N.A. \\
\hline
\end{tabular}


Appendix

Table A4: Tajima's D and Fu's FS neutrality tests of Steganacarus magnus COI protein sequences. Bold red letters show significance $(\mathrm{P}<0.05)$.

\begin{tabular}{|c|c|c|c|c|c|c|c|c|c|c|c|c|c|c|c|c|c|c|c|c|c|c|}
\hline Neutrality tests & A 1 & CHINA 1 & $\mathrm{CZ} 1$ & D 1 & D 2 & D 3 & D 4 & D 5 & D 7 & D 8 & D 9 & DK 1 & DK 2 & DK 3 & DK 4 & E 1 & E 3 & F 1 & F 2 & F 3 & F 4 & FIN 1 \\
\hline \multicolumn{23}{|l|}{ Tajima's D test } \\
\hline Samplesize & 2 & 2 & 5 & 14 & 9 & 3 & 9 & 5 & 4 & 3 & 4 & 3 & 5 & 3 & 3 & 2 & 4 & 6 & 4 & 5 & 2 & 3 \\
\hline S & 0 & 0 & 5 & 4 & 0 & 0 & 4 & 7 & 3 & 1 & 1 & 4 & 0 & 0 & 0 & 0 & 0 & 6 & 0 & 2 & 0 & 0 \\
\hline $\mathrm{Pi}$ & 0 & 0 & 1.9 & 0.67 & 0 & 0 & 2.11 & 3.5 & 1.83 & 0.67 & 0.50 & 2.67 & 0 & 0 & 0 & 0 & 0 & 2.53 & 0 & 0.8 & 0 & 0 \\
\hline Tajima's D & 0 & 0 & -1.4 & -1.54 & 0 & 0 & 1.77 & 0.29 & 1.09 & 0 & -0.61 & 0 & 0 & 0 & 0 & 0 & 0 & -0.21 & 0 & -0.97 & 0 & 0 \\
\hline Tajima's D p-value & 1 & 1 & $\mathbf{0}$ & 0.04 & 1 & 1 & 0.98 & 0.67 & 0.85 & 0.99 & 0.38 & 0.84 & 1 & 1 & 1 & 1 & 1 & 0.45 & 1 & 0.19 & 1 & 1 \\
\hline
\end{tabular}

\section{Fu's FS test}

Real no. of alleles

Orig. no. of alleles

Theta_pi

Exp. no. of alleles

FS

FS p-value

\begin{tabular}{|c|c|c|c|c|c|c|c|c|c|c|c|c|c|c|c|c|c|c|c|}
\hline 4 & 4 & 1 & 1 & 4 & 3 & 3 & 2 & 2 & 3 & 1 & 1 & 2 & 1 & 3 & 3 & 1 & 4 & 1 & 2 \\
\hline 4 & 4 & 1 & 1 & 4 & 3 & 3 & 2 & 2 & 3 & 1 & 1 & 2 & 1 & 3 & 3 & 1 & 4 & 1 & 2 \\
\hline 1.9 & 0.67 & 0 & 0 & 2.11 & 3.5 & 1.83 & 0.67 & 0.5 & 2.67 & 0 & 0 & 0 & 0 & 0 & 2.53 & 0 & 0.8 & 0 & 0 \\
\hline 2.85 & 2.65 & 0 & 0 & 3.95 & 3.42 & 2.5 & 1.65 & 1.68 & 2.3 & 0 & 0 & 0 & 0 & 0 & 3.46 & 0 & 2.11 & 0 & 0 \\
\hline 0.56 & -1.29 & 0 & 0 & 1.92 & 1.62 & 0.01 & 0.2 & 0.17 & -0.34 & 0 & 0 & 0 & 0 & 0 & 1.47 & 0 & 1.04 & 0 & 0 \\
\hline 0.54 & 0.06 & N.A. & N.A. & 0.86 & 0.77 & 0.29 & 0.39 & 0.34 & 0.19 & N.A. & N.A. & N.A. & N.A. & N.A. & 0.77 & N.A. & 0.62 & N.A. & N.A. \\
\hline
\end{tabular}

\begin{tabular}{|c|c|c|c|c|c|c|c|c|c|c|c|c|c|c|c|c|c|c|c|c|c|}
\hline Neutrality tests & GB 1 & GB 2 & GB 3 & I 1 & I 2 & IRL 1 & N 1 & NL 1 & PL 1 & PL 2 & RUM 1 & RUM 2 & 2 RUM 3 & 3 RUM & 4 RUM & 5 RUM 6 & RUS 1 & RUS 2 & S 2 & mean & s.d. \\
\hline \multicolumn{22}{|l|}{ Tajima's D test } \\
\hline Samplesize & 5 & 2 & 3 & 10 & 4 & 2 & 3 & 3 & 6 & 3 & 3 & 4 & 4 & 3 & 4 & 3 & 4 & 5 & 3 & 4.24 & 2.43 \\
\hline$S$ & 0 & 0 & 1 & 1 & 1 & 0 & 4 & 0 & 3 & 1 & 0 & 2 & 0 & 0 & 1 & 3 & 6 & 1 & 3 & 1.56 & 2.01 \\
\hline $\mathrm{Pi}$ & 0 & 0 & 0.67 & 0.44 & 0.33 & 0 & 2.67 & 0 & 1.6 & 0.67 & 0 & 1 & 0 & 0 & 0.5 & 2 & 3 & 0.4 & 2 & 0.79 & 1.03 \\
\hline Tajima's D & 0 & 0 & 0 & 0.66 & -2.86 & 0 & 0 & 0 & 1.12 & 0 & 0 & -0.71 & 0 & 0 & -0.61 & 0 & -0.81 & -0.82 & 0 & -0.14 & 0.73 \\
\hline Tajima's D p-value & 1 & 1 & 0.99 & 0.8 & 0 & 1 & 0.84 & 1 & 0.88 & 0.99 & 1 & 0.29 & 1 & 1 & 0.38 & 0.88 & 0.16 & 0.30 & 0.88 & 0.77 & 0.34 \\
\hline \multicolumn{22}{|l|}{ Fu's FS test } \\
\hline Real no. of alleles & 3 & 2 & 2 & 4 & 3 & 1 & 2 & 2 & 5 & 2 & 1 & 3 & 2 & 1 & 2 & 2 & 4 & 2 & 3 & 2.29 & 1.12 \\
\hline Orig. no. of alleles & 3 & 2 & 2 & 4 & 3 & 1 & 2 & 2 & 5 & 2 & 1 & 3 & 2 & 1 & 2 & 2 & 4 & 2 & 3 & 2.29 & 1.12 \\
\hline Theta_pi & 0 & 0 & 0.67 & 0.44 & 0.33 & 0 & 2.67 & 0 & 1.6 & 0.67 & 0 & 1 & 0 & 0 & 0.5 & 2 & 3 & 0.4 & 2 & 0.79 & 1.03 \\
\hline Exp. no. of alleles & 0 & 0 & 1.65 & 2.03 & 1.49 & 0 & 2.3 & 0 & 2.94 & 1.65 & 0 & 2.08 & 0 & 0 & 1.68 & 2.17 & 2.85 & 1.66 & 2.17 & 1.25 & 1.28 \\
\hline FS & 0 & 0 & 0.2 & 0.74 & -0.32 & 0 & 2.02 & 0 & 2.51 & 0.2 & 0 & 1.1 & 0 & 0 & 0.17 & 1.61 & 0.73 & 0.09 & 1.61 & 0.39 & 0.77 \\
\hline FS p-value & N.A. & N.A. & 0.39 & 0.46 & 0.04 & N.A. & 0.76 & N.A. & 0.87 & 0.39 & N.A. & 0.62 & N.A. & N.A. & 0.34 & 0.7 & 0.58 & 0.29 & 0.7 & N.A. & N.A. \\
\hline
\end{tabular}


Table A5: Results of the McDonald-Kreitman test for Steganacarus magnus. The differences between 90 populations are significant $(* 0.01<\mathrm{P}<0.05)$, between other 80 populations high significant $(* * 0.001<\mathrm{P}<0.01)$ and between 63 populations extremely high significant $(* * * \mathrm{P}<0.001)$. Number of fixed and polymorph synonymous and non-synonymous mutations are shown. Locations with less than two individuals were excluded.

\begin{tabular}{|c|c|c|c|c|c|c|c|c|c|c|c|c|c|}
\hline \multirow{2}{*}{$\begin{array}{l}\text { Population } \\
\text { CHINA } 1\end{array}$} & \multirow[b]{2}{*}{ fixed } & \multicolumn{3}{|c|}{$\begin{array}{c}\text { A1 } \\
\text { syn. nons. sign. }\end{array}$} & \multicolumn{3}{|c|}{$\begin{array}{l}\text { CHINA_1 } \\
\text { syn. nons. sign. }\end{array}$} & \multicolumn{3}{|c|}{$\begin{array}{c}\text { CZ_1 } \\
\text { syn. nons. sign. }\end{array}$} & \multicolumn{3}{|c|}{$\begin{array}{c}\text { D_1 } \\
\text { syn. nons. sign. }\end{array}$} \\
\hline & & 107 & 46 & $-n s$ & & & & & & & & & \\
\hline Nanjing & poly & 0 & 0 & & & & & & & & & & \\
\hline CZ_1 & fixed & 48 & 3 & $0.0443^{*}$ & 52 & 23 & $0.0286^{*}$ & & & & & & \\
\hline Decin & poly & 145 & 31 & & 145 & 31 & & & & & & & \\
\hline D_1 & fixed & 96 & 44 & $0.265 \mathrm{~ns}$ & 82 & 35 & $0.3596 \mathrm{~ns}$ & 30 & 17 & $0.0353^{*}$ & & & \\
\hline Kranichstein & poly & 15 & 12 & & 16 & 11 & & 156 & 41 & & & & \\
\hline D_2 & fixed & 97 & 45 & $0.1867 \mathrm{~ns}$ & 84 & 37 & $0.3204 \mathrm{~ns}$ & 32 & 17 & $0.0163^{*}$ & 0 & 0 & $-n s$ \\
\hline Goettingen & poly & 5 & 0 & & 5 & 0 & & 148 & 31 & & 17 & 11 & \\
\hline D_3 & fixed & 105 & 47 & $0.1055 \mathrm{~ns}$ & 88 & 37 & $0.1122 \mathrm{~ns}$ & 40 & 18 & $0.0383^{*}$ & 67 & 11 & $0.0375^{*}$ \\
\hline Ittendorf & poly & 7 & 0 & & 7 & 0 & & 151 & 31 & & 23 & 11 & \\
\hline D_4 & fixed & 40 & 5 & $0.6384 \mathrm{~ns}$ & 45 & 22 & $0.0039 * *$ & 6 & 0 & $0.5995 \mathrm{~ns}$ & 27 & 17 & $0.0082 * *$ \\
\hline Meckl. Seenpl. & poly & 161 & 29 & & 161 & 29 & & 243 & 41 & & 171 & 39 & \\
\hline D_5 & fixed & 53 & 6 & $0.0181^{*}$ & 46 & 16 & $0.8666 \mathrm{~ns}$ & 9 & 1 & $0.6929 \mathrm{~ns}$ & 20 & 5 & $0.8169 \mathrm{~ns}$ \\
\hline Moerfelden & poly & 148 & 48 & & 148 & 48 & & 229 & 57 & & 160 & 57 & \\
\hline D_7 & fixed & 61 & 10 & $1 \mathrm{~ns}$ & 62 & 37 & $0.0001^{* * *} *$ & 24 & 2 & $0.3919 \mathrm{~ns}$ & 52 & 29 & $0.0066^{* *}$ \\
\hline Uēzen & poly & 114 & 19 & & 114 & 19 & & 218 & 40 & & 124 & 29 & \\
\hline D_8 & fixed & 95 & 47 & $1 \mathrm{~ns}$ & 97 & 41 & $1 \mathrm{~ns}$ & 41 & 42 & $0.0083^{* *}$ & 81 & 13 & $0.0028 * *$ \\
\hline Cuxhaven & poly & 1 & 1 & & 1 & 1 & & 145 & 32 & & 17 & 12 & \\
\hline D_9 & fixed & 97 & 45 & $0.1746 \mathrm{~ns}$ & 82 & 36 & $0.1763 \mathrm{~ns}$ & 31 & 17 & $0.0095 * *$ & 0 & 0 & $-\mathrm{ns}$ \\
\hline Bonn & poly & 10 & 1 & & 10 & 1 & & 152 & 32 & & 17 & 11 & \\
\hline DK_1 & fixed & 69 & 24 & $0.3051 \mathrm{~ns}$ & 56 & 23 & $0.7602 \mathrm{~ns}$ & 9 & 2 & $1 \mathrm{~ns}$ & 0 & 0 & $-\mathrm{ns}$ \\
\hline Copenhagen & poly & 92 & 45 & & 93 & 44 & & 194 & 59 & & 106 & 55 & \\
\hline DK_2 & fixed & 96 & 15 & $-n s$ & 104 & 41 & $-\mathrm{ns}$ & 46 & 3 & $0.0458^{*}$ & 76 & 38 & $0.5044 \mathrm{~ns}$ \\
\hline Hjorring & poly & 0 & 0 & & 0 & 0 & & 145 & 31 & & 16 & 11 & \\
\hline DK_3 & fixed & 97 & 45 & $0.1781 \mathrm{~ns}$ & 84 & 37 & $0.1797 \mathrm{~ns}$ & 32 & 17 & $0.0163^{*}$ & 0 & 0 & $-n s$ \\
\hline Arhus Stadion & poly & 6 & 0 & & 6 & 0 & & 148 & 31 & & 18 & 11 & \\
\hline DK_4 & fixed & 98 & 45 & $0.3194 \mathrm{~ns}$ & 86 & 36 & $0.3008 \mathrm{~ns}$ & 33 & 17 & $0.0199^{*}$ & 0 & 0 & $-n s$ \\
\hline Arhus Mo & poly & 0 & 1 & & 0 & 1 & & 145 & 32 & & 16 & 12 & \\
\hline E_1 & fixed & 100 & 44 & $0.1807 \mathrm{~ns}$ & 100 & 38 & $0.195 \mathrm{~ns}$ & 41 & 18 & $0.0399^{*}$ & 76 & 14 & $0.0424^{*}$ \\
\hline Teno Mountains & poly & 6 & 0 & & 6 & 0 & & 149 & 31 & & 22 & 11 & \\
\hline E_3 & fixed & 95 & 48 & $0.4282 \mathrm{~ns}$ & 96 & 39 & $0.5968 \mathrm{~ns}$ & 27 & 19 & $0.0028 * *$ & 63 & 13 & $0.0419^{*}$ \\
\hline Anaga Mountains & poly & 14 & 4 & & 14 & 4 & & 154 & 35 & & 28 & 15 & \\
\hline F_1 & fixed & 49 & 4 & $0.0876 \mathrm{~ns}$ & 49 & 21 & $0.038^{*}$ & 0 & 0 & $-n s$ & 25 & 16 & $0.0263^{*}$ \\
\hline Mont Blanc & poly & 162 & 34 & & 162 & 34 & & 228 & 38 & & 170 & 45 & \\
\hline F_2 & fixed & 99 & 45 & $0.5706 \mathrm{~ns}$ & 84 & 37 & $0.5759 \mathrm{~ns}$ & 31 & 17 & $0.0175^{*}$ & 0 & 0 & $-n s$ \\
\hline Loire & poly & 2 & 0 & & 2 & 0 & & 147 & 31 & & 18 & 11 & \\
\hline F 3 & fixed & 77 & 45 & $0.0028 * *$ & 75 & 35 & $0.0225^{*}$ & 28 & 19 & $0.0017^{* *}$ & 64 & 10 & $0.114 \mathrm{~ns}$ \\
\hline Saint Isidore & poly & 57 & 11 & & 57 & 11 & & 180 & 40 & & 70 & 22 & \\
\hline$F_{4}$ & fixed & 96 & 12 & $-n s$ & 102 & 44 & $-n s$ & 44 & 2 & $0.021^{*}$ & 73 & 39 & $0.6561 \mathrm{~ns}$ \\
\hline Haute Loire & poly & 0 & 0 & & 0 & 0 & & 145 & 31 & & 16 & 11 & \\
\hline FIN_1 & fixed & 102 & 17 & $0.0039 * *$ & 98 & 43 & $0.0311^{*}$ & 60 & 3 & $0.1912 \mathrm{~ns}$ & 83 & 37 & $0.1313 \mathrm{~ns}$ \\
\hline Lahti & poly & 0 & 3 & & 0 & 3 & & 145 & 34 & & 16 & 14 & \\
\hline GB_1 & fixed & 78 & 45 & $0.0000^{* * *} *$ & 73 & 36 & $0.0002 * * *$ & 27 & 19 & $0.0004^{* * * *}$ & 62 & 10 & $0.5255 \mathrm{~ns}$ \\
\hline Bedford & poly & 61 & 6 & & 61 & 6 & & 186 & 35 & & 74 & 17 & \\
\hline GB_2 & fixed & 95 & 48 & $1 \mathrm{~ns}$ & 97 & 42 & $1 \mathrm{~ns}$ & 44 & 23 & $0.0097^{* *}$ & 85 & 14 & $0.003^{* *} *$ \\
\hline Ascot & poly & 1 & 1 & & 1 & 1 & & 145 & 32 & & 17 & 12 & \\
\hline GB_3 & fixed & 95 & 47 & $0.0493^{*}$ & 98 & 41 & $0.0332 *$ & 44 & 22 & $0.0265^{*}$ & 86 & 13 & $0.0003 * * *$ \\
\hline Braēmar & poly & 1 & 4 & & 1 & 4 & & 146 & 35 & & 17 & 15 & \\
\hline I_1 & fixed & 98 & 45 & $1 \mathrm{~ns}$ & 83 & 36 & $1 \mathrm{~ns}$ & 31 & 17 & $0.0176^{*}$ & 0 & 0 & $-n s$ \\
\hline Grosseto & poly & 7 & 3 & & 7 & 3 & & 151 & 34 & & 16 & 13 & \\
\hline I_2 & fixed & 97 & 44 & $0.3464 \mathrm{~ns}$ & 82 & 36 & $0.3448 \mathrm{~ns}$ & 31 & 16 & $0.0258^{*}$ & 0 & 0 & $-\mathrm{ns}$ \\
\hline Parma & poly & 11 & 2 & & 11 & 2 & & 154 & 33 & & 18 & 12 & \\
\hline IRL_1 & fixed & 94 & 47 & $0.5556 \mathrm{~ns}$ & 98 & 41 & $1 \mathrm{~ns}$ & 43 & 22 & $0.0086^{* *}$ & 85 & 13 & $0.0058 * *$ \\
\hline Swords & poly & 2 & 0 & & 2 & 0 & & 146 & 31 & & 18 & 11 & \\
\hline N_1 & fixed & 64 & 5 & $0.0099 * *$ & 67 & 28 & $0.2239 \mathrm{~ns}$ & 2 & 0 & $1 \mathrm{~ns}$ & 53 & 23 & $0.6446 \mathrm{~ns}$ \\
\hline Narvik & poly & 109 & 30 & & 108 & 31 & & 181 & 36 & & 118 & 44 & \\
\hline
\end{tabular}


Table A5 continue:

\begin{tabular}{|c|c|c|c|c|c|c|c|c|c|c|c|c|c|}
\hline \multirow{2}{*}{$\begin{array}{c}\text { Population } \\
\text { NL_1 }\end{array}$} & & \multicolumn{3}{|c|}{$\begin{array}{c}\text { A1 } \\
\text { syn. nons. sign. }\end{array}$} & \multicolumn{3}{|c|}{$\begin{array}{l}\text { CHINA_1 } \\
\text { syn. nons. sign. }\end{array}$} & \multicolumn{3}{|c|}{$\begin{array}{c}\mathrm{CZ}_{1} 1 \\
\text { syn. nons. sign. }\end{array}$} & \multicolumn{3}{|c|}{$\begin{array}{c}\text { D_1 } \\
\text { syn. nons. sign. }\end{array}$} \\
\hline & fixed & 97 & 45 & $0.1867 \mathrm{~ns}$ & 96 & 41 & $1 \mathrm{~ns}$ & 41 & 22 & $0.0084^{*}$ & 82 & 13 & $0.0057^{* *}$ \\
\hline Wageningen & poly & 5 & 0 & & 6 & 2 & & 149 & 33 & & 22 & 13 & \\
\hline PL_1 & fixed & 63 & 8 & $0.0289^{*}$ & 68 & 30 & $0.3813 \mathrm{~ns}$ & 1 & 0 & $1 \mathrm{~ns}$ & 39 & 21 & $0.3266 \mathrm{~ns}$ \\
\hline Krakow & poly & 107 & 35 & & 106 & 36 & & 193 & 39 & & 118 & 46 & \\
\hline PL_2 & fixed & 102 & 43 & $0.5574 \mathrm{~ns}$ & 79 & 39 & $0.3873 \mathrm{~ns}$ & 32 & 19 & $0.0069 * *$ & 56 & 18 & $0.2833 \mathrm{~ns}$ \\
\hline Warsaw & poly & 12 & 3 & & 12 & 3 & & 150 & 33 & & 27 & 14 & \\
\hline RUM_1 & fixed & 100 & 3 & $0.5799 \mathrm{~ns}$ & 89 & 40 & $0.5725 \mathrm{~ns}$ & 40 & 17 & $0.0581 \mathrm{~ns}$ & 68 & 14 & $0.0363^{*}$ \\
\hline Sibiu_1 & poly & 2 & 3 & & 2 & 0 & & 147 & 31 & & 18 & 11 & \\
\hline RUM_2 & fixed & 97 & 42 & $0.5094 \mathrm{~ns}$ & 87 & 39 & $0.505 \mathrm{~ns}$ & 40 & 17 & $0.0601 \mathrm{~ns}$ & 66 & 13 & $0.0557 \mathrm{~ns}$ \\
\hline Sibiu_2 & poly & 9 & 2 & & 9 & 2 & & 149 & 32 & & 25 & 13 & \\
\hline RUM_3 & fixed & 83 & 5 & $0.133 \mathrm{~ns}$ & 96 & 45 & $0.2759 \mathrm{~ns}$ & 23 & 1 & $0.1362 \mathrm{~ns}$ & 83 & 42 & $0.8541 \mathrm{~ns}$ \\
\hline Bagau & poly & 15 & 3 & & 15 & 3 & & 152 & 32 & & 31 & 14 & \\
\hline RUM_4 & fixed & 87 & 5 & $1 \mathrm{~ns}$ & 96 & 46 & $0.1 \mathrm{~ns}$ & 24 & 1 & $0.0903 \mathrm{~ns}$ & 88 & 43 & $1 \mathrm{~ns}$ \\
\hline Cluj & poly & 7 & 0 & & 7 & 0 & & 148 & 31 & & 23 & 11 & \\
\hline RUM_5 & fixed & 105 & 42 & $0.6769 \mathrm{~ns}$ & 96 & 38 & $0.6775 \mathrm{~ns}$ & 40 & 18 & $0.0409^{*}$ & 66 & 18 & $0.1055 \mathrm{~ns}$ \\
\hline Busteni & poly & 5 & 1 & & 5 & 1 & & 148 & 32 & & 21 & 12 & \\
\hline RUM_6 & fixed & 83 & 36 & $0.1571 \mathrm{~ns}$ & 72 & 29 & $0.3155 \mathrm{~ns}$ & 17 & 13 & $0.004^{* *}$ & 36 & 5 & $0.0827 \mathrm{~ns}$ \\
\hline Sinaīa & poly & 70 & 19 & & 70 & 19 & & 191 & 44 & & 84 & 29 & \\
\hline RUS_1 & fixed & 58 & 3 & $0.1278 \mathrm{~ns}$ & 66 & 14 & $0.0000^{* * *}$ & 20 & 2 & $0.5457 \mathrm{~ns}$ & 64 & 33 & $0.0065^{* *}$ \\
\hline Altai Moūntains & poly & 114 & 16 & & 114 & 16 & & 213 & 42 & & 124 & 28 & \\
\hline RUS_2 & fixed & 94 & 14 & $0.5628 \mathrm{~ns}$ & 96 & 48 & $0.0019^{* *}$ & 53 & 7 & $0.4178 \mathrm{~ns}$ & 95 & 42 & $0.3076 \mathrm{~ns}$ \\
\hline Novosibirsk & poly & 33 & 3 & & 33 & 3 & & 160 & 32 & & 47 & 14 & \\
\hline S_2 & fixed & 54 & 6 & $0.0398 *$ & 60 & 18 & $1 \mathrm{~ns}$ & 0 & 0 & $-n s$ & 28 & 14 & $0.251 \mathrm{~ns}$ \\
\hline Stroemstad & poly & 152 & 44 & & 152 & 44 & & 223 & 51 & & 163 & 53 & \\
\hline \multicolumn{2}{|l|}{ Population } & \multicolumn{3}{|c|}{$\begin{array}{c}\text { D_2 } \\
\text { syn. nons. sign. }\end{array}$} & \multicolumn{3}{|c|}{$\begin{array}{c}\text { D_3 } \\
\text { svn. nons. sign. }\end{array}$} & \multicolumn{3}{|c|}{$\begin{array}{c}D_{-4} \\
\text { syn. nons. sign. }\end{array}$} & \multicolumn{3}{|c|}{$\begin{array}{c}\text { D_5 } \\
\text { lons. sign. }\end{array}$} \\
\hline D_3 & fixed & 68 & 12 & $0.2133 \mathrm{~ns}$ & & & & & & & & & \\
\hline Ittendorf & poly & 12 & 0 & & & & & & & & & & \\
\hline D_4 & fixed & 28 & 17 & $0.0013^{* *}$ & 29 & 14 & $0.0143^{*}$ & & & & & & \\
\hline Meckl. Seenpl. & poly & 163 & 23 & & 165 & 29 & & & & & & & \\
\hline D_5 & fixed & 20 & 7 & $1 \mathrm{~ns}$ & 27 & 7 & $0.8275 \mathrm{~ns}$ & 0 & 0 & $-n s$ & & & \\
\hline Moerfelden & poly & 151 & 48 & & 151 & 48 & & 132 & 55 & & & & \\
\hline D_ 7 & fixed & 52 & 29 & $0.0003 * * *$ & 47 & 33 & $0.0000^{* * *}$ & 12 & 1 & $0.6989 \mathrm{~ns}$ & 17 & 1 & $0.1388 \mathrm{~ns}$ \\
\hline Uelzen & poly & 117 & 19 & & 120 & 19 & & 220 & 40 & & 218 & 57 & \\
\hline D_8 & fixed & 82 & 15 & $1 \mathrm{~ns}$ & 88 & 17 & $1 \mathrm{~ns}$ & 40 & 22 & $0.0018^{* *}$ & 39 & 9 & $0.4509 \mathrm{~ns}$ \\
\hline Cuxhaven & poly & 6 & 1 & & 8 & 1 & & 161 & 30 & & 148 & 49 & \\
\hline D_9 & fixed & 0 & 0 & $-n s$ & 67 & 11 & $0.4536 \mathrm{~ns}$ & 27 & 17 & $0.0012 * *$ & 20 & 6 & $1 \mathrm{~ns}$ \\
\hline Bonn & poly & 11 & 1 & & 17 & 1 & & 166 & 30 & & 155 & 48 & \\
\hline DK_1 & fixed & 0 & 0 & $-n s$ & 41 & 4 & $0.0018^{* *}$ & 0 & 0 & $-n s$ & 5 & 1 & $0.6891 \mathrm{~ns}$ \\
\hline Copenhagen & poly & 97 & 44 & & 97 & 45 & & 187 & 50 & & 184 & 67 & \\
\hline DK_2 & fixed & 78 & 38 & $0.1794 \mathrm{~ns}$ & 77 & 40 & $0.0952 \mathrm{~ns}$ & 0 & 0 & $-n s$ & 8 & 1 & $0.4627 \mathrm{~ns}$ \\
\hline Hjorring & poly & 5 & 0 & & 9 & 0 & & 161 & 29 & & 148 & 48 & \\
\hline DK_3 & fixed & 0 & 0 & $-n s$ & 68 & 12 & $0.2055 \mathrm{~ns}$ & 27 & 17 & $0.0011^{* *}$ & 20 & 7 & $0.8134 \mathrm{~ns}$ \\
\hline Arhus Stadion & poly & 6 & 0 & & 13 & 0 & & 164 & 29 & & 152 & 48 & \\
\hline DK_4 & fixed & 0 & 0 & $-n s$ & 70 & 12 & $1 \mathrm{~ns}$ & 28 & 17 & $0.0017^{* *}$ & 20 & 7 & $1 \mathrm{~ns}$ \\
\hline Arhus Mo & poly & 5 & 1 & & 7 & 1 & & 161 & 30 & & 148 & 49 & \\
\hline E_1 & fixed & 38 & 15 & $0.2116 \mathrm{~ns}$ & 89 & 13 & $0.3561 \mathrm{~ns}$ & 47 & 14 & $0.0583 \mathrm{~ns}$ & 34 & 7 & $0.4166 \mathrm{~ns}$ \\
\hline Teno Mountains & poly & 11 & 0 & & 13 & 0 & & 164 & 29 & & 152 & 48 & \\
\hline E_3 & fixed & 66 & 14 & $1 \mathrm{~ns}$ & 61 & 10 & $1 \mathrm{~ns}$ & 35 & 17 & $0.0109^{*}$ & 30 & 8 & $0.6863 \mathrm{~ns}$ \\
\hline Anaga Mountains & poly & 19 & 4 & & 21 & 4 & & 170 & 33 & & 158 & 52 & \\
\hline F_1 & fixed & 26 & 16 & $0.0129^{*}$ & 30 & 19 & $0.0016^{* *}$ & 6 & 0 & $0.5978 \mathrm{~ns}$ & 5 & 1 & $1 \mathrm{~ns}$ \\
\hline Mont Blanc & poly & 164 & 34 & & 167 & 34 & & 241 & 42 & & 240 & 59 & \\
\hline F_2 & fixed & 0 & 0 & $-n s$ & 68 & 12 & $0.353 \mathrm{~ns}$ & 29 & 17 & $0.0016^{* *}$ & 23 & 7 & $1 \mathrm{~ns}$ \\
\hline Loire & poly & 7 & 0 & & 9 & 0 & & 162 & 29 & & 150 & 48 & \\
\hline F_3 & fixed & 64 & 12 & $1 \mathrm{~ns}$ & 66 & 13 & $0.8271 \mathrm{~ns}$ & 23 & 18 & $0.0002 * * *$ & 23 & 6 & $0.8196 \mathrm{~ns}$ \\
\hline Saint Isidore & poly & 61 & 11 & & 63 & 11 & & 200 & 39 & & 185 & 58 & \\
\hline
\end{tabular}


Table A5 continue:

\begin{tabular}{|c|c|c|c|c|c|c|c|c|c|c|c|c|c|}
\hline \multirow{2}{*}{$\begin{array}{c}\text { Population } \\
\text { F } 4\end{array}$} & \multirow[b]{2}{*}{ fixed } & \multicolumn{3}{|c|}{$\begin{array}{c}\text { D_2 } \\
\text { syn. nons. sign. }\end{array}$} & \multicolumn{3}{|c|}{$\begin{array}{c}\text { D_3 } \\
\text { syn. nons. sign. }\end{array}$} & \multicolumn{3}{|c|}{$\begin{array}{c}\text { D_4 } \\
\text { syn. nons. sign. }\end{array}$} & \multicolumn{3}{|c|}{$\begin{array}{c}\text { D_5 } \\
\text { syn. nons. sign. }\end{array}$} \\
\hline & & 74 & 39 & $0.1692 \mathrm{~ns}$ & 75 & 41 & $0.0941 \mathrm{~ns}$ & 6 & 1 & $1 \mathrm{~ns}$ & 0 & 1 & $0.2487 \mathrm{~ns}$ \\
\hline Haute Loire & poly & 5 & 0 & & 7 & 0 & & 161 & 29 & & 148 & 48 & \\
\hline FIN_1 & fixed & 87 & 37 & $0.698 \mathrm{~ns}$ & 87 & 39 & $1 \mathrm{~ns}$ & 39 & 6 & $0.6596 \mathrm{~ns}$ & 43 & 4 & $0.0108 *$ \\
\hline Lahti & poly & 5 & 3 & & 7 & 3 & & 161 & 32 & & 148 & 51 & \\
\hline GB_1 & fixed & 62 & 12 & $0.2091 \mathrm{~ns}$ & 68 & 13 & $0.1515 \mathrm{~ns}$ & 22 & 18 & $0.0000^{* * *} *$ & 24 & 6 & $1 \mathrm{~ns}$ \\
\hline Bedford & poly & 65 & 6 & & 67 & 6 & & 201 & 34 & & 188 & 53 & \\
\hline GB 2 & fixed & 87 & 16 & $1 \mathrm{~ns}$ & 89 & 18 & $1 \mathrm{~ns}$ & 43 & 23 & $0.0023^{* *}$ & 42 & 9 & $0.3541 \mathrm{~ns}$ \\
\hline Ascot & poly & 5 & 1 & & 8 & 1 & & 161 & 30 & & 148 & 49 & \\
\hline GB_3 & fixed & 87 & 15 & $0.0644 \mathrm{~ns}$ & 90 & 17 & $0.2215 \mathrm{~ns}$ & 43 & 22 & $0.0079^{* *}$ & 42 & 8 & $0.194 \mathrm{~ns}$ \\
\hline Braemar & poly & 6 & 4 & & 8 & 4 & & 162 & 33 & & 149 & 52 & \\
\hline I_1 & fixed & 0 & 0 & $-n s$ & 67 & 11 & $0.711 \mathrm{~ns}$ & 27 & 17 & $0.0017^{* *}$ & 21 & 6 & $0.8192 \mathrm{~ns}$ \\
\hline Grosseto & poly & 10 & 3 & & 14 & 3 & & 165 & 32 & & 153 & 50 & \\
\hline I_2 & fixed & 0 & 0 & $-n s$ & 66 & 11 & $0.7317 \mathrm{~ns}$ & 27 & 17 & $0.0012^{* *}$ & 20 & 6 & $1 \mathrm{~ns}$ \\
\hline Parma & poly & 12 & 2 & & 18 & 2 & & 167 & 30 & & 155 & 48 & \\
\hline IRL_1 & fixed & 86 & 15 & $0.5899 \mathrm{~ns}$ & 89 & 17 & $0.352 \mathrm{~ns}$ & 42 & 22 & $0.0018^{* *} *$ & 42 & 8 & $0.2584 \mathrm{~ns}$ \\
\hline Swords & poly & 7 & 0 & & 9 & 0 & & 163 & 29 & & 150 & 48 & \\
\hline N_1 & fixed & 53 & 24 & $0.1424 \mathrm{~ns}$ & 59 & 23 & $0.3375 \mathrm{~ns}$ & 11 & 0 & $0.3702 \mathrm{~ns}$ & 15 & 0 & $0.0467^{*}$ \\
\hline Narvik & poly & 112 & 31 & & 112 & 32 & & 225 & 37 & & 214 & 61 & \\
\hline NL_ 1 & fixed & 83 & 15 & $1 \mathrm{~ns}$ & 86 & 17 & $1 \mathrm{~ns}$ & 40 & 22 & $0.0019^{* *}$ & 39 & 8 & $0.3392 \mathrm{~ns}$ \\
\hline Wageningen & poly & 11 & 2 & & 13 & 2 & & 165 & 31 & & 153 & 49 & \\
\hline PL_1 & fixed & 39 & 21 & $0.1209 \mathrm{~ns}$ & 40 & 23 & $0.0657 \mathrm{~ns}$ & 0 & 0 & $-n s$ & 0 & 0 & $-n s$ \\
\hline Krakow & poly & 112 & 35 & & 112 & 35 & & 205 & 40 & & 195 & 61 & \\
\hline PL_2 & fixed & 54 & 20 & $0.3825 \mathrm{~ns}$ & 72 & 17 & $0.7591 \mathrm{~ns}$ & 31 & 15 & $0.0107^{*}$ & 29 & 6 & $0.5137 \mathrm{~ns}$ \\
\hline Warsaw & poly & 17 & 3 & & 19 & 3 & & 166 & 30 & & 154 & 48 & \\
\hline RUM_1 & fixed & 71 & 16 & $0.351 \mathrm{~ns}$ & 78 & 16 & $0.3507 \mathrm{~ns}$ & 37 & 13 & $0.092 \mathrm{~ns}$ & 37 & 4 & $0.0394^{*}$ \\
\hline Sibiu_1 & poly & 7 & 0 & & 8 & 0 & & 163 & 29 & & 149 & 48 & \\
\hline RUM_2 & fixed & 69 & 15 & $0.7327 \mathrm{~ns}$ & 76 & 15 & $0.7363 \mathrm{~ns}$ & 37 & 13 & $0.0943 \mathrm{~ns}$ & 36 & 4 & $0.0584 \mathrm{~ns}$ \\
\hline Sibiu_2 & poly & 14 & 2 & & 15 & 2 & & 166 & 30 & & 153 & 49 & \\
\hline RUM_3 & fixed & 88 & 42 & $0.0817 \mathrm{~ns}$ & 78 & 45 & $0.0185^{*}$ & 29 & 3 & $0.5851 \mathrm{~ns}$ & 39 & 5 & $0.0726 \mathrm{~ns}$ \\
\hline Bagau & poly & 20 & 3 & & 22 & 3 & & 168 & 29 & & 157 & 49 & \\
\hline RUM_4 & fixed & 93 & 43 & $0.0189^{*}$ & 80 & 45 & $0.0049^{* *}$ & 28 & 3 & $0.5846 \mathrm{~ns}$ & 42 & 5 & $0.0486^{*}$ \\
\hline Cluj & poly & 12 & 0 & & 14 & 0 & & 166 & 29 & & 151 & 48 & \\
\hline RUM_5 & fixed & 67 & 20 & $0.454 \mathrm{~ns}$ & 74 & 18 & $0.4546 \mathrm{~ns}$ & 34 & 15 & $0.0227^{*}$ & 31 & 5 & $0.1984 \mathrm{~ns}$ \\
\hline Busteni & poly & 9 & 1 & & 12 & 1 & & 163 & 30 & & 149 & 49 & \\
\hline RUM_6 & fixed & 38 & 6 & $0.3566 \mathrm{~ns}$ & 44 & 7 & $0.3713 \mathrm{~ns}$ & 18 & 12 & $0.0057^{* *}$ & 11 & 2 & $0.534 \mathrm{~ns}$ \\
\hline Sinaìa & poly & 73 & 19 & & 74 & 19 & & 197 & 40 & & 185 & 60 & \\
\hline RUS_1 & fixed & 66 & 34 & $0.0001^{* * * *}$ & 51 & 36 & $0.0000^{*} * *$ & 16 & 1 & $0.4824 \mathrm{~ns}$ & 22 & 1 & $0.0572 \mathrm{~ns}$ \\
\hline Altai Moūntains & poly & 117 & 16 & & 120 & 16 & & 228 & 39 & & 222 & 60 & \\
\hline RUS_2 & fixed & 95 & 42 & $0.0019^{* *}$ & 91 & 46 & $0.0003^{* * * *}$ & 35 & 7 & $0.6351 \mathrm{~ns}$ & 47 & 6 & $0.0864 \mathrm{~ns}$ \\
\hline Novosibirsk & poly & 38 & 3 & & 40 & 3 & & 184 & 30 & & 170 & 49 & \\
\hline S_2 & fixed & 30 & 15 & $0.1236 \mathrm{~ns}$ & 29 & 13 & $0.2309 \mathrm{~ns}$ & 0 & 0 & $-n s$ & 2 & 0 & $1 \mathrm{~ns}$ \\
\hline Stroemstad & poly & 156 & 44 & & 157 & 44 & & 238 & 49 & & 219 & 64 & \\
\hline \multicolumn{2}{|l|}{ Population } & \multicolumn{3}{|c|}{$\begin{array}{c}D_{-}^{7} \\
\text { syn. nons. sign. }\end{array}$} & \multicolumn{3}{|c|}{ D_8 } & \multicolumn{3}{|c|}{$\begin{array}{c}\text { D_9 } \\
\text { syn. nons. sign. }\end{array}$} & \multicolumn{3}{|c|}{$\begin{array}{c}\text { DK_1 } \\
\text { ons.sign. }\end{array}$} \\
\hline D_8 & fixed & 53 & 32 & $0.0002 * * *$ & & & & & & & & & \\
\hline Cuxhaven & poly & 115 & 20 & & & & & & & & & & \\
\hline D_9 & fixed & 52 & 29 & $0.0004^{* * * *}$ & 82 & 14 & $1 \mathrm{~ns}$ & & & & & & \\
\hline Bonn & poly & 119 & 20 & & 11 & 2 & & & & & & & \\
\hline DK_1 & fixed & 31 & 12 & $0.7017 \mathrm{~ns}$ & 50 & 9 & $0.0098^{*} *$ & 0 & 0 & $-n s$ & & & \\
\hline Copenhagen & poly & 176 & 57 & & 93 & 46 & & 101 & 45 & & & & \\
\hline DK_2 & fixed & 50 & 4 & $0.2284 \mathrm{~ns}$ & 97 & 44 & $1 \mathrm{~ns}$ & 76 & 38 & $0.1704 \mathrm{~ns}$ & 44 & 17 & $0.5119 \mathrm{~ns}$ \\
\hline Hjorring & poly & 114 & 19 & & 1 & 1 & & 10 & 1 & & 92 & 45 & \\
\hline DK_3 & fixed & 52 & 29 & $0.0003^{* * *} *$ & 82 & 15 & $1 \mathrm{~ns}$ & 0 & 0 & $-n s$ & 0 & 0 & $-\mathrm{ns}$ \\
\hline Arhus Stadion & poly & 118 & 19 & & 7 & 1 & & 12 & 1 & & 97 & 44 & \\
\hline DK_4 & fixed & 54 & 29 & $0.0008 * * *$ & 85 & 15 & $0.07 \mathrm{~ns}$ & 0 & 0 & $-n s$ & 0 & 0 & $-n s$ \\
\hline Arhus Mo & poly & 114 & 20 & & 1 & 2 & & 10 & 2 & & 93 & 45 & \\
\hline
\end{tabular}


Table A5 continue:

\begin{tabular}{|c|c|c|c|c|c|c|c|c|c|c|c|c|c|}
\hline \multirow{2}{*}{$\begin{array}{c}\text { Population } \\
\text { E_1 }\end{array}$} & \multirow{3}{*}{$\begin{array}{l}\text { fixed } \\
\text { poly }\end{array}$} & \multicolumn{3}{|c|}{$\begin{array}{c}D_{-7}^{7} \\
\text { syn. nons. sign. }\end{array}$} & \multicolumn{3}{|c|}{$\begin{array}{c}\text { D_8 } \\
\text { syn. nons. sign. }\end{array}$} & \multicolumn{3}{|c|}{$\begin{array}{c}\text { D_9 } \\
\text { syn. nons. sign. }\end{array}$} & \multicolumn{3}{|c|}{$\begin{array}{c}\text { DK_1 } \\
\text { syn. nons. sign. }\end{array}$} \\
\hline & & 63 & 34 & $0.0002^{* * *} *$ & 97 & 17 & $1 \mathrm{~ns}$ & 76 & 14 & $0.4566 \mathrm{~ns}$ & 53 & 5 & $0.0005 * * *$ \\
\hline Teno Mountains & & 120 & 19 & & 7 & 1 & & 16 & 1 & & 97 & 45 & \\
\hline E_3 & fixed & 52 & 34 & $0.0007^{* * * *}$ & 82 & 14 & $0.3169 \mathrm{~ns}$ & 64 & 13 & $1 \mathrm{~ns}$ & 42 & 4 & $0.0011^{* *}$ \\
\hline Anaga Mountains & poly & 123 & 23 & & 15 & 5 & & 24 & 5 & & 103 & 49 & \\
\hline F_1 & fixed & 15 & 0 & $0.1382 \mathrm{~ns}$ & 44 & 24 & $0.004^{* *}$ & 27 & 16 & $0.0066^{* *}$ & 11 & 2 & $0.739 \mathrm{~ns}$ \\
\hline Mont Blanc & poly & 222 & 41 & & 162 & 35 & & 166 & 35 & & 207 & 62 & \\
\hline F_2 & fixed & 53 & 29 & $0.0004^{* * * *}$ & 83 & 15 & $1 \mathrm{~ns}$ & 0 & 0 & $-n s$ & 4 & 0 & $0.3108 \mathrm{~ns}$ \\
\hline Loire & poly & 116 & 19 & & 3 & 1 & & 12 & 1 & & 94 & 44 & \\
\hline F_3 & fixed & 43 & 30 & $0.0001 * * *$ & 2 & 0 & $1 \mathrm{~ns}$ & 65 & 11 & $1 \mathrm{~ns}$ & 38 & 6 & $0.0371^{*}$ \\
\hline Saint Isidore & poly & 154 & 30 & & 57 & 12 & & 64 & 12 & & 134 & 55 & \\
\hline F_4 & fixed & 52 & 3 & $0.0903 \mathrm{~ns}$ & 95 & 45 & $1 \mathrm{~ns}$ & 73 & 39 & $0.1011 \mathrm{~ns}$ & 42 & 17 & $0.6189 \mathrm{~ns}$ \\
\hline Haute Loire & poly & 114 & 19 & & 1 & 1 & & 10 & 1 & & 92 & 45 & \\
\hline FIN_1 & fixed & 54 & 5 & $0.1808 \mathrm{~ns}$ & 102 & 39 & $0.0265^{*}$ & 84 & 37 & $1 \mathrm{~ns}$ & 61 & 20 & $0.1756 \mathrm{~ns}$ \\
\hline Lahti & poly & 114 & 22 & & 1 & 4 & & 10 & 4 & & 93 & 47 & \\
\hline GB_1 & fixed & 43 & 30 & $0.0000^{* * *}$ & 0 & 0 & $-n s$ & 63 & 11 & $0.3263 \mathrm{~ns}$ & 36 & 6 & $0.1122 \mathrm{~ns}$ \\
\hline Bedford & poly & 160 & 25 & & 61 & 7 & & 68 & 7 & & 137 & 50 & \\
\hline GB_2 & fixed & 55 & 33 & $0.0002^{* * *}$ & 5 & 1 & $0.5 \mathrm{~ns}$ & 86 & 15 & $1 \mathrm{~ns}$ & 55 & 10 & $0.0073^{*} *$ \\
\hline Ascot & poly & 115 & 20 & & 2 & 2 & & 11 & 2 & & 92 & 46 & \\
\hline GB_3 & fixed & 55 & 32 & $0.0007^{* * *}$ & 5 & 0 & $0.0278^{*}$ & 87 & 14 & $0.1353 \mathrm{~ns}$ & 55 & 9 & $0.0025^{*} *$ \\
\hline Braemar & poly & 115 & 22 & & 2 & 5 & & 11 & 5 & & 93 & 49 & \\
\hline I_1 & fixed & 52 & 29 & $0.0009^{* * *}$ & 84 & 14 & $0.2092 \mathrm{~ns}$ & 0 & 0 & $-n s$ & 2 & 0 & $0.562 \mathrm{~ns}$ \\
\hline Grosseto & poly & 118 & 22 & & 8 & 4 & & 10 & 3 & & 98 & 47 & \\
\hline I_2 & fixed & 52 & 29 & $0.0004^{* * *}$ & 81 & 14 & $0.6998 \mathrm{~ns}$ & 0 & 0 & $-n s$ & 0 & 0 & $-\mathrm{ns}$ \\
\hline Parma & poly & 120 & 20 & & 12 & 3 & & 12 & 2 & & 101 & 45 & \\
\hline IRL_1 & fixed & 55 & 32 & $0.0001^{* * *}$ & 5 & 0 & $0.4444 \mathrm{~ns}$ & 86 & 14 & $0.6936 \mathrm{~ns}$ & 54 & 9 & $0.0064^{* *}$ \\
\hline Swords & poly & 116 & 19 & & 3 & 1 & & 12 & 1 & & 94 & 45 & \\
\hline N_1 & fixed & 32 & 5 & $0.6438 \mathrm{~ns}$ & 56 & 30 & $0.0448^{*}$ & 53 & 23 & $0.1952 \mathrm{~ns}$ & 25 & 3 & $0.0669 \mathrm{~ns}$ \\
\hline Narvik & poly & 198 & 43 & & 109 & 31 & & 116 & 33 & & 172 & 67 & \\
\hline NL_1 & fixed & 53 & 32 & $0.0002 * * *$ & 0 & 0 & $-n s$ & 83 & 14 & $1 \mathrm{~ns}$ & 51 & 9 & $0.01^{*}$ \\
\hline Wageningen & poly & 117 & 21 & & 7 & 3 & & 16 & 3 & & 97 & 47 & \\
\hline$P_{-} 1$ & fixed & 28 & 1 & $0.0239^{*}$ & 57 & 29 & $0.1759 \mathrm{~ns}$ & 39 & 21 & $0.1242 \mathrm{~ns}$ & 13 & 3 & $0.5674 \mathrm{~ns}$ \\
\hline Krakow & poly & 190 & 47 & & 107 & 36 & & 113 & 36 & & 168 & 67 & \\
\hline PL_2 & fixed & 43 & 29 & $0.0001^{* * *}$ & 81 & 20 & $0.7475 \mathrm{~ns}$ & 56 & 19 & $0.4191 \mathrm{~ns}$ & 32 & 8 & $0.1733 \mathrm{~ns}$ \\
\hline Warsaw & poly & 121 & 21 & & 13 & 4 & & 21 & 4 & & 101 & 47 & \\
\hline RUM_1 & fixed & 58 & 28 & $0.0014^{* *}$ & 81 & 15 & $1 \mathrm{~ns}$ & 68 & 15 & $0.4601 \mathrm{~ns}$ & 43 & 6 & $0.0054 * *$ \\
\hline Sibiu_1 & poly & 116 & 19 & & 3 & 1 & & 12 & 1 & & 94 & 45 & \\
\hline RUM_2 & fixed & 56 & 28 & $0.0012^{*} *$ & 79 & 15 & $0.6915 \mathrm{~ns}$ & 66 & 14 & $0.7598 \mathrm{~ns}$ & 42 & 6 & $0.0087^{* *}$ \\
\hline Sibiu_2 & poly & 122 & 20 & & 10 & 3 & & 19 & 3 & & 99 & 46 & \\
\hline RUM_3 & fixed & 50 & 8 & $1 \mathrm{~ns}$ & 85 & 46 & $0.2119 \mathrm{~ns}$ & 84 & 42 & $0.0662 \mathrm{~ns}$ & 54 & 23 & $0.8797 \mathrm{~ns}$ \\
\hline Bagau & poly & 125 & 22 & & 16 & 4 & & 24 & 4 & & 102 & 47 & \\
\hline RUM_4 & fixed & 53 & 10 & $0.829 \mathrm{~ns}$ & 91 & 47 & $0.272 \mathrm{~ns}$ & 89 & 43 & $0.0148^{*}$ & 56 & 23 & $0.7618 \mathrm{~ns}$ \\
\hline Cluj & poly & 119 & 19 & & 8 & 1 & & 17 & 1 & & 97 & 45 & \\
\hline RUM_5 & fixed & 53 & 29 & $0.0007^{* * *}$ & 92 & 17 & $0.6145 \mathrm{~ns}$ & 66 & 19 & $0.5131 \mathrm{~ns}$ & 41 & 9 & $0.0689 \mathrm{~ns}$ \\
\hline Busteni & poly & 118 & 20 & & 6 & 2 & & 15 & 2 & & 96 & 45 & \\
\hline RUM_6 & fixed & 36 & 25 & $0.0004^{* * *} *$ & 57 & 7 & $0.0876 \mathrm{~ns}$ & 37 & 5 & $0.2423 \mathrm{~ns}$ & 17 & 3 & $0.2922 \mathrm{~ns}$ \\
\hline Sinaia & poly & 160 & 35 & & 71 & 20 & & 77 & 20 & & 142 & 55 & \\
\hline RUS_1 & fixed & 31 & 4 & $0.6243 \mathrm{~ns}$ & 61 & 42 & $0.0000^{* * * *}$ & 64 & 34 & $0.0001^{* * *}$ & 43 & 14 & $1 \mathrm{~ns}$ \\
\hline Altai Mountains & poly & 192 & 34 & & 115 & 17 & & 118 & 17 & & 179 & 59 & \\
\hline RUS_2 & fixed & 50 & 8 & $1 \mathrm{~ns}$ & 95 & 43 & $0.0122 *$ & 94 & 42 & $0.0028 * *$ & 61 & 22 & $0.7668 \mathrm{~ns}$ \\
\hline Novosibirsk & poly & 136 & 22 & & 34 & 4 & & 42 & 4 & & 120 & 48 & \\
\hline S_2 & fixed & 20 & 1 & $0.141 \mathrm{~ns}$ & 46 & 17 & $0.501 \mathrm{~ns}$ & 29 & 14 & $0.1649 \mathrm{~ns}$ & 9 & 2 & $0.7362 \mathrm{~ns}$ \\
\hline Stroemstad & poly & 227 & 52 & & 152 & 45 & & 159 & 44 & & 200 & 68 & \\
\hline
\end{tabular}


Table A5 continue:

\begin{tabular}{|c|c|c|c|c|c|c|c|c|c|c|c|c|c|}
\hline \multirow{2}{*}{$\begin{array}{c}\text { Population } \\
\text { DK 3 }\end{array}$} & \multirow{2}{*}{ fixed } & \multicolumn{3}{|c|}{$\begin{array}{c}\text { DK_2 } \\
\text { syn. nons. sign. }\end{array}$} & \multicolumn{3}{|c|}{$\begin{array}{c}\text { DK_3 } \\
\text { syn. nons. sign. }\end{array}$} & \multicolumn{3}{|c|}{$\begin{array}{c}\mathrm{DK}_{-} 4 \\
\text { syn. nons. sign. }\end{array}$} & \multicolumn{3}{|c|}{$\begin{array}{c}\text { E_1 } \\
\text { syn. nons. sign. }\end{array}$} \\
\hline & & 78 & 38 & $0.1 / 53 \mathrm{~ns}$ & & & & & & & & & \\
\hline Arhus Stadion & poly & 6 & 0 & & & & & & & & & & \\
\hline DK_4 & fixed & 79 & 38 & $0.3305 \mathrm{~ns}$ & 0 & 0 & $-n s$ & & & & & & \\
\hline Arhus Mo & poly & 0 & 1 & & 6 & 1 & & & & & & & \\
\hline E_1 & fixed & 92 & 43 & $0.1775 \mathrm{~ns}$ & 76 & 15 & $0.2061 \mathrm{~ns}$ & 78 & 15 & $1 \mathrm{~ns}$ & & & \\
\hline Teno Mountains & poly & 6 & 0 & & 12 & 0 & & 6 & 1 & & & & \\
\hline E_3 & fixed & 81 & 45 & $0.3005 \mathrm{~ns}$ & 66 & 14 & $1 \mathrm{~ns}$ & 67 & 14 & $0.5155 \mathrm{~ns}$ & 61 & 5 & $0.2191 \mathrm{~ns}$ \\
\hline Anaga Mountains & poly & 14 & 4 & & 20 & 4 & & 14 & 5 & & 18 & 4 & \\
\hline F_1 & fixed & 33 & 1 & $0.0357^{*}$ & 29 & 16 & $0.0128 *$ & 30 & 16 & $0.0141^{*}$ & 41 & 22 & $0.0043^{* *}$ \\
\hline Mont Blanc & poly & 162 & 34 & & 165 & 34 & & 162 & 34 & & 166 & 34 & \\
\hline F_2 & fixed & 80 & 38 & $0.5636 \mathrm{~ns}$ & 0 & 0 & $-n s$ & 4 & 0 & $0.4286 \mathrm{~ns}$ & 78 & 15 & $0.3569 \mathrm{~ns}$ \\
\hline Loire & poly & 2 & 0 & & 8 & 0 & & 2 & 1 & & 8 & 0 & \\
\hline F_3 & fixed & 80 & 41 & $0.0106^{*}$ & 64 & 12 & $1 \mathrm{~ns}$ & 67 & 12 & $0.824 \mathrm{~ns}$ & 72 & 15 & $0.8305 \mathrm{~ns}$ \\
\hline Saint Isidore & poly & 57 & 11 & & 62 & 11 & & 57 & 12 & & 62 & 11 & \\
\hline$F_{-} 4$ & fixed & 17 & 3 & $-\mathrm{ns}$ & 74 & 39 & $0.1008 \mathrm{~ns}$ & 75 & 39 & $0.3478 \mathrm{~ns}$ & 95 & 42 & $0.1801 \mathrm{~ns}$ \\
\hline Haute Loire & poly & 0 & 0 & & 6 & 0 & & 0 & 1 & & 6 & 0 & \\
\hline FIN_1 & fixed & 91 & 15 & $0.0039 * *$ & 87 & 37 & $1 \mathrm{~ns}$ & 89 & 37 & $0.0089^{* *}$ & 108 & 42 & $0.7134 \mathrm{~ns}$ \\
\hline Lahti & poly & 0 & 3 & & 6 & 3 & & 0 & 4 & & 6 & 3 & \\
\hline GB_1 & fixed & 80 & 41 & $0.0001^{* * * *}$ & 62 & 12 & $0.2082 \mathrm{~ns}$ & 65 & 12 & $0.4609 \mathrm{~ns}$ & 70 & 15 & $0.105 \mathrm{~ns}$ \\
\hline Bedford & poly & 61 & 6 & & 66 & 6 & & 61 & 7 & & 65 & 6 & \\
\hline GB_2 & fixed & 100 & 45 & $1 \mathrm{~ns}$ & 87 & 16 & $1 \mathrm{~ns}$ & 89 & 16 & $0.0714 \mathrm{~ns}$ & 95 & 18 & $1 \mathrm{~ns}$ \\
\hline Ascot & poly & 1 & 1 & & 6 & 1 & & 1 & 2 & & 7 & 1 & \\
\hline GB_3 & fixed & 101 & 44 & $0.0364^{*}$ & 87 & 15 & $0.0874 \mathrm{~ns}$ & 90 & 15 & $0.0006^{*} * *$ & 93 & 18 & $0.2113 \mathrm{~ns}$ \\
\hline Braemar & poly & 1 & 4 & & 7 & 4 & & 1 & 5 & & 7 & 4 & \\
\hline I_1 & fixed & 76 & 38 & $1 \mathrm{~ns}$ & 0 & 0 & $-\mathrm{ns}$ & 2 & 0 & $0.5385 \mathrm{~ns}$ & 76 & 14 & $0.7185 \mathrm{~ns}$ \\
\hline Grosseto & poly & 7 & 3 & & 11 & 3 & & 7 & 4 & & 13 & 3 & \\
\hline I_2 & fixed & 76 & 37 & $0.2301 \mathrm{~ns}$ & 0 & 0 & $-\mathrm{ns}$ & 0 & 0 & $-\mathrm{ns}$ & 76 & 14 & $0.7331 \mathrm{~ns}$ \\
\hline Parma & poly & 11 & 2 & & 13 & 2 & & 11 & 3 & & 17 & 2 & \\
\hline IRL_1 & fixed & 101 & 44 & $0.5777 \mathrm{~ns}$ & 86 & 15 & $0.3708 \mathrm{~ns}$ & 89 & 15 & $0.3879 \mathrm{~ns}$ & 95 & 17 & $0.3677 \mathrm{~ns}$ \\
\hline Swords & poly & 2 & 0 & & 8 & 0 & & 2 & 1 & & 8 & 0 & \\
\hline N_1 & fixed & 73 & 5 & $0.0022 * *$ & 52 & 24 & $0.14 \mathrm{~ns}$ & 55 & 24 & $0.2594 \mathrm{~ns}$ & 68 & 25 & $0.2748 \mathrm{~ns}$ \\
\hline Narvik & poly & 108 & 31 & & 113 & 31 & & 108 & 32 & & 115 & 30 & \\
\hline NL_1 & fixed & 98 & 44 & $1 \mathrm{~ns}$ & 83 & 15 & $1 \mathrm{~ns}$ & 86 & 15 & $0.1634 \mathrm{~ns}$ & 94 & 17 & $1 \mathrm{~ns}$ \\
\hline Wageningen & poly & 6 & 2 & & 12 & 2 & & 6 & 3 & & 12 & 2 & \\
\hline PL_1 & fixed & 0 & 0 & $-\mathrm{ns}$ & 39 & 21 & $0.1201 \mathrm{~ns}$ & 40 & 21 & $0.2326 \mathrm{~ns}$ & 50 & 23 & $0.2553 \mathrm{~ns}$ \\
\hline Krakow & poly & 107 & 35 & & 113 & 35 & & 107 & 36 & & 113 & 35 & \\
\hline PL_2 & fixed & 84 & 34 & $0.5576 \mathrm{~ns}$ & 54 & 20 & $0.2663 \mathrm{~ns}$ & 56 & 2 & $1 \mathrm{~ns}$ & 80 & 17 & $1 \mathrm{~ns}$ \\
\hline Warsaw & poly & 12 & 3 & & 18 & 3 & & 12 & 4 & & 18 & 3 & \\
\hline RUM_1 & fixed & 88 & 36 & $1 \mathrm{~ns}$ & 71 & 16 & $0.344 \mathrm{~ns}$ & 72 & 16 & $0.4664 \mathrm{~ns}$ & 86 & 20 & $0.3469 \mathrm{~ns}$ \\
\hline Sibiu_1 & poly & 2 & 0 & & 8 & 0 & & 2 & 1 & & 8 & 0 & \\
\hline RUM_2 & fixed & 86 & 35 & $0.5163 \mathrm{~ns}$ & 69 & 15 & $0.7301 \mathrm{~ns}$ & 70 & 15 & $0.6908 \mathrm{~ns}$ & 84 & 15 & $0.7339 \mathrm{~ns}$ \\
\hline Sibiu_2 & poly & 15 & 3 & & 15 & 2 & & 9 & 3 & & 15 & 2 & \\
\hline RUM_3 & fixed & 85 & 11 & $0.6951 \mathrm{~ns}$ & 88 & 42 & $0.0539 \mathrm{~ns}$ & 88 & 42 & $0.4291 \mathrm{~ns}$ & 94 & 43 & $0.0844 \mathrm{~ns}$ \\
\hline Bagau & poly & 5 & 1 & & 21 & 3 & & 15 & 4 & & 20 & 3 & \\
\hline RUM_4 & fixed & 86 & 13 & $0.5934 \mathrm{~ns}$ & 93 & 43 & $0.0114^{*}$ & 93 & 43 & $0.4352 \mathrm{~ns}$ & 99 & 43 & $0.0201^{*}$ \\
\hline Cluj & poly & 7 & 0 & & 13 & 0 & & 7 & 1 & & 13 & 0 & \\
\hline RUM_5 & fixed & 92 & 35 & $0.6816 \mathrm{~ns}$ & 66 & 20 & $0.4471 \mathrm{~ns}$ & 68 & 20 & $1 \mathrm{~ns}$ & 89 & 14 & $1 \mathrm{~ns}$ \\
\hline Busteni & poly & 5 & 1 & & 10 & 1 & & 5 & 2 & & 11 & 1 & \\
\hline RUM_6 & fixed & 63 & 29 & $0.1845 \mathrm{~ns}$ & 38 & 6 & $0.3566 \mathrm{~ns}$ & 38 & 6 & $0.258 \mathrm{~ns}$ & 58 & 5 & $0.0425^{*}$ \\
\hline Sinaia & poly & 69 & 20 & & 73 & 19 & & 70 & 20 & & 75 & 19 & \\
\hline RUS_1 & fixed & 62 & 9 & $1 \mathrm{~ns}$ & 66 & 34 & $0.0001 * * *$ & 67 & 34 & $0.0002^{* * *} *$ & 76 & 36 & $0.0001^{* * *}$ \\
\hline Altai Mountains & poly & 114 & 16 & & 118 & 16 & & 114 & 17 & & 117 & 16 & \\
\hline RUS_2 & fixed & 96 & 21 & $0.1991 \mathrm{~ns}$ & 95 & 42 & $0.0019 * *$ & 97 & 43 & $0.013^{*}$ & 99 & 41 & $0.0021^{* *}$ \\
\hline Novosibirsk & poly & 33 & 3 & & 39 & 3 & & 33 & 4 & & 39 & 3 & \\
\hline S_2 & fixed & 0 & 0 & $-n s$ & 30 & 15 & $0.1226 \mathrm{~ns}$ & 31 & 15 & $0.1853 \mathrm{~ns}$ & 35 & 14 & $0.347 \mathrm{~ns}$ \\
\hline Stroemstad & poly & 152 & 44 & & 157 & 44 & & 152 & 45 & & 157 & 44 & \\
\hline
\end{tabular}


Table A5 continue:

\begin{tabular}{|c|c|c|c|c|c|c|c|c|c|c|c|c|c|}
\hline \multirow{2}{*}{$\begin{array}{c}\text { Population } \\
\text { F_1 }\end{array}$} & \multirow[b]{2}{*}{ fixed } & \multicolumn{3}{|c|}{$\begin{array}{c}\text { E_3 } \\
\text { syn. nons. sign. }\end{array}$} & \multicolumn{3}{|c|}{$\begin{array}{c}\text { F_t }_{-} \\
\text {syn. nons. sign. }\end{array}$} & \multicolumn{3}{|c|}{$\begin{array}{c}\text { F_2 } \\
\text { syn. nons. sign. }\end{array}$} & \multicolumn{3}{|c|}{$\begin{array}{c}\text { F_3 } \\
\text { syn. nons. sign. }\end{array}$} \\
\hline & & 32 & 21 & $0.0017^{* *}$ & & & & & & & & & \\
\hline Mont Blanc & poly & 169 & 38 & & & & & & & & & & \\
\hline F_2 & fixed & 68 & 14 & $0.7492 \mathrm{~ns}$ & 31 & 16 & $0.0153^{*}$ & & & & & & \\
\hline Loire & poly & 16 & 4 & & 163 & 34 & & & & & & & \\
\hline F 3 & fixed & 59 & 12 & $1 \mathrm{~ns}$ & 30 & 19 & $0.0038 * *$ & 65 & 12 & $1 \mathrm{~ns}$ & & & \\
\hline Saint Isidore & poly & 70 & 15 & & 194 & 44 & & 59 & 11 & & & & \\
\hline F_4 & fixed & 81 & 44 & $0.3043 \mathrm{~ns}$ & 33 & 1 & $0.0357^{*}$ & 76 & 39 & $0.5517 \mathrm{~ns}$ & 77 & 43 & $0.0044^{* *}$ \\
\hline Haute Loire & poly & 14 & 4 & & 162 & 34 & & 2 & 0 & & 57 & 11 & \\
\hline FIN_1 & fixed & 96 & 42 & $0.8028 \mathrm{~ns}$ & 35 & 1 & $0.0136^{*}$ & 88 & 37 & $0.3228 \mathrm{~ns}$ & 80 & 37 & $0.0911 \mathrm{~ns}$ \\
\hline Lahti & poly & 14 & 7 & & 162 & 37 & & 2 & 3 & & 57 & 14 & \\
\hline GB 1 & fixed & 60 & 12 & $0.4927 \mathrm{~ns}$ & 29 & 19 & $0.0006^{* * *} *$ & 63 & 12 & $0.2149 \mathrm{~ns}$ & 0 & 0 & $-n s$ \\
\hline Bedford & poly & 72 & 10 & & 200 & 39 & & 63 & 6 & & 67 & 11 & \\
\hline GB_2 & fixed & 85 & 15 & $0.3236 \mathrm{~ns}$ & 45 & 25 & $0.0042 * *$ & 87 & 16 & $1 \mathrm{~ns}$ & 0 & 0 & $-n s$ \\
\hline Ascot & poly & 15 & 5 & & 163 & 35 & & 3 & 1 & & 58 & 12 & \\
\hline GB_3 & fixed & 84 & 15 & $0.0401^{*}$ & 45 & 24 & $0.0122 *$ & 88 & 15 & $0.0082 * *$ & 0 & 0 & $-n s$ \\
\hline Braēmar & poly & 15 & 8 & & 162 & 38 & & 2 & 4 & & 58 & 15 & \\
\hline I 1 & fixed & 64 & 13 & $0.4023 \mathrm{~ns}$ & 27 & 16 & $0.0129^{*}$ & 0 & 0 & $-n s$ & 67 & 11 & $0.5171 \mathrm{~ns}$ \\
\hline Grosseto & poly & 21 & 7 & & 166 & 37 & & 9 & 3 & & 62 & 14 & \\
\hline I_2 & fixed & 64 & 13 & $0.7836 \mathrm{~ns}$ & 27 & 15 & $0.02 *$ & 0 & 0 & $-n s$ & 65 & 11 & $0.8246 \mathrm{~ns}$ \\
\hline Parma & poly & 25 & 6 & & 167 & 36 & & 13 & 2 & & 64 & 13 & \\
\hline IRL 1 & fixed & 85 & 14 & $0.5017 \mathrm{~ns}$ & 44 & 24 & $0.0034^{* *} *$ & 87 & 15 & $1 \mathrm{~ns}$ & 0 & 0 & $-n s$ \\
\hline Swords & poly & 16 & 4 & & 164 & 34 & & 4 & 0 & & 59 & 11 & \\
\hline N_1 & fixed & 50 & 28 & $0.0417^{*}$ & 9 & 1 & $0.704 \mathrm{~ns}$ & 55 & 24 & $0.1949 \mathrm{~ns}$ & 41 & 27 & $0.0033^{* *}$ \\
\hline Narvik & poly & 120 & 34 & & 227 & 46 & & 110 & 31 & & 151 & 39 & \\
\hline NL_1 & fixed & 83 & 14 & $0.3618 \mathrm{~ns}$ & 43 & 24 & $0.0034^{* *} *$ & 84 & 15 & $0.6536 \mathrm{~ns}$ & 0 & 0 & $-n s$ \\
\hline Wageningen & poly & 19 & 6 & & 166 & 35 & & 8 & 2 & & 60 & 12 & \\
\hline PL_1 & fixed & 38 & 26 & $0.0337^{*}$ & 15 & 1 & $0.3239 \mathrm{~ns}$ & 41 & 21 & $0.174 \mathrm{~ns}$ & 39 & 24 & $0.0213^{*}$ \\
\hline Krakow & poly & 117 & 39 & & 212 & 45 & & 109 & 35 & & 153 & 45 & \\
\hline PL_2 & fixed & 71 & 16 & $0.7963 \mathrm{~ns}$ & 30 & 18 & $0.0051^{* *}$ & 55 & 20 & $0.5468 \mathrm{~ns}$ & 59 & 17 & $0.5518 \mathrm{~ns}$ \\
\hline Warsaw & poly & 26 & 7 & & 167 & 35 & & 14 & 3 & & 63 & 14 & \\
\hline RUM 1 & fixed & 71 & 17 & $1 \mathrm{~ns}$ & 33 & 17 & $0.0174^{*}$ & 73 & 16 & $0.5991 \mathrm{~ns}$ & 59 & 12 & $1 \mathrm{~ns}$ \\
\hline Sibiu- 1 & poly & 16 & 4 & & 164 & 34 & & 4 & 0 & & 59 & 11 & \\
\hline RUM_2 & fixed & 69 & 17 & $1 \mathrm{~ns}$ & 32 & 17 & $0.0102 *$ & 71 & 15 & $1 \mathrm{~ns}$ & 57 & 12 & $1 \mathrm{~ns}$ \\
\hline Sibiu_2 & poly & 23 & 6 & & 168 & 35 & & 11 & 2 & & 71 & 14 & \\
\hline RUM_3 & fixed & 82 & 46 & $0.0711 \mathrm{~ns}$ & 38 & 2 & $0.0558 \mathrm{~ns}$ & 85 & 42 & $0.266 \mathrm{~ns}$ & 66 & 44 & $0.0005^{* * *}$ \\
\hline Bagaù & poly & 29 & 7 & & 170 & 34 & & 22 & 6 & & 62 & 11 & \\
\hline RUM 4 & fixed & 88 & 46 & $0.0993 \mathrm{~ns}$ & 41 & 2 & $0.0354^{*}$ & 93 & 43 & $0.0581 \mathrm{~ns}$ & 71 & 45 & $0.0006^{* * *}$ \\
\hline Cluj & poly & 21 & 4 & & 165 & 34 & & 9 & 0 & & 61 & 11 & \\
\hline RUM_5 & fixed & 70 & 15 & $0.7674 \mathrm{~ns}$ & 27 & 18 & $0.0022 * *$ & 69 & 20 & $0.6823 \mathrm{~ns}$ & 66 & 14 & $0.8273 \mathrm{~ns}$ \\
\hline Busteni & poly & 19 & 5 & & 165 & 35 & & 7 & 1 & & 62 & 11 & \\
\hline RUM_6 & fixed & 41 & 5 & $0.1145 \mathrm{~ns}$ & 17 & 13 & $0.0044 * *$ & 41 & 6 & $0.2551 \mathrm{~ns}$ & 37 & 6 & $0.3878 \mathrm{~ns}$ \\
\hline Sinaia & poly & 79 & 23 & & 200 & 47 & & 71 & 19 & & 115 & 29 & \\
\hline RUS_1 & fixed & 61 & 40 & $0.0000^{* * *}$ & 23 & 0 & $0.0317^{*}$ & 65 & 35 & $0.0000^{* * *}$ & 46 & 39 & $0.0000^{* * *}$ \\
\hline Altai Mountains & poly & 122 & 20 & & 224 & 44 & & 116 & 16 & & 159 & 27 & \\
\hline RUS_2 & fixed & 100 & 43 & $0.025^{*}$ & 38 & 7 & $1 \mathrm{~ns}$ & 96 & 42 & $0.0034 * *$ & 85 & 41 & $0.0078^{*}$ \\
\hline Novosibirsk & poly & 44 & 7 & & 179 & 35 & & 35 & 3 & & 67 & 15 & \\
\hline S_2 & fixed & 27 & 14 & $0.1651 \mathrm{~ns}$ & 6 & 0 & $0.5957 \mathrm{~ns}$ & 32 & 15 & $0.1849 \mathrm{~ns}$ & 31 & 14 & $0.2532 \mathrm{~ns}$ \\
\hline Stroemstad & poly & 161 & 48 & & 241 & 54 & & 154 & 44 & & 185 & 54 & \\
\hline Population & & \multicolumn{3}{|c|}{$F_{-} 4$} & \multicolumn{3}{|c|}{ FIN_1 } & \multicolumn{3}{|c|}{$\begin{array}{c}\text { GB_1 } \\
\text { syn. nons. sign. }\end{array}$} & & & \\
\hline FIN_1 & fixed & 91 & 14 & $0.0033^{* *} *$ & & & & & & & & & \\
\hline Lahti & poly & 0 & 3 & & & & & & & & & & \\
\hline GB_1 & fixed & 77 & 43 & $0.0000^{* * *}$ & 83 & 37 & $0.0051^{* *}$ & & & & & & \\
\hline Bedford & poly & 61 & 6 & & 61 & 9 & & & & & & & \\
\hline GB_2 & fixed & 97 & 46 & $1 \mathrm{~ns}$ & 101 & 40 & $0.0289^{*}$ & 0 & 0 & $-n s$ & & & \\
\hline Ascot & poly & 1 & 1 & & 1 & 4 & & 62 & 7 & & & & \\
\hline
\end{tabular}


Table A5 continue:

\begin{tabular}{|c|c|c|c|c|c|c|c|c|c|c|c|c|c|}
\hline \multicolumn{2}{|l|}{ Population } & \multicolumn{3}{|c|}{$\begin{array}{c}\text { F_4 }_{-} \\
\text {syn. nons. sign. }\end{array}$} & \multicolumn{3}{|c|}{$\begin{array}{r}\text { FIN_1 } \\
\text { syn. nons. sign. }\end{array}$} & \multicolumn{3}{|c|}{$\begin{array}{c}\text { GB_1 } \\
\text { syn. nons. sign. }\end{array}$} & \multicolumn{3}{|c|}{$\begin{array}{c}\text { GB_2 } \\
\text { syn. nons. sign. }\end{array}$} \\
\hline GB_3 & fixed & 98 & 45 & $0.0414^{*}$ & 101 & 39 & $0.0012^{* *}$ & 0 & 0 & $-n s$ & 0 & 1 & $1 \mathrm{~ns}$ \\
\hline Braemar & poly & 1 & 4 & & 1 & 7 & & 62 & 10 & & 2 & 5 & \\
\hline I_1 & fixed & 73 & 39 & $1 \mathrm{~ns}$ & 85 & 37 & $1 \mathrm{~ns}$ & 65 & 11 & $0.811 \mathrm{~ns}$ & 88 & 15 & $0.2117 \mathrm{~ns}$ \\
\hline Grosseto & poly & 7 & 3 & & 7 & 6 & & 66 & 9 & & 8 & 4 & \\
\hline I_2 & fixed & 72 & 38 & $0.2186 \mathrm{~ns}$ & 83 & 37 & $1 \mathrm{~ns}$ & 63 & 11 & $0.4694 \mathrm{~ns}$ & 85 & 15 & $0.7025 \mathrm{~ns}$ \\
\hline Parma & poly & 11 & 2 & & 11 & 5 & & 68 & 8 & & 12 & 3 & \\
\hline IRL_1 & fixed & 98 & 45 & $0.569 \mathrm{~ns}$ & 102 & 39 & $0.1434 \mathrm{~ns}$ & 0 & 0 & $-n s$ & 0 & 1 & $0.4 \mathrm{~ns}$ \\
\hline Swords & poly & 2 & 0 & & 2 & 3 & & 63 & 9 & & 3 & 1 & \\
\hline N_1 & fixed & 70 & 4 & $0.0016^{* *}$ & 80 & 10 & $0.0163^{*}$ & 40 & 27 & $0.0004^{* * *} *$ & 59 & 31 & $0.0475^{*}$ \\
\hline Narvik & poly & 109 & 30 & & 108 & 34 & & 157 & 34 & & 110 & 31 & \\
\hline NL_1 & fixed & 95 & 45 & $1 \mathrm{~ns}$ & 99 & 39 & $0.3022 \mathrm{~ns}$ & 0 & 0 & $-n s$ & 0 & 0 & $-n s$ \\
\hline Wageningen & poly & 6 & 2 & & 6 & 5 & & 62 & 7 & & 7 & 3 & \\
\hline PL_1 & fixed & 0 & 0 & $-n s$ & 60 & 8 & $0.0198 *$ & 38 & 24 & $0.0064^{* *} *$ & 60 & 30 & $0.1813 \mathrm{~ns}$ \\
\hline Krakow & poly & 107 & 35 & & 107 & 38 & & 157 & 40 & & 108 & 36 & \\
\hline PL 2 & fixed & 81 & 37 & $0.5516 \mathrm{~ns}$ & 92 & 38 & $0.7848 \mathrm{~ns}$ & 59 & 17 & $0.0887 \mathrm{~ns}$ & 80 & 21 & $1 \mathrm{~ns}$ \\
\hline Warsaw & poly & 12 & 3 & & 12 & 6 & & 68 & 9 & & 13 & 4 & \\
\hline RUM_1 & fixed & 89 & 39 & $0.5767 \mathrm{~ns}$ & 93 & 37 & $0.1542 \mathrm{~ns}$ & 60 & 12 & $0.2083 \mathrm{~ns}$ & 82 & 16 & $1 \mathrm{~ns}$ \\
\hline Sibiu_1 & poly & 2 & 0 & & 2 & 3 & & 63 & 6 & & 3 & 1 & \\
\hline RUM_2 & fixed & 87 & 38 & $0.5072 \mathrm{~ns}$ & 91 & 36 & $0.7574 \mathrm{~ns}$ & 58 & 12 & $0.2388 \mathrm{~ns}$ & 80 & 16 & $0.6957 \mathrm{~ns}$ \\
\hline Sibiu_2 & poly & 9 & 2 & & 9 & 5 & & 70 & 8 & & 10 & 3 & \\
\hline RUM_3 & fixed & 85 & 9 & $0.4054 \mathrm{~ns}$ & 96 & 16 & $0.1969 \mathrm{~ns}$ & 65 & 44 & $0.0000^{*} * *$ & 87 & 47 & $0.2125 \mathrm{~ns}$ \\
\hline Bagau & poly & 15 & 3 & & 15 & 6 & & 75 & 9 & & 16 & 4 & \\
\hline RUM_4 & fixed & 88 & 10 & $0.6182 \mathrm{~ns}$ & 95 & 17 & $0.3653 \mathrm{~ns}$ & 71 & 45 & $0.0000^{*} * *$ & 93 & 48 & $0.2723 \mathrm{~ns}$ \\
\hline Cluj & poly & 7 & 0 & & 7 & 3 & & 65 & 6 & & 8 & 1 & \\
\hline RUM_5 & fixed & 87 & 38 & $0.6687 \mathrm{~ns}$ & 89 & 38 & $0.4574 \mathrm{~ns}$ & 69 & 14 & $0.2425 \mathrm{~ns}$ & 92 & 18 & $0.6015 \mathrm{~ns}$ \\
\hline Busteni & poly & 5 & 1 & & 5 & 4 & & 65 & 7 & & 5 & 2 & \\
\hline RUM_6 & fixed & 58 & 32 & $0.0699 \mathrm{~ns}$ & 69 & 31 & $0.4224 \mathrm{~ns}$ & 40 & 5 & $0.4795 \mathrm{~ns}$ & 57 & 9 & $0.2134 \mathrm{~ns}$ \\
\hline Sinaia & poly & 69 & 20 & & 69 & 23 & & 117 & 24 & & 70 & 20 & \\
\hline RUS_1 & fixed & 57 & 6 & $0.6372 \mathrm{~ns}$ & 67 & 10 & $0.8388 \mathrm{~ns}$ & 46 & 39 & $0.0000^{* * * *}$ & 62 & 43 & $0.0000^{* * *} *$ \\
\hline Altai Mountains & poly & 114 & 16 & & 114 & 19 & & 162 & 22 & & 115 & 17 & \\
\hline RUS_2 & fixed & 97 & 18 & $0.4081 \mathrm{~ns}$ & 101 & 18 & $1 \mathrm{~ns}$ & 83 & 41 & $0.0002 * * *$ & 99 & 44 & $0.0126^{*}$ \\
\hline Novosibirsk & poly & 33 & 3 & & 33 & 6 & & 82 & 10 & & 34 & 4 & \\
\hline S_2 & fixed & 8 & 1 & $0.687 \mathrm{~ns}$ & 49 & 6 & $0.0406^{*}$ & 31 & 14 & $0.1696 \mathrm{~ns}$ & 49 & 18 & $0.5102 \mathrm{~ns}$ \\
\hline Stroemstad & poly & 152 & 44 & & 152 & 47 & & 190 & 49 & & 152 & 45 & \\
\hline Population & & syn. & nons & $\begin{array}{l}\text { B_3 } \\
\text { sign. }\end{array}$ & syn. & & sign. & syn. & nons: & $\begin{array}{l}2 \\
\text { sign. }\end{array}$ & syn. & $\begin{array}{r}I \\
\text { non: }\end{array}$ & $\begin{array}{l}\text { L_1 } \\
\text { sign. }\end{array}$ \\
\hline I_1 & fixed & 89 & 14 & $0.0055^{* *}$ & & & & & & & & & \\
\hline Grosseto & poly & 8 & 7 & & & & & & & & & & \\
\hline I_2 & fixed & 86 & 14 & $0.0801 \mathrm{~ns}$ & 0 & 0 & $-n s$ & & & & & & \\
\hline Parma & poly & 12 & 6 & & 11 & 4 & & & & & & & \\
\hline IRL_1 & fixed & 0 & 0 & $-\mathrm{ns}$ & 88 & 14 & $0.3336 \mathrm{~ns}$ & 85 & 14 & $1 \mathrm{~ns}$ & & & \\
\hline Swords & poly & 3 & 4 & & 114 & 35 & & 13 & 2 & & & & \\
\hline N_1 & fixed & 59 & 30 & $0.0991 \mathrm{~ns}$ & 53 & 23 & $0.1685 \mathrm{~ns}$ & 53 & 22 & $0.3267 \mathrm{~ns}$ & 58 & 30 & $0.0444^{*}$ \\
\hline Narvik & poly & 110 & 34 & & 13 & 5 & & 116 & 34 & & 110 & 30 & \\
\hline NL_1 & fixed & 0 & 0 & $-\mathrm{ns}$ & 85 & 14 & $0.1685 \mathrm{~ns}$ & 82 & 14 & $0.7381 \mathrm{~ns}$ & 0 & 0 & $-\mathrm{ns}$ \\
\hline Wageningen & poly & 7 & 6 & & 13 & 5 & & 17 & 4 & & 8 & 2 & \\
\hline PL_1 & fixed & 61 & 29 & $0.3764 \mathrm{~ns}$ & 39 & 21 & $0.1776 \mathrm{~ns}$ & 39 & 20 & $0.2265 \mathrm{~ns}$ & 61 & 29 & $0.2279 \mathrm{~ns}$ \\
\hline Krakow & poly & 108 & 39 & & 111 & 38 & & 114 & 37 & & 109 & 35 & \\
\hline PL_2 & fixed & 79 & 20 & $0.2394 \mathrm{~ns}$ & 56 & 19 & $1 \mathrm{~ns}$ & 55 & 18 & $0.6013 \mathrm{~ns}$ & 80 & 20 & $1 \mathrm{~ns}$ \\
\hline Warsaw & poly & 13 & 7 & & 18 & 6 & & 22 & 5 & & 14 & 3 & \\
\hline RUM_1 & fixed & 81 & 16 & $0.0244^{*}$ & 68 & 15 & $0.6929 \mathrm{~ns}$ & 66 & 14 & $1 \mathrm{~ns}$ & 83 & 15 & $1 \mathrm{~ns}$ \\
\hline Sibiu_1 & poly & 3 & 4 & & 9 & 3 & & 13 & 2 & & 4 & 0 & \\
\hline RUM_2 & fixed & 76 & 16 & $0.0847 \mathrm{~ns}$ & 66 & 14 & $0.5364 \mathrm{~ns}$ & 64 & 13 & $1 \mathrm{~ns}$ & 81 & 15 & $1 \mathrm{~ns}$ \\
\hline Sibiu_2 & poly & 10 & 6 & & 16 & 5 & & 20 & 4 & & 11 & 2 & \\
\hline RUM_3 & fixed & 87 & 46 & $0.8138 \mathrm{~ns}$ & 85 & 42 & $0.266 \mathrm{~ns}$ & 83 & 41 & $0.0795 \mathrm{~ns}$ & 87 & 46 & $0.1212 \mathrm{~ns}$ \\
\hline Bagaù & poly & 16 & 7 & & 22 & 6 & & 26 & 2 & & 17 & 3 & \\
\hline
\end{tabular}


Table A5 continue:

\begin{tabular}{|c|c|c|c|c|c|c|c|c|c|c|c|c|c|}
\hline \multirow{2}{*}{$\begin{array}{c}\text { Population } \\
\text { RUM } 4\end{array}$} & \multirow{3}{*}{$\begin{array}{l}\text { fixed } \\
\text { poly }\end{array}$} & \multicolumn{3}{|c|}{$\begin{array}{c}\text { GB_3 } \\
\text { syn. nons. sign. }\end{array}$} & \multicolumn{3}{|c|}{$\begin{array}{c}\text { I_1 } \\
\text { syn. nons. sign. }\end{array}$} & \multicolumn{3}{|c|}{$\begin{array}{c}\text { I_2 } \\
\text { syn. nons. sign. }\end{array}$} & \multicolumn{3}{|c|}{$\begin{array}{r}\text { IRL_1 } \\
\text { syn. nons. sign. }\end{array}$} \\
\hline & & 93 & 47 & $1 \mathrm{~ns}$ & 90 & 43 & $0.273 \mathrm{~ns}$ & 88 & 42 & $0.062 \mathrm{~ns}$ & 93 & 47 & $0.0576 \mathrm{~ns}$ \\
\hline Cluj & & 8 & 4 & & 14 & 3 & & 18 & 5 & & 9 & 0 & \\
\hline RUM_5 & fixed & 91 & 17 & $0.0299^{*}$ & 68 & 19 & $1 \mathrm{~ns}$ & 66 & 18 & $0.7571 \mathrm{~ns}$ & 92 & 17 & $1 \mathrm{~ns}$ \\
\hline Busteni & poly & 6 & 5 & & 12 & 4 & & 16 & 3 & & 7 & 1 & \\
\hline RUM_6 & fixed & 57 & 8 & $0.067 \mathrm{~ns}$ & 38 & 5 & $0.166 \mathrm{~ns}$ & 36 & 5 & $0.3358 \mathrm{~ns}$ & 56 & 8 & $0.2021 \mathrm{~ns}$ \\
\hline Sinaia & poly & 70 & 23 & & 76 & 22 & & 79 & 20 & & 72 & 19 & \\
\hline RUS_1 & fixed & 63 & 42 & $0.0000^{* * *}$ & 64 & 34 & $0.0002 * * *$ & 63 & 33 & $0.0002 * * *$ & 63 & 42 & $0.0000 * * *$ \\
\hline Altai Mountains & poly & 115 & 20 & & 117 & 19 & & 119 & 18 & & 116 & 16 & \\
\hline RUS_2 & fixed & 99 & 43 & $0.1131 \mathrm{~ns}$ & 95 & 42 & $0.0002 * * *$ & 94 & 42 & $0.0041^{* *}$ & 99 & 43 & $0.0035^{* *}$ \\
\hline Novosibirsk & poly & 34 & 7 & & 39 & 6 & & 43 & 5 & & 35 & 3 & \\
\hline S 2 & fixed & 49 & 17 & $0.8688 \mathrm{~ns}$ & 29 & 14 & $0.2402 \mathrm{~ns}$ & 29 & 14 & $0.1643 \mathrm{~ns}$ & 49 & 17 & $0.6134 \mathrm{~ns}$ \\
\hline Stroemstad & poly & 153 & 48 & & 157 & 46 & & 160 & 44 & & 154 & 44 & \\
\hline \multicolumn{2}{|l|}{ Population } & \multicolumn{3}{|c|}{$\begin{array}{c}\mathrm{N}_{-} 1 \\
\text { syn. nons. sign. }\end{array}$} & \multicolumn{3}{|c|}{$\begin{array}{c}\text { NL_1 } \\
\text { ons. sign. }\end{array}$} & \multicolumn{3}{|c|}{$\begin{array}{c}\text { PL_1 } \\
\text { syn. nons. sign. }\end{array}$} & \multicolumn{3}{|c|}{$\begin{array}{c}\text { PL_2 } \\
\text { syn. nons. sign. }\end{array}$} \\
\hline NL_1 & fixed & 56 & 30 & $0.0454^{*}$ & & & & & & & & & \\
\hline Wageningen & poly & 113 & 32 & & & & & & & & & & \\
\hline PL_1 & fixed & 26 & 0 & $0.0066^{* *}$ & 57 & 29 & $0.1747 \mathrm{~ns}$ & & & & & & \\
\hline Krakow & poly & 191 & 46 & & 113 & 37 & & & & & & & \\
\hline PL_2 & fixed & 50 & 24 & $0.1031 \mathrm{~ns}$ & 79 & 20 & $1 \mathrm{~ns}$ & 42 & 18 & $0.4882 \mathrm{~ns}$ & & & \\
\hline Warsaw & poly & 118 & 33 & & 17 & 4 & & 118 & 38 & & & & \\
\hline RUM_1 & fixed & 65 & 25 & $0.3468 \mathrm{~ns}$ & 79 & 15 & $1 \mathrm{~ns}$ & 47 & 20 & $0.4039 \mathrm{~ns}$ & 66 & 9 & $0.6893 \mathrm{~ns}$ \\
\hline Sibiu_1 & poly & 110 & 31 & & 8 & 2 & & 109 & 35 & & 14 & 3 & \\
\hline RUM_2 & fixed & 63 & 25 & $0.2731 \mathrm{~ns}$ & 77 & 15 & $0.7378 \mathrm{~ns}$ & 46 & 20 & $0.4 \mathrm{~ns}$ & 63 & 8 & $0.325 \mathrm{~ns}$ \\
\hline Sibiu_2 & poly & 115 & 32 & & 15 & 4 & & 113 & 36 & & 21 & 5 & \\
\hline RUM_3 & fixed & 44 & 1 & $0.0013^{* *}$ & 83 & 46 & $0.1156 \mathrm{~ns}$ & 47 & 8 & $0.1806 \mathrm{~ns}$ & 87 & 42 & $0.138 \mathrm{~ns}$ \\
\hline Bagau & poly & 120 & 33 & & 21 & 5 & & 115 & 36 & & 26 & 6 & \\
\hline RUM 4 & fixed & 46 & 1 & $0.0012^{* *} *$ & 89 & 47 & $0.1452 \mathrm{~ns}$ & 50 & 9 & $0.1913 \mathrm{~ns}$ & 90 & 43 & $0.0835 \mathrm{~ns}$ \\
\hline Cluj & poly & 114 & 30 & & 13 & 2 & & 110 & 35 & & 19 & 3 & \\
\hline RUM_5 & fixed & 55 & 24 & $0.1935 \mathrm{~ns}$ & 89 & 17 & $0.7019 \mathrm{~ns}$ & 50 & 19 & $0.7378 \mathrm{~ns}$ & 53 & 3 & $0.0833 \mathrm{~ns}$ \\
\hline Busteni & poly & 113 & 31 & & 11 & 3 & & 111 & 36 & & 17 & 4 & \\
\hline RUM_6 & fixed & 32 & 18 & $0.0674 \mathrm{~ns}$ & 55 & 7 & $0.1319 \mathrm{~ns}$ & 24 & 14 & $0.1598 \mathrm{~ns}$ & 35 & 3 & $0.1242 \mathrm{~ns}$ \\
\hline Sinaia & poly & 165 & 47 & & 74 & 20 & & 157 & 51 & & 79 & 20 & \\
\hline RUS_1 & fixed & 30 & 1 & $0.0376^{*}$ & 60 & 42 & $0.0000^{* * *} *$ & 33 & 4 & $0.2584 \mathrm{~ns}$ & 72 & 34 & $0.0004^{* * *} *$ \\
\hline Altai Mountains & poly & 194 & 43 & & 119 & 17 & & 196 & 47 & & 122 & 18 & \\
\hline RUS_2 & fixed & 66 & 10 & $0.2774 \mathrm{~ns}$ & 95 & 43 & $0.0102 *$ & 62 & 11 & $0.2924 \mathrm{~ns}$ & 91 & 43 & $0.0126^{*}$ \\
\hline Novosibirsk & poly & 136 & 33 & & 39 & 5 & & 134 & 37 & & 40 & 6 & \\
\hline S_2 & fixed & 15 & 0 & $0.051 \mathrm{~ns}$ & 45 & 17 & $0.4952 \mathrm{~ns}$ & 0 & 0 & $-\mathrm{ns}$ & 30 & 11 & $0.5422 \mathrm{~ns}$ \\
\hline Stroemstad & poly & 222 & 55 & & 158 & 46 & & 160 & 49 & & 159 & 45 & \\
\hline \multicolumn{2}{|l|}{ Population } & \multicolumn{3}{|c|}{$\begin{array}{r}\text { RUM_1 } \\
\text { syn. nons. sign. }\end{array}$} & \multicolumn{3}{|c|}{$\begin{array}{r}\text { RUM_2 } \\
\text { syn. nons. sign. }\end{array}$} & \multicolumn{3}{|c|}{$\begin{array}{r}\text { RUM_3 } \\
\text { syn. nons. sign. }\end{array}$} & syn. & $\begin{array}{r}\mathrm{RI} \\
\text { nons }\end{array}$ & $\begin{array}{l}\text { M_4 } \\
\text { sign. }\end{array}$ \\
\hline RUM_2 & fixed & 0 & 0 & $-n s$ & & & & & & & & & \\
\hline Sibiu_2 & poly & 9 & 2 & & & & & & & & & & \\
\hline RUM 3 & fixed & 96 & 41 & $0.1938 \mathrm{~ns}$ & 93 & 40 & $0.2516 \mathrm{~ns}$ & & & & & & \\
\hline Bagau & poly & 17 & 3 & & 22 & 5 & & & & & & & \\
\hline RUM_4 & fixed & 97 & 42 & $0.0609 \mathrm{~ns}$ & 94 & 41 & $0.1012 \mathrm{~ns}$ & 5 & 0 & $1 \mathrm{~ns}$ & & & \\
\hline Cluj & poly & 9 & 0 & & 16 & 2 & & 20 & 3 & & & & \\
\hline RUM_5 & fixed & 70 & 8 & $1 \mathrm{~ns}$ & 69 & 7 & $0.3828 \mathrm{~ns}$ & 101 & 42 & $0.3187 \mathrm{~ns}$ & 102 & 42 & $0.1156 \mathrm{~ns}$ \\
\hline Busteni & poly & 7 & 1 & & 14 & 3 & & 19 & 4 & & 12 & 1 & \\
\hline RUM_6 & fixed & 71 & 16 & $0.351 \mathrm{~ns}$ & 41 & 3 & $0.0319^{*}$ & 72 & 35 & $0.1226 \mathrm{~ns}$ & 73 & 36 & $0.0828 \mathrm{~ns}$ \\
\hline Sinaia & poly & 7 & 0 & & 77 & 21 & & 79 & 23 & & 74 & 20 & \\
\hline RUS_1 & fixed & 70 & 35 & $0.0001^{* * *}$ & 70 & 34 & $0.0004^{* * * *}$ & 27 & 0 & $0.0457^{*}$ & 33 & 1 & $0.2001 \mathrm{~ns}$ \\
\hline Altai Mountains & poly & 116 & 16 & & 120 & 18 & & 123 & 19 & & 118 & 16 & \\
\hline RUS_2 & fixed & 89 & 41 & $0.003 * *$ & 88 & 41 & $0.0038 * *$ & 84 & 15 & $0.4541 \mathrm{~ns}$ & 85 & 15 & $0.2728 \mathrm{~ns}$ \\
\hline Novosibirsk & poly & 35 & 3 & & 42 & 5 & & 45 & 5 & & 39 & 3 & \\
\hline S_2 & fixed & 27 & 10 & $0.5271 \mathrm{~ns}$ & 26 & 10 & $0.5195 \mathrm{~ns}$ & 38 & 7 & $0.4198 \mathrm{~ns}$ & 39 & 8 & $0.5527 \mathrm{~ns}$ \\
\hline Stroemstad & poly & 154 & 44 & & 158 & 45 & & 160 & 45 & & 155 & 44 & \\
\hline
\end{tabular}


Appendix

Table A5 continue:

\begin{tabular}{|c|c|c|c|c|c|c|c|c|c|c|c|c|c|}
\hline \multirow{2}{*}{$\begin{array}{c}\text { Population } \\
\text { RUM_6 }\end{array}$} & \multirow{3}{*}{$\begin{array}{l}\text { fixed } \\
\text { poly }\end{array}$} & \multicolumn{3}{|c|}{$\begin{array}{r}\text { RUM_5 } \\
\text { syn. nons. sign. }\end{array}$} & \multicolumn{3}{|c|}{$\begin{array}{r}\text { RUM_6 } \\
\text { syn. nons. sign. }\end{array}$} & \multicolumn{3}{|c|}{$\begin{array}{r}\text { RUS_1 } \\
\text { syn. nons. sign. }\end{array}$} & \multicolumn{3}{|c|}{$\begin{array}{r}\text { RUS_2 } \\
\text { syn. nons. sign. }\end{array}$} \\
\hline & & 0 & 0 & $-n s$ & & & & & & & & & \\
\hline Sinaia & & 72 & 20 & & & & & & & & & & \\
\hline RUS_1 & fixed & 77 & 35 & $0.0005^{* * *}$ & 50 & 30 & $0.0005^{* * *} *$ & & & & & & \\
\hline Altai Mountains & poly & 119 & 17 & & 165 & 34 & & & & & & & \\
\hline RUS 2 & fixed & 98 & 41 & $0.0127^{*}$ & 80 & 34 & $0.0929 \mathrm{~ns}$ & 50 & 11 & $0.2818 \mathrm{~ns}$ & & & \\
\hline Novosibirsk & poly & 37 & 4 & & 95 & 23 & & 135 & 19 & & & & \\
\hline S_2 & fixed & 35 & 9 & $0.8432 \mathrm{~ns}$ & 13 & 7 & $0.2646 \mathrm{~ns}$ & 26 & 2 & $0.1284 \mathrm{~ns}$ & 43 & 7 & $0.4244 \mathrm{~ns}$ \\
\hline Stroemstad & poly & 154 & 45 & & 194 & 54 & & 228 & 56 & & 175 & 44 & \\
\hline
\end{tabular}




\section{Appendix}

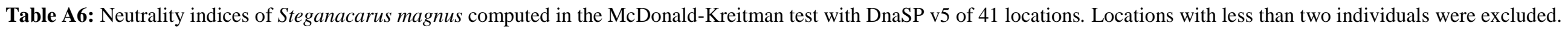

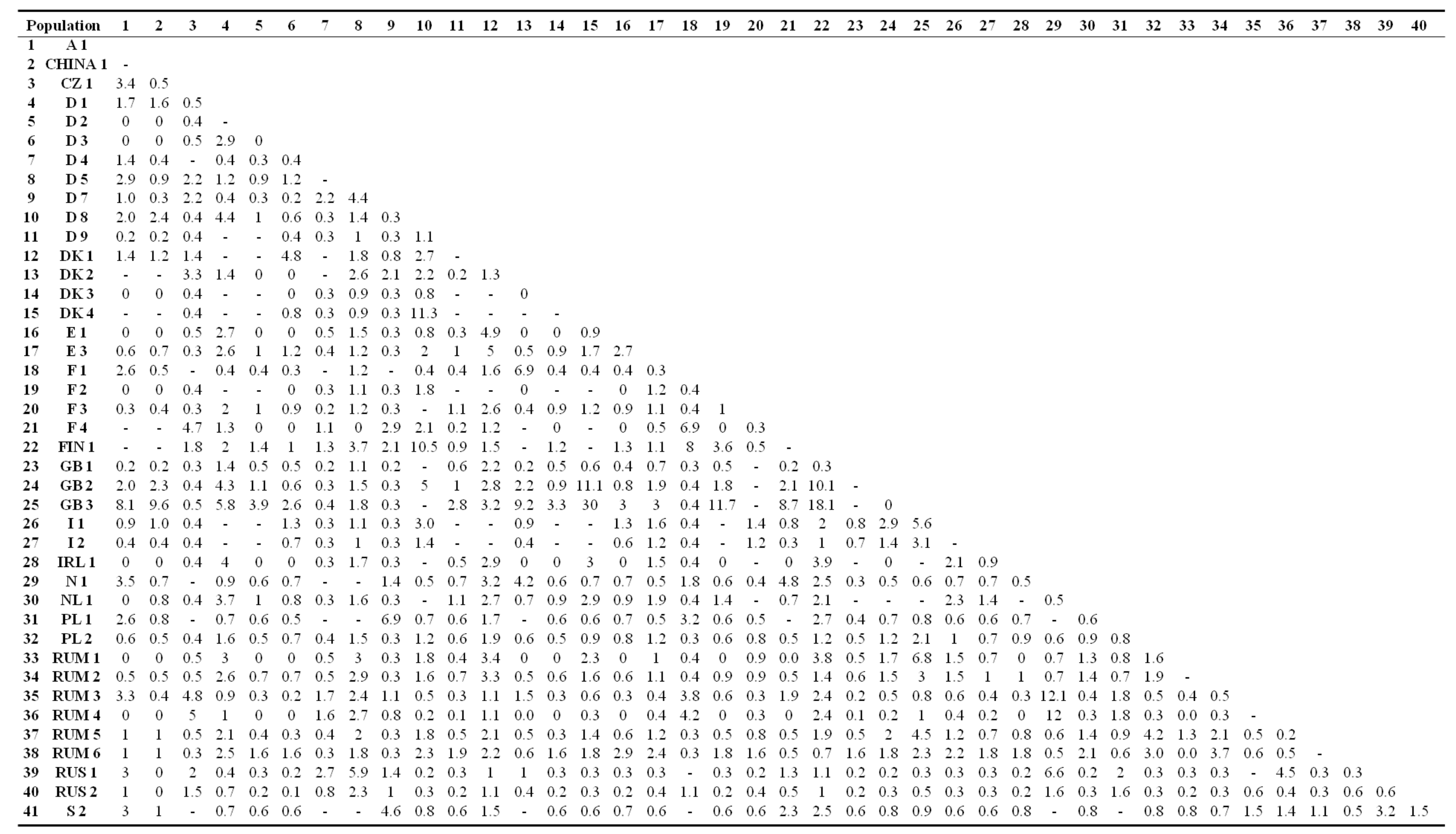


Appendix

Table A7: Standard diversity measures of the COI gene of Steganacarus magnus. Populations with less than two individuals were excluded (Chapter III).

\begin{tabular}{cccccccccc}
\hline population & $\begin{array}{c}\text { sample } \\
\text { size }\end{array}$ & $\begin{array}{c}\text { invarible } \\
\text { sites }\end{array}$ & $\begin{array}{c}\text { variable } \\
\text { sites }\end{array}$ & $\begin{array}{c}\text { parsimony } \\
\text { inform. sites } \\
\mathbf{N}_{\text {is }}\end{array}$ & $\begin{array}{c}\mathbf{N}_{\mathbf{v s}} \\
\mathbf{N}_{\text {pars }}\end{array}$ & $\begin{array}{c}\text { number of } \\
\text { singeltons } \\
\mathbf{N}_{\mathbf{s}}\end{array}$ & $\begin{array}{c}\text { number of } \\
\text { haplotypes } \\
\mathbf{N}_{\mathbf{h}}\end{array}$ & $\begin{array}{c}\text { haplotype } \\
\text { diversity } \\
\mathbf{H}_{\mathbf{d}}\end{array}$ & $\begin{array}{c}\text { nucleotide } \\
\text { variance } \\
\text { diversity }\end{array}$ \\
\hline A_1 & 2 & 597 & 0 & 0 & 0 & 1 & 0 & 0 & 0 \\
CZ_1 & 5 & 405 & 192 & 38 & 154 & 5 & 1 & 0.02 & 0.158 \\
D_1 & 14 & 562 & 35 & 16 & 19 & 9 & 0.84 & 0.01 & 0.02 \\
D_2 & 9 & 383 & 214 & 142 & 72 & 5 & 0.83 & 0.01 & 0.16 \\
DK_1 & 3 & 438 & 159 & 0 & 159 & 3 & 1 & 0.07 & 0.181 \\
F_1 & 4 & 595 & 2 & 0 & 2 & 3 & 0.83 & 0.05 & 0.002 \\
GB_1 & 5 & 521 & 76 & 6 & 70 & 4 & 0.9 & 0.03 & 0.053 \\
I_1 & 10 & 583 & 14 & 11 & 3 & 5 & 0.76 & 0.02 & 0.011 \\
PL_1 & 6 & 438 & 159 & 152 & 7 & 6 & 1 & 0.01 & 0.142 \\
RUM_1 & 4 & 577 & 20 & 0 & 20 & 3 & 0.83 & 0.05 & 0.017 \\
RUM_2 & 3 & 587 & 10 & 0 & 10 & 3 & 1 & 0.07 & 0.011 \\
all & 67 & 288 & 309 & 277 & 32 & 47 & 0.98 & 0 & 0.191 \\
\hline
\end{tabular}

Table A8: Standard diversity measures of the ef $1 \alpha$ gene of Steganacarus magnus. Populations with less than two individuals were excluded (Chapter III).

\begin{tabular}{cccccccccc}
\hline population & $\begin{array}{c}\text { sample } \\
\text { size }\end{array}$ & $\begin{array}{c}\text { invarible } \\
\text { sites }\end{array}$ & $\begin{array}{c}\text { variable } \\
\text { sites }\end{array}$ & $\begin{array}{c}\text { parsimony } \\
\text { inform. sites } \\
\mathbf{N}_{\mathbf{i s}}\end{array}$ & $\begin{array}{c}\mathbf{N}_{\mathbf{v s}} \\
\mathbf{N}_{\text {pars }}\end{array}$ & $\begin{array}{c}\text { number of } \\
\text { singeltons } \\
\mathbf{N}_{\mathbf{s}}\end{array}$ & $\begin{array}{c}\text { number of } \\
\text { haplotypes } \\
\mathbf{N}_{\mathbf{h}}\end{array}$ & $\begin{array}{c}\text { haplotype } \\
\text { diversity } \\
\mathbf{H}_{\mathbf{d}}\end{array}$ & $\begin{array}{c}\text { nucleotide } \\
\text { variance } \\
\text { diversity }\end{array}$ \\
\hline CZ_1 & 2 & 458 & 3 & 0 & 3 & 2 & 1 & 0.25 & 0.007 \\
D_1 & 6 & 423 & 38 & 19 & 19 & 6 & 1 & 0.01 & 0.037 \\
D_2 & 5 & 400 & 61 & 36 & 25 & 4 & 0.9 & 0.03 & 0.071 \\
F_1 & 2 & 396 & 65 & 0 & 65 & 2 & 1 & 0.25 & 0.14 \\
GB_1 & 5 & 453 & 8 & 1 & 7 & 4 & 0.9 & 0.03 & 0.007 \\
I_1 & 4 & 385 & 76 & 13 & 63 & 4 & 1 & 0.03 & 0.090 \\
PL_1 & 4 & 400 & 61 & 0 & 61 & 4 & 1 & 0.03 & 0.066 \\
RUM_1 & 2 & 426 & 35 & 0 & 35 & 2 & 1 & 0.25 & 0.076 \\
RUM_2 & 2 & 422 & 29 & 0 & 29 & 2 & 1 & 0.25 & 0.085 \\
all & 37 & 243 & 218 & 127 & 91 & 35 & 0.997 & 0 & 0.113 \\
\hline
\end{tabular}


Appendix

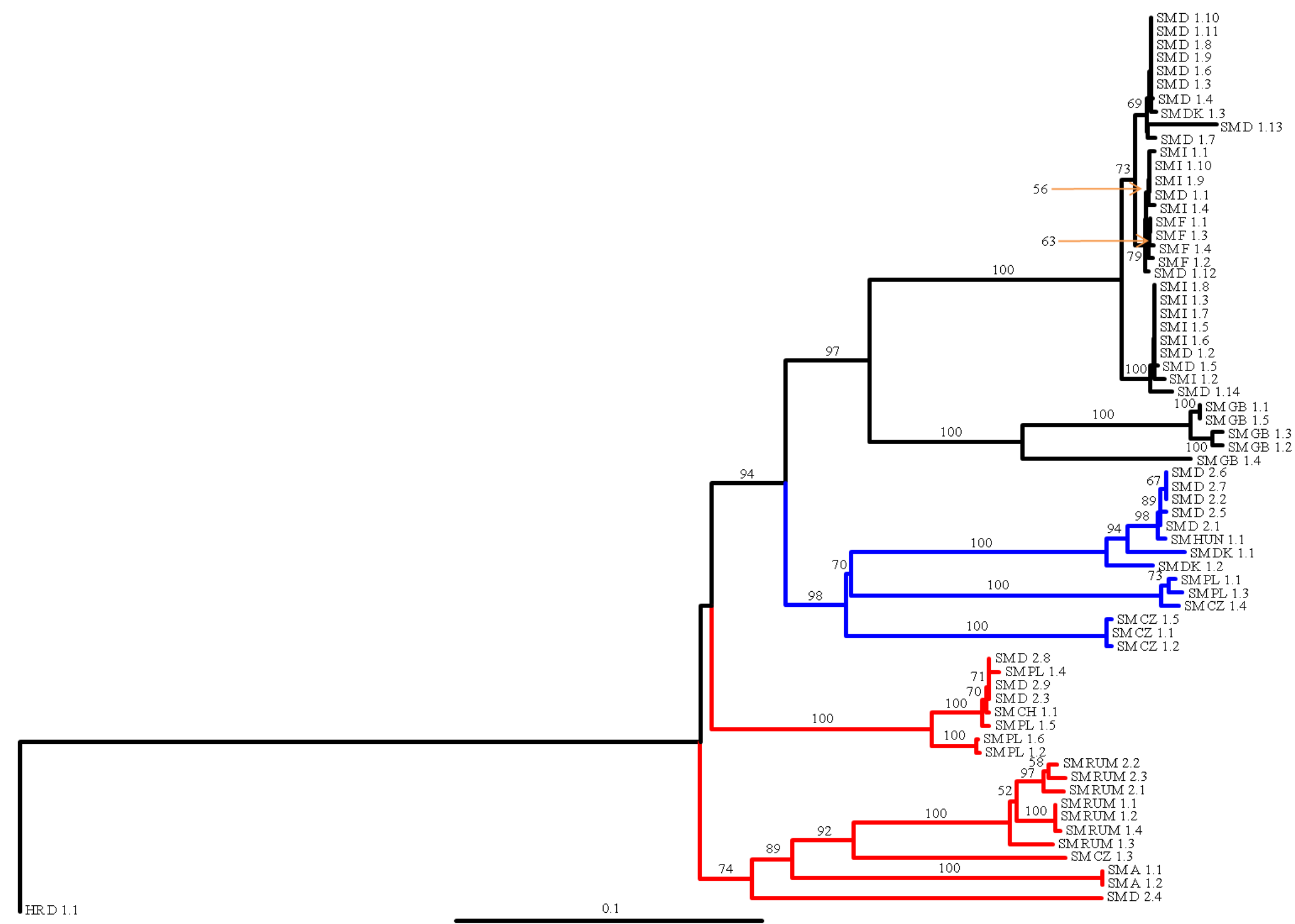

Figure A8: Neighbor-Joining tree of the 67 COI nucleotide sequences of Steganacarus magnus. Outgroup is Hypochthonius rufulus (HD_D_1.1). Numbers on the branches are bootstrap values after 100,000 pseudo-replicates computed in PAUP*. Branch colours show the different clades (black, blue, red). 
Appendix

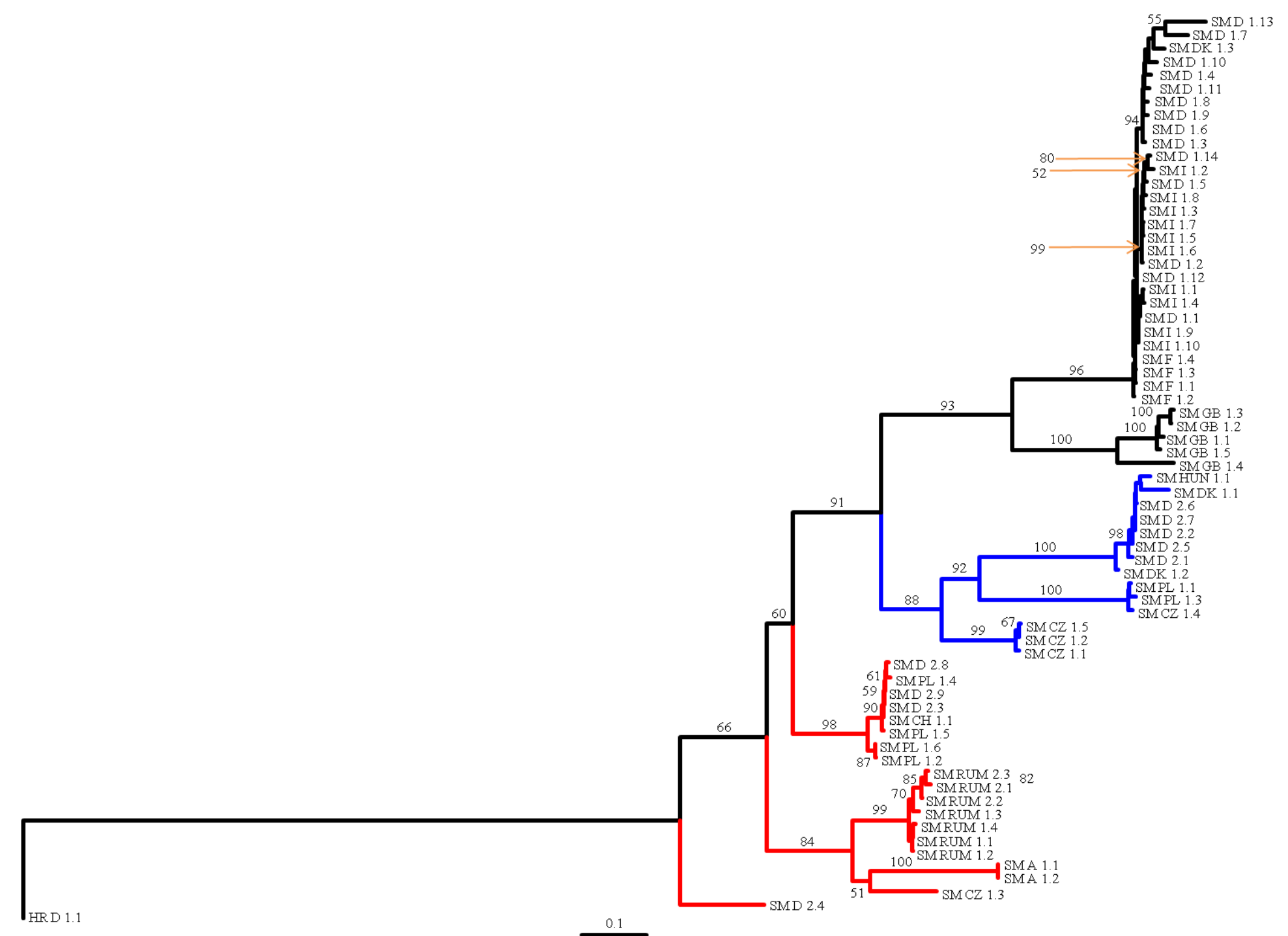

Figure A9: Neighbor-Joining tree with model of sequence evolution (TVM+I+G) of the 67 COI nucleotide sequences of Steganacarus magnus. Outgroup is Hypochthonius rufulus (HD_D_1.1). Numbers on the branches are bootstrap values after 100,000 pseudo-replicates computed in PAUP*. Branch colours show the different clades (black, blue, red). 
Appendix

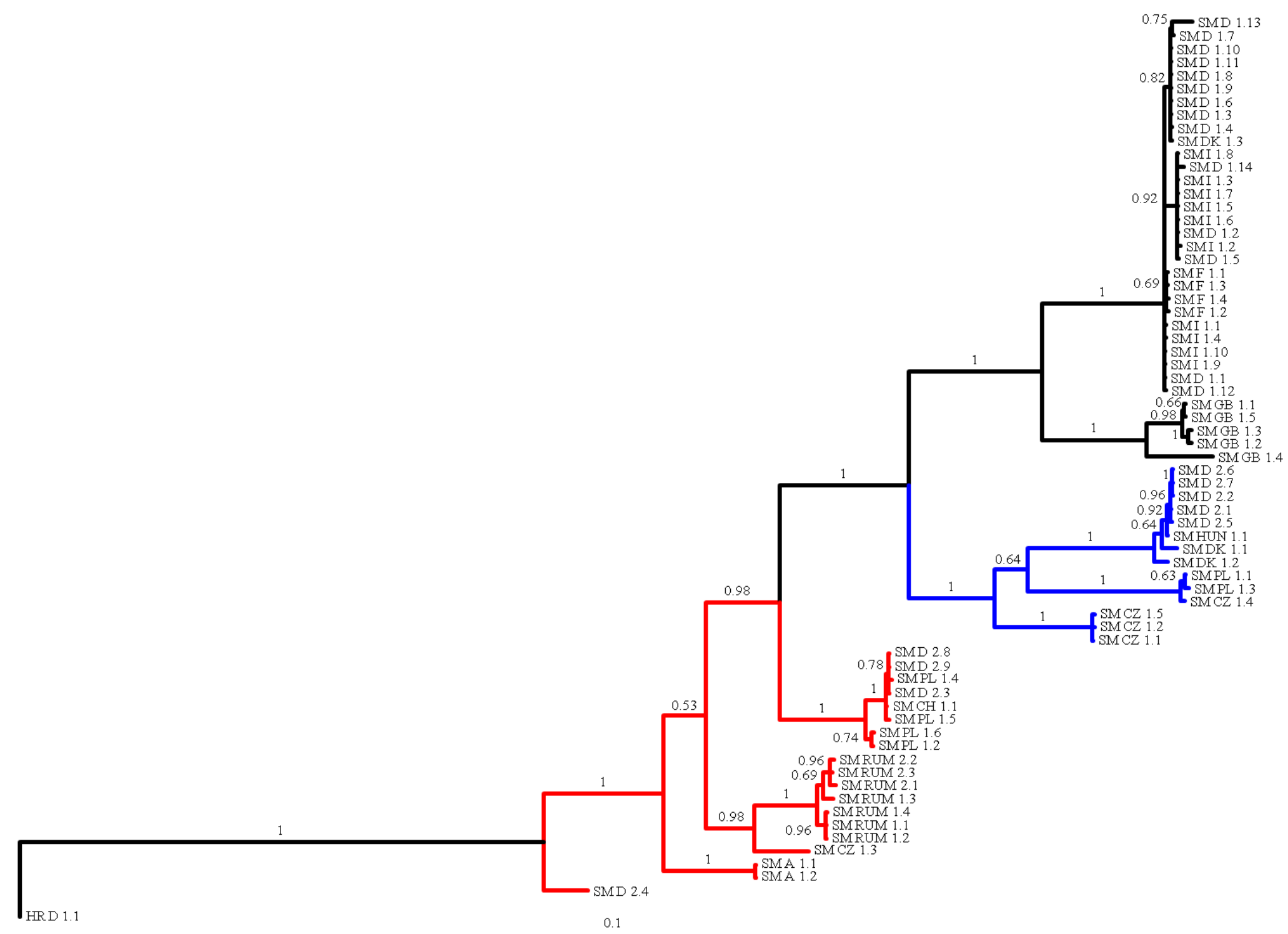

Figure A10: Bayesian phylogeny after 10x106 generations from the 67 COI nucleotide sequences of Steganacarus magnus. Outgroup is Hypochthonius rufulus (HD_D_1.1). Numbers on the branches are posterior probabilities from MrBayes. Branch colours show the different clades (black, blue, red). 
Appendix

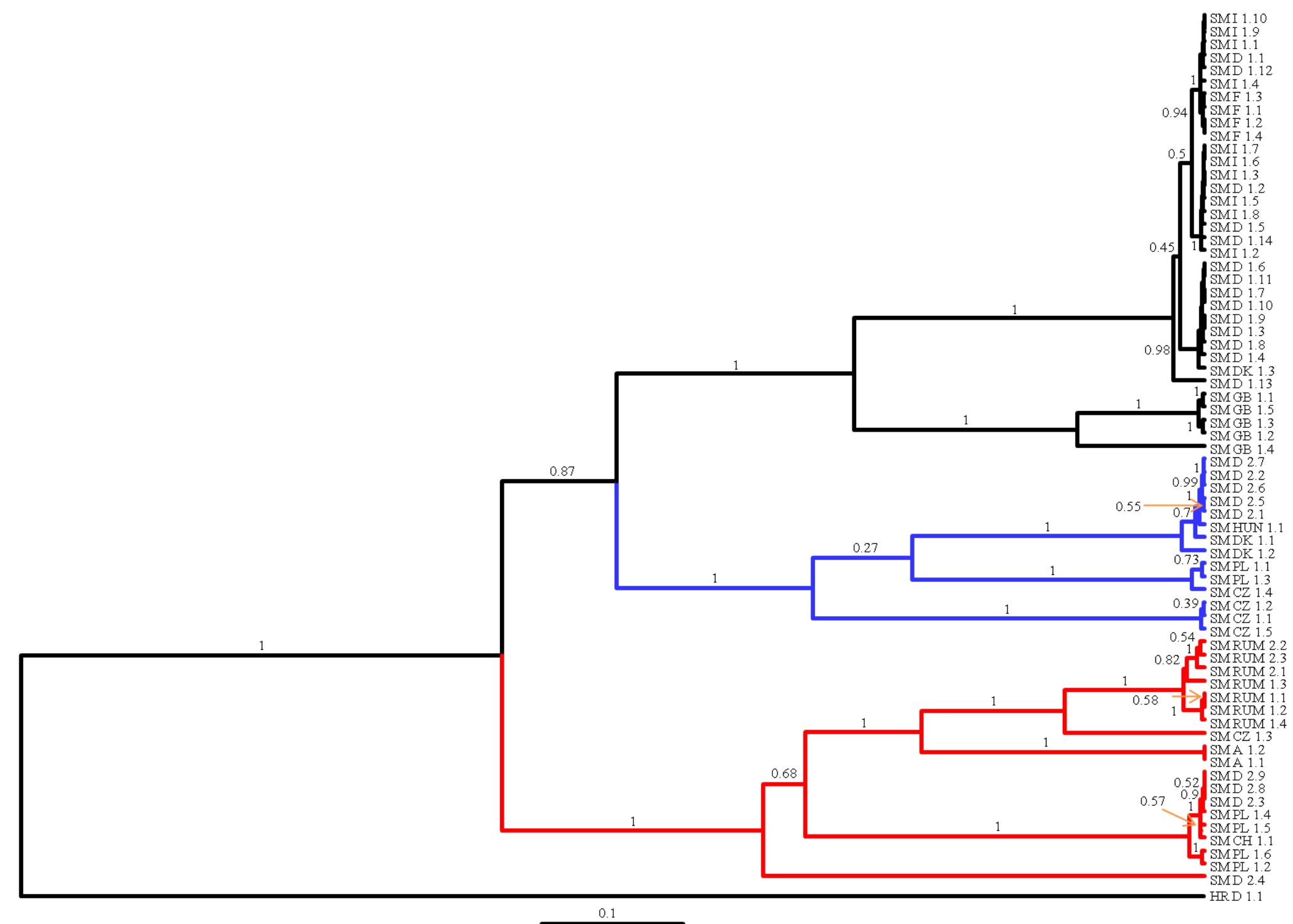

Figure A11: Bayesian phylogeny after 10x10 $10^{6}$ generations from the 67 COI nucleotide sequences of Steganacarus magnus. Outgroup is Hypochthonius rufulus (HD_D_1.1). Numbers on the branches are posterior probabilities from BEAST. Branch colours show the different clades (black, blue, red). 
Appendix

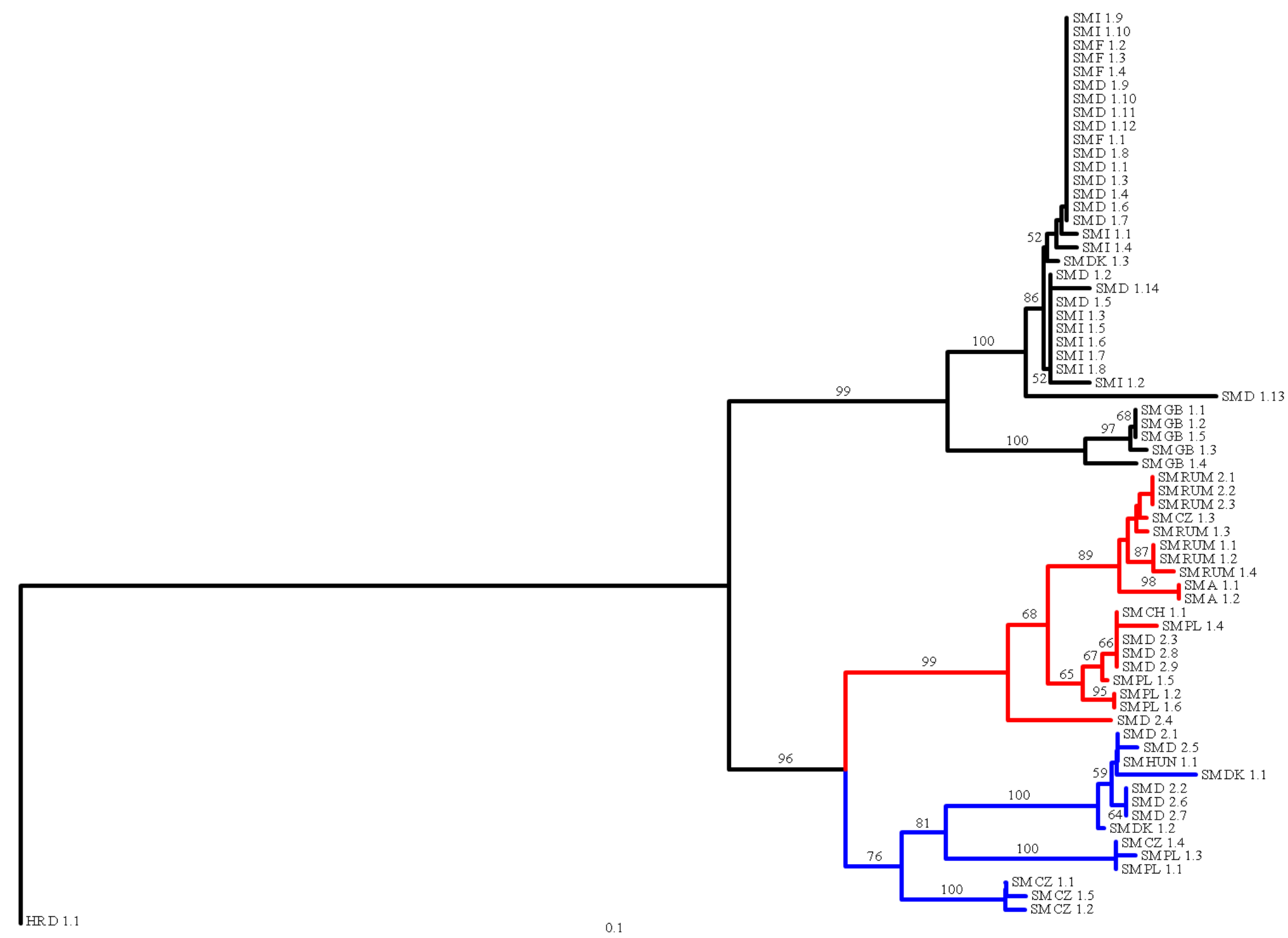

Figure A12: Neighbor-Joining tree from the 67 COI protein sequences of Steganacarus magnus. Outgroup is Hypochthonius rufulus (HD_D_1.1). Numbers on the branches are bootstrap values after 100,000 pseudo-replicates computed in PAUP*. Branch colours show the different clades (black, blue, red). 
Appendix

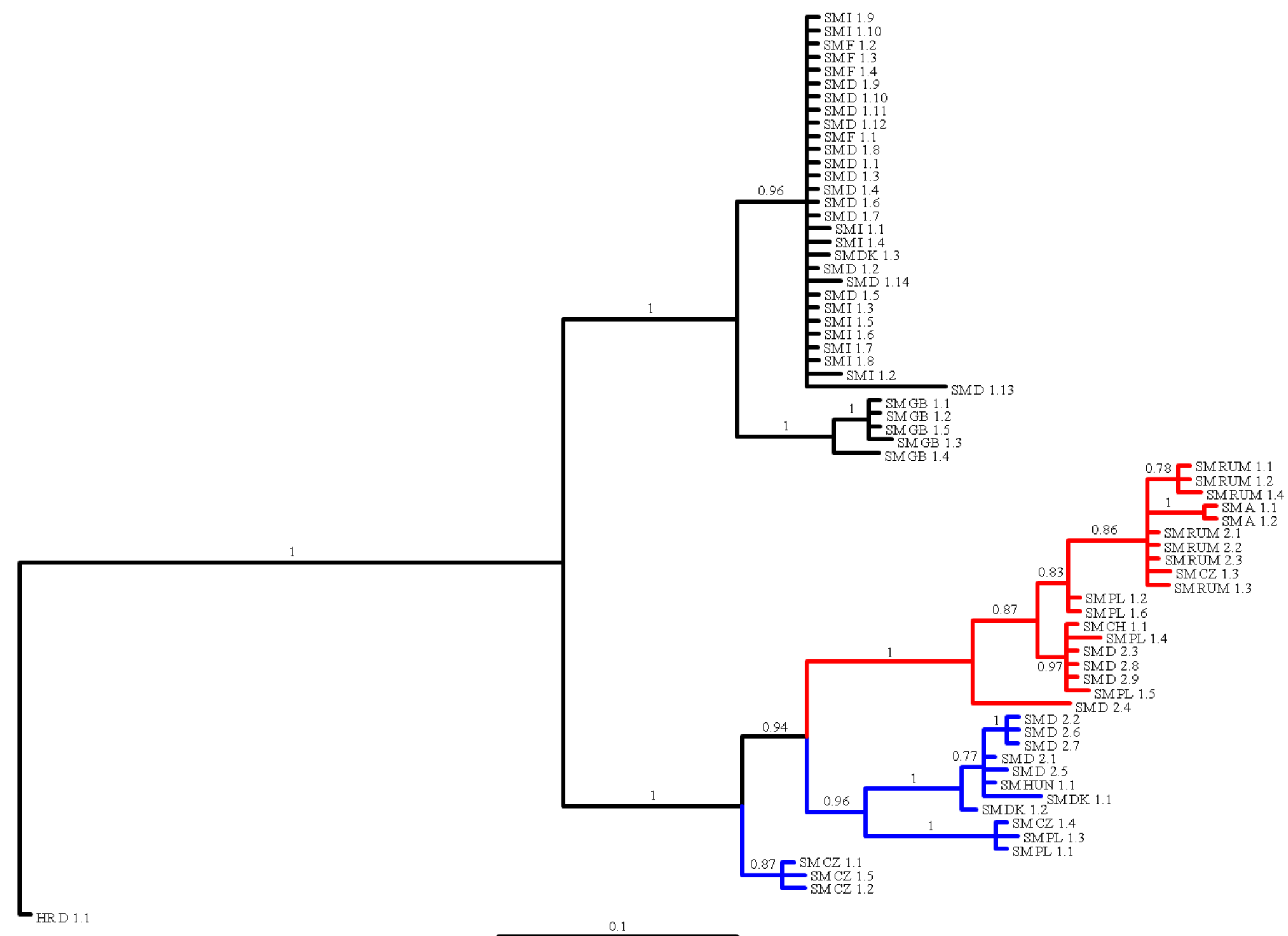

Figure A13: Bayesian phylogeny after 10x10 $10^{6}$ generations from the 67 COI protein sequences of Steganacarus magnus. Outgroup is Hypochthonius rufulus (HD_D_1.1). Numbers on the branches are posterior probabilities from MrBayes. Branch colours show the different clades (black, blue, red). 
Appendix

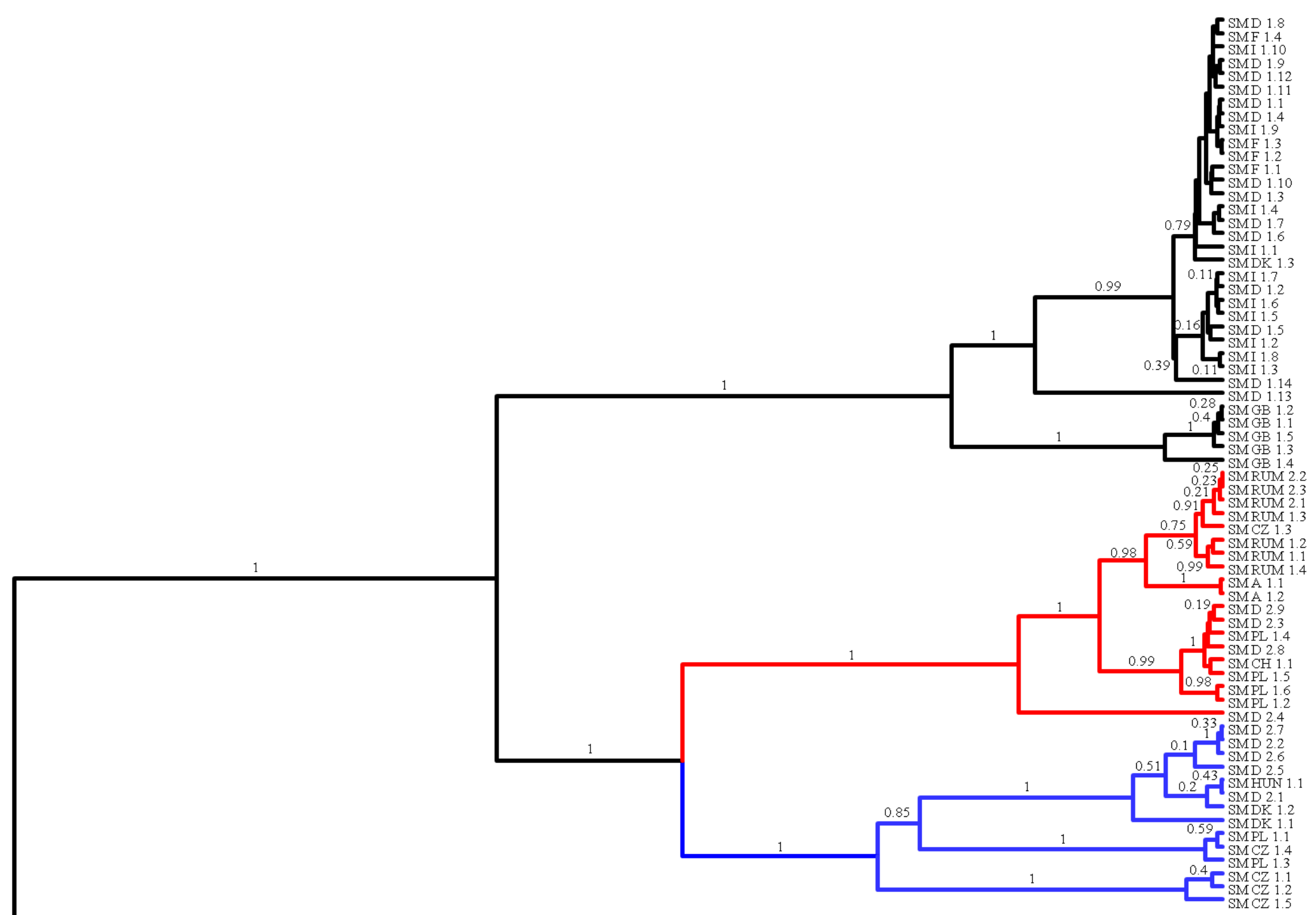

0.1

Figure A14: Bayesian phylogeny after $10 \times 10^{6}$ generations from the 67 COI protein sequences of Steganacarus magnus. Outgroup is Hypochthonius rufulus (HD_D_1.1). Numbers on the branches are posterior probabilities from BEAST. Branch colours show the different clades (black, blue, red). 
Appendix

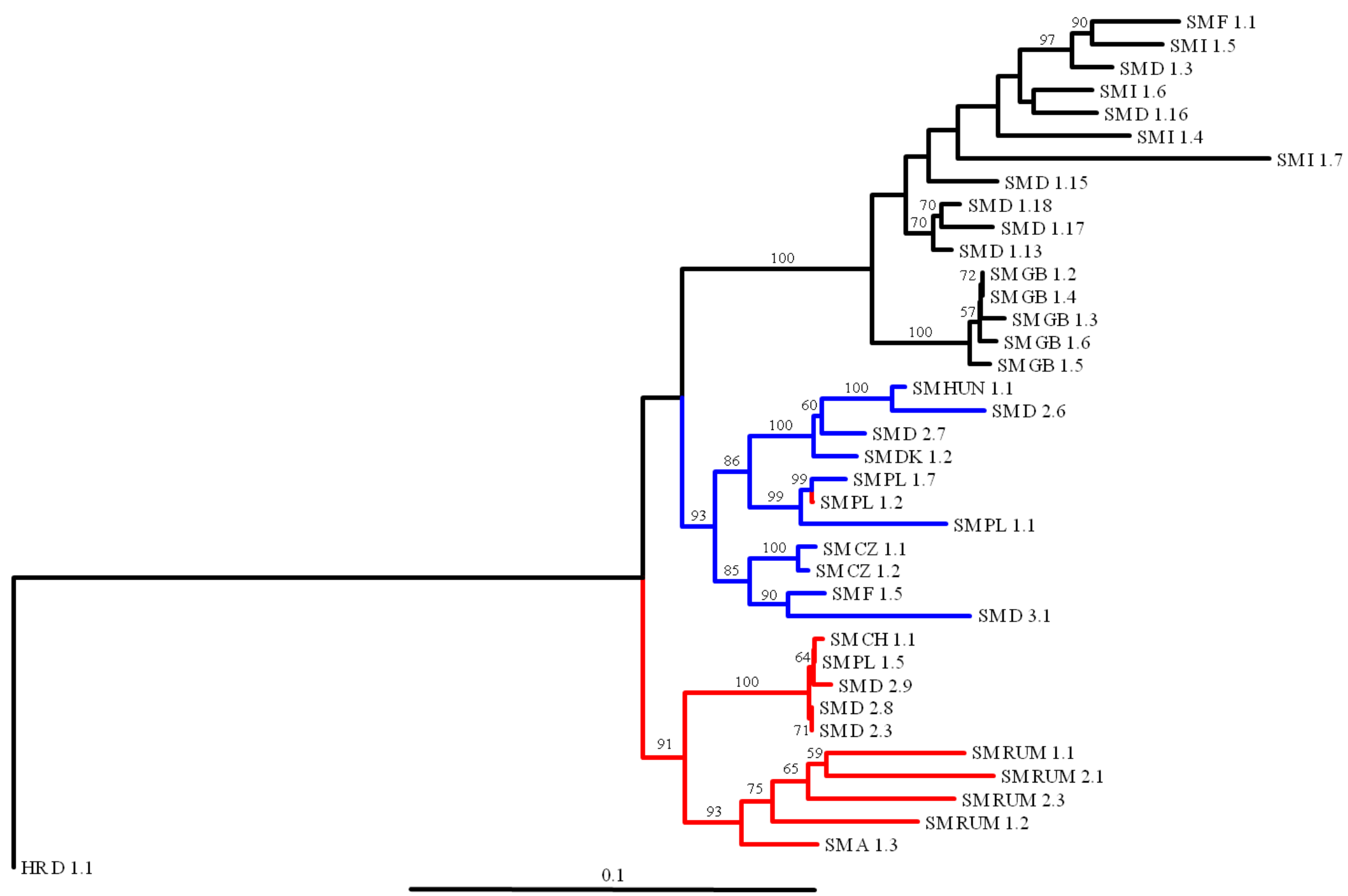

Figure A15: Neighbor-Joining tree of the ef $1 \alpha$ nucleotide sequences of Steganacarus magnus. Outgroup is Hypochthonius rufulus (HD_D_1.1). Numbers on the branches are bootstrap values after 100,000 pseudo-replicates computed in PAUP*. Branch colours show the different clades (black, blue, red). 
Appendix

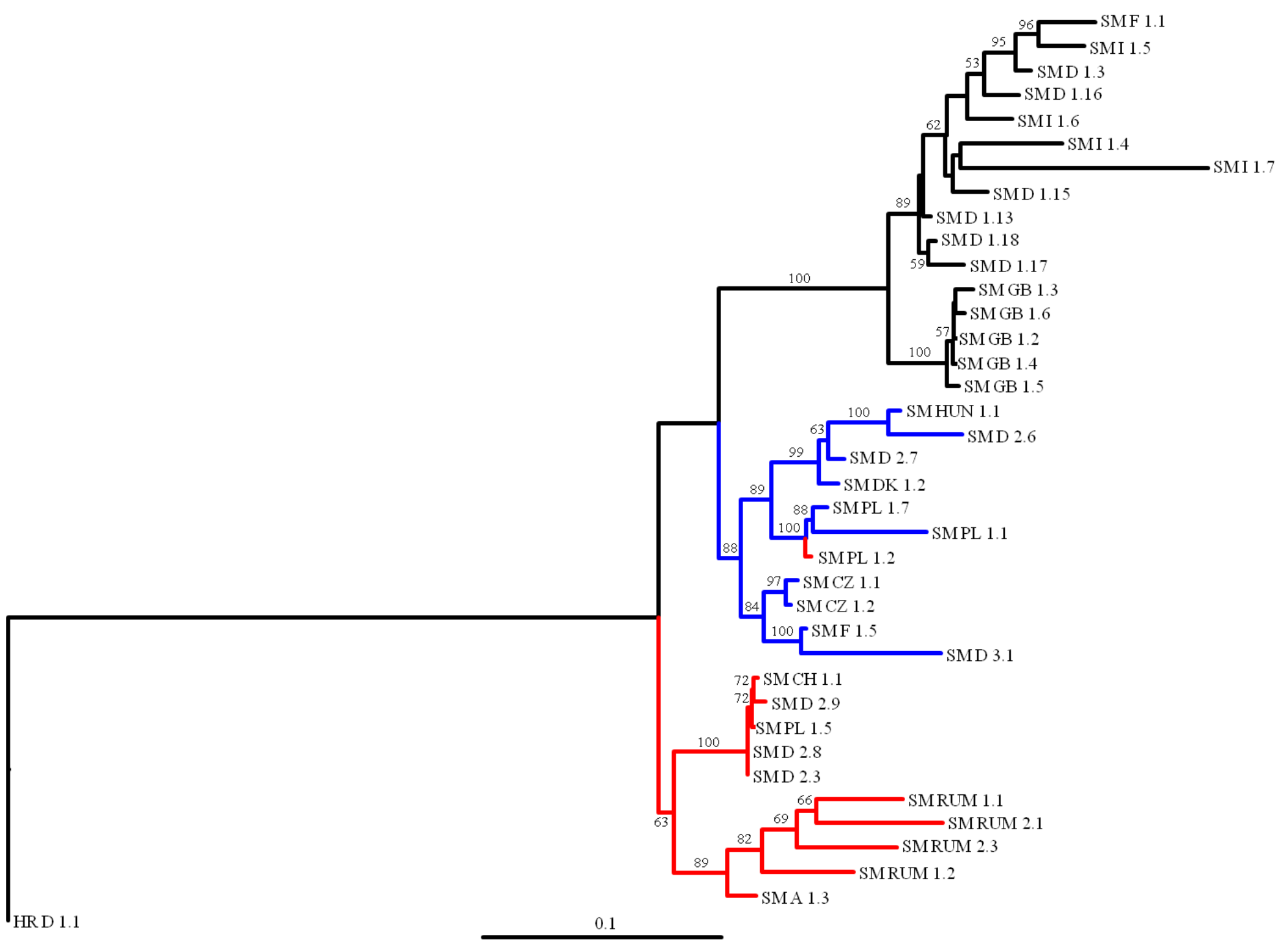

Figure A16: Neighbor-Joining tree with model of sequence evolution $(\operatorname{TrNef}+\mathrm{G})$ of the ef $1 \alpha$ nucleotide sequences of Steganacarus magnus. Outgroup is Hypochthonius rufulus (HD_D_1.1).. Numbers on the branches are bootstrap values after 100,000 pseudo-replicates computed in PAUP*. Branch colours show the different clades (black, blue, red). 
Appendix

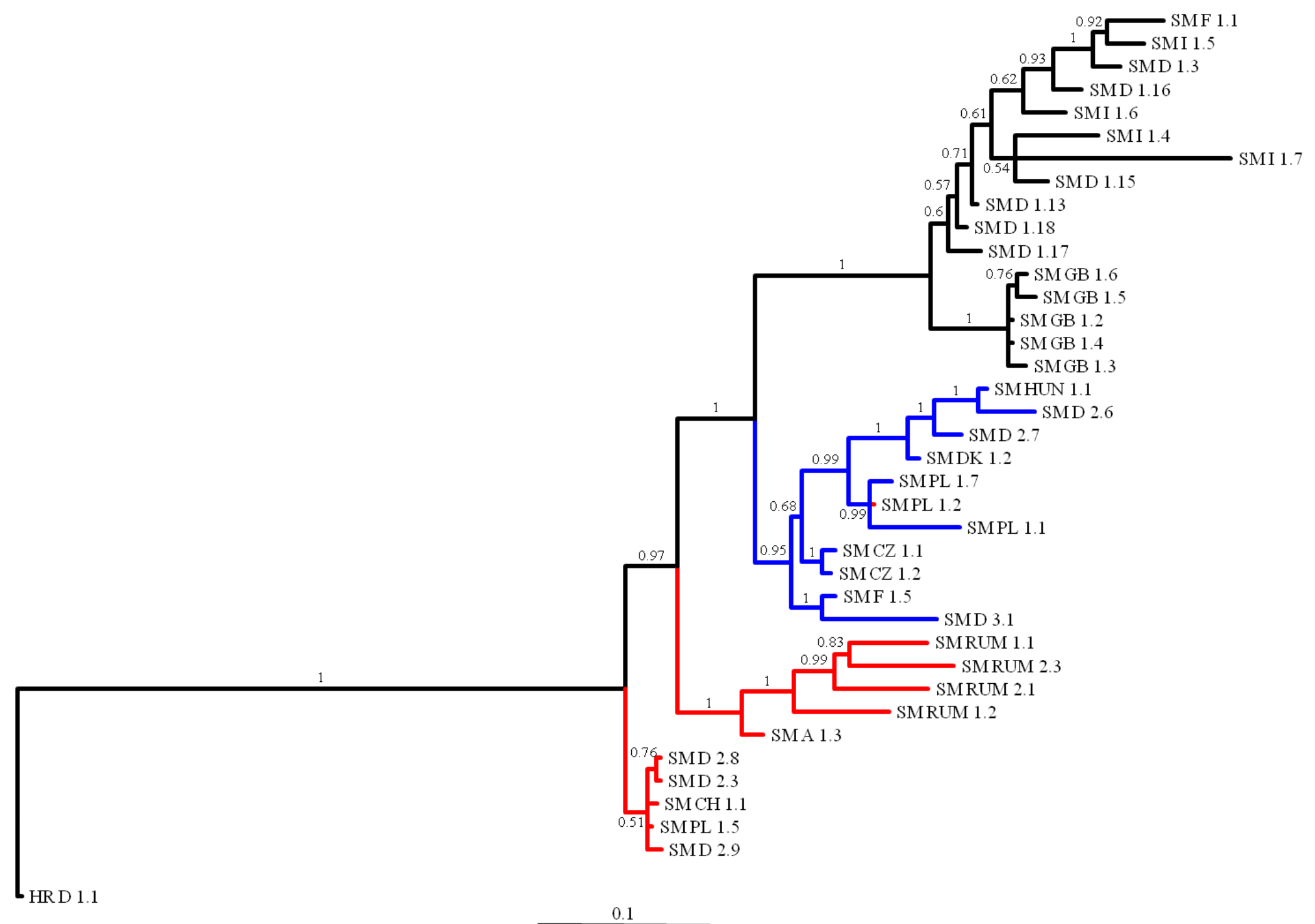

Figure A17: Bayesian phylogeny after 10x10 $0^{6}$ generations from the ef $1 \alpha$ nucleotide sequences of Steganacarus magnus. Outgroup is Hypochthonius rufulus (HD_D_1.1).. Numbers on the branches are posterior probabilities from MrBayes. Branch colours show the different clades (black, blue, red). 
Appendix

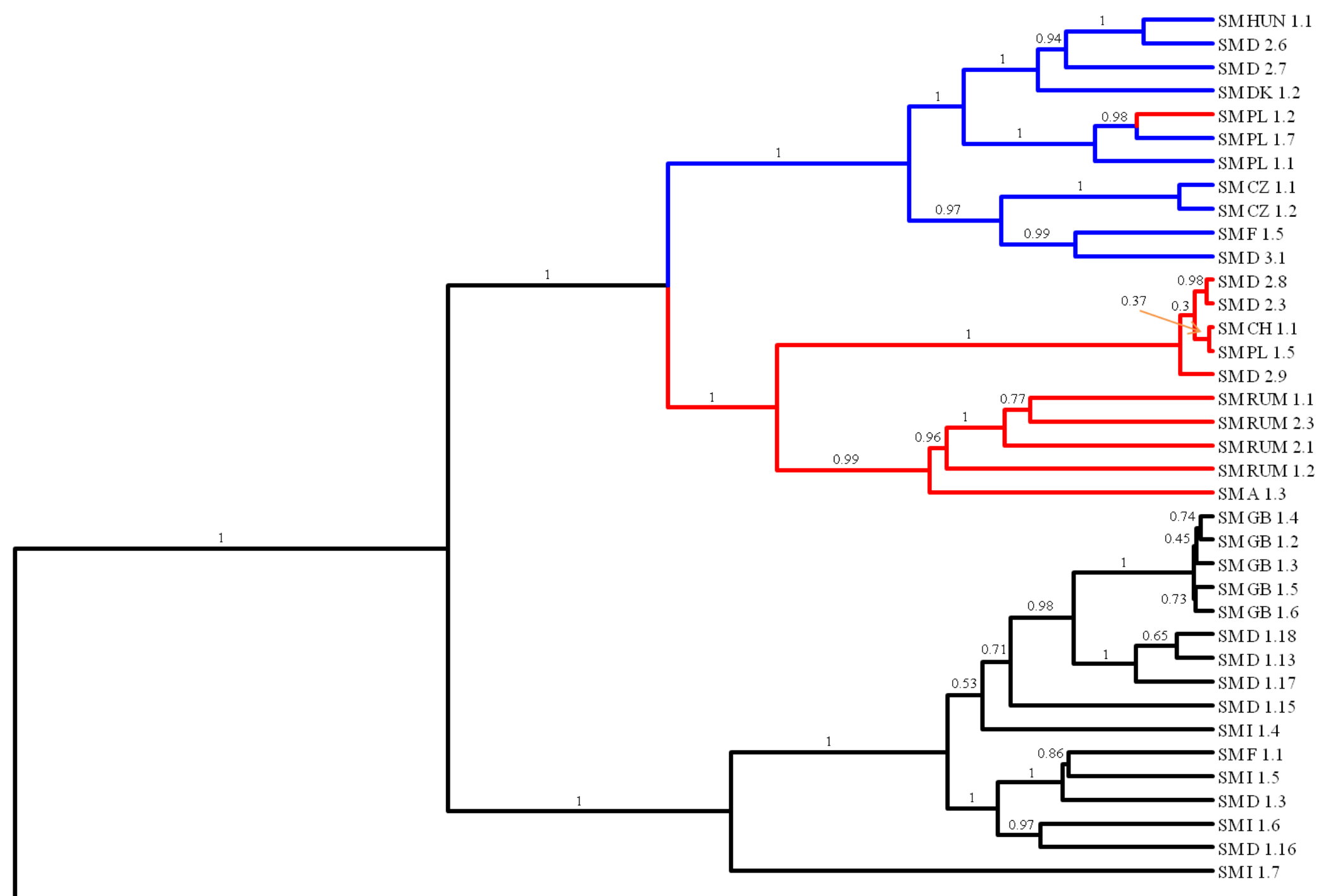

0.1

Figure A18: Bayesian phylogeny after 10x10 $0^{6}$ generations from the ef $1 \alpha$ nucleotide sequences of Steganacarus magnus. Outgroup is Hypochthonius rufulus (HD_D_1.1). Numbers on the branches are posterior probabilities from BEAST. Branch colours show the different clades (black, blue, red). 
Appendix

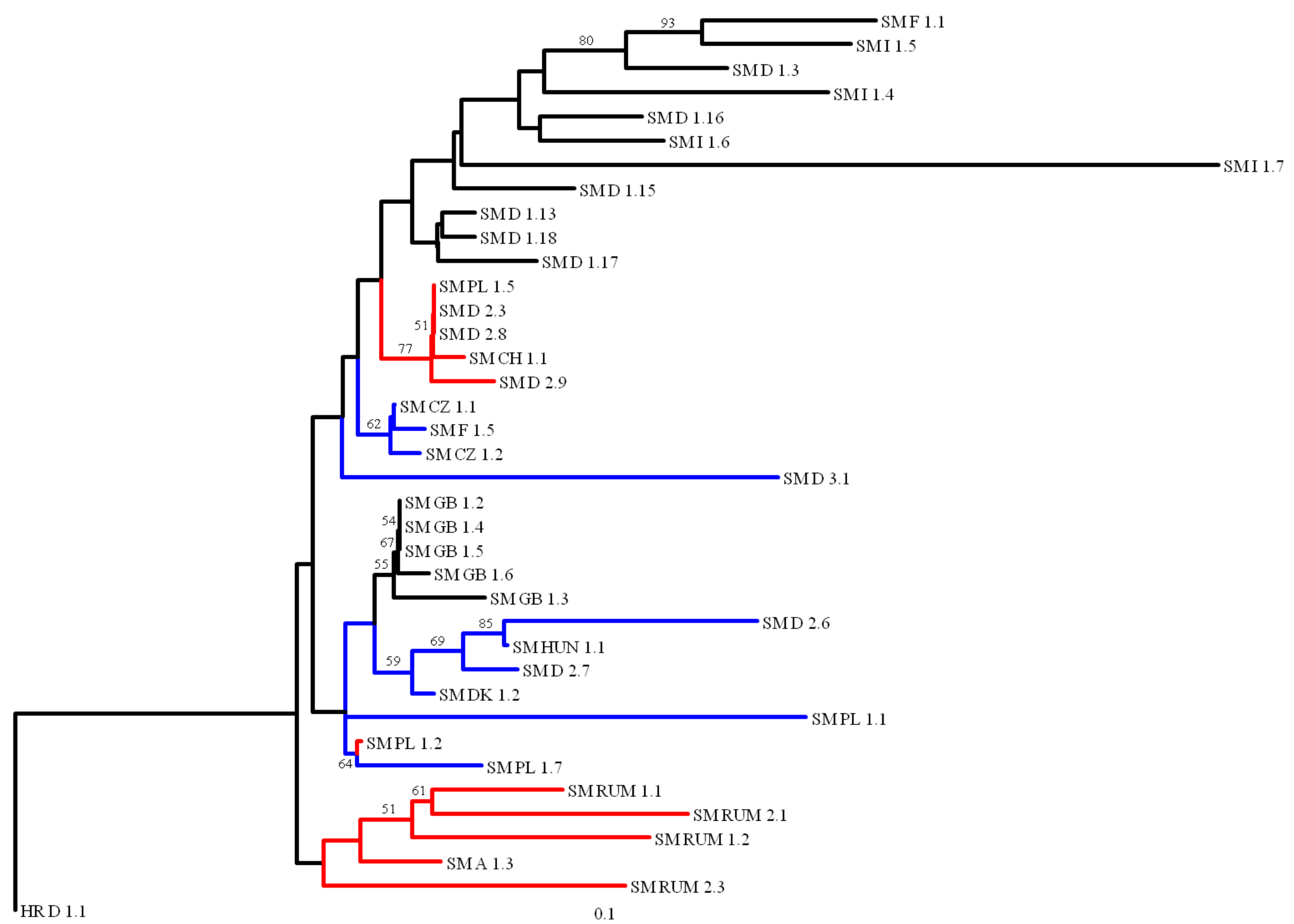

Figure A19: Neighbor-Joining tree of the ef $1 \alpha$ protein sequences of Steganacarus magnus. Outgroup is Hypochthonius rufulus (HD_D_1.1). Numbers on the branches are bootstrap values after 100,000 pseudo-replicates computed in PAUP*. Branch colours show the different clades (black, blue, red). 
Appendix

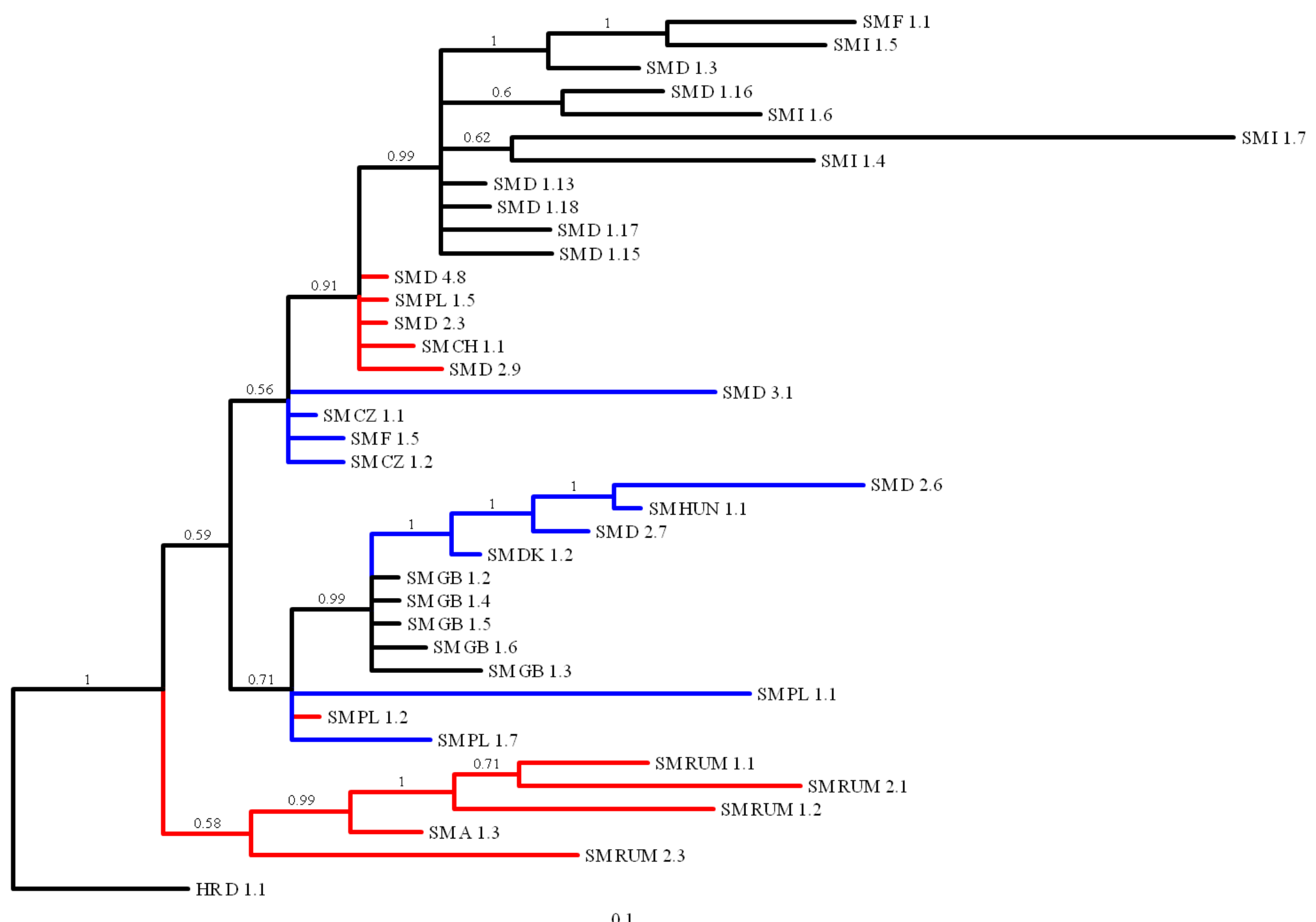

Figure A20: Bayesian phylogeny after $10 \times 10^{6}$ generations from the ef $1 \alpha$ protein sequences of Steganacarus magnus. Outgroup is Hypochthonius rufulus (HD_D_1.1). Numbers on the branches are posterior probabilities from MrBayes. Branch colours show the different clades (black, blue, red). 
Appendix

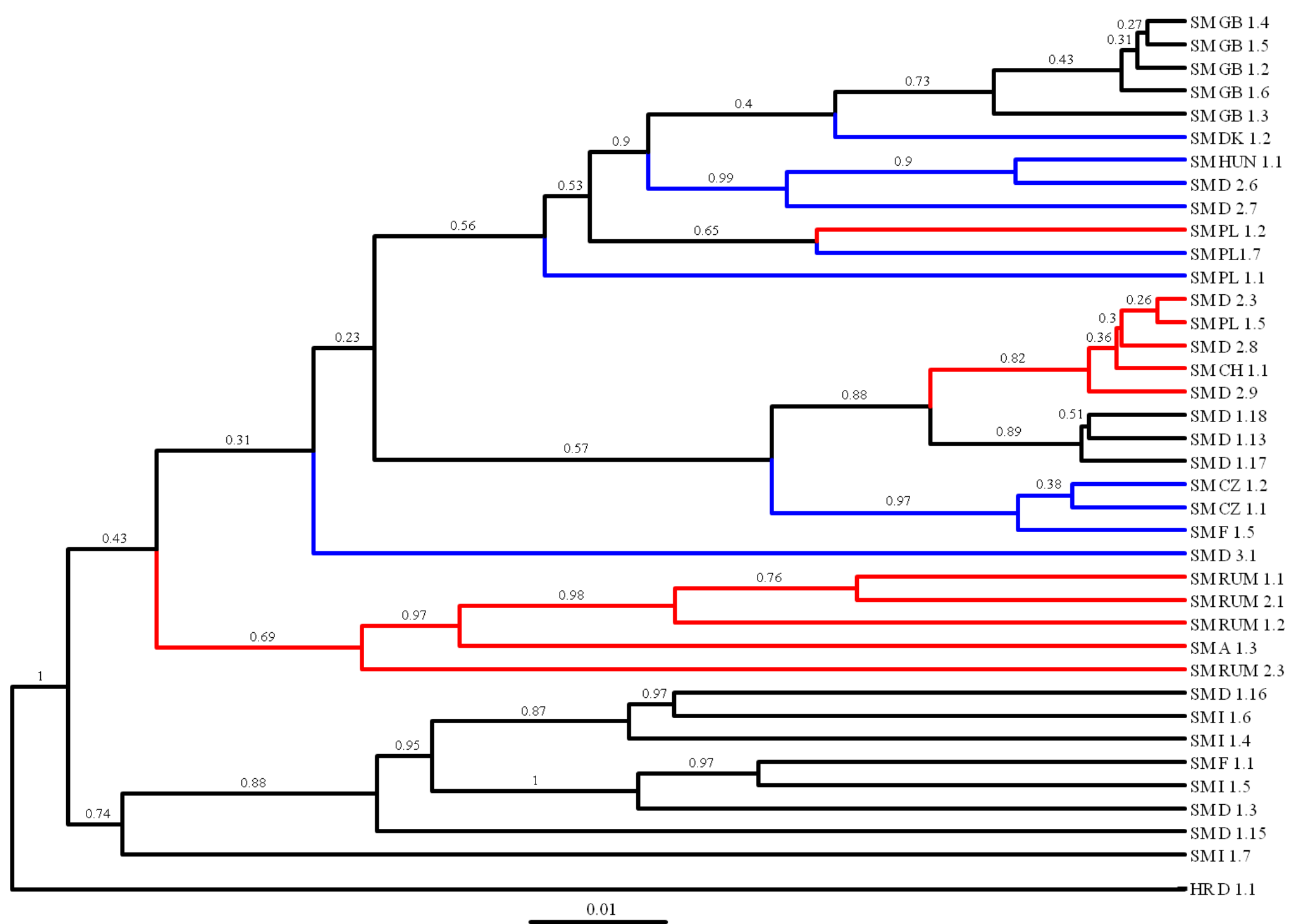

Figure A21: Bayesian phylogeny after 10x $10^{6}$ generations with Beast v1.5.4 from the ef $1 \alpha$ protein sequences of Steganacarus magnus. Outgroup is Hypochthonius rufulus (HD_D_1.1). Numbers on the branches are posterior probabilities from Beast. Branch colours show the different clades (black, blue, red). 


\section{Appendix}

Table A9: Mean pairwise percentage differences of uncorrected p-distances for the COI protein of Steganacarus magnus from eleven locations. The diagonal is the within population differences (bold) and below the diagonal the among population differences. Bold letters in red are the minimum and maximum differences within and among the populations. Locations with less than two individuals were excluded from the analysis.

\begin{tabular}{lcccccccccccc}
\hline Population & $\mathbf{1}$ & $\mathbf{2}$ & $\mathbf{3}$ & $\mathbf{4}$ & $\mathbf{5}$ & $\mathbf{6}$ & $\mathbf{7}$ & $\mathbf{8}$ & $\mathbf{9}$ & $\mathbf{1 0}$ & $\mathbf{1 1}$ \\
\hline $\mathbf{1}$ & A_1 & $\mathbf{0}$ & & & & & & & & & & \\
$\mathbf{2}$ & CZ_1 & 1.6 & $\mathbf{1}$ & & & & & & & & & \\
$\mathbf{3}$ & D_1 & $\mathbf{3 . 1}$ & 2.1 & $\mathbf{0 . 3}$ & & & & & & & & \\
$\mathbf{4}$ & D_2 & 1.5 & 1.1 & 2.5 & $\mathbf{1 . 1}$ & & & & & & & \\
$\mathbf{5}$ & DK_1 & 2.5 & 1.5 & 1.8 & 1.5 & $\mathbf{1 . 7}$ & & & & & & \\
$\mathbf{6}$ & F_1 & 3 & 1.9 & 0.2 & 2.4 & 1.7 & $\mathbf{0}$ & & & & & \\
$\mathbf{7}$ & GB_1 & 3 & 2.7 & 1.1 & 3.1 & 2.5 & 1 & $\mathbf{0}$ & & & & \\
$\mathbf{8}$ & I_1 & 3.1 & 2.1 & 0.4 & 2.5 & 1.6 & 0.3 & 1.1 & $\mathbf{0 . 3}$ & & & \\
$\mathbf{9}$ & PL_1 & 0.8 & 1.1 & 2.6 & 1.1 & 2 & 2.5 & 2.9 & 2.6 & $\mathbf{0 . 8}$ & & \\
$\mathbf{1 0}$ & RUM_1 & 0.1 & 1.7 & $\mathbf{3 . 1}$ & 1.4 & 2.5 & 3 & 3 & $\mathbf{3 . 1}$ & 0.9 & $\mathbf{0 . 3}$ & \\
$\mathbf{1 1}$ & RUM_2 & $\mathbf{0}$ & 1.6 & $\mathbf{3 . 1}$ & 1.4 & 2.5 & 3 & 3 & $\mathbf{3 . 1}$ & 0.9 & 0.1 & $\mathbf{0}$ \\
\hline
\end{tabular}

Table A10: Mean pairwise percentage differences of uncorrected p-distances for the ef $1 \alpha$ nucleotide of Steganacarus magnus from nine locations. The diagonal is the within population differences (bold) and below the diagonal the among population differences. Bold letters in red are the minimum and maximum differences within and among the populations. Locations with less than two individuals were excluded from the analysis.

\begin{tabular}{lcccccccccc}
\hline Population & $\mathbf{1}$ & $\mathbf{2}$ & $\mathbf{3}$ & $\mathbf{4}$ & $\mathbf{5}$ & $\mathbf{6}$ & $\mathbf{7}$ & $\mathbf{8}$ & $\mathbf{9}$ \\
\hline $\mathbf{1}$ & CZ_1 & $\mathbf{0}$ & & & & & & & & \\
$\mathbf{2}$ & D_1 & 0.9 & $\mathbf{0 . 4}$ & & & & & & & \\
$\mathbf{3}$ & D_2 & $\mathbf{0 . 5}$ & 1.7 & $\mathbf{0 . 9}$ & & & & & & \\
$\mathbf{4}$ & F_1 & 0.7 & 0.8 & 1.3 & $\mathbf{1 . 3}$ & & & & & \\
$\mathbf{5}$ & GB_1 & 1.6 & 1.1 & 1.4 & 1.6 & $\mathbf{0 . 5}$ & & & & \\
$\mathbf{6}$ & I_1 & 2.6 & 2.1 & 3.2 & 2.1 & 3 & $\mathbf{3 . 1}$ & & & \\
$\mathbf{7}$ & PL_1 & 1.1 & 2.1 & 1.2 & 1.9 & 1.7 & $\mathbf{3 . 7}$ & $\mathbf{1 . 6}$ & & \\
$\mathbf{8}$ & RUM_1 & 1 & 1.7 & 1.2 & 1.3 & 1.8 & 3 & 1.6 & $\mathbf{1 . 3}$ & \\
$\mathbf{9}$ & RUM_2 & 0.7 & 1.4 & 0.7 & 1 & 0.9 & 2.8 & 0.8 & 1 & $\mathbf{0}$ \\
\hline
\end{tabular}


Table A11: Tajima's D and Fu's FS neutrality tests of Steganacarus magnus COI nucleotide sequences from eleven locations. Bold red letters show significance (P<0.05).

\begin{tabular}{|c|c|c|c|c|c|c|c|c|c|c|c|c|c|}
\hline Neutrality tests & A_1 & $\mathrm{CZ}_{-} \mathbf{1}$ & D_1 & D_4 & DK_1 & F_2 & GB_1 & I_1 & PL_1 & RUM_3 & RUM_4 & Mean & s.d. \\
\hline \multicolumn{14}{|l|}{ Tajima's D test } \\
\hline Samplesize & 2 & 5 & 14 & 9 & 3 & 4 & 5 & 10 & 6 & 4 & 3 & 5.909 & 3.646 \\
\hline $\mathrm{S}$ & 0 & 193 & 35 & 215 & 159 & 2 & 76 & 14 & 160 & 20 & 10 & 80.364 & 84.270 \\
\hline $\mathrm{Pi}$ & 0 & 94.4 & 8.703 & 95.75 & 108.66667 & 1 & 31.6 & 6.644 & 85.2 & 10 & 6.667 & 40.785 & 44.848 \\
\hline Tajima's D & 0 & 0.145 & -0.905 & 1.094 & 6409109.877 & -0.710 & -1.014 & 1.569 & 1.4 & -0.854 & 0 & 582646.418 & 1932419.315 \\
\hline Tajima's D p-value & 1 & 0.584 & 0.185 & 0.906 & 0.999 & 0.284 & 0.137 & 0.958 & 0.954 & 0.07 & 0.72 & 0.618 & 0.38 \\
\hline \multicolumn{14}{|l|}{ Fu's FS test } \\
\hline Real no. of alleles & 1 & 5 & 9 & 5 & 3 & 3 & 4 & 5 & 6 & 3 & 3 & 4.273 & 2.102 \\
\hline Orig. no. of alleles & 1 & 5 & 9 & 5 & 3 & 3 & 4 & 5 & 6 & 3 & 3 & 4.273 & 2.102 \\
\hline Theta_pi & 0 & 94.4 & 8.703 & 95.75 & 108.667 & 1 & 31.6 & 6.644 & 85.2 & 10 & 6.667 & 40.785 & 44.848 \\
\hline Exp. no. of alleles & 0 & 4.897 & 8.661 & 8.645 & 2.973 & 2.083 & 4.711 & 6.412 & 5.831 & 3.512 & 2.639 & 4.579 & 2.7 \\
\hline FS & 0 & 2.208 & 0.171 & 12.039 & 3.584 & -0.887 & 3.581 & 2.352 & 1.671 & 2.624 & 0.703 & 2.55 & 3.471 \\
\hline FS p-value & N.A. & 0.54 & 0.534 & 1 & 0.583 & 0.089 & 0.895 & 0.866 & 0.495 & 0.84 & 0.414 & N.A. & N.A. \\
\hline
\end{tabular}

Table A12: Tajima's D and Fu's FS neutrality tests of Steganacarus magnus COI protein sequences from eleven locations. Bold red letters show significance (P $<0.05)$.

\begin{tabular}{|c|c|c|c|c|c|c|c|c|c|c|c|c|c|}
\hline Neutrality tests & A_1 & CZ_1 & D_1 & D_4 & DK_1 & F_2 & GB_1 & I_1 & PL_1 & RUM_3 & RUM_4 & Mean & s.d. \\
\hline \multicolumn{14}{|l|}{$\overline{\text { Tajima's D test }}$} \\
\hline Samplesize & 2 & 5 & 14 & 9 & 3 & 4 & 5 & 10 & 6 & 4 & 3 & 5.909 & 3.646 \\
\hline $\mathrm{S}$ & 0 & 5 & 4 & 4 & 5 & 0 & 0 & 2 & 3 & 1 & 0 & 2.182 & 2.089 \\
\hline $\mathrm{Pi}$ & 0 & 1.9 & 0.67 & 2.111 & 3.333 & 0 & 0 & 0.644 & 1.6 & 0.5 & 0 & 0.978 & 1.11 \\
\hline Tajima's D & 0 & -1.405 & -1.539 & 1.766 & 0 & 0 & 0 & -0.285 & 1.124 & -0.612 & 0 & -0.086 & 0.954 \\
\hline Tajima's D p-value & 1 & 0 & 0.043 & 0.977 & 0.809 & 1 & 1 & 0.325 & 0.881 & 0.386 & 1 & 0.675 & 0.404 \\
\hline \multicolumn{14}{|l|}{ Fu's FS test } \\
\hline Real no. of alleles & 1 & 5 & 4 & 4 & 3 & 1 & 3 & 5 & 5 & 3 & 1 & 3.182 & 1.601 \\
\hline Orig. no. of alleles & 1 & 5 & 4 & 4 & 3 & 1 & 3 & 5 & 5 & 3 & 1 & 3.182 & 1.601 \\
\hline Theta_pi & 0 & 1.9 & 0.67 & 2.111 & 3.333 & 0 & 0 & 0.644 & 1.6 & 0.5 & 0 & 0.978 & 1.11 \\
\hline Exp. no. of alleles & 0 & 2.852 & 2.653 & 3.948 & 2.394 & 0 & 3 & 2.388 & 2.936 & 1.676 & 0 & 1.986 & 1.387 \\
\hline FS & 0 & 0.558 & -1.29 & 1.919 & -0.077 & 0 & 0 & -0.332 & 2.506 & 0.172 & 0 & 0.314 & 1.049 \\
\hline FS p-value & N.A. & 0.54 & 0.072 & 0.848 & 0.235 & N.A. & N.A. & 0.29 & 0.865 & 0.34 & N.A. & N.A. & N.A. \\
\hline
\end{tabular}




\section{Appendix}

Table A13: Tajima's D and Fu's FS neutrality tests of Steganacarus magnus ef $1 \alpha$ nucleotide sequences from eleven locations. Bold red letters show significance $(\mathrm{P}<0.05)$.

\begin{tabular}{|c|c|c|c|c|c|c|c|c|c|c|c|}
\hline Neutrality tests & CZ_1 & D_1 & D_4 & F_2 & GB_1 & I_1 & PL_1 & RUM_3 & RUM_4 & Mean & s.d. \\
\hline \multicolumn{12}{|l|}{ Tajima's D test } \\
\hline Samplesize & 2 & 6 & 5 & 2 & 5 & 4 & 4 & 2 & 2 & 3.556 & 1.590 \\
\hline S & 3 & 38 & 61 & 65 & 8 & 77 & 61 & 35 & 39 & 43 & 25.539 \\
\hline $\mathrm{Pi}$ & 3 & 17.133 & 32.8 & 65 & 3.4 & 42 & 30.5 & 35 & 39 & 29.759 & 19.636 \\
\hline Tajima's D & 0 & 0.188 & 0.910 & 0 & -0.807 & 0 & -0.869 & 0 & 0 & -0.064 & 0.528 \\
\hline Tajima's D p-value & 1 & 0.581 & 0.808 & 1 & 0.304 & 0.662 & 0.027 & 1 & 1 & 0.709 & 0.353 \\
\hline \multicolumn{12}{|l|}{ Fu's FS test } \\
\hline Real no. of alleles & 2 & 6 & 4 & 2 & 4 & 4 & 4 & 2 & 2 & 3.333 & 1.414 \\
\hline Orig. no. of alleles & 2 & 6 & 4 & 2 & 4 & 4 & 4 & 2 & 2 & 3.333 & 1.414 \\
\hline Theta_pi & 3 & 17.133 & 32.8 & 65 & 3.4 & 42 & 30.5 & 35 & 39 & 29.759 & 19.636 \\
\hline Exp. no. of alleles & 1.75 & 5.276 & 4.720 & 1.985 & 3.393 & 3.865 & 3.817 & 1.972 & 1.975 & 3.195 & 1.326 \\
\hline FS & 1.099 & -0.193 & 3.648 & 4.174 & -0.128 & 1.903 & 1.567 & 3.555 & 3.664 & 2.143 & 1.688 \\
\hline FS p-value & 0.434 & 0.275 & 0.9 & 0.611 & 0.343 & 0.516 & 0.492 & 0.612 & 0.608 & 0.532 & 0.183 \\
\hline
\end{tabular}

Table A14: Tajima's D and Fu's FS neutrality tests of Steganacarus magnus ef $1 \alpha$ protein sequences from eleven locations. Bold red letters show significance $(\mathrm{P}<0.05)$.

\begin{tabular}{lccccccccccc}
\hline Neutrality tests & CZ_1 & D_1 & D_4 & F_2 & GB_1 & I_1 & PL_1 & RUM_3 & RUM_4 & Mean & s.d. \\
\hline Tajima's D test & & & & & & & & & & & \\
Samplesize & 2 & 6 & 5 & 2 & 5 & 4 & 4 & 2 & 2 & 3.556 & 1.590 \\
$\mathrm{~S}$ & 0 & 2 & 3 & 2 & 2 & 9 & 5 & 2 & 0 & 2.778 & 2.774 \\
Pi & 0 & 0.667 & 1.3 & 2 & 0.8 & 4.667 & 2.5 & 2 & 0 & 1.548 & 1.468 \\
Tajima's D & 0 & -1.132 & -0.612 & 0 & -0.973 & -0.492 & -0.797 & 0 & 0 & -0.445 & 0.460 \\
Tajima's D p-value & 1 & 0.154 & 0.388 & 1 & 0.191 & 0.463 & 0.170 & 1 & 1 & 0.596 & 0.396 \\
Fu's FS test & & & & & & & & & & & \\
Real no. of alleles & 2 & 6 & 4 & 2 & 3 & 4 & 4 & 2 & 2 & 3.222 & 1.394 \\
Orig. no. of alleles & 2 & 6 & 4 & 2 & 3 & 4 & 4 & 2 & 2 & 3.222 & 1.394 \\
Theta_pi & 0 & 0.667 & 1.3 & 2 & 0.8 & 4.667 & 2.5 & 2 & 0 & 1.548 & 1.468 \\
Exp. no. of alleles & 0 & 2.092 & 2.507 & 1.667 & 2.107 & 3.132 & 2.724 & 1.667 & 0 & 1.766 & 1.108 \\
FS & 0 & 0.952 & -1.786 & 0.693 & -0.829 & -0.615 & -1.514 & 0.693 & 0 & -0.267 & 0.986 \\
FS p-value & N.A. & 0.613 & $\mathbf{0 . 0 2 1}$ & 0.361 & 0.089 & 0.188 & 0.056 & 0.366 & N.A. & N.A. & N.A. \\
\hline
\end{tabular}


Table A15: Results of the McDonald-Kreitman test for COI of Steganacarus magnus. The differences between seven populations are significant $(* 0.01<\mathrm{P}<0.05)$, between other two populations high significant $(* * 0.001<\mathrm{P}<0.01)$ and between seven populations extremely high significant $(* * * \mathrm{P}<0.001)$. Number of fixed and polymorph synonymous and non-synonymous mutations are shown. Locations with less than two individuals were excluded.

\begin{tabular}{|c|c|c|c|c|c|c|c|c|c|c|c|c|c|c|c|c|c|c|c|}
\hline \multicolumn{2}{|l|}{ Population } & \multicolumn{3}{|c|}{$\begin{array}{c}\text { A } 1 \\
\text { syn. nons. sign. }\end{array}$} & \multicolumn{3}{|c|}{$\begin{array}{c}\text { CZ 1 } \\
\text { syn. nons. sign. } \\
\end{array}$} & \multicolumn{3}{|c|}{$\begin{array}{c}\text { D } 1 \\
\text { syn. nons. sign. } \\
\end{array}$} & \multicolumn{3}{|c|}{$\begin{array}{c}\text { D } 2 \\
\text { syn. nons. sign. }\end{array}$} & \multicolumn{3}{|c|}{$\begin{array}{c}\text { DK 1 } \\
\text { syn. nons. sign. } \\
\end{array}$} & \multicolumn{3}{|c|}{$\begin{array}{c}\text { F } 1 \\
\text { syn. nons. sign. }\end{array}$} \\
\hline CZ 1 & fixed & 55 & 3 & $0.0203^{*}$ & & & & & & & & & & & & & & & \\
\hline Decin & poly & 169 & 35 & & & & & & & & & & & & & & & & \\
\hline D 1 & fixed & 112 & 46 & $0.1653 \mathrm{~ns}$ & 38 & 19 & $0.0531 \mathrm{~ns}$ & & & & & & & & & & & & \\
\hline Kranichstein & poly & 21 & 15 & & 185 & 48 & & . & & & & & & & & & & & \\
\hline D 2 & fixed & 44 & 5 & $0.6424 \mathrm{~ns}$ & 7 & 0 & $0.5996 \mathrm{~ns}$ & 32 & 19 & $0.0038 * *$ & & & & & & & & & \\
\hline Meckl. Seenpl. & poly & 19 & 30 & & 284 & 46 & & 203 & 43 & & & & & & & & & & \\
\hline DK 1 & fixed & 82 & 24 & $0.2064 \mathrm{~ns}$ & 9 & 2 & $1 \mathrm{~ns}$ & 0 & 0 & $-\mathrm{ns}$ & 1 & 0 & $1 \mathrm{~ns}$ & & & & & & \\
\hline Copenhagen & poly & 112 & 49 & & 234 & 66 & & 128 & 62 & & 223 & 55 & & & & & & & \\
\hline F 1 & fixed & 11 & 2 & $1 \mathrm{~ns}$ & 42 & 19 & $0.0282 *$ & 1 & 0 & $1 \mathrm{~ns}$ & 34 & 19 & $0.0005^{* * *}$ & 5 & 0 & $0.3228 \mathrm{~ns}$ & & & \\
\hline Loire & poly & 2 & 0 & & 171 & 35 & & 24 & 14 & & 191 & 30 & & 114 & 48 & & & & \\
\hline GB 1 & fixed & 94 & 47 & $0.0000 * * *$ & 32 & 21 & $0.0002 * * *$ & 73 & 10 & $0.316 \mathrm{~ns}$ & 25 & 20 & $0.0000^{* * *}$ & 40 & 6 & $0.0866 \mathrm{~ns}$ & 76 & 12 & $0.3175 \mathrm{~ns}$ \\
\hline Bedford & poly & 70 & 6 & & 215 & 39 & & 89 & 20 & & 234 & 35 & & 164 & 54 & & 72 & 6 & \\
\hline I 1 & fixed & 11 & 47 & $0.7704 \mathrm{~ns}$ & 40 & 19 & $0.0308 *$ & 0 & 0 & $-\mathrm{ns}$ & 32 & 19 & $0.0007 * * *$ & 2 & 0 & $0.5739 \mathrm{~ns}$ & 1 & 0 & $1 \mathrm{~ns}$ \\
\hline Grosetto & poly & 10 & 5 & & 177 & 40 & & 22 & 18 & & 195 & 35 & & 119 & 53 & & 12 & 5 & \\
\hline PL 1 & fixed & 71 & 8 & $0.0148 *$ & 1 & 0 & $1 \mathrm{~ns}$ & 48 & 23 & $0.4414 \mathrm{~ns}$ & 0 & 0 & $-\mathrm{ns}$ & 17 & 3 & $0.3009 \mathrm{~ns}$ & 51 & 23 & $0.2003 \mathrm{~ns}$ \\
\hline Krakau & poly & 126 & 38 & & 224 & 43 & & 142 & 52 & & 240 & 44 & & 201 & 74 & & 128 & 38 & \\
\hline RUM 1 & fixed & 100 & 5 & $0.0359 *$ & 29 & 1 & $0.0577 \mathrm{~ns}$ & 99 & 44 & $0.866 \mathrm{~ns}$ & 34 & 3 & $0.4381 \mathrm{~ns}$ & 62 & 23 & $0.7706 \mathrm{~ns}$ & 105 & 44 & $0.4395 \mathrm{~ns}$ \\
\hline Bagau & poly & 16 & 4 & & 177 & 37 & & 38 & 18 & & 197 & 31 & & 123 & 52 & & 17 & 4 & \\
\hline RUM 2 & fixed & 104 & 5 & $1 \mathrm{~ns}$ & 31 & 1 & $0.0379 *$ & 105 & 45 & $1 \mathrm{~ns}$ & 33 & 3 & $0.5895 \mathrm{~ns}$ & 64 & 23 & $0.6634 \mathrm{~ns}$ & 110 & 45 & $0.0375^{*}$ \\
\hline Cluj & poly & 10 & 0 & & 173 & 35 & & 31 & 14 & & 196 & 30 & & 119 & 49 & & 12 & 0 & \\
\hline
\end{tabular}


Appendix

Table A15: continue

\begin{tabular}{|c|c|c|c|c|c|c|c|c|c|c|c|c|c|}
\hline \multicolumn{2}{|c|}{ Population } & \multicolumn{3}{|c|}{$\begin{array}{c}\text { GB 1 } \\
\text { syn. nons. sign. }\end{array}$} & \multicolumn{3}{|c|}{$\begin{array}{c}\text { I 1 } \\
\text { syn. nons. sign. }\end{array}$} & \multicolumn{3}{|c|}{$\begin{array}{c}\text { PL 1 } \\
\text { syn. nons. sign. }\end{array}$} & \multicolumn{3}{|c|}{$\begin{array}{c}\text { RUM 1 } \\
\text { syn. nons. sign. }\end{array}$} \\
\hline I 1 & fixed & 76 & 11 & $1 \mathrm{~ns}$ & & & & & & & & & \\
\hline Grosetto & poly & 78 & 11 & & & & & & & & & & \\
\hline PL 1 & fixed & 47 & 26 & $0.0062 * *$ & 49 & 23 & $0.2686 \mathrm{~ns}$ & & & & & & \\
\hline Krakau & poly & 182 & 43 & & 132 & 43 & & & & & & & \\
\hline RUM 1 & fixed & 75 & 46 & $0.0000^{* * *}$ & 101 & 44 & $0.6822 \mathrm{~ns}$ & 58 & 8 & $0.071 \mathrm{~ns}$ & & & \\
\hline Bagau & poly & 85 & 10 & & 26 & 9 & & 134 & 40 & & & & \\
\hline RUM 2 & fixed & 82 & 47 & $0.0000 * * *$ & 107 & 45 & $0.3534 \mathrm{~ns}$ & 60 & 9 & $0.1087 \mathrm{~ns}$ & 5 & 0 & $1 \mathrm{~ns}$ \\
\hline Cluj & poly & 75 & 6 & & 20 & 5 & & 131 & 38 & & 24 & 4 & \\
\hline
\end{tabular}


Table A16: Results of the McDonald-Kreitman test for ef $1 \alpha$ from Steganacarus magnus. The differences between two populations are significant $(* 0.01<\mathrm{P}<0.05)$, between other three populations high significant $(* * 0.001<\mathrm{P}<0.01)$ and between 14 populations extremely high significant $(* * * \mathrm{P}<0.001)$. Number of fixed and polymorph synonymous and non-synonymous mutations are shown. Locations with less than two individuals are excluded.

\begin{tabular}{|c|c|c|c|c|c|c|c|c|c|c|c|c|c|c|c|c|c|c|c|}
\hline \multicolumn{2}{|c|}{ Population } & \multicolumn{3}{|c|}{$\begin{array}{c}\text { CZ 1 } \\
\text { syn. nons. sign. }\end{array}$} & \multicolumn{3}{|c|}{$\begin{array}{c}\text { D } 1 \\
\text { syn. nons. sign. }\end{array}$} & \multicolumn{3}{|c|}{$\begin{array}{c}\text { D } 2 \\
\text { syn. nons. sign. }\end{array}$} & \multicolumn{3}{|c|}{$\begin{array}{c}\text { F } 1 \\
\text { syn. nons. sign. } \\
\end{array}$} & \multicolumn{3}{|c|}{$\begin{array}{c}\text { GB } 1 \\
\text { syn. nons. sign. }\end{array}$} & \multicolumn{3}{|c|}{$\begin{array}{c}\text { I } 1 \\
\text { syn. nons. sign. } \\
\end{array}$} \\
\hline$\overline{D 1}$ & fixed & 35 & 4 & $0.0000^{* * *}$ & & & & & & & & & & & & & & & \\
\hline Kranichstein & poly & 19 & 24 & & & & & & & & & & & & & & & & \\
\hline D 2 & fixed & 6 & 0 & $0.1684 \mathrm{~ns}$ & 27 & 2 & $0.0003 * * *$ & & & & & & & & & & & & \\
\hline Meckl. Seenpl. & poly & 43 & 21 & & 58 & 42 & & & & & & & & & & & & & \\
\hline F 1 & fixed & 6 & 0 & $0.1701 \mathrm{~ns}$ & 2 & 0 & $0.5204 \mathrm{~ns}$ & 6 & 0 & $0.176 \mathrm{~ns}$ & & & & & & & & & \\
\hline Loire & poly & 46 & 22 & & 52 & 34 & & 80 & 38 & & & & & & & & & & \\
\hline GB 1 & fixed & 46 & 4 & $0.0067 * *$ & 7 & 5 & $0.5196 \mathrm{~ns}$ & 33 & 1 & $0.0002 * * *$ & 13 & 2 & $0.1322 \mathrm{~ns}$ & & & & & & \\
\hline Bedford & poly & 6 & 5 & & 21 & 27 & & 44 & 24 & & 47 & 25 & & & & & & & \\
\hline I 1 & fixed & 32 & 4 & $0.0000 * * *$ & 0 & 0 & $-\mathrm{ns}$ & 23 & 2 & $0.0000 * * *$ & 1 & 0 & $1 \mathrm{~ns}$ & 8 & 5 & $0.1434 \mathrm{~ns}$ & & & \\
\hline Grosetto & poly & 32 & 51 & & 35 & 62 & & 69 & 66 & & 64 & 61 & & 34 & 53 & & & & \\
\hline PL 1 & fixed & 5 & 0 & $0.1487 \mathrm{~ns}$ & 27 & 2 & $0.0001 * * *$ & 0 & 0 & $-\mathrm{ns}$ & 5 & 0 & $0.1582 \mathrm{~ns}$ & 34 & 2 & $0.0000 * * *$ & 22 & 2 & $0.0000 * * *$ \\
\hline Krakau & poly & 38 & 26 & & 51 & 46 & & 51 & 39 & & 73 & 44 & & 38 & 29 & & 64 & 71 & \\
\hline RUM 1 & fixed & 28 & 10 & $0.4596 \mathrm{~ns}$ & 41 & 9 & $0.0005 * * *$ & 11 & 7 & $0.7934 \mathrm{~ns}$ & 23 & 8 & $0.3861 \mathrm{~ns}$ & 45 & 12 & $0.0491 *$ & 34 & 10 & $0.0003 * * *$ \\
\hline Bagau & poly & 24 & 14 & & 37 & 36 & & 56 & 31 & & 60 & 32 & & 26 & 17 & & 52 & 63 & \\
\hline RUM 2 & fixed & 37 & 8 & $0.0006^{* * *}$ & 48 & 7 & $0.0000 * * *$ & 16 & 2 & $0.0091 * *$ & 28 & 4 & $0.0027 * *$ & 52 & 8 & $0.0000 * * *$ & 43 & 7 & $0.0000 * * *$ \\
\hline Cluj & poly & 19 & 22 & & 34 & 44 & & 55 & 42 & & 61 & 43 & & 21 & 26 & & 47 & 72 & \\
\hline
\end{tabular}

\begin{tabular}{cccccccc}
\hline \multirow{2}{*}{ Population } & \multicolumn{4}{c}{ PL 1 } & \multicolumn{3}{c}{ RUM 1 } \\
& & syn. nons. sign. & syn. nons. sign. \\
\hline RUM 1 & fixed & 12 & 7 & $1 \mathrm{~ns}$ & & & \\
Bagau & poly & 54 & 36 & & & & \\
RUM 2 & fixed & 15 & 4 & $0.0422 *$ & 4 & 2 & $0.6773 \mathrm{~ns}$ \\
Cluj & poly & 51 & 47 & & 36 & 34 & \\
\hline
\end{tabular}


Appendix

Table A17: Neutrality indices of COI from Steganacarus magnus computed in the McDonald-Kreitman test with DnaSP v5 of eleven locations. Locations with less than two individuals were excluded.

\begin{tabular}{ccccccccccc}
\hline Population & A 1 & CZ 1 & D 1 & D 2 & DK 1 & F 1 & GB 1 & I 1 & PL 1 & RUM 1 \\
\hline CZ 1 & 3.797 & & & & & & & & & \\
D 1 & 1.739 & 0.519 & & & & & & & & \\
D 2 & 1.389 & - & 0.357 & & & & & & & \\
DK 1 & 1.495 & 1.269 & - & - & & & & & & \\
F 1 & 0 & 0.452 & - & 0.281 & - & & & & & \\
GB 1 & 0.171 & 0.276 & 1.64 & 0.187 & 2.195 & 0.528 & & & & \\
I 1 & 1.213 & 0.476 & - & 0.302 & - & - & 0.974 & & & \\
PL 1 & 2.677 & - & 0.764 & - & 2.086 & 0.658 & 0.427 & 0.694 & & \\
RUM 1 & 5 & 6.062 & 1.066 & 1.783 & 1.14 & 0.561 & 0.192 & 0.795 & 2.164 & \\
RUM 2 & 0 & 6.272 & 1.054 & 1.684 & 1.146 & 0 & 0.14 & 0.594 & 1.934 & - \\
\hline
\end{tabular}

Table A18: Neutrality indices of ef $1 \alpha$ from Steganacarus magnus computed in the McDonald-Kreitman test with DnaSP v5 of nine locations. Locations with less than two individuals were excluded.

\begin{tabular}{ccccccccc}
\hline Population & CZ 1 & D 1 & D 2 & F 1 & GB 1 & I 1 & PL 1 & RUM 1 \\
\hline D 1 & 11.053 & & & & & & & \\
D 2 & - & 9.776 & & & & & & \\
F 1 & - & - & - & & & & & \\
GB 1 & 9.583 & 1.8 & 18 & 3.457 & & & & \\
I 1 & 12.75 & - & 11 & - & 2.494 & & & \\
PL 1 & - & 12.176 & - & - & 12.974 & 12.203 & & \\
RUM 1 & 1.633 & 4.432 & 0.87 & 1.533 & 2.452 & 4.119 & 1.143 & \\
RUM 2 & 5.355 & 8.874 & 6.109 & 4.934 & 8.048 & 9.41 & 3.456 & 1.889 \\
\hline
\end{tabular}


Appendix

Table A19: Standard diversity measures of Nothrus silvestris with DnaSP v5. Populations with less than two individuals were excluded.

\begin{tabular}{|c|c|c|c|c|c|c|c|c|c|}
\hline population & $\begin{array}{c}\text { sample } \\
\text { size } \\
\mathbf{n}\end{array}$ & $\begin{array}{c}\text { invarible } \\
\text { sites } \\
\mathbf{N}_{\text {is }}\end{array}$ & $\begin{array}{c}\text { variable } \\
\text { sites } \\
\mathbf{N}_{\mathrm{vs}}\end{array}$ & $\begin{array}{c}\text { parsimony } \\
\text { inform. sites } \\
\mathbf{N}_{\text {pars }}\end{array}$ & $\begin{array}{c}\text { number of } \\
\text { singeltons } \\
\mathbf{N}_{\mathrm{s}}\end{array}$ & $\begin{array}{c}\text { number of } \\
\text { haplotypes } \\
N_{h}\end{array}$ & $\begin{array}{c}\text { haplotype } \\
\text { diversity } \\
\mathbf{H}_{\mathrm{d}}\end{array}$ & variance & $\begin{array}{c}\text { nucleotide } \\
\text { diversity } \\
\Pi_{n}\end{array}$ \\
\hline$\overline{\mathrm{CH}} \_2$ & 3 & 572 & 9 & 0 & 9 & 2 & 0.67 & 0.099 & 0.01 \\
\hline CH_3 & 2 & 579 & 2 & 0 & 2 & 2 & 1 & 0.25 & 0.003 \\
\hline D_1 & 4 & 581 & 0 & 0 & 0 & 1 & 0 & 0 & 0 \\
\hline D_2 & 4 & 570 & 11 & 1 & 10 & 4 & 1 & 0.031 & 0.010 \\
\hline D_4 & 8 & 561 & 20 & 10 & 10 & 6 & 0.93 & 0.007 & 0.014 \\
\hline D_6 & 2 & 571 & 10 & 0 & 10 & 2 & 1 & 0.25 & 0.017 \\
\hline D_7 & 4 & 581 & 0 & 0 & 0 & 1 & 0 & 0 & 0 \\
\hline D_8 & 3 & 581 & 0 & 0 & 0 & 1 & 0 & 0 & 0 \\
\hline D_9 & 4 & 581 & 0 & 0 & 0 & 1 & 0 & 0 & 0 \\
\hline D_11 & 4 & 578 & 3 & 0 & 3 & 2 & 0.5 & 0.070 & 0.003 \\
\hline D_16 & 4 & 580 & 1 & 0 & 1 & 2 & 0.5 & 0.070 & 0.001 \\
\hline D_17 & 3 & 568 & 13 & 0 & 13 & 3 & 1 & 0.074 & 0.015 \\
\hline D_18 & 4 & 581 & 0 & 0 & 0 & 1 & 0 & 0 & 0 \\
\hline F_3 & 4 & 572 & 9 & 0 & 9 & 3 & 0.83 & 0.049 & 0.008 \\
\hline FIN_1 & 4 & 581 & 0 & 0 & 0 & 1 & 0 & 0 & 0 \\
\hline GB_1 & 4 & 571 & 10 & 0 & 10 & 3 & 0.83 & 0.049 & 0.009 \\
\hline GB_2 & 4 & 568 & 13 & 11 & 2 & 3 & 0.83 & 0.049 & 0.014 \\
\hline NL_1 & 3 & 581 & 0 & 0 & 0 & 1 & 0 & 0 & 0 \\
\hline NL_2 & 2 & 572 & 9 & 0 & 9 & 2 & 1 & 0.25 & 0.015 \\
\hline PL_2 & 13 & 575 & 6 & 0 & 6 & 2 & 0.15 & 0.016 & 0.002 \\
\hline RUM_1 & 3 & 578 & 3 & 0 & 3 & 2 & 0.67 & 0.099 & 0.003 \\
\hline RUM_2 & 4 & 581 & 0 & 0 & 0 & 1 & 0 & 0 & 0 \\
\hline RUM_5 & 4 & 581 & 0 & 0 & 0 & 1 & 0 & 0 & 0 \\
\hline S_3 & 4 & 571 & 10 & 0 & 10 & 3 & 0.83 & 0.049 & 0.009 \\
\hline all & 98 & 536 & 45 & 21 & 24 & 25 & 0.67 & 0.003 & 0.009 \\
\hline
\end{tabular}


Appendix

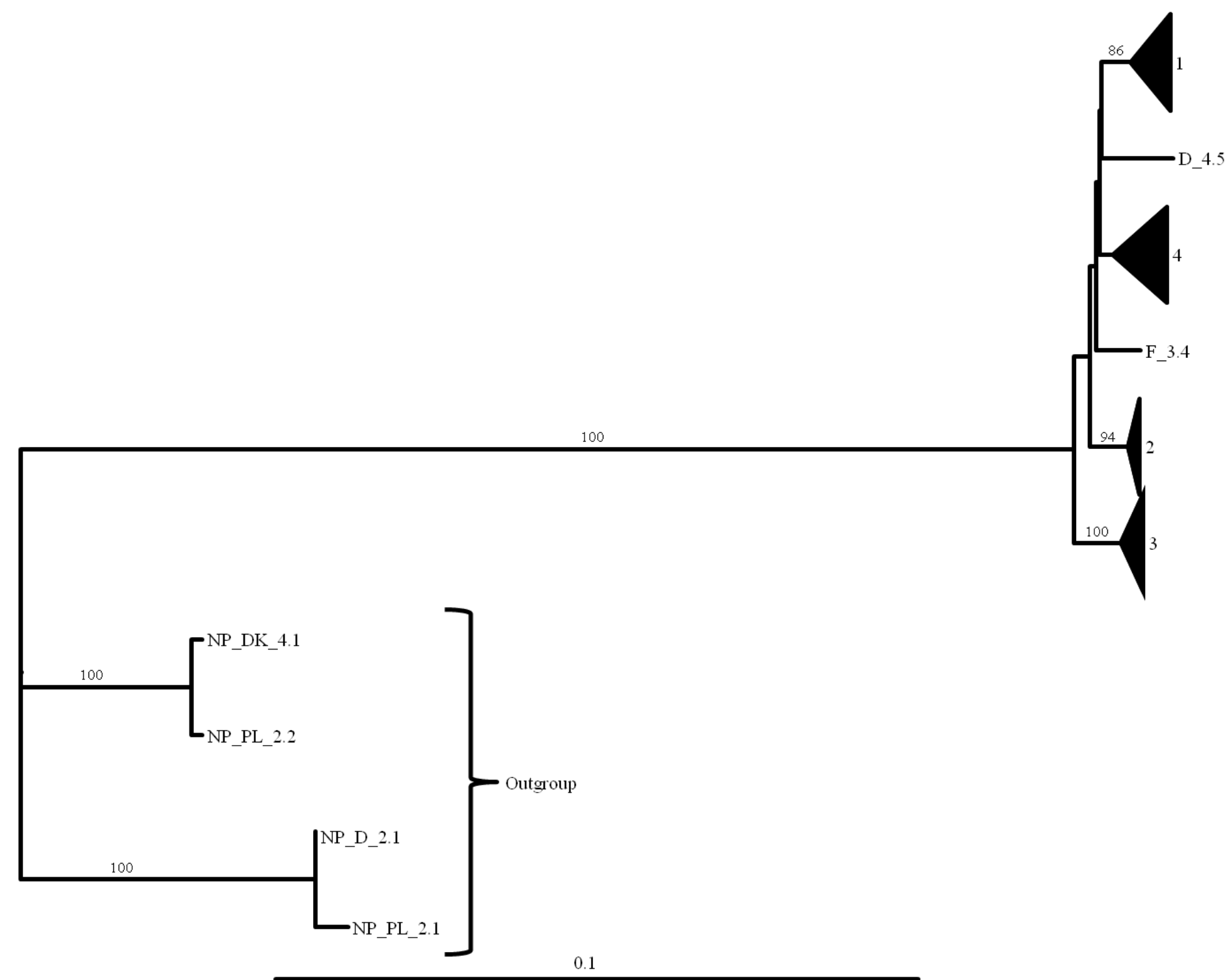

Figure A22: Neighbor-Joining tree of 100 COI nucleotide sequences of Nothrus silvestris. Outgroup are four individuals of Nothrus palustris from different locations. Numbers on the branches are bootstrap values of 100,000 pseudo-replicates. Tip numbers are the different subclades and explain in Table 15. 
Appendix

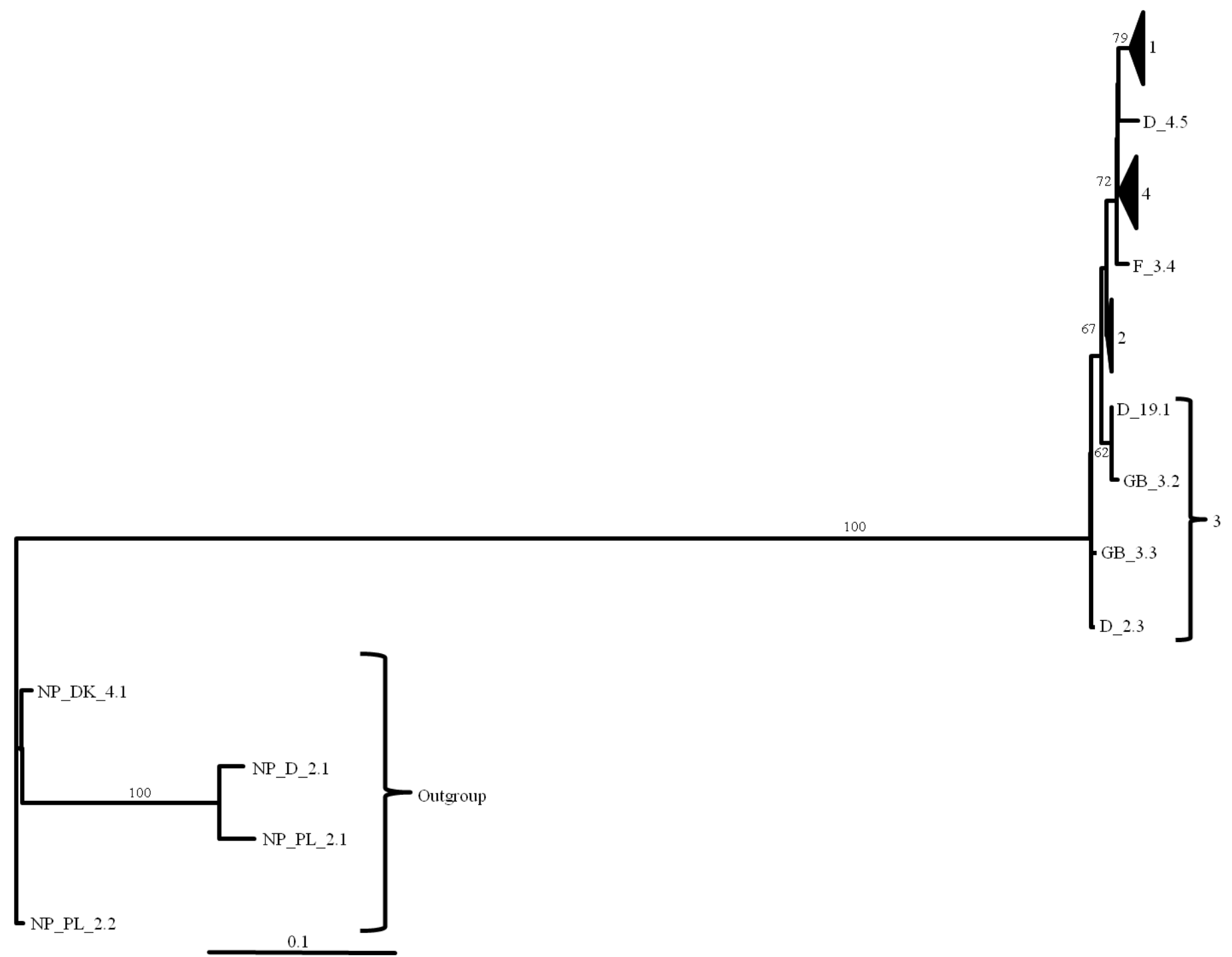

Figure A23: Neighbor-Joining tree of 100 COI nucleotide sequences of Nothrus silvestris with model of sequence evolution HKY+G. Outgroup are four individuals of Nothrus palustris from different locations. Numbers on the branches are bootstrap values of 100,000 pseudo-replicates. Tip numbers are the different subclades and explain in Table 15. 
Appendix

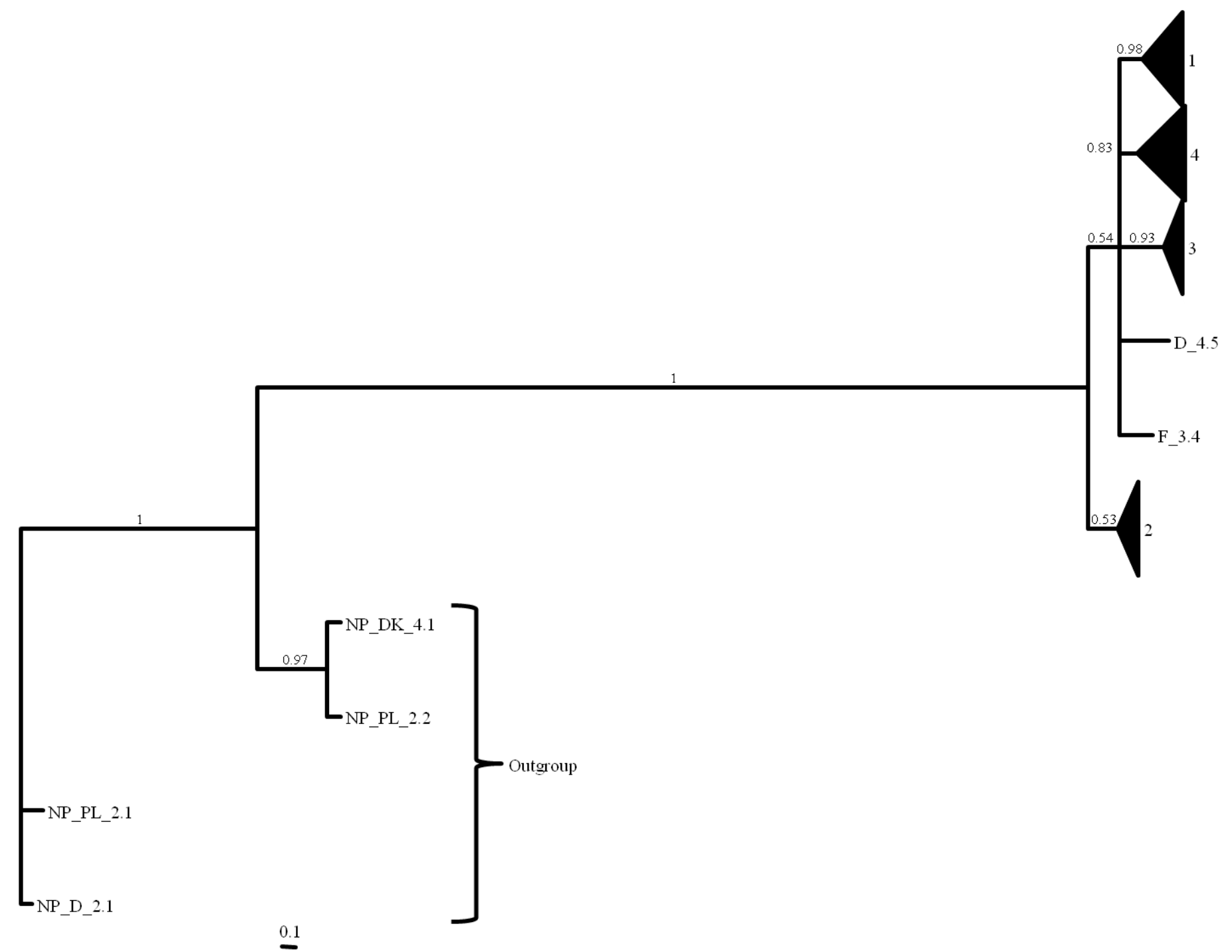

Figure A24: Bayesian tree after 10x $10^{6}$ generations from 100 COI nucleotide sequences of Nothrus silvestris with MrBayes. Split frequencies of 0.007767 and burnin of $25 \%$. Outgroup four individuals of Nothrus palustris from different locations. Numbers on the branches are posterior probabilities. Tip numbers are the different subclades and explain in Table 15. 
Appendix

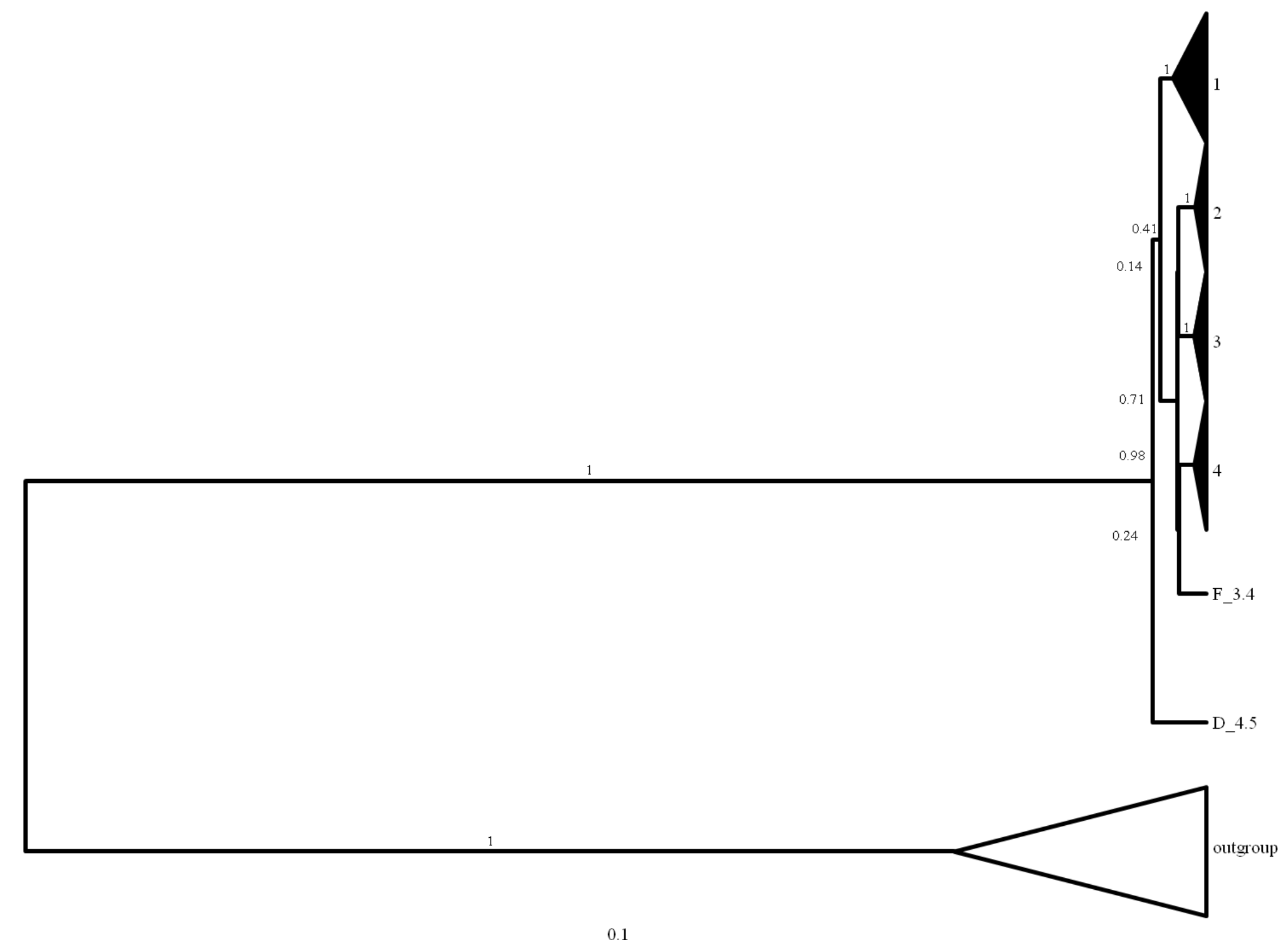

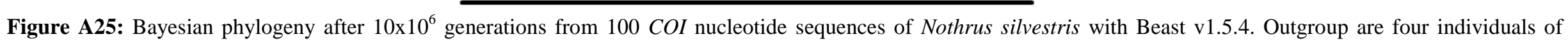
Nothrus palustris from different locations. Numbers on the branches are posterior probabilities. Tip numbers are the different subclades and explain in Table 15. 
Appendix

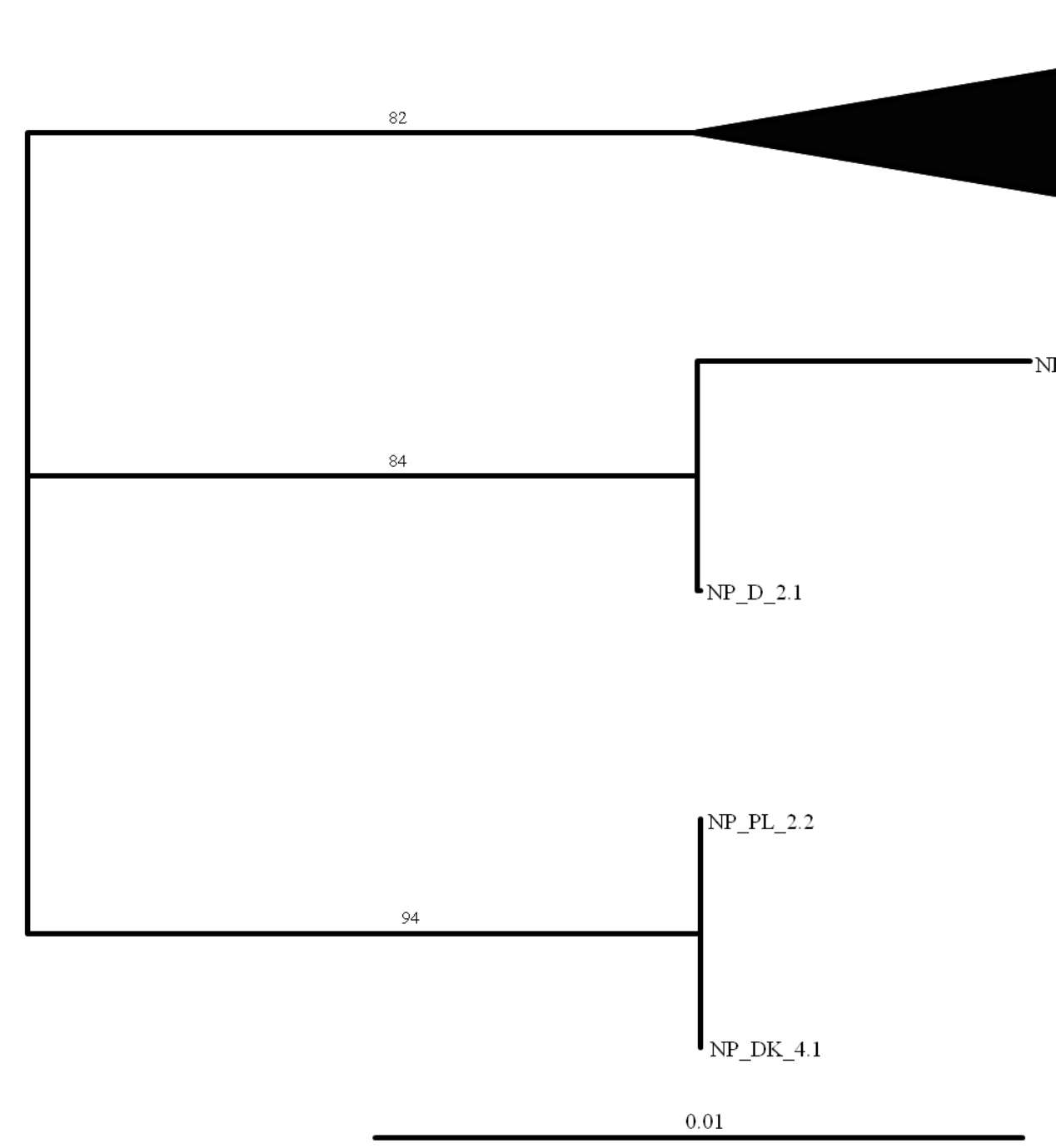

Figure A26: Neighbor-Joining tree of 100 COI protein sequences of Nothrus silvestris. Outgroup are four individuals of Nothrus palustris from different locations. Numbers on the branches are bootstrap values of 100,000 pseudo-replicates. Tip numbers are the different subclades and explain in Table 15 . 
Appendix

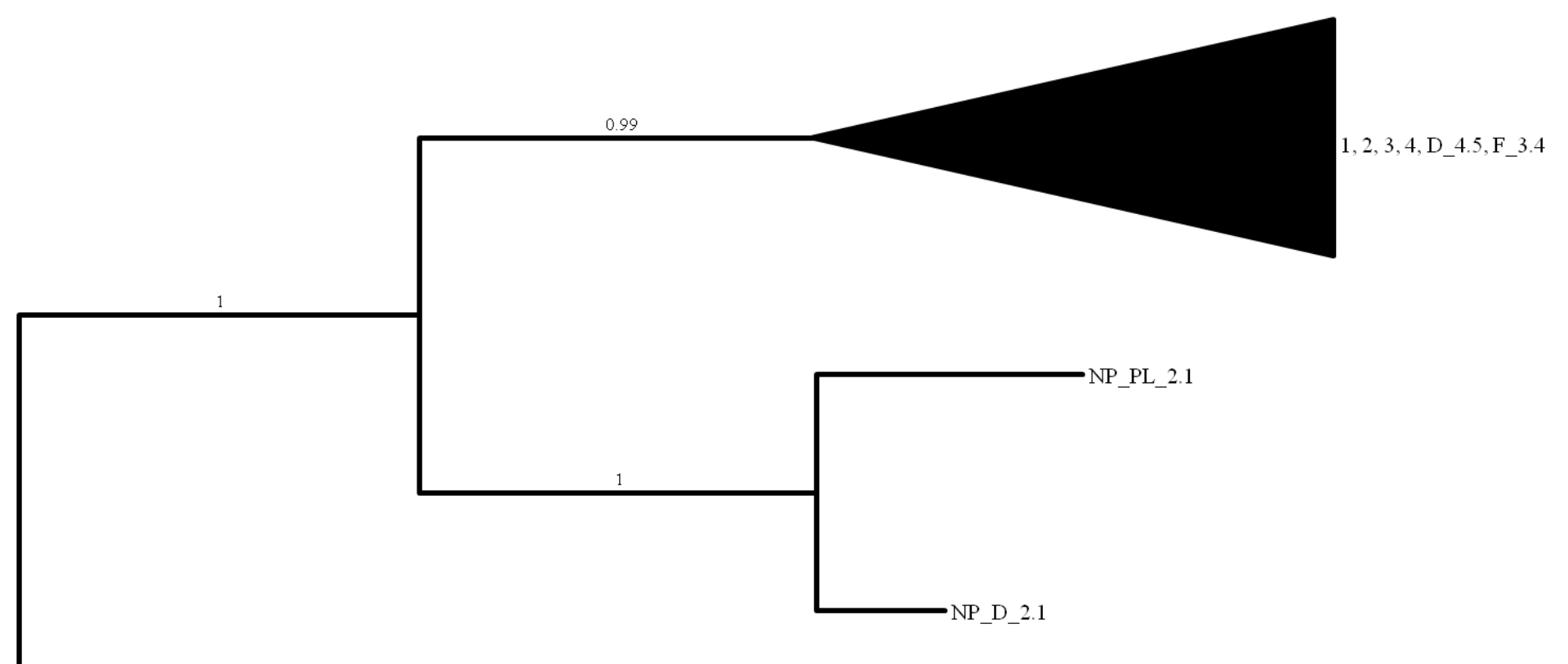

NP DK 4.1

NP_PL 2.2

Figure A27: Bayesian tree after 10x $10^{6}$ generations from 100 COI protein sequences of Nothrus silvestris. Split frequencies of 0.042324 and burnin of $25 \%$. Outgroup are four individuals of Nothrus palustris from different locations. Numbers on the branches are posterior probabilities. Tip numbers are the different subclades and explain in Table 15 . 
Appendix

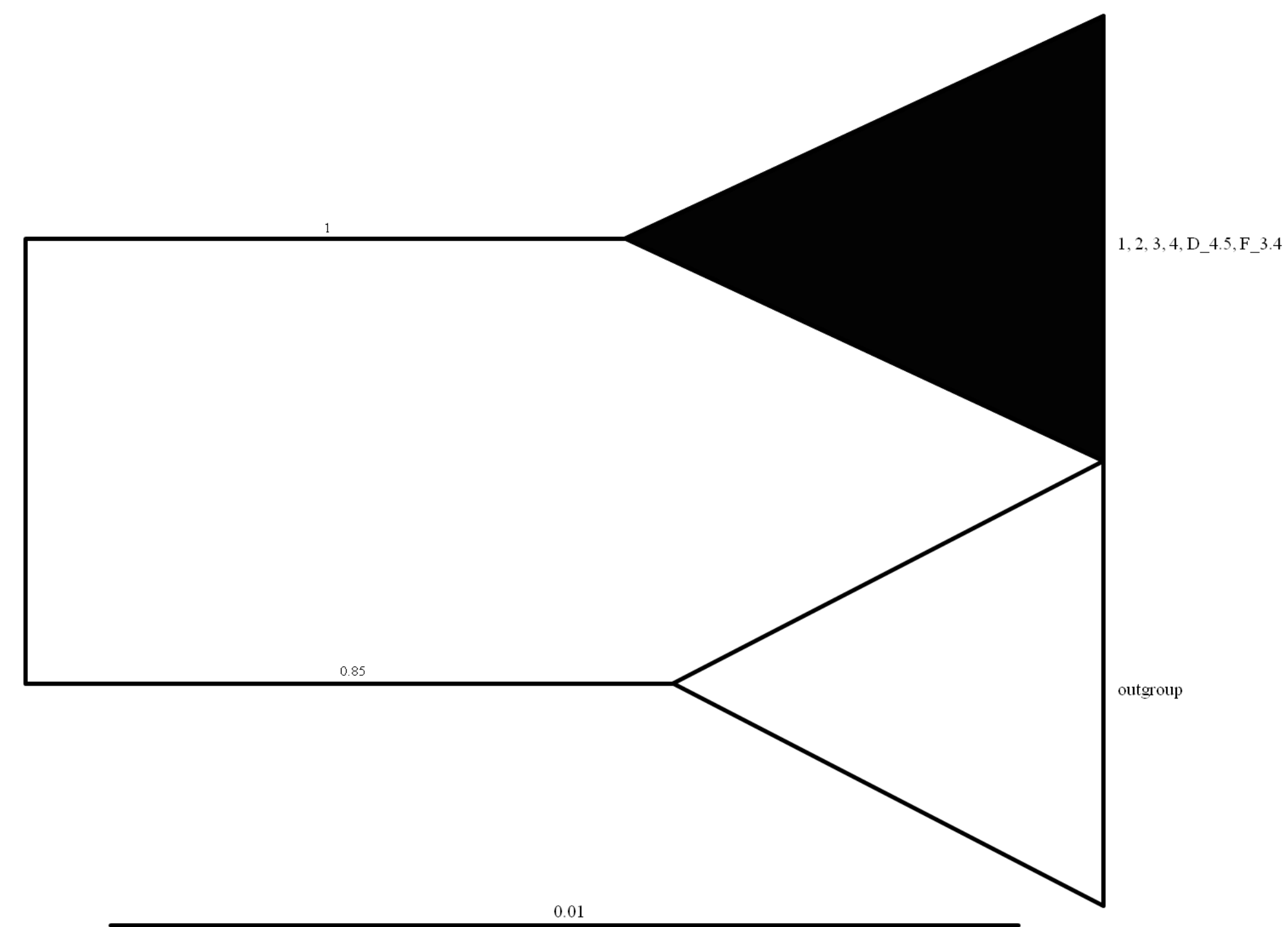

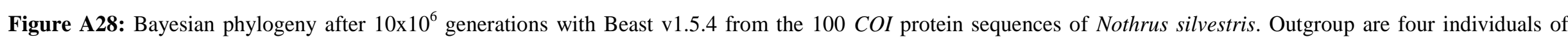
Nothrus palustris from different locations. Numbers on the branches are posterior probabilities. Tip numbers are the different subclades and explain in Table 15. 
Appendix

Table A20: Mean pairwise differences of uncorrected p-distances of the protein of Nothrus silvestris from 24 locations in percent. The diagonal is the within population differences (bold) and below the diagonal among populations. Bold letters in red are the minimum and maximum differences within and among the populations. Locations with less than two individuals were excluded from the analysis.

\begin{tabular}{|c|c|c|c|c|c|c|c|c|c|c|c|c|c|c|c|c|c|c|c|c|c|c|c|c|}
\hline Population & 1 & 2 & 3 & 4 & 5 & 6 & 7 & 8 & 9 & 10 & 11 & 12 & 13 & 14 & 15 & 16 & 17 & 18 & 19 & 20 & 21 & 22 & 23 & 24 \\
\hline $1 \mathrm{CH} 2$ & 0 & & & & & & & & & & & & & & & & & & & & & & & \\
\hline $2 \mathrm{CH} 3$ & 0 & 0 & & & & & & & & & & & & & & & & & & & & & & \\
\hline 3 D 1 & 0 & 0 & 0 & & & & & & & & & & & & & & & & & & & & & \\
\hline D 2 & 0 & 0 & 0 & 0 & & & & & & & & & & & & & & & & & & & & \\
\hline D 4 & 0 & 0 & 0 & 0 & 0 & & & & & & & & & & & & & & & & & & & \\
\hline D 6 & 0 & 0 & 0 & 0 & 0 & 0 & & & & & & & & & & & & & & & & & & \\
\hline D 7 & 0 & 0 & 0 & 0 & 0 & 0 & 0 & & & & & & & & & & & & & & & & & \\
\hline D 8 & 0 & 0 & 0 & 0 & 0 & 0 & 0 & 0 & & & & & & & & & & & & & & & & \\
\hline D 9 & 0 & 0 & 0 & 0 & 0 & 0 & 0 & 0 & 0 & & & & & & & & & & & & & & & \\
\hline 10 D 11 & 0 & 0 & 0 & 0 & 0 & 0 & 0 & 0 & 0 & 0 & & & & & & & & & & & & & & \\
\hline 11 D 16 & 0 & 0 & 0 & 0 & 0 & 0 & 0 & 0 & 0 & 0 & 0 & & & & & & & & & & & & & \\
\hline 12 D 17 & 0 & 0 & 0 & 0 & 0 & 0 & 0 & 0 & 0 & 0 & 0 & 0 & & & & & & & & & & & & \\
\hline 13 D 18 & 0 & 0 & 0 & 0 & 0 & 0 & 0 & 0 & 0 & 0 & 0 & 0 & 0 & & & & & & & & & & & \\
\hline 14 F 3 & 0 & 0 & 0 & 0 & 0 & 0 & 0 & 0 & 0 & 0 & 0 & 0 & 0 & 0 & & & & & & & & & & \\
\hline 15 FIN 1 & 0 & 0 & 0 & 0 & 0 & 0 & 0 & 0 & 0 & 0 & 0 & 0 & 0 & 0 & 0 & & & & & & & & & \\
\hline 16 GB 1 & 0 & 0 & 0 & 0 & 0 & 0 & 0 & 0 & 0 & 0 & 0 & 0 & 0 & 0 & 0 & 0 & & & & & & & & \\
\hline 17 GB 2 & 0.1 & 0.1 & 0.1 & 0.1 & 0.1 & 0.1 & 0.1 & 0.1 & 0.1 & 0.1 & 0.1 & 0.1 & 0.1 & 0.1 & 0.1 & 0.1 & 0.3 & & & & & & & \\
\hline 18 NL 1 & 0 & 0 & 0 & 0 & 0 & 0 & 0 & 0 & 0 & 0 & 0 & 0 & 0 & 0 & 0 & 0 & 0.1 & 0 & & & & & & \\
\hline 19 NL 2 & 0 & 0 & 0 & 0 & 0 & 0 & 0 & 0 & 0 & 0 & 0 & 0 & 0 & 0 & 0 & 0 & 0.1 & 0 & 0 & & & & & \\
\hline $20 \quad$ PL 2 & 0 & 0 & 0 & 0 & 0 & 0 & 0 & 0 & 0 & 0 & 0 & 0 & 0 & 0 & 0 & 0 & 0.1 & 0 & 0 & 0 & & & & \\
\hline 21 RUM 1 & 0 & 0 & 0 & 0 & 0 & 0 & 0 & 0 & 0 & 0 & 0 & 0 & 0 & 0 & 0 & 0 & 0.1 & 0 & 0 & 0 & 0 & & & \\
\hline 22 RUM 2 & 0 & 0 & 0 & 0 & 0 & 0 & 0 & 0 & 0 & 0 & 0 & 0 & 0 & 0 & 0 & 0 & 0.1 & 0 & 0 & 0 & 0 & 0 & & \\
\hline 23 RUM 5 & 0 & 0 & 0 & 0 & 0 & 0 & 0 & 0 & 0 & 0 & 0 & 0 & 0 & 0 & 0 & 0 & 0.1 & 0 & 0 & 0 & 0 & 0 & 0 & \\
\hline $24 \quad$ S 3 & 0 & 0 & 0 & 0 & 0 & 0 & 0 & 0 & 0 & 0 & 0 & 0 & 0 & 0 & 0 & 0 & 0.1 & 0 & 0 & 0 & 0 & 0 & 0 & 0 \\
\hline
\end{tabular}


Appendix

Table A21: Tajima's D and Fu's FS neutrality tests of Nothrus silvestris COI nucleotide sequences from 24 locations. Bold red letters show significance (P<0.05).

\begin{tabular}{|c|c|c|c|c|c|c|c|c|c|c|c|c|c|c|c|c|c|c|c|c|}
\hline Neutrality tests & $\mathrm{CH} 2$ & CH 3 & D 1 & D 2 & D 4 & D 6 & D 7 & D 8 & D 9 & D 11 & D 16 & D 17 & D 18 & F 3 & FIN 1 & GB 1 & GB 2 & NL 1 & NL 2 & PL 2 \\
\hline \multicolumn{21}{|l|}{ Tajima's D test } \\
\hline Samplesize & 3 & 2 & 4 & 4 & 8 & 2 & 4 & 3 & 4 & 4 & 4 & 3 & 4 & 4 & 4 & 3 & 4 & 3 & 2 & 13 \\
\hline S & 9 & 2 & 0 & 11 & 20 & 10 & 0 & 0 & 0 & 3 & 1 & 13 & 0 & 9 & 0 & 10 & 13 & 0 & 9 & 6 \\
\hline $\mathrm{Pi}$ & 6 & 2 & 0 & 5.67 & 7.93 & 10 & 0 & 0 & 0 & 1.5 & 0.5 & 8.67 & 0 & 4.5 & 0 & 6.67 & 8.33 & 0 & 9 & 0.92 \\
\hline Tajima's D & 0 & 0 & 0 & -0.56 & 0.15 & 0 & 0 & 0 & 0 & -0.75 & -0.61 & 0 & 0 & -0.83 & 0 & 0 & 1.77 & 0 & 0 & -1.93 \\
\hline Tajima's D p-value & 0.73 & 1 & 1 & 0.43 & 0.57 & 1 & 1 & 1 & 1 & 0.23 & 0.38 & 0.70 & 1 & 0.12 & 1 & 0.72 & 0.94 & 1 & 1 & 0.01 \\
\hline \multicolumn{21}{|l|}{ Fu's FS test } \\
\hline Real no. of alleles & 2 & 2 & 1 & 4 & 6 & 2 & 1 & 1 & 1 & 2 & 2 & 3 & 1 & 3 & 1 & 3 & 3 & 1 & 2 & 2 \\
\hline Orig. no. of alleles & 2 & 2 & 1 & 4 & 6 & 2 & 1 & 1 & 1 & 2 & 2 & 3 & 1 & 3 & 1 & 3 & 3 & 1 & 2 & 2 \\
\hline Theta_pi & 6 & 2 & 0 & 5.67 & 7.93 & 10 & 0 & 0 & 0 & 1.5 & 0.5 & 8.67 & 0 & 4.5 & 0 & 6.67 & 8.33 & 0 & 9 & 0.92 \\
\hline Exp.no. of alleles & 2.61 & 1.67 & 0 & 3.24 & 5.79 & 1.91 & 0 & 0 & 0 & 2.36 & 1.68 & 2.71 & 0 & 3.11 & 0 & 2.64 & 3.43 & 0 & 1.9 & 3.05 \\
\hline FS & 3.30 & 0.69 & 0 & -0.36 & 0.46 & 2.3 & 0 & 0 & 0 & 1.72 & 0.17 & 0.99 & 0 & 1.34 & 0 & 0.7 & 2.32 & 0 & 2.2 & 2.3 \\
\hline FS p-value & 0.89 & 0.37 & N.A. & 0.22 & 0.54 & 0.56 & N.A. & N.A. & N.A. & 0.75 & 0.34 & 0.45 & N.A. & 0.69 & N.A. & 0.42 & 0.81 & N.A. & 0.55 & 0.84 \\
\hline
\end{tabular}

\begin{tabular}{lcccccc}
\hline Neutrality tests & RUM 1 RUM 2 RUM 5 & S 3 & mean & s.d. \\
\hline Tajima's D test & & & & & & \\
Samplesize & 3 & 4 & 4 & 4 & 4.04 & 2.24 \\
$\mathrm{~S}$ & 3 & 0 & 0 & 10 & 5.38 & 5.78 \\
$\mathrm{Pi}$ & 2 & 0 & 0 & 5 & 3.28 & 3.61 \\
Tajima's D & 0 & 0 & 0 & -0.83 & -0.15 & 0.63 \\
Tajima's D p-value & 0.88 & 1 & 1 & 0.11 & 0.74 & 0.34 \\
& & & & & & \\
Fu's FS test & & & & & & \\
Real no. of alleles & 2 & 1 & 1 & 3 & 2.08 & 1.21 \\
Orig. no. of alleles & 2 & 1 & 1 & 3 & 2.08 & 1.21 \\
Theta_pi & 2 & 0 & 0 & 5 & 3.28 & 3.61 \\
Exp.no. of alleles & 2.17 & 0 & 0 & 3.17 & 1.73 & 1.58 \\
FS & 1.61 & 0 & 0 & 1.51 & 0.89 & 1.04 \\
FS p-value & 0.69 & N.A. & N.A. & 0.71 & N.A. & N.A. \\
\hline
\end{tabular}




\section{Appendix}

Table A22: Tajima's D and Fu's FS neutrality tests of Nothrus silvestris COI protein sequences from 24 locations. Bold red letters show significance ( $<<0.05)$.

\begin{tabular}{lccccccccccccccccccccc}
\hline Neutrality tests & CH 2 & CH 3 & D 1 & D 2 & D 4 & D 6 & D 7 & D 8 & D 9 & D 11 & D 16 & D 17 & D 18 & F 3 & FIN 1 & GB 1 & GB 2 & NL 1 & NL 2 & PL 2 \\
\hline Tajima's D test & & & & & & & & & & & & & & & & & & & & & \\
Samplesize & 3 & 2 & 4 & 4 & 8 & 2 & 4 & 3 & 4 & 4 & 4 & 3 & 4 & 4 & 4 & 3 & 4 & 3 & 2 & 13 \\
S & 0 & 0 & 0 & 0 & 0 & 0 & 0 & 0 & 0 & 0 & 0 & 0 & 0 & 0 & 0 & 0 & 1 & 0 & 0 & 0 \\
Pi & 0 & 0 & 0 & 0 & 0 & 0 & 0 & 0 & 0 & 0 & 0 & 0 & 0 & 0 & 0 & 0 & 0.5 & 0 & 0 & 0 \\
Tajima's D & 0 & 0 & 0 & 0 & 0 & 0 & 0 & 0 & 0 & 0 & 0 & 0 & 0 & 0 & 0 & 0 & -0.61 & 0 & 0 & 0 \\
Tajima's D p-value & 1 & 1 & 1 & 1 & 1 & 1 & 1 & 1 & 1 & 1 & 1 & 1 & 1 & 1 & 1 & 1 & 0.38 & 1 & 1 & 1
\end{tabular}

\section{Fu's FS test}

$\begin{array}{llllllllllllllllllllll}\text { Real no. of alleles } & 2 & 1 & 1 & 2 & 4 & 2 & 1 & 1 & 1 & 2 & 2 & 2 & 1 & 1 & 1 & 3 & 2 & 1 & 2 & 1\end{array}$ Orig. no. of alleles

Theta_pi

Exp. no. of alleles

FS

FS p-value N.A. N.A. N.A. N.A. N.A. N.A. N.A. N.A. N.A. N.A. N.A. N.A. N.A.

\begin{tabular}{lcccccc}
\hline Neutrality tests & RUM 1 & RUM 2 & RUM 5 & S 3 & mean & s.d. \\
\hline Tajima's D test & & & & & & \\
Samplesize & 3 & 4 & 4 & 4 & 4.04 & 2.24 \\
S & 0 & 0 & 0 & 0 & 0.04 & 0.20 \\
Pi & 0 & 0 & 0 & 0 & 0.02 & 0.10 \\
Tajima's D & 0 & 0 & 0 & 0 & -0.03 & 0.13 \\
Tajima's D p-value & 1 & 1 & 1 & 1 & 0.97 & 0.13
\end{tabular}

\section{Fu's FS test}

$\begin{array}{lllllll}\text { Real no. of alleles } & 1 & 1 & 1 & 2 & 1.58 & 0.78\end{array}$

$\begin{array}{lllllll}\text { Orig. no. of alleles } & 1 & 1 & 1 & 2 & 1.58 & 0.78\end{array}$

Theta_pi

Exp. no. of alleles

FS

FS p-value 
Table A23: Results of the McDonald-Kreitman test for Nothrus silvestris. The differences between the populations are not significant. Locations with less than two individuals were excluded.

\begin{tabular}{|c|c|c|c|c|c|c|c|c|c|c|c|c|c|}
\hline \multirow{2}{*}{$\begin{array}{c}\text { Population } \\
\mathrm{CH} 3\end{array}$} & \multicolumn{4}{|c|}{$\begin{array}{r}\text { CH 2 } \\
\text { syn.nons. sign. }\end{array}$} & \multicolumn{3}{|c|}{$\begin{array}{r}\mathrm{CH} 3 \\
\text { syn.nons. sign. }\end{array}$} & \multicolumn{3}{|c|}{$\begin{array}{c}\text { D 1 } \\
\text { syn.nons. sign. }\end{array}$} & \multicolumn{3}{|c|}{$\begin{array}{c}\text { D 2 } \\
\text { syn.nons. sign. }\end{array}$} \\
\hline & fixed & 0 & 0 & - & & & & & & & & & \\
\hline Rohrschach & poly & 10 & 1 & & & & & & & & & & \\
\hline D 1 & fixed & 0 & 0 & - & 0 & 0 & - & & & & & & \\
\hline Kranichstein & poly & 8 & 1 & & 2 & 0 & & & & & & & \\
\hline D 2 & fixed & 0 & 0 & - & 5 & 0 & $1 \mathrm{~ns}$ & 5 & 0 & $1 \mathrm{~ns}$ & & & \\
\hline Goettingen & poly & 18 & 2 & & 12 & 0 & & 10 & 1 & & & & \\
\hline D 4 & fixed & 0 & 0 & - & 0 & 0 & - & 0 & 0 & - & 0 & 0 & - \\
\hline Meckl. Seenpl. & poly & 17 & 3 & & 18 & 3 & & 17 & 3 & & 26 & 3 & \\
\hline D 6 & fixed & 0 & 0 & - & 0 & 0 & - & 0 & 0 & - & 0 & 0 & - \\
\hline Black For est & poly & 9 & 1 & & 11 & 1 & & 9 & 1 & & 19 & 2 & \\
\hline D 7 & fixed & 0 & 0 & - & 8 & 1 & $1 \mathrm{~ns}$ & 8 & 1 & - & 3 & 1 & $0.48 \mathrm{~ns}$ \\
\hline Uelzen & poly & 8 & 1 & & 2 & 0 & & 0 & 0 & & 10 & 1 & \\
\hline D 8 & fixed & 0 & 0 & - & 0 & 0 & - & 0 & 0 & - & 5 & 0 & $1 \mathrm{~ns}$ \\
\hline Cuxhaven & poly & 8 & 1 & & 2 & 0 & & 0 & 0 & & 10 & 1 & \\
\hline D 9 & fixed & 0 & 0 & - & 0 & 0 & - & 0 & 0 & - & 5 & 0 & $1 \mathrm{~ns}$ \\
\hline Bonn & poly & 8 & 1 & & 2 & 0 & & 0 & 0 & & 10 & 1 & \\
\hline D 11 & fixed & 3 & 0 & $1 \mathrm{~ns}$ & 8 & 0 & $0.38 \mathrm{~ns}$ & 8 & 2 & $0.27 \mathrm{~ns}$ & 0 & 0 & - \\
\hline Wittmoor & poly & 10 & 2 & & 4 & 1 & & 2 & 1 & & 12 & 1 & \\
\hline D 16 & fixed & 0 & 0 & - & 0 & 0 & - & 0 & 0 & - & 5 & 0 & $1 \mathrm{~ns}$ \\
\hline Solling & poly & 8 & 2 & & 2 & 1 & & 0 & 1 & & 10 & 1 & \\
\hline D 17 & fixed & 0 & 0 & - & 0 & 0 & - & 0 & 0 & - & 0 & 0 & - \\
\hline Rubi & poly & 15 & 2 & & 14 & 1 & & 12 & 1 & & 16 & 2 & \\
\hline D 18 & fixed & 0 & 0 & - & 0 & 0 & - & 0 & 0 & - & 5 & 0 & $1 \mathrm{~ns}$ \\
\hline Steinh. Meer & poly & 8 & 1 & & 2 & 0 & & 0 & 0 & & 10 & 1 & \\
\hline F 3 & fixed & 0 & 0 & - & 4 & 0 & - & 4 & 0 & - & 0 & 0 & - \\
\hline Saint Isidore & poly & 16 & 1 & & 11 & 0 & & 9 & 0 & & 17 & 1 & \\
\hline FIN 1 & fixed & 0 & 0 & - & 0 & 0 & - & 0 & 0 & - & 5 & 0 & $1 \mathrm{~ns}$ \\
\hline Lahti & poly & 8 & 1 & & 2 & 0 & & 0 & 0 & & 10 & 1 & \\
\hline GB 1 & fixed & 0 & 0 & - & 0 & 0 & - & 0 & 0 & - & 0 & 0 & - \\
\hline Ascot & poly & 8 & 2 & & 10 & 2 & & 8 & 2 & & 18 & 3 & \\
\hline GB 2 & fixed & 0 & 0 & - & 0 & 0 & - & 0 & 0 & - & 0 & 0 & - \\
\hline Braemar & poly & 14 & 3 & & 13 & 2 & & 11 & 2 & & 15 & 3 & \\
\hline NL 1 & fixed & 0 & 0 & - & 0 & 0 & - & 0 & 0 & - & 5 & 0 & $1 \mathrm{~ns}$ \\
\hline Wageningen & poly & 8 & 1 & & 2 & 0 & & 0 & 0 & & 10 & 1 & \\
\hline NL 2 & fixed & 0 & 0 & - & 0 & 0 & - & 0 & 0 & - & 0 & 0 & - \\
\hline Hoge Veluwe & poly & 8 & 1 & & 10 & 1 & & 8 & 1 & & 18 & 2 & \\
\hline PL 2 & fixed & 0 & 0 & - & 0 & 0 & - & 0 & 0 & - & 1 & 0 & $1 \mathrm{~ns}$ \\
\hline Warsaw & poly & 10 & 1 & & 8 & 0 & & 6 & 0 & & 15 & 1 & \\
\hline RUM 1 & fixed & 0 & 0 & - & 0 & 0 & - & 0 & 0 & - & 3 & 0 & $1 \mathrm{~ns}$ \\
\hline Sibiu 1 & poly & 9 & 1 & & 5 & 0 & & 3 & 0 & & 13 & 1 & \\
\hline RUM 2 & fixed & 0 & 0 & - & 0 & 0 & - & 0 & 0 & - & 5 & 0 & $1 \mathrm{~ns}$ \\
\hline Sibiu 2 & poly & 8 & 1 & & 2 & 0 & & 0 & 0 & & 10 & 1 & \\
\hline RUM 5 & fixed & 2 & 0 & $1 \mathrm{~ns}$ & 4 & 0 & - & 4 & 0 & - & 5 & 0 & $1 \mathrm{~ns}$ \\
\hline Busteni & poly & 8 & 1 & & 2 & 0 & & 0 & 0 & & 10 & 1 & \\
\hline S 3 & fixed & 0 & 0 & - & 0 & 0 & - & 0 & 0 & - & 0 & 0 & - \\
\hline Malmoe & poly & 9 & 1 & & 11 & 1 & & 9 & 1 & & 19 & 2 & \\
\hline \multicolumn{2}{|l|}{ Population } & \multicolumn{3}{|c|}{$\begin{array}{c}\text { D 4 } \\
\text { syn.nons. sign. }\end{array}$} & \multicolumn{3}{|c|}{ D 6} & \multicolumn{3}{|c|}{$\begin{array}{c}\text { D 7 } \\
\text { syn.nons. sign. }\end{array}$} & \multicolumn{3}{|c|}{$\begin{array}{c}\text { D 8 } \\
\text { svn.nons. sign. }\end{array}$} \\
\hline D 6 & fixed & 0 & 0 & - & & & & & & & & & \\
\hline Black Forest & poly & 18 & 3 & & & & & & & & & & \\
\hline D 7 & fixed & 0 & 0 & - & 0 & 0 & - & & & & & & \\
\hline Uelzen & poly & 17 & 3 & & 9 & 1 & & & & & & & \\
\hline D 8 & fixed & 0 & 0 & - & 0 & 0 & - & 8 & 1 & - & & & \\
\hline Cuxhaven & poly & 17 & 3 & & 9 & 1 & & 0 & 0 & & & & \\
\hline D 9 & fixed & 0 & 0 & - & 0 & 0 & - & 8 & 1 & - & 0 & 0 & - \\
\hline Bonn & poly & 17 & 3 & & 9 & 1 & & 0 & 0 & & 0 & 0 & \\
\hline
\end{tabular}


Table A23 continue:

\begin{tabular}{|c|c|c|c|c|c|c|c|c|c|c|c|c|c|}
\hline \multirow{2}{*}{$\begin{array}{c}\text { Population } \\
\text { D } 11\end{array}$} & \multirow[b]{2}{*}{ fixed } & \multicolumn{3}{|c|}{$\begin{array}{c}\text { D } 4 \\
\text { syn.nons. sign. }\end{array}$} & \multicolumn{3}{|c|}{$\begin{array}{c}\text { D } 6 \\
\text { syn.nons. sign. }\end{array}$} & \multicolumn{3}{|c|}{$\begin{array}{c}\text { D } 7 \\
\text { syn.nons. sign. }\end{array}$} & \multicolumn{3}{|c|}{$\begin{array}{c}\text { D } 8 \\
\text { syn.nons. sign. }\end{array}$} \\
\hline & & 2 & 0 & $1 \mathrm{~ns}$ & 3 & 0 & $1 \mathrm{~ns}$ & 6 & 1 & $1 \mathrm{~ns}$ & 8 & 0 & $0.27 \mathrm{~ns}$ \\
\hline Wittmoor & poly & 19 & 3 & & 11 & 2 & & 2 & 1 & & 2 & 1 & \\
\hline D 16 & fixed & 0 & 0 & - & 0 & 0 & - & 8 & 1 & $0.2 \mathrm{~ns}$ & 0 & 0 & - \\
\hline Solling & poly & 17 & 3 & & 9 & 2 & & 0 & 1 & & 0 & 1 & \\
\hline D 17 & fixed & 0 & 0 & - & 0 & 0 & - & 3 & 1 & $0.43 \mathrm{~ns}$ & 0 & 0 & - \\
\hline Rubi & poly & 23 & 4 & & 16 & 2 & & 12 & 1 & & 12 & 1 & \\
\hline D 18 & fixed & 0 & 0 & - & 0 & 0 & - & 8 & 1 & - & 0 & 0 & - \\
\hline Steinh. Meer & poly & 17 & 3 & & 9 & 1 & & 0 & 0 & & 0 & 0 & \\
\hline F 3 & fixed & 0 & 0 & - & 0 & 0 & - & 3 & 1 & $0.31 \mathrm{~ns}$ & 4 & 0 & - \\
\hline Saintisidore & poly & 24 & 3 & & 17 & 1 & & 9 & 0 & & 9 & 0 & \\
\hline FIN 1 & fixed & 0 & 0 & - & 0 & 0 & - & 8 & 1 & - & 0 & 0 & - \\
\hline Lahti & poly & 17 & 3 & & 9 & 1 & & 0 & 0 & & 0 & 0 & \\
\hline GB 1 & fixed & 0 & 0 & - & 0 & 0 & - & 0 & 0 & - & 0 & 0 & - \\
\hline Ascot & poly & 17 & 4 & & 9 & 2 & & 8 & 2 & & 8 & 2 & \\
\hline GB 2 & fixed & 0 & 0 & - & 0 & 0 & - & 3 & 1 & $1 \mathrm{~ns}$ & 0 & 0 & - \\
\hline Braemar & poly & 23 & 5 & & 15 & 3 & & 11 & 2 & & 11 & 2 & \\
\hline NL 1 & fixed & 0 & 0 & - & 0 & 0 & - & 0 & 0 & - & 0 & 0 & - \\
\hline Wageningen & poly & 17 & 3 & & 9 & 1 & & 2 & 0 & & 0 & 0 & \\
\hline NL 2 & fixed & 0 & 0 & - & 0 & 0 & - & 0 & 0 & - & 0 & 0 & - \\
\hline Hoge Veluwe & poly & 17 & 3 & & 9 & 1 & & 8 & 1 & & 8 & 1 & \\
\hline PL 2 & fixed & 0 & 0 & - & 0 & 0 & - & 4 & 1 & $0.45 \mathrm{~ns}$ & 0 & 0 & - \\
\hline Warsaw & poly & 18 & 3 & & 11 & 1 & & 6 & 0 & & 6 & 0 & \\
\hline RUM 1 & fixed & 0 & 0 & - & 0 & 0 & - & 6 & 1 & $1 \mathrm{~ns}$ & 0 & 0 & - \\
\hline Sibiu 1 & poly & 18 & 3 & & 10 & 1 & & 3 & 0 & & 3 & 0 & \\
\hline RUM 2 & fixed & 0 & 0 & - & 0 & 0 & - & 8 & 1 & - & 0 & 0 & - \\
\hline Sibiu 2 & poly & 17 & 3 & & 9 & 1 & & 0 & 0 & & 0 & 0 & \\
\hline RUM 5 & fixed & 2 & 0 & $1 \mathrm{~ns}$ & 2 & 0 & $1 \mathrm{~ns}$ & 8 & 1 & - & 4 & 0 & - \\
\hline Busteni & poly & 17 & 3 & & 9 & 1 & & 0 & 0 & & 0 & 0 & \\
\hline S 3 & fixed & 0 & 0 & - & 0 & 0 & - & 0 & 0 & - & 0 & 0 & - \\
\hline Malmoe & poly & 18 & 3 & & 10 & 1 & & 9 & 1 & & 9 & 1 & \\
\hline \multicolumn{2}{|l|}{ Population } & \multicolumn{3}{|c|}{$\begin{array}{c}\text { D 9 } \\
\text { syn.nons. sign. }\end{array}$} & \multicolumn{3}{|c|}{$\begin{array}{r}\text { D } 11 \\
\text { syn.nons. sign. }\end{array}$} & \multicolumn{3}{|c|}{$\begin{array}{r}\text { D } 16 \\
\text { syn.nons. sign. }\end{array}$} & \multicolumn{3}{|c|}{$\begin{array}{r}\text { D } 17 \\
\text { syn.nons sign. }\end{array}$} \\
\hline D 11 & fixed & 8 & 0 & $0.27 \mathrm{~ns}$ & & & & & & & & & \\
\hline Wittmoor & poly & 2 & 1 & & & & & & & & & & \\
\hline D 16 & fixed & 0 & 0 & - & 8 & 0 & $0.27 \mathrm{~ns}$ & & & & & & \\
\hline Solling & poly & 0 & 1 & & 2 & 1 & & & & & & & \\
\hline D 17 & fixed & 0 & 0 & - & 3 & 0 & $1 \mathrm{~ns}$ & 0 & 0 & - & & & \\
\hline Rubi & poly & 12 & 1 & & 14 & 2 & & 12 & 2 & & & & \\
\hline D 18 & fixed & 0 & 0 & - & 8 & 0 & $0.27 \mathrm{~ns}$ & 0 & 0 & - & 0 & 0 & - \\
\hline Steinh. Meer & poly & 0 & 0 & & 2 & 1 & & 0 & 1 & & 12 & 1 & \\
\hline F 3 & fixed & 4 & 0 & - & 1 & 0 & $1 \mathrm{~ns}$ & 4 & 0 & $1 \mathrm{~ns}$ & 0 & 0 & - \\
\hline Saint Isidore & poly & 9 & 0 & & 11 & 1 & & 9 & 1 & & 20 & 1 & \\
\hline FIN 1 & fixed & 0 & 0 & - & 8 & 0 & $0.27 \mathrm{~ns}$ & 0 & 0 & - & 0 & 0 & - \\
\hline Lahti & poly & 0 & 0 & & 2 & 1 & & 0 & 1 & & 12 & 1 & \\
\hline GB 1 & fixed & 0 & 0 & - & 3 & 0 & $1 \mathrm{~ns}$ & 0 & 0 & - & 0 & 0 & - \\
\hline Ascot & poly & 10 & 2 & & 10 & 3 & & 8 & 3 & & 15 & 3 & \\
\hline GB 2 & fixed & 0 & 0 & - & 3 & 0 & $1 \mathrm{~ns}$ & 0 & 0 & - & 0 & 0 & - \\
\hline Braemar & poly & 11 & 2 & & 13 & 3 & & 11 & 3 & & 12 & 3 & \\
\hline NL 1 & fixed & 0 & 0 & - & 8 & 0 & $0.27 \mathrm{~ns}$ & 0 & 0 & - & 0 & 0 & - \\
\hline Wageningen & poly & 0 & 0 & & 2 & 1 & & 0 & 1 & & 12 & 1 & \\
\hline NL 2 & fixed & 0 & 0 & - & 3 & 0 & $1 \mathrm{~ns}$ & 0 & 0 & - & 0 & 0 & - \\
\hline Hoge Veluwe & poly & 8 & 1 & & 10 & 2 & & 8 & 2 & & 11 & 2 & \\
\hline PL 2 & fixed & 0 & 0 & - & 3 & 0 & $1 \mathrm{~ns}$ & 0 & 0 & - & 0 & 0 & - \\
\hline Warsaw & poly & 6 & 0 & & 8 & 1 & & 6 & 1 & & 12 & 1 & \\
\hline RUM 1 & fixed & 0 & 0 & - & 6 & 0 & $1 \mathrm{~ns}$ & 0 & 0 & - & 0 & 0 & - \\
\hline Sibiu 1 & poly & 3 & 0 & & 5 & 1 & & 3 & 1 & & 13 & 1 & \\
\hline
\end{tabular}


Appendix

Table A23 continue:

\begin{tabular}{|c|c|c|c|c|c|c|c|c|c|c|c|c|c|}
\hline \multirow{2}{*}{$\begin{array}{c}\text { Population } \\
\text { RUM } 2\end{array}$} & \multirow[b]{2}{*}{ fixed } & \multicolumn{3}{|c|}{$\begin{array}{c}\text { D 9 } \\
\text { syn.nons. sign. }\end{array}$} & \multicolumn{3}{|c|}{$\begin{array}{r}\text { D } 11 \\
\text { syn.nons. sign. }\end{array}$} & \multicolumn{3}{|c|}{$\begin{array}{r}\text { D } 16 \\
\text { syn.nons. sign. }\end{array}$} & \multicolumn{3}{|c|}{$\begin{array}{r}\text { D } 17 \\
\text { syn.nons sign. }\end{array}$} \\
\hline & & 0 & 0 & - & 8 & 0 & $0.27 \mathrm{~ns}$ & 0 & 0 & - & 0 & 0 & - \\
\hline Sibiu 2 & poly & 0 & 0 & & 2 & 1 & & 0 & 1 & & 12 & 1 & \\
\hline RUM 5 & fixed & 4 & 0 & - & 8 & 0 & $0.27 \mathrm{~ns}$ & 4 & 0 & $0.2 \mathrm{~ns}$ & 2 & 0 & $1 \mathrm{~ns}$ \\
\hline Busteni & poly & 0 & 0 & & 2 & 1 & & 0 & 1 & & 12 & 1 & \\
\hline S 3 & fixed & 0 & 0 & - & 3 & 0 & $1 \mathrm{~ns}$ & 0 & 0 & - & 0 & 0 & - \\
\hline Malmoe & poly & 9 & 1 & & 11 & 2 & & 9 & 2 & & 16 & 2 & \\
\hline \multicolumn{2}{|l|}{ Population } & \multicolumn{3}{|c|}{$\begin{array}{r}\text { D } 18 \\
\text { ons. sign. }\end{array}$} & \multicolumn{3}{|c|}{$\begin{array}{c}\text { F } 3 \\
\text { syn.nons. sign. }\end{array}$} & \multicolumn{3}{|c|}{$\begin{array}{r}\text { FIN } 1 \\
\text { syn.nons. sign. }\end{array}$} & \multicolumn{3}{|c|}{$\begin{array}{r}\text { GB } 1 \\
\text { syn.nons. sign. }\end{array}$} \\
\hline F 3 & fixed & 4 & 0 & - & & & & & & & & & \\
\hline Saint Isidore & poly & 9 & 0 & & & & & & & & & & \\
\hline FIN 1 & fixed & 0 & 0 & - & 4 & 0 & - & & & & & & \\
\hline Lahti & poly & 0 & 0 & & 9 & 0 & & & & & & & \\
\hline GB 1 & fixed & 0 & 0 & - & 0 & 0 & - & 0 & 0 & - & & & \\
\hline Ascot & poly & 8 & 2 & & 16 & 2 & & 8 & 2 & & & & \\
\hline GB 2 & fixed & 0 & 0 & - & 0 & 0 & - & 0 & 0 & - & 0 & 0 & - \\
\hline Braemar & poly & 11 & 2 & & 19 & 2 & & 11 & 2 & & 14 & 4 & \\
\hline NL 1 & fixed & 0 & 0 & - & 4 & 0 & - & 0 & 0 & - & 0 & 0 & - \\
\hline Wageningen & poly & 0 & 0 & & 9 & 0 & & 0 & 0 & & 8 & 2 & \\
\hline NL 2 & fixed & 0 & 0 & - & 0 & 0 & - & 0 & 0 & - & 0 & 0 & - \\
\hline Hoge Veluwe & poly & 8 & 1 & & 16 & 1 & & 8 & 1 & & 8 & 2 & \\
\hline PL 2 & fixed & 0 & 0 & - & 0 & 0 & - & 0 & 0 & - & 0 & 0 & - \\
\hline Warsaw & poly & 6 & 0 & & 13 & 0 & & 6 & 0 & & 10 & 2 & \\
\hline RUM 1 & fixed & 0 & 0 & - & 3 & 0 & - & 0 & 0 & - & 0 & 0 & - \\
\hline Sibiu 1 & poly & 3 & 0 & & 11 & 0 & & 3 & 0 & & 9 & 2 & \\
\hline RUM 2 & fixed & 0 & 0 & - & 4 & 0 & - & 0 & 0 & - & 0 & 0 & - \\
\hline Sibiu 2 & poly & 0 & 0 & & 9 & 0 & & 0 & 0 & & 8 & 2 & \\
\hline RUM 5 & fixed & 4 & 0 & - & 5 & 0 & - & 4 & 0 & - & 2 & 0 & $1 \mathrm{~ns}$ \\
\hline Busteni & poly & 0 & 0 & & 9 & 0 & & 0 & 0 & & 8 & 2 & \\
\hline S 3 & fixed & 0 & 0 & - & 0 & 0 & - & 0 & 0 & - & 0 & 0 & - \\
\hline Malmoe & poly & 9 & 1 & & 17 & 1 & & 9 & 1 & & 9 & 2 & \\
\hline \multicolumn{2}{|l|}{ Population } & \multicolumn{3}{|c|}{$\begin{array}{r}\text { GB 2 } \\
\text { syn. nons. sign. }\end{array}$} & \multicolumn{3}{|c|}{$\begin{array}{r}\text { NL } 1 \\
\text { syn.nons. sign. }\end{array}$} & \multicolumn{3}{|c|}{$\begin{array}{r}\text { NL 2 } \\
\text { syn. nons. sign. }\end{array}$} & \multicolumn{3}{|c|}{$\begin{array}{r}\text { PL 2 } \\
\text { syn. nons. sign. }\end{array}$} \\
\hline NL 1 & fixed & 0 & 0 & - & & & & & & & & & \\
\hline Wageningen & poly & 11 & 2 & & & & & & & & & & \\
\hline NL 2 & fixed & 0 & 0 & - & 0 & 0 & - & & & & & & \\
\hline Hoge Veluwe & poly & 14 & 3 & & 8 & 1 & & & & & & & \\
\hline PL 2 & fixed & 0 & 0 & - & 0 & 0 & - & 0 & 0 & - & & & \\
\hline Warsaw & poly & 13 & 2 & & 6 & 0 & & 10 & 1 & & & & \\
\hline RUM 1 & fixed & 0 & 0 & - & 0 & 0 & - & 0 & 0 & - & 0 & 0 & - \\
\hline Sibiu 1 & poly & 12 & 2 & & 3 & 0 & & 9 & 1 & & 8 & 0 & \\
\hline RUM 2 & fixed & 0 & 0 & - & 0 & 0 & - & 0 & 0 & - & 0 & 0 & - \\
\hline Sibiu 2 & poly & 11 & 2 & & 0 & 0 & & 8 & 1 & & 6 & 0 & \\
\hline RUM 5 & fixed & 2 & 0 & $1 \mathrm{~ns}$ & 4 & 0 & - & 2 & 0 & $1 \mathrm{~ns}$ & 3 & 0 & - \\
\hline Busteni & poly & 11 & 2 & & 0 & 0 & & 8 & 1 & & 6 & 0 & \\
\hline S 3 & fixed & 0 & 0 & - & 0 & 0 & - & 0 & 0 & - & 0 & 0 & - \\
\hline Malmoe & poly & 15 & 3 & & 9 & 1 & & 9 & 1 & & 11 & 1 & \\
\hline Population & & syn & $\begin{array}{r}\mathbf{F} \\
\text { ons }\end{array}$ & $\begin{array}{l}\text { M } 1 \\
\text { ign. }\end{array}$ & syn. & I & $\begin{array}{l}\text { UM } 2 \\
\text { sign. }\end{array}$ & syn & & $\begin{array}{l}\text { UM } 5 \\
\text { ign. }\end{array}$ & & & \\
\hline RUM 2 & fixed & 0 & 0 & - & & & & & & & & & \\
\hline Sibiu 2 & poly & 3 & 0 & & & & & & & & & & \\
\hline RUM 5 & fixed & 1 & 0 & - & 4 & 0 & - & & & & & & \\
\hline Busteni & poly & 3 & 0 & & 0 & 0 & & & & & & & \\
\hline S 3 & fixed & 0 & 0 & - & 0 & 0 & - & 2 & 0 & $1 \mathrm{~ns}$ & & & \\
\hline Malmoe & poly & 10 & 1 & & 9 & 1 & & 9 & 1 & & & & \\
\hline
\end{tabular}


Table A24: Neutrality indices of Nothrus silvestris computed in the McDonald-Kreitman test with DnaSP v5. Locations with less than two individuals were excluded.

\begin{tabular}{|c|c|c|c|c|c|c|c|c|c|c|c|c|c|c|c|c|c|c|c|c|c|c|c|c|}
\hline \multicolumn{2}{|c|}{ Population } & 1 & 2 & 3 & 4 & 5 & 6 & 7 & 8 & 9 & 10 & 11 & 12 & 13 & 14 & 15 & 16 & 17 & 18 & 19 & 20 & 21 & 22 & 23 \\
\hline 1 & $\mathrm{CH} 2$ & & & & & & & & & & & & & & & & & & & & & & & \\
\hline 2 & $\mathrm{CH} 3$ & - & & & & & & & & & & & & & & & & & & & & & & \\
\hline 3 & D 1 & - & - & & & & & & & & & & & & & & & & & & & & & \\
\hline 4 & D 2 & - & - & - & & & & & & & & & & & & & & & & & & & & \\
\hline 5 & D 4 & - & - & - & - & & & & & & & & & & & & & & & & & & & \\
\hline 6 & D 6 & - & - & - & - & - & & & & & & & & & & & & & & & & & & \\
\hline 7 & D 7 & - & 0 & - & 0.3 & - & - & & & & & & & & & & & & & & & & & \\
\hline 8 & D 8 & - & - & - & - & - & - & - & & & & & & & & & & & & & & & & \\
\hline 9 & D 9 & - & - & - & - & - & - & - & - & & & & & & & & & & & & & & & \\
\hline 10 & D 11 & - & - & - & - & - & - & 3 & - & - & & & & & & & & & & & & & & \\
\hline 11 & D 16 & - & - & - & - & - & - & - & - & - & - & & & & & & & & & & & & & \\
\hline 12 & D 17 & - & - & - & - & - & - & 0.25 & - & - & - & - & & & & & & & & & & & & \\
\hline 13 & D 18 & - & - & - & - & - & - & - & - & - & - & - & - & & & & & & & & & & & \\
\hline 14 & F 3 & - & - & - & - & - & - & 0 & - & - & - & - & - & - & & & & & & & & & & \\
\hline 15 & FIN 1 & - & - & - & - & - & - & - & - & - & - & - & - & - & - & & & & & & & & & \\
\hline 16 & GB 1 & - & - & - & - & - & - & - & - & - & - & - & - & - & - & - & & & & & & & & \\
\hline 17 & GB 2 & - & - & - & - & - & - & 0.55 & - & - & - & - & - & - & - & - & - & & & & & & & \\
\hline 18 & NL 1 & - & - & - & - & - & - & - & - & - & - & - & - & - & - & - & - & - & & & & & & \\
\hline 19 & NL 2 & - & - & - & - & - & - & - & - & - & - & - & - & - & - & - & - & - & - & & & & & \\
\hline 20 & PL 2 & - & - & - & - & - & - & 0 & - & - & - & - & - & - & - & - & - & - & - & - & & & & \\
\hline 21 & RUM 1 & - & - & - & - & - & - & 0 & - & - & - & - & - & - & - & - & - & - & - & - & - & & & \\
\hline 22 & RUM 2 & - & - & - & - & - & - & - & - & - & - & - & - & - & - & - & - & - & - & - & - & - & & \\
\hline 23 & RUM 5 & - & - & - & - & - & - & - & - & - & - & - & - & - & - & - & - & - & - & - & - & - & - & \\
\hline 24 & S 3 & - & - & - & - & - & - & - & - & - & - & - & - & - & - & - & - & - & - & - & - & - & - & - \\
\hline
\end{tabular}




\section{Appendix}

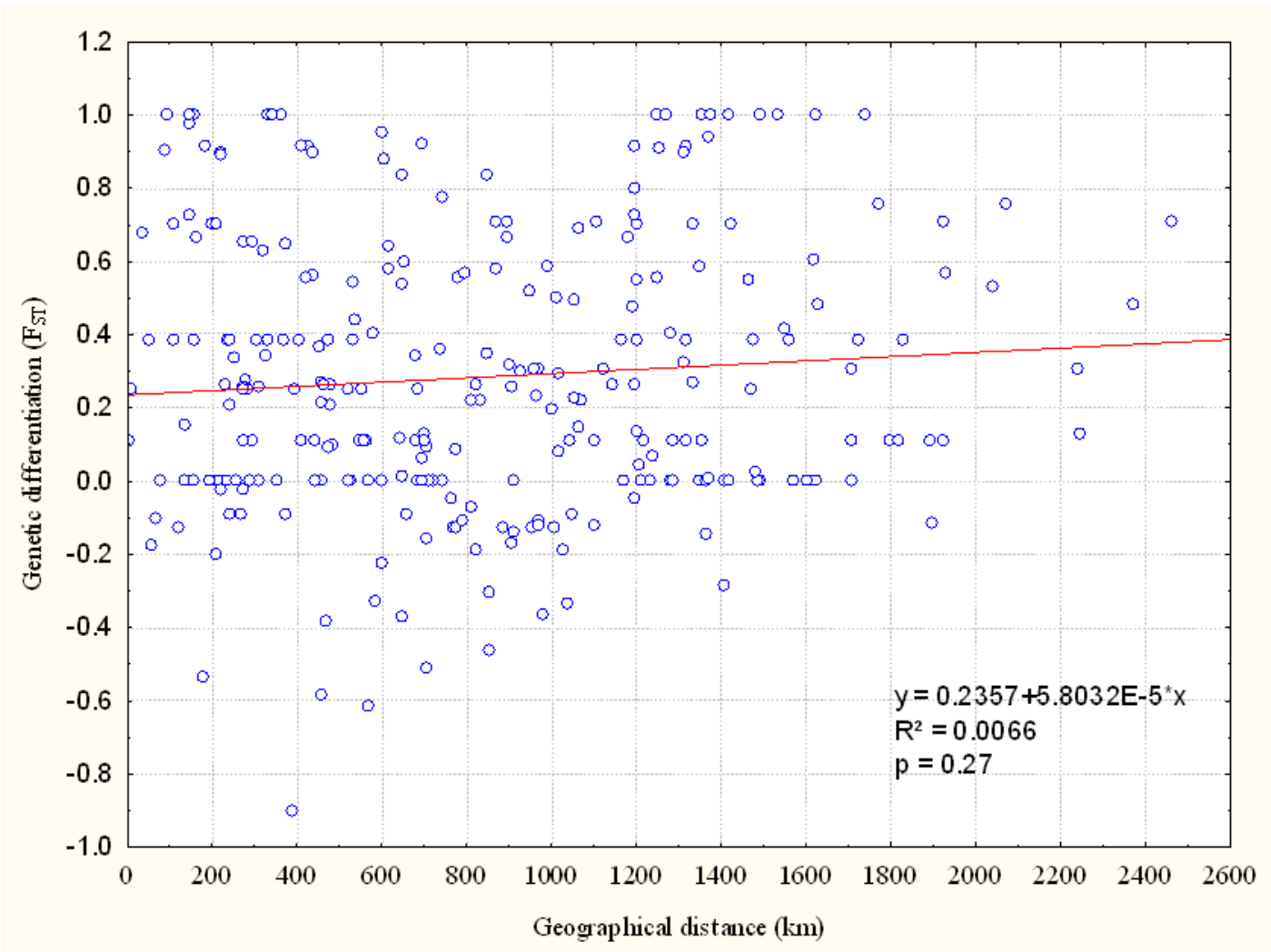

Figure A29: Linear regression of geographical distances (kilometers) vs. genetic differentiations $\left(\mathrm{F}_{\mathrm{ST}}\right)$ based on COI of Nothrus silvestris. Regression is not significant $(\mathrm{p}=0.27 ;(* 0.01<\mathrm{P}<0.05 ; * * 0.001<\mathrm{P}<0.01 ; * * * \mathrm{P}<0.001))$ using 1000 randomizations. 
Appendix

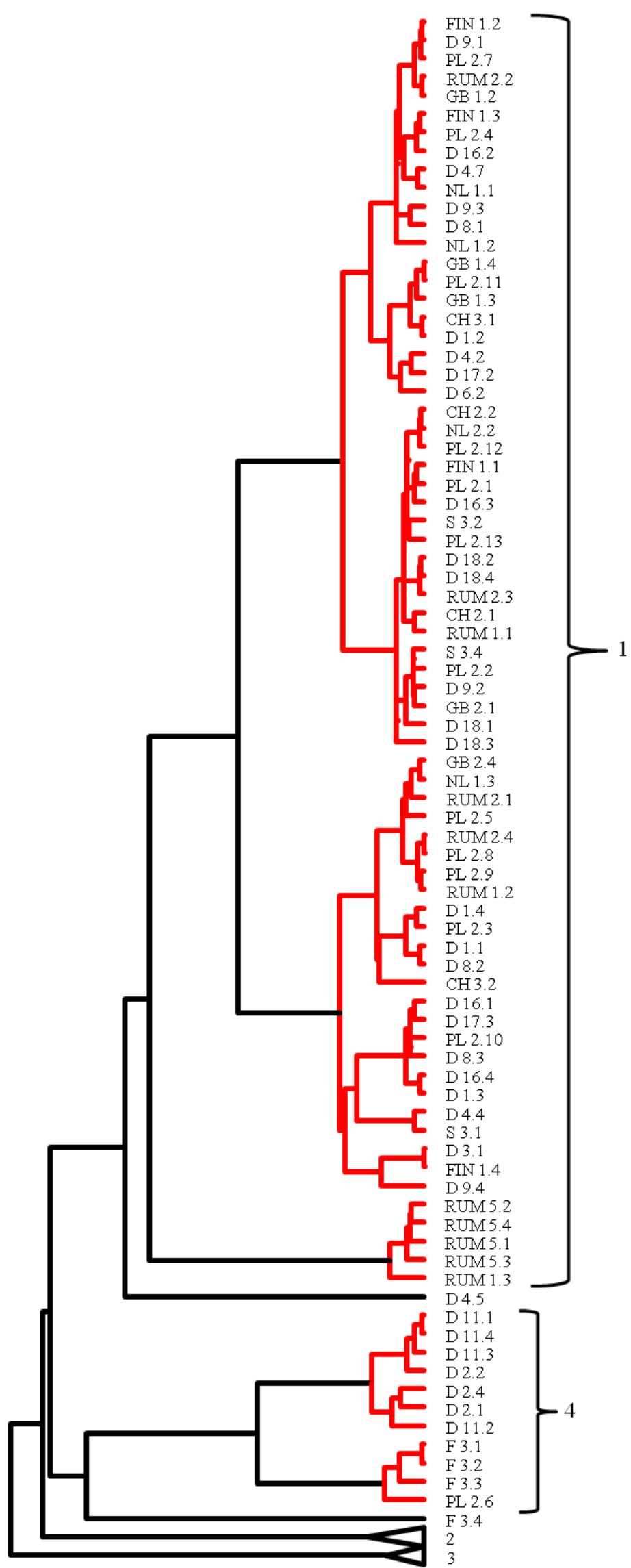

Figure A30: Cluster delimitation of COI nucleotide sequences from Nothrus silvestris after the single method of the generalized mixed Yule coalescent method (GMYC). Red branches are the different distinct clusters. Tip labels are explained in Table 15. 
Appendix

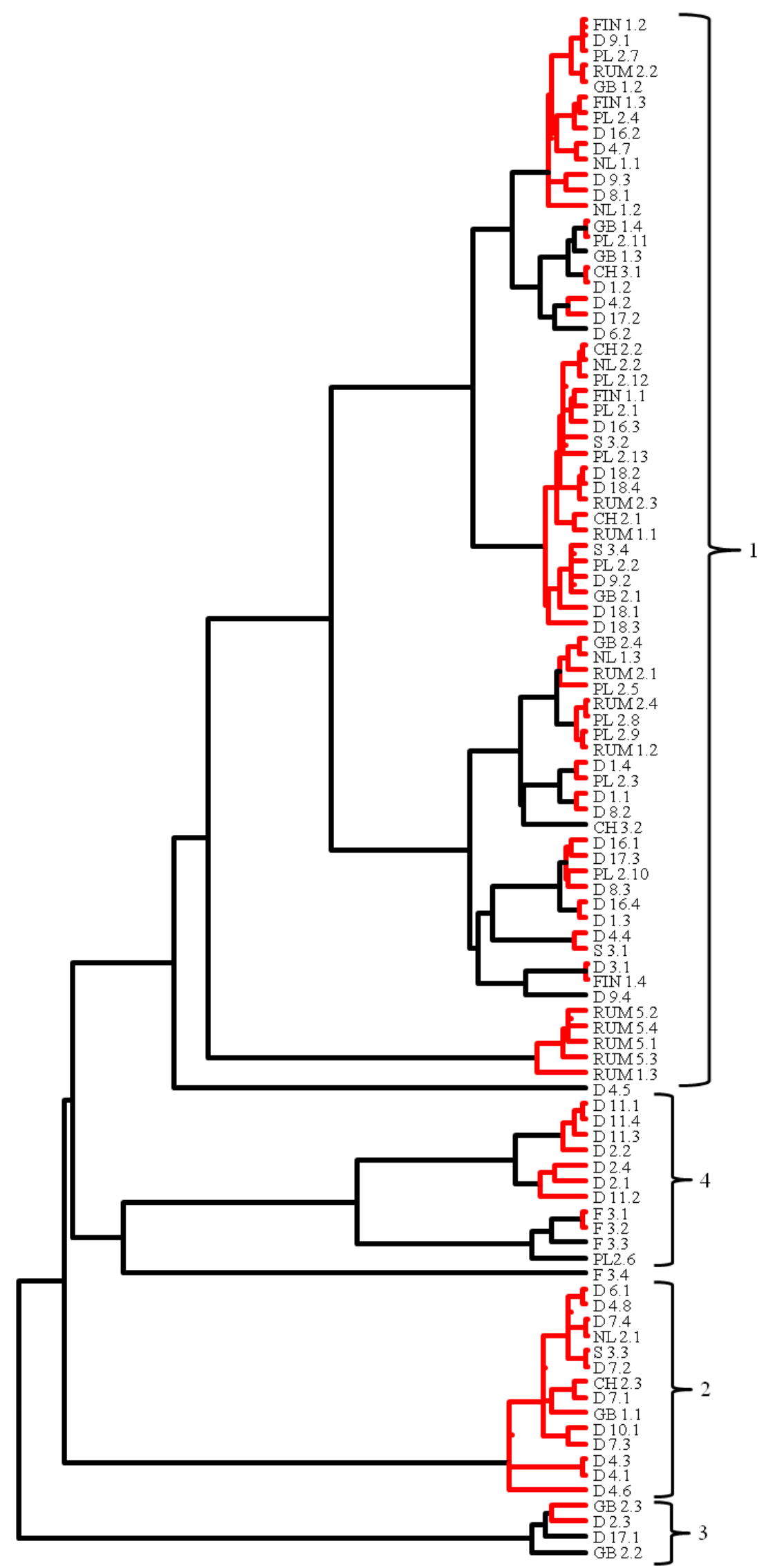

Figure A31 Cluster delimitation of COI nucleotide sequences from Nothrus silvestris after the multiple method of the generalized mixed Yule coalescent method (GMYC). Red branches are the different distinct clusters. Tip labels are explained in Table 15. 
Appendix

Table A25: Standard diversity measures of Platynothrus peltifer. Populations with less than two individuals were excluded.

\begin{tabular}{|c|c|c|c|c|c|c|c|c|c|}
\hline population & $\begin{array}{c}\text { sample } \\
\text { size } \\
\mathrm{n} \\
\end{array}$ & $\begin{array}{c}\text { invarible } \\
\text { sites } \\
\mathbf{N}_{\text {is }} \\
\end{array}$ & $\begin{array}{c}\text { variable } \\
\text { sites } \\
\mathbf{N}_{\mathrm{vs}} \\
\end{array}$ & $\begin{array}{c}\text { parsimony } \\
\text { inform. sites } \\
N_{\text {pars }} \\
\end{array}$ & $\begin{array}{c}\text { number of } \\
\text { singeltons } \\
\mathbf{N}_{\mathrm{s}} \\
\end{array}$ & $\begin{array}{c}\text { number of } \\
\text { haplotypes } \\
N_{h} \\
\end{array}$ & $\begin{array}{c}\text { haplotype } \\
\text { diversity } \\
\mathbf{H}_{\mathrm{d}} \\
\end{array}$ & variance & $\begin{array}{c}\text { nucleotide } \\
\text { diversity } \\
\Pi_{n} \\
\end{array}$ \\
\hline$\overline{A \_1}$ & 2 & 555 & 1 & 0 & 1 & 2 & 1 & 0.25 & 0.002 \\
\hline A_2 & 2 & 556 & 0 & 0 & 0 & 1 & 0 & 0 & 0 \\
\hline A_3 & 3 & 553 & 3 & 0 & 3 & 2 & 0.67 & 0.099 & 0.004 \\
\hline A_4 & 2 & 556 & 0 & 0 & 0 & 1 & 0 & 0 & 0 \\
\hline A_5 & 4 & 413 & 143 & 32 & 111 & 4 & 1 & 0.031 & 0.144 \\
\hline A_9 & 5 & 553 & 3 & 1 & 2 & 4 & 0.9 & 0.026 & 0.003 \\
\hline A_10 & 5 & 556 & 0 & 0 & 0 & 1 & 0 & 0 & 0 \\
\hline CH_3 & 5 & 554 & 2 & 0 & 2 & 3 & 0.7 & 0.048 & 0.001 \\
\hline D_1 & 4 & 456 & 100 & 6 & 94 & 4 & 1 & 0.031 & 0.093 \\
\hline D_2 & 2 & 556 & 0 & 0 & 0 & 1 & 0 & 0 & 0 \\
\hline D_3 & 4 & 549 & 7 & 2 & 5 & 4 & 1 & 0.031 & 0.007 \\
\hline D_4 & 8 & 455 & 101 & 99 & 2 & 4 & 0.64 & 0.034 & 0.078 \\
\hline D_5 & 4 & 546 & 10 & 1 & 9 & 4 & 1 & 0.031 & 0.009 \\
\hline D_6 & 5 & 555 & 1 & 0 & 1 & 2 & 0.4 & 0.056 & 0.001 \\
\hline D_7 & 3 & 556 & 0 & 0 & 0 & 1 & 0 & 0 & 0 \\
\hline D_8 & 4 & 443 & 113 & 104 & 9 & 4 & 1 & 0.031 & 0.132 \\
\hline D_9 & 3 & 456 & 100 & 0 & 100 & 2 & 0.67 & 0.099 & 0.12 \\
\hline D_11 & 2 & 454 & 102 & 0 & 102 & 2 & 1 & 0.25 & 0.183 \\
\hline D_12 & 5 & 551 & 5 & 2 & 3 & 3 & 0.7 & 0.048 & 0.004 \\
\hline D_15 & 3 & 444 & 112 & 0 & 112 & 3 & 1 & 0.074 & 0.134 \\
\hline D_17 & 3 & 446 & 110 & 0 & 110 & 3 & 1 & 0.074 & 0.132 \\
\hline D_18 & 7 & 527 & 29 & 25 & 4 & 4 & 0.71 & 0.033 & 0.024 \\
\hline DK_2 & 6 & 529 & 27 & 27 & 0 & 3 & 0.73 & 0.024 & 0.030 \\
\hline DK_3 & 6 & 556 & 0 & 0 & 0 & 1 & 0 & 0 & 0 \\
\hline F_1 & 4 & 412 & 144 & 62 & 82 & 3 & 0.83 & 0.049 & 0.157 \\
\hline F_3 & 2 & 556 & 0 & 0 & 0 & 1 & 0 & 0 & 0 \\
\hline F_5 & 3 & 553 & 3 & 0 & 3 & 3 & 1 & 0.074 & 0.004 \\
\hline FIN_1 & 4 & 555 & 1 & 0 & 1 & 2 & 0.5 & 0.07 & 0.001 \\
\hline GB_1 & 3 & 445 & 111 & 0 & 111 & 2 & 0.67 & 0.099 & 0.133 \\
\hline GB_2 & 4 & 530 & 26 & 0 & 26 & 2 & 0.5 & 0.07 & 0.024 \\
\hline N_1 & 2 & 554 & 2 & 0 & 2 & 2 & 1 & 0.25 & 0.004 \\
\hline NL_1 & 3 & 473 & 83 & 0 & 83 & 2 & 0.67 & 0.099 & 0.1 \\
\hline $\mathrm{NL} \_2$ & 4 & 553 & 3 & 1 & 2 & 3 & 0.83 & 0.049 & 0.003 \\
\hline PL_2 & 9 & 511 & 45 & 27 & 18 & 8 & 0.97 & 0.004 & 0.027 \\
\hline RUM_2 & 2 & 458 & 98 & 0 & 98 & 2 & 1 & 0.25 & 0.176 \\
\hline S_1 & 3 & 554 & 2 & 0 & 2 & 3 & 1 & 0.074 & 0.002 \\
\hline S_2 & 9 & 467 & 89 & 82 & 7 & 8 & 0.97 & 0.004 & 0.063 \\
\hline S_4 & 4 & 537 & 19 & 0 & 19 & 3 & 0.83 & 0.049 & 0.017 \\
\hline S_5 & 4 & 552 & 4 & 1 & 3 & 3 & 0.83 & 0.049 & 0.004 \\
\hline all & 157 & 360 & 196 & 172 & 24 & 74 & 0.98 & 0 & 0.113 \\
\hline
\end{tabular}


Appendix

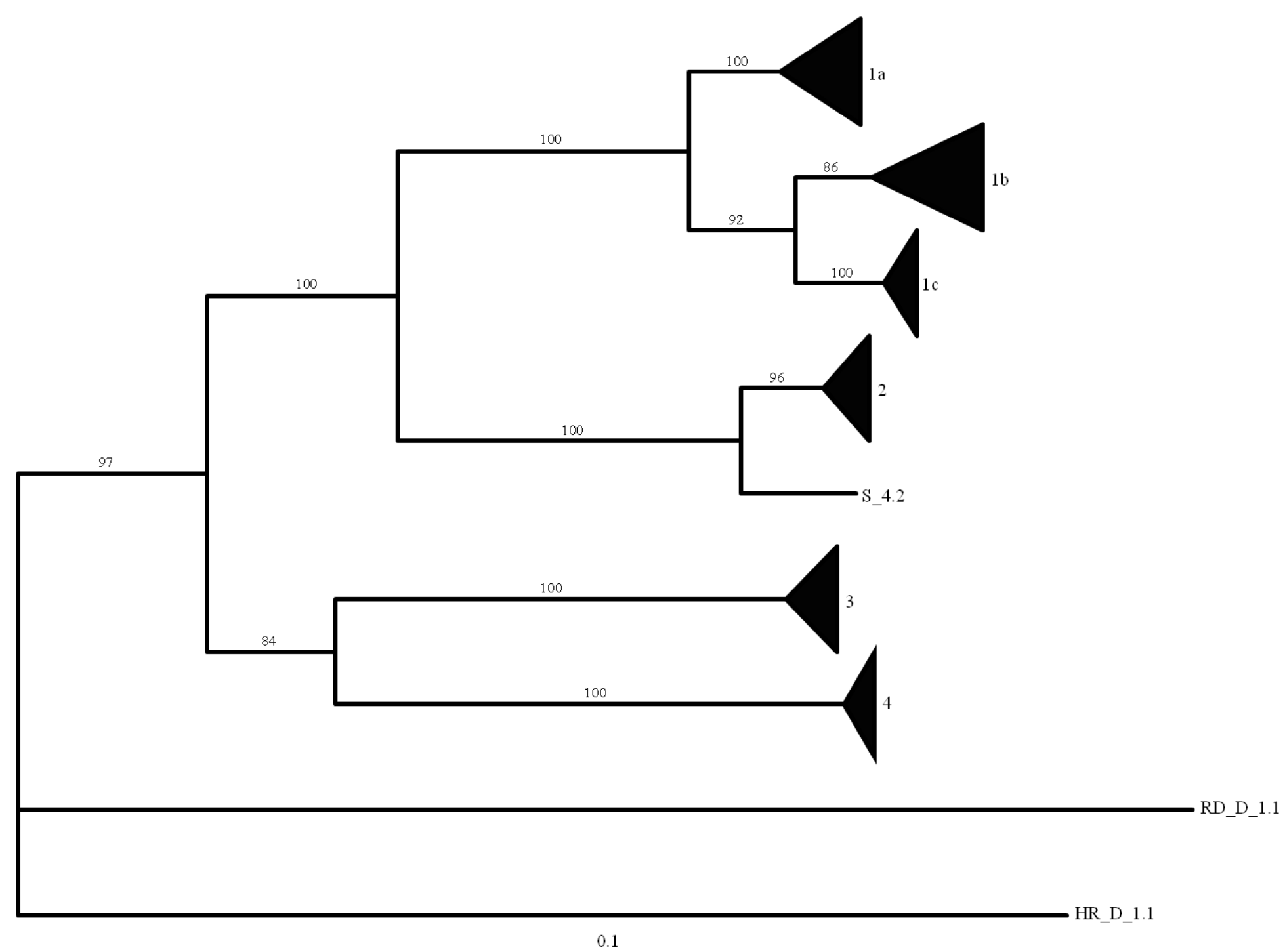

Figure A32: Neighbor-Joining tree of 160 COI nucleotide sequences of Platynothrus peltifer. Outgroups are one individual of Hypochthonius rufulus and Rhysotritia duplicata, respectively. Numbers on the branches are bootstrap values of 100,000 pseudo-replicates. Tip numbers are the different subclades and explain in Table 20. 
Appendix

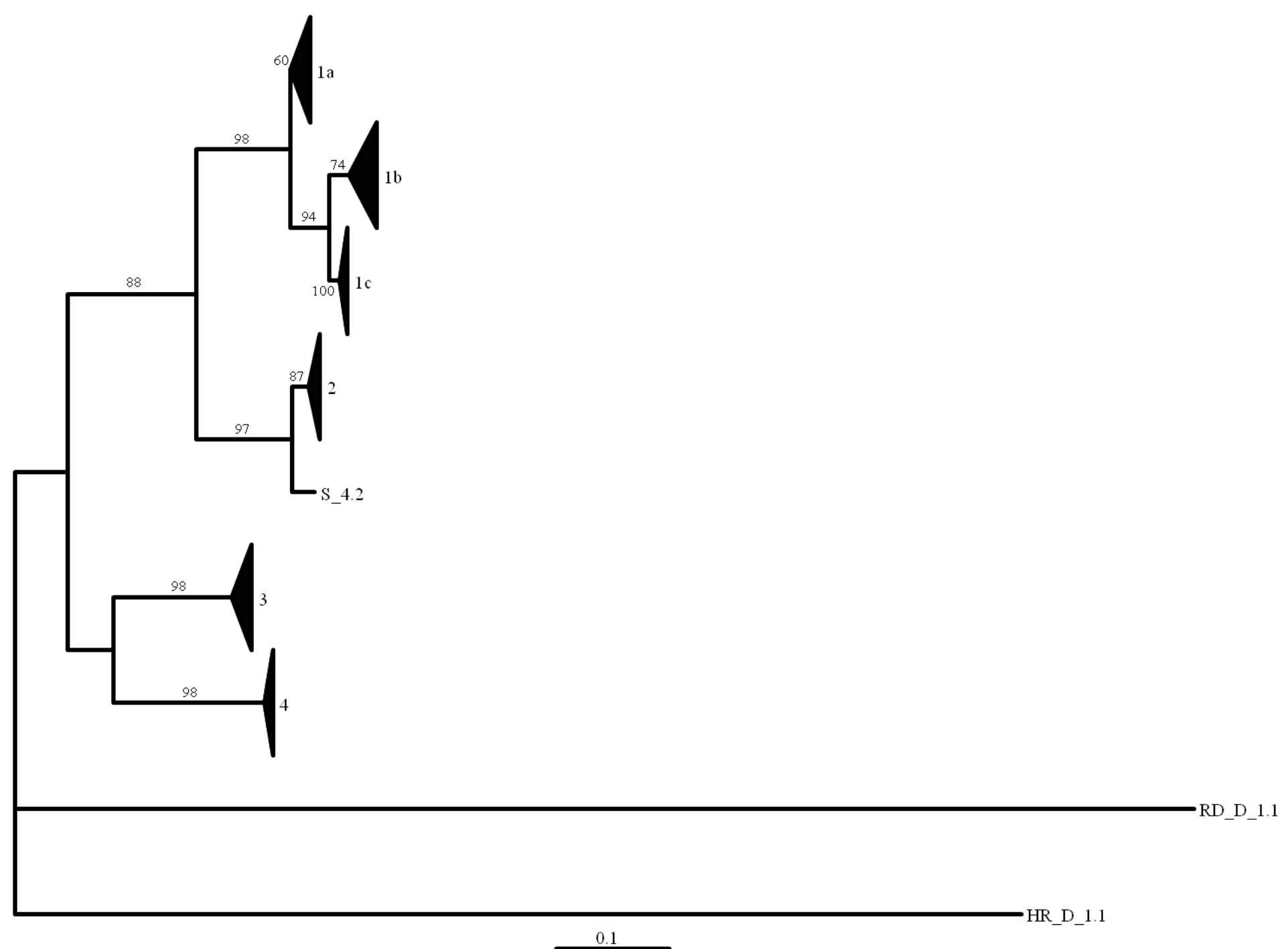

Figure A33: Neighbor-Joining tree of 160 COI nucleotide sequences of Platynothrus peltifer with model of sequence evolution HKY+G. Outgroups are one individual of Hypochthonius rufulus and Rhysotritia duplicata, respectively. Numbers on the branches are bootstrap values of 100,000 pseudo-replicates. Tip numbers are the different subclades and explain in Table 20. 
Appendix

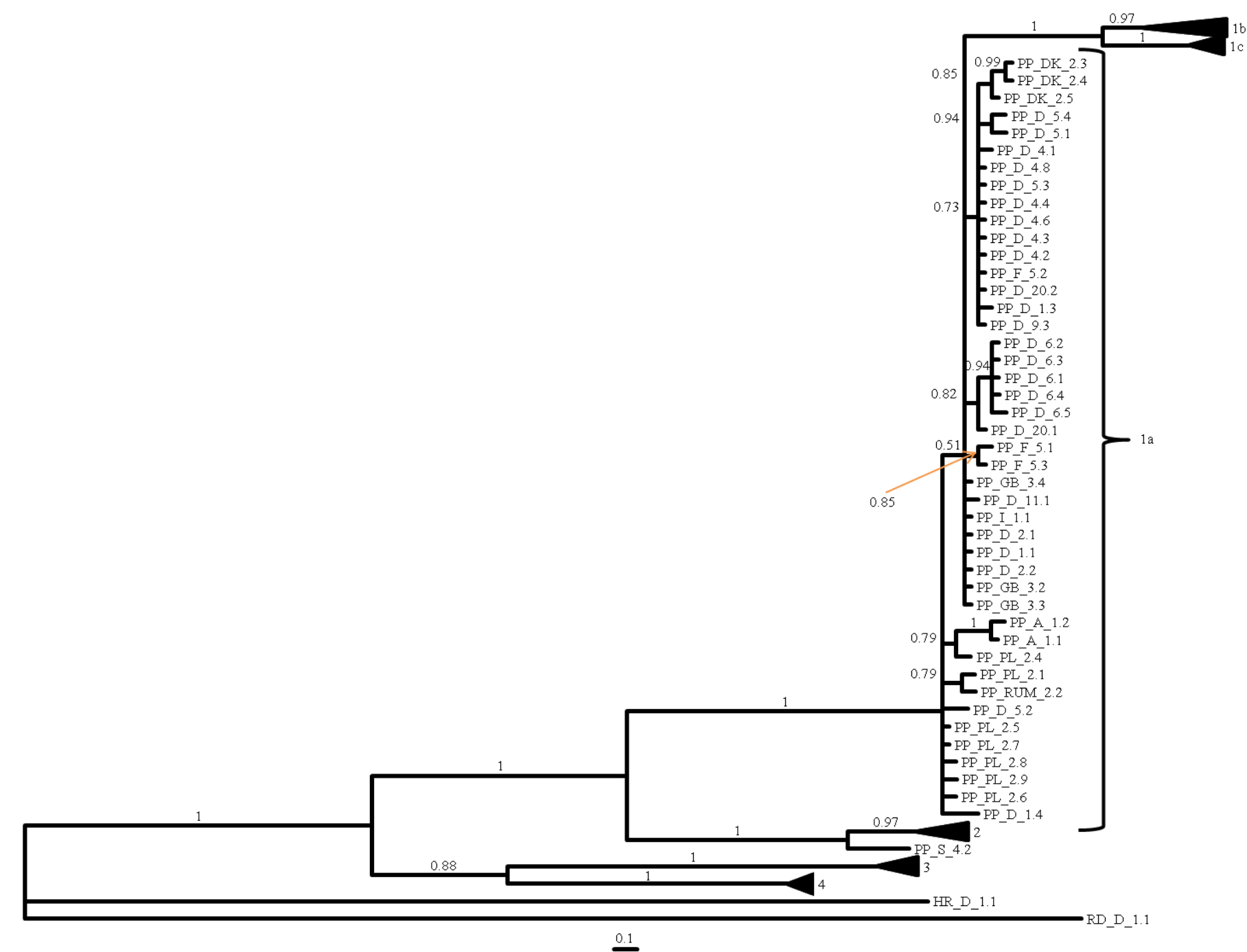

Figure A34: Bayesian tree after 10x10 $10^{6}$ generations from 160 COI nucleotide sequences from Platynothrus peltifer with MrBayes. Split frequencies of 0.01069 and burnin of $25 \%$. Outgroups are one individual of Hypochthonius rufulus and Rhysotritia duplicata, respectively. Numbers on the branches are posterior probabilities. Tip numbers are the different subclades and explain in Table 20. 
Appendix

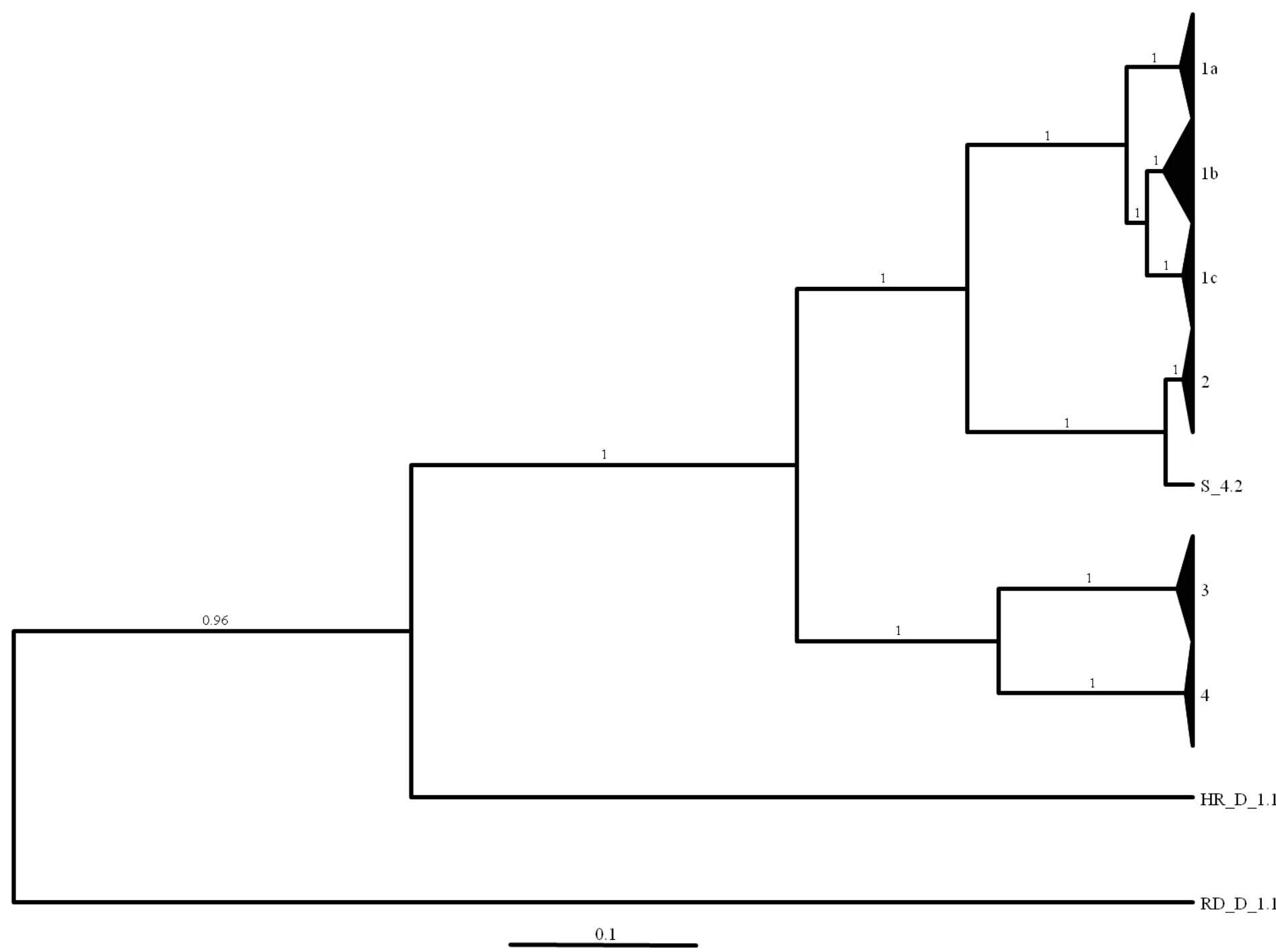

Figure A35: Bayesian phylogeny after 10x106 generations from the 160 COI nucleotide sequences of Platynothrus peltifer with Beast v1.5.4. Outgroups are one individual of Hypochthonius rufulus and Rhysotritia duplicata, respectively. Numbers on the branches are posterior probabilities. Tip numbers are the different subclades and explain in Table 20. 
Appendix

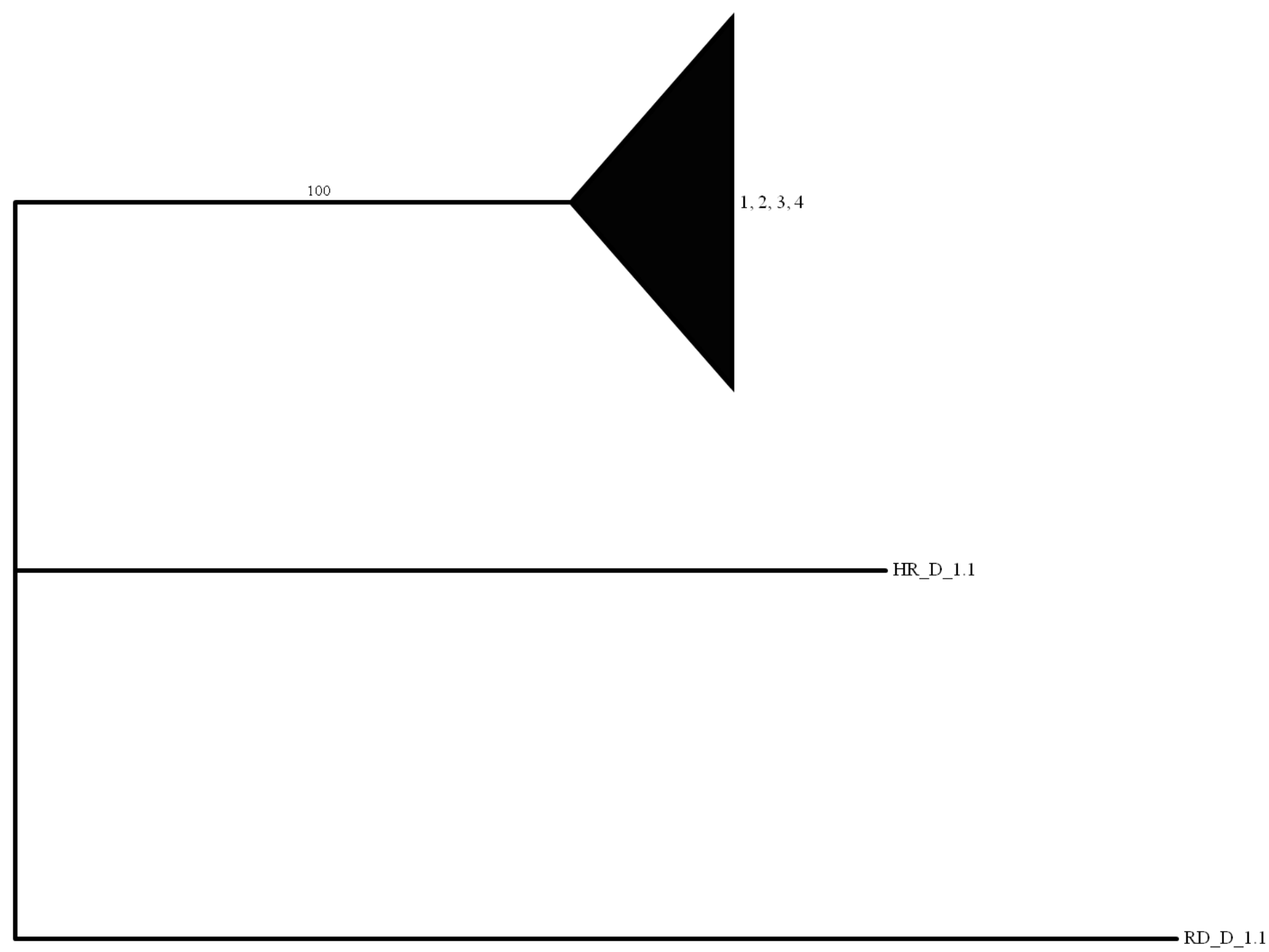

0.1

Figure A36: Neighbor-Joining tree of 160 COI protein sequences of Platynothrus peltifer. Outgroups are one individual of Hypochthonius rufulus and Rhysotritia duplicata, respectively. Numbers on the branches are bootstrap values of 100,000 pseudo-replicates. Tip numbers are the different subclades and explain in Table 20. 
Appendix

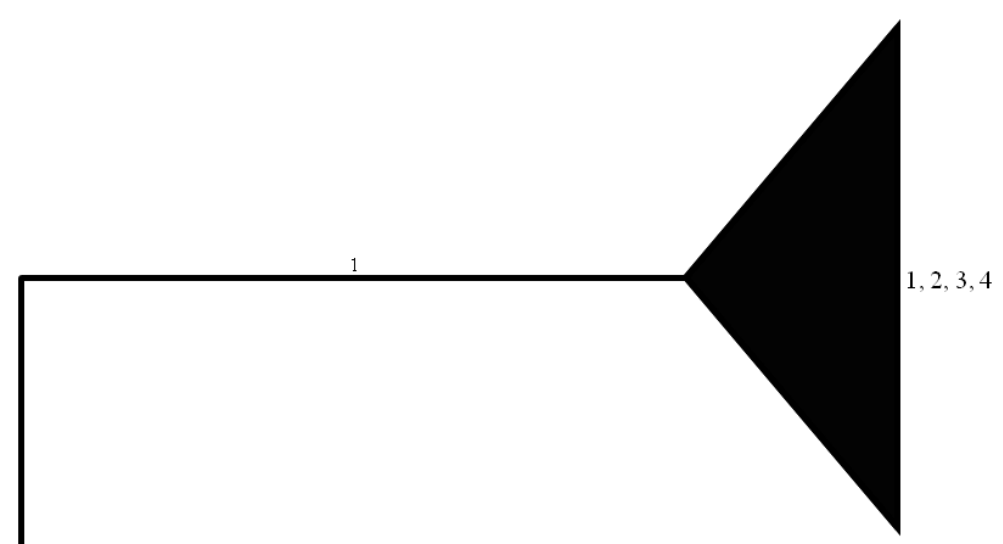

Figure A37: Bayesian tree after 10x1 $10^{6}$ generations from 160 COI protein sequences of Platynothrus peltifer. Split frequencies of 0.028819 and burnin of $25 \%$. Outgroups are one individual of Hypochthonius rufulus and Rhysotritia duplicata, respectively. Numbers on the branches are posterior probabilities. Tip numbers are the different subclades and explain in Table 20. 
Appendix

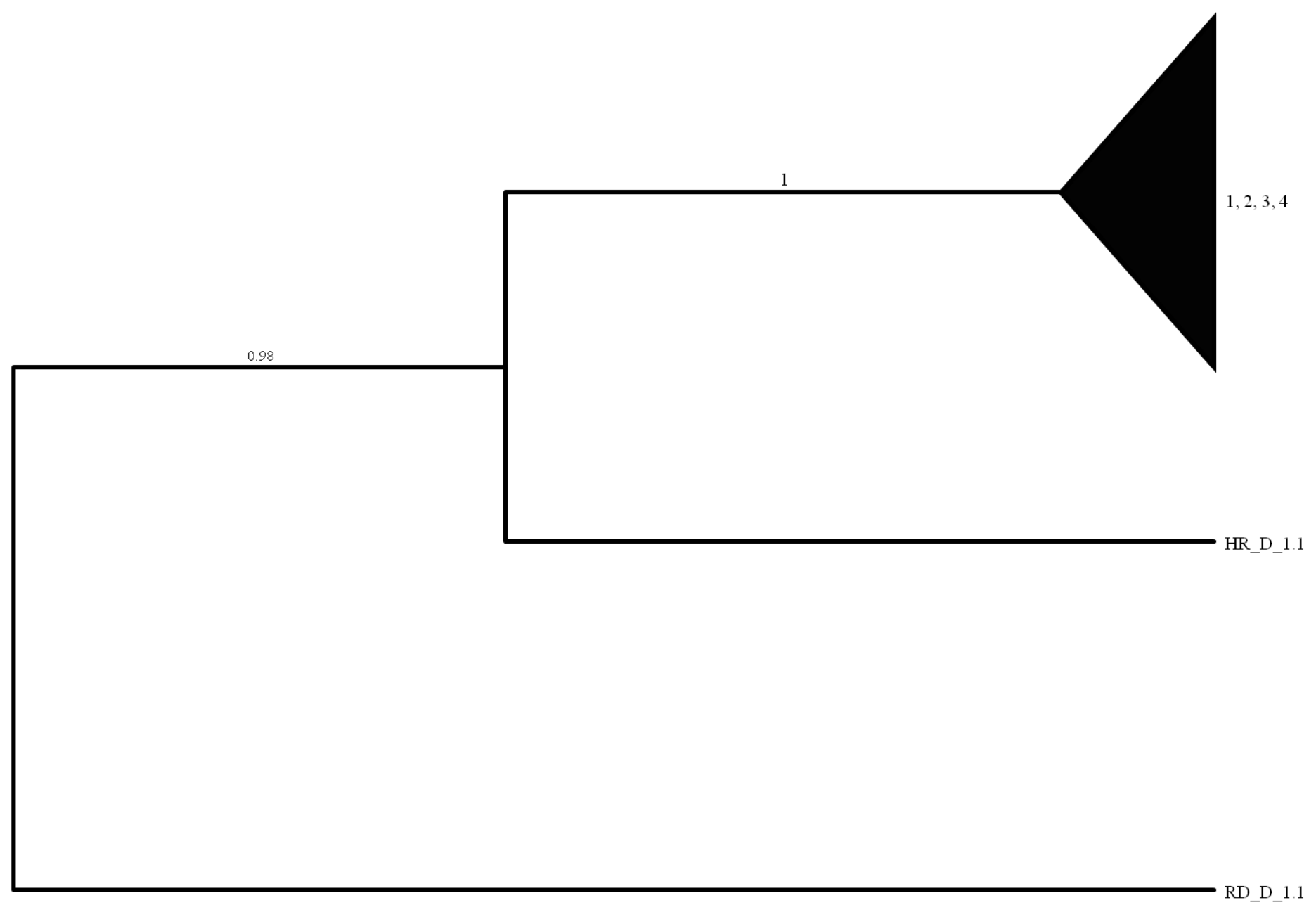

0.1

Figure A38: Bayesian phylogeny after 10x10 generations with Beast v1.5.4 from the 160 COI protein sequences of Platynothrus peltifer. Outgroups are one individual of Hypochthonius rufulus and Rhysotritia duplicata, respectively. Numbers on the branches are posterior probabilities. Tip numbers are the different subclades and explain in Table 20. 


\section{Appendix}

Table A26: Mean pairwise percentage differences of uncorrected p-distances of the protein of Platynothrus peltifer from 39 locations. The diagonal is the within population differences (bold) and below the diagonal the among population differences. Bold letters in red are the minimum and maximum differences within and among the populations. Locations with less than two individuals were excluded from the analysis.

\begin{tabular}{|c|c|c|c|c|c|c|c|c|c|c|c|c|c|c|c|c|c|c|c|c|c|c|c|c|}
\hline Population & A 1 & A2 & $\mathrm{A} 3$ & $\mathrm{~A} 4$ & A5 & A9 & A 10 & $\mathrm{CH} 3$ & D 1 & D 2 & D 3 & D 4 & D 5 & D 6 & D 7 & D 8 & D 9 & D 11 & D 12 & D 15 & D 17 & D 18 & DK2 & DK 3 \\
\hline A 1 & 0 & & & & & & & & & & & & & & & & & & & & & & & \\
\hline A 2 & 0 & 0 & & & & & & & & & & & & & & & & & & & & & & \\
\hline A 3 & 0 & 0 & 0 & & & & & & & & & & & & & & & & & & & & & \\
\hline A 4 & 0 & 0 & 0 & 0 & & & & & & & & & & & & & & & & & & & & \\
\hline A 5 & 0 & 0 & 0 & 0 & 0 & & & & & & & & & & & & & & & & & & & \\
\hline A 9 & 0 & 0 & 0 & 0 & 0 & 0 & & & & & & & & & & & & & & & & & & \\
\hline A 10 & 0 & 0 & 0 & 0 & 0 & 0 & 0 & & & & & & & & & & & & & & & & & \\
\hline $\mathrm{CH} 3$ & 0 & 0 & 0 & 0 & 0 & 0 & 0 & 0 & & & & & & & & & & & & & & & & \\
\hline D 1 & 0 & 0 & 0 & 0 & 0 & 0 & 0 & 0 & 0 & & & & & & & & & & & & & & & \\
\hline D 2 & 0 & 0 & 0 & 0 & 0 & 0 & 0 & 0 & 0 & 0 & & & & & & & & & & & & & & \\
\hline D 3 & 0 & 0 & 0 & 0 & 0 & 0 & 0 & 0 & 0 & 0 & 0 & & & & & & & & & & & & & \\
\hline D 4 & 0 & 0 & 0 & 0 & 0 & 0 & 0 & 0 & 0 & 0 & 0 & 0 & & & & & & & & & & & & \\
\hline D 5 & 0 & 0 & 0 & 0 & 0 & 0 & 0 & 0 & 0 & 0 & 0 & 0 & 0 & & & & & & & & & & & \\
\hline D 6 & 0 & 0 & 0 & 0 & 0 & 0 & 0 & 0 & 0 & 0 & 0 & 0 & 0 & 0 & & & & & & & & & & \\
\hline D 7 & 0 & 0 & 0 & 0 & 0 & 0 & 0 & 0 & 0 & 0 & 0 & 0 & 0 & 0 & 0 & & & & & & & & & \\
\hline D 8 & 0 & 0 & 0 & 0 & 0 & 0 & 0 & 0 & 0 & 0 & 0 & 0 & 0 & 0 & 0 & 0 & & & & & & & & \\
\hline D 9 & 0 & 0 & 0 & 0 & 0 & 0 & 0 & 0 & 0 & 0 & 0 & 0 & 0 & 0 & 0 & 0 & 0 & & & & & & & \\
\hline D 11 & 0 & 0 & 0 & 0 & 0 & 0 & 0 & 0 & 0 & 0 & 0 & 0 & 0 & 0 & 0 & 0 & 0 & 0 & & & & & & \\
\hline D 12 & 0.1 & 0.1 & 0.1 & 0.1 & 0.1 & 0.1 & 0.1 & 0.1 & 0.1 & 0.1 & 0.1 & 0.1 & 0.1 & 0.1 & 0.1 & 0.1 & 0.1 & 0.1 & 0.2 & & & & & \\
\hline D 15 & 0 & 0 & 0 & 0 & 0 & 0 & 0 & 0 & 0 & 0 & 0 & 0 & 0 & 0 & 0 & 0 & 0 & 0 & 0.1 & 0 & & & & \\
\hline D 17 & 0 & 0 & 0 & 0 & 0 & 0 & 0 & 0 & 0 & 0 & 0 & 0 & 0 & 0 & 0 & 0 & 0 & 0 & 0.1 & 0 & 0 & & & \\
\hline D 18 & 0 & 0 & 0 & 0 & 0 & 0 & 0 & 0 & 0 & 0 & 0 & 0 & 0 & 0 & 0 & 0 & 0 & 0 & 0.1 & 0 & 0 & 0 & & \\
\hline DK 2 & 0 & 0 & 0 & 0 & 0 & 0 & 0 & 0 & 0 & 0 & 0 & 0 & 0 & 0 & 0 & 0 & 0 & 0 & 0.1 & 0 & 0 & 0 & 0 & \\
\hline DK 3 & 0 & 0 & 0 & 0 & 0 & 0 & 0 & 0 & 0 & 0 & 0 & 0 & 0 & 0 & 0 & 0 & 0 & 0 & 0.1 & 0 & 0 & 0 & 0 & 0 \\
\hline F 1 & 0 & 0 & 0 & 0 & 0 & 0 & 0 & 0 & 0 & 0 & 0 & 0 & 0 & 0 & 0 & 0 & 0 & 0 & 0.1 & 0 & 0 & 0 & 0 & 0 \\
\hline F 3 & 0 & 0 & 0 & 0 & 0 & 0 & 0 & 0 & 0 & 0 & 0 & 0 & 0 & 0 & 0 & 0 & 0 & 0 & 0.1 & 0 & 0 & 0 & 0 & 0 \\
\hline F 5 & 0 & 0 & 0 & 0 & 0 & 0 & 0 & 0 & 0 & 0 & 0 & 0 & 0 & 0 & 0 & 0 & 0 & 0 & 0.1 & 0 & 0 & 0 & 0 & 0 \\
\hline FIN 1 & 0 & 0 & 0 & 0 & 0 & 0 & 0 & 0 & 0 & 0 & 0 & 0 & 0 & 0 & 0 & 0 & 0 & 0 & 0.1 & 0 & 0 & 0 & 0 & 0 \\
\hline GB 1 & 0 & 0 & 0 & 0 & 0 & 0 & 0 & 0 & 0 & 0 & 0 & 0 & 0 & 0 & 0 & 0 & 0 & 0 & 0.1 & 0 & 0 & 0 & 0 & 0 \\
\hline GB 2 & 0 & 0 & 0 & 0 & 0 & 0 & 0 & 0 & 0 & 0 & 0 & 0 & 0 & 0 & 0 & 0 & 0 & 0 & 0.1 & 0 & 0 & 0 & 0 & 0 \\
\hline N 1 & 0 & 0 & 0 & 0 & 0 & 0 & 0 & 0 & 0 & 0 & 0 & 0 & 0 & 0 & 0 & 0 & 0 & 0 & 0.1 & 0 & 0 & 0 & 0 & 0 \\
\hline NL 1 & 0 & 0 & 0 & 0 & 0 & 0 & 0 & 0 & 0 & 0 & 0 & 0 & 0 & 0 & 0 & 0 & 0 & 0 & 0.1 & 0 & 0 & 0 & 0 & 0 \\
\hline NL 2 & 0.1 & 0.1 & 0.1 & 0.1 & 0.1 & 0.1 & 0.1 & 0.1 & 0.1 & 0.1 & 0.1 & 0.1 & 0.1 & 0.1 & 0.1 & 0.1 & 0.1 & 0.1 & 0.2 & 0.1 & 0.1 & 0.1 & 0.1 & 0.1 \\
\hline PL 2 & 0.1 & 0.1 & 0.1 & 0.1 & 0.1 & 0.1 & 0.1 & 0.1 & 0.1 & 0.1 & 0.1 & 0.1 & 0.1 & 0.1 & 0.1 & 0.1 & 0.1 & 0.1 & 0.2 & 0.1 & 0.1 & 0.1 & 0.1 & 0.1 \\
\hline RUM 2 & 0.3 & 0.3 & 0.3 & 0.3 & 0.3 & 0.3 & 0.3 & 0.3 & 0.3 & 0.3 & 0.3 & 0.3 & 0.3 & 0.3 & 0.3 & 0.3 & 0.3 & 0.3 & 0.3 & 0.3 & 0.3 & 0.3 & 0.3 & 0.3 \\
\hline $\mathrm{S}_{1}$ & 0 & 0 & 0 & 0 & 0 & 0 & 0 & 0 & 0 & 0 & 0 & 0 & 0 & 0 & 0 & 0 & 0 & 0 & 0.1 & 0 & 0 & 0 & 0 & 0 \\
\hline $\mathrm{S} 2$ & 0 & 0 & 0 & 0 & 0 & 0 & 0 & 0 & 0 & 0 & 0 & 0 & 0 & 0 & 0 & 0 & 0 & 0 & 0.1 & 0 & 0 & 0 & 0 & 0 \\
\hline $\mathrm{S} 4$ & 0 & 0 & 0 & 0 & 0 & 0 & 0 & 0 & 0 & 0 & 0 & 0 & 0 & 0 & 0 & 0 & 0 & 0 & 0.1 & 0 & 0 & 0 & 0 & 0 \\
\hline S 5 & 0 & 0 & 0 & 0 & 0 & 0 & 0 & 0 & 0 & 0 & 0 & 0 & 0 & 0 & 0 & 0 & 0 & 0 & 0.1 & 0 & 0 & 0 & 0 & 0 \\
\hline
\end{tabular}


Appendix

Table A26 continue:

\begin{tabular}{cccccccccccccccc}
\hline Population & F 1 & F3 & F5 & FIN 1 & GB 1 & GB 2 & N1 & NL1 & NL2 & PL2 & RUM2 & S1 & S2 & S 4 & S 5 \\
\hline F 1 & 0 & & & & & & & & & & & & & & \\
F 3 & 0 & 0 & & & & & & & & & & & & & \\
F 5 & 0 & 0 & 0 & & & & & & & & & & & \\
FIN 1 & 0 & 0 & 0 & 0 & & & & & & & & & & \\
GB 1 & 0 & 0 & 0 & 0 & 0 & & & & & & & & & \\
GB 2 & 0 & 0 & 0 & 0 & 0 & 0 & & & & & & & & & \\
N 1 & 0 & 0 & 0 & 0 & 0 & 0 & 0 & & & & & & & \\
NL 1 & 0 & 0 & 0 & 0 & 0 & 0 & 0 & 0 & & & & & & \\
NL 2 & 0.1 & 0.1 & 0.1 & 0.1 & 0.1 & 0.1 & 0.1 & 0.1 & $\mathbf{0 . 3}$ & & & & & \\
PL 2 & 0.1 & 0.1 & 0.1 & 0.1 & 0.1 & 0.1 & 0.1 & 0.1 & 0.2 & $\mathbf{0 . 1}$ & & & & \\
RUM 2 & 0.3 & 0.3 & 0.3 & 0.3 & 0.3 & 0.3 & 0.3 & 0.3 & $\mathbf{0 . 4}$ & 0.3 & 0.5 & & & & \\
S 1 & 0 & 0 & 0 & 0 & 0 & 0 & 0 & 0 & 0.1 & 0.1 & 0.3 & 0 & & & \\
S 2 & 0 & 0 & 0 & 0 & 0 & 0 & 0 & 0 & 0.1 & 0.1 & 0.3 & 0 & 0 & & \\
S 4 & 0 & 0 & 0 & 0 & 0 & 0 & 0 & 0 & 0.1 & 0.1 & 0.3 & 0 & 0 & 0 & \\
S 5 & 0 & 0 & 0 & 0 & 0 & 0 & 0 & 0 & 0.1 & 0.1 & 0.3 & 0 & 0 & 0 & 0 \\
\hline
\end{tabular}

Table A27: Tajima's D and Fu's FS neutrality tests of Platynothrus peltifer COI nucleotide sequences from 39 locations. Bold red letters show significance (P<0.05).

\begin{tabular}{|c|c|c|c|c|c|c|c|c|c|c|c|c|c|c|c|c|c|c|c|c|c|c|c|}
\hline Neutrality tests & A 1 & A2 & A3 & A 4 & A5 & A9 & A 10 & CH 3 & D 1 & D 2 & D 3 & D 4 & D5 & D 6 & D 7 & D 8 & D 9 & D 11 & D 12 & D 15 & D 17 & D 18 & DK2 \\
\hline \multicolumn{24}{|c|}{ 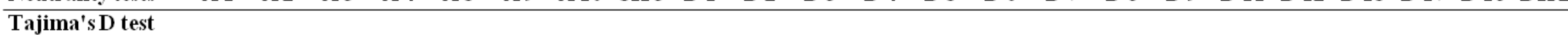 } \\
\hline Samplesize & 2 & 2 & 3 & 2 & 4 & 5 & 5 & 5 & 4 & 2 & 4 & 8 & 4 & 5 & 3 & 4 & 3 & 2 & 5 & 3 & 3 & 7 & 6 \\
\hline$S$ & 1 & 0 & 3 & 0 & 143 & 3 & 0 & 2 & 101 & 0 & 7 & 102 & 10 & 1 & 0 & 113 & 101 & 103 & 5 & 112 & 110 & 30 & 28 \\
\hline $\mathrm{Pi}$ & 1 & 0 & 2 & 0 & 80.5 & 1.4 & 0 & 0.8 & 51.83 & 0 & 3.83 & 43.36 & 5.17 & 0.4 & 0 & 73.83 & 67.33 & 103 & 2.4 & 74.67 & 73.33 & 13.62 & 216.73 \\
\hline Tajima's D & 0 & 0 & 0 & 0 & 0.34 & -0.17 & 0 & -0.97 & -0.62 & 0 & 0.04 & 0.56 & -0.53 & -0.82 & 0 & 2.07 & 0 & 0 & 0 & 0 & 0 & 0.64 & 2.3 \\
\hline Tajima's D p-value & 1 & 1 & 0.87 & 1 & 0.73 & 0.48 & 1 & 0.19 & 0.34 & 1 & 0.67 & 0.77 & 0.44 & 0.3 & 1 & 0.98 & 0.04 & 1 & 0.58 & 0.92 & 0.08 & 0.77 & 1 \\
\hline \multicolumn{24}{|l|}{ Fu's FS test } \\
\hline Real & 2 & 1 & 2 & 1 & 4 & 4 & 1 & 3 & 4 & 1 & 4 & A & 4 & 2 & 1 & F & 2 & 2 & 3 & 3 & 3 & 4 & 3 \\
\hline Orig. no. of alleles & 2 & 1 & 2 & 1 & 4 & 4 & 1 & 3 & 4 & 1 & 4 & 4 & 4 & 2 & 1 & 4 & 2 & 2 & 3 & 3 & 3 & 4 & 3 \\
\hline Theta_pi & 1 & 0 & 2 & 0 & 80.5 & 1.4 & 0 & 0.8 & 51.83 & 0 & 3.83 & 43.36 & 5.17 & 0.4 & 0 & 73.83 & 67.33 & 103 & 2.4 & 74.67 & 73.33 & 13.62 & 216.73 \\
\hline Exp. no. of alleles & 1.5 & 0 & 2.17 & 0 & 3.93 & 2.57 & 0 & 2.11 & 3.89 & 0 & 3.01 & 7.42 & 3.19 & 1.66 & 0 & 3.92 & 2.96 & 1.99 & 3.07 & 2.96 & 2.96 & 5.82 & 5.26 \\
\hline FS & 0 & 0 & 1.61 & 0 & 2.57 & -1.65 & 0 & -0.83 & 2.12 & 0 & -0.88 & 9.95 & -0.48 & 0.09 & 0 & 2.49 & 7.77 & 4.63 & 0.95 & 3.21 & 3.19 & 4.3 & 6.44 \\
\hline FS p-value & 0.26 & N.A. & 0.7 & N.A. & 0.55 & 0.05 & N.A. & 0.09 & 0.53 & N.A. & 0.14 & 1 & 0.21 & 0.3 & N.A. & 0.54 & 0.99 & 0.63 & 0.67 & 0.57 & 0.58 & 0.96 & 0.99 \\
\hline
\end{tabular}


Appendix

Table A27 continue:

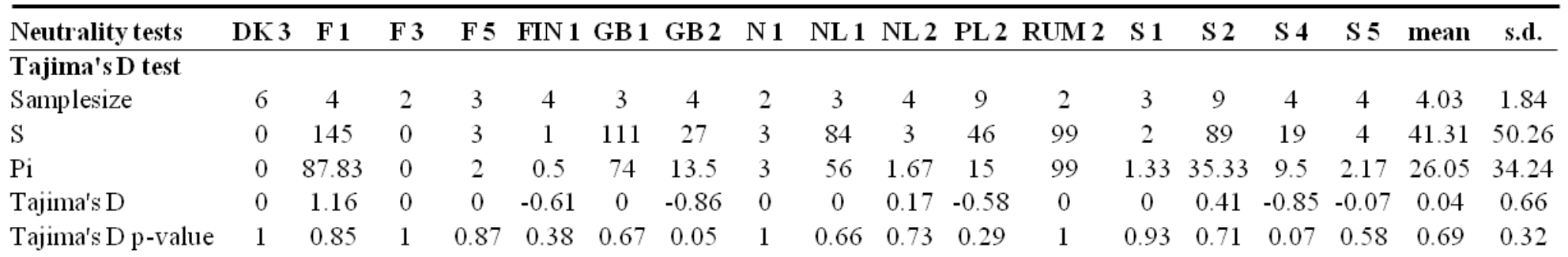

\section{Fu's FS test}

$\begin{array}{llllllllllllllllllll}\text { Real no. of alleles } & 1 & 3 & 1 & 3 & 2 & 2 & 2 & 2 & 2 & 3 & 8 & 2 & 3 & 8 & 3 & 3 & 2.82 & 1.59\end{array}$ $\begin{array}{lllllllllllllllllll}\text { Orig. no. of alleles } & 1 & 3 & 1 & 3 & 2 & 2 & 2 & 2 & 2 & 3 & 8 & 2 & 3 & 8 & 3 & 3 & 2.82 & 1.59\end{array}$ Theta pi Exp. no. of alleles

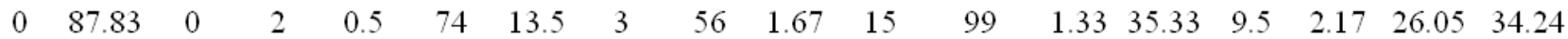

FS p-value

$\begin{array}{llllllllllllllllll}6.61 & 0 & -0.69 & 0.17 & 7.95 & 6.42 & 1.1 & 7.41 & -0.13 & -0.24 & 4.6 & -1.22 & 1.29 & 2.54 & 0.25 & 2.09 & 3.04\end{array}$
$\begin{array}{llllllllllllllllll}\text { N.A } & 0.98 & \text { N.A } & 0.13 & 0.35 & 0.99 & 0.99 & 0.43 & 0.99 & 0.26 & 0.37 & 0.62 & 0.07 & 0.66 & 0.83 & 0.46 & \text { N.A. NA. }\end{array}$

Table A28: Tajima's D and Fu's FS neutrality tests of Platynothrus peltifer COI protein sequences from 39 locations. Bold red letters show significance (P<0.05).

\begin{tabular}{|c|c|c|c|c|c|c|c|c|c|c|c|c|c|c|c|c|c|c|c|c|c|c|c|}
\hline Neutrality tests & A 1 & A2 & $\mathrm{A3}$ & $\mathrm{A4}$ & A5 & A9 & A 10 & $\mathrm{CH} 3$ & D 1 & D 2 & D 3 & D 4 & D 5 & D 6 & D 7 & D 8 & D 9 & D 11 & D 12 & D 15 & D 17 & D 18 & DK 2 \\
\hline \multicolumn{24}{|l|}{ Tajima's D test } \\
\hline Samplesize & 2 & 2 & 3 & 2 & 4 & 5 & 5 & 5 & 4 & 2 & 4 & 8 & 4 & 5 & 3 & 4 & 3 & 2 & 5 & 3 & 3 & 7 & 6 \\
\hline $\mathrm{S}$ & 0 & 0 & 0 & 0 & 0 & 0 & 0 & 0 & 0 & 0 & 0 & 0 & 0 & 0 & 0 & 0 & 0 & 0 & 1 & 0 & 0 & 0 & 0 \\
\hline $\mathrm{Pi}$ & 0 & 0 & 0 & 0 & 0 & 0 & 0 & 0 & 0 & 0 & 0 & 0 & 0 & 0 & 0 & 0 & 0 & 0 & 0.4 & 0 & 0 & 0 & 0 \\
\hline Tajima's D & 0 & 0 & 0 & 0 & 0 & 0 & 0 & 0 & 0 & 0 & 0 & 0 & 0 & 0 & 0 & 0 & 0 & 0 & -0.82 & 0 & 0 & 0 & 0 \\
\hline Tajima's D p-value & 1 & 1 & 1 & 1 & 1 & 1 & 1 & 1 & 1 & 1 & 1 & 1 & 1 & 1 & 1 & 1 & 1 & 1 & 0.31 & 1 & 1 & 1 & 1 \\
\hline \multicolumn{24}{|l|}{ Fu's FS test } \\
\hline Real no. of alleles & 1 & 1 & 1 & 1 & 1 & 1 & 1 & 1 & 1 & 1 & 2 & 1 & 1 & 2 & 1 & 2 & 1 & 2 & 2 & 1 & 1 & 1 & 2 \\
\hline Orig. no. of alleles & 1 & 1 & 1 & 1 & 1 & 1 & 1 & 1 & 1 & 1 & 2 & 1 & 1 & 2 & 1 & 2 & 1 & 2 & 2 & 1 & 1 & 1 & 2 \\
\hline Theta_pi & 0 & 0 & 0 & 0 & 0 & 0 & 0 & 0 & 0 & 0 & 0 & 0 & 0 & 0 & 0 & 0 & 0 & 0 & 0.4 & 0 & 0 & 0 & 0 \\
\hline Exp. no. of alleles & 0 & 0 & 0 & 0 & 0 & 0 & 0 & 0 & 0 & 0 & 2 & 0 & 0 & 2 & 0 & 2 & 0 & 0 & 1.66 & 0 & 0 & 0 & 2 \\
\hline FS & 0 & 0 & 0 & 0 & 0 & 0 & 0 & 0 & 0 & 0 & 0 & 0 & 0 & 0 & 0 & 0 & 0 & 0 & 0.09 & 0 & 0 & 0 & 0 \\
\hline FS p-value & N.A. & N.A. & N.A. & N.A. & N.A. & N.A. & N.A. & N.A. & N.A. & N.A. & N.A. & N.A. & N.A. & N.A. & N.A. & N.A. & N.A. & N.A. & 0.29 & N.A. & N.A. & N.A. & N.A. \\
\hline
\end{tabular}


Appendix

Table A28 continue:

\begin{tabular}{|c|c|c|c|c|c|c|c|c|c|c|c|c|c|c|c|c|c|c|}
\hline Neutrality tests & DK 3 & F 1 & F 3 & F 5 & FIN 1 & GB 1 & GB 2 & $\mathrm{~N} 1$ & NL 1 & NL 2 & PL 2 & RUM 2 & S 1 & S 2 & $\mathrm{~S} 4$ & S 5 & mean & s.d. \\
\hline \multicolumn{19}{|l|}{ Tajima's D test } \\
\hline Samplesize & 6 & 4 & 2 & 3 & 4 & 3 & 4 & 2 & 3 & 4 & 9 & 2 & 3 & 9 & 4 & 4 & 4.03 & 1.84 \\
\hline $\mathrm{s}$ & 0 & 0 & 0 & 0 & 0 & 0 & 0 & 0 & 0 & 1 & 1 & 1 & 0 & 0 & 0 & 0 & 0.10 & 0.31 \\
\hline $\mathrm{Pi}$ & 0 & 0 & 0 & 0 & 0 & 0 & 0 & 0 & 0 & 0.5 & 0.22 & 1 & 0 & 0 & 0 & 0 & 0.05 & 0.19 \\
\hline Tajima's D & 0 & 0 & 0 & 0 & 0 & 0 & 0 & 0 & 0 & -0.61 & -1.09 & 0 & 0 & 0 & 0 & 0 & -0.06 & 0.23 \\
\hline Tajima's D p-value & 1 & 1 & 1 & 1 & 1 & 1 & 1 & 1 & 1 & 0.38 & 0.20 & 1 & 1 & 1 & 1 & 1 & 0.95 & 0.19 \\
\hline \multicolumn{19}{|l|}{ Fu's FS test } \\
\hline Real no. of alleles & 1 & 1 & 1 & 1 & 1 & 1 & 1 & 2 & 1 & 2 & 3 & 2 & 1 & 5 & 2 & 1 & 1.41 & 0.79 \\
\hline Orig. no. of alleles & 1 & 1 & 1 & 1 & 1 & 1 & 1 & 2 & 1 & 2 & 3 & 2 & 1 & 5 & 2 & 1 & 1.41 & 0.79 \\
\hline Theta_pi & 0 & 0 & 0 & 0 & 0 & 0 & 0 & 0 & 0 & 0.5 & 0.22 & 1 & 0 & 0 & 0 & 0 & 0.05 & 0.19 \\
\hline Exp.no. of alleles & 0 & 0 & 0 & 0 & 0 & 0 & 0 & 0 & 0 & 1.68 & 1.54 & 1.5 & 0 & 5 & 2 & 0 & 0.55 & 1.07 \\
\hline FS & 0 & 0 & 0 & 0 & 0 & 0 & 0 & 0 & 0 & 0.17 & -0.26 & 0 & 0 & 0 & 0 & 0 & 0.00 & 0.05 \\
\hline FS p-value & N.A. & N.A. & N.A. & N.A. & N.A. & N.A. & N.A. & N.A. & N.A. & 0.35 & 0.17 & 0.24 & N.A. & N.A. & N.A. & N.A. & N.A. & N.A. \\
\hline
\end{tabular}


Table A29: Results of the McDonald-Kreitman test of Platynothrus peltifer. The differences between 64 populations are significant $(* 0.01<\mathrm{P}<0.05)$, between other 17 populations high significant $(* * 0.001<\mathrm{P}<0.01)$ and between 3 populations extremely high significant $(* * * \mathrm{P}<0.001)$. Locations with less than two individuals were excluded.

\begin{tabular}{|c|c|c|c|c|c|c|c|c|c|c|c|c|c|}
\hline \multirow{2}{*}{$\begin{array}{c}\text { Population } \\
\text { A } 2\end{array}$} & \multirow[b]{2}{*}{ fixed } & \multicolumn{3}{|c|}{$\begin{array}{c}\text { A } 1 \\
\text { syn. nons. sign. }\end{array}$} & \multicolumn{3}{|c|}{$\begin{array}{c}\text { A } 2 \\
\text { syn. nons. sign. }\end{array}$} & \multicolumn{3}{|c|}{$\begin{array}{c}\text { A } 3 \\
\text { syn. nons. sign. }\end{array}$} & \multicolumn{3}{|c|}{$\begin{array}{c}\text { A } 4 \\
\text { syn. nons. sign. }\end{array}$} \\
\hline & & 99 & 1 & $1 \mathrm{~ns}$ & & & & & & & & & \\
\hline Bregenz & poly & 1 & 0 & & & & & & & & & & \\
\hline A 3 & fixed & 98 & 1 & $1 \mathrm{~ns}$ & 0 & 0 & $-\mathrm{ns}$ & & & & & & \\
\hline Hittisau & poly & 4 & 0 & & 3 & 0 & & & & & & & \\
\hline A 4 & fixed & 99 & 1 & $1 \mathrm{~ns}$ & 2 & 0 & $-n s$ & 0 & 0 & $-n s$ & & & \\
\hline Holzgau & poly & 1 & 0 & & 0 & 0 & & 3 & 0 & & & & \\
\hline A 5 & fixed & 8 & 1 & $0.1084 \mathrm{~ns}$ & 0 & 0 & $-\mathrm{ns}$ & 0 & 0 & $-n s$ & 0 & 0 & $-\mathrm{ns}$ \\
\hline Memm. Huette & poly & 152 & 1 & & 152 & 1 & & 153 & 1 & & 152 & 1 & \\
\hline A 9 & fixed & 98 & 1 & $1 \mathrm{~ns}$ & 1 & 0 & $-n s$ & 0 & 0 & $-n s$ & 1 & 0 & $-\mathrm{ns}$ \\
\hline Glacier & poly & 4 & 0 & & 3 & 0 & & 6 & 0 & & 3 & 0 & \\
\hline A 10 & fixed & 101 & 1 & $1 \mathrm{~ns}$ & 2 & 0 & $-n s$ & 1 & 0 & $-n s$ & 2 & 0 & $-\mathrm{ns}$ \\
\hline Stuiben & poly & 1 & 0 & & 0 & 0 & & 3 & 0 & & 0 & 0 & \\
\hline CH 3 & fixed & 98 & 1 & $1 \mathrm{~ns}$ & 1 & 0 & $-n s$ & 0 & 0 & $-n s$ & 0 & 0 & $-\mathrm{ns}$ \\
\hline Rohrschach & poly & 3 & 0 & & 2 & 0 & & 4 & 0 & & 2 & 0 & \\
\hline D 1 & fixed & 4 & 1 & $0.0472 *$ & 2 & 0 & $-n s$ & 2 & 0 & $-n s$ & 2 & 0 & $-\mathrm{ns}$ \\
\hline Kranichstein & poly & 101 & 0 & & 101 & 0 & & 102 & 0 & & 101 & 0 & \\
\hline D 2 & fixed & 7 & 1 & $1 \mathrm{~ns}$ & 99 & 1 & $-n s$ & 98 & 0 & $-n s$ & 99 & 0 & $-n s$ \\
\hline Goettingen & poly & 1 & 0 & & 0 & 0 & & 3 & 0 & & 0 & 0 & \\
\hline D 3 & fixed & 97 & 1 & $0.0145^{*}$ & 0 & 0 & $-n s$ & 0 & 0 & $-n s$ & 1 & 0 & $1 \mathrm{~ns}$ \\
\hline Lake Constance & poly & 6 & 2 & & 5 & 2 & & 7 & 2 & & 5 & 2 & \\
\hline D 4 & fixed & 5 & 1 & $0.0561 \mathrm{~ns}$ & 0 & 0 & $-n s$ & 0 & 0 & $-n s$ & 0 & 0 & $-n s$ \\
\hline Meckl. Seenpl. & poly & 101 & 0 & & 101 & 0 & & 102 & 0 & & 101 & 0 & \\
\hline D 5 & fixed & 4 & 1 & $0.3125 \mathrm{~ns}$ & 96 & 0 & $-n s$ & 95 & 0 & $-n s$ & 96 & 0 & $-\mathrm{ns}$ \\
\hline Moerfelden & poly & 11 & 0 & & 10 & 0 & & 13 & 0 & & 10 & 0 & \\
\hline D 6 & fixed & 9 & 1 & $0.3182 \mathrm{~ns}$ & 97 & 0 & $0.0102 *$ & 96 & 0 & $0.04^{*}$ & 97 & 0 & $0.0102 *$ \\
\hline Black Forest & poly & 1 & 1 & & 0 & 1 & & 3 & 1 & & 0 & 1 & \\
\hline D 7 & fixed & 28 & 1 & $1 \mathrm{~ns}$ & 109 & 0 & $-\mathrm{ns}$ & 108 & 0 & $-n s$ & 109 & 0 & $-\mathrm{ns}$ \\
\hline Uelzen & poly & 1 & 0 & & 0 & 0 & & 3 & 0 & & 0 & 0 & \\
\hline D 8 & fixed & 12 & 1 & $0.2827 \mathrm{~ns}$ & 0 & 0 & $-\mathrm{ns}$ & 0 & 0 & $-n s$ & 0 & 0 & $-n s$ \\
\hline Cuxhaven & poly & 110 & 2 & & 110 & 2 & & 111 & 2 & & 110 & 2 & \\
\hline D 9 & fixed & 5 & 1 & $0.0566 \mathrm{~ns}$ & 0 & 0 & $-n s$ & 0 & 0 & $-n s$ & 0 & 0 & $-n s$ \\
\hline Bonn & poly & 100 & 0 & & 100 & 0 & & 101 & 0 & & 100 & 0 & \\
\hline D 11 & fixed & 4 & 1 & $0.0917 \mathrm{~ns}$ & 1 & 0 & $1 \mathrm{~ns}$ & 1 & 0 & $1 \mathrm{~ns}$ & 1 & 0 & $1 \mathrm{~ns}$ \\
\hline Wittmoor & poly & 101 & 1 & & 101 & 1 & & 102 & 1 & & 101 & 1 & \\
\hline D 12 & fixed & 98 & 1 & $0.1115 \mathrm{~ns}$ & 1 & 0 & $1 \mathrm{~ns}$ & 0 & 0 & $-n s$ & 0 & 0 & $-n s$ \\
\hline Sonthofen & poly & 5 & 1 & & 4 & 1 & & 5 & 1 & & 4 & 1 & \\
\hline D 15 & fixed & 12 & 1 & $0.1048 \mathrm{~ns}$ & 0 & 0 & $-n s$ & 0 & 0 & $-n s$ & 0 & 0 & $-n s$ \\
\hline Langenwang & poly & 111 & 0 & & 111 & 0 & & 112 & 0 & & 111 & 0 & \\
\hline D 17 & fixed & 13 & 1 & $0.1138 \mathrm{~ns}$ & 0 & 0 & $-n s$ & 0 & 0 & $-n s$ & 0 & 0 & $-\mathrm{ns}$ \\
\hline Rubi & poly & 109 & 0 & & 109 & 0 & & 110 & 0 & & 109 & 0 & \\
\hline D 18 & fixed & 6 & 1 & $0.1944 \mathrm{~ns}$ & 92 & 1 & $-\mathrm{ns}$ & 91 & 0 & $-n s$ & 92 & 0 & $-\mathrm{ns}$ \\
\hline Steinh. Meer & poly & 29 & 0 & & 28 & 0 & & 31 & 0 & & 28 & 0 & \\
\hline DK 2 & fixed & 5 & 1 & $0.3352 \mathrm{~ns}$ & 94 & 0 & $0.2167 \mathrm{~ns}$ & 93 & 0 & $0.2377 \mathrm{~ns}$ & 94 & 0 & $0.2167 \mathrm{~ns}$ \\
\hline Hjorring & poly & 26 & 1 & & 25 & 1 & & 28 & 1 & & 25 & 1 & \\
\hline DK 3 & fixed & 100 & 1 & $1 \mathrm{~ns}$ & 3 & 0 & $-\mathrm{ns}$ & 2 & 0 & $-\mathrm{ns}$ & 3 & 0 & $-\mathrm{ns}$ \\
\hline Arhus & poly & 1 & 0 & & 0 & 0 & & 3 & 0 & & 0 & 0 & \\
\hline F 1 & fixed & 10 & 1 & $0.0651 \mathrm{~ns}$ & 0 & 0 & $-n s$ & 0 & 0 & $-n s$ & 0 & 0 & $-n s$ \\
\hline Mont Blanc & poly & 158 & 0 & & 158 & 0 & & 158 & 0 & & 158 & 0 & \\
\hline F 3 & fixed & 28 & 1 & $1 \mathrm{~ns}$ & 109 & 1 & $-n s$ & 108 & 1 & $1 \mathrm{~ns}$ & 109 & 1 & $-n s$ \\
\hline Saint Isidore & poly & 1 & 0 & & 0 & 0 & & 3 & 0 & & 0 & 0 & \\
\hline F 5 & fixed & 7 & 1 & $1 \mathrm{~ns}$ & 98 & 0 & $-n s$ & 97 & 0 & $-n s$ & 98 & 0 & $-n s$ \\
\hline Bruenstatt & poly & 4 & 0 & & 3 & 0 & & 6 & 0 & & 3 & 0 & \\
\hline FIN 1 & fixed & 29 & 1 & $1 \mathrm{~ns}$ & 108 & 0 & $-\mathrm{ns}$ & 107 & 0 & $-n s$ & 108 & 0 & $-n s$ \\
\hline Lahti & poly & 2 & 0 & & 1 & 0 & & 4 & 0 & & 1 & 0 & \\
\hline GB 1 & fixed & 12 & 1 & $0.1057 \mathrm{~ns}$ & 0 & 0 & $-n s$ & 0 & 0 & $-n s$ & 0 & 0 & $-n s$ \\
\hline Ascot & poly & 110 & 0 & & 110 & 0 & & 111 & 0 & & 110 & 0 & \\
\hline
\end{tabular}


Table A29 continue:

\begin{tabular}{|c|c|c|c|c|c|c|c|c|c|c|c|c|c|}
\hline \multirow{2}{*}{$\begin{array}{c}\text { Population } \\
\text { GB 2 }\end{array}$} & \multicolumn{4}{|c|}{$\begin{array}{c}\text { A } 1 \\
\text { syn. nons. sign. }\end{array}$} & \multicolumn{3}{|c|}{$\begin{array}{c}\text { A } 2 \\
\text { syn. nons. sign. }\end{array}$} & \multicolumn{3}{|c|}{$\begin{array}{c}\text { A 3 } \\
\text { syn. nons. sign. }\end{array}$} & \multicolumn{3}{|c|}{$\begin{array}{c}\text { A 4 } \\
\text { syn. nons. sign. }\end{array}$} \\
\hline & fixed & 6 & 1 & $0.2121 \mathrm{~ns}$ & 95 & 1 & $-n s$ & 94 & 0 & $-n s$ & 95 & 0 & $-n s$ \\
\hline Braemar & poly & 26 & 0 & & 25 & 0 & & 28 & 0 & & 25 & 0 & \\
\hline N 1 & fixed & 71 & 1 & $0.0789 \mathrm{~ns}$ & 102 & 0 & $0.0192 *$ & 101 & 0 & $0.0472 *$ & 102 & 0 & $0.0192 *$ \\
\hline Narvik & poly & 2 & 1 & & 1 & 1 & & 4 & 1 & & 1 & 1 & \\
\hline NL 1 & fixed & 71 & 1 & $0.4645 \mathrm{~ns}$ & 2 & 0 & $-n s$ & 1 & 0 & $-n s$ & 1 & 0 & $-n s$ \\
\hline Wageningen & poly & 83 & 0 & & 82 & 0 & & 83 & 0 & & 82 & 0 & \\
\hline NL 2 & fixed & 28 & 1 & $0.2311 \mathrm{~ns}$ & 108 & 0 & $0.027^{*}$ & 107 & 0 & $0.0531 \mathrm{~ns}$ & 108 & 0 & $0.027^{*}$ \\
\hline Hoge Veluwe & poly & 3 & 1 & & 2 & 1 & & 5 & 1 & & 2 & 1 & \\
\hline PL 2 & fixed & 2 & 1 & $0.2865 \mathrm{~ns}$ & 88 & 0 & $0.0112 *$ & 87 & 0 & $0.0139^{*}$ & 88 & 0 & $0.0112 *$ \\
\hline Warsaw & poly & 41 & 4 & & 40 & 4 & & 43 & 4 & & 40 & 4 & \\
\hline RUM 2 & fixed & 5 & 1 & $0.1126 \mathrm{~ns}$ & 1 & 0 & $1 \mathrm{~ns}$ & 1 & 0 & $1 \mathrm{~ns}$ & 1 & 0 & $1 \mathrm{~ns}$ \\
\hline Sibiu 2 & poly & 97 & 1 & & 97 & 1 & & 98 & 1 & & 97 & 1 & \\
\hline S 1 & fixed & 71 & 1 & $1 \mathrm{~ns}$ & 102 & 0 & $-n s$ & 101 & 0 & $-n s$ & 102 & 0 & $-n s$ \\
\hline Umea & poly & 3 & 0 & & 2 & 0 & & 5 & 0 & & 2 & 0 & \\
\hline S 2 & fixed & 10 & 1 & $1 \mathrm{~ns}$ & 67 & 0 & $0.0709 \mathrm{~ns}$ & 66 & 0 & $0.0755 \mathrm{~ns}$ & 67 & 0 & $0.0709 \mathrm{~ns}$ \\
\hline Stroemstad & poly & 85 & 5 & & 84 & 5 & & 87 & 5 & & 84 & 5 & \\
\hline S 4 & fixed & 64 & 1 & $0.1366 \mathrm{~ns}$ & 97 & 0 & $0.0256^{*}$ & 96 & 0 & $0.0335^{*}$ & 97 & 0 & $0.0256^{*}$ \\
\hline Abisko & poly & 18 & 2 & & 17 & 2 & & 20 & 2 & & 17 & 2 & \\
\hline S 5 & fixed & 96 & 1 & $1 \mathrm{~ns}$ & 1 & 0 & $-n s$ & 0 & 0 & $-n s$ & 1 & 0 & $-n s$ \\
\hline Gothenburg & poly & 5 & 0 & & 4 & 0 & & 7 & 0 & & 4 & 0 & \\
\hline
\end{tabular}

\begin{tabular}{|c|c|c|c|c|c|c|c|c|c|c|c|c|}
\hline \multirow{2}{*}{$\begin{array}{c}\text { Population } \\
\text { A } 9\end{array}$} & \multicolumn{3}{|c|}{$\begin{array}{c}\text { A 5 } \\
\text { syn. nons. sign. }\end{array}$} & \multicolumn{3}{|c|}{$\begin{array}{c}\text { A 9 } \\
\text { syn. nons. sign. }\end{array}$} & \multicolumn{3}{|c|}{$\begin{array}{r}\text { A } 10 \\
\text { syn. nons. sign. }\end{array}$} & \multicolumn{3}{|c|}{$\begin{array}{r}\text { CH } 3 \\
\text { syn. nons. sign. }\end{array}$} \\
\hline & fixed 0 & 0 & $-n s$ & & & & & & & & & \\
\hline Glacier & poly 154 & 1 & & & & & & & & & & \\
\hline A 10 & fixed 1 & 0 & $1 \mathrm{~ns}$ & 1 & 0 & $-n s$ & & & & & & \\
\hline Stuiben & poly 152 & 1 & & 3 & 0 & & & & & & & \\
\hline CH 3 & fixed 0 & 0 & $-n s$ & 0 & 0 & $-n s$ & 1 & 0 & $-n s$ & & & \\
\hline Rohrschach & poly 152 & 1 & & 5 & 0 & & 2 & 0 & & & & \\
\hline D 1 & fixed 1 & 0 & $1 \mathrm{~ns}$ & 2 & 0 & $-\mathrm{ns}$ & 3 & 0 & $-n s$ & 2 & 0 & $-n s$ \\
\hline Kranichstein & poly 159 & 1 & & 102 & 0 & & 101 & 0 & & 101 & 0 & \\
\hline D 2 & fixed 7 & 0 & $1 \mathrm{~ns}$ & 98 & 0 & $-n s$ & 101 & 1 & $-\mathrm{ns}$ & 98 & 0 & $-n s$ \\
\hline Goettingen & poly 152 & 1 & & 3 & 0 & & 0 & 0 & & 2 & 0 & \\
\hline D 3 & fixed 0 & 0 & $-n s$ & 0 & 0 & $-n s$ & 1 & 0 & $1 \mathrm{~ns}$ & 0 & 0 & $-n s$ \\
\hline Lake Constance & poly 154 & 3 & & 8 & 2 & & 5 & 2 & & 6 & 2 & \\
\hline D 4 & fixed 0 & 0 & $-n s$ & 0 & 0 & $-n s$ & 1 & 0 & $-n s$ & 0 & 0 & $-n s$ \\
\hline Meckl. Seenpl. & poly 159 & 1 & & 102 & 0 & & 101 & 0 & & 101 & 0 & \\
\hline D 5 & fixed 7 & 0 & $1 \mathrm{~ns}$ & 95 & 0 & $-n s$ & 98 & 0 & $-n s$ & 95 & 0 & $-n s$ \\
\hline Moerfelden & poly 155 & 1 & & 13 & 0 & & 10 & 0 & & 12 & 0 & \\
\hline D 6 & fixed 7 & 0 & $1 \mathrm{~ns}$ & 96 & 0 & $0.04^{*}$ & 99 & 0 & $0.01^{*}$ & 96 & 0 & $0.0303^{*}$ \\
\hline Black Forest & poly 152 & 2 & & 3 & 1 & & 0 & 1 & & 2 & 1 & \\
\hline D 7 & fixed 0 & 0 & $-n s$ & 109 & 0 & $-n s$ & 111 & 0 & $-n s$ & 108 & 0 & $-n s$ \\
\hline Uelzen & poly 152 & 1 & & 3 & 0 & & 0 & 0 & & 2 & 0 & \\
\hline D 8 & fixed 0 & 0 & $-\mathrm{ns}$ & 0 & 0 & $-n s$ & 1 & 0 & $1 \mathrm{~ns}$ & 0 & 0 & $-n s$ \\
\hline Cuxhaven & poly 152 & 3 & & 112 & 2 & & 110 & 2 & & 110 & 2 & \\
\hline D 9 & fixed 0 & 0 & $-n s$ & 0 & 0 & $-n s$ & 1 & 0 & $-n s$ & 0 & 0 & $-n s$ \\
\hline Bonn & poly 159 & 1 & & 101 & 0 & & 100 & 0 & & 100 & 0 & \\
\hline D 11 & fixed 0 & 0 & $-n s$ & 1 & 0 & $1 \mathrm{~ns}$ & 2 & 0 & $1 \mathrm{~ns}$ & 1 & 0 & $1 \mathrm{~ns}$ \\
\hline Wittmoor & poly 160 & 2 & & 102 & 1 & & 101 & 1 & & 101 & 1 & \\
\hline D 12 & fixed 0 & 0 & $-n s$ & 0 & 0 & $-\mathrm{ns}$ & 1 & 0 & $1 \mathrm{~ns}$ & 0 & 0 & $-n s$ \\
\hline Sonthofen & poly 154 & 2 & & 7 & 1 & & 4 & 1 & & 5 & 1 & \\
\hline D 15 & fixed 0 & 0 & $-\mathrm{ns}$ & 0 & 0 & $-n s$ & 1 & 0 & $-n s$ & 0 & 0 & $-n s$ \\
\hline Langenwang & poly 153 & 1 & & 113 & 0 & & 111 & 0 & & 111 & 0 & \\
\hline D 17 & fixed 0 & 0 & $-\mathrm{ns}$ & 0 & 0 & $-n s$ & 1 & 0 & $-n s$ & 0 & 0 & $-n s$ \\
\hline Rubi & poly 152 & 1 & & 111 & 0 & & 109 & 0 & & 109 & 0 & \\
\hline
\end{tabular}


Table A29 continue:

\begin{tabular}{|c|c|c|c|c|c|c|c|c|c|c|c|c|}
\hline \multirow{2}{*}{$\begin{array}{c}\text { Population } \\
\text { D } 18\end{array}$} & \multicolumn{3}{|c|}{$\begin{array}{c}\text { A 5 } \\
\text { syn. nons. sign. }\end{array}$} & \multicolumn{3}{|c|}{$\begin{array}{c}\text { A 9 } \\
\text { syn. nons. sign. }\end{array}$} & \multicolumn{3}{|c|}{$\begin{array}{r}\text { A } 10 \\
\text { syn. nons. sign. }\end{array}$} & \multicolumn{3}{|c|}{$\begin{array}{r}\text { CH } 3 \\
\text { syn. nons. sign. }\end{array}$} \\
\hline & fixed & 0 & $-\mathrm{ns}$ & 91 & 0 & $-\mathrm{ns}$ & 94 & 0 & $-\mathrm{ns}$ & 91 & 0 & $-n s$ \\
\hline Steinh. Meer & poly 160 & 1 & & 31 & 0 & & 28 & 0 & & 30 & 0 & \\
\hline DK 2 & fixed 0 & 0 & $-n s$ & 93 & 0 & $0.2377 \mathrm{~ns}$ & 96 & 0 & $0.2131 \mathrm{~ns}$ & 93 & 0 & $0.2314 \mathrm{~ns}$ \\
\hline Hjorring & poly 159 & 2 & & 28 & 1 & & 25 & 1 & & 27 & 1 & \\
\hline DK 3 & fixed 1 & 0 & $1 \mathrm{~ns}$ & 2 & 0 & $-n s$ & 3 & 0 & $-n s$ & 2 & 0 & $-n s$ \\
\hline Arhus & poly 152 & 1 & & 3 & 0 & & 0 & 0 & & 2 & 0 & \\
\hline F 1 & fixed 0 & 0 & $-n s$ & 0 & 0 & $-n s$ & 0 & 0 & $-n s$ & 0 & 0 & $-n s$ \\
\hline Mont Blanc & poly 185 & 1 & & 160 & 0 & & 158 & 0 & & 158 & 0 & \\
\hline F 3 & fixed 0 & 1 & $0.013^{*}$ & 109 & 1 & $1 \mathrm{~ns}$ & 111 & 1 & $-n s$ & 108 & 1 & $1 \mathrm{~ns}$ \\
\hline Saint Isidore & poly 152 & 1 & & 3 & 0 & & 0 & 0 & & 2 & 0 & \\
\hline F 5 & fixed 6 & 0 & $1 \mathrm{~ns}$ & 97 & 0 & $-n s$ & 100 & 0 & $-n s$ & 97 & 0 & $-n s$ \\
\hline Bruenstatt & poly 154 & 1 & & 6 & 0 & & 3 & 0 & & 5 & 0 & \\
\hline FIN 1 & fixed 0 & 0 & $-n s$ & 108 & 0 & $-n s$ & 110 & 0 & $-n s$ & 107 & 0 & $-n s$ \\
\hline Lahti & poly 152 & 1 & & 4 & 0 & & 1 & 0 & & 3 & 0 & \\
\hline GB 1 & fixed 0 & 0 & $-n s$ & 0 & 0 & $-n s$ & 1 & 0 & $-n s$ & 0 & 0 & $-n s$ \\
\hline Ascot & poly 152 & 1 & & 112 & 0 & & 110 & 0 & & 110 & 0 & \\
\hline GB 2 & fixed 0 & 0 & $-n s$ & 94 & 0 & $-n s$ & 97 & 0 & $-n s$ & 94 & 0 & $-n s$ \\
\hline Braemar & poly 159 & 1 & & 28 & 0 & & 25 & 0 & & 27 & 0 & \\
\hline N 1 & fixed 0 & 0 & $-n s$ & 103 & 0 & $0.0463^{*}$ & 103 & 0 & $0.0191^{*}$ & 101 & 0 & $0.0381^{*}$ \\
\hline Narvik & poly 152 & 2 & & 4 & 1 & & 1 & 1 & & 3 & 1 & \\
\hline NL 1 & fixed 1 & 0 & $1 \mathrm{~ns}$ & 1 & 0 & $-n s$ & 1 & 0 & $-n s$ & 1 & 0 & $-n s$ \\
\hline Wageningen & poly 184 & 1 & & 84 & 0 & & 82 & 0 & & 82 & 0 & \\
\hline NL 2 & fixed 0 & 0 & $-n s$ & 108 & 0 & $0.0526^{*}$ & 110 & 0 & $0.0266^{*}$ & 107 & 0 & $0.0446^{*}$ \\
\hline Hoge Veluwe & poly 152 & 2 & & 5 & 1 & & 2 & 1 & & 4 & 1 & \\
\hline PL 2 & fixed 0 & 0 & $-n s$ & 87 & 0 & $0.0139^{*}$ & 90 & 0 & $0.0106^{*}$ & 87 & 0 & $0.0131^{*}$ \\
\hline Warsaw & poly 165 & 5 & & 43 & 4 & & 40 & 4 & & 42 & 4 & \\
\hline RUM 2 & fixed & 0 & $-n s$ & 1 & 0 & $1 \mathrm{~ns}$ & 2 & 0 & $1 \mathrm{~ns}$ & 0 & 0 & $-n s$ \\
\hline Sibiu 2 & poly 160 & 2 & & 98 & 1 & & 97 & 1 & & 98 & 1 & \\
\hline S 1 & fixed 0 & 0 & $-n s$ & 103 & 0 & $-n s$ & 103 & 0 & $-n s$ & 101 & 0 & $-n s$ \\
\hline Umea & poly 152 & 1 & & 5 & 0 & & 2 & 0 & & 4 & 0 & \\
\hline $\mathrm{S} 2$ & fixed 0 & 0 & $-n s$ & 68 & 0 & $0.0716 \mathrm{~ns}$ & 69 & 0 & $0.0685 \mathrm{~ns}$ & 66 & 0 & $0.0744 \mathrm{~ns}$ \\
\hline Stroemstad & poly 154 & 6 & & 86 & 5 & & 84 & 5 & & 86 & 5 & \\
\hline S 4 & fixed 0 & 0 & $-n s$ & 98 & 0 & $0.0324^{*}$ & 98 & 0 & $0.0252^{*}$ & 96 & 0 & $0.031 *$ \\
\hline Abisko & poly 155 & 2 & & 20 & 2 & & 17 & 2 & & 19 & 2 & \\
\hline S 5 & fixed 0 & 0 & $-n s$ & 0 & 0 & $-n s$ & 1 & 0 & $-n s$ & 0 & 0 & $-n s$ \\
\hline Gothenburg & poly 152 & 2 & & 6 & 0 & & 4 & 0 & & 5 & 0 & \\
\hline
\end{tabular}

\begin{tabular}{|c|c|c|c|c|c|c|c|c|c|c|c|c|c|}
\hline \multirow{2}{*}{$\begin{array}{c}\text { Population } \\
\text { D } 2\end{array}$} & \multicolumn{4}{|c|}{$\begin{array}{c}\text { D 1 } \\
\text { syn. nons. sign. }\end{array}$} & \multicolumn{3}{|c|}{$\begin{array}{c}\text { D } 2 \\
\text { syn. nons. sign. }\end{array}$} & \multicolumn{3}{|c|}{$\begin{array}{c}\text { D 3 } \\
\text { syn. nons. sign. }\end{array}$} & \multicolumn{3}{|c|}{$\begin{array}{c}\text { D 4 } \\
\text { syn. nons. sign. }\end{array}$} \\
\hline & fixed & 97 & 0 & $0.0039 * *$ & & & & & & & & & \\
\hline Goettingen & poly & 5 & 2 & & & & & & & & & & \\
\hline D 3 & fixed & 1 & 0 & $-n s$ & 1 & 0 & $-n s$ & & & & & & \\
\hline Lake Constance & poly & 101 & 0 & & 103 & 2 & & & & & & & \\
\hline D 4 & fixed & 0 & 0 & $-n s$ & 1 & 0 & $-n s$ & 1 & 0 & $-n s$ & & & \\
\hline Meckl. Seenpl. & poly & 104 & 0 & & 101 & 0 & & 103 & 2 & & & & \\
\hline D 5 & fixed & 0 & 0 & $-n s$ & 0 & 0 & $-\mathrm{ns}$ & 94 & 0 & $0.0223^{*}$ & 0 & 0 & $-n s$ \\
\hline Moerfelden & poly & 108 & 0 & & 10 & 0 & & 15 & 2 & & 108 & 0 & \\
\hline D 6 & fixed & 0 & 0 & -ns & 2 & 0 & $0.3333 \mathrm{~ns}$ & 95 & 0 & $0.0003^{* * * *}$ & 1 & 0 & $1 \mathrm{~ns}$ \\
\hline Black Forest & poly & 101 & 1 & & 0 & 1 & & 5 & 3 & & 101 & 1 & \\
\hline D 7 & fixed & 20 & 0 & $-n s$ & 23 & 0 & $-\mathrm{ns}$ & 107 & 0 & $0.0033^{* *}$ & 19 & 0 & $-\mathrm{ns}$ \\
\hline Uelzen & poly & 101 & 0 & & 0 & 0 & & 5 & 2 & & 101 & 0 & \\
\hline D 8 & fixed & 1 & 0 & $1 \mathrm{~ns}$ & 9 & 0 & $1 \mathrm{~ns}$ & 0 & 0 & $-n s$ & 0 & 0 & $-n s$ \\
\hline Cuxhaven & poly & 121 & 2 & & 110 & 2 & & 112 & 4 & & 120 & 2 & \\
\hline D 9 & fixed & 0 & 0 & $-n s$ & 1 & 0 & $-n s$ & 0 & 0 & $-n s$ & 0 & 0 & $-n s$ \\
\hline Bonn & poly & 103 & 0 & & 100 & 0 & & 102 & 2 & & 101 & 0 & \\
\hline
\end{tabular}


Table A29 continue:

\begin{tabular}{|c|c|c|c|c|c|c|c|c|c|c|c|c|}
\hline \multirow{2}{*}{$\begin{array}{c}\text { Population } \\
\text { D } 11\end{array}$} & \multicolumn{3}{|c|}{$\begin{array}{c}\text { D } 1 \\
\text { syn. nons. sign. }\end{array}$} & \multicolumn{3}{|c|}{$\begin{array}{c}\text { D } 2 \\
\text { syn. nons. sign. }\end{array}$} & \multicolumn{3}{|c|}{$\begin{array}{r}\text { D } 3 \\
\text { syn. nons. sign. }\end{array}$} & \multicolumn{3}{|c|}{$\begin{array}{c}\text { D } 4 \\
\text { syn. nons. sign. }\end{array}$} \\
\hline & fixed 0 & 0 & $-\mathrm{ns}$ & 0 & 0 & $-\mathrm{ns}$ & 1 & 0 & $1 \mathrm{~ns}$ & 0 & 0 & $-n s$ \\
\hline Wittmoor & poly 105 & 1 & & 101 & 1 & & 103 & 3 & & 104 & 1 & \\
\hline D 12 & fixed 1 & 0 & $1 \mathrm{~ns}$ & 98 & 0 & $0.0485^{*}$ & 0 & 0 & $-\mathrm{ns}$ & 0 & 0 & $-n s$ \\
\hline Sonthofen & poly 103 & 1 & & 4 & 1 & & 9 & 3 & & 103 & 1 & \\
\hline D 15 & fixed 0 & 0 & $-\mathrm{ns}$ & 9 & 0 & $-n s$ & 0 & 0 & $-\mathrm{ns}$ & 0 & 0 & $-n s$ \\
\hline Langenwang & poly 122 & 0 & & 111 & 0 & & 113 & 2 & & 121 & 0 & \\
\hline D 17 & fixed 1 & 0 & $-n s$ & 10 & 0 & $-n s$ & 0 & 0 & $-\mathrm{ns}$ & 0 & 0 & $-n s$ \\
\hline Rubi & poly 121 & 0 & & 109 & 0 & & 111 & 2 & & 120 & 0 & \\
\hline D 18 & fixed 0 & 0 & $-\mathrm{ns}$ & 0 & 0 & $-\mathrm{ns}$ & 90 & 0 & $0.0768 \mathrm{~ns}$ & 0 & 0 & $-n s$ \\
\hline Steinh.Meer & poly 122 & 0 & & 28 & 0 & & 33 & 2 & & 121 & 0 & \\
\hline DK 2 & fixed 0 & 0 & $-\mathrm{ns}$ & 0 & 0 & $-\mathrm{ns}$ & 92 & 0 & $0.0172^{*}$ & 0 & 0 & $-n s$ \\
\hline Hjorring & poly 121 & 1 & & 25 & 1 & & 30 & 3 & & 120 & 1 & \\
\hline DK 3 & fixed 1 & 0 & $-\mathrm{ns}$ & 100 & 0 & $-n s$ & 2 & 0 & $1 \mathrm{~ns}$ & 1 & 0 & $-n s$ \\
\hline Arhus & poly 101 & 0 & & 0 & 0 & & 5 & 2 & & 101 & 0 & \\
\hline F 1 & fixed 0 & 0 & $-\mathrm{ns}$ & 9 & 0 & $-n s$ & 0 & 0 & $-\mathrm{ns}$ & 0 & 0 & $-n s$ \\
\hline Mont Blanc & poly 168 & 0 & & 158 & 0 & & 159 & 2 & & 167 & 0 & \\
\hline F 3 & fixed 20 & 1 & $0.1721 \mathrm{~ns}$ & 23 & 1 & $-n s$ & 107 & 1 & $0.0093^{* *}$ & 19 & 1 & $0.1653 \mathrm{~ns}$ \\
\hline Saint Isidore & poly 101 & 0 & & 0 & 0 & & 5 & 2 & & 101 & 0 & \\
\hline F 5 & fixed 0 & 0 & $-n s$ & 0 & 0 & $-n s$ & 96 & 0 & $0.0081^{* *}$ & 0 & 0 & $-n s$ \\
\hline Bruenstatt & poly 103 & 1 & & 3 & 0 & & 8 & 2 & & 103 & 0 & \\
\hline FIN 1 & fixed 20 & 1 & $-n s$ & 24 & 0 & $-n s$ & 106 & 0 & $0.0044^{* *}$ & 19 & 0 & $-n s$ \\
\hline Lahti & poly 102 & 0 & & 1 & 0 & & 6 & 2 & & 102 & 0 & \\
\hline GB 1 & fixed 1 & 0 & $-n s$ & 9 & 0 & $-n s$ & 0 & 0 & $-\mathrm{ns}$ & 0 & 0 & $-n s$ \\
\hline Ascot & poly 121 & 0 & & 110 & 0 & & 112 & 2 & & 120 & 0 & \\
\hline GB 2 & fixed 0 & 0 & $-n s$ & 0 & 0 & $-n s$ & 93 & 0 & $0.064 \mathrm{~ns}$ & 1 & 0 & $-n s$ \\
\hline Braemar & poly 123 & 0 & & 25 & 0 & & 30 & 2 & & 121 & 0 & \\
\hline N 1 & fixed 44 & 0 & $1 \mathrm{~ns}$ & 74 & 0 & $0.0263^{*}$ & 100 & 0 & $0.0004 * * *$ & 44 & 0 & $1 \mathrm{~ns}$ \\
\hline Narvik & poly 101 & 1 & & 1 & 1 & & 6 & 3 & & 101 & 1 & \\
\hline NL 1 & fixed 3 & 0 & $-n s$ & 71 & 0 & $-n s$ & 1 & 0 & $1 \mathrm{~ns}$ & 1 & 0 & $-n s$ \\
\hline Wageningen & poly 152 & 0 & & 82 & 0 & & 85 & 2 & & 153 & 0 & \\
\hline NL 2 & fixed 20 & 1 & $-n s$ & 23 & 0 & $0.1154 \mathrm{~ns}$ & 106 & 0 & $0.0005 * * *$ & 19 & 0 & $1 \mathrm{~ns}$ \\
\hline Hoge Veluwe & poly 102 & 0 & & 2 & 1 & & 7 & 3 & & 102 & 1 & \\
\hline PL 2 & fixed 0 & 0 & $-n s$ & 0 & 0 & $-n s$ & 86 & 0 & $0.0022^{* *}$ & 1 & 0 & $1 \mathrm{~ns}$ \\
\hline Warsaw & poly 131 & 4 & & 40 & 4 & & 45 & 6 & & 129 & 4 & \\
\hline RUM 2 & fixed 0 & 0 & $-n s$ & 4 & 0 & $1 \mathrm{~ns}$ & 0 & 0 & $-n s$ & 0 & 0 & $-n s$ \\
\hline Sibiu 2 & poly 105 & 1 & & 97 & 1 & & 100 & 3 & & 104 & 1 & \\
\hline S 1 & fixed 44 & 0 & $-n s$ & 74 & 0 & $-n s$ & 100 & 0 & $0.0061^{* *}$ & 44 & 0 & $-n s$ \\
\hline Umea & poly 102 & 0 & & 2 & 0 & & 7 & 2 & & 102 & 0 & \\
\hline S 2 & fixed 6 & 0 & $1 \mathrm{~ns}$ & 9 & 0 & $1 \mathrm{~ns}$ & 65 & 0 & $0.0422^{*}$ & 5 & 0 & $1 \mathrm{~ns}$ \\
\hline Stroemstad & poly 153 & 5 & & 84 & 5 & & 89 & 7 & & 153 & 5 & \\
\hline S 4 & fixed 38 & 0 & $1 \mathrm{~ns}$ & 66 & 0 & $0.0479^{*}$ & 95 & 0 & $0.0018^{* *}$ & 38 & 0 & $1 \mathrm{~ns}$ \\
\hline Abisko & poly 114 & 2 & & 17 & 2 & & 22 & 4 & & 113 & 2 & \\
\hline S 5 & fixed 2 & 0 & $-n s$ & 96 & 0 & $-n s$ & 0 & 0 & $-n s$ & 0 & 0 & $-n s$ \\
\hline Gothenburg & poly 101 & 0 & & 4 & 0 & & 8 & 2 & & 101 & 0 & \\
\hline Population & \multicolumn{3}{|c|}{$\begin{array}{c}\text { D } 5 \\
\text { syn. nons. sign. }\end{array}$} & \multicolumn{3}{|c|}{$\begin{array}{c}\text { D } 6 \\
\text { syn. nons. sign. }\end{array}$} & \multicolumn{3}{|c|}{$\begin{array}{r}\text { D } 7 \\
\text { syn. nons. sign. }\end{array}$} & \multicolumn{3}{|c|}{$\begin{array}{c}\text { D } 8 \\
\text { ns. sign. }\end{array}$} \\
\hline D 6 & fixed 2 & 0 & $1 \mathrm{~ns}$ & & & & & & & & & \\
\hline Black Forest & poly 10 & 1 & & & & & & & & & & \\
\hline D 7 & fixed 23 & 0 & $-n s$ & 25 & 0 & $0.0385^{*}$ & & & & & & \\
\hline Uelzen & poly 10 & 0 & & 0 & 1 & & & & & & & \\
\hline D 8 & fixed 9 & 0 & $1 \mathrm{~ns}$ & 9 & 0 & $1 \mathrm{~ns}$ & 0 & 0 & $-\mathrm{ns}$ & & & \\
\hline Cuxhaven & poly 117 & 2 & & 110 & 3 & & 110 & 2 & & & & \\
\hline D 9 & fixed 0 & 0 & $-n s$ & 1 & 0 & $1 \mathrm{~ns}$ & 20 & 0 & $-\mathrm{ns}$ & 0 & 0 & $-n s$ \\
\hline Bonn & poly 107 & 0 & & 100 & 1 & & 100 & 0 & & 120 & 2 & \\
\hline
\end{tabular}


Table A29 continue:

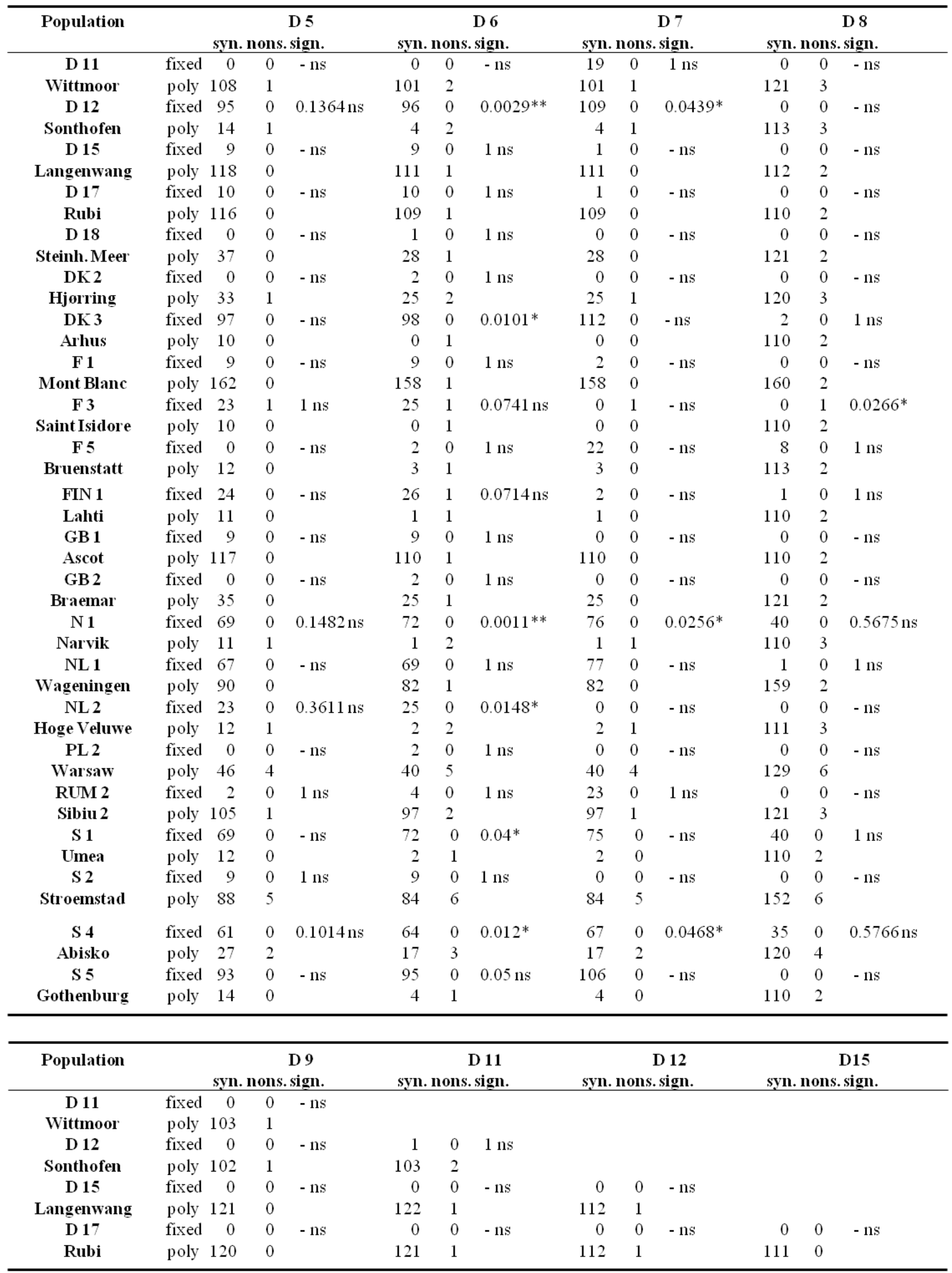


Table A29 continue:

\begin{tabular}{|c|c|c|c|c|c|c|c|c|c|c|c|c|c|}
\hline \multirow{2}{*}{$\begin{array}{c}\text { Population } \\
\text { D } 18\end{array}$} & \multicolumn{4}{|c|}{$\begin{array}{c}\text { D 9 } \\
\text { syn. nons. sign. }\end{array}$} & \multicolumn{3}{|c|}{$\begin{array}{r}\text { D } 11 \\
\text { syn. nons. sign. }\end{array}$} & \multicolumn{3}{|c|}{$\begin{array}{r}\text { D } 12 \\
\text { syn. nons. sign. }\end{array}$} & \multicolumn{3}{|c|}{$\begin{array}{r}\text { D15 } \\
\text { syn. nons. sign. }\end{array}$} \\
\hline & fixed & 0 & 0 & $-n s$ & 0 & 0 & $-n s$ & 92 & 0 & $0.2581 \mathrm{~ns}$ & 1 & 0 & $-n s$ \\
\hline Steinh. Meer & poly & 121 & 0 & & 122 & 1 & & 31 & 1 & & 121 & 0 & \\
\hline DK 2 & fixed & 0 & 0 & $-n s$ & 0 & 0 & $-n s$ & 94 & 0 & $0.057 \mathrm{~ns}$ & 1 & 0 & $1 \mathrm{~ns}$ \\
\hline Hjorring & poly & 120 & 1 & & 121 & 2 & & 28 & 2 & & 120 & 1 & \\
\hline DK 3 & fixed & 1 & 0 & $-n s$ & 2 & 0 & $1 \mathrm{~ns}$ & 0 & 0 & $-\mathrm{ns}$ & 0 & 0 & $-\mathrm{ns}$ \\
\hline Arhus & poly & 100 & 0 & & 101 & 1 & & 4 & 1 & & 111 & 0 & \\
\hline F 1 & fixed & 0 & 0 & $-n s$ & 0 & 0 & $-n s$ & 0 & 0 & $-n s$ & 0 & 0 & $-n s$ \\
\hline Mont Blanc & poly & 167 & 0 & & 167 & 1 & & 158 & 1 & & 160 & 0 & \\
\hline F 3 & fixed & 20 & 1 & $0.1736 \mathrm{~ns}$ & 19 & 1 & $0.3021 \mathrm{~ns}$ & 109 & 0 & $0.0854 \mathrm{~ns}$ & 1 & 1 & $0.0177^{*}$ \\
\hline Saint Isidore & poly & 100 & 0 & & 101 & 1 & & 4 & 1 & & 111 & 0 & \\
\hline F 5 & fixed & 0 & 0 & $-n s$ & 0 & 0 & $-n s$ & 98 & 0 & $0.0667 \mathrm{~ns}$ & 9 & 0 & $-n s$ \\
\hline Bruenstatt & poly & 102 & 1 & & 103 & 1 & & 6 & 1 & & 113 & 0 & \\
\hline FIN 1 & fixed & 20 & 0 & $-n s$ & 19 & 0 & $1 \mathrm{~ns}$ & 108 & 0 & $0.0526 \mathrm{~ns}$ & 1 & 0 & $-n s$ \\
\hline Lahti & poly & 101 & 0 & & 102 & 1 & & 5 & 1 & & 111 & 0 & \\
\hline GB 1 & fixed & 0 & 0 & $-n s$ & 0 & 0 & $-n s$ & 0 & 0 & $-\mathrm{ns}$ & 0 & 0 & $-\mathrm{ns}$ \\
\hline Ascot & poly & 120 & 0 & & 121 & 1 & & 113 & 1 & & 112 & 0 & \\
\hline GB 2 & fixed & 1 & 0 & $-n s$ & 0 & 0 & $-n s$ & 95 & 0 & $0.2339 \mathrm{~ns}$ & 1 & 0 & $-n s$ \\
\hline Braemar & poly & 121 & 0 & & 122 & 1 & & 28 & 1 & & 121 & 0 & \\
\hline N 1 & fixed & 44 & 0 & $1 \mathrm{~ns}$ & 44 & 0 & $0.5778 \mathrm{~ns}$ & 101 & 0 & $0.0036^{* *}$ & 40 & 0 & $1 \mathrm{~ns}$ \\
\hline Narvik & poly & 100 & 1 & & 101 & 2 & & 5 & 2 & & 111 & 1 & \\
\hline NL 1 & fixed & 1 & 0 & $-n s$ & 0 & 0 & $-n s$ & 1 & 0 & $1 \mathrm{~ns}$ & 1 & 0 & $-n s$ \\
\hline Wageningen & poly & 152 & 0 & & 153 & 1 & & 83 & 1 & & 159 & 0 & \\
\hline NL 2 & fixed & 20 & 0 & $1 \mathrm{~ns}$ & 19 & 0 & $1 \mathrm{~ns}$ & 108 & 0 & $0.0042 * *$ & 1 & 0 & $1 \mathrm{~ns}$ \\
\hline Hoge Veluwe & poly & 101 & 1 & & 102 & 2 & & 6 & 2 & & 112 & 1 & \\
\hline PL 2 & fixed & 1 & 0 & $1 \mathrm{~ns}$ & 0 & 0 & $-n s$ & 88 & 0 & $0.0048 * *$ & 0 & 0 & $-\mathrm{ns}$ \\
\hline Warsaw & poly & 129 & 4 & & 130 & 5 & & 43 & 5 & & 130 & 4 & \\
\hline RUM 2 & fixed & 0 & 0 & $-n s$ & 0 & 0 & $-n s$ & 1 & 0 & $1 \mathrm{~ns}$ & 0 & 0 & $-n s$ \\
\hline Sibiu 2 & poly & 103 & 1 & & 104 & 2 & & 99 & 2 & & 122 & 1 & \\
\hline S 1 & fixed & 44 & 0 & $-n s$ & 44 & 0 & $1 \mathrm{~ns}$ & 101 & 0 & $0.0648 \mathrm{~ns}$ & 40 & 0 & $-n s$ \\
\hline Umea & poly & 101 & 0 & & 102 & 1 & & 6 & 1 & & 111 & 0 & \\
\hline $\mathrm{S} 2$ & fixed & 5 & 0 & $1 \mathrm{~ns}$ & 5 & 0 & $1 \mathrm{~ns}$ & 67 & 0 & $0.0407^{*}$ & 1 & 0 & $1 \mathrm{~ns}$ \\
\hline Stroemstad & poly & 153 & 5 & & 153 & 6 & & 87 & 6 & & 152 & 5 & \\
\hline S 4 & fixed & 38 & 0 & $1 \mathrm{~ns}$ & 38 & 0 & $0.5764 \mathrm{~ns}$ & 96 & 0 & $0.0072 * *$ & 35 & 0 & $1 \mathrm{~ns}$ \\
\hline Abisko & poly & 113 & 2 & & 114 & 3 & & 21 & 3 & & 121 & 2 & \\
\hline S 5 & fixed & 0 & 0 & $-n s$ & 1 & 0 & $1 \mathrm{~ns}$ & 0 & 0 & $-n s$ & 0 & 0 & $-n s$ \\
\hline Gothenburg & poly & 100 & 0 & & 101 & 1 & & 8 & 1 & & 111 & 0 & \\
\hline \multicolumn{2}{|l|}{ Population } & \multicolumn{3}{|c|}{$\begin{array}{r}\text { D } 17 \\
\text { syn. nons. sign. }\end{array}$} & syn. & \multicolumn{2}{|c|}{$\begin{array}{r}\text { D } 18 \\
\text { ons. sign. }\end{array}$} & \multicolumn{3}{|c|}{$\begin{array}{r}\text { DK 2 } \\
\text { syn. nons. sign. }\end{array}$} & \multicolumn{3}{|c|}{$\begin{array}{r}\text { DK } 3 \\
\text { syn. nons. sign. }\end{array}$} \\
\hline D 18 & fixed & 1 & 0 & $-n s$ & & & & & & & & & \\
\hline Steinh. Meer & poly & 120 & 0 & & & & & & & & & & \\
\hline DK 2 & fixed & 1 & 0 & $1 \mathrm{~ns}$ & 0 & 0 & $-n s$ & & & & & & \\
\hline Hjorring & poly & 119 & 1 & & 29 & 1 & & & & & & & \\
\hline DK 3 & fixed & 2 & 0 & $-n s$ & 94 & 0 & $-n s$ & 96 & 0 & $0.2131 \mathrm{~ns}$ & & & \\
\hline Arhus & poly & 109 & 0 & & 28 & 0 & & 25 & 1 & & & & \\
\hline F 1 & fixed & 0 & 0 & $-n s$ & 2 & 0 & $-n s$ & 2 & 0 & $1 \mathrm{~ns}$ & 0 & 0 & $-\mathrm{ns}$ \\
\hline Mont Blanc & poly & 160 & 0 & & 166 & 0 & & 165 & 1 & & 158 & 0 & \\
\hline F 3 & fixed & 1 & 1 & $0.018^{*}$ & 0 & 1 & $0.0345^{*}$ & 1 & 0 & $0.0741 \mathrm{~ns}$ & 112 & 1 & $-n s$ \\
\hline Saint Isidore & poly & 109 & 0 & & 28 & 0 & & 25 & 1 & & 0 & 0 & \\
\hline F 5 & fixed & 9 & 0 & $-n s$ & 0 & 0 & $-n s$ & 0 & 0 & $-n s$ & 100 & 0 & $-n s$ \\
\hline Bruenstatt & poly & 112 & 0 & & 29 & 0 & & 26 & 1 & & 3 & 0 & \\
\hline FIN 1 & fixed & 1 & 0 & $-n s$ & 2 & 0 & $-n s$ & 2 & 0 & $1 \mathrm{~ns}$ & 1 & 0 & $1 \mathrm{~ns}$ \\
\hline Lahti & poly & 109 & 0 & & 28 & 0 & & 25 & 1 & & 152 & 1 & \\
\hline GB 1 & fixed & 0 & 0 & $-n s$ & 0 & 0 & $-n s$ & 0 & 0 & $-\mathrm{ns}$ & 2 & 0 & $-n s$ \\
\hline Ascot & poly & 110 & 0 & & 121 & 0 & & 120 & 1 & & 110 & 0 & \\
\hline
\end{tabular}


Table A29 continue:

\begin{tabular}{|c|c|c|c|c|c|c|c|c|c|c|c|c|}
\hline \multirow{2}{*}{$\begin{array}{c}\text { Population } \\
\text { GB 2 }\end{array}$} & \multicolumn{3}{|c|}{$\begin{array}{c}\text { D } 17 \\
\text { syn. nons. sign. }\end{array}$} & \multicolumn{3}{|c|}{$\begin{array}{c}\text { D } 18 \\
\text { syn. nons. sign. }\end{array}$} & \multicolumn{3}{|c|}{$\begin{array}{r}\text { DK2 } \\
\text { syn. nons. sign. }\end{array}$} & \multicolumn{3}{|c|}{$\begin{array}{r}\text { DK 3 } \\
\text { syn. nons. sign. }\end{array}$} \\
\hline & fixed 1 & 0 & $-n s$ & 0 & 0 & $-n s$ & 0 & 0 & $-n s$ & 97 & 0 & $-n s$ \\
\hline Braemar & poly 120 & 0 & & 30 & 0 & & 27 & 1 & & 25 & 0 & \\
\hline N 1 & fixed 41 & 0 & $1 \mathrm{~ns}$ & 62 & 0 & $0.3261 \mathrm{~ns}$ & 62 & 0 & $0.0944 \mathrm{~ns}$ & 103 & 0 & $0.0191^{*}$ \\
\hline Narvik & poly 109 & 1 & & 29 & 1 & & 26 & 2 & & 1 & 1 & \\
\hline NL 1 & fixed 1 & 0 & $-n s$ & 64 & 0 & $-n s$ & 66 & 0 & $1 \mathrm{~ns}$ & 2 & 0 & $-n s$ \\
\hline Wageningen & poly 158 & 0 & & 104 & 0 & & 101 & 1 & & 82 & 0 & \\
\hline NL 2 & fixed & 0 & $1 \mathrm{~ns}$ & 0 & 0 & $-n s$ & 0 & 0 & $-n s$ & 111 & 0 & $0.0263 *$ \\
\hline Hoge Veluwe & poly 110 & 1 & & 29 & 1 & & 27 & 2 & & 2 & 1 & \\
\hline PL 2 & fixed 0 & 0 & $-n s$ & 0 & 0 & $-n s$ & 0 & 0 & $-n s$ & 90 & 0 & $0.0106^{*}$ \\
\hline Warsaw & poly 129 & 4 & & 45 & 4 & & 41 & 5 & & 40 & 4 & \\
\hline RUM 2 & fixed 0 & 0 & $-n s$ & 4 & 0 & $1 \mathrm{~ns}$ & 3 & 0 & $1 \mathrm{~ns}$ & 2 & 0 & $1 \mathrm{~ns}$ \\
\hline Sibiu 2 & poly 121 & 1 & & 118 & 1 & & 118 & 2 & & 97 & 1 & \\
\hline S 1 & fixed 41 & 0 & $-n s$ & 62 & 0 & $-n s$ & 62 & 0 & $0.3034 \mathrm{~ns}$ & 103 & 0 & $-n s$ \\
\hline Umea & poly 109 & 0 & & 29 & 0 & & 26 & 1 & & 2 & 0 & \\
\hline $\mathrm{S} 2$ & fixed 1 & 0 & $1 \mathrm{~ns}$ & 0 & 0 & $-n s$ & 0 & 0 & $-n s$ & 69 & 0 & $0.0685 \mathrm{~ns}$ \\
\hline Stroemstad & poly 151 & 5 & & 96 & 5 & & 93 & 6 & & 84 & 5 & \\
\hline S 4 & fixed 36 & 0 & $1 \mathrm{~ns}$ & 55 & 0 & $0.1951 \mathrm{~ns}$ & 55 & 0 & $0.0779 \mathrm{~ns}$ & 98 & 0 & $0.0252 *$ \\
\hline Abisko & poly 119 & 2 & & 42 & 2 & & 39 & 3 & & 17 & 2 & \\
\hline S 5 & fixed 0 & 0 & $-n s$ & 89 & 0 & $-n s$ & 91 & 0 & $0.2479 \mathrm{~ns}$ & 2 & 0 & $-n s$ \\
\hline Gothenburg & poly 109 & 0 & & 32 & 0 & & 29 & 1 & & 4 & 0 & \\
\hline
\end{tabular}

\begin{tabular}{|c|c|c|c|c|c|c|c|c|c|c|c|c|}
\hline \multirow{2}{*}{$\begin{array}{c}\text { Population } \\
\text { F } 3\end{array}$} & \multicolumn{3}{|c|}{$\begin{array}{c}\text { F } 1 \\
\text { syn. nons. sign. }\end{array}$} & \multicolumn{3}{|c|}{$\begin{array}{c}\text { F 3 } \\
\text { syn. nons. sign. }\end{array}$} & \multicolumn{3}{|c|}{$\begin{array}{c}\text { F } 5 \\
\text { syn. nons. sign. }\end{array}$} & \multicolumn{3}{|c|}{$\begin{array}{r}\text { FIN } 1 \\
\text { syn. nons. sign. }\end{array}$} \\
\hline & fixed & 1 & $0.0186^{*}$ & & & & & & & & & \\
\hline Saint Isidore & poly 158 & 0 & & & & & & & & & & \\
\hline F 5 & fixed & 0 & $-n s$ & 22 & 1 & $1 \mathrm{~ns}$ & & & & & & \\
\hline Bruenstatt & poly 159 & 0 & & 3 & 0 & & & & & & & \\
\hline FIN 1 & fixed & 0 & $-\mathrm{ns}$ & 2 & 1 & $1 \mathrm{~ns}$ & 23 & 0 & $-\mathrm{ns}$ & & & \\
\hline Lahti & poly 158 & 0 & & 1 & 0 & & 4 & 0 & & & & \\
\hline GB 1 & fixed 0 & 1 & $0.009 * *$ & 0 & 1 & $0.009^{* *}$ & 8 & 0 & $-n s$ & 1 & 0 & $-n s$ \\
\hline Ascot & poly 110 & 0 & & 110 & 0 & & 113 & 0 & & 110 & 0 & \\
\hline GB 2 & fixed & 0 & $-\mathrm{ns}$ & 0 & 1 & $0.0385^{*}$ & 0 & 0 & $-n s$ & 2 & 0 & $-n s$ \\
\hline Braemar & poly 165 & 0 & & 25 & 0 & & 27 & 0 & & 25 & 0 & \\
\hline N 1 & fixed 24 & 0 & $1 \mathrm{~ns}$ & 76 & 1 & $0.0503 \mathrm{~ns}$ & 72 & 0 & $0.0649 \mathrm{~ns}$ & 75 & 0 & $0.0385^{*}$ \\
\hline Narvik & poly 158 & 1 & & 1 & 1 & & 4 & 1 & & 2 & 1 & \\
\hline NL 1 & fixed 0 & 0 & $-n s$ & 77 & 1 & $0.4875 \mathrm{~ns}$ & 69 & 0 & $-n s$ & 75 & 0 & $-n s$ \\
\hline Wageningen & poly 161 & 0 & & 82 & 0 & & 85 & 0 & & 83 & 0 & \\
\hline NL 2 & fixed & 0 & $1 \mathrm{~ns}$ & 0 & 1 & $1 \mathrm{~ns}$ & 22 & 0 & $0.2143 \mathrm{~ns}$ & 2 & 0 & $1 \mathrm{~ns}$ \\
\hline Hoge Veluwe & poly 158 & 1 & & 2 & 1 & & 5 & 1 & & 3 & 1 & \\
\hline PL 2 & fixed & 0 & $1 \mathrm{~ns}$ & 0 & 1 & $0.1111 \mathrm{~ns}$ & 0 & 0 & $-n s$ & 0 & 0 & $-n s$ \\
\hline Warsaw & poly 172 & 4 & & 40 & 4 & & 42 & 4 & & 40 & 4 & \\
\hline RUM 2 & fixed & 0 & $-n s$ & 23 & 1 & $0.3561 \mathrm{~ns}$ & 4 & 0 & $1 \mathrm{~ns}$ & 23 & 0 & $1 \mathrm{~ns}$ \\
\hline Sibiu 2 & poly 168 & 1 & & 97 & 1 & & 99 & 1 & & 98 & 1 & \\
\hline S 1 & fixed 24 & 0 & $-\mathrm{ns}$ & 75 & 1 & $1 \mathrm{~ns}$ & 72 & 0 & $-\mathrm{ns}$ & 74 & 0 & $-n s$ \\
\hline Umea & poly 158 & 0 & & 2 & 0 & & 5 & 0 & & 3 & 0 & \\
\hline S 2 & fixed & 1 & $0.0667 \mathrm{~ns}$ & 0 & 1 & $0.0667 \mathrm{~ns}$ & 8 & 0 & $1 \mathrm{~ns}$ & 1 & 0 & $1 \mathrm{~ns}$ \\
\hline Stroemstad & poly 84 & 5 & & 84 & 5 & & 86 & 5 & & 84 & 5 & \\
\hline $\mathrm{S} 4$ & fixed 20 & 0 & $1 \mathrm{~ns}$ & 67 & 1 & $0.1189 \mathrm{~ns}$ & 64 & 0 & $0.0632 \mathrm{~ns}$ & 66 & 0 & $0.052 \mathrm{~ns}$ \\
\hline Abisko & poly 167 & 2 & & 17 & 2 & & 20 & 2 & & 18 & 2 & \\
\hline S 5 & fixed & 0 & $-n s$ & 106 & 1 & $1 \mathrm{~ns}$ & 95 & 0 & $-n s$ & 105 & 0 & $-n s$ \\
\hline Gothenburg & poly 158 & 0 & & 4 & 0 & & 7 & 0 & & 5 & 0 & \\
\hline
\end{tabular}


Appendix

Table A29 continue:

\begin{tabular}{|c|c|c|c|c|c|c|c|c|c|c|c|c|}
\hline \multirow{2}{*}{$\begin{array}{c}\text { Population } \\
\text { GB } 2\end{array}$} & \multicolumn{3}{|c|}{$\begin{array}{r}\text { GB 1 } \\
\text { syn. nons. sign. }\end{array}$} & \multicolumn{3}{|c|}{$\begin{array}{r}\text { GB 2 } \\
\text { syn. nons. sign. }\end{array}$} & \multicolumn{3}{|c|}{$\begin{array}{c}\text { N } 1 \\
\text { syn. nons. sign. }\end{array}$} & \multicolumn{3}{|c|}{$\begin{array}{r}\text { NL 1 } \\
\text { syn. nons. sign. }\end{array}$} \\
\hline & fixed & 0 & $-n s$ & & & & & & & & & \\
\hline & poly 121 & 0 & & & & & & & & & & \\
\hline N 1 & fixed 40 & 0 & $1 \mathrm{~ns}$ & 62 & 0 & $0.3034 \mathrm{~ns}$ & & & & & & \\
\hline Narvik & poly 110 & 1 & & 26 & 1 & & & & & & & \\
\hline NL 1 & fixed 1 & 0 & $-n s$ & 67 & 0 & $-n s$ & 63 & 0 & $1 \mathrm{~ns}$ & & & \\
\hline Wageningen & poly 159 & 0 & & 100 & 0 & & 83 & 1 & & & & \\
\hline NL 2 & fixed 0 & 0 & $-n s$ & 0 & 0 & $-n s$ & 75 & 0 & $0.0032 * *$ & 77 & 0 & $1 \mathrm{~ns}$ \\
\hline Hoge Veluwe & poly 111 & 1 & & 26 & 1 & & 3 & 2 & & 82 & 1 & \\
\hline PL 2 & fixed 0 & 0 & $-n s$ & 0 & 0 & $-n s$ & 57 & 0 & $0.0157^{*}$ & 64 & 0 & $0.2982 \mathrm{~ns}$ \\
\hline Warsaw & poly 129 & 4 & & 42 & 4 & & 41 & 5 & & 110 & 4 & \\
\hline RUM 2 & fixed 0 & 0 & $-n s$ & 4 & 0 & $1 \mathrm{~ns}$ & 45 & 0 & $0.5673 \mathrm{~ns}$ & 1 & 0 & $1 \mathrm{~ns}$ \\
\hline Sibiu 2 & poly 121 & 1 & & 118 & 1 & & 97 & 2 & & 151 & 1 & \\
\hline S 1 & fixed 40 & 0 & $-n s$ & 62 & 0 & $-n s$ & 0 & 0 & $-n s$ & 63 & 0 & $-n s$ \\
\hline Umea & poly 110 & 0 & & 26 & 0 & & 2 & 1 & & 84 & 0 & \\
\hline $\mathrm{S} 2$ & fixed 0 & 0 & $-n s$ & 0 & 0 & $-n s$ & 0 & 0 & $-n s$ & 42 & 0 & $0.3529 \mathrm{~ns}$ \\
\hline Stroemstad & poly 152 & 5 & & 93 & 5 & & 84 & 6 & & 141 & 5 & \\
\hline S 4 & fixed 35 & 0 & $1 \mathrm{~ns}$ & 55 & 0 & $0.1798 \mathrm{~ns}$ & 0 & 0 & $-n s$ & 59 & 0 & $0.5266 \mathrm{~ns}$ \\
\hline Abisko & poly 120 & 2 & & 39 & 2 & & 18 & 3 & & 95 & 2 & \\
\hline S 5 & fixed 0 & 0 & $-n s$ & 92 & 0 & -ns & 101 & 0 & $0.0561 \mathrm{~ns}$ & 1 & 0 & $-n s$ \\
\hline Gothenburg & poly 110 & 0 & & 29 & 0 & & 5 & 1 & & 84 & 0 & \\
\hline
\end{tabular}

\begin{tabular}{|c|c|c|c|c|c|c|c|}
\hline \multirow{2}{*}{$\begin{array}{c}\text { Population } \\
\text { PL } 2\end{array}$} & \multicolumn{4}{|c|}{$\begin{array}{r}\text { NL } 2 \\
\text { syn. nons. sign. }\end{array}$} & \multicolumn{3}{|c|}{$\begin{array}{r}\text { PL 2 } \\
\text { syn. nons. sign. }\end{array}$} \\
\hline & fixed & 0 & 0 & $-n s$ & & & \\
\hline Warsaw & poly & 42 & 5 & & & & \\
\hline RUM 2 & fixed & 23 & 0 & $1 \mathrm{~ns}$ & 0 & 0 & $-n s$ \\
\hline Sibiu 2 & poly & 98 & 2 & & 128 & 5 & \\
\hline S 1 & fixed & 74 & 0 & $0.0633 \mathrm{~ns}$ & 57 & 0 & $0.0351^{*}$ \\
\hline Umea & poly & 4 & 1 & & 41 & 4 & \\
\hline S 2 & fixed & 0 & 0 & $-n s$ & 0 & 0 & $-n s$ \\
\hline Stroemstad & poly & 85 & 6 & & 103 & 8 & \\
\hline S 4 & fixed & 66 & 0 & $0.014^{*}$ & 50 & 0 & $0.0308^{*}$ \\
\hline Abisko & poly & 19 & 3 & & 54 & 6 & \\
\hline S 5 & fixed & 105 & 0 & $0.0625 \mathrm{~ns}$ & 85 & 0 & $0.0156^{*}$ \\
\hline Gothenburg & poly & 6 & 1 & & 44 & 4 & \\
\hline \multicolumn{2}{|l|}{ Population } & \multicolumn{3}{|c|}{$\begin{array}{c}\text { S } 2 \\
\text { syn. nons. sign. }\end{array}$} & syn. & \multicolumn{2}{|c|}{$\begin{array}{c}\text { S } 4 \\
\text { ons. sign. }\end{array}$} \\
\hline S 4 & fixed & 0 & 0 & $-n s$ & & & \\
\hline Abisko & poly & 92 & 7 & & & & \\
\hline S 5 & fixed & 66 & 0 & $0.0744 \mathrm{~ns}$ & 96 & 0 & $0.036^{*}$ \\
\hline Gothenburg & poly & 86 & 5 & & 21 & 2 & \\
\hline
\end{tabular}




\section{Appendix}

Table A30: Neutrality indices of Platynothrus peltifer computed in the McDonald-Kreitman test with DnaSP v5. Locations with less than two individuals were excluded.

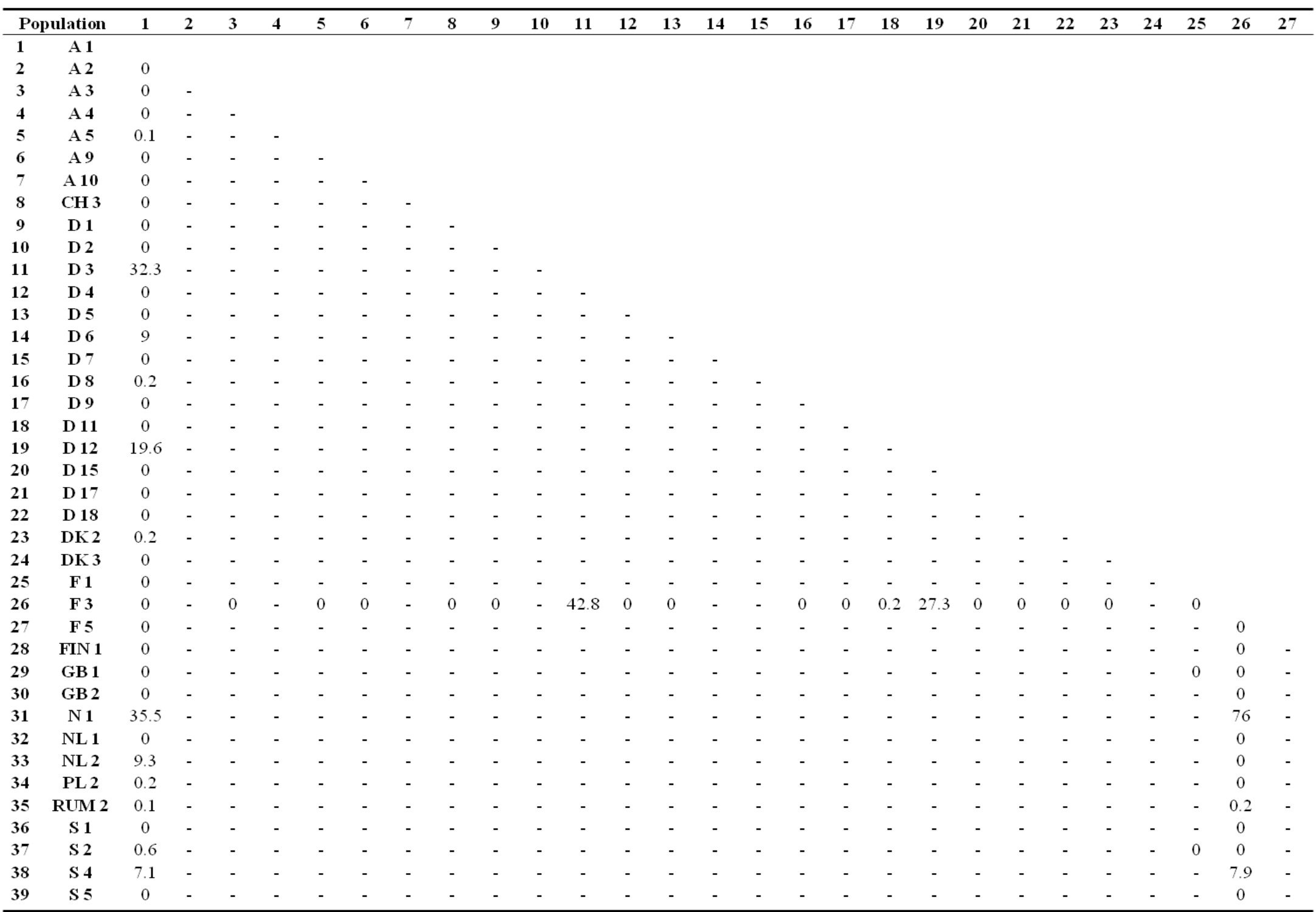


Appendix

Table A30 continue:

\begin{tabular}{ccccccccccccc}
\hline \multicolumn{2}{c}{ Population } & 28 & 29 & 30 & 31 & 32 & 33 & 34 & 35 & 36 & 37 & 38 \\
\hline 29 & GB 1 & - & & & & & & & & & & \\
30 & GB 2 & - & - & & & & & & & & & \\
31 & N 1 & - & - & - & & & & & & & & \\
32 & NL 1 & - & - & - & - & & & & & & & \\
33 & NL 2 & - & - & - & - & - & & & & & & \\
34 & PL 2 & - & - & - & - & - & - & & & & & \\
35 & RUM 2 & - & - & - & - & - & - & - & & & & \\
36 & S 1 & - & - & - & - & - & - & - & - & & & \\
37 & S 2 & - & - & - & - & - & - & - & - & - & & \\
38 & S 4 & - & - & - & - & - & - & - & - & - & - & \\
39 & S 5 & - & - & - & - & - & - & - & - & - & - & - \\
\hline
\end{tabular}

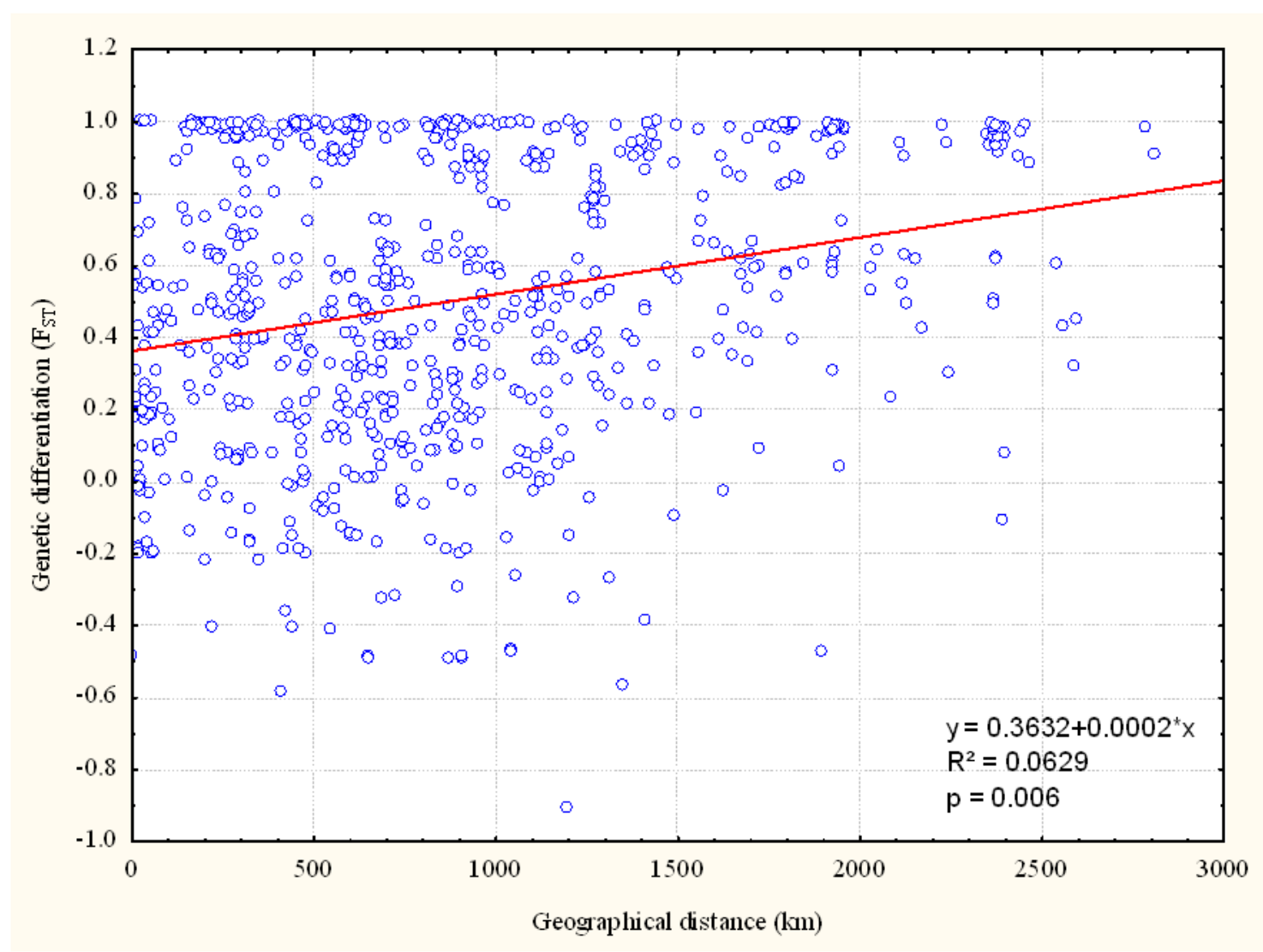

Figure A39: Linear regression of geographical distances (kilometers) vs. genetic differentiations $\left(\mathrm{F}_{\mathrm{ST}}\right)$ based on COI of Platynothrus peltifer. Regression is significant $(\mathrm{p}=0.006 * * ; \quad(* 0.01<\mathrm{P}<0.05 ; \quad * * 0.001<\mathrm{P}<0.01$; $* * * \mathrm{P}<0.001)$ ) using 1000 randomizations. 
Appendix

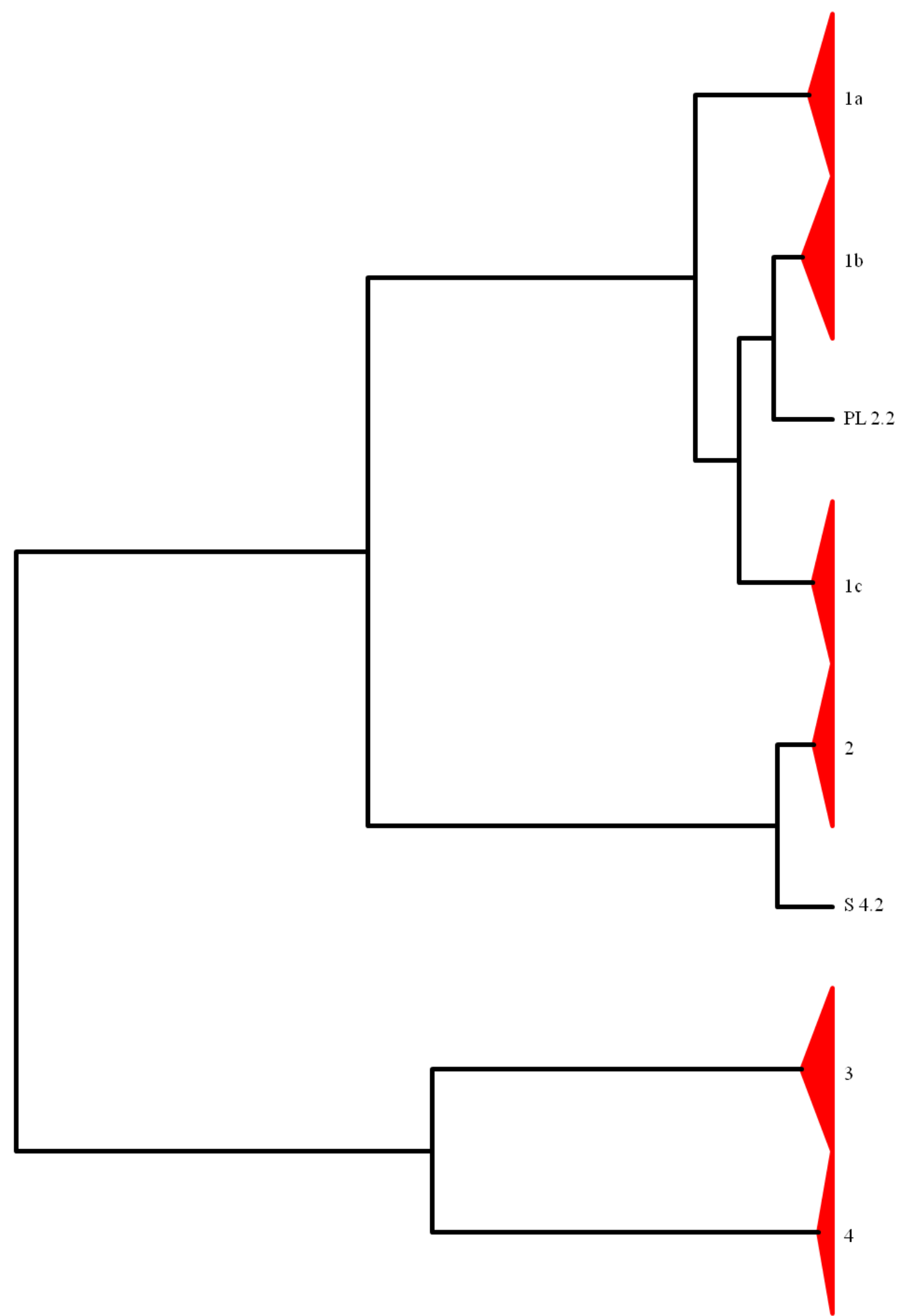

Figure A40: Cluster delimitation of $\mathrm{COI}$ nucleotide sequences from Platynothrus peltifer after the single method of the generalized mixed Yule coalescent method (GMYC). Red branches are the different distinct clusters. Tip labels are explained in Table 20. 
Appendix

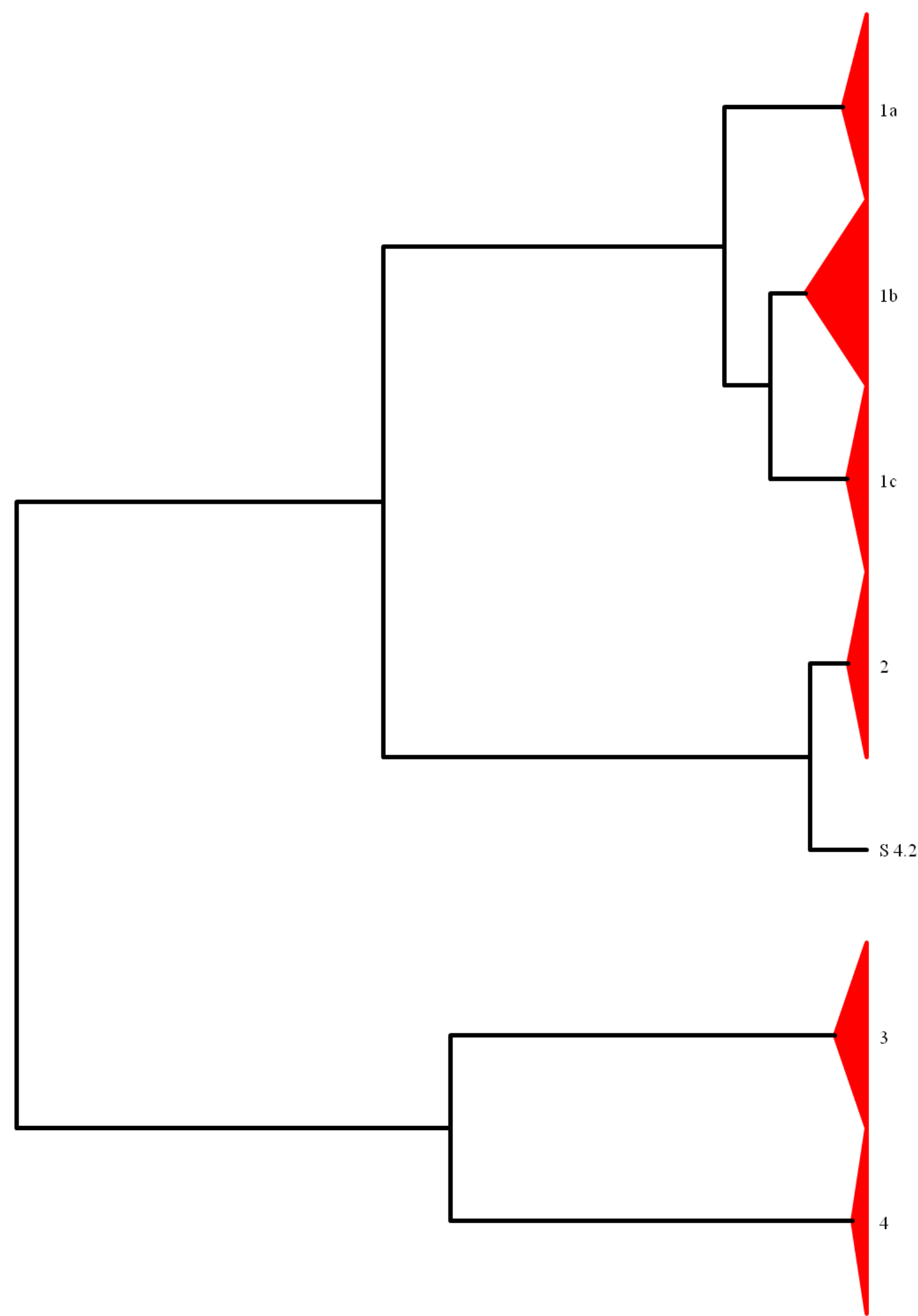

Figure A41: Cluster delimitation of COI nucleotide sequences from Platynothrus peltifer after the multiple method of the generalized mixed Yule coalescent method (GMYC). Red branches are the different distinct clusters. Tip labels are explained in Table 20. 
Appendix

Table A31: Standard diversity measures of Achipteria coleoptrata. Populations with less than two individuals were excluded.

\begin{tabular}{|c|c|c|c|c|c|c|c|c|c|}
\hline population & $\begin{array}{c}\text { sample } \\
\text { size } \\
\mathbf{n} \\
\end{array}$ & $\begin{array}{c}\text { invarible } \\
\text { sites } \\
\mathbf{N}_{\text {is }} \\
\end{array}$ & $\begin{array}{c}\text { variable } \\
\text { sites } \\
\mathbf{N}_{\mathrm{vs}} \\
\end{array}$ & $\begin{array}{c}\text { parsimony } \\
\text { inform. sites } \\
\mathbf{N}_{\text {pars }} \\
\end{array}$ & $\begin{array}{c}\text { number of } \\
\text { singeltons } \\
\mathrm{N}_{\mathrm{s}} \\
\end{array}$ & $\begin{array}{c}\text { number of } \\
\text { haplotypes } \\
N_{h} \\
\end{array}$ & $\begin{array}{c}\text { haplotype } \\
\text { diversity } \\
\mathbf{H}_{\mathbf{d}} \\
\end{array}$ & variance & $\begin{array}{c}\text { nucleotide } \\
\text { diversity } \\
\Pi_{n}\end{array}$ \\
\hline A_5 & 4 & 415 & 97 & 4 & 93 & 4 & 1 & 0.031 & 0.097 \\
\hline A_7 & 2 & 487 & 25 & 0 & 25 & 2 & 1 & 0.250 & 0.049 \\
\hline A_8 & 3 & 420 & 92 & 0 & 92 & 3 & 1 & 0.074 & 0.12 \\
\hline CH_1 & 5 & 503 & 9 & 2 & 7 & 5 & 1 & 0.016 & 0.008 \\
\hline CH_3 & 2 & 506 & 6 & 0 & 6 & 2 & 1 & 0.250 & 0.012 \\
\hline D_1 & 4 & 510 & 2 & 0 & 2 & 3 & 0.83 & 0.049 & 0.002 \\
\hline D_2 & 2 & 509 & 3 & 0 & 3 & 2 & 1 & 0.250 & 0.006 \\
\hline D_3 & 4 & 500 & 12 & 0 & 12 & 3 & 0.83 & 0.049 & 0.012 \\
\hline D_5 & 4 & 508 & 4 & 0 & 4 & 2 & 0.5 & 0.07 & 0.004 \\
\hline D_8 & 6 & 509 & 3 & 1 & 2 & 3 & 0.73 & 0.024 & 0.002 \\
\hline D_9 & 3 & 506 & 6 & 0 & 6 & 2 & 0.67 & 0.099 & 0.008 \\
\hline D_10 & 5 & 499 & 13 & 11 & 2 & 4 & 0.9 & 0.026 & 0.014 \\
\hline D_11 & 2 & 512 & 0 & 0 & 0 & 1 & 0 & 0 & 0 \\
\hline D_12 & 3 & 470 & 42 & 0 & 42 & 3 & 1 & 0.074 & 0.055 \\
\hline D_14 & 2 & 511 & 1 & 0 & 1 & 2 & 1 & 0.250 & 0.002 \\
\hline D_15 & 3 & 428 & 84 & 0 & 84 & 3 & 1 & 0.074 & 0.11 \\
\hline DK_3 & 4 & 503 & 9 & 1 & 8 & 4 & 1 & 0.031 & 0.009 \\
\hline EST_1 & 4 & 490 & 22 & 6 & 16 & 4 & 1 & 0.031 & 0.024 \\
\hline$F_{-} 1$ & 2 & 492 & 20 & 0 & 20 & 2 & 1 & 0.250 & 0.039 \\
\hline F_2 & 6 & 477 & 35 & 25 & 10 & 5 & 0.93 & 0.015 & 0.034 \\
\hline F_4 & 3 & 511 & 1 & 0 & 1 & 2 & 0.67 & 0.099 & 0.001 \\
\hline FIN_1 & 4 & 482 & 30 & 0 & 30 & 2 & 0.667 & 0.042 & 0.039 \\
\hline GB_1 & 4 & 471 & 41 & 3 & 38 & 4 & 1.00 & 0.031 & 0.042 \\
\hline GB_2 & 3 & 499 & 13 & 0 & 13 & 2 & 0.67 & 0.099 & 0.017 \\
\hline GR_1 & 6 & 504 & 8 & 4 & 4 & 3 & 0.6 & 0.046 & 0.007 \\
\hline I_1 & 8 & 504 & 8 & 7 & 1 & 3 & 0.61 & 0.027 & 0.006 \\
\hline I_2 & 4 & 504 & 8 & 4 & 4 & 3 & 0.83 & 0.049 & 0.004 \\
\hline PL_1 & 4 & 470 & 42 & 1 & 41 & 3 & 0.83 & 0.049 & 0.041 \\
\hline PL_2 & 8 & 405 & 107 & 36 & 71 & 6 & 0.89 & 0.012 & 0.074 \\
\hline RUM_1 & 4 & 496 & 16 & 2 & 14 & 3 & 0.83 & 0.049 & 0.016 \\
\hline RUM_2 & 4 & 503 & 9 & 0 & 9 & 2 & 0.5 & 0.07 & 0.009 \\
\hline RUM_5 & 3 & 508 & 4 & 0 & 4 & 3 & 1 & 0.074 & 0.005 \\
\hline RUM_6 & 2 & 506 & 6 & 0 & 6 & 2 & 1 & 0.250 & 0.012 \\
\hline RUS_2 & 3 & 509 & 3 & 0 & 3 & 2 & 0.67 & 0.099 & 0.004 \\
\hline S_2 & 6 & 511 & 1 & 0 & 1 & 2 & 0.33 & 0.046 & 0.001 \\
\hline all & 136 & 293 & 219 & 197 & 22 & 88 & 0.98 & 0 & 0.114 \\
\hline
\end{tabular}


Appendix

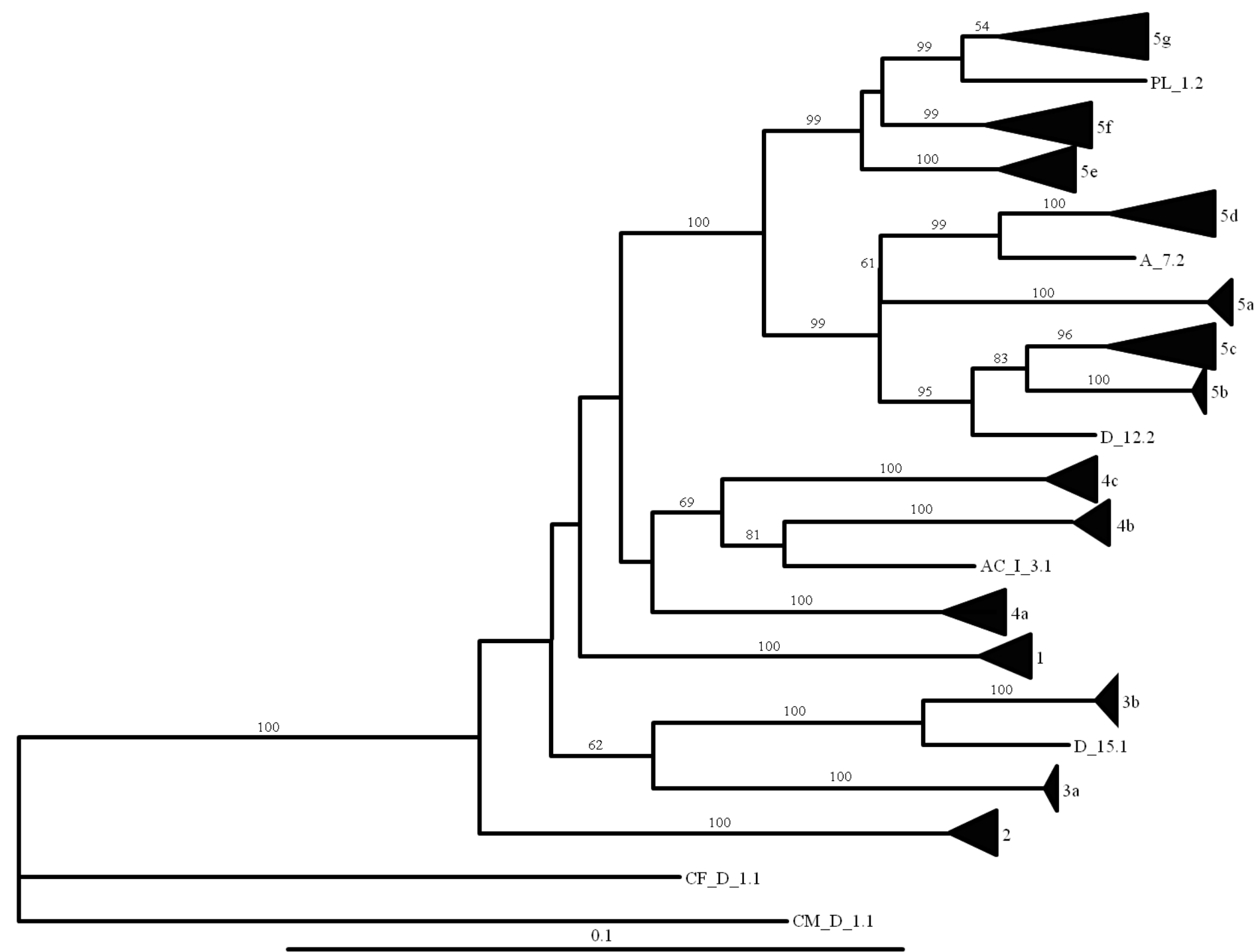

Figure A42: Neighbor-Joining tree of 141 COI nucleotide sequences of Achipteria coleoptrata. Outgroups are Carabodes femoralis (CF_D_1.1) and C. marginatus (CM_D 1.1). Numbers on the branches are bootstrap values of 100,000 pseudo-replicates. Tip numbers are the different subclades and explain in Table 24. 
Appendix

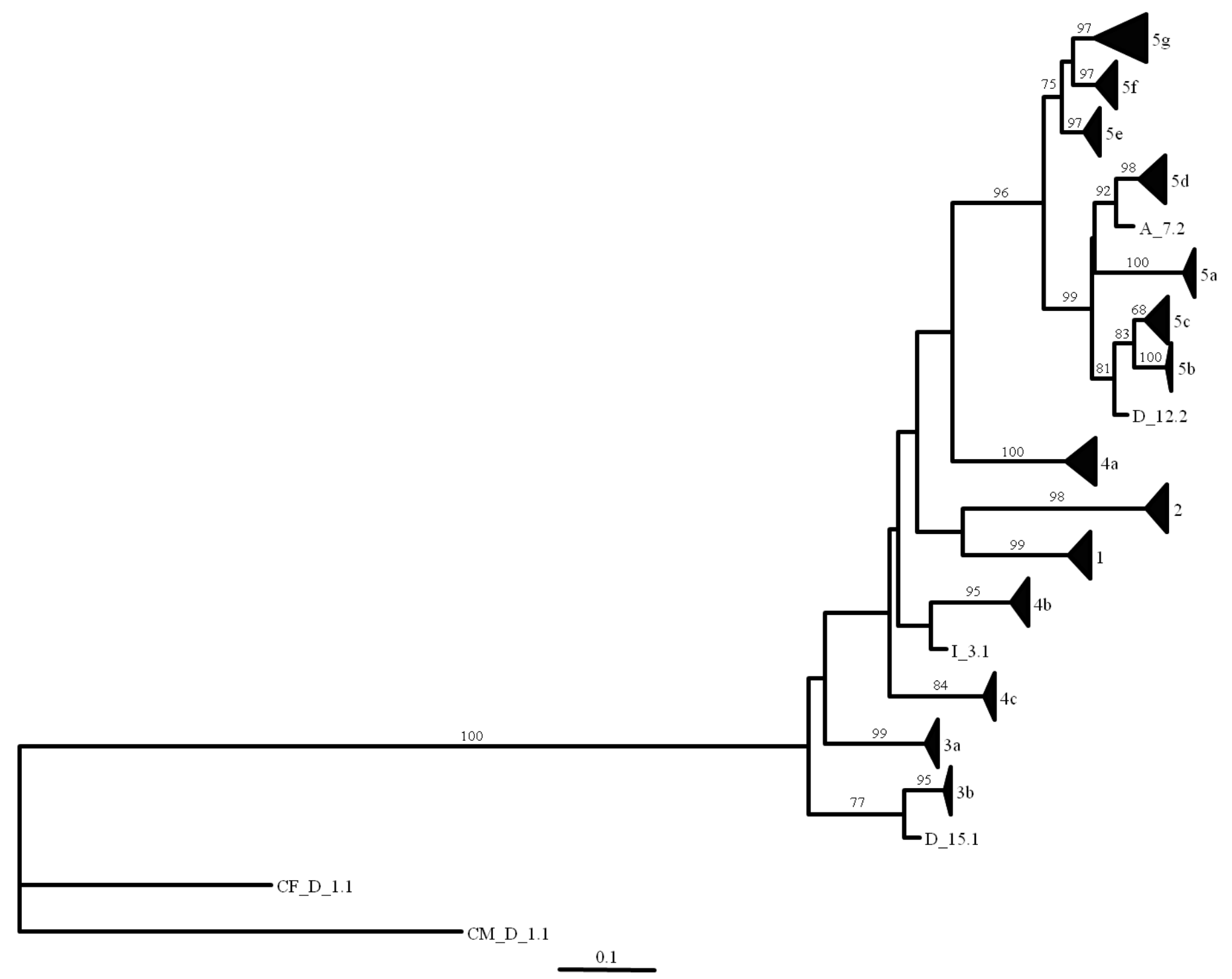

Figure A43: Neighbor-Joining tree of 141 COI nucleotide sequences of Achipteria coleoptrata with model of sequence evolution TVM+I+G. Outgroups are Carabodes femoralis (CF_D_1.1) and C. marginatus (CM_D_1.1). Numbers on the branches are bootstrap values of 100,000 pseudo-replicates. Tip numbers are the different subclades and explain in Table 24. 
Appendix

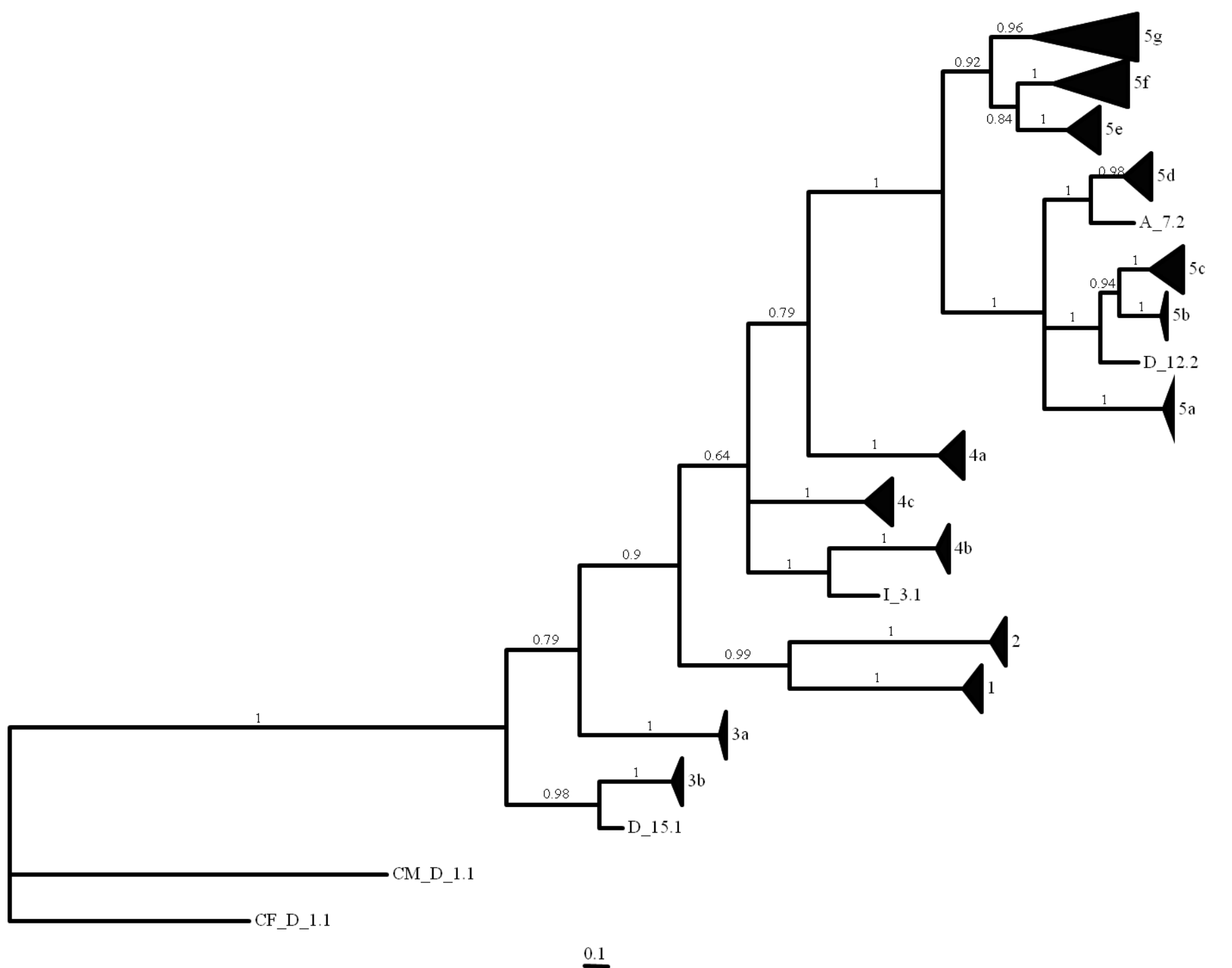

Figure A44: Bayesian tree after 10x10 6 generations from 141 COI nucleotide sequences from Achipteria coleoptrata with MrBayes. Split frequencies of 0.012879 and burnin of 25\%. Outgroups are Carabodes femoralis (CF_D_1.1) and C. marginatus (CM_D_1.1). Numbers on the branches are posterior probabilities. Tip numbers are the different subclades and explain in Table 24. 
Appendix

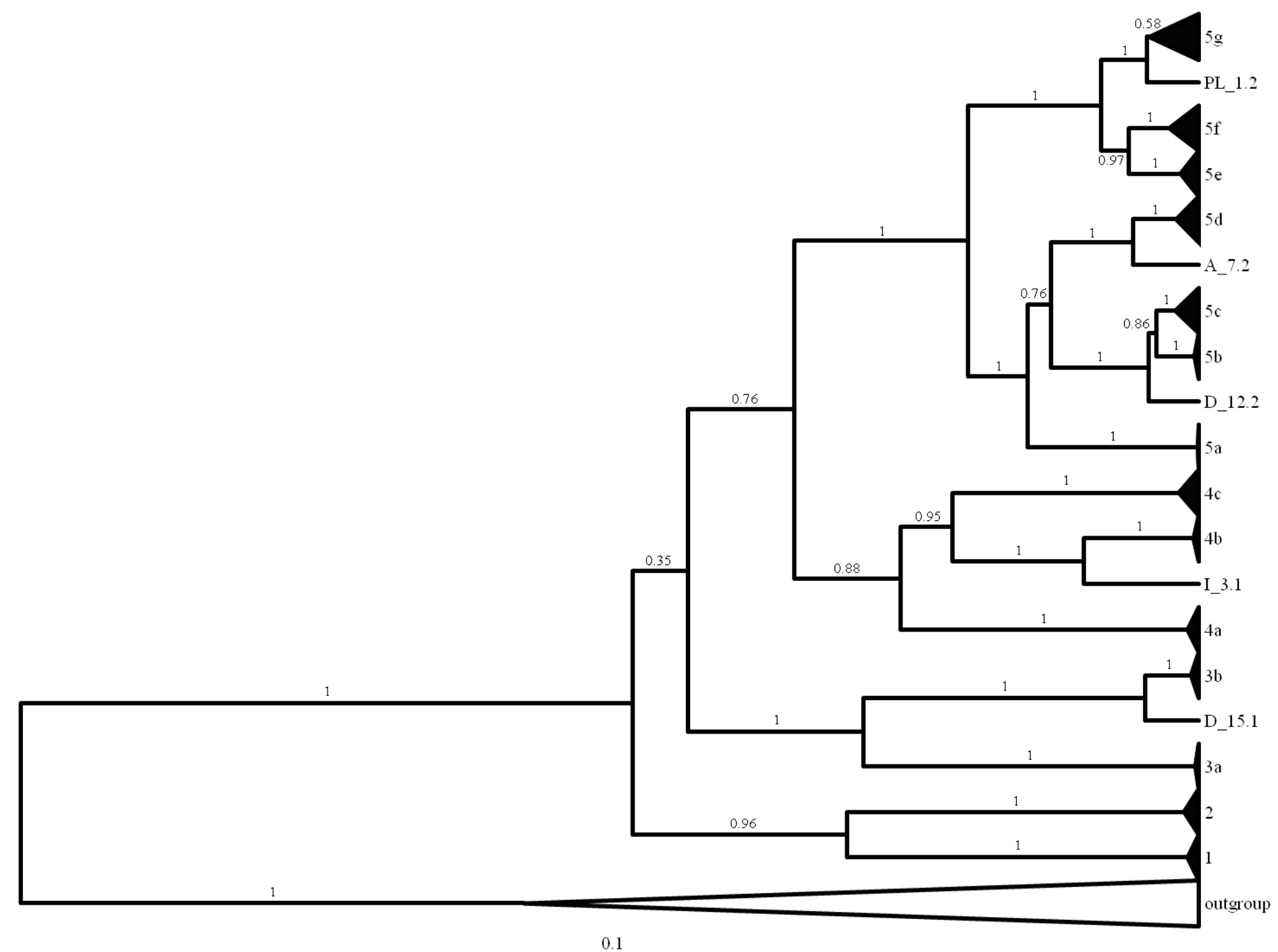

Figure A45: Bayesian phylogeny after 10x10 $0^{6}$ generations from 141 COI nucleotide sequences from Achipteria coleoptrata with Beast v1.5.4. Outgroups are Carabodes femoralis (CF_D_1.1) and C. marginatus (CM_D_1.1).. Numbers on the branches are posterior probabilities. Tip numbers are the different subclades and explain in Table 24. 
Appendix

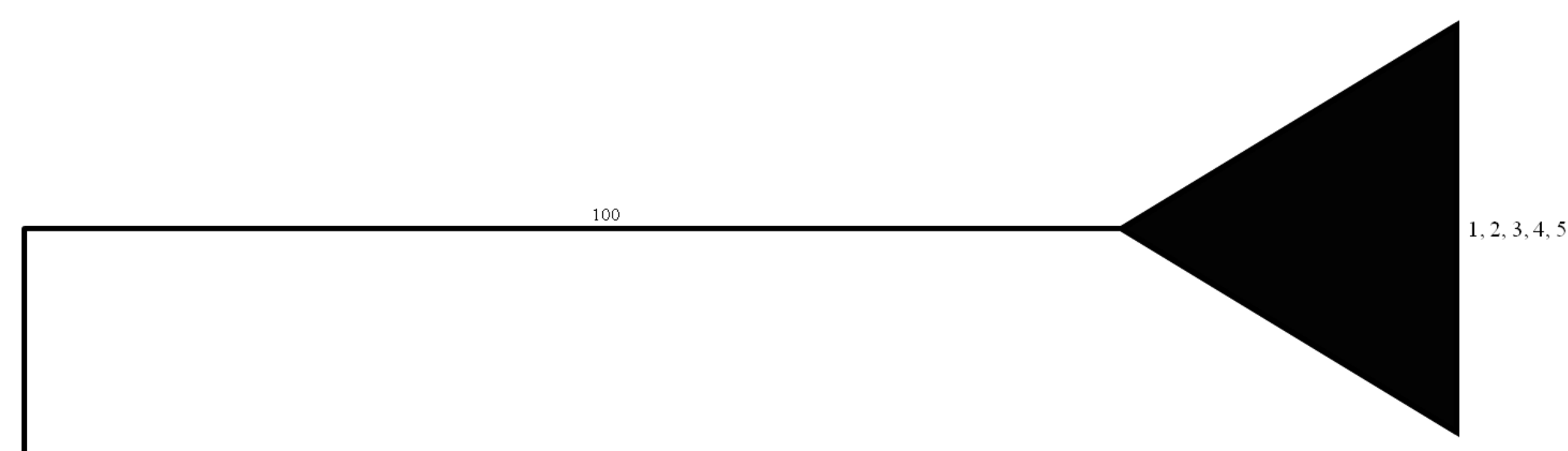

CF_D_1.1

CM_D_1.1

0.1

Figure A46: Neighbor-Joining tree of 141 COI protein sequences of Achipteria coleoptrata. Outgroups are Carabodes femoralis (CF_D_1.1) and C. marginatus (CM_D_1.1). Numbers on the branches are bootstrap values of 100,000 pseudo-replicates. Tip numbers are the different subclades and explain in Table 24. 
Appendix

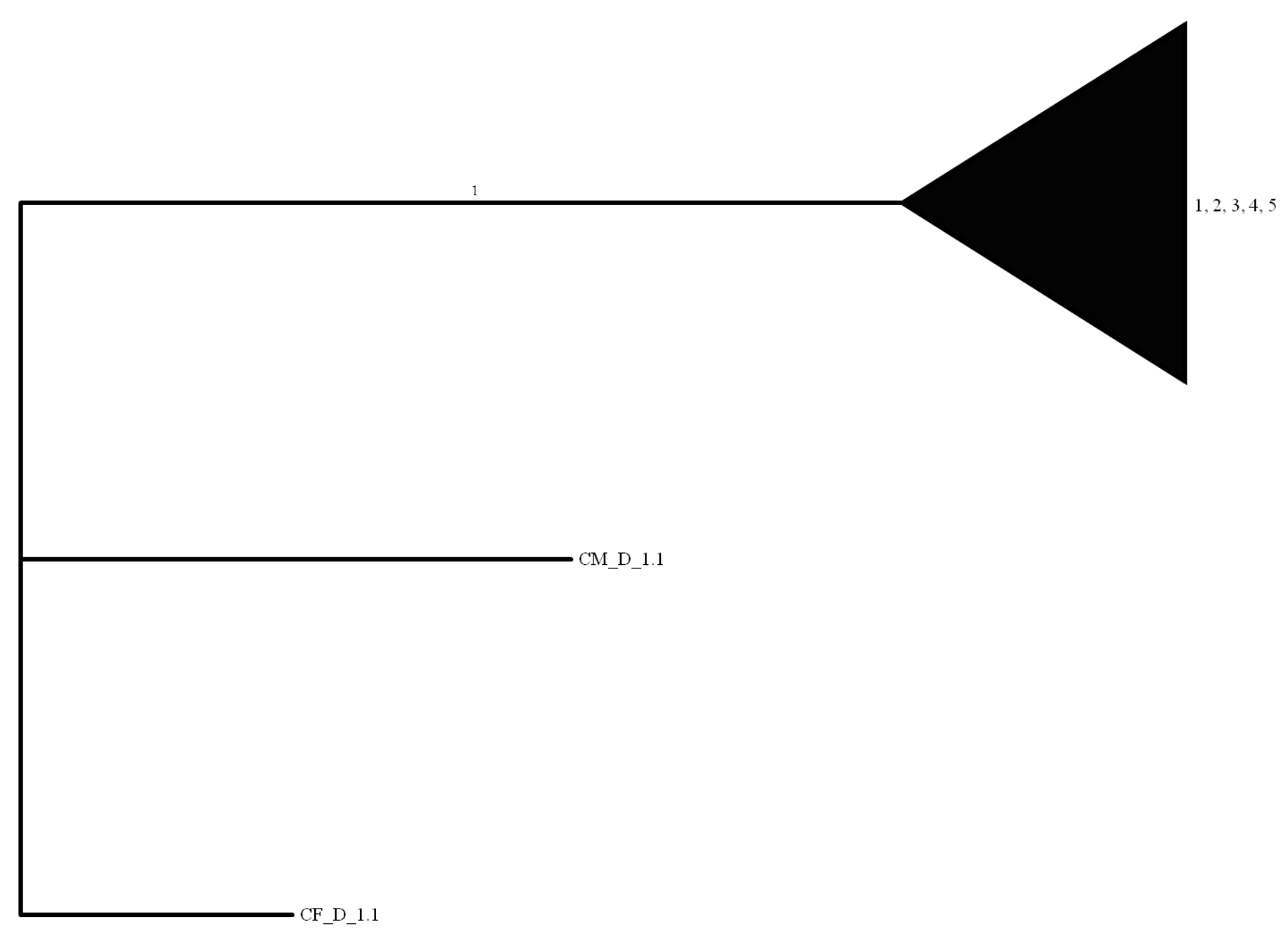

Figure A47: Bayesian tree after $10 \times 10^{6}$ generations from 141 COI protein sequences of Achipteria coleoptrata. Outgroups are Carabodes femoralis (CF_D_1.1) and C. marginatus (CM_D_1.1). Split frequencies of 0.031752 and burnin of $25 \%$. Numbers on the branches are posterior probabilities. Tip numbers are the different subclades and explain in Table 24. 
Appendix

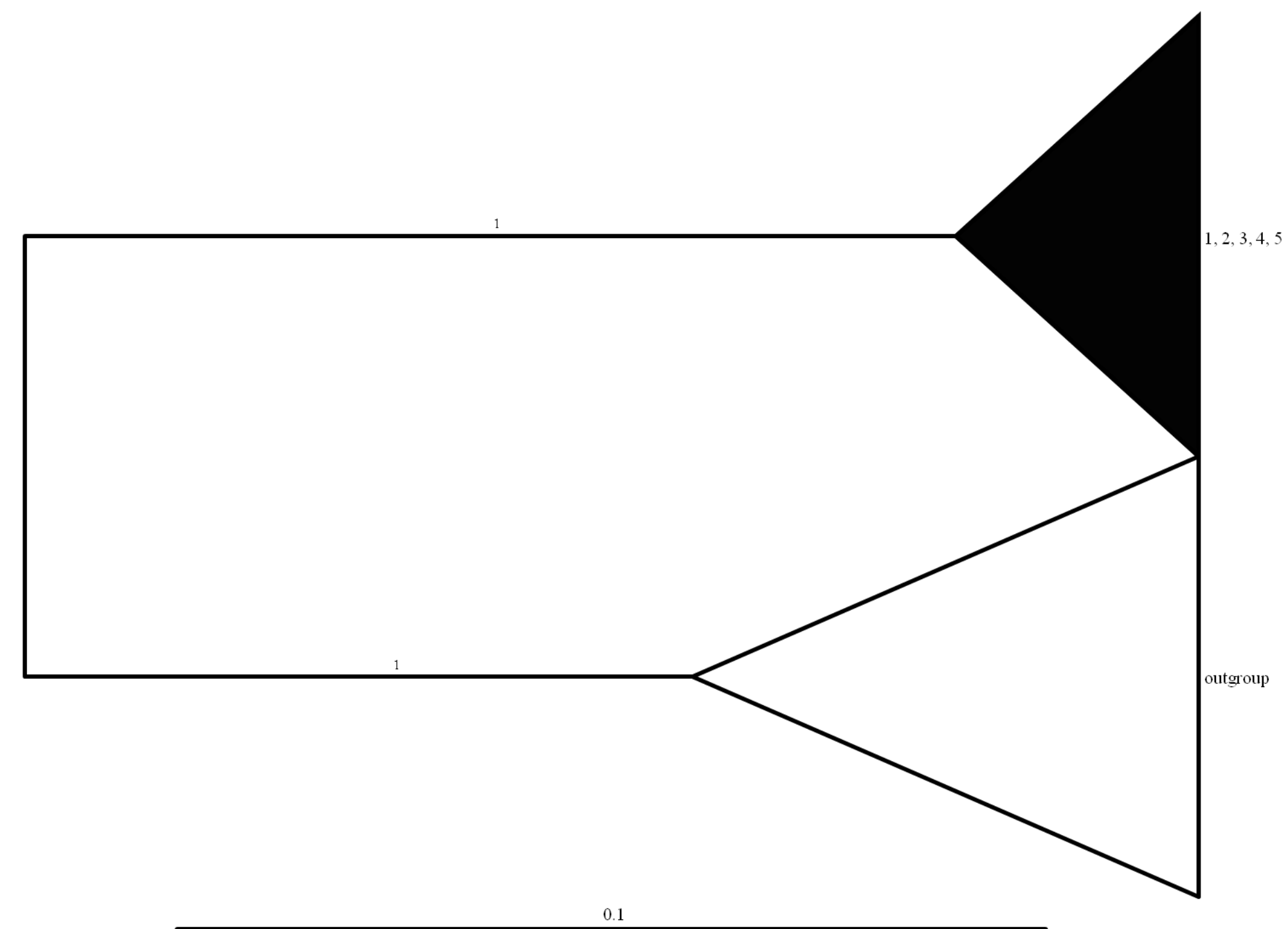

Figure A48: Bayesian phylogeny after 10x10 generations with Beast v1.5.4 from the 141 COI protein sequences of Achipteria coleoptrata. Outgroups are Carabodes femoralis (CF_D_1.1) and C. marginatus (CM_D_1.1). Numbers on the branches are posterior probabilities. Tip numbers are the different subclades and explain in Table 24 . 


\section{Appendix}

Table A32: Mean pairwise percentage differences of uncorrected p-distances of the protein of Achipteria coleoptrata from 35 locations. The diagonal is the within population differences (bold) and below the diagonal the among population differences. Bold letters in red are the minimum and maximum differences within and among the populations. Locations with less than two individuals were excluded from the analysis.

\begin{tabular}{|c|c|c|c|c|c|c|c|c|c|c|c|c|c|c|c|c|c|c|c|c|c|c|c|c|c|}
\hline \multicolumn{2}{|c|}{ Population } & 1 & 2 & 3 & 4 & 5 & 6 & 7 & 8 & 9 & 10 & 11 & 12 & 13 & 14 & 15 & 16 & 17 & 18 & 19 & 20 & 21 & 22 & 23 & 24 \\
\hline 1 & A 5 & 0.3 & & & & & & & & & & & & & & & & & & & & & & & \\
\hline 2 & A 7 & 0.2 & 0 & & & & & & & & & & & & & & & & & & & & & & \\
\hline 3 & A 8 & 0.2 & 0 & 0 & & & & & & & & & & & & & & & & & & & & & \\
\hline 4 & CH 1 & 0.2 & 0 & 0 & 0 & & & & & & & & & & & & & & & & & & & & \\
\hline 5 & $\mathrm{CH} 3$ & 0.4 & 0.3 & 0.3 & 0.3 & 0.6 & & & & & & & & & & & & & & & & & & & \\
\hline 6 & D 1 & 0.2 & 0 & 0 & 0 & 0.3 & 0 & & & & & & & & & & & & & & & & & & \\
\hline 7 & D 2 & 0.7 & 0.6 & 0.6 & 0.6 & 0.9 & 0.6 & 1.2 & & & & & & & & & & & & & & & & & \\
\hline 8 & D 3 & 0.2 & 0 & 0 & 0 & 0.3 & 0 & 0.6 & 0 & & & & & & & & & & & & & & & & \\
\hline 9 & D 5 & 0.3 & 0.2 & 0.2 & 0.2 & 0.4 & 0.2 & 0.7 & 0.2 & 0.3 & & & & & & & & & & & & & & & \\
\hline 10 & D 8 & 0.3 & 0.2 & 0.2 & 0.2 & 0.5 & 0.2 & 0.8 & 0.2 & 0.3 & 0.3 & & & & & & & & & & & & & & \\
\hline 11 & D 9 & 0.2 & 0 & 0 & 0 & 0.3 & 0 & 0.6 & 0 & 0.2 & 0.2 & 0 & & & & & & & & & & & & & \\
\hline 12 & D 10 & 0.3 & 0.1 & 0.1 & 0.1 & 0.4 & 0.1 & 0.6 & 0.1 & 0.3 & 0.3 & 0.1 & 0.2 & & & & & & & & & & & & \\
\hline 13 & D 11 & 0.2 & 0 & 0 & 0 & 0.3 & 0 & 0.6 & 0 & 0.2 & 0.2 & 0 & 0.1 & 0 & & & & & & & & & & & \\
\hline 14 & D 12 & 0.2 & 0 & 0 & 0 & 0.3 & 0 & 0.6 & 0 & 0.2 & 0.2 & 0 & 0.1 & 0 & 0 & & & & & & & & & & \\
\hline 15 & D 14 & 0.4 & 0.3 & 0.3 & 0.3 & 0.6 & 0.3 & 0.9 & 0.3 & 0.4 & 0.5 & 0.3 & 0.4 & 0.3 & 0.3 & 0.6 & & & & & & & & & \\
\hline 16 & D 15 & 2.1 & 2 & 2 & 2 & 1.9 & 2.0 & 2.6 & 2.0 & 2.1 & 2.2 & 2 & 2.1 & 2 & 2 & 1.9 & 2.4 & & & & & & & & \\
\hline 17 & DK 3 & 0.3 & 0.2 & 0.2 & 0.2 & 0.3 & 0.2 & 0.7 & 0.2 & 0.3 & 0.3 & 0.2 & 0.3 & 0.2 & 0.2 & 0.4 & 1.9 & 0.3 & & & & & & & \\
\hline 18 & EST 1 & 0.2 & 0 & 0 & 0 & 0.3 & 0 & 0.6 & 0 & 0.2 & 0.2 & 0 & 0.1 & 0 & 0 & 0.3 & 2 & 0.2 & 0 & & & & & & \\
\hline 19 & F 1 & 0.2 & 0 & 0 & 0 & 0.3 & 0 & 0.6 & 0 & 0.2 & 0.2 & 0 & 0.1 & 0 & 0 & 0.3 & 2 & 0.2 & 0 & 0 & & & & & \\
\hline 20 & F 2 & 0.2 & 0 & 0 & 0 & 0.3 & 0 & 0.6 & 0 & 0.2 & 0.2 & 0 & 0.1 & 0 & 0 & 0.3 & 2 & 0.2 & 0 & 0 & 0 & & & & \\
\hline 21 & F 4 & 0.2 & 0 & 0 & 0 & 0.3 & 0 & 0.6 & 0 & 0.2 & 0.2 & 0 & 0.1 & 0 & 0 & 0.3 & 2 & 0.2 & 0 & 0 & 0 & 0 & & & \\
\hline 22 & FIN 1 & 0.2 & 0 & 0 & 0 & 0.3 & 0 & 0.6 & 0 & 0.2 & 0.2 & 0 & 0.1 & 0 & 0 & 0.3 & 2 & 0.2 & 0 & 0 & 0 & 0 & 0 & & \\
\hline 23 & GB 1 & 0.3 & 0.2 & 0.2 & 0.2 & 0.4 & 0.2 & 0.7 & 0.2 & 0.3 & 0.3 & 0.2 & 0.3 & 0.2 & 0.2 & 0.3 & 1.9 & 0.3 & 0.2 & 0.2 & 0.2 & 0.2 & 0.2 & 0.3 & \\
\hline 24 & GB 2 & 0.2 & 0 & 0 & 0 & 0.3 & 0 & 0.6 & 0 & 0.2 & 0.2 & 0 & 0.1 & 0 & 0 & 0.3 & 2 & 0.2 & 0 & 0 & 0 & 0 & 0 & 0.2 & 0 \\
\hline 25 & GR 1 & 0.2 & 0 & 0 & 0 & 0.3 & 0 & 0.6 & 0 & 0.2 & 0.2 & 0 & 0.1 & 0 & 0 & 0.3 & 2 & 0.2 & 0 & 0 & 0 & 0 & 0 & 0.2 & 0 \\
\hline 26 & I 1 & 0.2 & 0 & 0 & 0 & 0.3 & 0 & 0.6 & 0 & 0.2 & 0.2 & 0 & 0.1 & 0 & 0 & 0.3 & 2 & 0.2 & 0 & 0 & 0 & 0 & 0 & 0.2 & 0 \\
\hline 27 & I 2 & 0.4 & 0.3 & 0.3 & 0.3 & 0.6 & 0.6 & 0.9 & 0.3 & 0.4 & 0.5 & 0.3 & 0.4 & 0.3 & 0.3 & 0.6 & 2.3 & 0.4 & 0.3 & 0.3 & 0.3 & 0.3 & 0.3 & 0.4 & 0.3 \\
\hline 28 & PL 1 & 0.6 & 0.4 & 0.4 & 0.4 & 0.7 & 0.4 & 1 & 0.4 & 0.6 & 0.6 & 0.4 & 0.6 & 0.4 & 0.4 & 0.6 & 2.2 & 0.6 & 0.4 & 0.4 & 0.4 & 0.4 & 0.4 & 0.5 & 0.4 \\
\hline 29 & PL 2 & 0.2 & 0 & 0 & 0 & 0.3 & 0 & 0.6 & 0 & 0.2 & 0.2 & 0 & 0.1 & 0 & 0 & 0.3 & 2 & 0.2 & 0 & 0 & 0 & 0 & 0 & 0.2 & 0 \\
\hline 30 & RUM 1 & 0.7 & 0.6 & 0.6 & 0.6 & 0.9 & 0.5 & 1.2 & 0.6 & 0.7 & 0.8 & 0.6 & 0.7 & 0.6 & 0.6 & 0.9 & 2.6 & 0.7 & 0.6 & 0.6 & 0.6 & 0.6 & 0.6 & 0.7 & 0.6 \\
\hline 31 & RUM 2 & 0.6 & 0.4 & 0.4 & 0.4 & 0.7 & 0.4 & 1.0 & 0.4 & 0.6 & 0.6 & 0.4 & 0.6 & 0.4 & 0.4 & 0.7 & 2.4 & 0.6 & 0.4 & 0.4 & 0.4 & 0.4 & 0.4 & 0.6 & 0.4 \\
\hline 32 & RUM 5 & 0.5 & 0.4 & 0.4 & 0.4 & 0.7 & 0.4 & 1.0 & 0.4 & 0.5 & 0.6 & 0.4 & 0.5 & 0.4 & 0.4 & 0.7 & 2.4 & 0.5 & 0.4 & 0.4 & 0.4 & 0.4 & 0.4 & 0.5 & 0.4 \\
\hline 33 & RUM 6 & 0.2 & 0 & 0 & 0 & 0.3 & 0 & 0.6 & 0 & 0.2 & 0.2 & 0 & 0.1 & 0 & 0 & 0.3 & 2 & 0.2 & 0 & 0 & 0 & 0 & 0 & 0.2 & 0 \\
\hline 34 & RUS 2 & 0.5 & 0.4 & 0.4 & 0.4 & 0.7 & 0.4 & 1.0 & 0.4 & 0.5 & 0.6 & 0.4 & 0.5 & 0.4 & 0.4 & 0.3 & 1.8 & 0.5 & 0.4 & 0.4 & 0.4 & 0.4 & 0.4 & 0.3 & 0.4 \\
\hline 35 & S 2 & 0.2 & 0 & 0 & 0 & 0.3 & 0 & 0.6 & 0 & 0.2 & 0.2 & 0 & 0.1 & 0 & 0 & 0.3 & 2 & 0.2 & 0 & 0 & 0 & 0 & 0 & 0.2 & 0 \\
\hline
\end{tabular}

\section{Table A32 continue:}


Appendix

\begin{tabular}{lcccccccccccc}
\hline Population & $\mathbf{2 5}$ & $\mathbf{2 6}$ & $\mathbf{2 7}$ & $\mathbf{2 8}$ & $\mathbf{2 9}$ & $\mathbf{3 0}$ & $\mathbf{3 1}$ & $\mathbf{3 2}$ & $\mathbf{3 3}$ & $\mathbf{3 4}$ & $\mathbf{3 5}$ \\
\hline $\mathbf{2 5}$ & GR 1 & $\mathbf{0}$ & & & & & & & & & & \\
$\mathbf{2 6}$ & I 1 & $\mathbf{0}$ & 0 & & & & & & & & & \\
$\mathbf{2 7}$ & I 2 & 0.3 & 0.3 & $\mathbf{0 . 6}$ & & & & & & & & \\
$\mathbf{2 8}$ & PL 1 & 0.4 & 0.4 & 0.7 & $\mathbf{0 . 7}$ & & & & & & & \\
$\mathbf{2 9}$ & PL 2 & 0 & 0 & 0.3 & 0.4 & 0 & & & & & & \\
$\mathbf{3 0}$ & RUM 1 & 0.6 & 0.6 & 0.9 & 0.9 & 0.6 & $\mathbf{1}$ & & & & & \\
31 & RUM 2 & 0.4 & 0.4 & 0.7 & 0.9 & 0.4 & 0.8 & $\mathbf{0 . 3}$ & & & & \\
$\mathbf{3 2}$ & RUM 5 & 0.4 & 0.4 & 0.7 & 0.9 & 0.4 & 0.9 & 0.7 & $\mathbf{0 . 8}$ & & & \\
33 & RUM 6 & 0 & 0 & 0.3 & 0.4 & 0 & 0.6 & 0.4 & 0.4 & 0 & & \\
$\mathbf{3 4}$ & RUS 2 & 0.4 & 0.4 & 0.7 & 0.6 & 0.4 & 1 & 0.8 & 0.8 & 0.4 & $\mathbf{0 . 4}$ & \\
35 & S 2 & $\mathbf{0}$ & $\mathbf{0}$ & 0.3 & 0.4 & $\mathbf{0}$ & 0.6 & 0.4 & 0.4 & 0 & 0.4 & 0 \\
\hline
\end{tabular}


Appendix

Table A33: Tajima's D and Fu's FS neutrality tests of Achipteria coleoptrata COI nucleotide sequences from 35 locations. Bold red letters show significance $(\mathrm{P}<0.05)$.

\begin{tabular}{|c|c|c|c|c|c|c|c|c|c|c|c|c|c|c|c|c|c|c|c|}
\hline Neutrality tests & A 5 & A 7 & A 8 & CH $1 \mathrm{C}$ & CH 3 D 1 & D 2 & D 3 & D 5 & D 8 & D 10 D 11 & 1 D 12 & D 14 & D 15 & \multicolumn{2}{|c|}{ DK 3 EST 1} & $1 F 1$ & F 2 & F 4 & FIN 1 \\
\hline \multicolumn{20}{|l|}{ Tajima's D test } \\
\hline Samplesize & 4 & 2 & 3 & 5 & 4 & 2 & 4 & 4 & 3 & 2 & 3 & 2 & 3 & 4 & 4 & 2 & 6 & 3 & 4 \\
\hline S & 97 & 25 & 92 & 9 & 6 & 3 & 12 & 4 & 6 & 13 & 42 & 1 & 84 & 9 & 22 & 20 & 35 & 1 & 30 \\
\hline $\mathrm{Pi}$ & 49.83 & 25 & 61.33 & 4 & 6 & 3 & 6 & 2 & 1.2 & 7.4 & 28 & 1 & 56.33 & 4.67 & 712.33 & 320 & 17.53 & 0.67 & 20 \\
\hline Tajima's D & -0.61 & 0 & 0 & -0.53 & -0.71 & 0 & -0.84 & $-0.78-$ & -0.45 & 1.35 & 0 & $\begin{array}{ll}0 & 153\end{array}$ & 36564.26 & -0.49 & 90.29 & 0 & 0.91 & 0 & 2.30 \\
\hline Tajima's D p-value & 0.34 & 1 & 0.68 & 0.41 & 0.27 & 1 & 0.1 & 0.2 & $\begin{array}{ll}0.36 & 0.78\end{array}$ & 0.91 & 0.68 & 1 & 1 & 0.46 & $5 \quad 0.72$ & 1 & 0.84 & 0.99 & 1 \\
\hline \multicolumn{20}{|l|}{ Fu's FS test } \\
\hline Real no. of alleles & 4 & 2 & 3 & 5 & 3 & 2 & 3 & 2 & 2 & 1 & 3 & 2 & 3 & 4 & 4 & 2 & 5 & 2 & 2 \\
\hline Orig. no. of alleles & 4 & 2 & 3 & 5 & 2 & 2 & 3 & 2 & 2 & 1 & 3 & 2 & 3 & 4 & 4 & 2 & 5 & 2 & 2 \\
\hline Theta_pi & 49.83 & 25 & 61.33 & 4 & 6 & 3 & 6 & 2 & 1.2 & 7.4 & 28 & 1 & 56.33 & 4.67 & 712.33 & 320 & 17.53 & 0.67 & 20 \\
\hline Exp.no. of alleles & 3.88 & 1.96 & 2.95 & $3.54 \quad 1$ & $\begin{array}{ll}1.86 & 2.08\end{array}$ & 1.75 & 3.27 & 2.572 & $2.63 \quad 2.47$ & 4.03 & 2.9 & 1.5 & 2.95 & 3.13 & 3.59 & 1.95 & 5.29 & 1.65 & 3.73 \\
\hline FS & 2.08 & 3.22 & 3.01 & $\begin{array}{ll}-1.72 & 1\end{array}$ & $1.79-0.89$ & $9 \quad 1.1$ & 1.79 & 2.20 & $0.12 \quad 2.64$ & 1.12 & 2.21 & 0 & 2.92 & -0.62 & 20.58 & 3 & 1.75 & 0.2 & 7.48 \\
\hline FS p-value & 0.53 & 0.6 & 0.57 & 0.060 & $0.51 \quad 0.09$ & 0.43 & 0.76 & 0.83 & $0.45 \quad 0.83$ & 0.63 N.A. & 1. 0.55 & 0.25 & 0.57 & 0.19 & 0.38 & 0.61 & 0.73 & 0.38 & 1 \\
\hline Neutrality tests & & GB 1 & GB 2 & GR 1 & I 1 & I 2 & PL 1 & PL 2 & RUM 1 & RUM 2 I & RUM 5 & RUM 6 & 6 RUS 2 & & S 2 & mean & & s.d & \\
\hline \multicolumn{20}{|l|}{ Tajima's D test } \\
\hline Samplesize & & 4 & 3 & 6 & 8 & 4 & 4 & 8 & 4 & 4 & 3 & 2 & 3 & & 6 & 3.89 & & 1.5 & \\
\hline$S$ & & 41 & 13 & 8 & 8 & 4 & 42 & 107 & 16 & 9 & 4 & 6 & 3 & & 1 & 22.23 & & 29.3 & 32 \\
\hline $\mathrm{Pi}$ & & 21.33 & 8.67 & 3.73 & 3.25 & 2 & 21.17 & 37.64 & 8.33 & 4.5 & 2.67 & 6 & 2 & & 0.33 & 12.94 & & 16.2 & 26 \\
\hline Tajima's D & & -0.48 & 0 & 0.39 & $0.26-$ & -0.78 & -0.79 & -0.48 & -0.46 & -0.83 & 0 & 0 & 0 & & -0.93 & 43901. & .73 & 25972 & 26.78 \\
\hline Tajima's D p-value & & 0.46 & 0.71 & 0.66 & 0.62 & 0.2 & 0.17 & 0.33 & 0.47 & 0.12 & 0.83 & 1 & 0.88 & & 0.26 & 0.64 & & 0.3 & 32 \\
\hline \multicolumn{20}{|l|}{ Fu's FS test } \\
\hline Real no. of alleles & & 4 & 2 & 3 & 3 & 3 & 3 & 6 & 3 & 2 & 3 & 2 & 2 & & 2 & 2.89 & & 1.0 & 98 \\
\hline Orig. no. of alleles & & 4 & 2 & 3 & 3 & 3 & 3 & 6 & 3 & 2 & 3 & 2 & 2 & & 2 & 2.89 & & 1.0 & 08 \\
\hline Theta_pi & & 21.33 & 8.67 & 3.73 & 3.25 & 2 & 21.17 & 37.64 & 8.33 & 4.5 & 2.67 & 6 & 2 & & 0.33 & 12.94 & & 16.2 & \\
\hline Exp.no. of alleles & & 3.75 & 2.71 & 3.9 & 4.41 & 2.57 & 3.74 & 7.34 & 3.43 & 3.11 & 2.30 & 1.86 & 2.17 & & 1.63 & 2.93 & & 1.2 & 28 \\
\hline FS & & 1.18 & 3.92 & 2.28 & $3 \quad 2.75$ & 0.13 & 3.93 & 3.89 & 2.32 & 3.78 & -0.34 & 1.79 & 1.61 & & 0.00 & 1.75 & & 1.7 & \\
\hline FS p-value & & 0.45 & 0.92 & 0.87 & 0.91 & 0.33 & 0.92 & 0.94 & 0.81 & 0.94 & 0.19 & 0.51 & 0.7 & & 0.27 & N.A. & & N.A & \\
\hline
\end{tabular}


Appendix

Table A34: Tajima's D and Fu's FS neutrality tests of Achipteria coleoptrata COI protein sequences from 35 locations. Bold red letters show significance (P<0.05).

\begin{tabular}{|c|c|c|c|c|c|c|c|c|c|c|c|c|c|c|c|c|c|c|c|c|c|}
\hline Neutrality tests & A 5 & A 7 & A 8 & CH 1 & $\mathrm{CH} 3$ & D 1 & D 3 & D 5 & D 8 & D 9 & D 10 & D 11 D & D 12 & D 14 & D 15 & DK: & 3 EST 1 & 1 F 1 & F 2 & F 4 & FIN 1 \\
\hline \multicolumn{22}{|l|}{ Tajima's D test } \\
\hline Samplesize & 4 & 2 & 3 & 5 & 2 & 2 & 4 & 4 & 6 & 3 & 5 & 2 & 3 & 2 & 3 & 4 & 4 & 2 & 6 & 3 & 4 \\
\hline$S$ & 1 & 0 & 0 & 0 & 1 & 2 & 0 & 1 & 1 & 0 & 1 & 0 & 0 & 1 & 6 & 1 & 0 & 0 & 0 & 0 & 0 \\
\hline $\mathrm{Pi}$ & 0.5 & 0 & 0 & 0 & 1 & 0 & 0 & 0.5 & 0.53 & 0 & 0.4 & 0 & 0 & 1 & 4 & 0.5 & 0 & 0 & 0 & 0 & 0 \\
\hline Tajima's D & -0.61 & 0 & 0 & 0 & 0 & 0 & 0 & -0.61 & 0.85 & 0 & -0.82 & 0 & 0 & 0 & 0 & -0.61 & 0 & 0 & 0 & 0 & 0 \\
\hline Tajima's D p-value & 0.38 & 1 & 1 & 1 & 1 & 1 & 1 & 0.38 & 0.88 & 1 & 0.3 & 1 & 1 & 1 & 0.78 & 0.38 & 1 & 1 & 1 & 1 & 1 \\
\hline \multicolumn{22}{|l|}{ Fu's FS test } \\
\hline Real no. of alleles & 3 & 1 & 2 & 2 & 2 & 2 & 1 & 2 & 2 & 1 & 2 & 1 & 2 & 2 & 3 & 3 & 1 & 1 & 3 & 1 & 1 \\
\hline Orig. no. of alleles & 3 & 1 & 2 & 2 & 2 & 2 & 1 & 2 & 2 & 1 & 2 & 1 & 2 & 2 & 3 & 3 & 1 & 1 & 3 & 1 & 1 \\
\hline Theta_pi & 0.5 & 0 & 0 & 0 & 1 & 2 & 0 & 0.5 & 0.53 & 0 & 0.4 & 0 & 0 & 1 & 4 & 0.5 & 0 & 0 & 0 & 0 & 0 \\
\hline Exp. no. of alleles & 1.68 & 0 & 2 & 2 & 1.5 & $1.6^{\circ}$ & 0 & 1.68 & 1.92 & 0 & 1.66 & 0 & 2 & 1.5 & 2.47 & 1.68 & 0 & 0 & 3 & 0 & 0 \\
\hline FS & 0.17 & 0 & 0 & 0 & 0 & 0.6 & 0 & 0.17 & 0.63 & 0 & 0.09 & 0 & 0 & 0 & 0.13 & 0.17 & 0 & 0 & 0 & 0 & 0 \\
\hline FS p-value & 0.35 & N.A. & N.A. & N.A. & $0.24 \mathrm{I}$ & N.A. $\quad 0.3$ & 7 N.A. & 0.34 & 0.47 & N.A. & 0.3 & N.A. $\quad \mathrm{N}$ & N.A. & 0.25 & 0.27 & 0.34 & 4 N.A. & N.A. I & N.A. & N.A. & N.A. \\
\hline Neutrality tests & & GB 1 & GB 2 & GR 1 & I 1 & I 2 & PL 1 & PL 2 & RUM & & RUM 2 & RUM 5 & $15 \mathrm{R}$ & RUM 6 & RUS & & $\mathrm{S} 2$ & mean & & s.d. & \\
\hline \multicolumn{22}{|l|}{ Tajima's D test } \\
\hline Samplesize & & 4 & 3 & 6 & 8 & 4 & 4 & 8 & 4 & & 4 & 3 & & 2 & 3 & & 6 & 3.89 & & 1.59 & \\
\hline$S$ & & 41 & 13 & 8 & 8 & 4 & 42 & 107 & 16 & & 9 & 4 & & 6 & 3 & & 1 & 22.23 & & 29.32 & \\
\hline $\mathrm{Pi}$ & & 21.33 & 8.67 & 3.73 & 3.25 & 2 & 21.17 & 37.64 & 8.33 & & 4.5 & 2.67 & & 6 & 2 & & 0.33 & 12.94 & & 16.2 & \\
\hline Tajima's D & & -0.48 & 0 & 0.39 & $9 \quad 0.26$ & $6 \quad-0.78$ & -0.79 & -0.48 & -0.46 & & -0.83 & 0 & & 0 & 0 & & -0.93 & 43901.7 & & 259726 & 6.78 \\
\hline Tajima's D p-value & & 0.46 & 0.71 & 0.66 & $\begin{array}{ll}6 & 0.62\end{array}$ & 0.2 & 0.17 & 0.33 & 0.47 & & 0.12 & 0.83 & & 1 & 0.88 & & 0.26 & 0.64 & & 0.32 & \\
\hline \multicolumn{22}{|l|}{ Fu's FS test } \\
\hline Real no. of alleles & & 4 & 2 & 3 & 3 & 3 & 3 & 6 & 3 & & 2 & 3 & & 2 & 2 & & 2 & 2.89 & & 1.08 & \\
\hline Orig. no. of alleles & & 4 & 2 & 3 & 3 & 3 & 3 & 6 & 3 & & 2 & 3 & & 2 & 2 & & 2 & 2.89 & & 1.08 & \\
\hline Theta_pi & & 21.33 & 8.67 & 3.73 & 3.25 & 2 & 21.17 & 37.64 & 8.33 & & 4.5 & 2.67 & & 6 & 2 & & 0.33 & 12.94 & & 16.2 & \\
\hline Exp.no. of alleles & & 3.75 & 2.71 & 3.9 & 4.41 & 2.57 & 3.74 & 7.34 & 3.43 & & 3.11 & 2.30 & & 1.86 & 2.1 & & 1.63 & 2.93 & & 1.28 & \\
\hline $\mathrm{FS}$ & & 1.18 & 3.92 & 2.28 & 2.75 & 0.13 & 3.93 & 3.89 & 2.32 & & 3.78 & -0.34 & & 1.79 & 1.6 & & 0.00 & 1.75 & & 1.79 & \\
\hline FS p-value & & 0.45 & 0.92 & 0.87 & 0.91 & 0.33 & 0.92 & 0.94 & 0.81 & & 0.94 & 0.19 & & 0.51 & 0.7 & & 0.27 & N.A. & & N.A. & \\
\hline
\end{tabular}


Table A35: Results of the McDonald-Kreitman test for Achipteria coleoptrata. The differences between 58 populations are significant $(* 0.01<\mathrm{P}<0.05)$, between other 41 populations high significant $(* * 0.001<\mathrm{P}<0.01)$ and between 37 populations extremely high significant $(* * * \mathrm{P}<0.001)$. Number of fixed and polymorph synonymous and non-synonymous mutations are shown. Locations with less than two individuals were excluded.

\begin{tabular}{|c|c|c|c|c|c|c|c|c|c|c|c|c|c|}
\hline \multirow{2}{*}{$\begin{array}{c}\text { Population } \\
\text { A } 7\end{array}$} & \multirow[b]{2}{*}{ fixed } & \multicolumn{3}{|c|}{$\begin{array}{c}\text { A } 5 \\
\text { syn. nons. sign. }\end{array}$} & \multicolumn{3}{|c|}{$\begin{array}{c}\text { A } 7 \\
\text { syn. nons. sign. }\end{array}$} & \multicolumn{3}{|c|}{$\begin{array}{c}\text { A } 8 \\
\text { syn. nons. sign. }\end{array}$} & \multicolumn{3}{|c|}{$\begin{array}{r}\text { CH } 1 \\
\text { syn. nons. sign. }\end{array}$} \\
\hline & & 0 & 0 & $-n s$ & & & & & & & & & \\
\hline Roggendorf & poly & 107 & 4 & & & & & & & & & & \\
\hline A 8 & fixed & 0 & 0 & $-n s$ & 3 & 0 & $1 \mathrm{~ns}$ & & & & & & \\
\hline Imst & poly & 98 & 4 & & 102 & 3 & & & & & & & \\
\hline CH 1 & fixed & 0 & 0 & $-n s$ & 1 & 0 & $1 \mathrm{~ns}$ & 0 & 0 & $-n s$ & & & \\
\hline Basel & poly & 100 & 4 & & 32 & 1 & & 95 & 3 & & & & \\
\hline CH 3 & fixed & 0 & 0 & $-\mathrm{ns}$ & 1 & 0 & $1 \mathrm{~ns}$ & 3 & 0 & $1 \mathrm{~ns}$ & 0 & 0 & $-n s$ \\
\hline Rohrschach & poly & 98 & 5 & & 28 & 1 & & 92 & 4 & & 10 & 2 & \\
\hline D 1 & fixed & 50 & 0 & $0.1709 \mathrm{~ns}$ & 76 & 3 & $1 \mathrm{~ns}$ & 49 & 0 & $0.299 \mathrm{~ns}$ & 84 & 2 & $0.0622 \mathrm{~ns}$ \\
\hline Kranichstein & poly & 96 & 5 & & 25 & 1 & & 90 & 4 & & 9 & 2 & \\
\hline D 2 & fixed & 26 & 1 & $0.6899 \mathrm{~ns}$ & 46 & 1 & $0.1435 \mathrm{~ns}$ & 27 & 1 & $0.6916 \mathrm{~ns}$ & 58 & 1 & $0.0023^{* *}$ \\
\hline Goettingen & poly & 95 & 7 & & 25 & 3 & & 89 & 6 & & 8 & 4 & \\
\hline D 3 & fixed & 23 & 1 & $1 \mathrm{~ns}$ & 43 & 1 & $1 \mathrm{~ns}$ & 24 & 1 & $1 \mathrm{~ns}$ & 54 & 1 & $0.4649 \mathrm{~ns}$ \\
\hline Lake Constance & poly & 103 & 4 & & 34 & 0 & & 96 & 3 & & 19 & 1 & \\
\hline D 5 & fixed & 51 & 0 & $0.1007 \mathrm{~ns}$ & 76 & 3 & $0.6091 \mathrm{~ns}$ & 50 & 0 & $0.1655 \mathrm{~ns}$ & 85 & 2 & $0.0151^{*}$ \\
\hline Moerfelden & poly & 97 & 6 & & 27 & 2 & & 91 & 5 & & 10 & 3 & \\
\hline D 8 & fixed & 24 & 1 & $1 \mathrm{~ns}$ & 44 & 1 & $1 \mathrm{~ns}$ & 25 & 1 & $1 \mathrm{~ns}$ & 56 & 1 & $0.076 \mathrm{~ns}$ \\
\hline Cuxhaven & poly & 97 & 5 & & 27 & 1 & & 91 & 4 & & 10 & 2 & \\
\hline D 9 & fixed & 25 & 1 & $1 \mathrm{~ns}$ & 45 & 1 & $1 \mathrm{~ns}$ & 26 & 1 & $1 \mathrm{~ns}$ & 57 & 1 & $0.371 \mathrm{~ns}$ \\
\hline Bonn & poly & 97 & 4 & & 29 & 0 & & 91 & 3 & & 14 & 1 & \\
\hline D 10 & fixed & 22 & 1 & $1 \mathrm{~ns}$ & 43 & 1 & $1 \mathrm{~ns}$ & 23 & 1 & $1 \mathrm{~ns}$ & 54 & 1 & $0.1947 \mathrm{~ns}$ \\
\hline Cologne & poly & 104 & 5 & & 33 & 1 & & 98 & 4 & & 20 & 2 & \\
\hline D 11 & fixed & 28 & 0 & $-n s$ & 43 & 0 & $-n s$ & 31 & 0 & $0.5712 \mathrm{~ns}$ & 56 & 0 & $0.1385 \mathrm{~ns}$ \\
\hline Wittmoor & poly & 95 & 4 & & 25 & 0 & & 89 & 3 & & 8 & 1 & \\
\hline D 12 & fixed & 0 & 0 & $-\mathrm{ns}$ & 1 & 0 & $1 \mathrm{~ns}$ & 1 & 0 & $1 \mathrm{~ns}$ & 1 & 0 & $1 \mathrm{~ns}$ \\
\hline Sonthofen & poly & 118 & 5 & & 58 & 1 & & 114 & 4 & & 48 & 2 & \\
\hline D 14 & fixed & 26 & 1 & $1 \mathrm{~ns}$ & 33 & 1 & $1 \mathrm{~ns}$ & 29 & 1 & $1 \mathrm{~ns}$ & 41 & 1 & $0.091 \mathrm{~ns}$ \\
\hline Steineberg & poly & 95 & 5 & & 25 & 1 & & 89 & 4 & & 8 & 2 & \\
\hline D 15 & fixed & 6 & 0 & $1 \mathrm{~ns}$ & 20 & 1 & $0.69 \mathrm{~ns}$ & 6 & 0 & $1 \mathrm{~ns}$ & 27 & 0 & $0.0683 \mathrm{~ns}$ \\
\hline Langenwang & poly & 140 & 14 & & 96 & 10 & & 136 & 13 & & 82 & 11 & \\
\hline DK 3 & fixed & 24 & 1 & $1 \mathrm{~ns}$ & 46 & 1 & $0.5626 \mathrm{~ns}$ & 25 & 1 & $1 \mathrm{~ns}$ & 56 & 1 & $0.0408^{*}$ \\
\hline Arhus & poly & 99 & 6 & & 30 & 2 & & 92 & 5 & & 15 & 3 & \\
\hline EST 1 & fixed & 22 & 0 & $0.6157 \mathrm{~ns}$ & 37 & 0 & $-n s$ & 24 & 0 & $1 \mathrm{~ns}$ & 50 & 0 & $0.375 \mathrm{~ns}$ \\
\hline Tallin & poly & 113 & 4 & & 46 & 0 & & 107 & 3 & & 29 & 1 & \\
\hline F 1 & fixed & 20 & 1 & $1 \mathrm{~ns}$ & 42 & 1 & $1 \mathrm{~ns}$ & 21 & 1 & $1 \mathrm{~ns}$ & 54 & 1 & $1 \mathrm{~ns}$ \\
\hline Mont Blanc & poly & 115 & 4 & & 42 & 0 & & 109 & 3 & & 28 & 1 & \\
\hline F 2 & fixed & 18 & 1 & $1 \mathrm{~ns}$ & 40 & 1 & $1 \mathrm{~ns}$ & 19 & 1 & $1 \mathrm{~ns}$ & 46 & 1 & $0.3559 \mathrm{~ns}$ \\
\hline Loire & poly & 118 & 6 & & 49 & 2 & & 112 & 5 & & 42 & 3 & \\
\hline F 4 & fixed & 28 & 1 & $1 \mathrm{~ns}$ & 46 & 1 & $1 \mathrm{~ns}$ & 29 & 1 & $1 \mathrm{~ns}$ & 58 & 1 & $0.2707 \mathrm{~ns}$ \\
\hline Haute Loire & poly & 95 & 4 & & 25 & 0 & & 89 & 3 & & 9 & 1 & \\
\hline FIN 1 & fixed & 17 & 0 & $1 \mathrm{~ns}$ & 1 & 0 & $1 \mathrm{~ns}$ & 18 & 0 & $1 \mathrm{~ns}$ & 42 & 0 & $0.4615 \mathrm{~ns}$ \\
\hline Lahti & poly & 116 & 4 & & 50 & 0 & & 110 & 3 & & 35 & 1 & \\
\hline GB 1 & fixed & 18 & 0 & $0.596 \mathrm{~ns}$ & 34 & 0 & $0.2924 \mathrm{~ns}$ & 19 & 0 & $0.5932 \mathrm{~ns}$ & 43 & 0 & $0.0584 \mathrm{~ns}$ \\
\hline Ascot & poly & 118 & 8 & & 55 & 4 & & 113 & 7 & & 44 & 5 & \\
\hline GB 2 & fixed & 21 & 1 & $1 \mathrm{~ns}$ & 41 & 1 & $1 \mathrm{~ns}$ & 22 & 1 & $1 \mathrm{~ns}$ & 51 & 1 & $1 \mathrm{~ns}$ \\
\hline Braemar & poly & 102 & 4 & & 35 & 0 & & 95 & 3 & & 21 & 1 & \\
\hline GR 1 & fixed & 33 & 0 & $0.3402 \mathrm{~ns}$ & 64 & 0 & $0.3402 \mathrm{~ns}$ & 33 & 0 & $0.5717 \mathrm{~ns}$ & 70 & 0 & $0.04^{*}$ \\
\hline Thessaloniki & poly & 102 & 5 & & 32 & 1 & & 96 & 4 & & 16 & 2 & \\
\hline I 1 & fixed & 37 & 0 & $0.5725 \mathrm{~ns}$ & 72 & 1 & $1 \mathrm{~ns}$ & 38 & 0 & $0.5611 \mathrm{~ns}$ & 81 & 0 & $0.1735 \mathrm{~ns}$ \\
\hline Grosseto & poly & 103 & 4 & & 32 & 0 & & 97 & 3 & & 16 & 1 & \\
\hline I 2 & fixed & 38 & 0 & $0.1895 \mathrm{~ns}$ & 70 & 2 & $0.141 \mathrm{~ns}$ & 37 & 0 & $0.1856 \mathrm{~ns}$ & 80 & 1 & $0.0011^{* *}$ \\
\hline Parma & poly & 96 & 7 & & 26 & 3 & & 90 & 6 & & 9 & 4 & \\
\hline PL 1 & fixed & 16 & 0 & $0.5978 \mathrm{~ns}$ & 32 & 0 & $0.1639 \mathrm{~ns}$ & 16 & 0 & $0.5976 \mathrm{~ns}$ & 43 & 0 & $0.0296^{*}$ \\
\hline Krakow & poly & 123 & 9 & & 58 & 5 & & 119 & 8 & & 45 & 6 & \\
\hline PL 2 & fixed & 7 & 0 & $1 \mathrm{~ns}$ & 19 & 0 & $1 \mathrm{~ns}$ & 7 & 0 & $1 \mathrm{~ns}$ & 25 & 0 & $0.6047 \mathrm{~ns}$ \\
\hline Warsaw & poly & 173 & 7 & & 125 & 4 & & 168 & 3 & & 118 & 4 & \\
\hline
\end{tabular}


Table A35 continue:

\begin{tabular}{|c|c|c|c|c|c|c|c|c|c|c|c|c|c|}
\hline \multirow{2}{*}{$\begin{array}{c}\text { Population } \\
\text { RUM } 1\end{array}$} & & \multicolumn{3}{|c|}{$\begin{array}{c}\text { A 5 } \\
\text { syn. nons. sign. }\end{array}$} & \multicolumn{3}{|c|}{$\begin{array}{c}\text { A } 7 \\
\text { syn. nons. sign. }\end{array}$} & \multicolumn{3}{|c|}{$\begin{array}{c}\text { A } 8 \\
\text { syn. nons. sign. }\end{array}$} & \multicolumn{3}{|c|}{$\begin{array}{r}\text { CH } 1 \\
\text { syn. nons. sign. }\end{array}$} \\
\hline & fixed & 24 & 0 & $0.2144 \mathrm{~ns}$ & 32 & 0 & $0.0614 \mathrm{~ns}$ & 27 & 0 & $0.2073 \mathrm{~ns}$ & 41 & 0 & $0.0016^{* *}$ \\
\hline Sibiu 1 & poly & 102 & 9 & & 35 & 5 & & 98 & 8 & & 18 & 6 & \\
\hline RUM 2 & fixed & 25 & 0 & $0.3562 \mathrm{~ns}$ & 33 & 0 & $0.4923 \mathrm{~ns}$ & 28 & 0 & $0.3558 \mathrm{~ns}$ & 42 & 0 & $0.0209^{*}$ \\
\hline Sibiu 2 & poly & 100 & 6 & & 31 & 2 & & 96 & 5 & & 14 & 3 & \\
\hline RUM 5 & fixed & 42 & 1 & $0.447 \mathrm{~ns}$ & 67 & 2 & $0.5794 \mathrm{~ns}$ & 42 & 1 & $0.6654 \mathrm{~ns}$ & 76 & 2 & $0.0197^{*}$ \\
\hline Busteni & poly & 96 & 6 & & 27 & 2 & & 90 & 5 & & 10 & 3 & \\
\hline RUM 6 & fixed & 41 & 1 & $1 \mathrm{~ns}$ & 65 & 2 & $0.563 \mathrm{~ns}$ & 41 & 1 & $1 \mathrm{~ns}$ & 76 & 2 & $0.3942 \mathrm{~ns}$ \\
\hline Sinaia & poly & 100 & 4 & & 31 & 0 & & 95 & 3 & & 13 & 1 & \\
\hline RUS 2 & fixed & 40 & 1 & $0.6732 \mathrm{~ns}$ & 75 & 3 & $0.6059 \mathrm{~ns}$ & 39 & 1 & $0.6694 \mathrm{~ns}$ & 84 & 2 & $0.0125^{*}$ \\
\hline Novosibirsk & poly & 96 & 6 & & 26 & 2 & & 90 & 5 & & 9 & 3 & \\
\hline S 2 & fixed & 26 & 1 & $1 \mathrm{~ns}$ & 46 & 1 & $1 \mathrm{~ns}$ & 27 & 1 & $1 \mathrm{~ns}$ & 58 & 1 & $0.053 \mathrm{~ns}$ \\
\hline Stroemstad & poly & 95 & 5 & & 25 & 1 & & 89 & 4 & & 8 & 2 & \\
\hline \multicolumn{2}{|l|}{ Population } & \multicolumn{3}{|c|}{$\begin{array}{r}\text { CH } 3 \\
\text { syn. nons. sign. }\end{array}$} & \multicolumn{3}{|c|}{$\begin{array}{c}\text { D } 1 \\
\text { ns. sign. }\end{array}$} & \multicolumn{3}{|c|}{$\begin{array}{c}\text { D } 2 \\
\text { syn. nons. sign. }\end{array}$} & \multicolumn{3}{|c|}{$\begin{array}{c}\text { D } 3 \\
\text { syn. nons. sign. }\end{array}$} \\
\hline D 1 & fixed & 86 & 3 & $0.0528 \mathrm{~ns}$ & & & & & & & & & \\
\hline Kranichstein & poly & 6 & 2 & & & & & & & & & & \\
\hline D 2 & fixed & 58 & 1 & $0.0007^{* * *}$ & 81 & 4 & $0.0001^{* * *} *$ & & & & & & \\
\hline Goettingen & poly & 5 & 4 & & 1 & 4 & & & & & & & \\
\hline D 3 & fixed & 54 & 1 & $0.4190 \mathrm{~ns}$ & 78 & 4 & $1 \mathrm{~ns}$ & 1 & 0 & $1 \mathrm{~ns}$ & & & \\
\hline Lake Constance & poly & 16 & 1 & & 12 & 1 & & 12 & 3 & & & & \\
\hline D 5 & fixed & 87 & 3 & $0.0126^{*}$ & 0 & 0 & $-n s$ & 82 & 4 & $0.0001^{* * *} *$ & 78 & 4 & $0.2526 \mathrm{~ns}$ \\
\hline Moerfelden & poly & 7 & 3 & & 3 & 3 & & 2 & 5 & & 14 & 2 & \\
\hline D 8 & fixed & 56 & 1 & $0.0467^{*}$ & 81 & 4 & $0.0341^{*}$ & 0 & 0 & $-n s$ & 1 & 0 & $1 \mathrm{~ns}$ \\
\hline Cuxhaven & poly & 7 & 2 & & 3 & 2 & & 2 & 4 & & 13 & 1 & \\
\hline D 9 & fixed & 57 & 1 & $0.3155 \mathrm{~ns}$ & 80 & 4 & $0.3722 \mathrm{~ns}$ & 0 & 0 & $-\mathrm{ns}$ & 0 & 0 & $-n s$ \\
\hline Bonn & poly & 11 & 2 & & 7 & 1 & & 6 & 3 & & 18 & 0 & \\
\hline D 10 & fixed & 54 & 1 & $0.16 \mathrm{~ns}$ & 77 & 4 & $0.2354 \mathrm{~ns}$ & 0 & 0 & $-n s$ & 0 & 0 & $-n s$ \\
\hline Cologne & poly & 17 & 2 & & 13 & 2 & & 12 & 3 & & 23 & 1 & \\
\hline D 11 & fixed & 55 & 0 & $0.0984 \mathrm{~ns}$ & 80 & 3 & $0.0884 \mathrm{~ns}$ & 30 & 1 & $0.0007^{* * *}$ & 26 & 1 & $1 \mathrm{~ns}$ \\
\hline Wittmoor & poly & 5 & 1 & & 1 & 1 & & 0 & 3 & & 12 & 0 & \\
\hline D 12 & fixed & 1 & 0 & $1 \mathrm{~ns}$ & 67 & 3 & $1 \mathrm{~ns}$ & 36 & 0 & $0.125 \mathrm{~ns}$ & 36 & 0 & $1 \mathrm{~ns}$ \\
\hline Sonthofen & poly & 45 & 2 & & 41 & 2 & & 41 & 4 & & 48 & 1 & \\
\hline D 14 & fixed & 41 & 1 & $0.0498 *$ & 90 & 4 & $0.0094^{* *}$ & 60 & 2 & $0.0000^{* * * *}$ & 58 & 2 & $0.4498 \mathrm{~ns}$ \\
\hline Steineberg & poly & 5 & 2 & & 1 & 2 & & 0 & 4 & & 12 & 1 & \\
\hline D 15 & fixed & 28 & 1 & $0.2896 \mathrm{~ns}$ & 54 & 0 & $0.0068 * *$ & 39 & 2 & $0.1423 \mathrm{~ns}$ & 38 & 2 & $0.3493 \mathrm{~ns}$ \\
\hline Langenwang & poly & 79 & 10 & & 76 & 11 & & 75 & 13 & & 84 & 10 & \\
\hline DK 3 & fixed & 57 & 1 & $0.0946 \mathrm{~ns}$ & 77 & 4 & $0.0347^{*}$ & 0 & 0 & $-n s$ & 0 & 0 & $-n s$ \\
\hline Arhus & poly & 12 & 2 & & 8 & 3 & & 7 & 5 & & 19 & 2 & \\
\hline EST 1 & fixed & 49 & 0 & $0.3636 \mathrm{~ns}$ & 78 & 3 & $1 \mathrm{~ns}$ & 20 & 1 & $0.6174 \mathrm{~ns}$ & 16 & 1 & $0.34 \mathrm{~ns}$ \\
\hline Tallin & poly & 27 & 1 & & 24 & 1 & & 23 & 3 & & 33 & 0 & \\
\hline F 1 & fixed & 54 & 1 & $1 \mathrm{~ns}$ & 77 & 4 & $1 \mathrm{~ns}$ & 5 & 0 & $1 \mathrm{~ns}$ & 4 & 0 & $-n s$ \\
\hline Mont Blanc & poly & 25 & 1 & & 21 & 1 & & 20 & 3 & & 31 & 0 & \\
\hline F 2 & fixed & 46 & 1 & $0.3399 \mathrm{~ns}$ & 71 & 5 & $1 \mathrm{~ns}$ & 0 & 0 & $-n s$ & 0 & 0 & $-n s$ \\
\hline Loire & poly & 39 & 3 & & 35 & 3 & & 34 & 5 & & 44 & 2 & \\
\hline F 4 & fixed & 58 & 1 & $0.2023 \mathrm{~ns}$ & 78 & 4 & $0.1682 \mathrm{~ns}$ & 3 & 0 & $0.1429 \mathrm{~ns}$ & 3 & 0 & $-n s$ \\
\hline Haute Loire & poly & 6 & 1 & & 2 & 1 & & 1 & 3 & & 13 & 0 & \\
\hline FIN 1 & fixed & 41 & 0 & $0.4605 \mathrm{~ns}$ & 68 & 3 & $1 \mathrm{~ns}$ & 15 & 1 & $1 \mathrm{~ns}$ & 12 & 1 & $0.2453 \mathrm{~ns}$ \\
\hline Lahti & poly & 34 & 1 & & 31 & 1 & & 30 & 3 & & 40 & 0 & \\
\hline GB 1 & fixed & 42 & 0 & $0.0575 \mathrm{~ns}$ & 69 & 3 & $0.2558 \mathrm{~ns}$ & 0 & 0 & $-n s$ & 0 & 0 & $-n s$ \\
\hline Ascot & poly & 42 & 5 & & 39 & 5 & & 38 & 7 & & 46 & 4 & \\
\hline GB 2 & fixed & 52 & 1 & $0.4609 \mathrm{~ns}$ & 76 & 4 & $1 \mathrm{~ns}$ & 0 & 0 & $-n s$ & 0 & 0 & $-n s$ \\
\hline Braemar & poly & 18 & 1 & & 14 & 1 & & 13 & 3 & & 22 & 0 & \\
\hline GR 1 & fixed & 72 & 0 & $0.0281^{*}$ & 76 & 3 & $0.1111 \mathrm{~ns}$ & 68 & 1 & $0.0014^{* *}$ & 62 & 1 & $0.4398 \mathrm{~ns}$ \\
\hline Thessaloniki & poly & 13 & 2 & & 9 & 2 & & 8 & 4 & & 20 & 1 & \\
\hline I 1 & fixed & 83 & 1 & $0.2666 \mathrm{~ns}$ & 82 & 2 & $0.2892 \mathrm{~ns}$ & 73 & 2 & $0.0139 *$ & 69 & 2 & $1 \mathrm{~ns}$ \\
\hline Grosseto & poly & 13 & 1 & & 9 & 1 & & 8 & 3 & & 20 & 0 & \\
\hline
\end{tabular}


Table A35 continue:

\begin{tabular}{|c|c|c|c|c|c|c|c|c|c|c|c|c|c|}
\hline \multirow{2}{*}{$\begin{array}{c}\text { Population } \\
\text { I } 2\end{array}$} & \multicolumn{4}{|c|}{$\begin{array}{r}\text { CH } 3 \\
\text { syn. nons. sign. }\end{array}$} & \multicolumn{3}{|c|}{$\begin{array}{r}\text { D } 1 \\
\text { syn. nons. sign. }\end{array}$} & \multicolumn{3}{|c|}{$\begin{array}{r}\text { D } 2 \\
\text { syn. nons. sign. }\end{array}$} & \multicolumn{3}{|c|}{$\begin{array}{r}\text { D } 3 \\
\text { syn. nons. sign. }\end{array}$} \\
\hline & fixed & 80 & 2 & $0.001^{* *}$ & 79 & 1 & $0.0000^{*} * *$ & 74 & 3 & $0.0000^{* * * *}$ & 68 & 3 & $0.073 \mathrm{~ns}$ \\
\hline Parma & poly & 6 & 4 & & 2 & 4 & & 1 & 6 & & 13 & 3 & \\
\hline PL 1 & fixed & 43 & 0 & $0.0276^{*}$ & 72 & 3 & $0.0747 \mathrm{~ns}$ & 7 & 0 & $0.3506 \mathrm{~ns}$ & 6 & 0 & $1 \mathrm{~ns}$ \\
\hline Krakow & poly & 42 & 6 & & 38 & 6 & & 37 & 8 & & 45 & 5 & \\
\hline PL 2 & fixed & 26 & 0 & $0.5864 \mathrm{~ns}$ & 1 & 0 & $1 \mathrm{~ns}$ & 8 & 1 & $0.4487 \mathrm{~ns}$ & 6 & 1 & $0.2452 \mathrm{~ns}$ \\
\hline Warsaw & poly & 116 & 5 & & 113 & 5 & & 113 & 7 & & 119 & 4 & \\
\hline RUM 1 & fixed & 41 & 0 & $0.0009 * * *$ & 84 & 4 & $0.0015^{* *} *$ & 50 & 0 & $0.0000^{* * * *}$ & 48 & 0 & $0.0053^{* *}$ \\
\hline Sibiu 1 & poly & 15 & 6 & & 12 & 6 & & 11 & 8 & & 23 & 5 & \\
\hline RUM 2 & fixed & 42 & 0 & $0.0131 *$ & 88 & 3 & $0.0159^{*}$ & 51 & 0 & $0.0001^{* * *} *$ & 49 & 0 & $0.087 \mathrm{~ns}$ \\
\hline Sibiu 2 & poly & 11 & 3 & & 8 & 3 & & 7 & 5 & & 19 & 2 & \\
\hline RUM 5 & fixed & 76 & 2 & $0.0086^{* *}$ & 73 & 1 & $0.0001^{* * *} *$ & 75 & 3 & $0.0000^{* * *} *$ & 72 & 3 & $0.2107 \mathrm{~ns}$ \\
\hline Busteni & poly & 7 & 3 & & 3 & 3 & & 2 & 5 & & 14 & 2 & \\
\hline RUM 6 & fixed & 75 & 2 & $0.3559 \mathrm{~ns}$ & 70 & 1 & $0.1934 \mathrm{~ns}$ & 72 & 3 & $0.0148^{*}$ & 69 & 3 & $1 \mathrm{~ns}$ \\
\hline Sinaia & poly & 11 & 1 & & 7 & 1 & & 6 & 3 & & 18 & 0 & \\
\hline RUS 2 & fixed & 84 & 3 & $0.0101^{*}$ & 93 & 2 & $0.0001^{* * * *}$ & 81 & 4 & $0.0000^{* * * *}$ & 79 & 4 & $0.2277 \mathrm{~ns}$ \\
\hline Novosibirsk & poly & 6 & 3 & & 2 & 3 & & 1 & 5 & & 13 & 2 & \\
\hline S 2 & fixed & 58 & 1 & $0.0278^{*}$ & 81 & 3 & $0.0078^{* *}$ & 0 & 0 & $-\mathrm{ns}$ & 1 & 0 & $1 \mathrm{~ns}$ \\
\hline Stroemstad & poly & 5 & 2 & & 1 & 2 & & 0 & 4 & & 12 & 1 & \\
\hline \multirow[t]{2}{*}{ Population } & & \multirow{2}{*}{\multicolumn{3}{|c|}{$\begin{array}{r}\text { D 5 } \\
\text { syn. nons. sign. }\end{array}$}} & \multirow{2}{*}{\multicolumn{3}{|c|}{$\begin{array}{c}\text { D 8 } \\
\text { syn. nons. sign. }\end{array}$}} & \multirow{2}{*}{\multicolumn{3}{|c|}{$\begin{array}{c}\text { D 9 } \\
\text { syn. nons. sign. }\end{array}$}} & \multirow{2}{*}{\multicolumn{3}{|c|}{$\begin{array}{r}\text { D } 10 \\
\text { syn. nons. sign. }\end{array}$}} \\
\hline & & & & & & & & & & & & & \\
\hline D 8 & fixed & 82 & 4 & $0.0082 \mathrm{~ns}$ & & & & & & & & & \\
\hline Cuxhaven & poly & 4 & 3 & & . & & & & & & & & \\
\hline D 9 & fixed & 81 & 4 & $0.1194 \mathrm{~ns}$ & 0 & 0 & $-\mathrm{ns}$ & & & & & & \\
\hline Bonn & poly & 8 & 2 & & 8 & 1 & & & & & & & \\
\hline D 10 & fixed & 78 & 4 & $0.0955 \mathrm{~ns}$ & 0 & 0 & $-n s$ & 0 & 0 & $-n s$ & & & \\
\hline Cologne & poly & 14 & 3 & & 14 & 2 & & 15 & 1 & & & & \\
\hline D 11 & fixed & 85 & 3 & $0.0137^{*}$ & 29 & 1 & $0.1761 \mathrm{~ns}$ & 27 & 1 & $1 \mathrm{~ns}$ & 25 & 1 & $1 \mathrm{~ns}$ \\
\hline Wittmoor & poly & 2 & 2 & & 2 & 1 & & 6 & 0 & & 12 & 1 & \\
\hline D 12 & fixed & 67 & 3 & $0.6802 \mathrm{~ns}$ & 34 & 0 & $0.5034 \mathrm{~ns}$ & 36 & 0 & $1 \mathrm{~ns}$ & 33 & 0 & $0.5215 \mathrm{~ns}$ \\
\hline Sonthofen & poly & 43 & 3 & & 43 & 1 & & 46 & 1 & & 51 & 2 & \\
\hline D 14 & fixed & 90 & 4 & $0.0021^{* *} *$ & 58 & 2 & $0.0171^{*}$ & 59 & 2 & $0.2819 \mathrm{~ns}$ & 57 & 2 & $0.1637 \mathrm{~ns}$ \\
\hline Steineberg & poly & 2 & 3 & & 2 & 2 & & 6 & 1 & & 12 & 2 & \\
\hline D 15 & fixed & 55 & 0 & $0.0035^{* *} *$ & 38 & 2 & $0.2257 \mathrm{~ns}$ & 38 & 2 & $0.3415 \mathrm{~ns}$ & 27 & 2 & $0.3474 \mathrm{~ns}$ \\
\hline Langenwang & poly & 7 & 12 & & 76 & 11 & & 80 & 10 & & 86 & 11 & \\
\hline DK 3 & fixed & 78 & 4 & $0.0113^{*}$ & 0 & 0 & $-\mathrm{ns}$ & 0 & 0 & $-\mathrm{ns}$ & 0 & 0 & $-\mathrm{ns}$ \\
\hline Arhus & poly & 9 & 4 & & 9 & 3 & & 12 & 2 & & 17 & 3 & \\
\hline EST 1 & fixed & 79 & 3 & $0.5958 \mathrm{~ns}$ & 19 & 1 & $1 \mathrm{~ns}$ & 18 & 1 & $0.413 \mathrm{~ns}$ & 15 & 1 & $1 \mathrm{~ns}$ \\
\hline Tallin & poly & 25 & 2 & & 25 & 1 & & 27 & 0 & & 33 & 1 & \\
\hline F 1 & fixed & 78 & 4 & $0.6161 \mathrm{~ns}$ & 5 & 0 & $1 \mathrm{~ns}$ & 3 & 0 & $-n s$ & 1 & 0 & $1 \mathrm{~ns}$ \\
\hline Mont Blanc & poly & 22 & 2 & & 21 & 1 & & 25 & 0 & & 28 & 1 & \\
\hline F 2 & fixed & 72 & 5 & $0.7164 \mathrm{~ns}$ & 0 & 0 & $-\mathrm{ns}$ & 0 & 0 & $-\mathrm{ns}$ & 0 & 0 & $-n s$ \\
\hline Loire & poly & 36 & 4 & & 35 & 3 & & 37 & 2 & & 36 & 3 & \\
\hline F 4 & fixed & 79 & 4 & $0.0356^{*}$ & 3 & 0 & $1 \mathrm{~ns}$ & 3 & 0 & $-\mathrm{ns}$ & 2 & 0 & $1 \mathrm{~ns}$ \\
\hline Haute Loire & poly & 3 & 2 & & 3 & 1 & & 6 & 0 & & 12 & 1 & \\
\hline FIN 1 & fixed & 69 & 3 & $1 \mathrm{~ns}$ & 14 & 1 & $1 \mathrm{~ns}$ & 14 & 1 & $0.3125 \mathrm{~ns}$ & 12 & 1 & $0.4412 \mathrm{~ns}$ \\
\hline Lahti & poly & 32 & 2 & & 32 & 1 & & 33 & 0 & & 38 & 1 & \\
\hline GB 1 & fixed & 70 & 3 & $0.1508 \mathrm{~ns}$ & 0 & 0 & $-\mathrm{ns}$ & 0 & 0 & $-\mathrm{ns}$ & 0 & 0 & $-\mathrm{ns}$ \\
\hline Ascot & poly & 40 & 6 & & 39 & 5 & & 38 & 4 & & 44 & 5 & \\
\hline GB 2 & fixed & 77 & 4 & $0.2779 \mathrm{~ns}$ & 0 & 0 & $-\mathrm{ns}$ & 0 & 0 & $-\mathrm{ns}$ & 0 & 0 & $-\mathrm{ns}$ \\
\hline Braemar & poly & 15 & 2 & & 14 & 1 & & 18 & 0 & & 22 & 1 & \\
\hline GR 1 & fixed & 77 & 3 & $0.03392^{*}$ & 67 & 1 & $0.0573 \mathrm{~ns}$ & 65 & 1 & $0.338 \mathrm{~ns}$ & 62 & 1 & $0.1629 \mathrm{~ns}$ \\
\hline Thessaloniki & poly & 10 & 3 & & 10 & 2 & & 14 & 1 & & 20 & 2 & \\
\hline I 1 & fixed & 83 & 2 & $0.0735 \mathrm{~ns}$ & 72 & 2 & $0.3437 \mathrm{~ns}$ & 70 & 2 & $1 \mathrm{~ns}$ & 68 & 2 & $1 \mathrm{~ns}$ \\
\hline Grosseto & poly & 10 & 2 & & 10 & 1 & & 14 & 0 & & 20 & 1 & \\
\hline I 2 & fixed & 80 & 1 & $0.0000^{* * * *}$ & 73 & 3 & $0.0006^{* * * *}$ & 71 & 3 & $0.0206^{*}$ & 69 & 3 & $0.023^{*}$ \\
\hline Parma & poly & 3 & 5 & & 3 & 4 & & 14 & 1 & & 13 & 4 & \\
\hline
\end{tabular}


Table A35 continue:

\begin{tabular}{|c|c|c|c|c|c|c|c|c|c|c|c|c|c|}
\hline \multirow{2}{*}{$\begin{array}{c}\text { Population } \\
\text { PL } 1\end{array}$} & \multicolumn{4}{|c|}{$\begin{array}{c}\text { D } 5 \\
\text { syn. nons. sign. }\end{array}$} & \multicolumn{3}{|c|}{$\begin{array}{c}\text { D 8 } \\
\text { syn. nons. sign. }\end{array}$} & \multicolumn{3}{|c|}{$\begin{array}{c}\text { D 9 } \\
\text { syn. nons. sign. }\end{array}$} & \multicolumn{3}{|c|}{$\begin{array}{r}\text { D } 10 \\
\text { syn. nons. sign. }\end{array}$} \\
\hline & fixed & 73 & 3 & $0.0403^{*}$ & 7 & 0 & $0.5779 \mathrm{~ns}$ & 5 & 0 & $1 \mathrm{~ns}$ & 4 & 0 & $1 \mathrm{~ns}$ \\
\hline Krakow & poly & 39 & 7 & & 38 & 6 & & 41 & 5 & & 42 & 6 & \\
\hline PL 2 & fixed & 1 & 0 & $1 \mathrm{~ns}$ & 7 & 1 & $0.3265 \mathrm{~ns}$ & 8 & 1 & $0.3097 \mathrm{~ns}$ & 6 & 1 & $0.2894 \mathrm{~ns}$ \\
\hline Warsaw & poly & 115 & 6 & & 115 & 5 & & 115 & 4 & & 117 & 5 & \\
\hline RUM 1 & fixed & 84 & 4 & $0.0006^{* * *}$ & 48 & 0 & $0.0003^{* * *}$ & 50 & 0 & $0.0019^{* *}$ & 47 & 0 & $0.0022^{* *}$ \\
\hline Sibiu 1 & poly & 13 & 7 & & 13 & 6 & & 17 & 5 & & 23 & 6 & \\
\hline RUM 2 & fixed & 88 & 3 & $0.0043^{* *}$ & 49 & 0 & $0.0061^{* *}$ & 51 & 0 & $0.049^{*}$ & 48 & 0 & $0.0281^{*}$ \\
\hline Sibiu 2 & poly & 9 & 4 & & 9 & 3 & & 13 & 2 & & 19 & 3 & \\
\hline RUM 5 & fixed & 74 & 1 & $0.0002^{* * *}$ & 75 & 3 & $0.0063^{* *}$ & 73 & 3 & $0.1011 \mathrm{~ns}$ & 67 & 3 & $0.086 \mathrm{~ns}$ \\
\hline Busteni & poly & 4 & 4 & & 4 & 3 & & 8 & 2 & & 14 & 3 & \\
\hline RUM 6 & fixed & 71 & 1 & $0.0379^{*}$ & 72 & 3 & $0.3701 \mathrm{~ns}$ & 70 & 3 & $1 \mathrm{~ns}$ & 64 & 3 & $1 \mathrm{~ns}$ \\
\hline Sinaia & poly & 8 & 2 & & 8 & 1 & & 12 & 0 & & 18 & 1 & \\
\hline RUS 2 & fixed & 94 & 0 & $0.0000^{* * *}$ & 81 & 4 & $0.0052 * *$ & 79 & 4 & $0.1042 \mathrm{~ns}$ & 78 & 4 & $0.0836 \mathrm{~ns}$ \\
\hline Novosibirsk & poly & 3 & 4 & & 3 & 3 & & 7 & 2 & & 13 & 3 & \\
\hline $\mathrm{S} 2$ & fixed & 82 & 3 & $0.0016^{* *}$ & 0 & 0 & $-n s$ & 0 & 0 & $-n s$ & 0 & 0 & $-n s$ \\
\hline Stroemstad & poly & 2 & 3 & & 2 & 2 & & 6 & 1 & & 12 & 2 & \\
\hline Population & & \multicolumn{3}{|c|}{$\begin{array}{r}\text { D } 11 \\
\text { syn. nons. sign. }\end{array}$} & \multicolumn{3}{|c|}{$\begin{array}{r}\text { D } 12 \\
\text { ns. sign. }\end{array}$} & \multicolumn{3}{|c|}{$\begin{array}{r}\text { D } 14 \\
\text { syn. nons. sign. }\end{array}$} & \multicolumn{3}{|c|}{$\begin{array}{r}\text { D } 15 \\
\text { syn. nons. sign. }\end{array}$} \\
\hline D 12 & fixed & 37 & 0 & $1 \mathrm{~ns}$ & & & & & & & & & \\
\hline Sonthofen & poly & 41 & 1 & & & & & & & & & & \\
\hline D 14 & fixed & 59 & 1 & $0.0328 *$ & 15 & 1 & $1 \mathrm{~ns}$ & & & & & & \\
\hline Steineberg & poly & 0 & 1 & & 41 & 2 & & & & & & & \\
\hline D 15 & fixed & 34 & 1 & $0.1726 \mathrm{~ns}$ & 18 & 1 & $0.6975 \mathrm{~ns}$ & 38 & 2 & $0.335 \mathrm{~ns}$ & & & \\
\hline Langenwang & poly & 75 & 10 & & 104 & 11 & & 75 & 10 & & & & \\
\hline DK 3 & fixed & 29 & 1 & $0.1274 \mathrm{~ns}$ & 35 & 0 & $0.2674 \mathrm{~ns}$ & 58 & 2 & $0.0186^{*}$ & 38 & 2 & $0.3422 \mathrm{~ns}$ \\
\hline Arhus & poly & 7 & 2 & & 48 & 3 & & 7 & 3 & & 80 & 11 & \\
\hline EST 1 & fixed & 0 & 0 & $-n s$ & 31 & 0 & $1 \mathrm{~ns}$ & 51 & 1 & $1 \mathrm{~ns}$ & 29 & 1 & $0.4548 \mathrm{~ns}$ \\
\hline Tallin & poly & 23 & 0 & & 60 & 1 & & 23 & 1 & & 94 & 10 & \\
\hline F 1 & fixed & 26 & 1 & $1 \mathrm{~ns}$ & 33 & 0 & $1 \mathrm{~ns}$ & 55 & 2 & $1 \mathrm{~ns}$ & 38 & 2 & $0.5096 \mathrm{~ns}$ \\
\hline Mont Blanc & poly & 20 & 0 & & 58 & 1 & & 20 & 1 & & 92 & 10 & \\
\hline F 2 & fixed & 21 & 1 & $1 \mathrm{~ns}$ & 29 & 0 & $0.5527 \mathrm{~ns}$ & 49 & 2 & $0.646 \mathrm{~ns}$ & 32 & 2 & $0.5249 \mathrm{~ns}$ \\
\hline Loire & poly & 34 & 2 & & 66 & 3 & & 34 & 3 & & 103 & 12 & \\
\hline F 4 & fixed & 28 & 1 & $1 \mathrm{~ns}$ & 35 & 0 & $1 \mathrm{~ns}$ & 59 & 2 & $0.0937 \mathrm{~ns}$ & 39 & 2 & $0.3348 \mathrm{~ns}$ \\
\hline Haute Loire & poly & 1 & 0 & & 42 & 1 & & 1 & 1 & & 76 & 10 & \\
\hline FIN 1 & fixed & 6 & 0 & $-n s$ & 25 & 0 & $1 \mathrm{~ns}$ & 47 & 1 & $1 \mathrm{~ns}$ & 22 & 1 & $0.6898 \mathrm{~ns}$ \\
\hline Lahti & poly & 30 & 0 & & 66 & 1 & & 30 & 1 & & 103 & 10 & \\
\hline GB 1 & fixed & 2 & 0 & $1 \mathrm{~ns}$ & 24 & 0 & $0.5704 \mathrm{~ns}$ & 45 & 1 & $0.1881 \mathrm{~ns}$ & 23 & 1 & $0.4687 \mathrm{~ns}$ \\
\hline Ascot & poly & 38 & 4 & & 74 & 4 & & 38 & 4 & & 111 & 13 & \\
\hline GB 2 & fixed & 24 & 1 & $1 \mathrm{~ns}$ & 33 & 0 & $1 \mathrm{~ns}$ & 57 & 2 & $0.4773 \mathrm{~ns}$ & 34 & 2 & $0.509 \mathrm{~ns}$ \\
\hline Braemar & poly & 13 & 0 & & 51 & 1 & & 13 & 1 & & 84 & 10 & \\
\hline GR 1 & fixed & 66 & 0 & $0.12 \mathrm{~ns}$ & 57 & 0 & $0.216 \mathrm{~ns}$ & 79 & 1 & $0.0317^{*}$ & 43 & 1 & $0.1028 \mathrm{~ns}$ \\
\hline Thessaloniki & poly & 8 & 1 & & 48 & 2 & & 8 & 2 & & 82 & 11 & \\
\hline I 1 & fixed & 68 & 1 & $1 \mathrm{~ns}$ & 70 & 1 & $1 \mathrm{~ns}$ & 87 & 2 & $0.2533 \mathrm{~ns}$ & 46 & 0 & $0.0175^{*}$ \\
\hline Grosseto & poly & 8 & 0 & & 49 & 1 & & 8 & 1 & & 83 & 10 & \\
\hline I 2 & fixed & 70 & 2 & $0.0006^{* * *}$ & 66 & 2 & $0.2189 \mathrm{~ns}$ & 81 & 3 & $0.0001^{* * * *}$ & 48 & 0 & $0.0042^{* *} *$ \\
\hline Parma & poly & 1 & 3 & & 42 & 4 & & 1 & 4 & & 76 & 13 & \\
\hline PL 1 & fixed & 7 & 0 & $0.5891 \mathrm{~ns}$ & 26 & 0 & $0.3252 \mathrm{~ns}$ & 45 & 1 & $0.0992 \mathrm{~ns}$ & 25 & 1 & $0.3108 \mathrm{~ns}$ \\
\hline Krakow & poly & 37 & 5 & & 71 & 5 & & 37 & 5 & & 107 & 14 & \\
\hline PL 2 & fixed & 3 & 0 & $1 \mathrm{~ns}$ & 12 & 0 & $1 \mathrm{~ns}$ & 27 & 1 & $1 \mathrm{~ns}$ & 7 & 0 & $1 \mathrm{~ns}$ \\
\hline Warsaw & poly & 113 & 4 & & 139 & 5 & & 113 & 5 & & 170 & 12 & \\
\hline RUM 1 & fixed & 52 & 0 & $0.0004^{* * *}$ & 15 & 0 & $0.3462 \mathrm{~ns}$ & 20 & 1 & $0.0314^{*}$ & 33 & 1 & $0.0692 \mathrm{~ns}$ \\
\hline Sibiu 1 & poly & 11 & 5 & & 49 & 5 & & 11 & 6 & & 83 & 15 & \\
\hline RUM 2 & fixed & 53 & 0 & $0.019^{*}$ & 15 & 0 & $1 \mathrm{~ns}$ & 20 & 1 & $0.0868 \mathrm{~ns}$ & 35 & 1 & $0.1088 \mathrm{~ns}$ \\
\hline Sibiu 2 & poly & 7 & 2 & & 46 & 2 & & 7 & 3 & & 80 & 12 & \\
\hline RUM 5 & fixed & 69 & 2 & $0.0125^{*}$ & 61 & 2 & $0.6482 \mathrm{~ns}$ & 81 & 3 & $0.0017^{* *}$ & 51 & 1 & $0.0313^{*}$ \\
\hline Busteni & poly & 2 & 2 & & 43 & 3 & & 2 & 3 & & 76 & 12 & \\
\hline
\end{tabular}


Table A35 continue:

\begin{tabular}{|c|c|c|c|c|c|c|c|c|c|c|c|c|c|}
\hline \multirow{2}{*}{$\begin{array}{c}\text { Population } \\
\text { RUM } 6\end{array}$} & & \multicolumn{3}{|c|}{$\begin{array}{r}\text { D } 11 \\
\text { syn. nons. sign. }\end{array}$} & \multicolumn{3}{|c|}{$\begin{array}{r}\text { D } 12 \\
\text { lons. sign. }\end{array}$} & \multicolumn{3}{|c|}{$\begin{array}{r}\text { D } 14 \\
\text { syn. nons. sign. }\end{array}$} & \multicolumn{3}{|c|}{$\begin{array}{r}\text { D } 15 \\
\text { syn. nons. sign. }\end{array}$} \\
\hline & fixed & 67 & 2 & $1 \mathrm{~ns}$ & 60 & 2 & $1 \mathrm{~ns}$ & 80 & 3 & $0.2808 \mathrm{~ns}$ & 49 & 1 & $0.0968 \mathrm{~ns}$ \\
\hline Sinaia & poly & 6 & 0 & & 46 & 1 & & 6 & 1 & & 81 & 10 & \\
\hline RUS 2 & fixed & 81 & 3 & $0.0078^{* *}$ & 67 & 3 & $0.6774 \mathrm{~ns}$ & 94 & 4 & $0.0087^{* *}$ & 22 & 0 & $0.1159 \mathrm{~ns}$ \\
\hline Novosibirsk & poly & 1 & 2 & & 42 & 3 & & 1 & 2 & & 76 & 11 & \\
\hline S 2 & fixed & 30 & 1 & $0.0625 \mathrm{~ns}$ & 36 & 0 & $0.4976 \mathrm{~ns}$ & 50 & 2 & $0.003^{* *}$ & 39 & 2 & $0.3343 \mathrm{~ns}$ \\
\hline Stroemstad & poly & 0 & 1 & & 41 & 2 & & 0 & 2 & & 75 & 10 & \\
\hline Population & & \multicolumn{3}{|c|}{$\begin{array}{r}\text { DK 3 } \\
\text { syn. nons. sign. }\end{array}$} & \multicolumn{3}{|c|}{$\begin{array}{r}\text { EST 1 } \\
\text { syn. nons. sign. }\end{array}$} & \multicolumn{3}{|c|}{$\begin{array}{c}\text { F } 1 \\
\text { syn. nons. sign. }\end{array}$} & \multicolumn{3}{|c|}{$\begin{array}{c}\text { F } 2 \\
\text { syn. nons. sign. }\end{array}$} \\
\hline EST 1 & fixed & 19 & 1 & $1 \mathrm{~ns}$ & & & & & & & & & \\
\hline Tallin & poly & 28 & 2 & & & & & & & & & & \\
\hline F 1 & fixed & 2 & 0 & $1 \mathrm{~ns}$ & 19 & 1 & $0.3509 \mathrm{~ns}$ & & & & & & \\
\hline Mont Blanc & poly & 26 & 2 & & 37 & 0 & & & & & & & \\
\hline F 2 & fixed & 0 & 0 & $-n s$ & 15 & 1 & $1 \mathrm{~ns}$ & 0 & 0 & $-n s$ & & & \\
\hline Loire & poly & 37 & 4 & & 48 & 2 & & 39 & 2 & & & & \\
\hline F 4 & fixed & 3 & 0 & $1 \mathrm{~ns}$ & 18 & 1 & $0.4419 \mathrm{~ns}$ & 4 & 0 & $-n s$ & 1 & 0 & $1 \mathrm{~ns}$ \\
\hline Haute Loire & poly & 7 & 2 & & 24 & 0 & & 21 & 0 & & 34 & 2 & \\
\hline FIN 1 & fixed & 14 & 1 & $1 \mathrm{~ns}$ & 0 & 0 & $-n s$ & 15 & 1 & $0.254 \mathrm{~ns}$ & 9 & 1 & $0.3843 \mathrm{~ns}$ \\
\hline Lahti & poly & 35 & 2 & & 42 & 0 & & 47 & 0 & & 56 & 2 & \\
\hline GB 1 & fixed & 0 & 0 & $-\mathrm{ns}$ & 0 & 0 & $-\mathrm{ns}$ & 1 & 0 & $1 \mathrm{~ns}$ & 0 & 0 & $-\mathrm{ns}$ \\
\hline Ascot & poly & 42 & 6 & & 48 & 4 & & 54 & 4 & & 60 & 6 & \\
\hline GB 2 & fixed & 0 & 0 & $-n s$ & 15 & 1 & $0.3333 \mathrm{~ns}$ & 3 & 0 & $-n s$ & 0 & 0 & $-n s$ \\
\hline Braemar & poly & 15 & 2 & & 32 & 0 & & 31 & 0 & & 39 & 2 & \\
\hline GR 1 & fixed & 63 & 1 & $0.0316^{*}$ & 58 & 0 & $0.3483 \mathrm{~ns}$ & 63 & 1 & $1 \mathrm{~ns}$ & 57 & 1 & $0.31588 \mathrm{~ns}$ \\
\hline Thessaloniki & poly & 15 & 3 & & 30 & 1 & & 28 & 1 & & 42 & 3 & \\
\hline I 1 & fixed & 69 & 2 & $0.1667 \mathrm{~ns}$ & 61 & 1 & $1 \mathrm{~ns}$ & 69 & 2 & $1 \mathrm{~ns}$ & 64 & 3 & $1 \mathrm{~ns}$ \\
\hline Grosseto & poly & 15 & 2 & & 29 & 0 & & 26 & 0 & & 40 & 2 & \\
\hline I 2 & fixed & 69 & 3 & $0.0017^{* * *}$ & 61 & 2 & $0.157 \mathrm{~ns}$ & 67 & 3 & $0.3324 \mathrm{~ns}$ & 63 & 4 & $0.2897 \mathrm{~ns}$ \\
\hline Parma & poly & 5 & 8 & & 24 & 3 & & 21 & 3 & & 35 & 5 & \\
\hline PL 1 & fixed & 6 & 0 & $0.5889 \mathrm{~ns}$ & 0 & 0 & $-n s$ & 5 & 0 & $1 \mathrm{~ns}$ & 3 & 0 & $1 \mathrm{~ns}$ \\
\hline Krakow & poly & 43 & 7 & & 50 & 5 & & 52 & 5 & & 60 & 7 & \\
\hline PL 2 & fixed & 7 & 1 & $0.3683 \mathrm{~ns}$ & 0 & 0 & $-n s$ & 8 & 1 & $0.2899 \mathrm{~ns}$ & 6 & 1 & $0.3036 \mathrm{~ns}$ \\
\hline Warsaw & poly & 115 & 6 & & 117 & 4 & & 125 & 4 & & 129 & 6 & \\
\hline RUM 1 & fixed & 48 & 0 & $0.0003^{* * * *}$ & 45 & 0 & $0.0187^{*}$ & 47 & 0 & $0.013^{*}$ & 41 & 0 & $0.0309^{*}$ \\
\hline Sibiu 1 & poly & 18 & 7 & & 34 & 5 & & 31 & 5 & & 44 & 6 & \\
\hline RUM 2 & fixed & 49 & 0 & $0.004^{* *}$ & 46 & 0 & $0.1652 \mathrm{~ns}$ & 48 & 0 & $0.1388 \mathrm{~ns}$ & 42 & 0 & $0.1166 \mathrm{~ns}$ \\
\hline Sibiu 2 & poly & 14 & 4 & & 30 & 2 & & 27 & 2 & & 40 & 4 & \\
\hline RUM 5 & fixed & 73 & 3 & $0.0079 * *$ & 64 & 2 & $0.5768 \mathrm{~ns}$ & 70 & 3 & $0.5947 \mathrm{~ns}$ & 65 & 3 & $0.4184 \mathrm{~ns}$ \\
\hline Busteni & poly & 9 & 4 & & 25 & 2 & & 22 & 2 & & 35 & 4 & \\
\hline RUM 6 & fixed & 70 & 3 & $0.1998 \mathrm{~ns}$ & 62 & 2 & $1 \mathrm{~ns}$ & 67 & 3 & $0.5605 \mathrm{~ns}$ & 61 & 4 & $1 \mathrm{~ns}$ \\
\hline Sinaia & poly & 13 & 2 & & 26 & 0 & & 26 & 0 & & 39 & 2 & \\
\hline RUS 2 & fixed & 79 & 4 & $0.0081^{* *}$ & 76 & 3 & $0.5954 \mathrm{~ns}$ & 75 & 4 & $0.615 \mathrm{~ns}$ & 74 & 4 & $0.438 \mathrm{~ns}$ \\
\hline Novosibirsk & poly & 8 & 4 & & 24 & 2 & & 21 & 2 & & 35 & 4 & \\
\hline S 2 & fixed & 0 & 0 & $-n s$ & 20 & 1 & $1 \mathrm{~ns}$ & 5 & 0 & $1 \mathrm{~ns}$ & 0 & 0 & $-n s$ \\
\hline Stroemstad & poly & 7 & 3 & & 23 & 1 & & 20 & 1 & & 34 & 3 & \\
\hline FIN 1 & fixed & 14 & 1 & $0.3333 \mathrm{~ns}$ & & & & & & & & & \\
\hline Lahti & poly & 30 & 0 & & & & & & & & & & \\
\hline GB 1 & fixed & 1 & 0 & $1 \mathrm{~ns}$ & 1 & 0 & $1 \mathrm{~ns}$ & & & & & & \\
\hline Ascot & poly & 38 & 4 & & 51 & 4 & & & & & & & \\
\hline GB 2 & fixed & 3 & 0 & $-n s$ & 9 & 1 & $0.1961 \mathrm{~ns}$ & 0 & 0 & $-n s$ & & & \\
\hline Braemar & poly & 13 & 0 & & 41 & 0 & & 43 & 4 & & & & \\
\hline GR 1 & fixed & 68 & 1 & $0.2386 \mathrm{~ns}$ & 52 & 0 & $0.4286 \mathrm{~ns}$ & 52 & 0 & $0.0254^{*}$ & 61 & 1 & $0.4576 \mathrm{~ns}$ \\
\hline Thessaloniki & poly & 9 & 1 & & 38 & 1 & & 45 & 5 & & 21 & 1 & \\
\hline I 1 & fixed & 72 & 2 & $1 \mathrm{~ns}$ & 58 & 1 & $1 \mathrm{~ns}$ & 58 & 1 & $0.1712 \mathrm{~ns}$ & 68 & 2 & $1 \mathrm{~ns}$ \\
\hline Grosseto & poly & 9 & 0 & & 37 & 0 & & 44 & 4 & & 21 & 0 & \\
\hline I 2 & fixed & 75 & 3 & 0.0021 ** & 61 & 2 & $0.3354 \mathrm{~ns}$ & 58 & 2 & $0.0385^{*}$ & 65 & 3 & $0.0913 \mathrm{~ns}$ \\
\hline Parma & poly & 2 & 3 & & 30 & 3 & & 39 & 7 & & 14 & 3 & \\
\hline
\end{tabular}


Table A35 continue:

\begin{tabular}{|c|c|c|c|c|c|c|c|c|c|c|c|c|c|}
\hline \multirow{2}{*}{$\begin{array}{c}\text { Population } \\
\text { PL } 1\end{array}$} & \multicolumn{4}{|c|}{$\begin{array}{c}\text { F } 4 \\
\text { syn. nons. sign. }\end{array}$} & \multicolumn{3}{|c|}{$\begin{array}{r}\text { FIN } 1 \\
\text { syn. nons. sign. }\end{array}$} & \multicolumn{3}{|c|}{$\begin{array}{r}\text { GB 1 } \\
\text { syn. nons. sign. }\end{array}$} & \multicolumn{3}{|c|}{$\begin{array}{r}\text { GB 2 } \\
\text { syn. nons. sign. }\end{array}$} \\
\hline & fixed & 5 & 0 & $1 \mathrm{~ns}$ & 1 & 0 & $1 \mathrm{~ns}$ & 0 & 0 & $-n s$ & 6 & 0 & $1 \mathrm{~ns}$ \\
\hline Krakow & poly & 38 & 5 & & 56 & 5 & & 56 & 4 & & 44 & 5 & \\
\hline PL 2 & fixed & 7 & 1 & $0.2856 \mathrm{~ns}$ & 0 & 0 & $-n s$ & 0 & 0 & $-n s$ & 4 & 1 & $0.1833 \mathrm{~ns}$ \\
\hline Warsaw & poly & 113 & 4 & & 113 & 4 & & 126 & 8 & & 119 & 4 & \\
\hline RUM 1 & fixed & 51 & 1 & $0.0006^{* * *}$ & 41 & 0 & $0.0574 \mathrm{~ns}$ & 37 & 0 & $0.0205^{*}$ & 46 & 0 & $0.0069^{* *}$ \\
\hline Sibiu 1 & poly & 12 & 5 & & 41 & 5 & & 49 & 8 & & 24 & 5 & \\
\hline RUM 2 & fixed & 52 & 0 & $0.0238^{*}$ & 42 & 0 & $0.2287 \mathrm{~ns}$ & 38 & 0 & $0.0669 \mathrm{~ns}$ & 47 & 0 & $0.0985 \mathrm{~ns}$ \\
\hline Sibiu 2 & poly & 8 & 2 & & 37 & 2 & & 45 & 5 & & 20 & 2 & \\
\hline RUM 5 & fixed & 74 & 3 & $0.0279^{*}$ & 56 & 2 & $0.6245 \mathrm{~ns}$ & 59 & 2 & $0.0724 \mathrm{~ns}$ & 70 & 3 & $0.2372 \mathrm{~ns}$ \\
\hline Busteni & poly & 3 & 2 & & 32 & 2 & & 40 & 6 & & 15 & 2 & \\
\hline RUM 6 & fixed & 71 & 3 & $1 \mathrm{~ns}$ & 54 & 2 & $0.5214 \mathrm{~ns}$ & 57 & 2 & $0.4007 \mathrm{~ns}$ & 68 & 3 & $1 \mathrm{~ns}$ \\
\hline Sinaia & poly & 7 & 0 & & 35 & 0 & & 42 & 4 & & 18 & 0 & \\
\hline RUS 2 & fixed & 81 & 4 & $0.0216^{*}$ & 72 & 3 & $0.6402 \mathrm{~ns}$ & 69 & 3 & $0.2558 \mathrm{~ns}$ & 77 & 4 & $0.2567 \mathrm{~ns}$ \\
\hline Novosibirsk & poly & 2 & 2 & & 31 & 2 & & 39 & 5 & & 14 & 2 & \\
\hline S 2 & fixed & 3 & 0 & $0.4 \mathrm{~ns}$ & 15 & 1 & $1 \mathrm{~ns}$ & 0 & 0 & $-\mathrm{ns}$ & 0 & 0 & $-n s$ \\
\hline Stroemstad & poly & 1 & 1 & & 30 & 1 & & 38 & 5 & & 13 & 1 & \\
\hline Population & & \multicolumn{3}{|c|}{$\begin{array}{r}\text { GR 1 } \\
\text { syn. nons. sign. }\end{array}$} & \multicolumn{3}{|c|}{$\begin{array}{c}\text { I } 1 \\
\text { syn. nons. sign. }\end{array}$} & \multicolumn{3}{|c|}{$\begin{array}{c}\text { I } 2 \\
\text { syn. nons. sign. }\end{array}$} & \multicolumn{3}{|c|}{$\begin{array}{r}\text { PL } 1 \\
\text { ns. sign. }\end{array}$} \\
\hline I 1 & fixed & 59 & 1 & $0.3951 \mathrm{~ns}$ & & & & & & & & & \\
\hline Grosseto & poly & 16 & 1 & & & & & & & & & & \\
\hline I 2 & fixed & 64 & 2 & $0.0058^{* *}$ & 54 & 1 & $0.0164^{*}$ & & & & & & \\
\hline Parma & poly & 9 & 4 & & 9 & 3 & & & & & & & \\
\hline PL 1 & fixed & 55 & 0 & $0.0099 * *$ & 53 & 1 & $0.097 \mathrm{~ns}$ & 59 & 2 & $0.0182 *$ & & & \\
\hline Krakow & poly & 44 & 6 & & 43 & 5 & & 38 & 8 & & & & \\
\hline PL 2 & fixed & 29 & 0 & $0.5842 \mathrm{~ns}$ & 32 & 0 & $0.5807 \mathrm{~ns}$ & 34 & 0 & $0.2104 \mathrm{~ns}$ & 59 & 1 & $0.3951 \mathrm{~ns}$ \\
\hline Warsaw & poly & 120 & 5 & & 118 & 4 & & 113 & 7 & & 16 & 1 & \\
\hline RUM 1 & fixed & 76 & 0 & $0.0001^{* * *}$ & 85 & 2 & $0.005^{* *}$ & 82 & 3 & 0.0001 *** & 64 & 2 & $0.0058^{* *} *$ \\
\hline Sibiu 1 & poly & 19 & 6 & & 19 & 5 & & 12 & 8 & & 9 & 4 & \\
\hline RUM 2 & fixed & 78 & 0 & $0.0057^{* *}$ & 88 & 1 & $0.0663 \mathrm{~ns}$ & 85 & 2 & $0.0003 * * *$ & 55 & 0 & $0.0099 * *$ \\
\hline Sibiu 2 & poly & 15 & 3 & & 15 & 2 & & 8 & 5 & & 44 & 6 & \\
\hline RUM 5 & fixed & 80 & 2 & $0.0174^{*}$ & 70 & 3 & $0.1439 \mathrm{~ns}$ & 77 & 2 & $0.0000 * * *$ & 29 & 0 & $0.5842 \mathrm{~ns}$ \\
\hline Busteni & poly & 10 & 3 & & 10 & 2 & & 3 & 5 & & 120 & 5 & \\
\hline RUM 6 & fixed & 78 & 2 & $0.4064 \mathrm{~ns}$ & 69 & 3 & $1 \mathrm{~ns}$ & 75 & 2 & $0.00997 * *$ & 76 & 0 & $0.0001^{* * *}$ \\
\hline Sinaia & poly & 14 & 1 & & 14 & 0 & & 7 & 3 & & 19 & 6 & \\
\hline RUS 2 & fixed & 76 & 3 & $0.0285^{*}$ & 79 & 2 & $0.0687 \mathrm{~ns}$ & 88 & 1 & $0.0000^{*} * *$ & 78 & 0 & $0.0057^{* *}$ \\
\hline Novosibirsk & poly & 9 & 3 & & 9 & 2 & & 2 & 5 & & 15 & 3 & \\
\hline $\mathrm{S} 2$ & fixed & 68 & 1 & $0.0408^{*}$ & 73 & 2 & $0.2913 \mathrm{~ns}$ & 74 & 3 & $0.0001^{* * *}$ & 80 & 2 & $0.0174^{*}$ \\
\hline Stroemstad & poly & 8 & 2 & & 8 & 1 & & 1 & 4 & & 10 & 3 & \\
\hline Population & & \multicolumn{3}{|c|}{$\begin{array}{r}\text { PL 2 } \\
\text { syn. nons. sign. }\end{array}$} & \multicolumn{3}{|c|}{$\begin{array}{l}\text { RUM } 1 \\
\text { ns. sign. }\end{array}$} & \multicolumn{3}{|c|}{$\begin{array}{r}\text { RUM 2 } \\
\text { syn. nons. sign. }\end{array}$} & \multicolumn{3}{|c|}{ RUM 5} \\
\hline RUM 1 & fixed & 23 & 0 & $0.3567 \mathrm{~ns}$ & & & & & & & & & \\
\hline Sibiu 1 & poly & 121 & 9 & & & & & & & & & & \\
\hline RUM 2 & fixed & 24 & 0 & $0.5899 \mathrm{~ns}$ & 0 & 0 & $-\mathrm{ns}$ & & & & & & \\
\hline Sibiu 2 & poly & 119 & 6 & & 11 & 5 & & & & & & & \\
\hline RUM 5 & fixed & 28 & 4 & $1 \mathrm{~ns}$ & 82 & 2 & $0.0001^{* * * *}$ & 83 & 2 & $0.0025^{* *}$ & & & \\
\hline Busteni & poly & 114 & 6 & & 12 & 7 & & 8 & 4 & & & & \\
\hline RUM 6 & fixed & 25 & 0 & $0.6026 \mathrm{~ns}$ & 78 & 3 & $0.0105^{*}$ & 81 & 2 & $0.1098 \mathrm{~ns}$ & 1 & 0 & $1 \mathrm{~ns}$ \\
\hline Sinaia & poly & 116 & 4 & & 17 & 5 & & 13 & 2 & & 8 & 2 & \\
\hline RUS 2 & fixed & 51 & 1 & $0.4439 \mathrm{~ns}$ & 86 & 3 & $0.0002 * * *$ & 89 & 3 & $0.0031^{* *}$ & 78 & 1 & $0.0001^{* * *}$ \\
\hline Novosibirsk & poly & 114 & 6 & & 12 & 7 & & 8 & 4 & & 3 & 4 & \\
\hline S 2 & fixed & 8 & 1 & $0.314 \mathrm{~ns}$ & 50 & 0 & $0.0001 * * *$ & 51 & 0 & $0.0033^{* *}$ & 75 & 2 & $0.0011^{* *}$ \\
\hline Stroemstad & poly & 113 & 4 & & 11 & 6 & & 7 & 3 & & 2 & 3 & \\
\hline
\end{tabular}


Appendix

Table A35 continue:

\begin{tabular}{ccrccccc}
\hline Population & \multicolumn{5}{c}{ RUM 6 } & \multicolumn{2}{c}{ RUS 2 } \\
& \multicolumn{5}{c}{ syn. nons. sign. } & syn. nons. sign. \\
\hline RUS 2 & fixed & 79 & 1 & $0.0261^{*}$ & & & \\
Novosibirsk & poly & 7 & 2 & & & & \\
S 2 & fixed & 72 & 2 & 0.2402 ns & 81 & 1 & $0.0007^{* * * *}$ \\
Stroemstad & poly & 6 & 1 & & 1 & 3 & \\
\hline
\end{tabular}




\section{Appendix}

Table A36: Neutrality indices of Achipteria coleoptrata computed in the McDonald-Kreitman test with DnaSP v5 for 35 locations. Locations with less than two individuals were excluded.

\begin{tabular}{|c|c|c|c|c|c|c|c|c|c|c|c|c|c|c|c|c|c|c|c|c|c|c|c|c|c|c|c|c|c|c|c|c|c|c|c|}
\hline \multicolumn{2}{|c|}{ Population } & \multirow[t]{2}{*}{1} & \multirow[t]{2}{*}{2} & \multirow[t]{2}{*}{3} & \multirow[t]{2}{*}{4} & \multirow[t]{2}{*}{5} & \multirow[t]{2}{*}{6} & \multirow[t]{2}{*}{7} & \multirow[t]{2}{*}{8} & \multirow[t]{2}{*}{9} & \multirow[t]{2}{*}{10} & \multirow[t]{2}{*}{11} & \multirow[t]{2}{*}{12} & 13 & 14 & 15 & 16 & 17 & 18 & 19 & 20 & 21 & 22 & 23 & 24 & 25 & 26 & 27 & 28 & 29 & 30 & 31 & 32 & 33 & 34 \\
\hline 1 & A 5 & & & & & & & & & & & & & & & & & & & & & & & & & & & & & & & & & & \\
\hline 2 & A 7 & - & & & & & & & & & & & & & & & & & & & & & & & & & & & & & & & & & \\
\hline 3 & A 8 & - & - & & & & & & & & & & & & & & & & & & & & & & & & & & & & & & & & \\
\hline 4 & CH 1 & - & - & - & & & & & & & & & & & & & & & & & & & & & & & & & & & & & & & \\
\hline 5 & $\mathrm{CH} 3$ & - & - & - & - & & & & & & & & & & & & & & & & & & & & & & & & & & & & & & \\
\hline 6 & D 1 & - & 1 & - & 9.3 & 9.6 & & & & & & & & & & & & & & & & & & & & & & & & & & & & & \\
\hline 7 & D 2 & 1.9 & 5.5 & 1.8 & 29 & 46.4 & 81 & & & & & & & & & & & & & & & & & & & & & & & & & & & & \\
\hline 8 & D 3 & 0.9 & 0 & 0.8 & 2.8 & 3.4 & 1.6 & - & & & & & & & & & & & & & & & & & & & & & & & & & & & \\
\hline 9 & D 5 & - & 1.9 & - & 12.8 & 12.4 & - & 51.3 & 2.8 & & & & & & & & & & & & & & & & & & & & & & & & & & \\
\hline 10 & D 8 & 1.2 & 1.6 & 1.1 & 11.2 & 16 & 13.5 & - & - & 15.4 & & & & & & & & & & & & & & & & & & & & & & & & & \\
\hline 11 & D 9 & 1.0 & 0 & 0.9 & 4.1 & 5.2 & 2.9 & - & - & 5.1 & - & & & & & & & & & & & & & & & & & & & & & & & & \\
\hline 12 & D 10 & 1.1 & 1.3 & 0.9 & 5.4 & 6.4 & 3.0 & - & - & 4.2 & - & - & & & & & & & & & & & & & & & & & & & & & & & \\
\hline 13 & D 11 & - & - & - & - & - & 28 & - & 0 & 28.3 & 14.5 & 0 & 2.1 & & & & & & & & & & & & & & & & & & & & & & \\
\hline 14 & D 12 & - & - & - & - & - & 1.1 & - & - & 1.6 & - & - & - & - & & & & & & & & & & & & & & & & & & & & & \\
\hline 15 & D 14 & 1.4 & 1.3 & 1.3 & 10.3 & 16.4 & 45 & - & 2.4 & 33.8 & 29 & 4.9 & 1.2 & - & 0.0 & & & & & & & & & & & & & & & & & & & & \\
\hline 16 & D 15 & - & 2.1 & - & - & 3.5 & - & 3.4 & 2.3 & - & 2.8 & 2.4 & 4.8 & - & 0.7 & 2.5 & & & & & & & & & & & & & & & & & & & \\
\hline 17 & DK 3 & 1.5 & 3.1 & 1.4 & 11.2 & 9.5 & 7.2 & - & - & 8.7 & - & - & 2.4 & 4.5 & 1.9 & 12.4 & 2.6 & & & & & & & & & & & & & & & & & & \\
\hline 18 & EST 1 & - & - & - & - & - & 1.1 & 2.6 & 0 & 2.1 & 0.76 & 0 & - & 8.3 & - & 2.2 & 3.1 & 1.4 & & & & & & & & & & & & & & & & & \\
\hline 19 & F 1 & 0.7 & 0 & 0.6 & 1.9 & 2.2 & 0.9 & - & - & 1.8 & - & - & 0.5 & - & - & 1.4 & 2.1 & - & 0 & & & & & & & & & & & & & & & & \\
\hline 20 & F 2 & 0.9 & 1.6 & 0.8 & 3.3 & 3.5 & 1.2 & - & - & 1.6 & - & - & - & 0 & - & 2.2 & 1.9 & - & 0.6 & - & & & & & & & & & & & & & & & \\
\hline 21 & F 4 & 1.2 & 0 & 1.0 & 6.4 & 9.7 & 9.8 & - & - & 13.2 & - & - & - & 1.2 & - & 29.5 & 2.6 & - & 0 & - & - & & & & & & & & & & & & & & \\
\hline 22 & FIN 1 & - & - & - & - & - & 0.7 & 1.5 & 0 & 1.4 & 0.4 & 0 & - & 0 & - & 1.6 & 2.1 & 0.8 & - & 0 & 0.3 & 0 & & & & & & & & & & & & & \\
\hline 23 & GB 1 & - & - & - & - & - & 2.9 & - & - & 3.5 & - & - & 0.3 & - & - & 4.7 & 2.7 & - & - & - & - & - & - & & & & & & & & & & & & \\
\hline 24 & GB 2 & 0.8 & 0 & 0.7 & 2.4 & 2.9 & 1.4 & - & - & 2.6 & - & - & - & - & - & 2.2 & 2 & - & 0 & - & - & - & 0 & - & & & & & & & & & & & \\
\hline 25 & GR 1 & - & - & - & - & - & 5.63 & 34 & 3.1 & 7.7 & 13.4 & 4.6 & - & 0 & - & 19.8 & 5.8 & 12.6 & - & 2.3 & 4.1 & 7.6 & - & - & 2.9 & & & & & & & & & & \\
\hline 26 & I 1 & - & 0 & - & - & 6.4 & 4.6 & 13.7 & 0 & 8.3 & 3.6 & 0 & 6.2 & - & - & 5.4 & - & 4.6 & 0 & 0 & 1.1 & 0 & 0 & 5.3 & 0 & 3.7 & & & & & & & & & \\
\hline 27 & I 2 & - & 4.0 & - & 35.6 & 26.7 & 158 & 148 & 5.2 & 133.3 & 32.4 & 10.1 & 1.7 & 0 & 3.1 & 5.4 & - & 4.6 & 0 & 0 & 1.1 & 0 & 3.1 & 5.2 & 4.6 & 14.2 & 18 & & & & & & & & \\
\hline 28 & PL 1 & - & - & - & - & - & 3.8 & - & - & 4.4 & - & - & 7.1 & 105 & - & 108 & - & 14.4 & 3.8 & 3.2 & 2.3 & 37.5 & - & - & - & - & 6.2 & 6.2 & & & & & & & \\
\hline 29 & PL 2 & - & - & - & - & - & - & 0.5 & 0.2 & - & 0.3 & 0.3 & - & - & - & 6.1 & 3.3 & - & - & - & - & - & - & - & 0.1 & - & - & - & - & & & & & & \\
\hline 30 & RUM 1 & - & - & - & - & - & 10.5 & - & - & 11.3 & - & - & 0.3 & - & - & 1.2 & - & 0.4 & - & 0.3 & 0.3 & 0.2 & - & - & - & - & 11.2 & 18.2 & - & - & & & & & \\
\hline 31 & RUM 2 & - & - & - & - & - & 11 & - & - & 13.0 & - & - & - & - & - & 10.9 & 6 & - & - & - & - & - & - & - & - & - & 11.7 & 26.6 & - & - & - & & & & \\
\hline 32 & RUM 5 & 2.6 & 2.5 & 2.3 & 11.4 & 16.3 & 73 & 62.5 & 3.4 & 74 & 18.8 & 6.1 & - & - & & 8.6 & 5.3 & - & - & - & - & - & 1.8 & 4.4 & 3.1 & 12 & 4.7 & 64.2 & 5.2 & 1.5 & 23.9 & 18.4 & & & \\
\hline 33 & RUM 6 & 1.6 & 0 & 1.3 & 2.9 & 3.4 & 10 & 12 & 0 & 17.8 & 3 & 0 & 4.8 & 34.5 & 2.1 & 40.5 & 8.1 & 10.8 & 2.6 & 2.1 & 2.5 & 16.4 & 0 & 2.7 & 0 & 2.8 & 0 & 16.1 & 3.3 & - & 7.6 & 6.2 & - & & \\
\hline 34 & RUS 2 & 2.5 & 1.9 & 2.2 & 14 & 14 & - & 101.3 & 3.0 & - & 20.3 & 5.6 & 1.2 & 0 & 0.7 & 4.4 & 6 & 3.6 & 0 & 0 & 0.8 & 0 & 1.5 & 2.9 & 2.8 & 8.4 & 8.8 & 220 & 3.7 & 2.7 & 16.7 & 14.8 & 104 & 22.6 & \\
\hline 35 & $\mathrm{~S}_{2}$ & 1.4 & 1.8 & 1.2 & 14.5 & 23.2 & 54 & - & - & 41 & - & - & 4.5 & 54 & 1.6 & 47 & - & 9.9 & 2.1 & 1.8 & 2.1 & 20.3 & 0.5 & - & - & 17 & 4.6 & 98.7 & - & 0.3 & - & - & 56.3 & 6 & 81 \\
\hline
\end{tabular}




\section{Appendix}

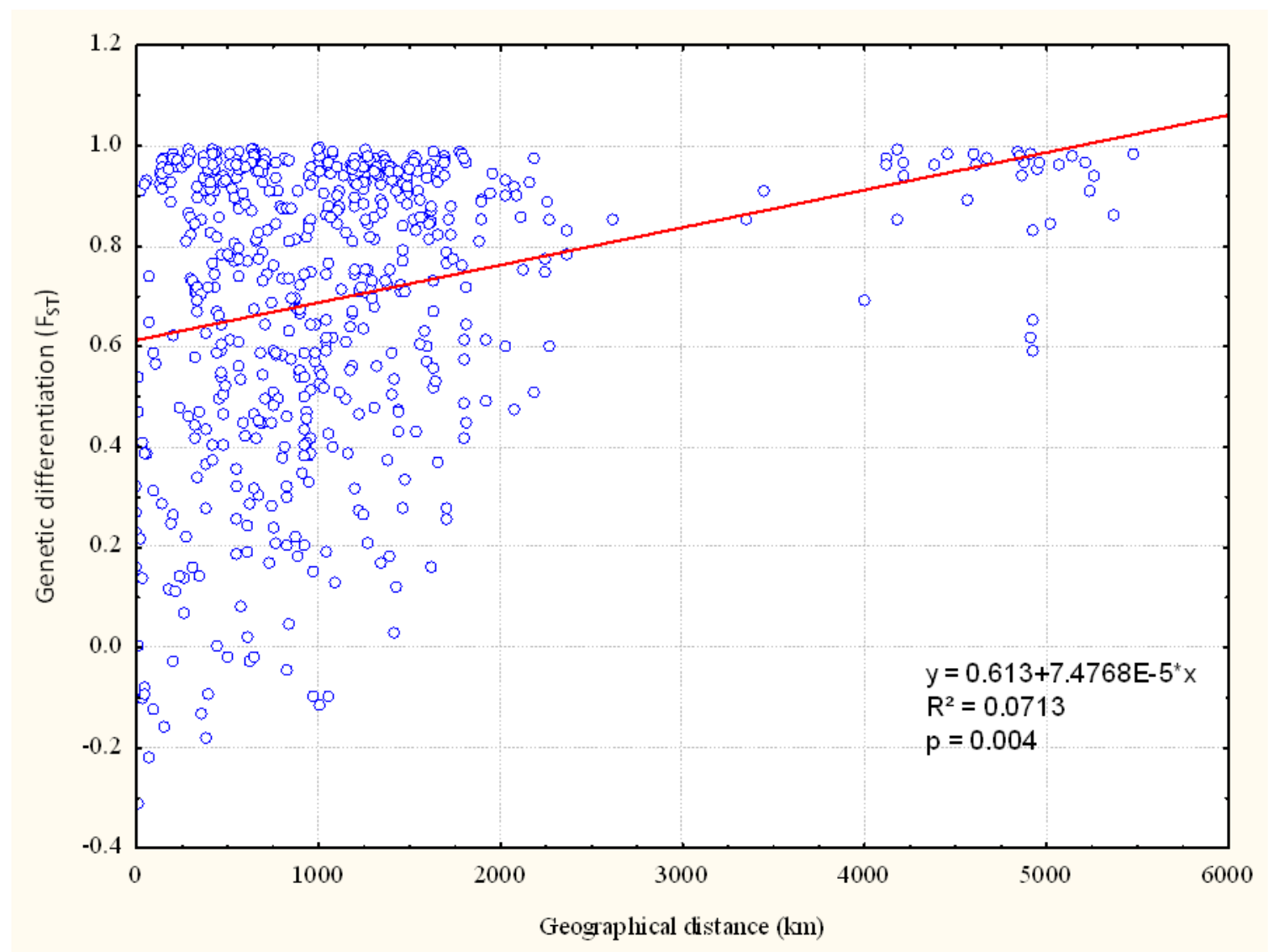

Figure A49: Linear regression of geographical distances (kilometers) vs. genetic differentiations $\left(\mathrm{F}_{\mathrm{ST}}\right)$ based on COI of Achipteria coleoptrata. Regression is significant $(\mathrm{p}=0.004 * * ; \quad(* 0.01<\mathrm{P}<0.05 ; \quad * * 0.001<\mathrm{P}<0.01$; $* * * \mathrm{P}<0.001)$ ) using 1000 randomizations. 
Appendix

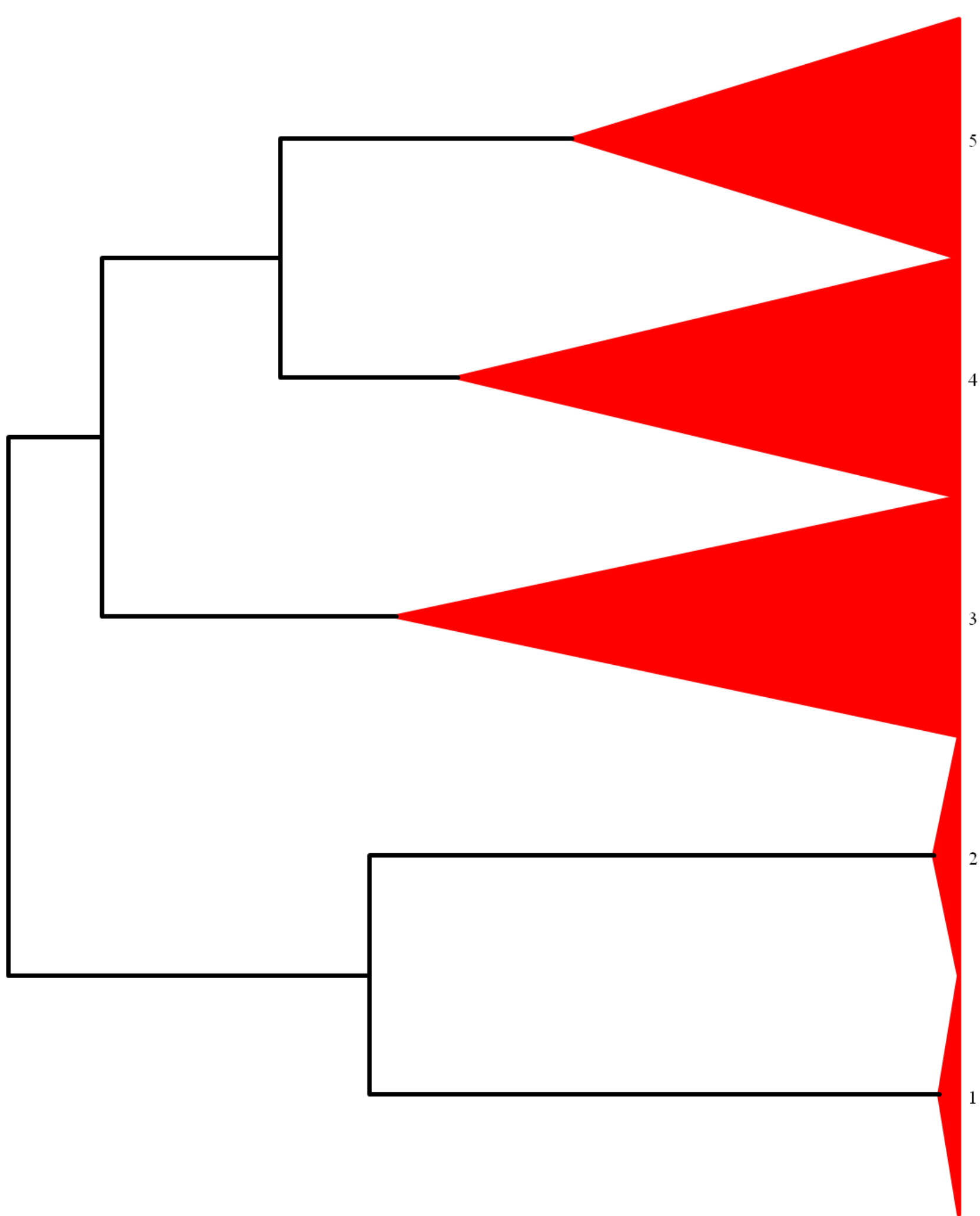

Figure A50: Cluster delimitation of $\mathrm{COI}$ nucleotide sequences from Achipteria coleoptrata after the single method of the generalized mixed Yule coalescent method (GMYC). Red branches are the different distinct clusters. Tip labels are explained in Table 24. 
Appendix

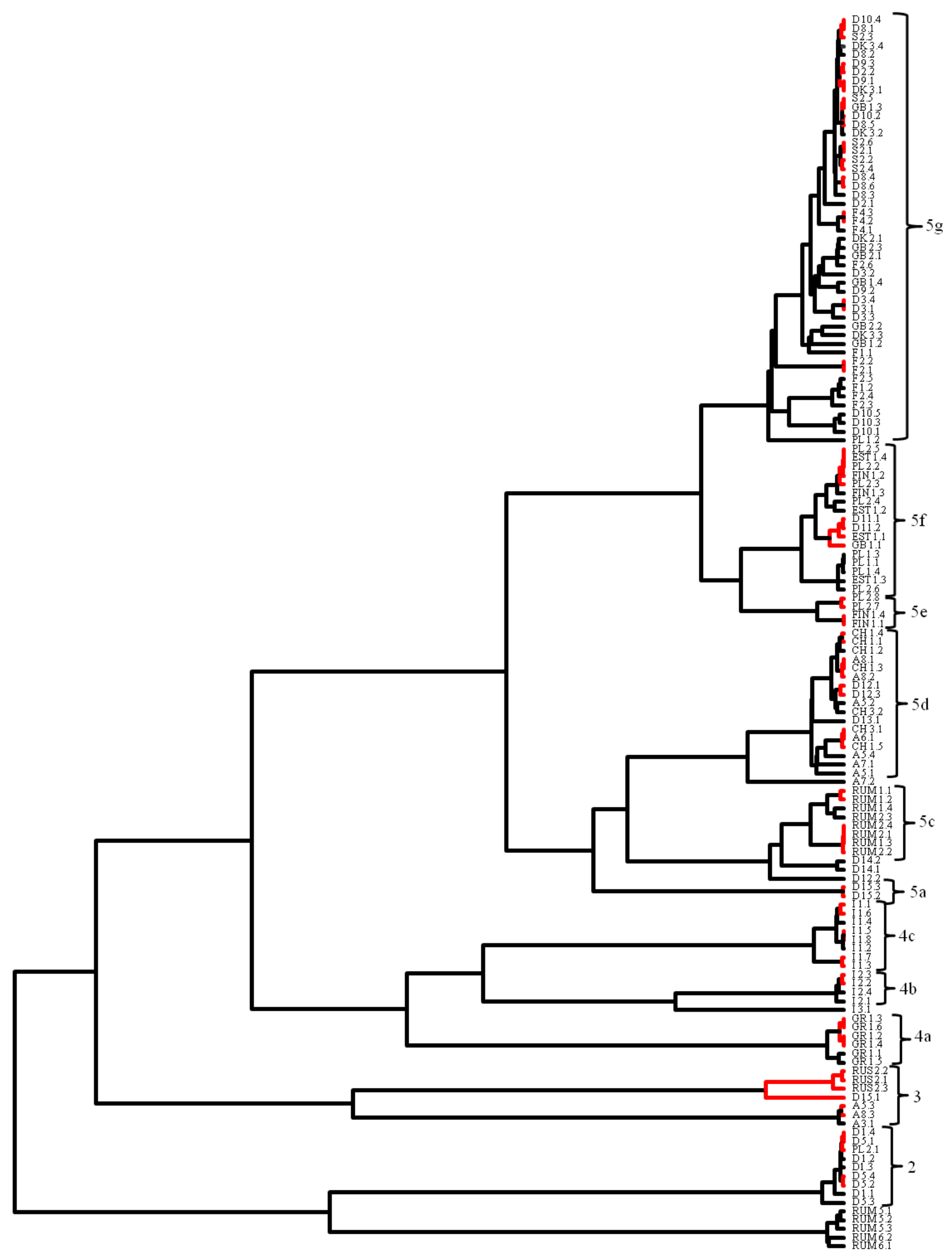

Figure A51: Cluster delimitation of COI nucleotide sequences from Achipteria coleoptrata after the multiple method of the generalized mixed Yule coalescent method (GMYC). Red branches are the different distinct clusters. Tip labels are explained in Table 24. 
Appendix

Table A37: Standard diversity measures of Steganacarus magnus. Populations with less than two individuals were excluded.

\begin{tabular}{|c|c|c|c|c|c|c|c|c|c|}
\hline population & $\begin{array}{c}\text { sample } \\
\text { size } \\
\mathbf{n} \\
\end{array}$ & $\begin{array}{c}\text { invarible } \\
\text { sites } \\
\mathbf{N}_{\text {is }} \\
\end{array}$ & $\begin{array}{c}\text { variable } \\
\text { sites } \\
\mathbf{N}_{\mathrm{vs}} \\
\end{array}$ & $\begin{array}{c}\text { parsimony } \\
\text { inform. sites } \\
\mathbf{N}_{\text {pars }} \\
\end{array}$ & $\begin{array}{c}\text { number of } \\
\text { singeltons } \\
\mathrm{N}_{\mathrm{s}} \\
\end{array}$ & $\begin{array}{c}\text { number of } \\
\text { haplotypes } \\
N_{h} \\
\end{array}$ & $\begin{array}{c}\text { haplotype } \\
\text { diversity } \\
\mathbf{H}_{\mathrm{d}} \\
\end{array}$ & variance & $\begin{array}{c}\text { nucleotide } \\
\text { diversity } \\
\Pi_{n} \\
\end{array}$ \\
\hline A_1 & 2 & 530 & 0 & 0 & 0 & 1 & 0 & 0 & 0 \\
\hline CZ_1 & 5 & 358 & 172 & 35 & 137 & 4 & 0.9 & 0.026 & 0.161 \\
\hline D_1 & 14 & 503 & 27 & 12 & 15 & 8 & 0.77 & 0.014 & 0.012 \\
\hline D_2 & 9 & 524 & 6 & 1 & 5 & 5 & 0.69 & 0.022 & 0.003 \\
\hline D_3 & 3 & 522 & 8 & 0 & 8 & 3 & 1 & 0.074 & 0.01 \\
\hline D_4 & 9 & 336 & 194 & 128 & 66 & 5 & 0.83 & 0.01 & 0.163 \\
\hline D_5 & 5 & 336 & 194 & 51 & 143 & 3 & 0.7 & 0.048 & 0.183 \\
\hline D_7 & 4 & 397 & 133 & 96 & 37 & 3 & 0.83 & 0.049 & 0.159 \\
\hline D_8 & 3 & 528 & 2 & 0 & 2 & 2 & 0.67 & 0.099 & 0.003 \\
\hline D_9 & 4 & 518 & 12 & 2 & 10 & 3 & 0.833 & 0.049 & 0.012 \\
\hline DK_1 & 3 & 391 & 139 & 0 & 139 & 3 & 1 & 0.074 & 0.179 \\
\hline DK_2 & 5 & 530 & 0 & 0 & 0 & 1 & 0 & 0 & 0 \\
\hline DK_3 & 3 & 529 & 1 & 0 & 1 & 2 & 0.67 & 0.099 & 0.001 \\
\hline F_1 & 6 & 339 & 191 & 50 & 151 & 3 & 0.6 & 0.046 & 0.154 \\
\hline F_2 & 4 & 528 & 2 & 0 & 2 & 3 & 0.83 & 0.049 & 0.002 \\
\hline F_3 & 5 & 459 & 71 & 0 & 71 & 4 & 0.9 & 0.026 & 0.054 \\
\hline F_4 & 2 & 530 & 0 & 0 & 0 & 1 & 0 & 0 & 0 \\
\hline FIN_1 & 3 & 527 & 3 & 0 & 3 & 2 & 0.67 & 0.099 & 0.004 \\
\hline GB_1 & 2 & 528 & 2 & 0 & 2 & 2 & 1 & 0.25 & 0.004 \\
\hline GB_2 & 3 & 525 & 5 & 0 & 5 & 2 & 0.67 & 0.099 & 0.006 \\
\hline I_1 & 10 & 520 & 10 & 8 & 2 & 4 & 0.71 & 0.014 & 0.009 \\
\hline I_2 & 4 & 517 & 13 & 2 & 11 & 4 & 1 & 0.031 & 0.013 \\
\hline N_1 & 3 & 387 & 143 & 0 & 143 & 2 & 0.67 & 0.099 & 0.18 \\
\hline NL_1 & 3 & 521 & 9 & 0 & 9 & 3 & 1 & 0.074 & 0.011 \\
\hline PL_1 & 6 & 387 & 143 & 136 & 7 & 6 & 1 & 0.009 & 0.143 \\
\hline PL_2 & 3 & 515 & 15 & 0 & 15 & 2 & 0.67 & 0.099 & 0.019 \\
\hline RUM_1 & 3 & 527 & 3 & 0 & 3 & 2 & 0.67 & 0.099 & 0.004 \\
\hline RUM_2 & 4 & 517 & 13 & 3 & 10 & 4 & 1 & 0.031 & 0.013 \\
\hline RUM_3 & 4 & 512 & 18 & 0 & 18 & 2 & 0.5 & 0.073 & 0.017 \\
\hline RUM_4 & 3 & 523 & 7 & 0 & 7 & 3 & 1 & 0.074 & 0.009 \\
\hline RUM_5 & 4 & 523 & 7 & 1 & 6 & 4 & 1 & 0.031 & 0.007 \\
\hline RUM_6 & 3 & 437 & 93 & 0 & 93 & 3 & 1 & 0.074 & 0.118 \\
\hline RUS_1 & 4 & 402 & 128 & 4 & 124 & 4 & 1 & 0.031 & 0.125 \\
\hline RUS_2 & 4 & 530 & 0 & 0 & 0 & 1 & 0 & 0 & 0 \\
\hline S_2 & 3 & 337 & 193 & 0 & 193 & 3 & 1 & 0.074 & 0.257 \\
\hline all & 155 & 216 & 314 & 276 & 38 & 93 & 0.98 & 0 & 0.21 \\
\hline
\end{tabular}


Appendix

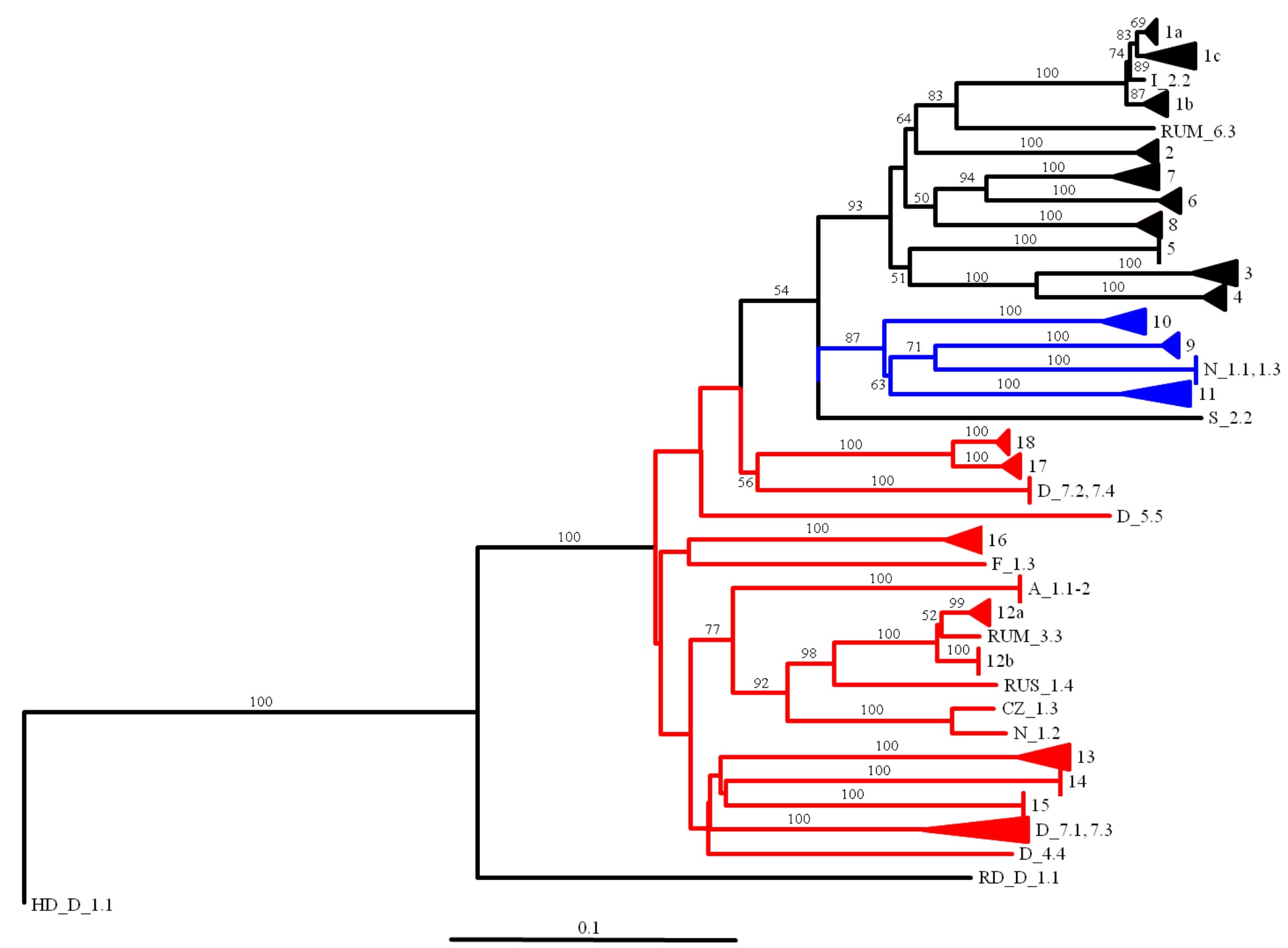

Figure A52: Neighbor-Joining tree of 157 COI nucleotide sequences of Steganacarus magnus. Outgroups are Hypochthonius rufulus (HD_D_1) and Rhysotritia duplicata (RD_D_1.1). Numbers on the branches are bootstrap values of 100,000 pseudo-replicates. Coloured branches show the three different main clades (black, blue, red). Tip numbers are the different subclades and explain in Table 28. 
Appendix

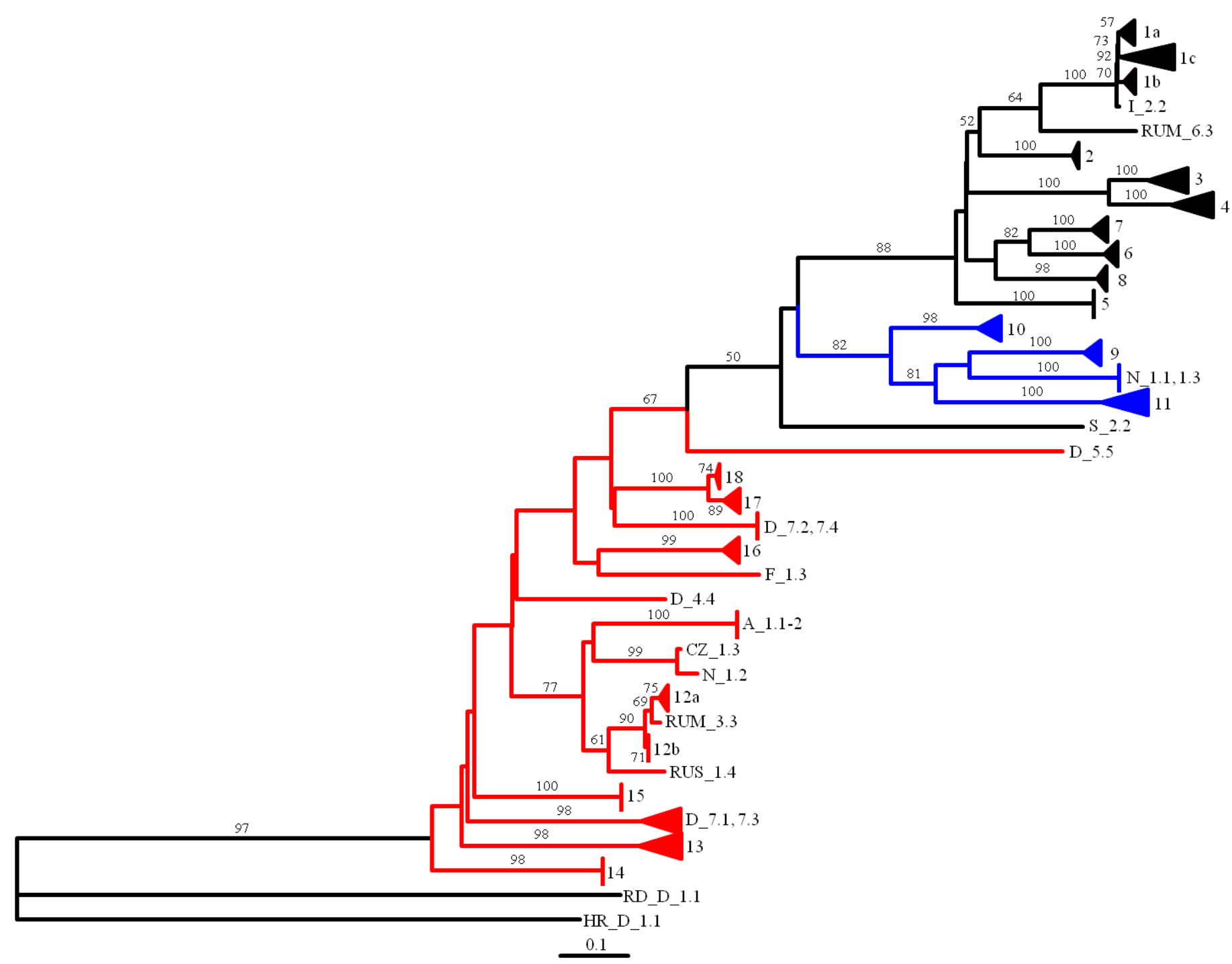

Figure A53: Neighbor-Joining tree of 157 COI nucleotide sequences of Steganacarus magnus with model of sequence evolution GTR+I+G. Outgroups are Hypochthonius rufulus (HD_D_1) and Rhysotritia duplicata (RD_D_1.1). Numbers on the branches are bootstrap values of 100,000 pseudo-replicates. Coloured branches show the three different main clades (black, blue, red). Tip numbers are the different subclades and explain in Table 28. 
Appendix

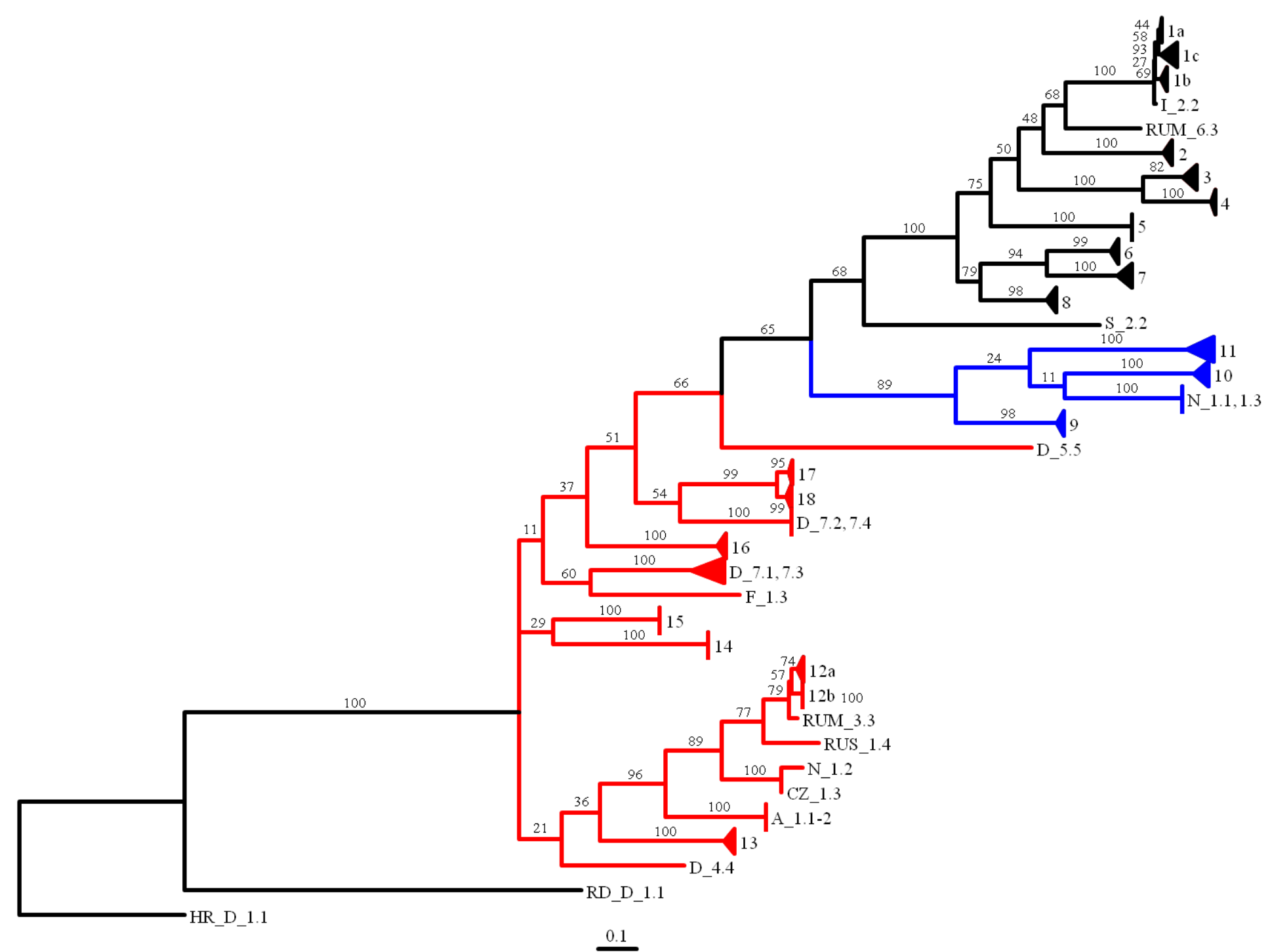

Figure A54: Maximum likelihood tree of 157 COI nucleotide sequences of Steganacarus magnus with model of sequence evolution GTR+I+G. Outgroups are Hypochthonius rufulus (HD_D_1) and Rhysotritia duplicata (RD_D_1.1) Numbers on the branches are bootstrap values of 100,000 pseudo-replicates. Coloured branches show the three different main clades (black, blue, red). Tip numbers are the different subclades and explain in Table 28. 
Appendix

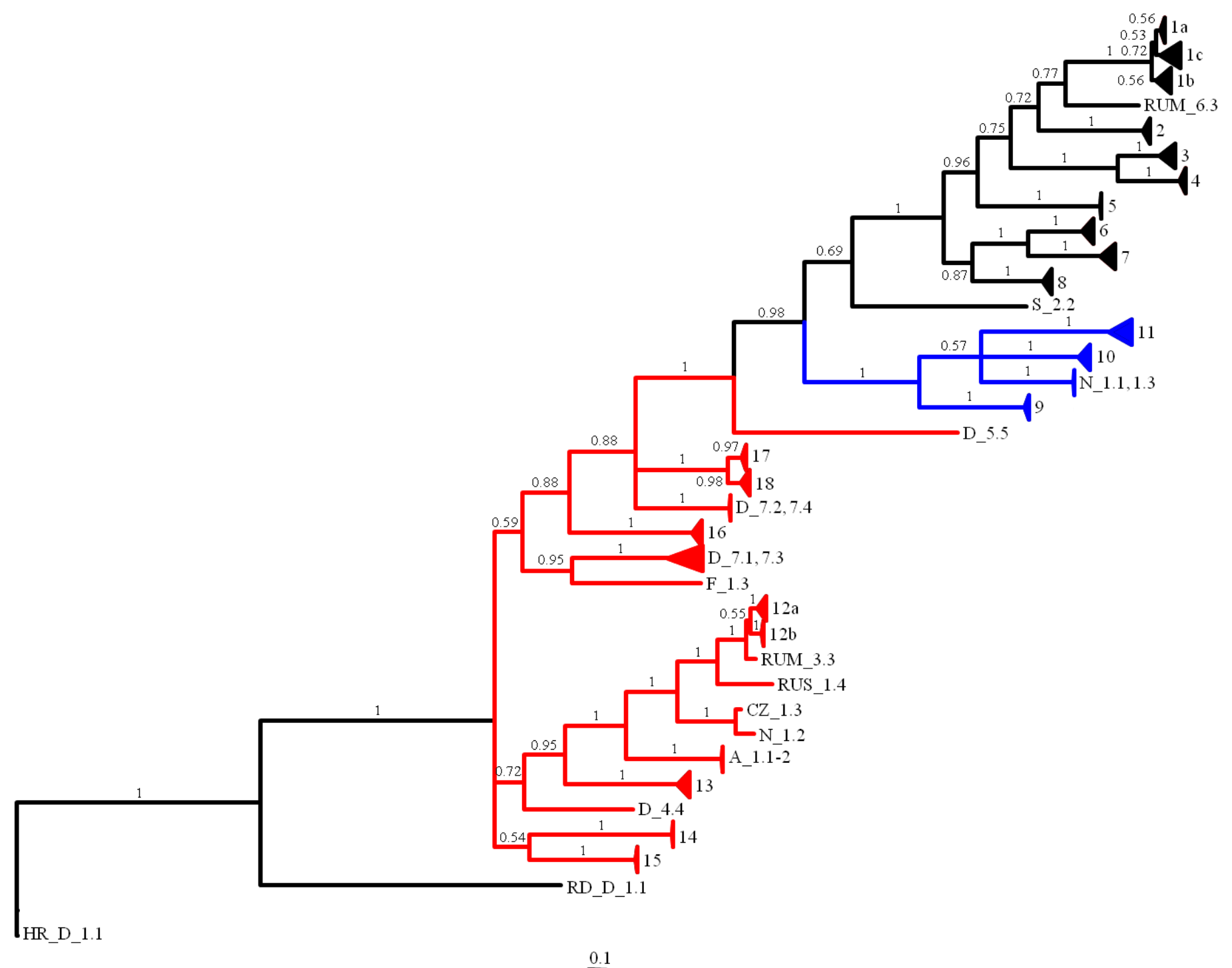

Figure A55: Bayesian tree from the nucleotide of Steganacarus magnus after $10 \times 10^{6}$ generations. Split frequencies of 0.024252 and burnin of $25 \%$. Outgroups are Hypochthonius rufulus (HD_D_1) and Rhysotritia duplicata (RD_D_1.1). Numbers on the branches are posterior probabilities. Coloured branches show the three different main clades (black, blue, red). Tip numbers are the different subclades and explain in Table 28. 
Appendix

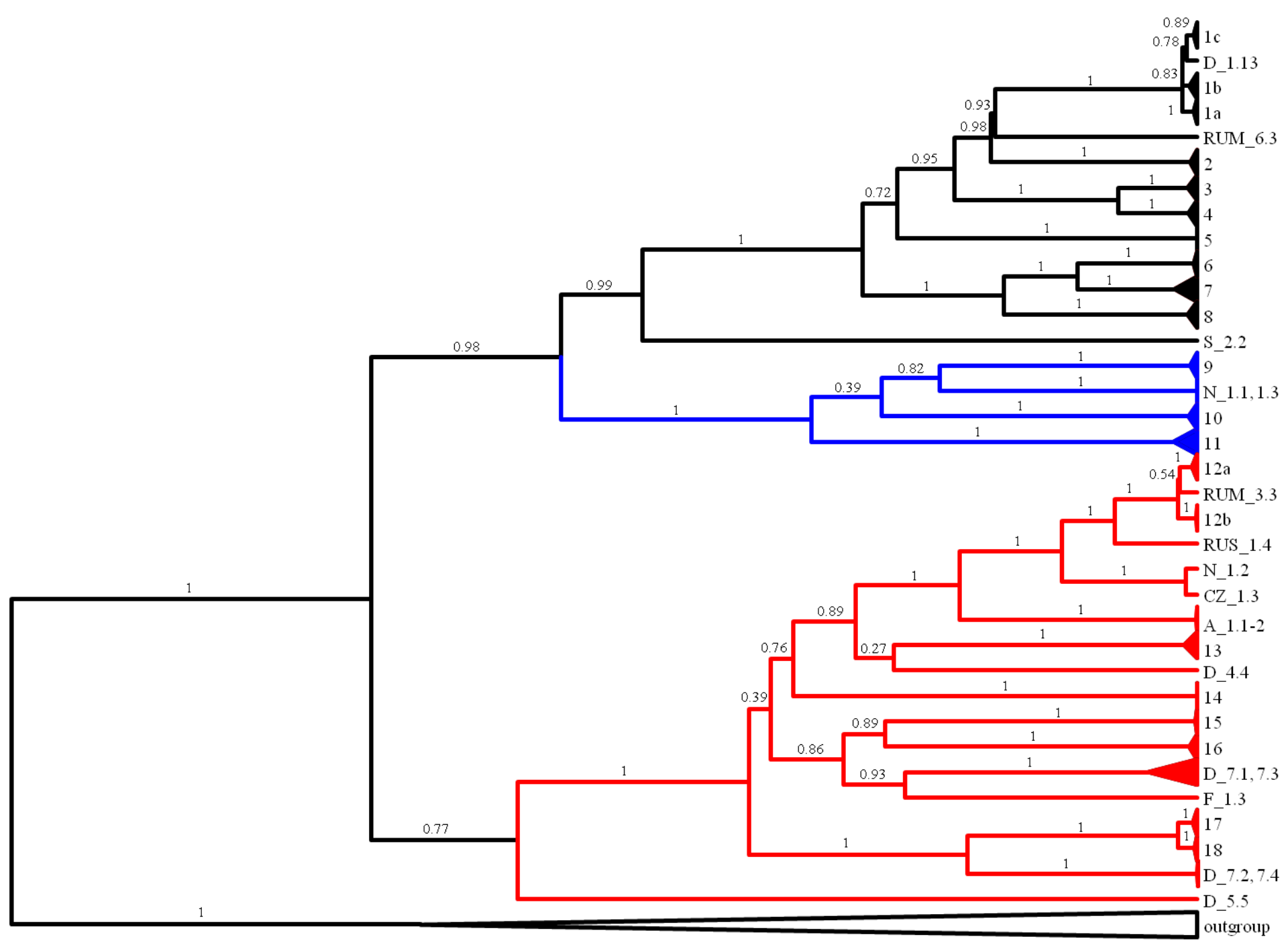

Figure A56: Bayesian phylogeny approach from the nucleotide of Steganacarus magnus after 10x10 $0^{6}$ generations with Beast v1.5.4. Outgroups are Hypochthonius rufulus (HD_D_1) and Rhysotritia duplicata (RD_D_1.1). Numbers on the branches are posterior probabilities. Coloured branches show the three different main clades (black, blue, red). Tip numbers are the different subclades and explain in Table 28. 
Appendix

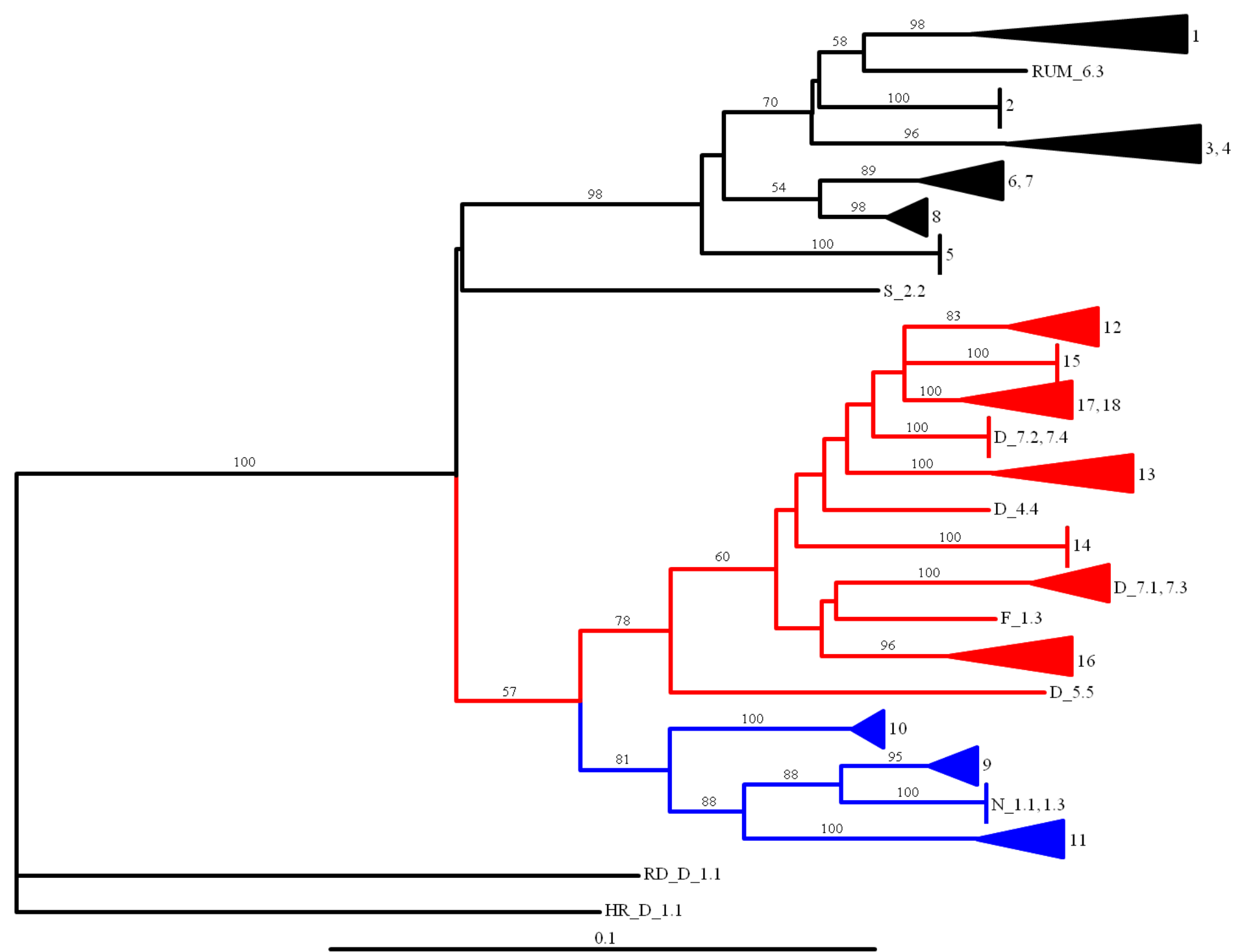

Figure A57: Neighbor-Joining tree of 157 COI protein sequences of Steganacarus magnus. Outgroups are Hypochthonius rufulus (HD_D_1) and Rhysotritia duplicata (RD_D_1.1). Numbers on the branches are bootstrap values of 100,000 pseudo-replicates. Coloured branches show the three different main clades (black, blue, red). Tip numbers are the different subclades and explain in Table 28. 
Appendix

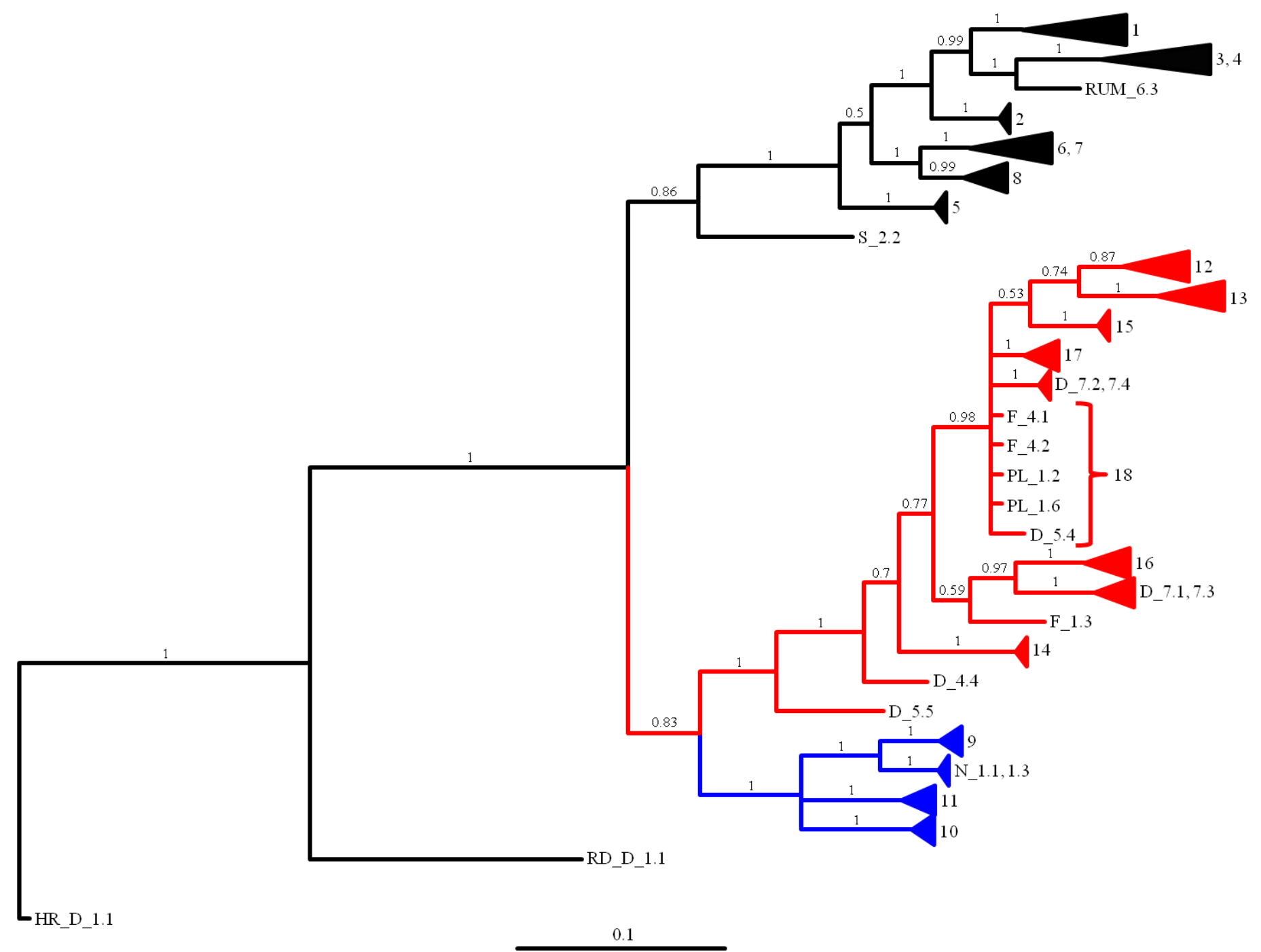

Figure A58: Bayesian tree from the protein of Steganacarus magnus after 10x10 generations with the model equalin, split frequencies of 0.006509 and burnin of $25 \%$. are Hypochthonius rufulus (HD_D_1) and Rhysotritia duplicata (RD_D_1.1). Numbers show the posterior probabilities. Coloured branches show the three different main clades (black, blue, red). Tip numbers are the different subclades and explain in Table 28. 
Appendix

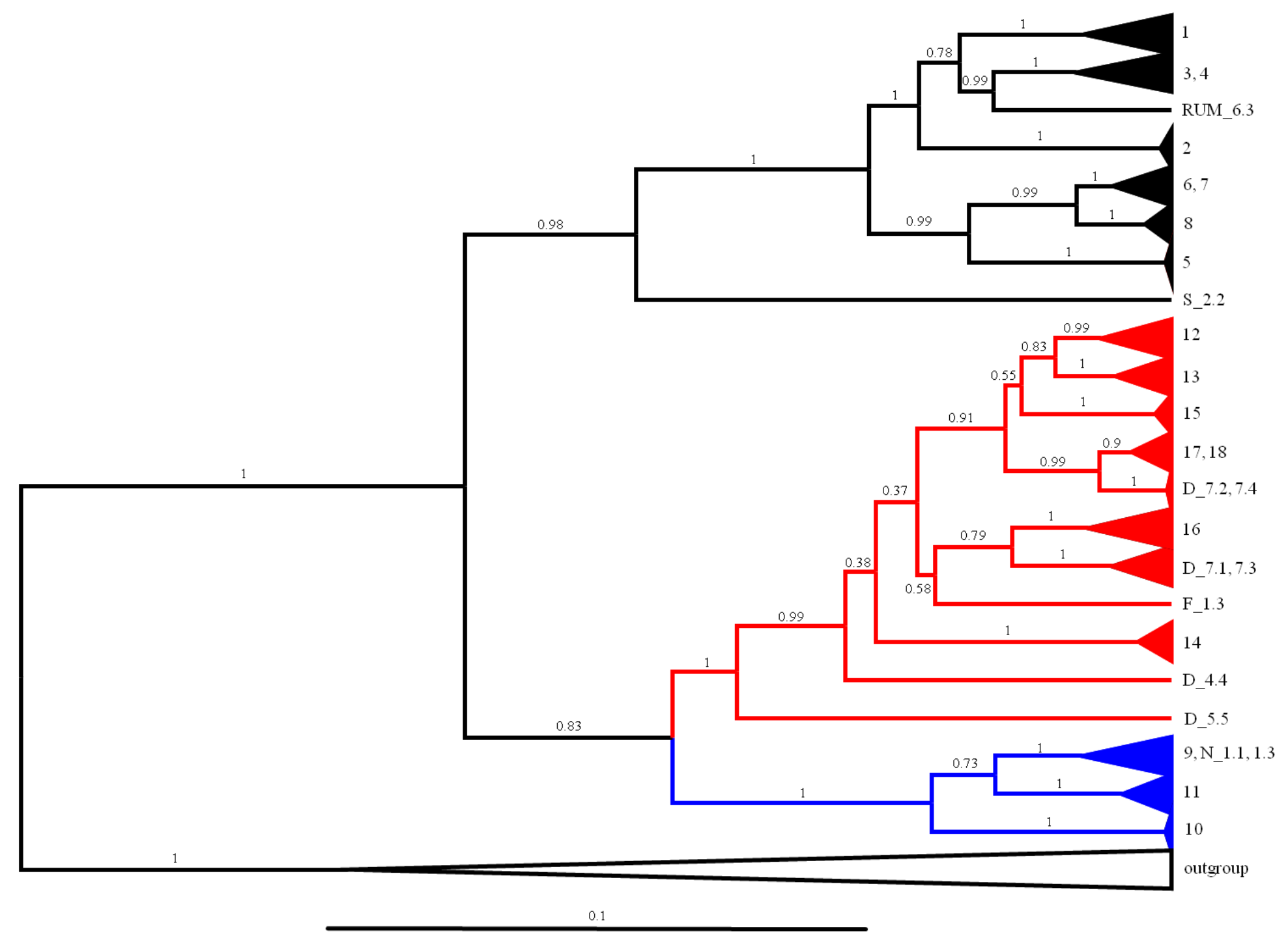

Figure A59: Bayesian tree from the protein of Steganacarus magnus after $10 \times 10^{6}$ generations. Split frequencies of 0.024252 and burnin of $25 \%$. Outgroups are Hypochthonius rufulus (HD_D_1) and Rhysotritia duplicata (RD_D_1.1). Numbers on the branches are posterior probabilities. Coloured branches show the three different main clades (black, blue, red). Tip numbers are the different subclades and explain in Table 28. 


\section{Appendix}

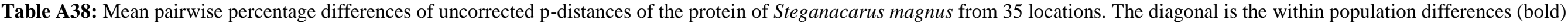

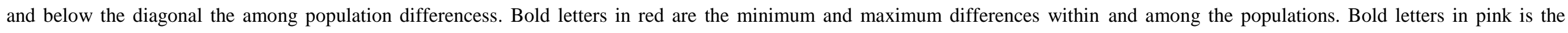
maximum divergences, if Russia is excluded. Locations with less than two individuals were excluded from the analysis.

\begin{tabular}{|c|c|c|c|c|c|c|c|c|c|c|c|c|c|c|c|c|c|c|c|c|c|c|c|c|c|c|c|c|c|c|c|c|c|c|c|c|}
\hline \multicolumn{2}{|c|}{ Population } & \multirow{2}{*}{$\frac{1}{0}$} & \multirow[t]{2}{*}{2} & \multirow[t]{2}{*}{3} & \multirow[t]{2}{*}{4} & \multirow[t]{2}{*}{5} & \multirow[t]{2}{*}{6} & \multirow[t]{2}{*}{7} & \multirow[t]{2}{*}{8} & \multirow[t]{2}{*}{9} & \multirow[t]{2}{*}{10} & \multirow[t]{2}{*}{11} & \multirow[t]{2}{*}{12} & 13 & 14 & 15 & 16 & 17 & 18 & 19 & 20 & 21 & 22 & 23 & 24 & 25 & 26 & 27 & 28 & 29 & 30 & 31 & 32 & 33 & 34 & 35 \\
\hline 1 & A 1 & & & & & & & & & & & & & & & & & & & & & & & & & & & & & & & & & & & \\
\hline 2 & $\mathrm{CZ}_{1}$ & 1.8 & 1.1 & & & & & & & & & & & & & & & & & & & & & & & & & & & & & & & & & \\
\hline 3 & D 1 & 2.9 & 1.7 & 0.2 & & & & & & & & & & & & & & & & & & & & & & & & & & & & & & & & \\
\hline 4 & D 2 & 2.8 & 1.6 & 0.1 & 0 & & & & & & & & & & & & & & & & & & & & & & & & & & & & & & & \\
\hline 5 & D 3 & 4 & 2.7 & 1.3 & 1.1 & 0 & & & & & & & & & & & & & & & & & & & & & & & & & & & & & & \\
\hline 6 & D 4 & 1.6 & 1.2 & 2.3 & 2.2 & 2.7 & 1.2 & & & & & & & & & & & & & & & & & & & & & & & & & & & & & \\
\hline 7 & D 5 & 2.5 & 1.7 & 1.5 & 1.4 & 2.7 & 2 & 2 & & & & & & & & & & & & & & & & & & & & & & & & & & & & \\
\hline 8 & D 7 & 2.1 & 1.6 & 2.8 & 2.7 & 3.8 & 1.5 & 2.1 & 1 & & & & & & & & & & & & & & & & & & & & & & & & & & & \\
\hline 9 & D 8 & 3 & 2.7 & 1.5 & 1.3 & 1.3 & 3.1 & 2.6 & 3.5 & 0.4 & & & & & & & & & & & & & & & & & & & & & & & & & & \\
\hline 10 & D 9 & 2.8 & 1.6 & 0.1 & 0 & 1.1 & 2.2 & 1.4 & 2.7 & 1.3 & 0 & & & & & & & & & & & & & & & & & & & & & & & & & \\
\hline 11 & DK 1 & 2.7 & 1.4 & 1.5 & 1.3 & 1.7 & 1.5 & 1.9 & 2.3 & 2.7 & 1.3 & 1.5 & & & & & & & & & & & & & & & & & & & & & & & & \\
\hline 12 & DK 2 & 0.6 & 1.6 & 2.9 & 2.8 & 4 & 1.4 & 2.3 & 1.3 & 3 & 2.8 & 2.7 & 0 & & & & & & & & & & & & & & & & & & & & & & & \\
\hline 13 & DK 3 & 2.8 & 1.6 & 0.1 & 0 & 1.1 & 2.2 & 1.4 & 2.7 & 1.3 & 0 & 1.3 & 2.8 & 0 & & & & & & & & & & & & & & & & & & & & & & \\
\hline 14 & F 1 & 1.5 & 1.9 & 2.6 & 2.5 & 4 & 1.7 & 2.3 & 2.1 & 3.8 & 2.5 & 2.5 & 1 & 2.5 & 1.4 & & & & & & & & & & & & & & & & & & & & & \\
\hline 15 & F 2 & 2.8 & 1.6 & 0.1 & 0 & 1.1 & 2.2 & 1.4 & 2.7 & 1.3 & 0 & 1.3 & 2.8 & 0 & 2.5 & 0 & & & & & & & & & & & & & & & & & & & & \\
\hline 16 & F 3 & 3.1 & 2.7 & 1.5 & 1 & 1.4 & 3.1 & 2.6 & 3.5 & 0.4 & 1.4 & 2.7 & 3.1 & 1.4 & 3.8 & 1.4 & 0.5 & & & & & & & & & & & & & & & & & & & \\
\hline 17 & F 4 & 0.6 & 1.6 & 2.9 & 2.8 & 4 & 1.4 & 2.3 & 1.3 & 3 & 2.8 & 2.7 & 0 & 2.8 & 1 & 2.8 & 3.1 & 0 & & & & & & & & & & & & & & & & & & \\
\hline 18 & FIN 1 & 1.1 & 1.5 & 2.4 & 2.3 & 3.4 & 1.3 & 1.9 & 1 & 2.5 & 2.3 & 2.1 & 0.6 & 2.3 & 1.5 & 2.3 & 2.5 & 0.6 & 0 & & & & & & & & & & & & & & & & & \\
\hline 19 & GB 1 & 2.8 & 2.5 & 1.3 & 1.1 & 1.1 & 2.9 & 2.4 & 3.3 & 0.2 & 1.1 & 2.5 & 2.8 & 1.1 & 3.6 & 1.1 & 0.2 & 2.8 & 2.3 & 0 & & & & & & & & & & & & & & & & \\
\hline 20 & GB 2 & 3 & 2.7 & 1.5 & 1.3 & 1.3 & 3.1 & 2.6 & 3.5 & 0.3 & 1.3 & 2.7 & 3 & 1.3 & 3.8 & 1.3 & 0.4 & 3 & 2.5 & 0.2 & 0.4 & & & & & & & & & & & & & & & \\
\hline 21 & I 1 & 2.8 & 1.6 & 0.1 & 0 & 1.1 & 2.2 & 1.4 & 2.7 & 1.3 & 0 & 1.3 & 2.8 & 0 & 2.5 & 0 & 1.4 & 2.8 & 2.3 & 1.1 & 1.3 & 0 & & & & & & & & & & & & & & \\
\hline 22 & I 2 & 2.8 & 1.6 & 0.1 & 0 & 1.1 & 2.2 & 1.4 & 2.7 & 1.3 & 0 & 1.3 & 2.8 & 0 & 2.5 & 0 & 1.4 & 2.8 & 2.3 & 1.1 & 1.3 & 0 & 0 & & & & & & & & & & & & & \\
\hline 23 & N 1 & 1.5 & 1.3 & 2.2 & 2.1 & 3.2 & 1.5 & 2.1 & 1.8 & 3 & 2.1 & 1.9 & 1.7 & 2.1 & 1.9 & 2.8 & 3.1 & 1.7 & 1.5 & 2.8 & 3 & 2.1 & 2.1 & 1.5 & & & & & & & & & & & & \\
\hline 24 & NL 1 & 3 & 2.5 & 1.3 & 1.1 & 1.3 & 3 & 2.5 & 3.5 & 0.4 & 1.1 & 2.5 & 3 & 1.1 & 3.5 & 1.1 & 0.4 & 3 & 2.5 & 0.2 & 0.4 & 1.1 & 1.1 & 2.9 & 0.8 & & & & & & & & & & & \\
\hline 25 & PL 1 & 1 & 1.2 & 2.4 & 2.3 & 3.4 & 1.3 & 1.9 & 1.4 & 2.8 & 2.3 & 2.1 & 0.6 & 2.3 & 1.2 & 2.3 & 2.9 & 0.6 & 0.8 & 2.7 & 2.8 & 2.3 & 2.3 & 1.5 & 2.8 & 0.9 & & & & & & & & & & \\
\hline 26 & PL 2 & 3.6 & 2.2 & 0.7 & 0.6 & 0.8 & 2.1 & 2.2 & 3.2 & 2.1 & 0.6 & 1.1 & 3.6 & 0.6 & 3.3 & 0.6 & 2.1 & 3.6 & 3 & 1.9 & 2.1 & 0.6 & 0.6 & 2.7 & 2 & 3 & 0.4 & & & & & & & & & \\
\hline 27 & RUM 1 & 2.8 & 1.6 & 0.1 & 0 & 1.1 & 2.1 & 1.5 & 2.4 & 1.3 & 0 & 1.3 & 2.8 & 0 & 2.8 & 0 & 1.4 & 2.8 & 2.3 & 1.1 & 1.3 & 0 & 0 & 2.1 & 1.3 & 2.3 & 0.8 & 0 & & & & & & & & \\
\hline 28 & RUM 2 & 2.8 & 1.6 & 0.1 & 0 & 1.1 & 2.1 & 1.5 & 2.4 & 1.3 & 0 & 1.3 & 2.8 & 0 & 2.8 & 0 & 1.4 & 2.8 & 2.3 & 1.1 & 1.3 & 0 & 0 & 2.1 & 1.3 & 2.3 & 0.8 & 0 & 0 & & & & & & & \\
\hline 29 & RUM 3 & 0.4 & 2.0 & 2.9 & 2.8 & 4 & 1.6 & 2.5 & 1.9 & 3 & 2.8 & 2.7 & 0.6 & 2.8 & 1.9 & 2.8 & 3.1 & 0.6 & 1.1 & 2.8 & 3 & 2.8 & 2.8 & 1.9 & 3 & 1.1 & 3.6 & 2.8 & 2.8 & 0.3 & & & & & & \\
\hline 30 & RUM 4 & 0 & 1.8 & 2.9 & 2.8 & 4 & 1.6 & 2.5 & 1.9 & 3 & 2.8 & 2.7 & 0.6 & 2.8 & 1.9 & 2.8 & 3.1 & 0.6 & 1.1 & 2.8 & 3 & 2.8 & 2.8 & 1.5 & 3 & 1 & 3.6 & 2.8 & 2.8 & 0.4 & 0 & & & & & \\
\hline 31 & RUM 5 & 3 & 1.7 & 0.2 & 0.1 & 1.3 & 2.2 & 1.5 & 2.6 & 1.5 & 0.1 & 1.5 & 3 & 0.1 & 2.9 & 0.1 & 1.5 & 3 & 2.4 & 1.3 & 1.5 & 0.1 & 0.1 & 2.2 & 1.5 & 2.4 & 0.9 & 0.1 & 0.1 & 3 & 3 & 0.3 & & & & \\
\hline 32 & RUM 6 & 2.7 & 1.6 & 0.5 & 0.4 & 1.7 & 2.1 & 1.6 & 2.5 & 1.5 & 0.4 & 1.5 & 2.7 & 0.4 & 2.5 & 0.4 & 1.6 & 2.7 & 2.1 & 1.3 & 1.5 & 0.4 & 0.4 & 2 & 1.4 & 2.2 & 1.2 & 0.6 & 0.6 & 2.7 & 2.7 & 0.7 & 1.1 & & & \\
\hline 33 & RUS 1 & 1.9 & 2.4 & 2.4 & 2.3 & 3.8 & 2.1 & 2.6 & 2.8 & 4.2 & 2.3 & 2.3 & 2.4 & 2.3 & 2.4 & 2.3 & 4.2 & 2.4 & 2.6 & 4 & 4.2 & 2.3 & 2.3 & 2.1 & 3.9 & 2.2 & 3.2 & 2.7 & 2.7 & 2.7 & 2.3 & 2.8 & 2.5 & 1.7 & & \\
\hline 34 & RUS 2 & 3.4 & 2.1 & 2.4 & 2.3 & 4 & 2.8 & 2.4 & 1.6 & 3.6 & 2.3 & 2.8 & 2.8 & 2.3 & 2.8 & 2.3 & 3.6 & 2.8 & 2.3 & 3.4 & 3.6 & 2.3 & 2.3 & 2.7 & 3.6 & 2.3 & 3 & 2.3 & 2.3 & 3.4 & 3.4 & 2.4 & 2.5 & 3.6 & 0 & \\
\hline 35 & S 2 & 1.3 & 0.8 & 1.8 & 1.7 & 2.8 & 1.1 & 1.6 & 1.5 & 2.7 & 1.7 & 2.7 & 1.1 & 1.7 & 1.5 & 1.7 & 2.7 & 1.1 & 1 & 2.5 & 2.7 & 1.7 & 1.7 & 1.2 & 2.6 & 1 & 2.4 & 1.7 & 1.7 & 1.6 & 1.3 & 1.9 & 1.7 & 1.9 & 2.3 & 1.1 \\
\hline
\end{tabular}


Appendix

Table A39: Tajima's D and Fu's FS neutrality tests of Steganacarus magnus COI nucleotide sequences from 35 locations. Bold red letters show significance (P<0.05).

\begin{tabular}{|c|c|c|c|c|c|c|c|c|c|c|c|c|c|c|c|c|c|c|c|c|c|c|}
\hline Neutrality tests & A 1 & $\mathrm{CZ} 1$ & D 1 & D 2 & D 3 & D 4 & D 5 & D 7 & D 8 & D 9 & DK 1 & DK 2 & DK 3 & F 1 & F 2 & F 3 & F 4 & FIN 1 & GB 1 & GB 2 & I 1 & I 2 \\
\hline \multicolumn{23}{|l|}{ Tajima's D test } \\
\hline Samplesize & 2 & 5 & 14 & 9 & 3 & 9 & 5 & 4 & 3 & 4 & 3 & 5 & 3 & 6 & 4 & 5 & 2 & 3 & 2 & 3 & 10 & 4 \\
\hline$S$ & 0 & 173 & 27 & 7 & 8 & 195 & 195 & 134 & 2 & 12 & 139 & 0 & 1 & 191 & 2 & 71 & 0 & 3 & 2 & 5 & 10 & 14 \\
\hline $\mathrm{Pi}$ & 0 & 85.5 & 6.44 & 1.72 & 5.33 & 86.78 & 97.7 & 84.67 & 1.33 & 6.33 & 95 & 0 & 0.67 & 81.67 & 1 & 28.4 & 0 & 2 & 2 & 3.33 & 4.67 & 7.33 \\
\hline Tajima's D & 0 & 0.23 & -1.03 & -1.48 & 0 & 1.09 & 0.33 & 1.66 & 0 & -0.33 & 6428365.59 & 0 & 0 & -0.15 & -0.71 & -1.26 & 0 & 0 & 0 & 0 & 1.42 & -0.4 \\
\hline Tajima's D p-value & 0.76 & 0.62 & 0.15 & 0.07 & 0.75 & 0.9 & 0.64 & 0.93 & 0.94 & 0.53 & 1 & 1 & 0.99 & 0.46 & 0.28 & 0 & 1 & 0.88 & 1 & 0.8 & 0.94 & 0.5 \\
\hline \multicolumn{23}{|l|}{ Fu's FS test } \\
\hline Real no. of alleles & 1 & 4 & 8 & 5 & 3 & 5 & 3 & 3 & 2 & 3 & 3 & 1 & 2 & 3 & 3 & 4 & 1 & 2 & 2 & 2 & 4 & 4 \\
\hline Orig. no. of alleles & 1 & 4 & 8 & 5 & 3 & 5 & 3 & 3 & 2 & 3 & 3 & 1 & 2 & 3 & 3 & 4 & 1 & 2 & 2 & 2 & 4 & 4 \\
\hline Theta_pi & 0 & 85.5 & 6.44 & 1.72 & 5.33 & 86.78 & 97.7 & 84.67 & 1.33 & 6.33 & 95 & 0 & 0.67 & 81.67 & 1 & 28.4 & 0 & 2 & 2 & 3.33 & 4.67 & 7.33 \\
\hline Exp. no. of alleles & 0 & 4.89 & 7.79 & 3.61 & 2.57 & 8.61 & 4.9 & 3.93 & 1.97 & 3.3 & 2.97 & 0 & 1.65 & 5.82 & 2.08 & 4.68 & 0 & 2.17 & 1.67 & 2.39 & 5.7 & 3.38 \\
\hline FS & 0 & 5.44 & 0.27 & -1.19 & 0.46 & 11.58 & 9.93 & 6.54 & 1.06 & 1.88 & 3.45 & 0 & 0.2 & 12.17 & -0.89 & 3.39 & 0 & 1.61 & 0.69 & 2.36 & 2.77 & -0.04 \\
\hline FS p-value & N.A. & 0.96 & 0.55 & 0.13 & 0.4 & 1 & 1 & 0.98 & 0.6 & 0.76 & 0.58 & N.A. & 0.38 & 1 & 0.09 & 0.89 & N.A. & 0.7 & 0.38 & 0.8 & 0.91 & 0.28 \\
\hline
\end{tabular}

\begin{tabular}{|c|c|c|c|c|c|c|c|c|c|c|c|c|c|c|c|}
\hline Neutrality tests & N 1 & NL 1 & PL 1 & PL 2 & RUM 1 & RUM 2 & RUM 3 & $\overline{\text { RUM } 4}$ & RUM 5 & RUM 6 & RUS 1 & RUS 2 & S 2 & mean & s. d. \\
\hline \multicolumn{16}{|l|}{ Tajima's D test } \\
\hline Samplesize & 3 & 3 & 6 & 3 & 3 & 4 & 4 & 3 & 4 & 3 & 4 & 4 & 3 & 4.43 & 2.52 \\
\hline$S$ & 143 & 9 & 144 & 15 & 3 & 13 & 18 & 7 & 7 & 93 & 129 & 0 & 194 & 56.17 & 72.90 \\
\hline $\mathrm{Pi}$ & 95.33 & 6 & 76.47 & 10 & 2 & 7 & 9 & 4.67 & 3.67 & 62.33 & 66.5 & 0 & 137 & 30.91 & 41.00 \\
\hline Tajima's D & 0 & 0 & 1.38 & 0 & 0 & -0.13 & -0.85 & 0 & -0.39 & 1384076.56 & -0.58 & 0 & 15062332.57 & 653564.96 & 2739489.35 \\
\hline Tajima's D p-value & 0.2 & 0.73 & 0.96 & 0.7 & 0.88 & 0.61 & 0.08 & 0.76 & 0.49 & 1 & 0.38 & 1 & 1.00 & 0.69 & 0.32 \\
\hline \multicolumn{16}{|l|}{ Fu's FS test } \\
\hline Real no. of alleles & 2 & 3 & 6 & 2 & 2 & 4 & 2 & 3 & 4 & 3 & 4 & 1 & 3 & 3.06 & 1.47 \\
\hline Orig. no. of alleles & 2 & 3 & 6 & 2 & 2 & 4 & 2 & 3 & 4 & 3 & 4 & 1 & 3 & 3.06 & 1.47 \\
\hline Theta_pi & 95.33 & 6 & 76.47 & 10 & 2 & 7 & 9 & 4.67 & 3.67 & 62.33 & 66.5 & 0 & 137 & 30.91 & 41.00 \\
\hline Exp. no. of alleles & 2.97 & 2.61 & 5.81 & 2.74 & 2.17 & 3.35 & 3.47 & 2.52 & 2.98 & 2.95 & 3.91 & 0 & 2.98 & 3.22 & 1.97 \\
\hline FS & 8.45 & 0.59 & 1.55 & 4.17 & 1.61 & -0.09 & 5.39 & 0.31 & -0.95 & 3.02 & 2.38 & 0 & 3.82 & 2.63 & 3.48 \\
\hline FS p-value & 0.99 & 0.4 & 0.48 & 0.93 & 0.71 & 0.27 & 0.98 & 0.37 & 0.11 & 0.57 & 0.53 & N.A. & 0.59 & N.A. & N.A. \\
\hline
\end{tabular}


Appendix

Table A40: Tajima's D and Fu's FS neutrality tests of Steganacarus magnus COI protein sequences from 35 locations. Bold red letters show significance $(\mathrm{P}<0.05)$.

\begin{tabular}{|c|c|c|c|c|c|c|c|c|c|c|c|c|c|c|c|c|c|c|c|c|c|c|c|}
\hline Neutrality tests & A 1 & $\mathrm{CZ} 1$ & D 1 & D 2 & D 3 & D 4 & D 5 & D 7 & D 8 & D 9 & DK 1 & DK 2 & DK 3 & F 1 & F 2 & F 3 & F 4 & FIN 1 & GB 1 & GB 2 & I 1 & I 2 & N 1 \\
\hline \multicolumn{24}{|l|}{ Tajima's D test } \\
\hline Samplesize & 2 & 5 & 14 & 9 & 3 & 9 & 5 & 4 & 3 & 4 & 3 & 5 & 3 & 6 & 4 & 5 & 2 & 3 & 2 & 3 & 10 & 4 & 3 \\
\hline S & 0 & 5 & 3 & 0 & 0 & 4 & 7 & 3 & 1 & 0 & 4 & 0 & 0 & 5 & 0 & 2 & 0 & 0 & 0 & 1 & 0 & 0 & 4 \\
\hline $\mathrm{Pi}$ & 0 & 1.9 & 0.43 & 0 & 0 & 2.11 & 3.5 & 1.83 & 0.67 & 0 & 2.67 & 0 & 0 & 2.53 & 0 & 0.8 & 0 & 0 & 0 & 0.67 & 0 & 0 & 2.67 \\
\hline Tajima's D & 0 & -1.4 & -1.67 & 0 & 0 & 1.77 & 0.29 & 1.09 & 0 & 0 & 0 & 0 & 0 & 0.88 & 0 & -0.97 & 0 & 0 & 0 & 0 & 0 & 0 & 0 \\
\hline Tajima's D p-value & 1 & 0 & 0.03 & 1 & 1 & 0.98 & 0.66 & 0.85 & 0.99 & 1 & 0.84 & 1 & 1 & 0.81 & 1 & 0.2 & 1 & 1 & 1 & 0.99 & 1 & 1 & 0.84 \\
\hline \multicolumn{24}{|l|}{ Fu's FS test } \\
\hline Real no. of alleles & 1 & 4 & 3 & 1 & 1 & 5 & 3 & 3 & 2 & 1 & 3 & 1 & 1 & 3 & 1 & 4 & 1 & 2 & 2 & 2 & 2 & 1 & 2 \\
\hline Orig. no. of alleles & 1 & 4 & 3 & 1 & 1 & 5 & 3 & 3 & 2 & 1 & 3 & 1 & 1 & 3 & 1 & 4 & 1 & 2 & 2 & 2 & 2 & 1 & 2 \\
\hline Theta_pi & 0 & 1.9 & 0.43 & 0 & 0 & 2.11 & 3.5 & 1.83 & 0.67 & 0 & 2.67 & 0 & 0 & 2.53 & 0 & 0.8 & 0 & 0 & 0 & 0.67 & 0 & 0 & 2.67 \\
\hline Exp. no. of alleles & 0 & 2.85 & 2.14 & 0 & 0 & 3.95 & 3.42 & 2.5 & 1.65 & 0 & 2.3 & 0 & 0 & 3.46 & 0 & 2.11 & 0 & 2 & 0 & 1.65 & 2 & 0 & 2.3 \\
\hline FS & 0 & 0.56 & -0.76 & 0 & 0 & 1.92 & 1.62 & 0.01 & 0.2 & 0 & -0.34 & 0 & 0 & 1.47 & 0 & 1.04 & 0 & 0 & 0 & 0.2 & 0 & 0 & 2.02 \\
\hline FS p-value & N.A. & 0.54 & 0.08 & N.A. & N.A. & 0.85 & 0.76 & 0.3 & 0.39 & N.A. & 0.19 & N.A. & N.A. & 0.77 & N.A. & 0.62 & N.A. & N.A. & N.A. & 0.39 & N.A. & N.A. & 0.76 \\
\hline
\end{tabular}

\begin{tabular}{|c|c|c|c|c|c|c|c|c|c|c|c|c|c|c|}
\hline Neutrality tests & NL 1 & PL 1 & PL 2 & RUM 1 & RUM 2 & RUM 3 & RUM 4 & RUM 5 & RUM 6 & RUS 1 & RUS 2 & S 2 & mean & s. d. \\
\hline \multicolumn{15}{|l|}{ Tajima's D test } \\
\hline Samplesize & 3 & 6 & 3 & 3 & 4 & 4 & 3 & 4 & 3 & 4 & 4 & 3 & 4.43 & 2.52 \\
\hline S & 1 & 3 & 1 & 0 & 0 & 1 & 0 & 1 & 3 & 6 & 0 & 3 & 1.66 & 2.04 \\
\hline $\mathrm{Pi}$ & 0.67 & 1.6 & 0.67 & 0 & 0 & 0.5 & 0 & 0.5 & 2 & 3 & 0 & 2 & 0.88 & 1.09 \\
\hline Tajima's D & 0 & 1.12 & 0 & 0 & 0 & -0.61 & 0 & -0.61 & 0 & -0.81 & 0 & 0 & -0.03 & 0.63 \\
\hline Tajima's D p-value & 0.99 & 0.88 & 0.98 & 1 & 1 & 0.38 & 1 & 0.38 & 0.88 & 0.15 & 1 & 0.88 & 0.82 & 0.31 \\
\hline \multicolumn{15}{|l|}{ Fu's FS test } \\
\hline Real no. of alleles & 2 & 5 & 2 & 1 & 2 & 2 & 1 & 2 & 2 & 4 & 1 & 3 & 2.17 & 1.18 \\
\hline Orig. no. of alleles & 2 & 5 & 2 & 1 & 2 & 2 & 1 & 2 & 2 & 4 & 1 & 3 & 2.17 & 1.18 \\
\hline Theta_pi & 0.67 & 1.6 & 0.67 & 0 & 0 & 0.5 & 0 & 0.5 & 2 & 3 & 0 & 2 & 0.88 & 1.09 \\
\hline Exp.no. of alleles & 1.65 & 2.94 & 1.65 & 0 & 2 & 1.68 & 0 & 1.68 & 2.17 & 2.85 & 0 & 2.17 & 1.46 & 1.25 \\
\hline FS & 0.2 & 2.51 & 0.2 & 0 & 0 & 0.17 & 0 & 0.17 & 1.61 & 0.73 & 0 & 1.61 & 0.43 & 0.77 \\
\hline FS p-value & 0.37 & 0.87 & 0.39 & N.A. & N.A. & 0.34 & N.A. & 0.35 & 0.69 & 0.57 & N.A. & 0.7 & N.A. & N.A. \\
\hline
\end{tabular}


Table A41: Results of the McDonald-Kreitman test for Steganacarus magnus. The differences between 64 populations are significant $(* 0.01<\mathrm{P}<0.05)$, between other 64 populations high significant $(* * 0.001<\mathrm{P}<0.01)$ and between 41 populations extremely high significant $(* * * \mathrm{P}<0.001)$. Number of fixed and polymorph synonymous and non-synonymous mutations are shown. Locations with less than two individuals were excluded.

\begin{tabular}{|c|c|c|c|c|c|c|c|c|c|c|c|c|c|}
\hline \multirow{2}{*}{$\begin{array}{c}\text { Population } \\
\text { CZ } 1\end{array}$} & & \multicolumn{3}{|c|}{$\begin{array}{c}\text { A 1 } \\
\text { syn. nons. sign. }\end{array}$} & \multicolumn{3}{|c|}{$\begin{array}{c}\text { CZ 1 } \\
\text { syn. nons. sign. }\end{array}$} & \multicolumn{3}{|c|}{$\begin{array}{c}\text { D 1 } \\
\text { syn. nons. sign. }\end{array}$} & \multicolumn{3}{|c|}{$\begin{array}{c}\text { D } 2 \\
\text { syn. nons. sign. }\end{array}$} \\
\hline & fixed & 50 & 3 & $0.0303 *$ & & & & & & & & & \\
\hline Decin & poly & 151 & 32 & & & & & & & & & & \\
\hline D 1 & fixed & 98 & 45 & $0.2658 \mathrm{~ns}$ & 32 & 18 & $0.0263 *$ & & & & & & \\
\hline Kranichstein & poly & 15 & 12 & & 162 & 42 & & & & & & & \\
\hline D 2 & fixed & 99 & 46 & $0.1782 \mathrm{~ns}$ & 33 & 18 & $0.0107^{*}$ & 0 & 0 & $-n s$ & & & \\
\hline Goettingen & poly & 6 & 0 & & 155 & 32 & & 18 & 11 & & & & \\
\hline D 3 & fixed & 109 & 48 & $0.1063 \mathrm{~ns}$ & 40 & 19 & $0.0157^{*}$ & 70 & 11 & $0.0375^{*}$ & 70 & 12 & $0.2038 \mathrm{~ns}$ \\
\hline Lake Constance & poly & 8 & 0 & & 158 & 32 & & 24 & 11 & & 14 & 0 & \\
\hline D 4 & fixed & 42 & 5 & $0.4955 \mathrm{~ns}$ & 6 & 0 & $0.599 \mathrm{~ns}$ & 27 & 18 & $0.0028^{* *}$ & 28 & 18 & $0.0007^{* * *}$ \\
\hline Meckl. Seenpl. & poly & 167 & 30 & & 252 & 43 & & 177 & 40 & & 169 & 30 & \\
\hline D 5 & fixed & 55 & 6 & $0.0186^{*}$ & 9 & 1 & $0.693 \mathrm{~ns}$ & 21 & 7 & $1 \mathrm{~ns}$ & 20 & 8 & $0.6394 \mathrm{~ns}$ \\
\hline Moerfelden & poly & 153 & 49 & & 237 & 59 & & 165 & 58 & & 157 & 49 & \\
\hline D 7 & fixed & 64 & 10 & $0.8399 \mathrm{~ns}$ & 24 & 2 & $0.3923 \mathrm{~ns}$ & 55 & 30 & $0.0081^{* *}$ & 54 & 30 & $0.0004^{* * *}$ \\
\hline Uelzen & poly & 115 & 20 & & 224 & 42 & & 125 & 30 & & 119 & 20 & \\
\hline D 8 & fixed & 100 & 48 & $1 \mathrm{~ns}$ & 43 & 23 & $0.0092 * *$ & 87 & 13 & $0.0022 * *$ & 87 & 15 & $1 \mathrm{~ns}$ \\
\hline Cuxhaven & poly & 1 & 1 & & 151 & 33 & & 17 & 12 & & 7 & 1 & \\
\hline D 9 & fixed & 99 & 46 & $0.1098 \mathrm{~ns}$ & 33 & 18 & $0.011 *$ & 0 & 0 & $-n s$ & 0 & 0 & $-n s$ \\
\hline Bonn & poly & 11 & 1 & & 158 & 33 & & 18 & 11 & & 13 & 1 & \\
\hline DK 1 & fixed & 72 & 24 & $0.2458 \mathrm{~ns}$ & 9 & 2 & $1 \mathrm{~ns}$ & 0 & 0 & $-n s$ & 0 & 0 & $-n s$ \\
\hline Copenhagen & poly & 94 & 46 & & 202 & 61 & & 108 & 56 & & 99 & 45 & \\
\hline DK 2 & fixed & 101 & 15 & $-n s$ & 47 & 3 & $0.0457^{*}$ & 79 & 39 & $0.5033 \mathrm{~ns}$ & 80 & 39 & $0.1756 \mathrm{~ns}$ \\
\hline Hjorring & poly & 0 & 0 & & 151 & 32 & & 16 & 11 & & 6 & 0 & \\
\hline DK 3 & fixed & 100 & 46 & $0.3197 \mathrm{~ns}$ & 35 & 18 & $0.0216^{*}$ & 0 & 0 & $-n s$ & 0 & 0 & $-n s$ \\
\hline Arhus & poly & 0 & 1 & & 151 & 33 & & 16 & 12 & & 6 & 1 & \\
\hline F 1 & fixed & 51 & 4 & $0.0878 \mathrm{~ns}$ & 0 & 0 & $-n s$ & 26 & 17 & $0.0103^{*}$ & 30 & 17 & $0.0047^{* *}$ \\
\hline Mont Blanc & poly & 167 & 34 & & 237 & 39 & & 175 & 45 & & 169 & 34 & \\
\hline F 2 & fixed & 101 & 46 & $0.5703 \mathrm{~ns}$ & 35 & 18 & $0.0125^{*}$ & 0 & 0 & $-n s$ & 0 & 0 & $-n s$ \\
\hline Loire & poly & 2 & 0 & & 153 & 32 & & 18 & 11 & & 8 & 0 & \\
\hline F 3 & fixed & 80 & 46 & $0.0018^{* *}$ & 29 & 20 & $0.001^{* *}$ & 68 & 10 & $0.1147 \mathrm{~ns}$ & 67 & 12 & $1 \mathrm{~ns}$ \\
\hline Saint Isidore & poly & 60 & 11 & & 187 & 41 & & 73 & 22 & & 65 & 11 & \\
\hline F 4 & fixed & 101 & 12 & $-n s$ & 45 & 2 & $0.021 *$ & 76 & 40 & $0.6561 \mathrm{~ns}$ & 76 & 40 & $0.1009 \mathrm{~ns}$ \\
\hline Haute Loire & poly & 0 & 0 & & 151 & 32 & & 16 & 11 & & 6 & 0 & \\
\hline FIN 1 & fixed & 106 & 18 & $0.004^{* *} *$ & 60 & 9 & $0.352 \mathrm{~ns}$ & 89 & 37 & $0.0843 \mathrm{~ns}$ & 92 & 37 & $1 \mathrm{~ns}$ \\
\hline Lahti & poly & 0 & 3 & & 151 & 35 & & 16 & 14 & & 6 & 3 & \\
\hline GB 1 & fixed & 101 & 49 & $1 \mathrm{~ns}$ & 46 & 24 & $0.0071^{* *}$ & 92 & 14 & $0.0023^{* *}$ & 93 & 16 & $1 \mathrm{~ns}$ \\
\hline Ascot & poly & 1 & 1 & & 151 & 33 & & 17 & 12 & & 6 & 1 & \\
\hline GB 2 & fixed & 101 & 48 & $0.0447^{*}$ & 46 & 23 & $0.0287^{*}$ & 93 & 13 & $0.0001^{* * *}$ & 93 & 15 & $0.0741 \mathrm{~ns}$ \\
\hline Braemar & poly & 1 & 4 & & 152 & 36 & & 17 & 15 & & 7 & 4 & \\
\hline I 1 & fixed & 100 & 46 & $1 \mathrm{~ns}$ & 33 & 18 & $0.0127^{*}$ & 0 & 0 & $-n s$ & 0 & 0 & $-n s$ \\
\hline Grosseto & poly & 7 & 3 & & 157 & 35 & & 16 & 13 & & 11 & 3 & \\
\hline I 2 & fixed & 99 & 45 & $0.3467 \mathrm{~ns}$ & 33 & 17 & $0.018^{*}$ & 0 & 0 & $-n s$ & 0 & 0 & $-n s$ \\
\hline Parma & poly & 11 & 2 & & 160 & 34 & & 18 & 12 & & 13 & 2 & \\
\hline $\mathrm{N} 1$ & fixed & 69 & 5 & $0.0041^{* *}$ & 2 & 0 & $1 \mathrm{~ns}$ & 56 & 24 & $0.6517 \mathrm{~ns}$ & 55 & 14 & $0.1488 \mathrm{~ns}$ \\
\hline Narvik & poly & 112 & 31 & & 187 & 37 & & 121 & 45 & & 116 & 32 & \\
\hline NL 1 & fixed & 98 & 48 & $0.7192 \mathrm{~ns}$ & 43 & 23 & $0.0094^{* *}$ & 88 & 13 & $0.0052^{* *}$ & 88 & 15 & $1 \mathrm{~ns}$ \\
\hline Wageningen & poly & 7 & 2 & & 155 & 34 & & 23 & 13 & & 13 & 2 & \\
\hline PL 1 & fixed & 66 & 8 & $0.0197^{*}$ & 1 & 0 & $1 \mathrm{~ns}$ & 41 & 22 & $0.3333 \mathrm{~ns}$ & 40 & 22 & $0.0899 \mathrm{~ns}$ \\
\hline Krakow & poly & 111 & 36 & & 200 & 40 & & 122 & 47 & & 117 & 36 & \\
\hline PL 2 & fixed & 106 & 44 & $0.559 \mathrm{~ns}$ & 32 & 20 & $0.0026^{* *}$ & 62 & 18 & $0.1947 \mathrm{~ns}$ & 59 & 20 & $0.3876 \mathrm{~ns}$ \\
\hline Warsaw & poly & 12 & 3 & & 156 & 34 & & 27 & 14 & & 18 & 3 & \\
\hline RUM 1 & fixed & 104 & 44 & $0.5564 \mathrm{~ns}$ & 40 & 18 & $0.039^{*}$ & 72 & 14 & $0.0366^{*}$ & 74 & 16 & $0.3476 \mathrm{~ns}$ \\
\hline Sibiu 1 & poly & 3 & 0 & & 154 & 32 & & 19 & 11 & & 9 & 0 & \\
\hline RUM 2 & fixed & 101 & 43 & $0.5108 \mathrm{~ns}$ & 40 & 18 & $0.04^{*}$ & 70 & 13 & $0.0338^{*}$ & 72 & 15 & $0.7207 \mathrm{~ns}$ \\
\hline Sibiu 2 & poly & 10 & 2 & & 156 & 33 & & 26 & 13 & & 16 & 2 & \\
\hline RUM 3 & fixed & 88 & 5 & $0.119 \mathrm{~ns}$ & 24 & 1 & $0.0911 \mathrm{~ns}$ & 87 & 43 & $0.8555 \mathrm{~ns}$ & 91 & 43 & $0.0548 \mathrm{~ns}$ \\
\hline Bagau & poly & 15 & 3 & & 158 & 33 & & 31 & 14 & & 21 & 3 & \\
\hline
\end{tabular}


Table A41 continue:

\begin{tabular}{|c|c|c|c|c|c|c|c|c|c|c|c|c|c|}
\hline \multicolumn{2}{|l|}{ Population } & \multicolumn{3}{|c|}{$\begin{array}{c}\text { A } 1 \\
\text { svn. nons. sign. }\end{array}$} & \multicolumn{3}{|c|}{ CZ 1} & \multicolumn{3}{|c|}{$\begin{array}{c}\text { D 1 } \\
\text { syn. nons. sign. }\end{array}$} & \multicolumn{3}{|c|}{$\begin{array}{c}\text { D 2 } \\
\text { syn. nons. sign. }\end{array}$} \\
\hline RUM 4 & fixed & 92 & 5 & $1 \mathrm{~ns}$ & 42 & 2 & $0.0358^{*}$ & 92 & 44 & $1 \mathrm{~ns}$ & 96 & 44 & $0.0117^{*}$ \\
\hline Cluj Nap oca & poly & 7 & 0 & & 170 & 34 & & 23 & 11 & & 13 & 0 & \\
\hline RUM 5 & fixed & 108 & 43 & $1 \mathrm{~ns}$ & 42 & 19 & $0.0464 *$ & 70 & 18 & $0.0624 \mathrm{~ns}$ & 71 & 20 & $1 \mathrm{~ns}$ \\
\hline Busteni & poly & 5 & 2 & & 154 & 34 & & 21 & 13 & & 10 & 2 & \\
\hline RUM 6 & fixed & 85 & 37 & $0.1172 \mathrm{~ns}$ & 17 & 13 & $0.0034^{* *}$ & 37 & 5 & $0.1229 \mathrm{~ns}$ & 39 & 6 & $0.4792 \mathrm{~ns}$ \\
\hline Sinaia & poly & 75 & 19 & & 201 & 45 & & 89 & 29 & & 78 & 19 & \\
\hline RUS 1 & fixed & 60 & 3 & $0.119 \mathrm{~ns}$ & 20 & 2 & $0.5461 \mathrm{~ns}$ & 66 & 34 & $0.0068^{*}$ & 67 & 35 & $0.0001^{* * *}$ \\
\hline Altai Mountains & poly & 116 & 16 & & 221 & 43 & & 126 & 28 & & 120 & 16 & \\
\hline RUS 2 & fixed & 112 & 17 & $-n s$ & 63 & 8 & $0.2544 \mathrm{~ns}$ & 110 & 44 & $0.2565 \mathrm{~ns}$ & 110 & 44 & $0.1890 \mathrm{~ns}$ \\
\hline Novosibirsk & poly & 0 & 0 & & 151 & 32 & & 16 & 11 & & 6 & 0 & \\
\hline S 2 & fixed & 55 & 6 & $0.02788^{*}$ & 0 & 0 & $-n s$ & 29 & 15 & $0.2598 \mathrm{~ns}$ & 30 & 16 & $0.0873 \mathrm{~ns}$ \\
\hline Stroemstad & poly & 157 & 46 & & 229 & 54 & & 168 & 55 & & 162 & 46 & \\
\hline
\end{tabular}

\begin{tabular}{|c|c|c|c|c|c|c|c|c|c|c|c|c|c|}
\hline \multirow{2}{*}{$\begin{array}{c}\text { Population } \\
\text { D } 4\end{array}$} & \multicolumn{4}{|c|}{$\begin{array}{c}\text { D 3 } \\
\text { ns. sign. }\end{array}$} & \multicolumn{3}{|c|}{$\begin{array}{c}\text { D 4 } \\
\text { syn. nons. sign. }\end{array}$} & \multicolumn{3}{|c|}{$\begin{array}{c}\text { D 5 } \\
\text { syn. nons. sign. }\end{array}$} & \multicolumn{3}{|c|}{$\begin{array}{c}\text { D 7 } \\
\text { syn. nons. sign. }\end{array}$} \\
\hline & fixed & 29 & 15 & $0.0048^{* *}$ & & & & & & & & & \\
\hline Meckl. Seenpl. & poly & 172 & 30 & & & & & & & & & & \\
\hline D 5 & fixed & 27 & 7 & $0.8279 \mathrm{~ns}$ & 0 & 0 & $-\mathrm{ns}$ & & & & & & \\
\hline Moerfelden & poly & 147 & 49 & & 240 & 57 & & & & & & & \\
\hline D 7 & fixed & 48 & 34 & $0.0000^{* * *} *$ & 13 & 1 & $0.4884 \mathrm{~ns}$ & 17 & 1 & $0.1395 \mathrm{~ns}$ & & & \\
\hline Uelzen & poly & 122 & 20 & & 226 & 42 & & 223 & 58 & & & & \\
\hline D 8 & fixed & 90 & 17 & $1 \mathrm{~ns}$ & 42 & 23 & $0.0012 * *$ & 40 & 9 & $0.4528 \mathrm{~ns}$ & 55 & 32 & $0.0004^{* * *} *$ \\
\hline Cuxhaven & poly & 9 & 1 & & 167 & 31 & & 153 & 50 & & 116 & 21 & \\
\hline D 9 & fixed & 69 & 11 & $0.4505 \mathrm{~ns}$ & 27 & 18 & $0.0006^{* * * *}$ & 21 & 7 & $1 \mathrm{~ns}$ & 55 & 30 & $0.0005^{* * * *}$ \\
\hline Bonn & poly & 19 & 1 & & 172 & 31 & & 160 & 49 & & 120 & 21 & \\
\hline DK 1 & fixed & 42 & 4 & $0.0018^{* *}$ & 0 & 0 & $-n s$ & 5 & 1 & $0.6895 \mathrm{~ns}$ & 32 & 12 & $0.8502 \mathrm{~ns}$ \\
\hline Copenhagen & poly & 100 & 46 & & 193 & 52 & & 190 & 69 & & 179 & 59 & \\
\hline DK 2 & fixed & 79 & 41 & $0.0541 \mathrm{~ns}$ & 0 & 0 & $-n s$ & 8 & 1 & $0.4647 \mathrm{~ns}$ & 53 & 4 & $0.1583 \mathrm{~ns}$ \\
\hline Hjorring & poly & 8 & 0 & & 167 & 30 & & 153 & 49 & & 115 & 20 & \\
\hline DK 3 & fixed & 73 & 12 & $1 \mathrm{~ns}$ & 28 & 18 & $0.0008 * * *$ & 21 & 8 & $0.8188 \mathrm{~ns}$ & 57 & 30 & $0.0017^{* *}$ \\
\hline Arhus & poly & 8 & 1 & & 167 & 31 & & 153 & 50 & & 115 & 21 & \\
\hline F 1 & fixed & 31 & 20 & $0.0009^{* * *} *$ & 6 & 0 & $0.5979 \mathrm{~ns}$ & 6 & 1 & $1 \mathrm{~ns}$ & 16 & 0 & $0.1408 \mathrm{~ns}$ \\
\hline Mont Blanc & poly & 173 & 34 & & 247 & 43 & & 247 & 60 & & 227 & 42 & \\
\hline F 2 & fixed & 71 & 12 & $0.3498 \mathrm{~ns}$ & 29 & 18 & $0.0008 * * *$ & 24 & 8 & $1 \mathrm{~ns}$ & 56 & 30 & $0.0008^{* * *} *$ \\
\hline Loire & poly & 10 & 0 & & 168 & 30 & & 155 & 49 & & 117 & 20 & \\
\hline F 3 & fixed & 67 & 13 & $0.8254 \mathrm{~ns}$ & 24 & 19 & $0.0001^{* * *}$ & 24 & 6 & $0.82 \mathrm{~ns}$ & 45 & 30 & $0.0002^{* * * *}$ \\
\hline Saint Isidore & poly & 67 & 11 & & 207 & 40 & & 190 & 59 & & 156 & 31 & \\
\hline F 4 & fixed & 77 & 42 & $0.0517 \mathrm{~ns}$ & 6 & 1 & $1 \mathrm{~ns}$ & 0 & 1 & $0.2463 \mathrm{~ns}$ & 55 & 3 & $0.0586 \mathrm{~ns}$ \\
\hline Haute Loire & poly & 8 & 0 & & 167 & 30 & & 153 & 49 & & 115 & 20 & \\
\hline FIN 1 & fixed & 92 & 39 & $1 \mathrm{~ns}$ & 40 & 7 & $0.8315 \mathrm{~ns}$ & 43 & 4 & $0.0111^{*}$ & 55 & 5 & $0.1294 \mathrm{~ns}$ \\
\hline Lahti & poly & 8 & 3 & & 167 & 33 & & 153 & 52 & & 115 & 23 & \\
\hline GB 1 & fixed & 92 & 18 & $1 \mathrm{~ns}$ & 45 & 24 & $0.0016^{* *}$ & 43 & 9 & $0.2784 \mathrm{~ns}$ & 58 & 33 & $0.0004^{* * * *}$ \\
\hline Ascot & poly & 9 & 1 & & 167 & 31 & & 153 & 50 & & 116 & 21 & \\
\hline GB 2 & fixed & 93 & 17 & $0.2337 \mathrm{~ns}$ & 45 & 23 & $0.0055^{* *}$ & 43 & 8 & $0.1462 \mathrm{~ns}$ & 58 & 32 & $0.0014^{* *}$ \\
\hline Braemar & poly & 9 & 4 & & 168 & 34 & & 154 & 53 & & 116 & 23 & \\
\hline I 1 & fixed & 70 & 11 & $0.7153 \mathrm{~ns}$ & 27 & 18 & $0.0008^{* *}$ & 22 & 7 & $1 \mathrm{~ns}$ & 55 & 30 & $0.0019^{* *}$ \\
\hline Grosseto & poly & 15 & 3 & & 171 & 33 & & 158 & 51 & & 119 & 23 & \\
\hline I 2 & fixed & 69 & 11 & $0.7317 \mathrm{~ns}$ & 27 & 18 & $0.0006^{* * *}$ & 21 & 7 & $1 \mathrm{~ns}$ & 55 & 30 & $0.0005^{* * *} *$ \\
\hline Parma & poly & 19 & 2 & & 173 & 31 & & 160 & 49 & & 121 & 21 & \\
\hline N 1 & fixed & 60 & 24 & $0.341 \mathrm{~ns}$ & 11 & 0 & $0.3716 \mathrm{~ns}$ & 15 & 0 & $0.0469^{*}$ & 34 & 5 & $0.5013 \mathrm{~ns}$ \\
\hline Narvik & poly & 116 & 33 & & 232 & 39 & & 221 & 63 & & 201 & 45 & \\
\hline NL 1 & fixed & 88 & 17 & $0.7403 \mathrm{~ns}$ & 42 & 23 & $0.0013^{* *}$ & 40 & 8 & $0.3404 \mathrm{~ns}$ & 55 & 32 & $0.0004^{* * * *}$ \\
\hline Wageningen & poly & 15 & 2 & & 171 & 32 & & 158 & 50 & & 119 & 22 & \\
\hline PL 1 & fixed & 41 & 24 & $0.048^{*}$ & 0 & 0 & $-n s$ & 0 & 0 & $-n s$ & 30 & 1 & $0.0151^{*}$ \\
\hline Krakow & poly & 117 & 36 & & 213 & 42 & & 202 & 63 & & 194 & 49 & \\
\hline
\end{tabular}


Table A41 continue:

\begin{tabular}{|c|c|c|c|c|c|c|c|c|c|c|c|c|c|}
\hline \multirow{2}{*}{$\begin{array}{c}\text { Population } \\
\text { PL } 2\end{array}$} & \multirow[b]{2}{*}{ fixed } & \multicolumn{3}{|c|}{$\begin{array}{c}\text { D 3 } \\
\text { syn. nons. sign. }\end{array}$} & \multicolumn{3}{|c|}{$\begin{array}{c}\text { D } 4 \\
\text { syn. nons. sign. }\end{array}$} & \multicolumn{3}{|c|}{$\begin{array}{c}\text { D 5 } \\
\text { syn. nons. sign. }\end{array}$} & \multicolumn{3}{|c|}{$\begin{array}{c}\text { D } 7 \\
\text { syn. nons. sign. }\end{array}$} \\
\hline & & 75 & 17 & $0.7601 \mathrm{~ns}$ & 32 & 13 & $0.0068 * *$ & 29 & 6 & $0.5146 \mathrm{~ns}$ & 43 & 29 & $0.0001^{* * *} *$ \\
\hline Warsaw & poly & 20 & 3 & & 172 & 32 & & 159 & 49 & & 122 & 22 & \\
\hline RUM 1 & fixed & 80 & 16 & $0.3532 \mathrm{~ns}$ & 37 & 14 & $0.0614 \mathrm{~ns}$ & 37 & 4 & $0.0403 *$ & 59 & 28 & $0.0024^{* *}$ \\
\hline Sibiu 1 & poly & 10 & 0 & & 169 & 30 & & 154 & 49 & & 118 & 20 & \\
\hline RUM 2 & fixed & 78 & 15 & $0.7326 \mathrm{~ns}$ & 37 & 14 & $0.0627 \mathrm{~ns}$ & 36 & 4 & $0.059 \mathrm{~ns}$ & 57 & 28 & $0.0014^{* *}$ \\
\hline Sibiu 2 & poly & 17 & 2 & & 172 & 31 & & 158 & 50 & & 124 & 21 & \\
\hline RUM 3 & fixed & 81 & 46 & $0.0123^{*}$ & 30 & 3 & $0.4387 \mathrm{~ns}$ & 40 & 5 & $0.0726 \mathrm{~ns}$ & 52 & 8 & $0.8308 \mathrm{~ns}$ \\
\hline Bagau & poly & 23 & 3 & & 174 & 30 & & 162 & 50 & & 126 & 23 & \\
\hline RUM 4 & fixed & 83 & 46 & $0.0028^{* *}$ & 29 & 3 & $0.586 \mathrm{~ns}$ & 43 & 5 & $0.0493^{*}$ & 55 & 10 & $0.8342 \mathrm{~ns}$ \\
\hline Cluj Nap oca & poly & 15 & 0 & & 172 & 30 & & 156 & 49 & & 120 & 20 & \\
\hline RUM 5 & fixed & 79 & 18 & $0.7365 \mathrm{~ns}$ & 35 & 16 & $0.0165^{*}$ & 32 & 5 & $0.1448 \mathrm{~ns}$ & 55 & 29 & $0.0016^{* *}$ \\
\hline Busteni & poly & 13 & 2 & & 169 & 32 & & 154 & 51 & & 119 & 22 & \\
\hline RUM 6 & fixed & 44 & 7 & $0.4977 \mathrm{~ns}$ & 18 & 12 & $0.0052 * *$ & 11 & 2 & $0.5373 \mathrm{~ns}$ & 36 & 25 & $0.0004^{* * *}$ \\
\hline Sinaia & poly & 80 & 19 & & 206 & 41 & & 192 & 61 & & 165 & 36 & \\
\hline RUS 1 & fixed & 53 & 37 & $0.0000^{* * *} *$ & 17 & 1 & $0.4845 \mathrm{~ns}$ & 22 & 1 & $0.0576 \mathrm{~ns}$ & 31 & 4 & $0.6231 \mathrm{~ns}$ \\
\hline Altai Mountains & poly & 123 & 16 & & 235 & 40 & & 228 & 61 & & 195 & 35 & \\
\hline RUS 2 & fixed & 105 & 48 & $0.1065 \mathrm{~ns}$ & 46 & 8 & $1 \mathrm{~ns}$ & 57 & 7 & $0.0226^{*}$ & 61 & 9 & $0.8336 \mathrm{~ns}$ \\
\hline Novosibirsk & poly & 8 & 0 & & 167 & 30 & & 153 & 49 & & 115 & 20 & \\
\hline $\mathrm{S} 2$ & fixed & 29 & 14 & $0.1682 \mathrm{~ns}$ & 0 & 0 & $-n s$ & 2 & 0 & $1 \mathrm{~ns}$ & 20 & 1 & $0.1411 \mathrm{~ns}$ \\
\hline Stroemstad & poly & 163 & 46 & & 246 & 52 & & 225 & 67 & & 232 & 55 & \\
\hline
\end{tabular}

\begin{tabular}{|c|c|c|c|c|c|c|c|c|c|c|c|c|c|}
\hline \multirow{2}{*}{$\begin{array}{c}\text { Population } \\
\text { D } 9\end{array}$} & & \multicolumn{3}{|c|}{$\begin{array}{c}\text { D 8 } \\
\text { syn. nons. sign. }\end{array}$} & \multicolumn{3}{|c|}{$\begin{array}{c}\text { D 9 } \\
\text { syn. nons. sign. }\end{array}$} & \multicolumn{3}{|c|}{$\begin{array}{r}\text { DK 1 } \\
\text { syn. nons. sign. }\end{array}$} & \multicolumn{3}{|c|}{$\begin{array}{r}\text { DK 2 } \\
\text { syn. nons. sign. }\end{array}$} \\
\hline & fixed & 87 & 14 & $1 \mathrm{~ns}$ & & & & & & & & & \\
\hline Bonn & poly & 12 & 2 & & & & & & & & & & \\
\hline DK 1 & fixed & 54 & 9 & $0.0062 * *$ & 0 & 0 & $-n s$ & & & & & & \\
\hline Copenhagen & poly & 95 & 47 & & 104 & 46 & & & & & & & \\
\hline DK 2 & fixed & 101 & 45 & $1 \mathrm{~ns}$ & 78 & 39 & $0.103 \mathrm{~ns}$ & 46 & 17 & $0.4187 \mathrm{~ns}$ & & & \\
\hline Hjorring & poly & 1 & 1 & & 11 & 1 & & 94 & 46 & & & & \\
\hline DK 3 & fixed & 91 & 15 & $0.0628 \mathrm{~ns}$ & 0 & 0 & $-n s$ & 0 & 0 & $-n s$ & 82 & 39 & $0.3279 \mathrm{~ns}$ \\
\hline Arhus & poly & 1 & 2 & & 11 & 2 & & 95 & 46 & & 0 & 1 & \\
\hline F 1 & fixed & 46 & 25 & $0.0026^{* *}$ & 28 & 17 & $0.0038^{* *}$ & 12 & 2 & $0.5379 \mathrm{~ns}$ & 34 & 1 & $0.0362 *$ \\
\hline Mont Blanc & poly & 167 & 35 & & 171 & 35 & & 212 & 63 & & 167 & 34 & \\
\hline F 2 & fixed & 89 & 15 & $0.4786 \mathrm{~ns}$ & 0 & 0 & $-n s$ & 4 & 0 & $0.3107 \mathrm{~ns}$ & 83 & 39 & $0.5653 \mathrm{~ns}$ \\
\hline Loire & poly & 3 & 1 & & 13 & 1 & & 96 & 45 & & 2 & 0 & \\
\hline F 3 & fixed & 2 & 0 & $1 \mathrm{~ns}$ & 69 & 11 & $0.8252 \mathrm{~ns}$ & 40 & 6 & $0.0265^{*}$ & 82 & 42 & $0.0071^{* *}$ \\
\hline Saint Isidore & poly & 60 & 12 & & 67 & 12 & & 139 & 56 & & 60 & 11 & \\
\hline F 4 & fixed & 99 & 46 & $1 \mathrm{~ns}$ & 75 & 40 & $0.1015 \mathrm{~ns}$ & 44 & 17 & $0.513 \mathrm{~ns}$ & 17 & 3 & $-n s$ \\
\hline Haute Loire & poly & 1 & 1 & & 11 & 1 & & 94 & 46 & & 0 & 0 & \\
\hline FIN 1 & fixed & 106 & 39 & $0.024 *$ & 90 & 37 & $1 \mathrm{~ns}$ & 63 & 21 & $0.183 \mathrm{~ns}$ & 94 & 16 & $0.0041^{* *} *$ \\
\hline Lahti & poly & 1 & 4 & & 11 & 4 & & 95 & 48 & & 0 & 3 & \\
\hline GB 1 & fixed & 6 & 1 & $0.4909 \mathrm{~ns}$ & 92 & 15 & $1 \mathrm{~ns}$ & 60 & 10 & $0.0031^{* *}$ & 103 & 46 & $1 \mathrm{~ns}$ \\
\hline Ascot & poly & 2 & 2 & & 12 & 2 & & 94 & 47 & & 1 & 1 & \\
\hline GB 2 & fixed & 6 & 0 & $0.021 *$ & 93 & 14 & $0.1377 \mathrm{~ns}$ & 60 & 9 & $0.00098 * * *$ & 104 & 45 & $0.0357^{*}$ \\
\hline Braemar & poly & 2 & 5 & & 12 & 5 & & 95 & 50 & & 1 & 4 & \\
\hline I 1 & fixed & 90 & 14 & $0.0904 \mathrm{~ns}$ & 0 & 0 & $-n s$ & 2 & 0 & $0.5619 \mathrm{~ns}$ & 76 & 39 & $1 \mathrm{~ns}$ \\
\hline Grosseto & poly & 8 & 4 & & 11 & 3 & & 100 & 48 & & 7 & 3 & \\
\hline I 2 & fixed & 87 & 14 & $0.6944 \mathrm{~ns}$ & 0 & 0 & $-n s$ & 0 & 0 & $-n s$ & 79 & 38 & $0.3422 \mathrm{~ns}$ \\
\hline Parma & poly & 12 & 3 & & 13 & 2 & & 103 & 46 & & 11 & 2 & \\
\hline N 1 & fixed & 59 & 31 & $0.049^{*}$ & 56 & 24 & $0.2047 \mathrm{~ns}$ & 26 & 3 & $0.0444^{*}$ & 74 & 5 & $0.0023^{* *}$ \\
\hline Narvik & poly & 112 & 32 & & 119 & 34 & & 177 & 69 & & 111 & 32 & \\
\hline NL 1 & fixed & 0 & 0 & $-n s$ & 88 & 14 & $1 \mathrm{~ns}$ & 55 & 9 & $0.0066 * *$ & 101 & 45 & $0.7238 \mathrm{~ns}$ \\
\hline Wageningen & poly & 8 & 3 & & 18 & 3 & & 100 & 48 & & 7 & 2 & \\
\hline PL 1 & fixed & 60 & 30 & $0.1827 \mathrm{~ns}$ & 41 & 22 & $0.1299 \mathrm{~ns}$ & 14 & 3 & $0.413 \mathrm{~ns}$ & 0 & 0 & $-n s$ \\
\hline Krakow & poly & 111 & 37 & & 117 & 37 & & 173 & 69 & & 111 & 36 & \\
\hline
\end{tabular}


Table A41 continue:

\begin{tabular}{|c|c|c|c|c|c|c|c|c|c|c|c|c|c|}
\hline \multirow{2}{*}{$\begin{array}{c}\text { Population } \\
\text { PL } 2\end{array}$} & \multirow[b]{2}{*}{ fixed } & \multicolumn{3}{|c|}{$\begin{array}{c}\text { D 8 } \\
\text { syn. nons. sign. }\end{array}$} & \multicolumn{3}{|c|}{$\begin{array}{c}\text { D 9 } \\
\text { syn. nons. sign. }\end{array}$} & \multicolumn{3}{|c|}{$\begin{array}{r}\text { DK } 1 \\
\text { syn. nons. sign. } \\
\end{array}$} & \multicolumn{3}{|c|}{$\begin{array}{r}\text { DK 2 } \\
\text { syn. nons. sign. }\end{array}$} \\
\hline & & 83 & 20 & $0.745 \mathrm{~ns}$ & 61 & 19 & $0.4264 \mathrm{~ns}$ & 34 & 9 & $0.1887 \mathrm{~ns}$ & 87 & 35 & $0.5589 \mathrm{~ns}$ \\
\hline Warsaw & poly & 13 & 4 & & 22 & 4 & & 103 & 48 & & 12 & 3 & \\
\hline RUM 1 & fixed & 83 & 15 & $1 \mathrm{~ns}$ & 71 & 15 & $0.4544 \mathrm{~ns}$ & 44 & 7 & $0.0105^{*}$ & 89 & 37 & $0.5569 \mathrm{~ns}$ \\
\hline Sibiu 1 & poly & 4 & 1 & & 14 & 1 & & 96 & 46 & & 3 & 0 & \\
\hline RUM 2 & fixed & 81 & 15 & $0.6978 \mathrm{~ns}$ & 69 & 14 & $0.7578 \mathrm{~ns}$ & 43 & 7 & $0.0166^{*}$ & 87 & 36 & $0.5086 \mathrm{~ns}$ \\
\hline Sibiu 2 & poly & 11 & 3 & & 21 & 3 & & 101 & 47 & & 10 & 2 & \\
\hline RUM 3 & fixed & 88 & 47 & $0.2143 \mathrm{~ns}$ & 88 & 43 & $0.0447^{*}$ & 56 & 23 & $0.7647 \mathrm{~ns}$ & 87 & 11 & $0.693 \mathrm{~ns}$ \\
\hline Bagau & poly & 16 & 4 & & 25 & 4 & & 104 & 48 & & 15 & 3 & \\
\hline RUM 4 & fixed & 94 & 48 & $0.2725 \mathrm{~ns}$ & 93 & 44 & $0.0143^{*}$ & 58 & 23 & $0.6531 \mathrm{~ns}$ & 89 & 13 & $0.5955 \mathrm{~ns}$ \\
\hline Cluj Napoca & poly & 8 & 1 & & 18 & 1 & & 99 & 46 & & 7 & 0 & \\
\hline RUM 5 & fixed & 96 & 17 & $0.1649 \mathrm{~ns}$ & 69 & 19 & $0.758 \mathrm{~ns}$ & 43 & 10 & $0.0764 \mathrm{~ns}$ & 95 & 36 & $1 \mathrm{~ns}$ \\
\hline Busteni & poly & 6 & 3 & & 16 & 3 & & 98 & 47 & & 5 & 2 & \\
\hline RUM 6 & fixed & 59 & 7 & $0.0921 \mathrm{~ns}$ & 37 & 5 & $0.3389 \mathrm{~ns}$ & 18 & 3 & $0.2082 \mathrm{~ns}$ & 64 & 29 & $0.137 \mathrm{~ns}$ \\
\hline Sinaia & poly & 76 & 20 & & 83 & 20 & & 146 & 56 & & 74 & 20 & \\
\hline RUS 1 & fixed & 64 & 43 & $0.0000 * * *$ & 66 & 35 & $0.0001^{* * *}$ & 44 & 14 & $1 \mathrm{~ns}$ & 65 & 9 & $1 \mathrm{~ns}$ \\
\hline Altai Mountains & poly & 117 & 17 & & 121 & 17 & & 182 & 60 & & 116 & 16 & \\
\hline RUS 2 & fixed & 110 & 44 & $0.495 \mathrm{~ns}$ & 108 & 44 & $0.1824 \mathrm{~ns}$ & 78 & 22 & $0.0812 \mathrm{~ns}$ & 112 & 22 & $-n s$ \\
\hline Novosibirsk & poly & 1 & 1 & & 11 & 1 & & 94 & 46 & & 0 & 0 & \\
\hline S 2 & fixed & 48 & 18 & $0.5094 \mathrm{~ns}$ & 30 & 15 & $0.1233 \mathrm{~ns}$ & 9 & 2 & $0.7356 \mathrm{~ns}$ & 0 & 0 & $-\mathrm{ns}$ \\
\hline Stroemstad & poly & 157 & 47 & & 164 & 46 & & 206 & 71 & & 157 & 46 & \\
\hline
\end{tabular}

\begin{tabular}{|c|c|c|c|c|c|c|c|c|c|c|c|c|c|}
\hline \multirow{2}{*}{$\begin{array}{c}\text { Population } \\
\text { F } 1\end{array}$} & \multicolumn{4}{|c|}{$\begin{array}{r}\text { DK 3 } \\
\text { syn. nons. sign. }\end{array}$} & \multicolumn{3}{|c|}{$\begin{array}{c}\text { F } 1 \\
\text { syn. nons. sign. }\end{array}$} & \multicolumn{3}{|c|}{$\begin{array}{c}\text { F } 2 \\
\text { syn. nons. sign. }\end{array}$} & \multicolumn{3}{|c|}{$\begin{array}{c}\text { F 3 } \\
\text { syn. nons. sign. }\end{array}$} \\
\hline & fixed & 31 & 17 & $0.0086^{* *}$ & & & & & & & & & \\
\hline Mont Blanc & poly & 167 & 34 & & & & & & & & & & \\
\hline F 2 & fixed & 4 & 0 & $0.4286 \mathrm{~ns}$ & 32 & 17 & $0.0092 * *$ & & & & & & \\
\hline Loire & poly & 2 & 1 & & 168 & 34 & & & & & & & \\
\hline F 3 & fixed & 71 & 12 & $0.8245 \mathrm{~ns}$ & 31 & 20 & $0.0023^{* *}$ & 69 & 12 & $1 \mathrm{~ns}$ & & & \\
\hline Saint Isidore & poly & 60 & 12 & & 200 & 44 & & 62 & 11 & & & & \\
\hline F 4 & fixed & 78 & 40 & $0.3445 \mathrm{~ns}$ & 34 & 1 & $0.0362 *$ & 79 & 40 & $0.5537 \mathrm{~ns}$ & 79 & 44 & $0.0028^{* *}$ \\
\hline Haute Loire & poly & 0 & 1 & & 167 & 34 & & 2 & 0 & & 60 & 11 & \\
\hline FIN 1 & fixed & 95 & 37 & $0.0074^{* *}$ & 36 & 2 & $0.054 \mathrm{~ns}$ & 94 & 37 & $0.1514 \mathrm{~ns}$ & 82 & 37 & $0.067 \mathrm{~ns}$ \\
\hline Lahti & poly & 0 & 4 & & 167 & 37 & & 2 & 3 & & 60 & 14 & \\
\hline GB 1 & fixed & 96 & 16 & $0.0634 \mathrm{~ns}$ & 47 & 26 & $0.0018^{* *}$ & 94 & 16 & $0.4808 \mathrm{~ns}$ & 0 & 0 & $-n s$ \\
\hline Ascot & poly & 1 & 2 & & 168 & 35 & & 3 & 1 & & 61 & 12 & \\
\hline GB 2 & fixed & 97 & 15 & $0.0005^{* * * *} *$ & 47 & 25 & $0.0084^{* *}$ & 95 & 15 & $0.0065^{* *} *$ & 0 & 0 & $-n s$ \\
\hline Braemar & poly & 1 & 5 & & 167 & 38 & & 2 & 4 & & 61 & 15 & \\
\hline I 1 & fixed & 2 & 0 & $0.5385 \mathrm{~ns}$ & 28 & 17 & $0.0048^{* *}$ & 0 & 0 & $-\mathrm{ns}$ & 71 & 11 & $0.5171 \mathrm{~ns}$ \\
\hline Grosseto & poly & 7 & 4 & & 171 & 37 & & 9 & 3 & & 65 & 14 & \\
\hline I 2 & fixed & 0 & 0 & $-n s$ & 28 & 16 & $0.0073^{* *}$ & 0 & 0 & $-n s$ & 69 & 11 & $0.8252 \mathrm{~ns}$ \\
\hline Parma & poly & 11 & 3 & & 172 & 36 & & 13 & 2 & & 67 & 13 & \\
\hline N 1 & fixed & 58 & 25 & $0.2693 \mathrm{~ns}$ & 9 & 1 & $0.705 \mathrm{~ns}$ & 58 & 25 & $0.2043 \mathrm{~ns}$ & 42 & 28 & $0.0022 * *$ \\
\hline Narvik & poly & 111 & 33 & & 234 & 47 & & 113 & 32 & & 156 & 40 & \\
\hline NL 1 & fixed & 92 & 15 & $0.357 \mathrm{~ns}$ & 45 & 25 & $0.0022 * *$ & 90 & 15 & $1 \mathrm{~ns}$ & 0 & 0 & $-n s$ \\
\hline Wageningen & poly & 7 & 3 & & 171 & 35 & & 9 & 2 & & 63 & 12 & \\
\hline PL 1 & fixed & 42 & 22 & $0.1831 \mathrm{~ns}$ & 16 & 1 & $0.3225 \mathrm{~ns}$ & 43 & 22 & $0.1806 \mathrm{~ns}$ & 40 & 25 & $0.0149^{*}$ \\
\hline Krakow & poly & 111 & 37 & & 219 & 46 & & 113 & 36 & & 159 & 46 & \\
\hline PL 2 & fixed & 62 & 20 & $1 \mathrm{~ns}$ & 31 & 19 & $0.0018 * *$ & 61 & 20 & $0.7548 \mathrm{~ns}$ & 61 & 17 & $0.5512 \mathrm{~ns}$ \\
\hline Warsaw & poly & 12 & 4 & & 172 & 35 & & 14 & 3 & & 66 & 14 & \\
\hline RUM 1 & fixed & 76 & 16 & $1 \mathrm{~ns}$ & 34 & 18 & $0.0067^{* *}$ & 77 & 16 & $0.5879 \mathrm{~ns}$ & 61 & 12 & $0.824 \mathrm{~ns}$ \\
\hline Sibiu 1 & poly & 3 & 1 & & 169 & 34 & & 5 & 0 & & 63 & 11 & \\
\hline RUM 2 & fixed & 74 & 15 & $0.6962 \mathrm{~ns}$ & 33 & 18 & $0.0061^{* *}$ & 75 & 15 & $1 \mathrm{~ns}$ & 59 & 12 & $1 \mathrm{~ns}$ \\
\hline Sibiu 2 & poly & 10 & 3 & & 173 & 35 & & 12 & 2 & & 70 & 13 & \\
\hline RUM 3 & fixed & 92 & 43 & $0.4312 \mathrm{~ns}$ & 39 & 2 & $0.0563 \mathrm{~ns}$ & 93 & 43 & $0.1893 \mathrm{~ns}$ & 66 & 45 & $0.0002 * * *$ \\
\hline Bagau & poly & 15 & 4 & & 175 & 34 & & 16 & 3 & & 74 & 14 & \\
\hline
\end{tabular}


Table A41 continue:

\begin{tabular}{|c|c|c|c|c|c|c|c|c|c|c|c|c|c|}
\hline \multirow{2}{*}{$\begin{array}{c}\text { Population } \\
\text { RUM } 4\end{array}$} & \multirow{3}{*}{$\begin{array}{l}\text { fixed } \\
\text { poly }\end{array}$} & \multicolumn{3}{|c|}{$\begin{array}{r}\text { DK 3 } \\
\text { syn. nons. sign. }\end{array}$} & \multicolumn{3}{|c|}{$\begin{array}{c}\text { F } 1 \\
\text { syn. nons. sign. }\end{array}$} & \multicolumn{3}{|c|}{$\begin{array}{c}\text { F } 2 \\
\text { syn. nons. sign. }\end{array}$} & \multicolumn{3}{|c|}{$\begin{array}{c}\text { F 3 } \\
\text { syn. nons. sign. }\end{array}$} \\
\hline & & 97 & 44 & $0.436 \mathrm{~ns}$ & 42 & 2 & $0.0358^{*}$ & 97 & 44 & $0.0588 \mathrm{~ns}$ & 71 & 46 & $0.0003^{* * *}$ \\
\hline Cluj Nap oca & & 7 & 1 & & 170 & 34 & & 9 & 0 & & 64 & 11 & \\
\hline RUM 5 & fixed & 72 & 20 & $0.3804 \mathrm{~ns}$ & 28 & 19 & $0.0014^{* *}$ & 73 & 20 & $1 \mathrm{~ns}$ & 70 & 14 & $1 \mathrm{~ns}$ \\
\hline Busteni & poly & 5 & 3 & & 170 & 36 & & 7 & 2 & & 65 & 12 & \\
\hline RUM 6 & fixed & 39 & 6 & $0.3544 \mathrm{~ns}$ & 18 & 13 & $0.0046^{* *}$ & 42 & 6 & $0.3527 \mathrm{~ns}$ & 39 & 6 & $0.3908 \mathrm{~ns}$ \\
\hline Sinaia & poly & 75 & 20 & & 208 & 47 & & 76 & 19 & & 122 & 29 & \\
\hline RUS 1 & fixed & 69 & 35 & $0.0001^{* * *}$ & 24 & 0 & $0.0323 *$ & 67 & 36 & $0.0000^{* * *}$ & 47 & 40 & $0.0000^{* * *}$ \\
\hline Altai Mountains & poly & 116 & 17 & & 230 & 44 & & 118 & 16 & & 164 & 27 & \\
\hline RUS 2 & fixed & 113 & 45 & $0.2893 \mathrm{~ns}$ & 46 & 8 & $0.8375 \mathrm{~ns}$ & 112 & 44 & $1 \mathrm{~ns}$ & 94 & 42 & $0.0313^{*}$ \\
\hline Novosibirsk & poly & 0 & 1 & & 167 & 34 & & 2 & 0 & & 59 & 12 & \\
\hline $\mathrm{S} 2$ & fixed & 32 & 16 & $0.1938 \mathrm{~ns}$ & 7 & 0 & $0.3586 \mathrm{~ns}$ & 33 & 16 & $0.142 \mathrm{~ns}$ & 32 & 15 & $0.1941 \mathrm{~ns}$ \\
\hline Stroemstad & poly & 157 & 47 & & 248 & 56 & & 159 & 46 & & 191 & 56 & \\
\hline
\end{tabular}

\begin{tabular}{|c|c|c|c|c|c|c|c|c|c|c|c|c|c|}
\hline \multirow{2}{*}{$\begin{array}{c}\text { Population } \\
\text { FIN } 1\end{array}$} & \multicolumn{4}{|c|}{$\begin{array}{c}\text { F } 4 \\
\text { syn. nons. sign. }\end{array}$} & \multicolumn{3}{|c|}{$\begin{array}{r}\text { FIN } 1 \\
\text { syn. nons. sign. } \\
\end{array}$} & \multicolumn{3}{|c|}{$\begin{array}{r}\text { GB 1 } \\
\text { syn. nons. sign. } \\
\end{array}$} & \multicolumn{3}{|c|}{$\begin{array}{r}\text { GB 2 } \\
\text { syn. nons. sign. }\end{array}$} \\
\hline & fixed & 94 & 15 & $0.0036^{* *} *$ & & & & & & & & & \\
\hline Lahti & poly & 0 & 3 & & & & & & & & & & \\
\hline GB 1 & fixed & 100 & 47 & $1 \mathrm{~ns}$ & 104 & 40 & $0.0268 *$ & & & & & & \\
\hline Ascot & poly & 1 & 1 & & 1 & 4 & & & & & & & \\
\hline GB 2 & fixed & 101 & 46 & $0.0405^{*}$ & 104 & 39 & $0.0011^{* *}$ & 0 & 1 & $1 \mathrm{~ns}$ & & & \\
\hline Braemar & poly & 1 & 4 & & 1 & 7 & & 2 & 5 & & & & \\
\hline I 1 & fixed & 76 & 40 & $1 \mathrm{~ns}$ & 91 & 37 & $0.216 \mathrm{~ns}$ & 95 & 15 & $0.0921 \mathrm{~ns}$ & 96 & 14 & $0.0038^{* *}$ \\
\hline Grosseto & poly & 0 & 3 & & 0 & 3 & & 8 & 4 & & 8 & 7 & \\
\hline I 2 & fixed & 75 & 39 & $0.2207 \mathrm{~ns}$ & 89 & 37 & $1 \mathrm{~ns}$ & 92 & 15 & $0.696 \mathrm{~ns}$ & 93 & 14 & $0.0748 \mathrm{~ns}$ \\
\hline Parma & poly & 11 & 2 & & 11 & 5 & & 12 & 3 & & 12 & 6 & \\
\hline N 1 & fixed & 71 & 4 & $0.0016^{* *}$ & 81 & 11 & $0.0279^{*}$ & 61 & 32 & $0.0509 \mathrm{~ns}$ & 61 & 31 & $0.1028 \mathrm{~ns}$ \\
\hline Narvik & poly & 112 & 31 & & 111 & 35 & & 113 & 32 & & 113 & 35 & \\
\hline NL 1 & fixed & 98 & 46 & $0.7206 \mathrm{~ns}$ & 102 & 39 & $0.3276 \mathrm{~ns}$ & 0 & 0 & $-n s$ & 0 & 0 & $-n s$ \\
\hline Wageningen & poly & 7 & 2 & & 7 & 5 & & 8 & 3 & & 8 & 6 & \\
\hline PL 1 & fixed & 0 & 0 & $-n s$ & 61 & 9 & $0.0349^{*}$ & 62 & 31 & $0.1858 \mathrm{~ns}$ & 63 & 30 & $0.3821 \mathrm{~ns}$ \\
\hline Krakow & poly & 111 & 36 & & 111 & 39 & & 112 & 37 & & 112 & 40 & \\
\hline PL 2 & fixed & 84 & 38 & $0.5522 \mathrm{~ns}$ & 94 & 38 & $0.7834 \mathrm{~ns}$ & 83 & 21 & $0.7509 \mathrm{~ns}$ & 82 & 20 & $0.1461 \mathrm{~ns}$ \\
\hline Warsaw & poly & 12 & 3 & & 12 & 6 & & 13 & 4 & & 13 & 7 & \\
\hline RUM 1 & fixed & 90 & 40 & $0.5536 \mathrm{~ns}$ & 96 & 37 & $0.3544 \mathrm{~ns}$ & 85 & 16 & $1 \mathrm{~ns}$ & 84 & 16 & $0.0374^{*}$ \\
\hline Sibiu 1 & poly & 3 & 0 & & 3 & 3 & & 4 & 1 & & 4 & 4 & \\
\hline RUM 2 & fixed & 88 & 39 & $0.3535 \mathrm{~ns}$ & 94 & 36 & $0.7627 \mathrm{~ns}$ & 83 & 16 & $0.7022 \mathrm{~ns}$ & 82 & 16 & $0.0919 \mathrm{~ns}$ \\
\hline Sibiu 2 & poly & 10 & 2 & & 10 & 5 & & 11 & 3 & & 11 & 6 & \\
\hline RUM 3 & fixed & 88 & 9 & $0.3976 \mathrm{~ns}$ & 97 & 17 & $0.2014 \mathrm{~ns}$ & 89 & 48 & $0.2132 \mathrm{~ns}$ & 89 & 47 & $0.8141 \mathrm{~ns}$ \\
\hline Bagau & poly & 15 & 3 & & 15 & 6 & & 16 & 4 & & 16 & 7 & \\
\hline RUM 4 & fixed & 91 & 10 & $0.6225 \mathrm{~ns}$ & 96 & 18 & $0.371 \mathrm{~ns}$ & 95 & 49 & $0.2726 \mathrm{~ns}$ & 95 & 48 & $1 \mathrm{~ns}$ \\
\hline Cluj Napoca & poly & 7 & 0 & & 7 & 3 & & 8 & 1 & & 8 & 4 & \\
\hline RUM 5 & fixed & 90 & 39 & $1 \mathrm{~ns}$ & 93 & 38 & $0.283 \mathrm{~ns}$ & 97 & 18 & $0.1359 \mathrm{~ns}$ & 96 & 17 & $0.009^{*} *$ \\
\hline Busteni & poly & 5 & 2 & & 5 & 5 & & 5 & 3 & & 6 & 6 & \\
\hline RUM 6 & fixed & 59 & 32 & $0.0492 *$ & 71 & 31 & $0.3395 \mathrm{~ns}$ & 60 & 9 & $0.2172 \mathrm{~ns}$ & 60 & 8 & $0.0691 \mathrm{~ns}$ \\
\hline Sinaia & poly & 74 & 20 & & 74 & 23 & & 75 & 20 & & 75 & 23 & \\
\hline RUS 1 & fixed & 60 & 6 & $0.6351 \mathrm{~ns}$ & 68 & 11 & $1 \mathrm{~ns}$ & 66 & 44 & $0.0000^{*} * *$ & 67 & 43 & $0.0000^{* * *}$ \\
\hline Altai Mountains & poly & 116 & 16 & & 116 & 19 & & 117 & 17 & & 117 & 20 & \\
\hline RUS 2 & fixed & 113 & 19 & $-n s$ & 117 & 21 & $0.0044^{* *}$ & 113 & 45 & $0.4936 \mathrm{~ns}$ & 113 & 44 & $0.0274^{*}$ \\
\hline Novosibirsk & poly & 0 & 0 & & 0 & 3 & & 1 & 1 & & 1 & 4 & \\
\hline S 2 & fixed & 8 & 1 & $0.6872 \mathrm{~ns}$ & 49 & 7 & $0.0694 \mathrm{~ns}$ & 51 & 19 & $0.5185 \mathrm{~ns}$ & 51 & 18 & $0.7482 \mathrm{~ns}$ \\
\hline Stroemstad & poly & 157 & 46 & & 157 & 49 & & 157 & 47 & & 158 & 50 & \\
\hline
\end{tabular}


Table A41 continue:

\begin{tabular}{|c|c|c|c|c|c|c|c|c|c|c|c|c|c|}
\hline \multirow{2}{*}{$\begin{array}{c}\text { Population } \\
\text { I } 2\end{array}$} & \multirow[b]{2}{*}{ fixed } & \multicolumn{3}{|c|}{$\begin{array}{c}\text { I } 1 \\
\text { syn. nons. sign. }\end{array}$} & \multicolumn{3}{|c|}{$\begin{array}{c}\text { I } 2 \\
\text { syn. nons. sign. }\end{array}$} & \multicolumn{3}{|c|}{$\begin{array}{c}\text { N } 1 \\
\text { syn. nons. sign. }\end{array}$} & \multicolumn{3}{|c|}{$\begin{array}{r}\text { NL } 1 \\
\text { syn. nons. sign. }\end{array}$} \\
\hline & & 0 & 0 & $-n s$ & & & & & & & & & \\
\hline Parma & poly & 11 & 4 & & & & & & & & & & \\
\hline $\begin{array}{c}\text { N } 1 \\
\text { Narvik }\end{array}$ & $\begin{array}{l}\text { fixed } \\
\text { poly }\end{array}$ & $\begin{array}{r}56 \\
117\end{array}$ & $\begin{array}{l}24 \\
36\end{array}$ & $0.3439 \mathrm{~ns}$ & $\begin{array}{r}56 \\
119\end{array}$ & $\begin{array}{l}23 \\
35\end{array}$ & $0.3372 \mathrm{~ns}$ & & & & & & \\
\hline $\begin{array}{c}\text { NL } 1 \\
\text { Wageningen }\end{array}$ & $\begin{array}{l}\text { fixed } \\
\text { poly }\end{array}$ & $\begin{array}{l}91 \\
14\end{array}$ & $\begin{array}{r}14 \\
5\end{array}$ & $0.1688 \mathrm{~ns}$ & $\begin{array}{l}88 \\
18\end{array}$ & $\begin{array}{r}14 \\
4\end{array}$ & $0.7383 \mathrm{~ns}$ & $\begin{array}{r}58 \\
117\end{array}$ & $\begin{array}{l}31 \\
33\end{array}$ & $0.0349^{*}$ & & & \\
\hline $\begin{array}{c}\text { PL } 1 \\
\text { Krakow }\end{array}$ & $\begin{array}{l}\text { fixed } \\
\text { poly }\end{array}$ & $\begin{array}{r}41 \\
115\end{array}$ & $\begin{array}{l}22 \\
39\end{array}$ & $0.1835 \mathrm{~ns}$ & $\begin{array}{c}41 \\
118\end{array}$ & $\begin{array}{l}21 \\
38\end{array}$ & $0.1773 \mathrm{~ns}$ & $\begin{array}{r}26 \\
196\end{array}$ & $\begin{array}{r}0 \\
47\end{array}$ & $0.0067^{* *}$ & $\begin{array}{r}59 \\
118\end{array}$ & $\begin{array}{l}30 \\
38\end{array}$ & $0.1382 \mathrm{~ns}$ \\
\hline $\begin{array}{c}\text { PL 2 } \\
\text { Warsaw }\end{array}$ & $\begin{array}{l}\text { fixed } \\
\text { poly }\end{array}$ & $\begin{array}{l}62 \\
18\end{array}$ & $\begin{array}{r}19 \\
6\end{array}$ & $1 \mathrm{~ns}$ & $\begin{array}{l}61 \\
22\end{array}$ & $\begin{array}{r}18 \\
5\end{array}$ & $0.7895 \mathrm{~ns}$ & $\begin{array}{r}52 \\
121\end{array}$ & $\begin{array}{l}25 \\
34\end{array}$ & $0.1088 \mathrm{~ns}$ & $\begin{array}{l}81 \\
18\end{array}$ & $\begin{array}{r}20 \\
4\end{array}$ & $1 \mathrm{~ns}$ \\
\hline $\begin{array}{l}\text { RUM } 1 \\
\text { Sibiu } 1\end{array}$ & $\begin{array}{l}\text { fixed } \\
\text { poly }\end{array}$ & $\begin{array}{l}72 \\
10\end{array}$ & $\begin{array}{r}15 \\
3\end{array}$ & $0.6987 \mathrm{~ns}$ & $\begin{array}{l}70 \\
14\end{array}$ & $\begin{array}{r}14 \\
2\end{array}$ & $1 \mathrm{~ns}$ & $\begin{array}{r}66 \\
114\end{array}$ & $\begin{array}{l}26 \\
32\end{array}$ & $0.2811 \mathrm{~ns}$ & $\begin{array}{l}81 \\
10\end{array}$ & $\begin{array}{r}15 \\
2\end{array}$ & $1 \mathrm{~ns}$ \\
\hline $\begin{array}{l}\text { RUM } 2 \\
\text { Sibiu } 2\end{array}$ & $\begin{array}{l}\text { fixed } \\
\text { poly }\end{array}$ & $\begin{array}{l}70 \\
17\end{array}$ & $\begin{array}{r}14 \\
5\end{array}$ & $0.5377 \mathrm{~ns}$ & $\begin{array}{l}68 \\
21\end{array}$ & $\begin{array}{r}13 \\
4\end{array}$ & $1 \mathrm{~ns}$ & $\begin{array}{r}64 \\
119\end{array}$ & $\begin{array}{l}26 \\
33\end{array}$ & $0.2187 \mathrm{~ns}$ & $\begin{array}{r}79 \\
17\end{array}$ & $\begin{array}{r}15 \\
4\end{array}$ & $0.7483 \mathrm{~ns}$ \\
\hline $\begin{array}{c}\text { RUM } 3 \\
\text { Bagaul }\end{array}$ & $\begin{array}{l}\text { fixed } \\
\text { poly }\end{array}$ & $\begin{array}{l}89 \\
22\end{array}$ & $\begin{array}{r}43 \\
6\end{array}$ & $0.2703 \mathrm{~ns}$ & $\begin{array}{l}87 \\
26\end{array}$ & $\begin{array}{r}42 \\
5\end{array}$ & $0.0817 \mathrm{~ns}$ & $\begin{array}{r}45 \\
123\end{array}$ & $\begin{array}{r}1 \\
34\end{array}$ & $0.0008^{* * *} *$ & $\begin{array}{l}85 \\
22\end{array}$ & $\begin{array}{r}47 \\
5\end{array}$ & $0.1147 \mathrm{~ns}$ \\
\hline $\begin{array}{c}\text { RUM } 4 \\
\text { Cluj Napoca }\end{array}$ & $\begin{array}{l}\text { fixed } \\
\text { poly }\end{array}$ & $\begin{array}{l}94 \\
14\end{array}$ & $\begin{array}{r}44 \\
3\end{array}$ & $0.2761 \mathrm{~ns}$ & $\begin{array}{l}92 \\
18\end{array}$ & $\begin{array}{r}43 \\
2\end{array}$ & $0.0624 \mathrm{~ns}$ & $\begin{array}{r}47 \\
117\end{array}$ & $\begin{array}{r}1 \\
31\end{array}$ & $0.0012^{* *}$ & $\begin{array}{l}91 \\
14\end{array}$ & $\begin{array}{r}48 \\
2\end{array}$ & $0.0929 \mathrm{~ns}$ \\
\hline $\begin{array}{l}\text { RUM 5 } \\
\text { Busteni }\end{array}$ & $\begin{array}{l}\text { fixed } \\
\text { poly }\end{array}$ & $\begin{array}{l}72 \\
12\end{array}$ & $\begin{array}{r}19 \\
5\end{array}$ & $0.5254 \mathrm{~ns}$ & $\begin{array}{l}70 \\
16\end{array}$ & $\begin{array}{r}18 \\
4\end{array}$ & $1 \mathrm{~ns}$ & $\begin{array}{r}59 \\
116\end{array}$ & $\begin{array}{l}25 \\
33\end{array}$ & $0.2097 \mathrm{~ns}$ & $\begin{array}{l}93 \\
12\end{array}$ & $\begin{array}{r}17 \\
4\end{array}$ & $0.47 \mathrm{~ns}$ \\
\hline $\begin{array}{c}\text { RUM } 6 \\
\text { Sinaia }\end{array}$ & $\begin{array}{l}\text { fixed } \\
\text { poly }\end{array}$ & $\begin{array}{l}39 \\
81\end{array}$ & $\begin{array}{r}5 \\
22\end{array}$ & $0.1713 \mathrm{~ns}$ & $\begin{array}{l}37 \\
84\end{array}$ & $\begin{array}{r}5 \\
20\end{array}$ & $0.3404 \mathrm{~ns}$ & $\begin{array}{r}33 \\
173\end{array}$ & $\begin{array}{l}18 \\
48\end{array}$ & $0.0687 \mathrm{~ns}$ & $\begin{array}{l}57 \\
80\end{array}$ & $\begin{array}{r}7 \\
20\end{array}$ & $0.138 \mathrm{~ns}$ \\
\hline RUS 1 & fixed & 66 & 35 & $0.0003 * * *$ & 65 & 34 & $0.0001 * * *$ & 32 & 1 & $0.0239^{*}$ & 63 & 43 & $0.0000^{* * *}$ \\
\hline Altai Mountains & poly & 119 & 19 & & 121 & 18 & & 199 & 44 & & 122 & 17 & \\
\hline $\begin{array}{c}\text { RUS 2 } \\
\text { Novosibirsk }\end{array}$ & $\begin{array}{l}\text { fixed } \\
\text { poly }\end{array}$ & $\begin{array}{r}110 \\
7\end{array}$ & $\begin{array}{r}44 \\
3\end{array}$ & $1 \mathrm{~ns}$ & $\begin{array}{c}109 \\
11\end{array}$ & $\begin{array}{r}44 \\
2\end{array}$ & $0.3589 \mathrm{~ns}$ & $\begin{array}{r}84 \\
112\end{array}$ & $\begin{array}{l}13 \\
31\end{array}$ & $0.1265 \mathrm{~ns}$ & $\begin{array}{r}109 \\
7\end{array}$ & $\begin{array}{r}44 \\
2\end{array}$ & $1 \mathrm{~ns}$ \\
\hline $\begin{array}{c}\text { S 2 } \\
\text { Stroemstad }\end{array}$ & $\begin{array}{l}\text { fixed } \\
\text { poly }\end{array}$ & $\begin{array}{r}30 \\
162\end{array}$ & $\begin{array}{l}15 \\
48\end{array}$ & $0.1813 \mathrm{~ns}$ & $\begin{array}{r}30 \\
165\end{array}$ & $\begin{array}{l}15 \\
46\end{array}$ & $0.1225 \mathrm{~ns}$ & $\begin{array}{r}15 \\
228\end{array}$ & $\begin{array}{r}0 \\
58\end{array}$ & $0.0495^{*}$ & $\begin{array}{r}47 \\
163\end{array}$ & $\begin{array}{l}18 \\
48\end{array}$ & $0.5061 \mathrm{~ns}$ \\
\hline
\end{tabular}

\begin{tabular}{|c|c|c|c|c|c|c|c|c|c|c|c|c|}
\hline \multirow{2}{*}{$\begin{array}{c}\text { Population } \\
\text { PL 2 }\end{array}$} & \multirow[b]{2}{*}{ fixed } & \multicolumn{2}{|c|}{$\begin{array}{r}\text { PL 1 } \\
\text { syn. nons. sign. }\end{array}$} & \multicolumn{3}{|c|}{$\begin{array}{r}\text { PL 2 } \\
\text { syn. nons. sign. }\end{array}$} & \multicolumn{3}{|c|}{$\begin{array}{r}\text { RUM 1 } \\
\text { syn. nons. sign. }\end{array}$} & \multicolumn{3}{|c|}{$\begin{array}{r}\text { RUM } 2 \\
\text { syn. nons. sign. }\end{array}$} \\
\hline & & 19 & $0.3978 \mathrm{~ns}$ & & & & & & & & & \\
\hline Warsaw & poly 122 & 39 & & & & & & & & & & \\
\hline RUM 1 & fixed 48 & 21 & $0.4075 \mathrm{~ns}$ & 67 & 9 & $0.6942 \mathrm{~ns}$ & & & & & & \\
\hline Sibiu 1 & poly 113 & 36 & & 15 & 3 & & & & & & & \\
\hline RUM 2 & fixed 47 & 21 & $0.321 \mathrm{~ns}$ & 64 & 8 & $0.505 \mathrm{~ns}$ & 0 & 0 & $-n s$ & & & \\
\hline Sibiu 2 & poly 117 & 37 & & 22 & 5 & & 10 & 2 & & & & \\
\hline RUM 3 & fixed 48 & 8 & $0.1823 \mathrm{~ns}$ & 90 & 43 & $0.1401 \mathrm{~ns}$ & 99 & 42 & $0.1929 \mathrm{~ns}$ & 96 & 23 & $0.2503 \mathrm{~ns}$ \\
\hline Bagau & poly 119 & 37 & & 26 & 6 & & 18 & 3 & & 23 & 5 & \\
\hline RUM 4 & fixed 51 & 9 & $0.193 \mathrm{~ns}$ & 90 & 44 & $0.0848 \mathrm{~ns}$ & 100 & 43 & $0.0627 \mathrm{~ns}$ & 97 & 42 & $0.1007 \mathrm{~ns}$ \\
\hline Cluj Napoca & poly 114 & 36 & & 19 & 3 & & 10 & 0 & & 17 & 2 & \\
\hline RUM 5 & fixed 53 & 20 & $0.7451 \mathrm{~ns}$ & 55 & 3 & $0.0326^{*}$ & 72 & 8 & $0.5946 \mathrm{~ns}$ & 71 & 7 & $0.2177 \mathrm{~ns}$ \\
\hline Busteni & poly 115 & 38 & & 17 & 5 & & 8 & 2 & & 15 & 4 & \\
\hline RUM 6 & fixed 25 & 14 & $0.1624 \mathrm{~ns}$ & 35 & 3 & $0.1273 \mathrm{~ns}$ & 41 & 4 & $0.1423 \mathrm{~ns}$ & 41 & 3 & $0.051 \mathrm{~ns}$ \\
\hline Sinaia & poly 165 & 52 & & 84 & 20 & & 77 & 19 & & 82 & 21 & \\
\hline RUS 1 & fixed 35 & 4 & $0.19 \mathrm{~ns}$ & 73 & 35 & $0.0003 * * *$ & 72 & 36 & $0.0001 * * *$ & 72 & 35 & $0.0003^{* * * *}$ \\
\hline Altai Mountains & poly 201 & 48 & & 124 & 18 & & 118 & 16 & & 122 & 18 & \\
\hline RUS 2 & fixed 76 & 12 & $0.047^{*}$ & 107 & 43 & $0.5621 \mathrm{~ns}$ & 104 & 43 & $0.5577 \mathrm{~ns}$ & 103 & 43 & $0.5112 \mathrm{~ns}$ \\
\hline Novosibirsk & poly 111 & 36 & & 12 & 3 & & 3 & 0 & & 10 & 2 & \\
\hline S 2 & fixed & 0 & $-n s$ & 30 & 12 & $0.4246 \mathrm{~ns}$ & 95 & 36 & $0.5636 \mathrm{~ns}$ & 94 & 35 & $0.5185 \mathrm{~ns}$ \\
\hline Stroemstad & poly 165 & 52 & & 164 & 47 & & 3 & 0 & & 10 & 2 & \\
\hline
\end{tabular}


Appendix

Table A41 continue:

\begin{tabular}{|c|c|c|c|c|c|c|c|c|c|c|c|c|c|}
\hline \multirow{3}{*}{$\begin{array}{c}\text { Population } \\
\text { RUM } 4\end{array}$} & & \multirow{2}{*}{\multicolumn{3}{|c|}{$\begin{array}{r}\text { RUM 3 } \\
\text { syn. nons. sign. }\end{array}$}} & \multirow{2}{*}{\multicolumn{3}{|c|}{$\begin{array}{r}\text { RUM } 4 \\
\text { syn. nons. sign. }\end{array}$}} & \multirow{2}{*}{\multicolumn{3}{|c|}{$\begin{array}{r}\text { RUM } 5 \\
\text { syn. nons. sign. }\end{array}$}} & \multirow{2}{*}{\multicolumn{3}{|c|}{$\begin{array}{r}\text { RUM } 6 \\
\text { syn. nons. sign. }\end{array}$}} \\
\hline & \multirow{3}{*}{$\begin{array}{l}\text { fixed } \\
\text { poly }\end{array}$} & & & & & & & & & & & & \\
\hline & & \multirow{2}{*}{$\begin{array}{r}5 \\
20\end{array}$} & \multirow{2}{*}{$\begin{array}{l}0 \\
3\end{array}$} & \multirow{2}{*}{$1 \mathrm{~ns}$} & & & & & & & & & \\
\hline Cluj Napoca & & & & & & & & & & & & & \\
\hline RUM 5 & fixed & 106 & 42 & $0.4764 \mathrm{~ns}$ & 107 & 46 & $0.3541 \mathrm{~ns}$ & & & & & & \\
\hline Busteni & poly & 19 & 5 & & 12 & 2 & & & & & & & \\
\hline RUM 6 & fixed & 75 & 35 & $0.0935 \mathrm{~ns}$ & 76 & 36 & $0.0609 \mathrm{~ns}$ & 0 & 0 & $-n s$ & & & \\
\hline Sinaia & poly & 84 & 23 & & 79 & 20 & & 77 & 21 & & & & \\
\hline RUS 1 & fixed & 29 & 0 & $0.0461^{*}$ & 35 & 1 & $0.1283 \mathrm{~ns}$ & 79 & 36 & $0.0006^{* * *}$ & 51 & 30 & $0.0004^{* * *} *$ \\
\hline Altai Mountains & poly & 125 & 19 & & 120 & 16 & & 121 & 18 & & 171 & 34 & \\
\hline RUS 2 & fixed & 99 & 17 & $1 \mathrm{~ns}$ & 99 & 18 & $0.5921 \mathrm{~ns}$ & 114 & 42 & $1 \mathrm{~ns}$ & 91 & 35 & $0.3451 \mathrm{~ns}$ \\
\hline Novosibirsk & poly & 15 & 3 & & 7 & 0 & & 5 & 2 & & 74 & 20 & \\
\hline S 2 & fixed & 39 & 7 & $0.3261 \mathrm{~ns}$ & 40 & 8 & $0.4397 \mathrm{~ns}$ & 36 & 10 & $1 \mathrm{~ns}$ & 13 & 7 & $0.2649 \mathrm{~ns}$ \\
\hline Stroemstad & poly & 165 & 47 & & 160 & 46 & & 159 & 48 & & 202 & 56 & \\
\hline \multirow[t]{2}{*}{ Population } & \multicolumn{4}{|c|}{ RUS 1} & \multicolumn{3}{|c|}{ RUS 2} & & & & & & \\
\hline & \multicolumn{4}{|c|}{ syn. nons. sign. } & \multicolumn{3}{|c|}{ syn. nons. sign. } & & & & & & \\
\hline RUS 2 & fixed & 59 & 14 & $0.2156 \mathrm{~ns}$ & & & & & & & & & \\
\hline Novosibirsk & poly & 116 & 16 & & & & & & & & & & \\
\hline S 2 & fixed & 26 & 2 & $0.1287 \mathrm{~ns}$ & 54 & 7 & $0.0679 \mathrm{~ns}$ & & & & & & \\
\hline Stroemstad & poly & 234 & 58 & & 157 & 46 & & & & & & & \\
\hline
\end{tabular}




\section{Appendix}

Table A42: Neutrality indices of Steganacarus magnus computed in the McDonald-Kreitman test with DnaSP v5. Locations with less than two individuals were excluded.

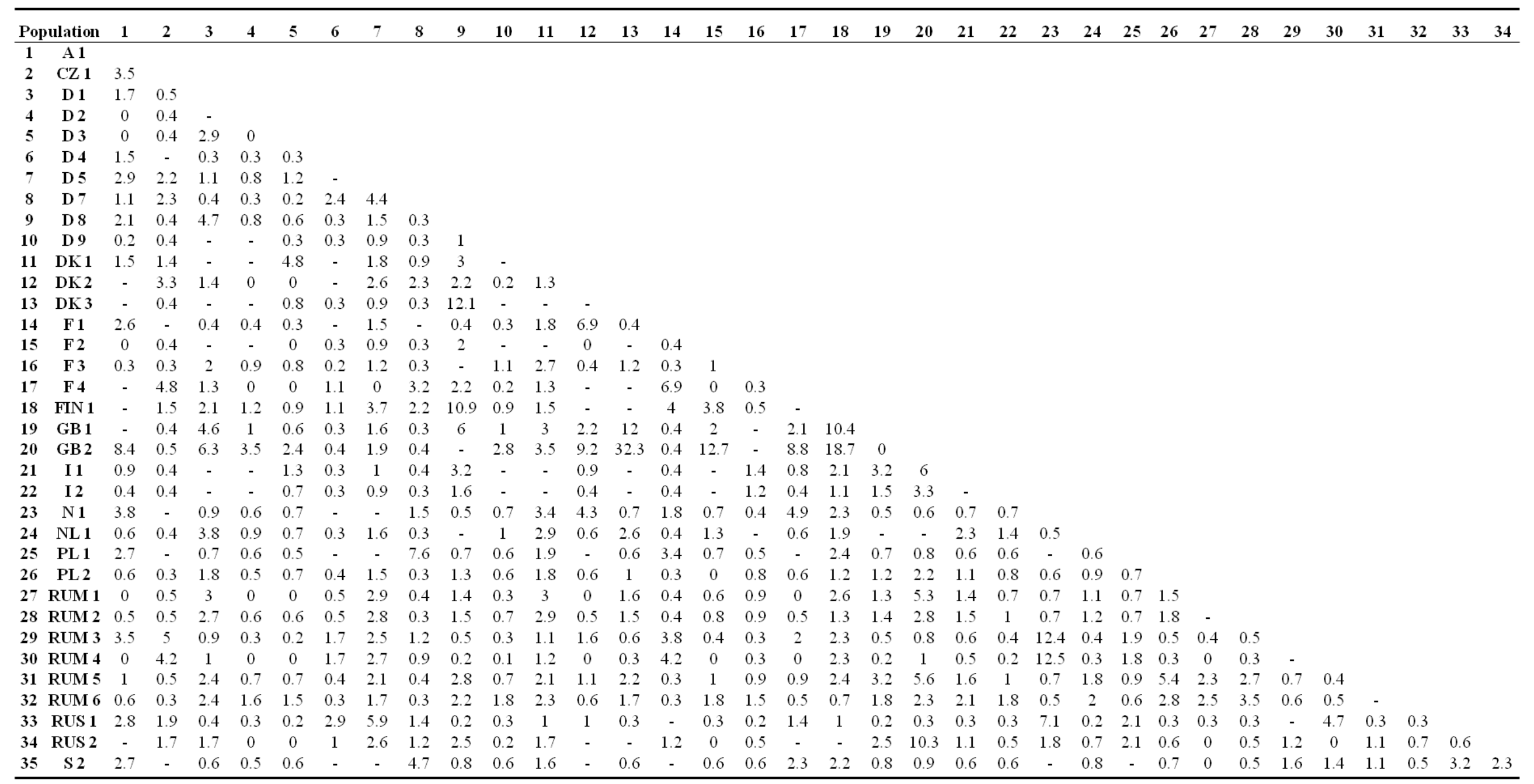




\section{Appendix}

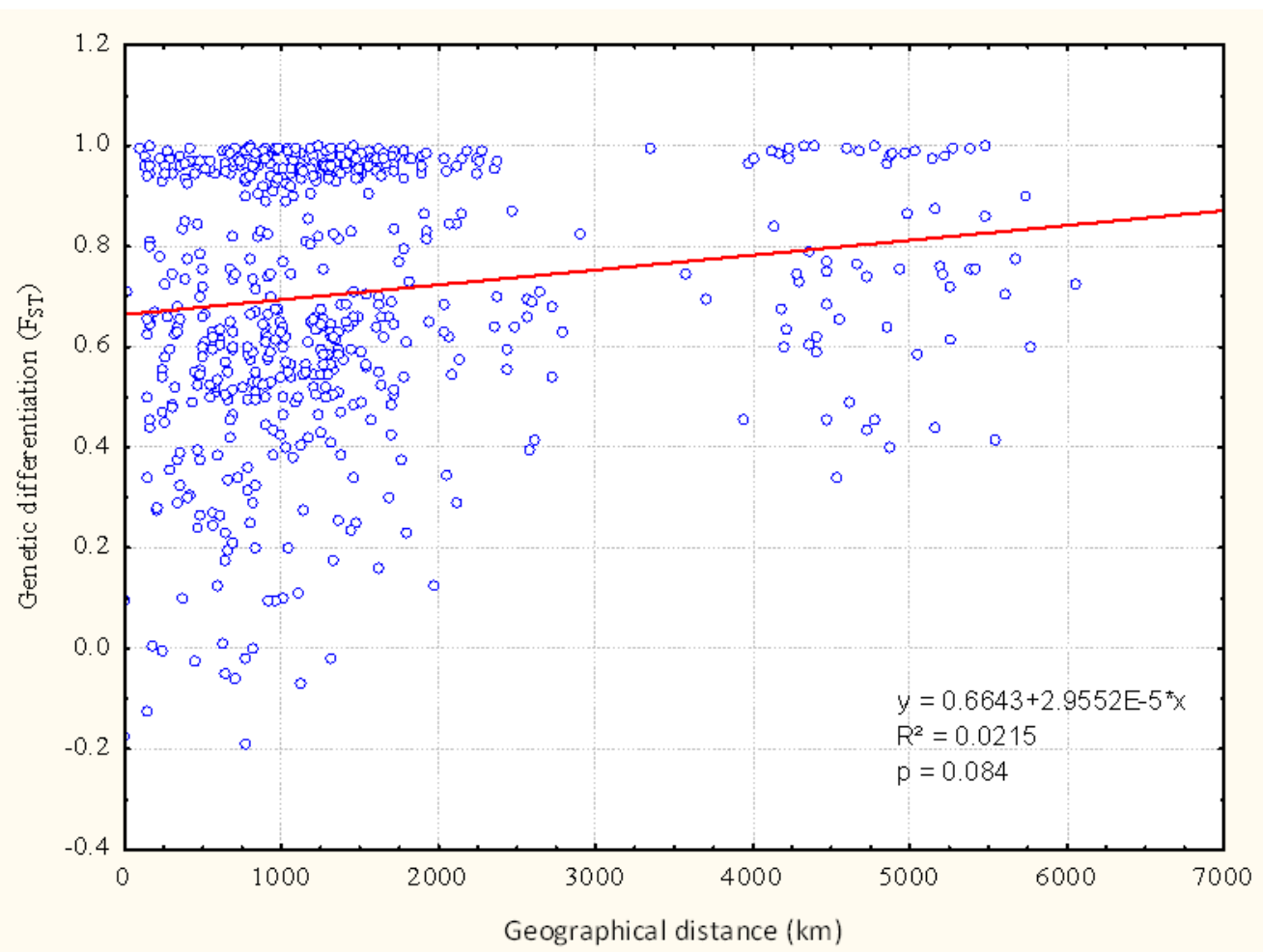

Figure A60: Linear regression of geographical distances (kilometers) vs. genetic differentiations $\left(\mathrm{F}_{\mathrm{ST}}\right)$ based on COI of Steganacarus magnus. Regression is not significant $(\mathrm{p}=0.084 ; \quad(* 0.01<\mathrm{P}<0.05 ; * * 0.001<\mathrm{P}<0.01$; $* * * \mathrm{P}<0.001)$ ) using 1000 randomizations.

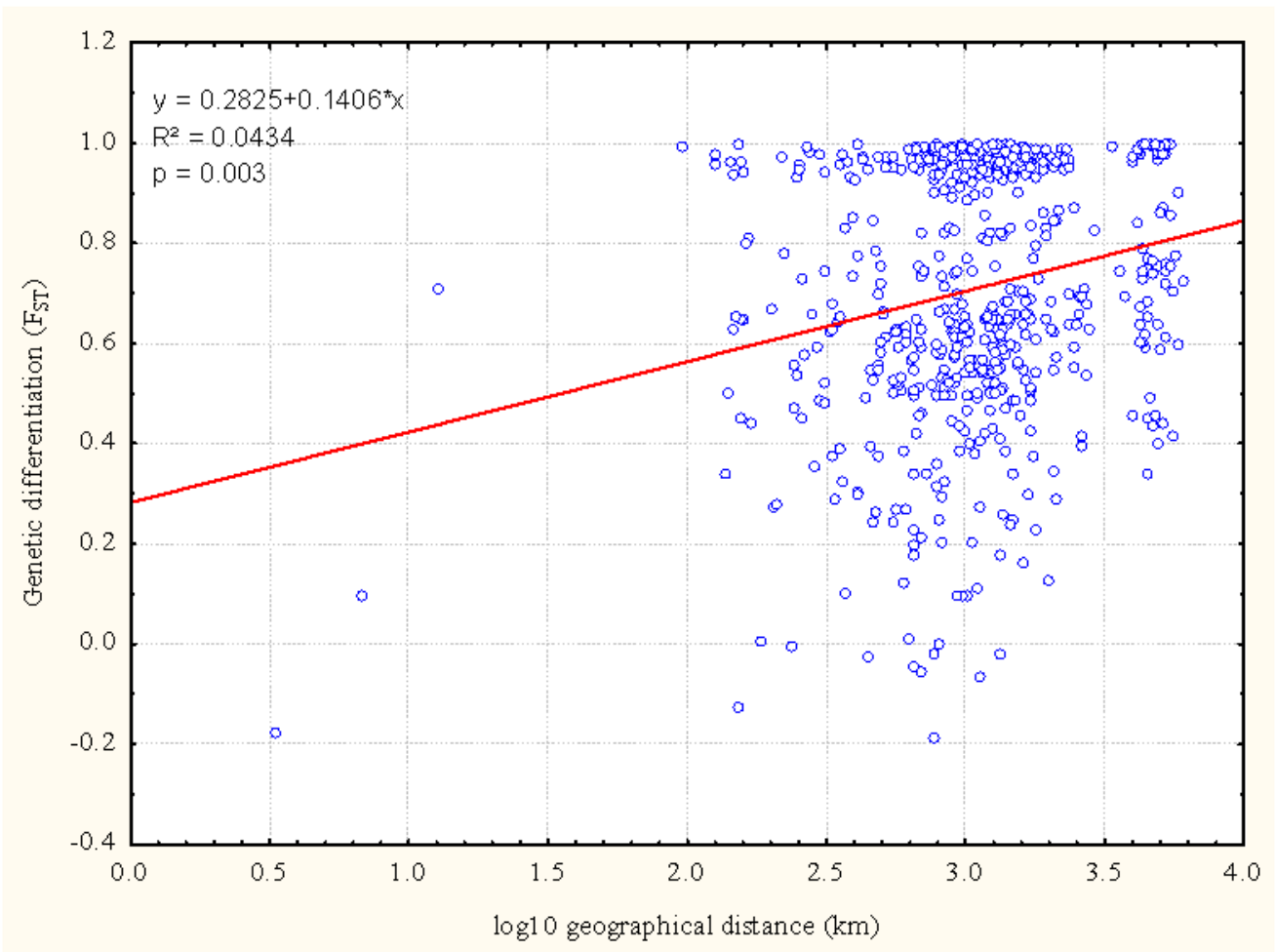

Figure A61: Linear regression of $\log 10$ geographical distances (kilometers) vs. genetic differentiations $\left(\mathrm{F}_{\mathrm{ST}}\right)$ based on COI of Steganacarus magnus. Regression is significant $\left(\mathrm{p}=0.003^{* *}\right)$ using 1000 randomizations. 
Appendix

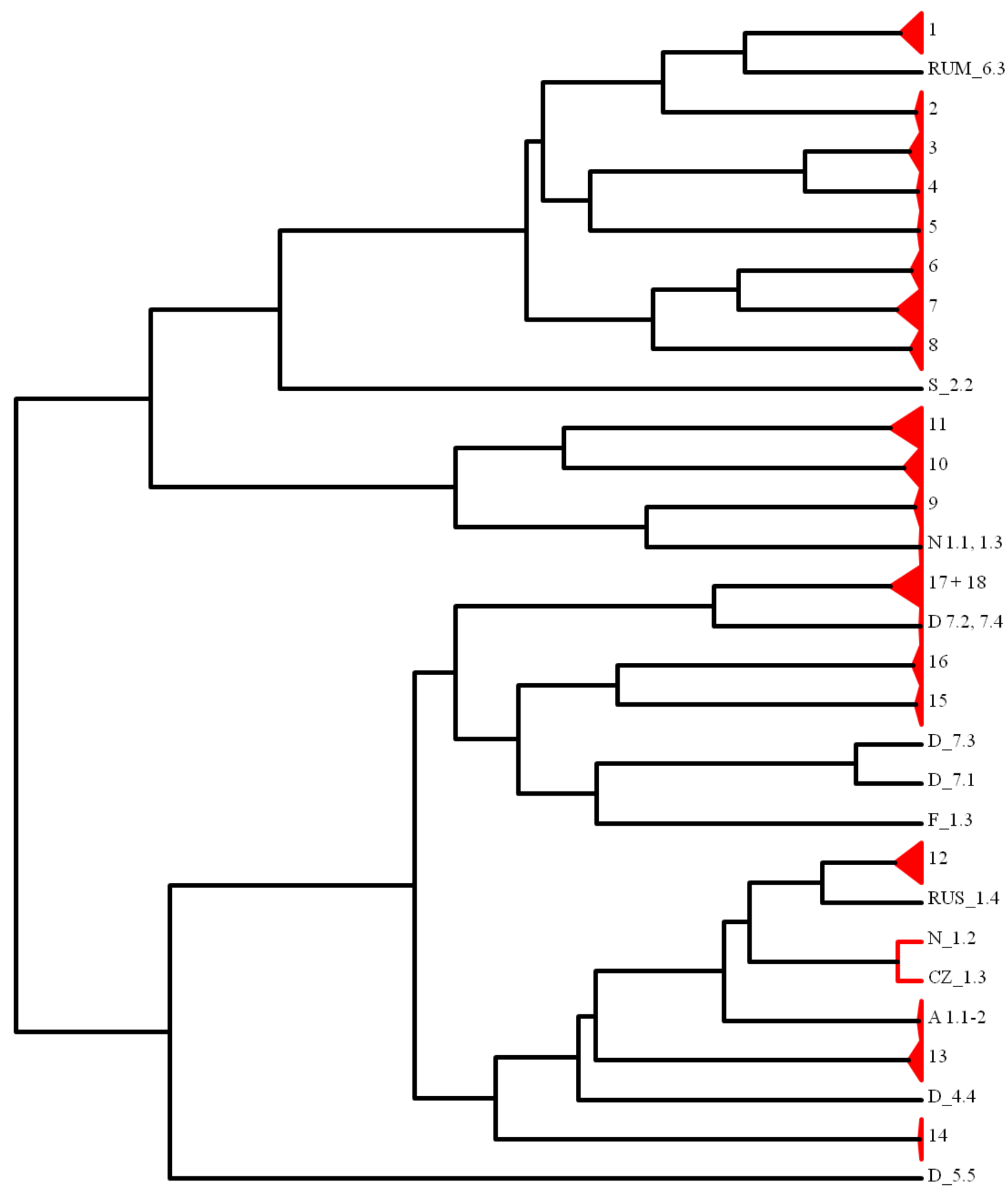

Figure A62: Cluster delimitation of COI nucleotide sequences from Steganacarus magnus after the single method of the generalized mixed Yule coalescent method (GMYC). Red branches are the different distinct clusters. Tip labels are explained in Table 28. 
Appendix

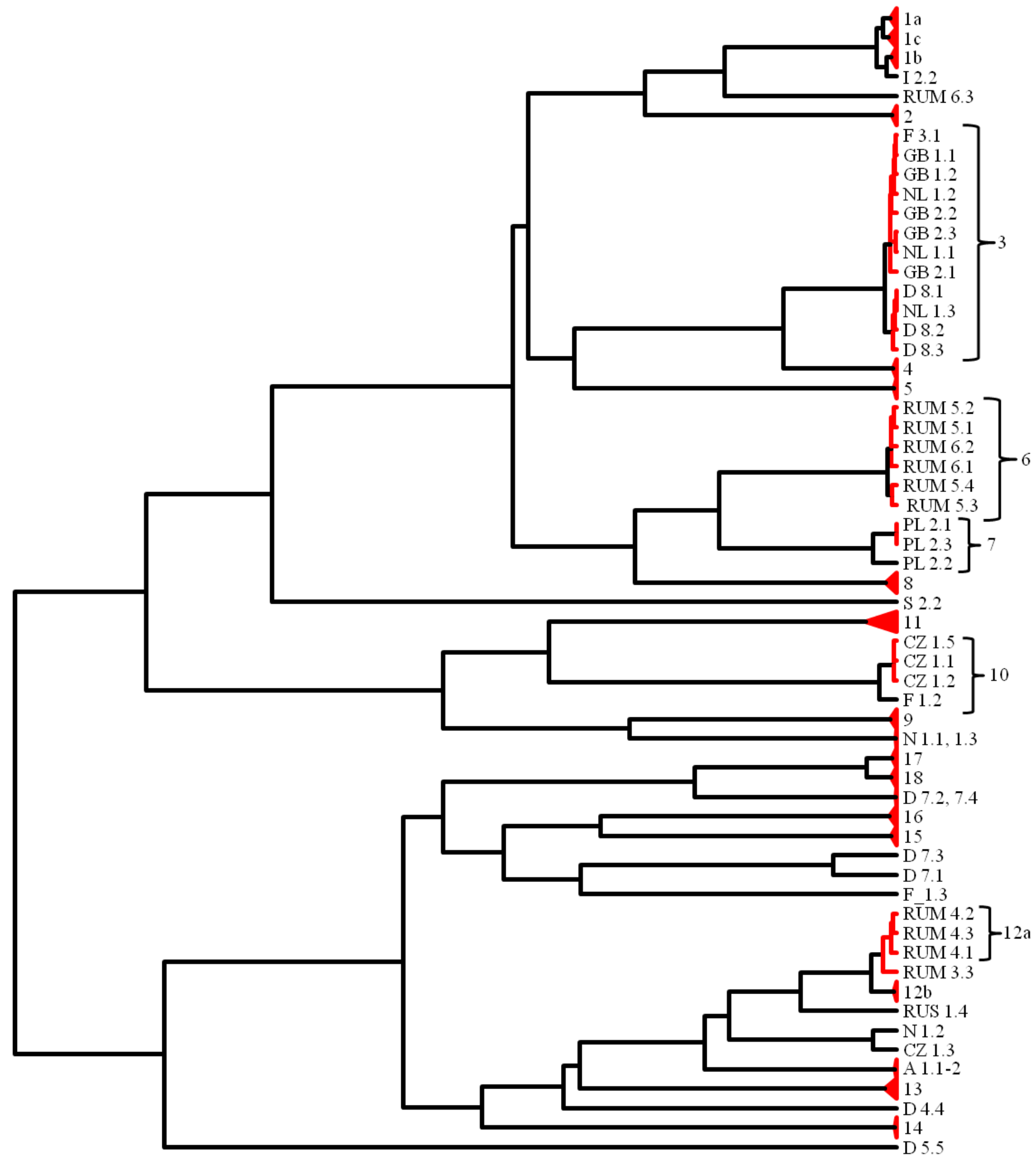

Figure A63: Cluster delimitation of COI nucleotide sequences from Steganacarus magnus after the multiple method of the generalized mixed Yule coalescent method (GMYC). Red branches are the different distinct clusters. Tip labels are explained in Table 28. 


\section{Acknowledgments}

I thank:

Prof. Dr. Stefan Scheu my thesis supervisor. He offered me the opportunity to work in his molecular lab and for his ideas and knowledge to explain strange data.

PD Dr. Mark Maraun to know so many facts about oribatid mites, phylogeny and for being the co-supervisor of my thesis.

Prof. Dr. Ulrich Brose for gladly accepting to be in my examination committee.

Dr. Ina Schäfer for the lab supervising, discussing problems with analyses, let me learning new computer programs and reviewing my thesis.

Dr. Katja Domes-Wehner for introducing me in oribatid mites and supervising me and the beginning of my work.

For the nice atmosphere in the Molli-lab during my work; Ina Schäfer, Kerstin Heidemann, Patrick Pachl, Helge von Saltzwedel, Jens Bast, Bernhard Eitzinger and Guido Humpert.

The whole AG Scheu for working with so many different characters in an unstressed atmosphere and the social events in the evenings.

Jens Bast, Marcel Graf, Diana Grubert, Bernhard Klarner, Kerstin Heidemann, Ellen Latz, Franca Marian, Patrick Pachl, Nils Peter, Dorothee Sandmann, Nicole Scheunemann, Garvin Schultz and Tobias Strahl for the funny evenings while playing at Göttingen.

All my litter collectors, who brought litter samples from their holidays and trips or asking their friends and family: Michael Ackermann; Julio Arcoyo; Trine Bilde; Olaf Butenschön; Csaba Csujdi; Christoph Digl; Katja Domes-Wehner; Nico Eisenhauer; Bernhard Eitzinger; Babara Fischer; Kerstin Heidemann; Guido Humpert; Cristina Ivanescu; Alexandre Jousset; Gregor Kalinkat; Robert Koller; Ellen Latz; Benedikt Lerp; Niklas Lindberg; Mark and Melanie Maraun; Alexandra Micic; Alexandru Milcu Patrick Pachl; T. Pasca; Nils Peter; Christian Platner; Daniela, Marcus, Matthias and Stefanie Rosenberger; Helge von Saltzwedel (Treptow); Ina Schaefer; Max and Stefan Scheu; Nicole Scheunemann; Katja Schneider; Heikki Setala; Juan Shan; Maria Tsiafaoli; Alexei Uvarov; Olivera Vucic-Pestic and Christof Wulff.

Isabell Reiffenstein, Christopher Schmidt, Stefanie Schmitz, Fabian Schreiber and Steffen Zühlke for their friendship.

My family for raising me up, give me the chance to study Biology and always believing in me.

Carlo Pedersoli and Mario Girotti for their awesome entertainment.

All I have forgotten. 


\section{Eidesstattliche Erklärung}

Ich erkläre hiermit an Eides statt, dass ich die vorliegende Dissertation selbständig und nur mit den angegebenen Hilfsmitteln angefertigt habe. Ich habe noch keinen Promotionsversuch unternommen.

Egelsbach, den 21 Oktober 2010

Martin Julien Rosenberger 